INTERIM REPORT

U.S. Department of Energy

Acoustic Probe for Solid-Gas-Liquid Suspension

Principle Investigator: Dr. L.L. Tavlarides

Co-Principle Investigator: Dr. Ashok Sangani

Institution: Syracuse University

Collaborators: Dr. P. Spelt ${ }^{+}$

Dr. M.A. Norato ${ }^{+}$

Dr. M. Greenwood ${ }^{*}$

Mr. M. Hedges ${ }^{+}$

Institutions: Syracuse University ${ }^{+}$

Pacific Northwest National Laboratory*

Grant Number: DE-FG07-96-ER14729

Grant Project Officers: Dr. Oscar P. Manley

(Review and Evaluation of Research)

Dr. Gordon Roesler Massey

(Review and Evaluation of Application of Research)

Project Duration: First Funding Period $\quad 9-15-96$ to 9-14-2000

Second Funding Period 9-15-2000 to 9-14-2003 
Table of Contents

3. Executive Summary ..................................................................

4. Research and Objectives ................................................................ 2

5. Methods and Results .......................................................................

6. Relevance, Impact and Technology Transfer....................................

7. Project Productivity ..................................................................... 14

8. Personnel Supported .................................................................. 14

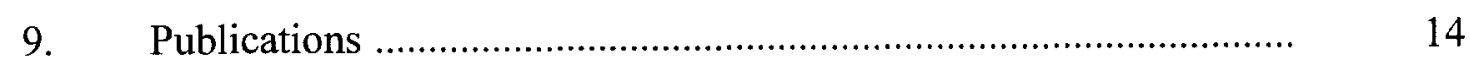

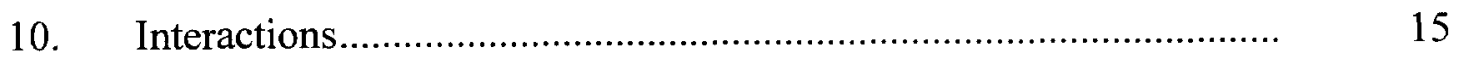

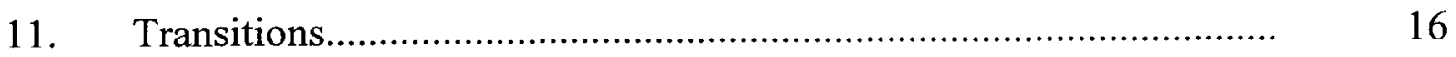

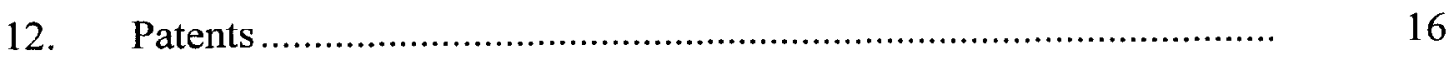

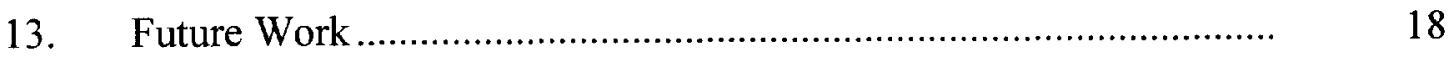

14. Literature Cited ......................................................................... 16

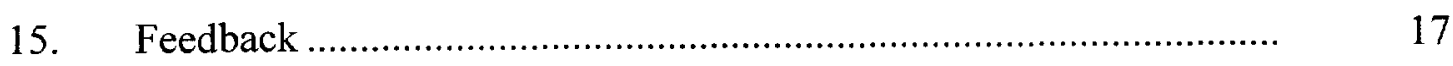

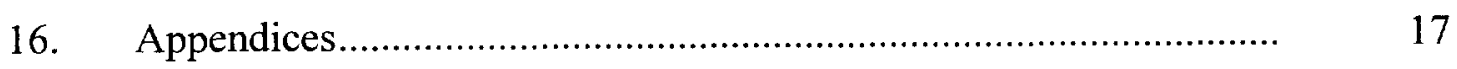

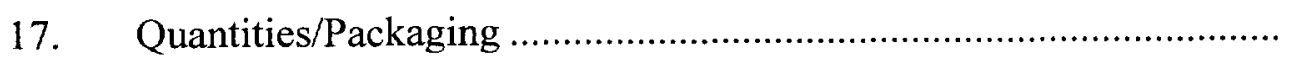




\section{Executive Summary}

The main objective of the project during the first period of funding is to develop an acoustic probe for monitoring particle size and volume fraction in slurries in the absence and presence of gas bubbles. The goals are to commission and verify the probe components and system operation, develop theory for the forward and inverse problems for acoustic wave propagation through a three phase medium, and experimentally verify the theoretical analysis. The acoustic probe will permit measurement of solid content in gas-liquid-solid waste slurries in tanks and pipelines across the Department of Energy complex. Particularly, in the second funding period, a prototype probe will be fabricated, commissioned and tested to demonstrate the capability to accurately measure slurries of one to five weight percent solids.

Our research work has established a solid theoretical foundation for predicting attenuation and phase speed of acoustic waves propagating through solid-liquid suspensions, both in the presence as well as absence of gas bubbles. The theory is based on ensemble averaging of the equations of motion in the solid and liquid phases to obtain expressions for the "effective properties" of the slurry mixture in terms of coefficients which appear in the equations of motion for the solid particle. The attenuation theory accounts for losses due to viscous dissipation, nonadiabatic thermal effects, and incoherent scattering, and as a result can cover a wide range of frequencies and particle sizes. The theory also applies to polydispersed suspensions of spherical particles. The theory agrees with results obtained by previous investigations who examined limiting cases of thermal attenuation at small volume fraction (Allegra and Hawley, 1972) and viscous attenuation at large frequencies (Sangani, Zhang and Prosperetti, 1991). The comprehensive theory developed allows us to interrogate a relatively large range of particle sizes and physical properties. The attenuations predicted from theory are in generally good agreement with experimental data obtained by Pulse/FFT data acquisition methods for solid-liquid slurries of soda-lime glass particles of 14.9 microns and 65 microns mean radius and polystyene particles of 79 microns mean radius at concentrations ranging from 5 to 50 percent solids by volume in water. The primary attenuation mechanisms for the former system are due to viscous and scattering losses, whereas, for the latter system, thermal and scattering losses dominate. Good comparisons are also obtained for 0.11 micron radius polystyrene particles in water from 5 to 50 percent solids by volume (Allegra and Hawley, 1992) where attenuation is dominated by thermal affects.

Another goal of the project was to devise a technique to remove the noise introduced by the presence of a small amount of gas bubbles in the suspension to infer the properties of the solid-liquid suspension. Experiments and analyses were made for the solid-gas-liquid slurries of soda lime glass particles of 14.9 micron mean radius at 5 and 10 percent by volume in water with gas bubbles from 25 to 150 micron radius at low volume fractions. The primary conclusion is that the noise is significant at low frequencies near the bubble resonance frequencies and the noise is minimal at high frequencies. We show it is possible to estimate the effects of bubbles and eliminate the slight noise produced by bubbles at higher frequencies to yield the volume fraction of the particles. 
An inverse theory was also developed to determine the concentration and solids particle size distribution in a solid-liquid slurry given the attenuation as a function of frequency using regularization techniques that have been successful for bubbly liquids. We have found that the success of solving the inverse problem is limited since it depends strongly on the physical properties of the particles and the frequency range used in the inverse calculations. We have determined bounds necessary for determining the particle size distribution.

The first six months of the new funding period focused on demonstrating the capability to accurately measure volume fractions of dilute suspensions in the range of 0.004 to 0.050 percent. The Pulse/FFT method accurately measures attenuation for soda lime glass beads (14.9 micron radius), clays in water, and a Hanford surrogate salt simulant in this rangc. A lincar relationship is obtained for attenuation versus volume fraction, and the theory accurately predicts the monodispersed soda-lime glass bead data. The linear relationship should readily permit application of the acoustic monitor to dilute slurries.

The results of this project has relevance to the DOE mission of mobilizing, transporting and processing solid-liquid slurries by providing a reliable and safe monitor of percent solids in these slurries. Significant impact is expected for application as an accurate, safe and reliable monitor which is non-invasive is required to quantify across site transfer of dilute and concentrated slurries from storage tanks to processing facilities for high level waste treatment. Transfer of this technology to the DOE complex is the primary objective of the second funding period of this project whereby a proto-type acoustic monitor will be designed, commissioned and demonstrated to accurately measure low weight percent slurries in a flow loop and on a test transfer line.

\section{Research Objectives}

The primary objective of the research project during the first funding period was to develop an acoustic probe to measure volume percent solids in solid-liquid slurries in the presence of small amounts of gas bubbles. This problem was addressed because of the great need for a non-invasive, accurate and reliable method for solids monitoring in liquid slurries in the presence of radiolytically generated gases throughout the DOE complex. These measurements are necessary during mobilization of salts and sediments in tanks, transport of these slurries in transfer lines to processing facilities across a site, and, in some instances, during high level waste processing. Although acoustic probes have been commonly used for monitoring flows in single-phase fluids (McLeod, 1967), their application to monitor two-phase mixtures has not yet fully realized its potential. A number of investigators in recent years have therefore been involved in developing probes for measuring the volume fractions in liquid solid suspensions (Atkinson and Kytomaa, 1993; Greenwood et al., 1993; Martin et al., 1995) and in liquid-liquid suspensions (Bonnet and Tavlarides, 1987; Tavlarides and Bonnet, 1988, Yi and Tavlarides, 1990; Tsouris and Tavlarides, 1993, Tsouris et al., 1995). In particular, Atkinson and Kytomaa (1993) showed that the acoustic technique can be used to determine both the velocity and the volume fraction of solids while Martin et al. (1995) and Spelt et al. (1999) showed that the acoustic probe can also be used to obtain information on the size distribution of the particles. In a recent testing of in-line slurry monitors with radioactive slurries suspended with Pulsair Mixers (Hylton \& Bayne, 1999), an acoustic probe did not compare well with other instruments most 
probably due to presence of entrained gases and improper acoustic frequency range of interrogation.

The work of the investigators cited has established the potential of the acoustic probe for characterizing/monitoring two-phase flows in relatively ideal, well-characterized suspensions. Two major factors which we judge has prevented its wide-spread use in the processing industry, particularly for dilute suspensions, is careful selection of the frequency range for interrogation and quantification and removal of the noise introduced by bubbles from the acoustic signal obtained from the suspension.

Our research during the first funding period to develop an acoustic probe for solid-gasliquid suspensions has resulted in a theory, supported by our experiments, to describe smallamplitude dilute suspensions (Norato, 1999, Spelt et al., 1999, Spelt et al., 2001). The theory agrees well with experimental data of sound attenuation up to $45 \mathrm{vol} \%$ suspensions of 0.11 and 77 micron radius polystyrene particles in water and 0.4 to $40 \mathrm{vol} \%$, suspensions of 32 micron soda-lime glass particles in water. Also, analyses of our attenuation experiments for solid-gasliquid experiments suggest the theory can be applied to correct for signal interference due to the presence of bubbles over a selected frequency range to permit determination of the solid-liquid volume fraction. Further, we show experimentally that a reliable linear dependency of weight percent solids with attenuation is obtained for low weight fractions at high frequencies of interrogation where bubble interference is minimal.

There was a collaborative effort during the first funding period with the Pacific Northwest National Laboratories in that Dr. Margaret Greenwood was a co-investigator on the project. Dr. Greenwood provided a high level of experimental knowledge and techniques on ultrasound propagation, measurement and data processing. During the second funding period the slurry test loop at Oak Ridge National Laboratories under the direction of Mr. Tom Hylton will be employed to demonstrate the measurement capabilities of the proto-type acoustic monitor.

\section{Methods and Results}

Our research during the last three and one-half years has established a solid theoretical foundation for predicting attenuation and phase speed of acoustic waves propagating through solid-liquid suspensions, both in the presence as well as absence of gas bubbles (Spelt et al., 1998; Spelt et al., 2001). The attenuation theory accounts for losses due to viscous dissipation, nonadiabatic thermal effects, and incoherent scattering, and as a result can cover a wide range of frequencies and particle sizes. The theory also applies to polydisperse suspensions of spherical particles. The theory agrees with the results obtained by previous investigators who examined limiting cases. For example, our theory agrees with that of Allegra and Hawley (1972) who considered only the case of thermal attenuation at small volume fractions and with Sangani, Zhang and Proseperetti (1991) who considered only the case of viscous attenuation at large frequencies. The comprehensive theory developed allows us to interrogate a relatively large range of particle sizes or particle physical properties. The theory was also tested against the experimental data obtained by previous investigators (e.g. (Allegra and Hawley, 1972)) and in our laboratory. 
Demonstration of our theory is accomplished experimentally with the set-up shown in Figure 1. An ultrasonic pulse generator (Panametrics 5052 PR) generates an electric pulse and sends it to the emitting transducer. The actuated emitting transducer transmits an acoustic pulse through the sample actuating the receiving transducer, where the signal is transmitted to and read by an oscilloscope (Lecroy 9310A). An FFT analysis of the amplitude is performed on the spike pulse, outputting voltage as a function of frequencies of the pulse. This procedure is performed on the suspending liquid, and the solid-liquid or solid-gas bubble-liquid slurry. The voltages are used to calculate attenuation.

Comparison between the theory and the experiments is shown in Figures 2-6. Figure 2 shows attenuation as a function of frequency for glass particles with about 15 micron radius at 5 and 10 percent volume fractions. For these particles the viscous and scattering losses are the primary attenuation mechanisms for the range of frequency considered in the figure. Figure 3 shows the comparison for about 77 micron radius polystyrene particles in water at 5 percent volume fractions. For this system the thermal attenuation is important at smaller frequencies and scattering at higher frequencies. The two theoretical curves correspond to assuming that (i) the suspension is monodisperse and (ii) the particle size distribution is Gaussian with a standard deviation of 1 micron. The peaks seen in the figure correspond to resonances in shape oscillations. Figure 4 shows attenuation as a function of volume fraction for 63 micron glass particles in water at various frequencies. The viscous and scattering losses are important in these relatively dense suspensions. Good agreement here suggests that the theory is reasonably accurate in predicting the volume fraction dependence even at high volume fractions. Figure 5 shows comparison with the data for 0.11 micron polystyrene particles in water obtained by Allegra and Hawley (1972). For this system the attenuation is dominated by the thermal effects.

In Spelt et al. (1999) we investigated in detail the inverse problem so determining the particle size distribution of the particles given the attenuation as a function of frequency. We devised and compared various analytical techniques for solving the inverse problem and determined the conditions, e.g. the particle size and frequency range, necessary for determining the particle size distribution.

As mentioned earlier, one of the aims of our project was to devise a technique whereby the noise introduced by the presence of small amount of gas bubbles in the suspension can be removed to infer the properties of solid-liquid suspension. We have done detailed analysis of the noise introduced by bubbles. The primary conclusion is that the noise is significant at frequencies that are not much greater than the resonance frequencies of bubbles. Beyond the resonance frequency of the bubbles, the attenuation due to the presence of bubbles decrease with the increasing frequency while the attenuation due to solids increase with the increasing frequency. Thus the noise is minimal at sufficiently high frequencies. It is possible then to use the data at low frequencies to estimate the effects of bubbles and eliminate the slight noise produced by the bubbles at higher frequencies to yield volume fraction of the particles. We have illustrated this through our experiments on solid-liquid suspensions sparged with bubbles. The 


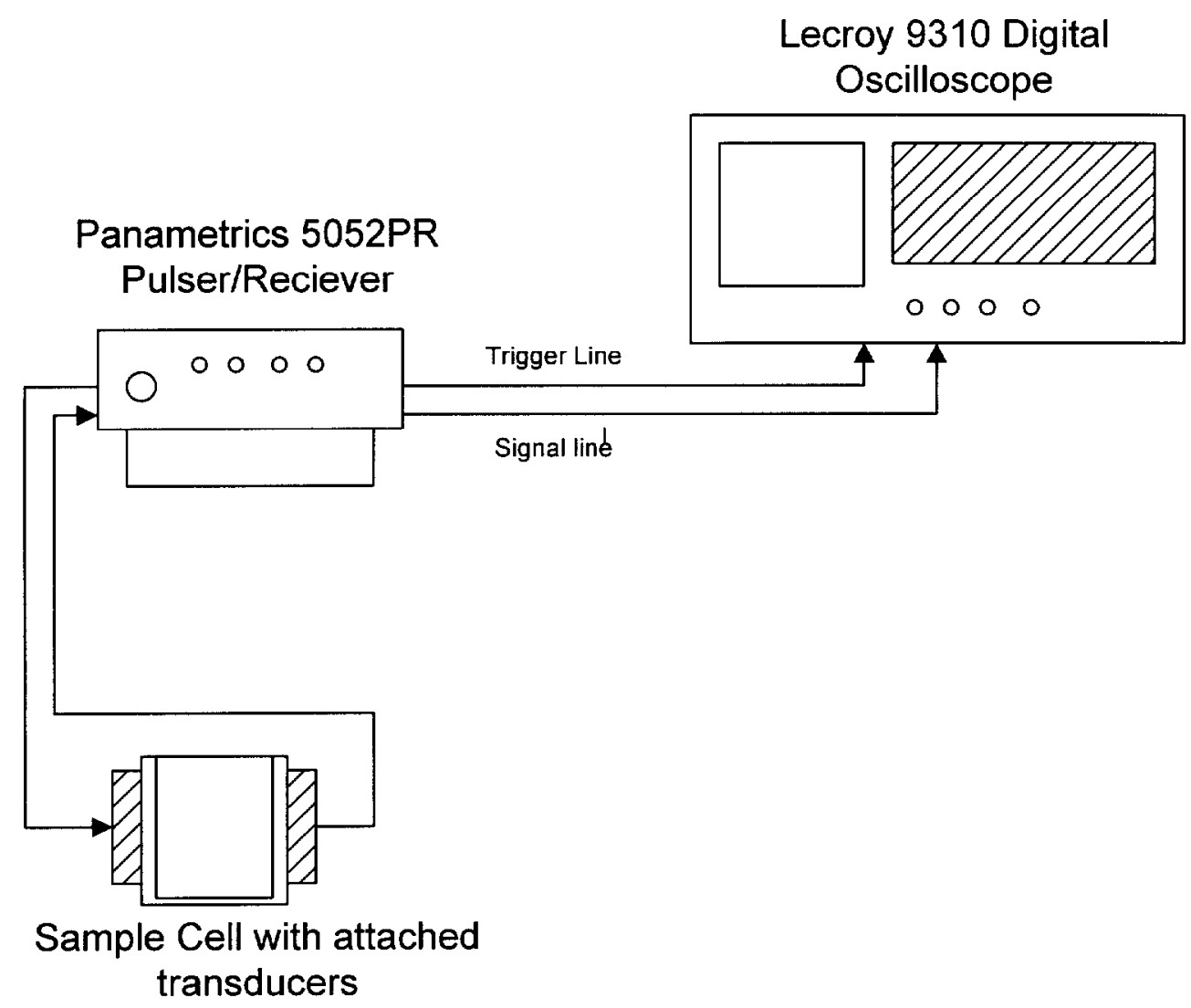

Figure 1. Schematic diagram of Pulse/FFT setup used to measure attenuation. A spike pulse is generated by the pulser/receiver and is transmitted to the transmitting transducer which is in contact with the sample. After traveling through the sample and being acquired by the receiving transducer the pulse is routed through the pulser/receiver to the oscilloscope. 


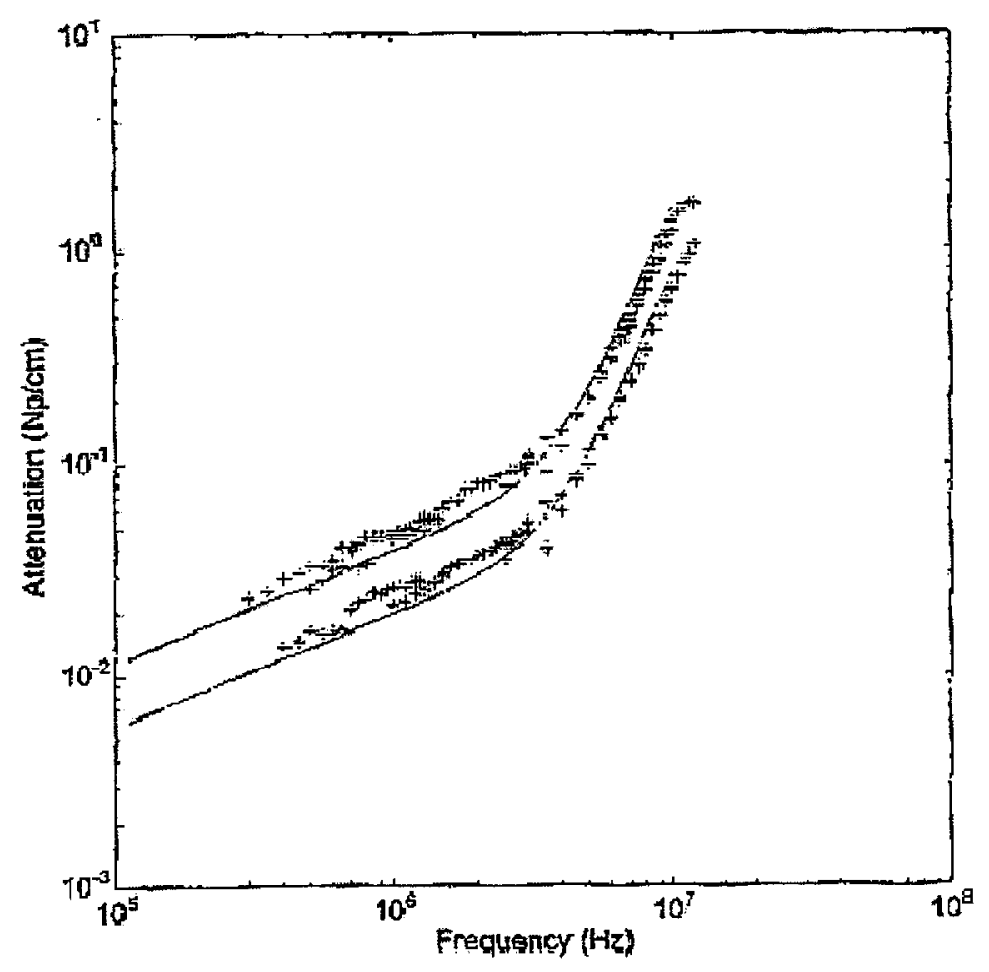

Figure 2. Comparison between experimental results and forward problem theory predictions for the attenuation versus frequency curves for soda-lime glass slurries at $5 \%$ and $10 \%$ solids by volume. The experimental solids size distribution has a mean radius of 14.9 microns with standard deviation of 3.56 microns. The forward theory predictions are based on a log-normal distribution with a mean radius of 14 microns and standard deviation of 7 microns.

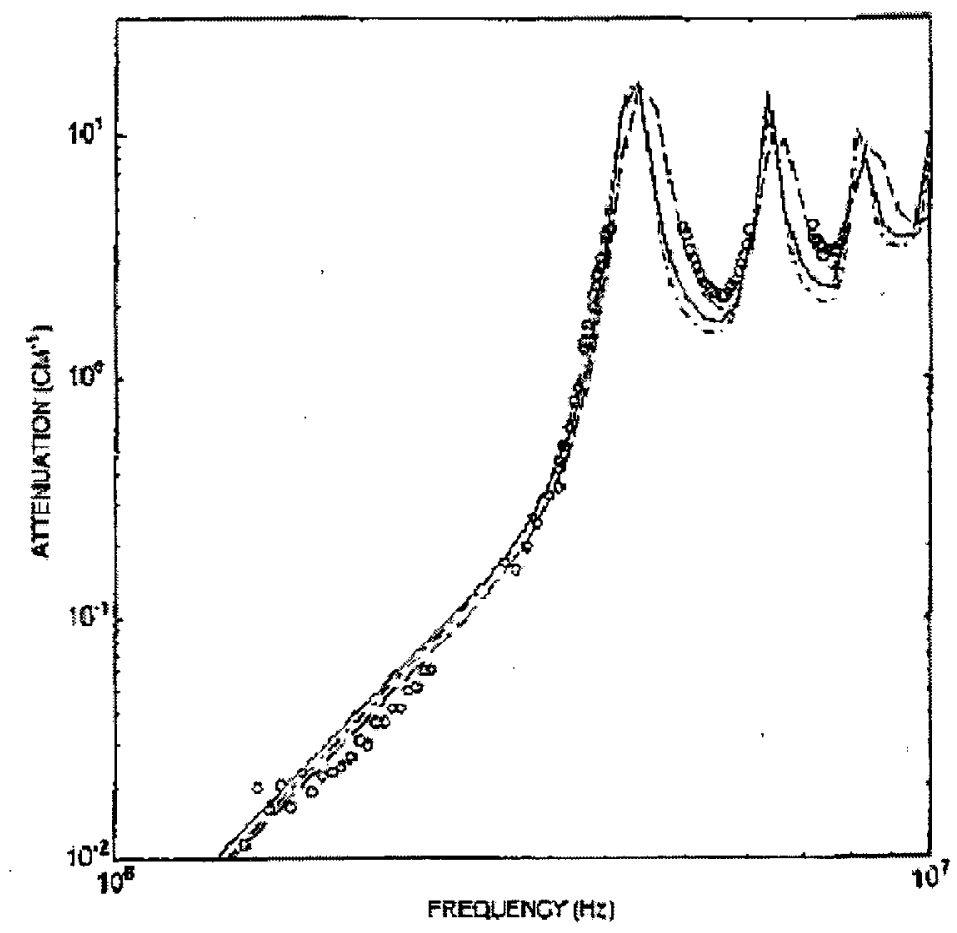

Figure 3. Experimental and theoretical results for the attenuation in a mixture of polystyrene particles (mean radius $79 \pm 3$ micron and 1.8 micron standard deviation) in water at 0.05 volume fraction. Circles are experiments, solid and broken lines are the theory for monodispersed particles of 79 microns and 77 microns radius, respectively. 


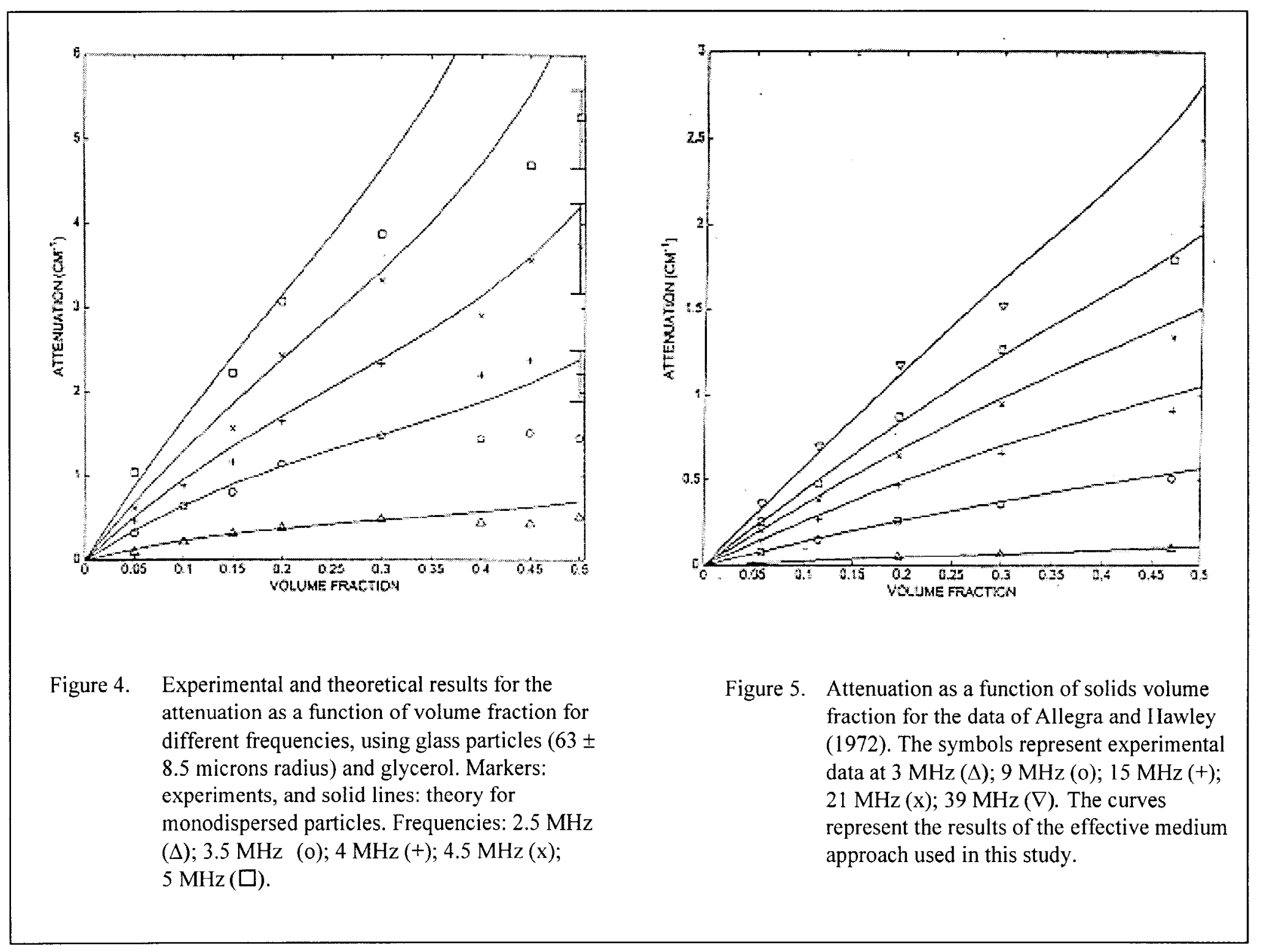




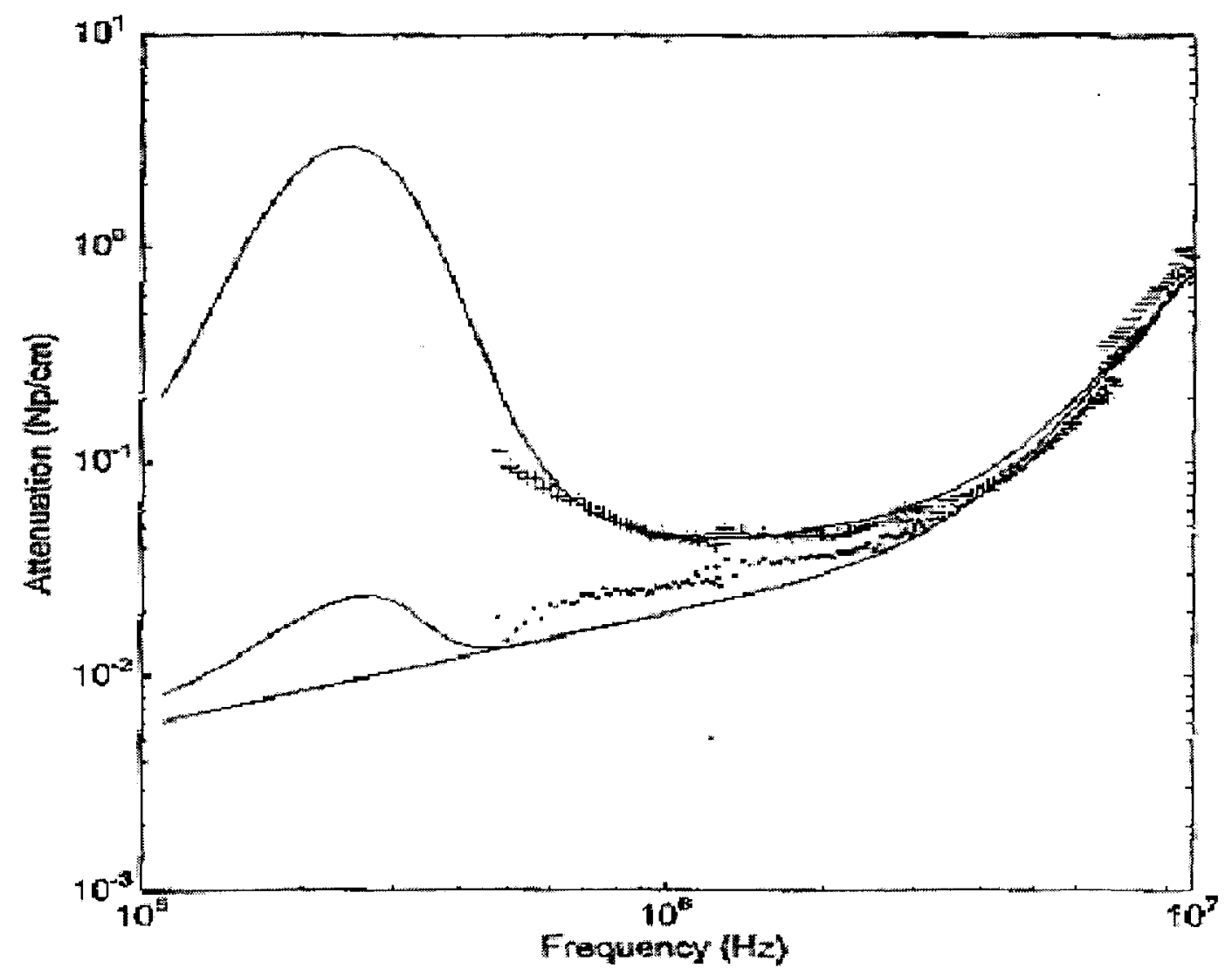

Figure 6. Results of predicting the attenuation due to the presence of bubbles in a solidgas-liquid slurry and simply subtracting that attenuation from the total attenuation. The symbols represent experimental data for the solid-liquid and solid-gas-liquid slurries and the difference after subtracting the bubble attenuation. 
preliminary results have been presented in the $\mathrm{PhD}$ dissertation of the student supported by the research (Norato, 1999); a more complete work will be submitted for publication in the near future. Figure 6 shows a comparison between theory and experiments for a gas-solid-liquid system. Note that the attenuation as a function of frequency goes through a minimum as the attenuation due to bubbles diminish while that due to solids increase with increasing frequency.

The above mentioned work deals with solids weight fraction above 5 percent. Our current activities extend the method to dilute suspensions in the weight percent range of 1-5 percent. We have conducted experiments for these low weight percents. The results are shown in Figures 7-9. We note from Figure 7 that the experimental data for soda-lime glass beads are in a reasonably good agreement with the theory based on monodispersed suspensions with no adjustable parameters. Figures 8 and 9 show that attenuation is significant for the entire range of volume fractions. Figure 9 shows results for a crystallized salt solution prepared according to surrogate protocol procedures for average Hanford supernate containing suspended salt particles (Glocar et al., 2000). These results show that with suitable calibration of the particle size, it would be possible to determine the volume fraction of particles from such attenuation frequency data. In principle only one calibration point would be needed, as a linear relationship appears to hold.

\section{Relevance, Impact and Technology Transfer}

The following answers the nine questions posed for this section.

a. This scientific work has direct application to monitor, in real time, solid-liquid slurry suspensions in the presence of gas bubbles at volume fractions of solids from 0.005 to 0.50 . Monitors can potentially be installed both on transfer lines, in a non-invasive manner, or in-tanks through riser entry ports.

b. This new technology has the potential to improve cleanup approaches and significantly reduce future costs, schedules and risks and meet DOE compliance requirements in the following ways:

b.1) By assuring uniform suspensions of solids through a tank during mobilization, sluicing, or emptying of tank salts and sediments. Time and costs to execute these operations will be minimized.

b.2) Transfer of low weight percent slurries in transfer lines from DOE operated tank farms to a contractor staging tanks for processing can be done in an accurate manner. This assurance will permit compliance of schedule requirements and appropriate guarantee of required and expected material transfers.

b.3) The monitor can be employed to determine onset of transfer line plugging to prevent such occurrences and alert personnel for quick and appropriate response 


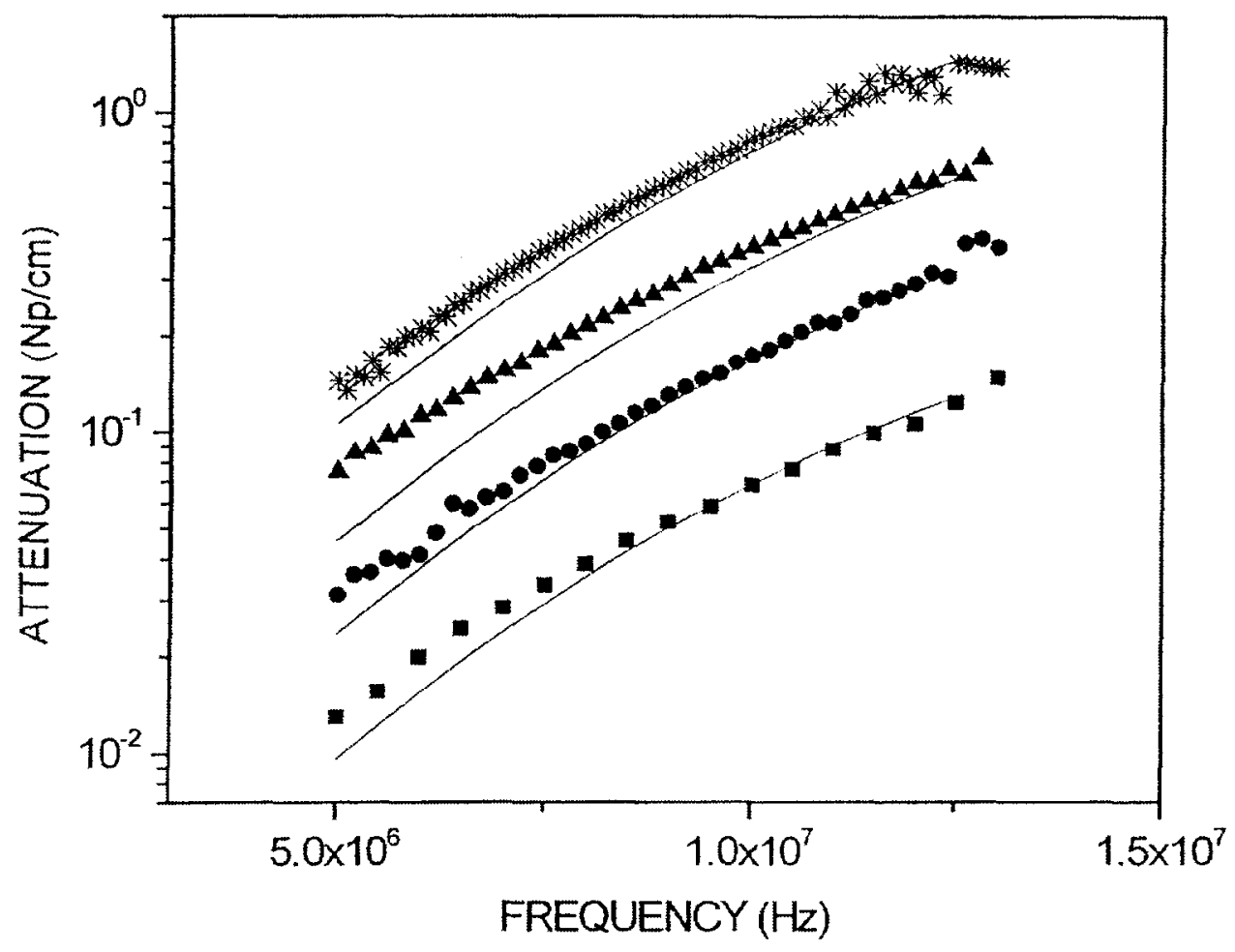

Figure 7. Attenuation versus frequency for slurries of 15 micron glass particles in water at various weight percent solids. The comparisons of theory (solid lines) with experiments (symbols) are given in ascending order for 1.0, 2.5, 5.0 and 12.0 weight percent solids. 


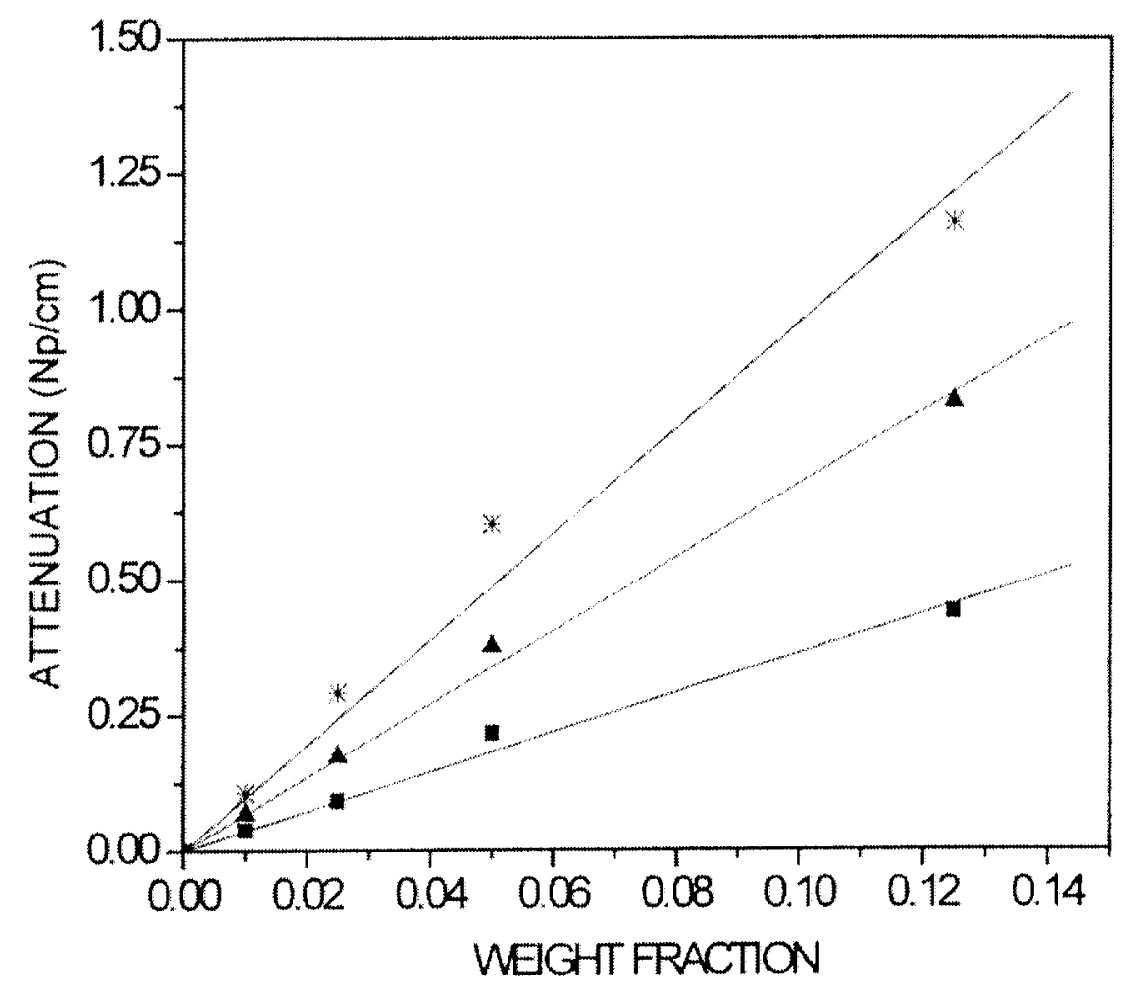

Figure 8. Attenuation versus weight fraction for slurries of 16 micron glass particles in water at three frequencies. Data shown with symbols are compared with a linear fit shown as solid lines. The curves in ascending order are for frequencies of 8 , 10 , and $12 \mathrm{MHz}$. 


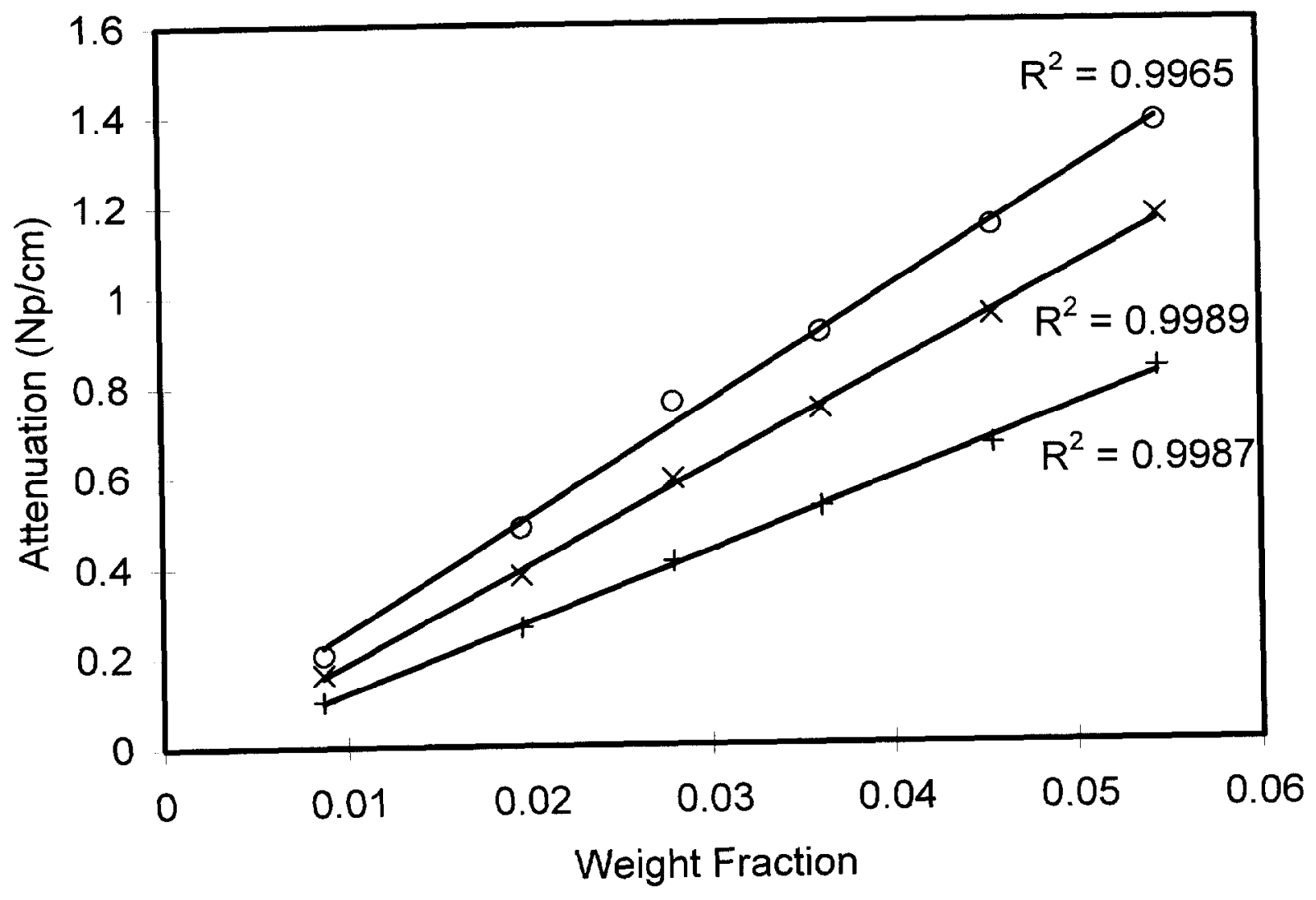

Figure 9 Attenuation versus weight fraction for the crystallized salt solution at three frequencies. The frequencies studied are (o) $12 \mathrm{MHz}(\mathrm{x})$, $10 \mathrm{MHz}$, and (+) $8 \mathrm{MHz}$. 
b.4) The monitor can be used to assure homogeneous suspensions of a mixture of solids in processing vessels during HLW and LAW treatment, for example, to guarantee glass former slurries uniformity for feeding melters.

c. The continuation of the project during the second funding period will result in a proto-type monitor which will be demonstrated at the ORNL flow loop. Also, efforts will be made to test it at the Hanford site. This effort should be completed by 9-30-03. Rapid deployment could follow subsequently pending success and availability of funds.

d. The project impact at Syracuse University resulted in continuation of this scientific effort leading to the proto-type development. The University infrastructure to conduct this research has improved through equipment acquisition and laboratory development. We have graduated one $\mathrm{PhD}$, one $\mathrm{MS}$ and trained during the first funding period. We have had a collaboration with Dr. Margaret Greenwood from PNNL during the first funding period and a two-way transfer of knowledge was accomplished. We have an agreement with ORNL to test the prototype monitor on the ORNL two phase flow loop during the second funding period, and this should provide a quality test facility and personnel for this aspect of the project. See section $c$ above for answers to the remaining questions.

e. Larger scale proto-type monitor development and testing are warranted, required and in progress with the second funding period of this project. The knowledge attained during the first period of funding is the basis for the proto-type monitor development and expected success in demonstration and future deployment.

f. Our collaboration with PNNL scientist Dr. Margaret Greenwood resulted in training Dr. Michael Norato (during his Ph.D. thesis work on the project) and transfer of knowledge. Our subsequent improvements of the monitor using the Pulse/FFT provides another technique PNNL can employ for their acoustic ultrasound investigations. Our particle size measurement and estimation techniques from attenuation measurements also should be of benefit to PNNL activities in this application.

g. We have increased out understanding in this area by improving our knowledge to monitor (experimental developments) and interpret attenuation signals (theoretical developments) heretofore not as accurately possible. These results provide the basis for continued development of the acoustic monitor.

h. The hurdles to overcome are the topics of the current funding period. These include:

h.1) Development of software and data acquisition system to determine volume fraction of particles.

h.2) Construct, test and commission in-line/at-tank acoustic monitor.

h.3) Conduct flow loop tests of monitor at ORNL and refine as needed. 
h.4) Test refined monitor at Hanford.

i. Ms. Judith Ann Bamberger (509-375-3898-FON; Judith.bamberger@pnl.gov) offered to collaborate on the project. Should we have success on h.3 above she offered to assist in implementation/testing at a Hanford test loop. Dr. Rajiv Srivastava, Florida International University, also offered to collaborate in ways yet to be determined.

\section{Project Productivity}

The project accomplished most of the targeted goals. We requested and received a oneyear no cost extension on the project due to typical delays in progress. We were not able to develop a theory to invert the acoustic signal to permit unambiguous prediction of the solid particle size distribution, along with the volume fraction. We show regions of physical properties and signal interrogation ranges where this may be possible. The results we have obtained were, however, quite valuable and are the basis for the continuation as described above.

\section{Personnel supported:}

8.1. P.I. Prof. Lawrence L. Tavlarides, Syracuse University

Co-P.I.: Professor Ashok Sangani, Syracuse, University

Co-Investigator: Dr. Margaret Greenwood

Post-Doctoral: Dr. Peter Spelt, currently at Imperial College, London, UK

Ph.D. Student: Dr. Michael A. Norato, currently at Westinghouse SRTC

M.S. Student: Mr. Mark Hedges, currently at Kionex, Inc.

Research Associate: Mr. Alexander Shcherbakov, on leave N.T.U. Kiev, Ukraine

\section{Publications}

9.1 Noratok, M.A., "Acoustic Probe for the Characterization of Solid-Gas-Liquid Slurries," Ph.D. Thesis, Syracuse University, May 1999.

9.2 Hedges, M., "Ultrasound Characterization of Low Volume Fraction Solid-Liquid Slurries," M.S. Thesis, Syracuse University, January 2001.

9.3 Spelt, P.D.M., Norato, M.A., Sangani, A.S., and Tavlarides, L.L., "Determination of Particle Size Distributions from Acoustic Wave Propagation," Physics of Fluids, Vol. 11 ns, 1065-1080 (1999).

9.4 Spelt, P.D.M., Norato, M.A., Sangani, A.S., Greenwood, M.S., and Tavlarides, L.L., "Attenuation of Sound in Concentrated Suspensions: Theory and Experiments," Jl. of Fluid Mechanics, 420, 1-36 (2001).

9.5 In preparation from Norato's Thesis.

9.6 In preparation from Hedges' Thesis. 


\section{Interactions}

10.1 M. Norato, P. Spelt, A. Sangani, M. Greenwood, L.L Tavlarides, "An Acoustic Probe for the Characterization of Three-Phase Slurries," 1997 Annual AIChE Meeting, Los Angeles, CA, November 20, 1997, paper $148 f$.

10.2 L.L. Tavlarides, "Acoustic Probe for Solid-Gas-Liquid Suspensions," Argonne National Laboratory, Argonne, IL, Ultrasonics/Acoustics Workshop, October 25, 1997.

10.3 M.A. Norato, A.S. Sangani, L.L. Tavlarides, M. Greenwood, "Acoustic Wave Propagation in Solid-Liquid Suspension," $16^{\text {th }}$ Biennial North American Mixing Conference, MIXING XVI, Williamsburg, VA, June 16, 1997.

10.4 M.S. Greenwood, M.A. Norato, A.S. Sangani, P.D.M. Spelt, L.L. Tavlarides, "Ultrasound Attenuation Measurements in Solid-Gas-Liquid Slurries and the Solution of the Inverse Problem," Annual AIChE Meeting, Miami Beach, paper 187f, November 17, 1998.

10.5 M.S. Norato, A.S. Sangani, P.D.M. Spelt, L.L. Tavlarides, "Acoustics of Slurries Containing Small Amounts of Bubbles: Measurement and Theory for Forward and Inverse Problems," Annual AIChE Meeting, Miami Beach, paper 126b, November 18, 1998.

10.6 M. Norato, P. Spelt, M. Greenwood, A. Sangani, L.L. Tavlarides, "Acoustic Probe for Solid/Gas Liquid Suspension, $18^{\text {th }}$ Biennial North American Mixing Conference, Banff, Canada. August 15-20, 1999.

10.7 M.S. Greenwood, M.A. Norato, A.S. Sangani, P.D.M. Spelt, L.L. Tavlarides, "Attenuation of Sound in Concentrated Suspension: Theory and Experiments," AIChE Meeting, Dallas, TX, Oct. 31-Nov. 5, 1999.

10.8 L.L. Tavlarides, "Acoustic Probe for Solid.Gas/Liquid Suspensions," DOE Characterization and Monitor Workshop, Atlanta, Georgia, Dec. 14-15, 1999.

10.9 L.L. Tavlarides, (with P. Spelt, M.A. Norato, M. Greenwood, A. Sangani), "Acoustic Probe for Characterization of Solid-Gas-Liquid Slurries," US DOE EMSP National Workshop, April 24-27, 2000, Atlanta, GA.

10.10 L.L. Tavlarides, A. Sangani, P. Spelt, M. Hedges, M.A. Norato and M. Greenwood, "Acoustic Probe for Characterization of Solid-Gas-Liquid Slurries," Tank Focus Area Midyear Review, Salt Lake City, UT, March 11-15, 2001. 


\section{Transitions}

The work is being transitioned through the second funding period.

\section{Patents}

None

13. Future Work

Continuation of funding in progress.

\section{Literature Cited}

Atkinson, C.M. and Kytomaa, H.K., "Acoustic Properties of Solid-Liquid Mixtures and the Limits of Ultrasound Diagnostics-I. Experiments. J. Fluids Eng. 115, 665 (1993).

Bonnet, J.C. and Tavlarides, L.L., "Ultasonic Technique for Dispersed Phase Hold-Up Measurements," I\&EC. Res. 26, 811 (1987).

Golcar, G.R., Colton, N.G., Durab, J.G., and Smith, H.D., "Hanford Tank Waste Simulants Specifications and Their Applicability for the Retrieval, Pretreatment, and Vitrification Processes," PNWD-2455/BNFL-RPT-012 Rev. 0, 2000.

Greenwood, M.S., Mai, J.L., and Good, M.S., "Attenuation Measurements of Ultrasound in a Kaolin-Water Slurry: A Linear Dependence Upon Frequency, J. Acoust. Soc. Am. 94, 908 (1993).

Hylton, T.D. and Bayne, C.K., "Testing of In-Line Slurry Monitors and Pulsair Mixers with Radioactive Slurries," ORNL/TM-1999/111, July, 1999.

Martin, J., Rakotomalala, N., and Salin, D., Phys. Rev. Lett. 74, 1457 (1995).

McLeod, F., "Directional Doppler Flowmeter," Intl. Conf. Med. Bio. Eng., Stockholm, 213 (1967).

Norato, M.A., "Acoustic Probe for the Characterization of Solid-Gas-Liquid Slurries," Ph.D. Thesis, Syracuse University, May 1999.

Spelt, P.D.M., Norato, M.A., Sangani, A.S., and Tavlarides, L.L., "Determination of Particle Size Distributions from Acoustic Wave Propagation," Physics of Fluids, Vol. $11 \mathrm{~ns}, 1065-1080$ (1999). 
Spelt, P.D.M., Norato, M.A., Sangani, A.S., Greenwood, M.S., and Tavlarides, L.L., "Attenuation of Sound in Concentrated Suspensions: Theory and Experiments," Jl. of Fluid Mechanics, 420, 1-36 (2001).

Tavlarides, L.L. and Bonnet, J.C., "An Ultrasonic Technique for Dispersed Phase Holdup Measurements," US Patent No. 4,726,221 (1988).

Tsouris, C. and Tavlarides, L.L., "Volume Fraction Measurement of Water in Oil by an Ultrasonic Technique," I\&EC Res. 32, 998 (1993).

Tsouris, C., Norato, M.A., and Tavlarides, L.L., "A Pulse-Echo Ultrasonic Probe for Local Volume Fraction Measurements in Liquid-Liquid Dispersions," I\&Res. 34, 3154 (1995).

Yi, J.H. and Tavlarides, L.L., "Model for Hold-Up Measurements in Liquid-Liquid Dispersions Using an Ultrasonic Technique," I\&EC Res. 29, 475 (1990).

15. Feedback

None

16. Appendices

A1 - Publication 9.1
A2 - Publication 9.2
A3 - Publication 9.3
A4 - Publication 9.4 


\title{
Attenuation of sound in concentrated suspensions: theory and experiments
}

\author{
By PETER D. M. SPELT $T^{1} \dagger$, MICHAEL A. NORATO ${ }^{1}$, \\ ASHOK S. SANGANI ${ }^{1} \ddagger$, MARGARET S. GREENWOOD \\ AND LAWRENCE L. TAVI.ARIDES \\ ${ }^{1}$ Department of Chemical Engineering and Materials Science, Syracuse University, Syracuse, \\ NY 13244, LSA \\ ${ }^{2}$ Pacific Northwest National Laboratory, Battelle Memorial Institute, Richland, WA 99352, USA
}

(Received 26 February 1999 and in revised form 6 September 2000)

Ensemble-averaged equations are derived for small-amplitude acoustic wave propagation through non-dilute suspensions. The equations are closed by introducing effective properties of the suspension such as the compressibility, density, viscoelasticity, heat capacity, and conductivity. These effective properties are estimated as a function of frequency, particle volume fraction, and physical properties of the individual phases using a self-consistent, effective-medium approximation. The theory is shown to be in excellent agreement with various rigorous analytical results accounting for multiparticle interactions. The theory is also shown to agree well with the experimental data on concentrated suspensions of small polystyrene particles in water obtained by Allegra \& Hawley and for glass particles in water obtained in the present study.

\section{Introduction}

We consider the problem of predicting the attenuation of sound waves propagating through suspensions. When the particle volume fraction in the suspension is very small the particle interactions may be neglected and the attenuation can be determined as a function of the sound wave frequency by examining the interaction of a single particle with the incident wave as has been done by a number of investigators in the past. For example, Carstensen \& Foldy (1947) examined the problem of dilute bubbly liquids while Epstein \& Carhart (1953) and Allegra \& Hawley (1972) examined, respectively, the case of dilute emulsions and dilute slurries. Since the attenuation behaviour is strongly dependent on the particle radius, the attenuation-frequency data for dilute suspensions may be used for determining the particle size distribution as shown by Duraiswami, Prabhukumar \& Chahine (1998), who considered the case of bubbly liquids. The corresponding problem for dilute suspensions has been examined by Spelt et al. (1999).

The particle interactions can have a significant effect on the acoustic behaviour of non-dilute suspensions and at present rigorous calculations accounting for these interactions are lacking. Direct attack on the problem, i.e. solving the linearized

$\dagger$ Present address: Centre for Composite Materials, Imperial College, Prince Consort Road, London SW7 2BY, UK.

¥ Author to whom correspondence should be addressed: e-mail asangani@syr.edu 
energy, momentum, and continuity equations for multiparticle systems, appears to be a daunting task even with the development of efficient computers. Thus, it is necessary to develop a suitable approximate theory and to assess its validity by comparison with the experimental data obtained with different kinds of suspensions.

We use the method of ensemble averaging to derive linearized continuity, momentum, and energy equations for the suspensions. These equations are closed by introducing effective properties of the suspensions, namely the effective conductivity, viscosity (or viscoelasticity), compressibility, and density. To estimate these properties as a function of frequency and physical properties and volume fractions of the individual phases, we must determine the relation between the conditionally averaged temperature and velocity fields inside a test particle and the temperature and velocity fields of the suspension. A self-consistent, effective-medium approximation is used for this purpose.

The predictions of the theory are compared with several known rigorous analytical calculations accounting for multiparticle interactions in dense suspensions in the limiting case of relatively small frequencies for which the acoustic wavelength is large compared with the particle radius. At very low frequencies, for which the thermal and viscous (Stokes) lengths become large compared with the particle radius, we expect the velocity and temperature fields to satisfy, respectively, the Stokes and Laplace equations. The effective properties such as the viscosity, conductivity, and permeability, for monodisperse suspensions in this limit are well-established (e.g. Ladd 1990; Mo \& Sangani 1994). It is shown that the effective-medium approximation is in excellent agreement with these results. For moderate frequencies, at which the Stokes layer is very small compared with the particle radius and the wavelength is large compared with the radius, the velocity field satisfies the Laplace equation outside the Stokes layers. Added mass and Basset force coefficients, which contribute to the effective density of the suspension, have been determined by Sangani, Zhang \& Prosperetti (1991) for this limiting case. Once again, the effective-medium predictions are shown to be in excellent agreement with these rigorous calculations.

We also compare the predictions of the theory with the experimental data on attenuation. Probably the best data in the literature are due to Allegra \& Hawley (1972) who measured attenuation in a polystyrene-water system at frequencies for which the thermal effects contribute most significantly to the attenuation. Our theory is shown to be in excellent agreement with their data. To test the theory for the cases in which the attenuation due to viscous and scattering effects is significant, we have measured attenuation in glass-water and glass-water/glycerol systems at small to intermediate frequencies. For smaller particles, for which the viscous attenuation dominates, the theory and experiments are in very good agreement with each other. For larger particles, for which the scattering dominates, the agreement is very good only up to about $30 \%$ volume fractions.

The organization of the rest of the paper is as follows. In $\$ 2$ we derive rigorous average equations for linear acoustics and introduce effective properties of the suspensions. In $\S 3$ we compare the predictions of the effective-medium theory with various analytical results and show how the effective properties vary with the frequency and particle volume fraction. Section 4 describes the experimental set-up used for obtaining attenuation data. Section 5 gives a comparison between the theory and various experimental data. In $\S 6$ we present some results on the phase speed of sound waves, and discuss the possibility of using phase speed measurements for measuring particle volume fractions. Finally, $\S 7$ summarizes important findings of the study. 


\section{Theory}

\subsection{Linearized equations}

Let us consider a small-amplitude plane acoustic wave with frequency $\omega$ propagating through a uniform, monodisperse suspension of solid particles of radius $a$. We write the density as $\rho+\rho^{\prime} \mathrm{e}^{-\mathrm{i} \omega t}$, the temperature as $T+T^{\prime} \mathrm{e}^{-\mathrm{i} \omega t}$, and the velocity as $\boldsymbol{u} \mathrm{e}^{-\mathrm{i} \omega t}$. A note regarding the notation: both the equilibrium and small fluctuation values are important for the density and temperature and we therefore use primes to denote the amplitudes of the fluctuations in these quantities. Only the amplitudes of the velocity and the other field variables (stress, heat flux, etc.) will be needed, and we denote the amplitudes of these quantities without a prime so that the resulting equations look less cluttered. When the amplitudes $\rho^{\prime}, T^{\prime}$ and $\boldsymbol{u}$ are small, the terms involving the products of these quantities can be neglected from the continuity, momentum, and energy equations to obtain the following linearized equations:

$$
\begin{gathered}
-\mathrm{i} \omega \rho^{\prime}+\rho \nabla \cdot \boldsymbol{u}=0 \\
-\mathrm{i} \omega \rho u_{i}=\frac{\partial \sigma_{i j}}{\partial x_{j}} \\
-\mathrm{i} \omega \rho C_{v} T^{\prime}=-\frac{\partial q_{j}}{\partial x_{j}}-\rho C_{v} \beta^{-1}(\gamma-1) \nabla \cdot \boldsymbol{u} .
\end{gathered}
$$

In writing the last equation, we have made use of the linearized equation of state to eliminate the pressure from the usual energy equation. The stress tensor amplitude $\sigma_{i j}$ for a Newtonian fluid is given by

$$
\sigma_{i j}=\left[\left\{-\frac{c^{2} \rho}{i \omega \gamma}+\mu_{i}\right\} \nabla \cdot u-\left\{\frac{\rho(\gamma-1) C_{v}}{\beta T}\right\} T^{\prime}\right] \delta_{i j}+d_{i j}
$$

where $d_{i j}$ is the deviatoric stress amplitude

$$
d_{i j}=\mu\left[\frac{\partial u_{i}}{\partial x_{j}}+\frac{\partial u_{j}}{\partial x_{i}}-\frac{2}{3} \delta_{i j} \nabla \cdot u\right] .
$$

$C_{v}$ is the constant volume specific heat, $\gamma=C_{p} / C_{v}$ is the ratio of specific heats, $\mu$ and $\mu_{v}$ are, respectively, the shear and bulk coefficients of viscosity, $c$ is the adiabatic sound speed through the fluid, and $\beta$ is the coefficient of thermal expansion. Note that the first and the third terms inside the square brackets on the right-hand side of (4) are related to the thermodynamic pressure amplitude:

$$
p^{\prime}=\left(\frac{\partial p}{\partial \rho}\right)_{T} \rho^{\prime}+\left(\frac{\partial p}{\partial T}\right)_{\rho} T^{\prime}=\frac{c^{2}}{\gamma} \rho^{\prime}+\frac{\rho(\gamma-1) C_{v}}{\beta T} T^{\prime}
$$

Finally, $q_{j}=-\kappa \partial T^{\prime} / \partial x_{j}$ in (3) is the heat flux amplitude, $\kappa$ being the thermal conductivity.

Inside the solid particles equations similar to (1)-(3) apply with the stress tensor given by (Landau \& Lifschitz 1986)

$$
\sigma_{i j}=\left[\left\{\frac{\tilde{\lambda}+\frac{2}{3} \tilde{\mu}}{-\mathrm{i} \omega}\right\} \nabla \cdot \boldsymbol{u}-\left\{\frac{\rho(\gamma-1) C_{v}}{\beta T}\right\} T^{\prime}\right] \delta_{i j}+\tilde{d}_{i j},
$$

where $\tilde{\lambda}$ and $\tilde{\mu}$ are the Lame constants for the particles which are assumed to be perfectly elastic. Note that for solids it is customary to write the stress in terms of displacement instead of velocity. For small-amplitude oscillatory motions the 
amplitudes of the two are, of course, related by a factor of $1 /(-\mathrm{i} \omega)$, and this fact has been used in writing the first term on the right-hand side of the above equation. Note also that the factor $\tilde{\lambda}+(2 / 3) \tilde{\mu}$ is the bulk modulus of the solid. Thus, the isotropic part of the stress tensor represented by the terms inside the square brackets in the above equation arises from the density and temperature changes in the solid. The deviatoric stress tensor $\tilde{d}_{i j}$ is defined in the manner similar to (5), with the fluid viscosity replaced by the 'particle viscosity', $\mu_{p}=\tilde{\mu} /(-\mathrm{i} \omega)$. Note that the Lamé constant $\tilde{\mu}$ is sometimes referred to as the shear modulus.

The above linearized equations must be solved subject to the boundary conditions of continuity of velocity, temperature, heat flux, and traction $\left(\sigma_{i j} n_{j}, n_{j}\right.$ being the unit outward normal at the particle surface) at the interface between the particles and the fluid. In concentrated suspensions particle interactions are significant and the rigorous evaluation of sound speed and attenuation through the suspension would require the very difficult task of solving the above set of equations in a domain containing many particles.

The problem as outlined here involves a number of variables. It may be possible to simplify it in some limiting cases of small or large frequencies or when the physical properties (e.g. density and compressibility) of the two phases are widely different as in the case of acoustic propagation in bubbly liquids (Prosperetti 1984). However, it is desirable to measure the attenuation over a wide range of frequencies in order to characterize the suspension, and for most solid-liquid suspensions the ratio of physical properties does not differ significantly from unity. Thus, it is necessary to solve the full problem as described above.

\subsection{Ensemble-averaged linearized equations for suspensions}

In this subsection we ensemble-average the equations for the amplitudes of density, velocity, and temperature in the fluid and solid phases, and obtain thereby the linearized continuity, momentum, and energy equations for the suspension. It will be shown that the resulting equations have a form similar to the equations for a single phase provided that the suspension is assigned suitable properties, which we refer to as the effective properties of the suspensions. An important outcome of the averaging process will be that it will yield rigorous expressions for various effective properties of the suspension. Unlike the case of single-phase fluids, the effective properties will be seen to be functions of the wave frequency, and the equations we derive are therefore restricted to small-amplitude sinusoidal acoustic waves.

Let us denote by $g(x)$ the particle indicator function defined to be unity when the point $\boldsymbol{x}$ is inside any of the particles and zero when $\boldsymbol{x}$ is in the fluid. The properties and field variables of the liquid and particles will be denoted by subscripts $l$ and $p$, respectively. The ensemble-averaged variables will be denoted by angular brackets.

Multiplying the continuity equation for the liquid by the liquid indicator function $1-g$ and for the particle by $g$, adding the two, and averaging the resulting equation we obtain the continuity equation for the suspension:

$$
-\mathrm{i} \omega\left\langle\rho^{\prime}\right\rangle+\rho_{l}\left\langle(1-g) \nabla \cdot \boldsymbol{u}_{l}\right\rangle+\rho_{p}\left\langle g \nabla \cdot \boldsymbol{u}_{p}\right\rangle=0 .
$$

The last two terms on the left-hand side of the above equation must now be expressed in terms of the divergence of the average velocity, i.e. $\nabla \cdot\langle\boldsymbol{u}\rangle$, so that the resulting equation resembles the continuity equation of a single-phase medium (cf. (1)). We begin with the identity

$$
\rho_{l}\left\langle(1-g) \nabla \cdot \boldsymbol{u}_{l}\right\rangle+\rho_{p}\left\langle g \nabla \cdot \boldsymbol{u}_{p}\right\rangle=\rho_{l} \boldsymbol{\nabla} \cdot\langle\boldsymbol{u}\rangle+\left(\rho_{p}-\rho_{l}\right)\left\langle g \nabla \cdot \boldsymbol{u}_{p}\right\rangle+\rho_{l}\left\langle\left(\boldsymbol{u}_{l}-\boldsymbol{u}_{p}\right) \cdot \nabla g\right\rangle .
$$


The gradient of the indicator function is zero at all points except at the particle-fluid interface where it is proportional to the Dirac delta function owing to the step jump in $g$ across the particle-fluid interface. More specifically,

$$
\nabla g=-n \dot{\delta}\left(\boldsymbol{x}-\boldsymbol{x}_{s}\right),
$$

where $\boldsymbol{x}=\boldsymbol{x}_{5}$ represents the surface of the particles, $\delta$ is the Dirac delta function, and $\boldsymbol{n}$ is the unit normal vector, pointing into the fluid, at the particle surface.

Because the velocity is continuous across the solid-fluid interfaces, the last term in (9) vanishes; the second term on the right-hand side contains an as yet unknown quantity, $\left\langle g \nabla \cdot \boldsymbol{u}_{p}\right\rangle$, which is related to the average amplitude of the dilatation rate inside the particles. We shall restrict our analysis to the suspensions which are isotropic on a macroscale. For such suspensions the above quantity will be expected to be proportional to the amplitude of other scalar quantities such as $\nabla \cdot\langle\boldsymbol{u}\rangle$, the average amplitude for the mixture dilatation rate. We therefore introduce the closure relation

$$
\left\langle g(\boldsymbol{x})\left(\boldsymbol{\nabla} \cdot \boldsymbol{u}_{p}\right)(\boldsymbol{x})\right\rangle=\phi \lambda_{\rho} \nabla \cdot\langle\boldsymbol{u}\rangle(\boldsymbol{x}),
$$

where $\phi$ is the volume fraction of the solids. The passage of a wave will induce non-zero amplitudes of other scalar quantities such as $\left\langle T^{\prime}\right\rangle$ and $\left\langle\sigma_{k k}\right\rangle$ also, and one may write a more general expression in which the average particle dilatation rate is expressed as a linear combination of all these scalar variables. In that case one must determine separately how variation in temperature, pressure and density affect separately the dilatation inside the particles. However, since all these scalar variables will be related to each other through algebraic relations that depend on the frequency and effective wavenumbers for the special case of sinusoidal acoustic waves, it is unnecessary to decompose the particle dilatation into various terms. Likewise, the dilatation rate for particles may also depend on the higher-order scalar derivatives such as $V^{2} \nabla \cdot\langle\boldsymbol{u}\rangle$. Since the average equations for the suspension are expected to obey wave equations, the Laplacian of the average dilatation rate can always be written in terms of the dilatation rate and the effective wavenumbers. Thus, it will suffice to use (11) for the dilatation rate inside the particles keeping in mind that $\lambda_{\beta}$, must be evaluated such that it accounts for not only the first derivative of the suspension velocity, but also its higher-order derivatives and temperature and pressure. The calculation for $\lambda_{\rho}$ to be presented in the next section does account for all these effects.

We note that in the present study we are interested in deriving a dispersion relation for the passage of small-amplitude acoustic waves through a suspension, and not a set of average equations valid for all suspension flows. The latter can indeed be a daunting task as equations such as (11) will not apply to the general case for which, as mentioned above, the effects of temperature, pressure, etc. must all be written separately, and the closure relation will possibly also include the higher-order derivatives. The procedure, however, is general enough in the sense that it can be used to determine the dispersion relation for other small-amplitude acoustic problems. For example, it can also be used for determining the dispersion relation for fluid-saturated porous media, which are sometimes modelled as fixed beds. Note that for the fixed bed case although the average particle velocity $\left\langle\boldsymbol{u}_{p}\right\rangle \equiv\left\langle g \boldsymbol{u}_{p}\right\rangle / \phi$ is zero, the left-hand side of (11), and hence $\lambda_{\rho}$, are non-zero. The radial oscillations of the fixed particles will contribute to $\lambda_{p}$ in such a situation. Note that $\nabla \cdot\langle\boldsymbol{u}\rangle$ is non-zero in all acoustic problems.

Substituting for $\left\langle g \nabla \cdot \boldsymbol{u}_{p}\right\rangle$ from (11) into (9) yields the continuity equation for the 
suspension given by

$$
-\mathrm{i} \omega\left\langle\rho^{\prime}\right\rangle+\rho_{c, e} \nabla \cdot\langle\boldsymbol{u}\rangle=0,
$$

with the effective equilibrium density of the suspension to be used in the suspension continuity equation, i.e. $\rho_{c, e}$, given by

$$
\rho_{c, e}=\rho_{l}+\left(\rho_{p}-\rho_{l}\right) \phi \hat{i}_{p} .
$$

Physically, $\lambda_{\rho}$ represents the ratio of average dilatation amplitude in the particle phase to that in the fluid-particle mixture or the suspension. This coefficient will depend, in addition to wave frequency, on the compressibilities of both phases, volume fraction, spatial distribution of the particles, and other variables appearing in the governing equations listed in the previous subsection. Thus, we see that, in general, the effective equilibrium density of the suspension to be used in the suspension continuity equation cannot be given by some arbitrary mixture rule, e.g. the volume-averaged density or the mass-averaged density. An approximate scheme for estimating $\lambda_{\rho}$ will be described in $\$ 2.4$.

We now proceed to derive the momentum equation for the suspension starting from (2) and its counterpart for the particles. Using the same procedure as in the continuity equation we obtain

$$
-i \omega \rho_{m, e}\left\langle u_{i}\right\rangle=\left\langle g \frac{\partial \sigma_{i j, p}}{\partial x_{j}}\right\rangle+\left\langle(1-g) \frac{\partial \sigma_{i j, l}}{\partial x_{j}}\right\rangle .
$$

The effective (equilibrium) density of the suspension to be used in the momentum equation, $\rho_{m, e}$, is given by

$$
\rho_{m, e}=\rho_{l}+\left(\rho_{p}-\rho_{l}\right) \phi \lambda_{1}
$$

with the coefficient $\lambda_{v}$ defined by

$$
\phi \lambda_{i}\langle\boldsymbol{u}\rangle(\boldsymbol{x})=\left\langle g(\boldsymbol{x}) \boldsymbol{u}_{p}(\boldsymbol{x})\right\rangle .
$$

Physically, $\lambda_{i}$ represents the ratio of average velocity amplitude inside the particles to that in the suspension. Once again this coefficient, and other such coefficients to be introduced in this subsection, will, in general, depend on complex multiparticle interactions, and the details of its evaluation will be described later.

The right-hand side of (14) can be simplified using the identity

$$
\begin{aligned}
\frac{\partial\left\langle\sigma_{i j}\right\rangle}{\partial x_{j}} & \equiv \frac{\partial}{\partial x_{j}}\left\langle g \sigma_{i j, p}+(1-g) \sigma_{i j, l}\right\rangle \\
& =\left\langle g \frac{\partial \sigma_{i j, p}}{\partial x_{j}}\right\rangle+\left\langle(1-g) \frac{\partial \sigma_{i j, l}}{\partial x_{j}}\right\rangle+\left\langle\left(\sigma_{i j, p}-\sigma_{i j, l}\right) \frac{\partial g}{\partial x_{j}}\right\rangle .
\end{aligned}
$$

The last term in the above equation, being related to the jump in the traction across the interface, vanishes owing to the boundary condition $\sigma_{i j, p} n_{j}=\sigma_{i, l, l} n_{j}$ at the particle-fluid interface. Thus, we see that the right-hand side of (14) simply equals the divergence of the average stress in the suspension, i.e. the momentum equation for the suspension is given by

$$
-\mathrm{i} \omega \rho_{m, e}\left\langle u_{i}\right\rangle=\frac{\partial\left\langle\sigma_{i j}\right\rangle}{\partial x_{j}}
$$

We must supplement the above momentum equation with an expression for the average stress. The linearity of the equations implies that the stress amplitude will be linear in the gradient of average velocity amplitude and $\left\langle T^{\prime}\right\rangle$. 
Let us first consider the isotropic part of the average stress or, equivalently, the stress trace. Multiplying the isotropic part in (4) by $1-g$ and that in (7) by $g$ and averaging, we obtain

$$
\frac{1}{3}\left\langle\sigma_{k k}\right\rangle=\left\{\frac{\left\langle\boldsymbol{c}^{2} \rho \gamma^{-1}\right\rangle_{e}}{-\mathrm{i} \omega}+\mu_{1, e}\right\} \nabla \cdot\langle\boldsymbol{u}\rangle-T^{-1}\left\langle\rho(\gamma-1) C_{l} \beta^{-1}\right\rangle_{m, e}\left\langle T^{\prime}\right\rangle
$$

with

$$
\begin{gathered}
\left\langle c^{2} \rho \gamma^{-1}\right\rangle_{e}=c_{l}^{2} \rho_{l} / \gamma_{l}+\phi \lambda_{\mu}\left[\{\tilde{\lambda}+2 \tilde{\mu} / 3\}-c_{l}^{2} \rho_{i} / \gamma_{l}\right], \\
\mu_{l, e}=\mu_{v}\left(1-\phi \lambda_{\rho}\right),
\end{gathered}
$$

and

$$
\left\langle\rho(\gamma-1) C_{v} \beta^{-1}\right\rangle_{m, e}=\rho_{l}\left(\gamma_{l}-1\right) C_{v, l} \beta_{l}^{-1}+\phi \lambda_{T}\left(\rho_{p}\left(\gamma_{p}-1\right) C_{t, p} \beta_{p}^{-1}-\rho_{l}\left(\gamma_{l}-1\right) C_{v, l} \beta_{l}^{-1}\right) .
$$

The coefficient $\lambda_{\rho}$ was defined earlier (cf. (11)). $\lambda_{T}$, on the other hand, is a new coefficient which is defined as the ratio of average temperature amplitude inside the particles to that in the mixture, i.e.

$$
\phi \lambda_{T}\left\langle T^{\prime}\right\rangle(\boldsymbol{x})=\left\langle g(x) T_{p}^{\prime}(x)\right\rangle .
$$

Both the effective $c^{2} \rho / \gamma$ and the bulk viscosity of the suspension depend on the coefficient $\lambda_{p}$. This is not surprising since both depend on the average dilatation amplitude inside the particles. The result that the effective bulk viscosity $\mu_{t, e}$ of the suspension depends only on the bulk viscosity of the fluid may appear strange at first sight, but it is really a consequence of the way the isotropic part of the stress is defined for the liquid and solids (cf. (4) and (7)). The stress arising from the thermal expansion or, equivalently, temperature fluctuations depends on $\rho(\gamma-1) C_{v} / \beta T$ of the two phases and the relative temperature fluctuations in the two phases.

Since the deviatoric stress amplitudes in the individual phases depend only on the velocity gradient amplitude, we expect the average deviatoric stress to be linear in the gradient of average velocity amplitude. It also must be traceless. If we further assume that the suspension is macroscopically isotropic, then the average deviatoric stress is characterized by a single effective (shear) viscosity, $\mu_{\ell}$. Thus, we write

$$
\left\langle d_{i j}\right\rangle=\mu_{e}\left(\frac{\partial\left\langle u_{i}\right\rangle}{\partial x_{j}}+\frac{\partial\left\langle u_{j}\right\rangle}{\partial x_{i}}-\frac{2}{3} \delta_{i j} \nabla \cdot\langle\boldsymbol{u}\rangle\right)
$$

To obtain an expression for the effective viscosity we need to evaluate only one component of the average deviatoric stress. We shall lake, without loss of generality, the mean velocity amplitude to be given by

$$
\langle\boldsymbol{u}\rangle(\boldsymbol{x})=-\nabla \mathrm{e}^{\mathrm{i} \boldsymbol{k}_{i \cdot} \cdot \boldsymbol{x}}=-\mathrm{i} \boldsymbol{k}_{i \mathrm{e}} \mathrm{e}^{\mathrm{i} \boldsymbol{k}_{c e} \cdot \boldsymbol{x}},
$$

where $\boldsymbol{k}_{c e}$ is the effective wavenumber vector for the compressional wave through the suspension. We shall choose this vector to be aligned along the $x_{1}$-axis. The 11-component of the deviatoric stress is given by

$$
\left\langle d_{11}\right\rangle=2\left\langle\mu \frac{\partial u_{1}}{\partial x_{1}}\right\rangle-\frac{2}{3}\left\langle\mu \frac{\partial u_{k}}{\partial x_{k}}\right\rangle
$$

The last term on the right-hand side of the above equation, being related to the dilatation amplitudes, can be readily related to the coefficient $\lambda_{\mu}$ introduced earlier. 
The first term on the right-hand side can be expressed in terms of a coefficient $\lambda_{d}$ defined by

$$
\phi \lambda_{d} \underset{\partial x_{1}}{\partial\left\langle u_{1}\right\rangle}=\left\langle g(x) \frac{\partial u_{1}^{p}}{\partial x_{1}}(x)\right\rangle
$$

With this definition it is straightforward now to relate $\left\langle d_{11}\right\rangle$ to the gradient in velocity amplitude:

$$
\left\langle d_{11}\right\rangle=2\left[\mu_{l}+\phi \lambda_{d}\left(\mu_{p}-\mu_{l}\right)\right] \frac{\partial\left\langle u_{1}\right\rangle}{\partial x_{1}}-\frac{2}{3}\left[\mu_{l}+\phi \lambda_{\rho}\left(\mu_{p}-\mu_{l}\right)\right] \frac{\partial\left\langle u_{k}\right\rangle}{\partial x_{k}} .
$$

Substituting for $\langle\boldsymbol{u}\rangle$ from (25) in (28) and in (24) with $i=j=1$ and comparing the resulting expressions yields the following expression for the effective viscosity:

$$
\mu_{e}=\mu_{i}+\frac{1}{2} \phi\left(\mu_{p}-\mu_{i}\right)\left(3 \lambda_{d}-\lambda_{p}\right) \text {. }
$$

Finally, the energy equation for the suspension, obtained by averaging $1-g$ times the energy equation for the liquid plus $g$ times that for the solid, is given by

$$
-\mathrm{i} \omega\left\langle\rho C_{n}\right\rangle_{e}\left\langle T^{\prime}\right\rangle=-\frac{\partial\left\langle q_{j}\right\rangle}{\partial x_{j}}-\left\langle\rho C_{n} \beta^{-1}(\gamma-1)\right\rangle_{e, e} \nabla \cdot\langle\boldsymbol{u}\rangle .
$$

Here an argument similar to (17) has been used to simplify the energy-flux term (thereby using the boundary condition at the particle surface that the heat flux is continuous). In (30) the effective heat capacity of the suspension is given by

$$
\left\langle\rho C_{v}\right\rangle_{e}=\rho_{l} C_{v, l}+\phi \lambda_{T}\left(\rho_{p} C_{v, p}-\rho_{l} C_{v, l}\right)
$$

with $\lambda_{T}$ defined by (23). The effective property $\left\langle\rho C_{v}(\gamma-1) \beta^{-1}\right\rangle_{e, e}$ appearing in the last term on the right-hand side of (30) is related to $\lambda_{\rho}$, and the expression for evaluating it is obtained by replacing $\lambda_{T}$ in (22) by $\lambda_{\rho}$.

The average heat flux amplitude is written as

$$
\left\langle q_{j}\right\rangle=-\kappa_{e} \frac{\partial\left\langle T^{\prime}\right\rangle}{\partial x_{j}}
$$

with the effective conductivity

$$
\kappa_{e}=\kappa_{l}+\phi \lambda_{\kappa}\left(\kappa_{p}-\kappa_{l}\right),
$$

where the coefficient $\lambda_{\kappa}$ is the ratio of the average temperature gradient amplitude inside the particles to that in the suspension, i.e.

$$
\phi \lambda_{k} \frac{\partial\left\langle T^{\prime}\right\rangle}{\partial x_{i}}=\left\langle g(x) \frac{\partial T_{p}^{\prime}}{\partial x_{i}}(x)\right\rangle \text {. }
$$

In summary, the continuity, momentum, and energy equations for the suspension are given by (8), (18) and (30), the average stress tensor by (19) and (24), and the average heat flux by (32). These equations resemble the equations for the single phase given in $\$ 2.1$ with suitably defined effective properties of the suspension. It must be noted that these equations are rigorous for small-amplitude sinusoidal waves through any suspension. The effective properties of the suspension will be functions of frequency and physical properties of the two phases as well as the microstructure of the suspension. Note also that properties such as $\rho_{c, e}$, the effective density to be used in the suspension continuity equation, will not depend only on the density and compressibility of the two phases but also on their thermal properties since its determination will require solving all the microscale equations simultaneously. 


\subsection{Wave equations for the suspension}

To find an expression for the attenuation of sound waves in a suspension it is necessary to derive wave equations from the linearized acoustic equations for the suspension as was done by Epstein \& Carhart (1953) for pure liquid. We shall follow that derivation closely here. As shown by these investigators the acoustics equations permit three waves: a thermal wave, a shear or rotational wave, and a compressional wave. The last one is the most significant as far as the attenuation of a plane acoustic wave is concerned. The other waves are important in determining the disturbance produced by a test particle in the suspension as we shall see in the next subsection. by

We decompose the average velocity amplitude in scalar and vector potentials, given

$$
\langle\boldsymbol{u}\rangle=-\nabla \Phi+\nabla \times A .
$$

Since the curl of a gradient of any scalar function is zero, $\boldsymbol{A}$ can be specified to within a gradient of an arbitrary scalar function. To remove this arbitrariness an additional restriction is imposed that $\boldsymbol{A}$ be divergence free, i.e. $\nabla \cdot A=0$. It may be noted that the vorticity amplitude equals $-\nabla^{2} \boldsymbol{A}$.

Introducing the decomposition in the momentum equation for the suspension (18), and rearranging, we obtain

$$
\begin{aligned}
\nabla\left[\mathrm{i} \omega \rho_{m, e} \Phi+\left\{\frac{\left\langle c^{2} \rho \gamma^{1}\right\rangle_{e}}{-\mathrm{i} \omega}+\mu_{r, e}+{ }_{3}^{4} \mu_{e^{\prime}}\right\}\right. & \left.\nabla^{2} \Phi+\frac{1}{T}\left\langle\rho(\gamma-1) C_{e} \beta^{-1}\right\rangle_{e}\left\langle T^{\prime}\right\rangle\right] \\
& =\nabla \times\left[\mathrm{i} \omega \rho_{m, e} \boldsymbol{A}-\mu_{e} \nabla \times(\nabla \times \boldsymbol{A})\right] .
\end{aligned}
$$

Here, we have used the vector identity $\nabla^{2} a=\nabla(\nabla \cdot a)-\nabla \times(\nabla \times a)$. The energy equation (30) becomes

$$
-\mathrm{i} \omega\left\langle\rho C_{v}\right\rangle_{e}\left\langle T^{\prime}\right\rangle=\kappa_{e} \nabla^{2}\left\langle T^{\prime}\right\rangle+\left\langle\rho C_{v} \beta^{-1}(\gamma-1)\right\rangle_{e, e} \nabla^{2} \Phi
$$

Both sides of (36) must vanish separately because a rotational vector field cannot balance an irrotational field. Hence the right-hand side being zero gives, after using the above-mentioned vector identity and $\nabla \cdot A=0$,

$$
\nabla^{2} A+k_{s e}^{2} A=\mathbf{0}
$$

with $k_{s e}^{2} \equiv \mathrm{i} \omega \rho_{m, e} / \mu_{e} ; k_{s e}$ is the effective wavenumber for shear waves through the suspension.

The left-hand side of (36) being zero gives an expression for $\left\langle T^{\prime}\right\rangle$ in terms of the velocity potential:

$$
\left\langle T^{\prime}\right\rangle=T\left[-\mathrm{i} \omega \rho_{m, e} \Phi-\left\{\frac{\mathrm{i}}{\omega}\left\langle c^{2} \rho \gamma^{-1}\right\rangle_{e}+\left(\mu_{v, e}+\frac{4}{3} \mu_{e}\right)\right\} \nabla^{2} \Phi\right] /\left\langle\rho(\gamma-1) C_{r} \beta^{-1}\right\rangle_{m, e} .
$$

Eliminating $\left\langle T^{\prime}\right\rangle$ from the energy equation for the suspension (37) by substituting for $\left\langle T^{\prime}\right\rangle$ from the above yields

$$
\Phi+(E-F+G) \nabla^{2} \Phi-E F \nabla^{4} \Phi=0,
$$

with

$$
\begin{gathered}
E=\frac{\left\langle c^{2} \rho \gamma^{-1}\right\rangle_{e}}{\rho_{m, e} \omega^{2}}-\frac{\mathrm{i}}{\rho_{m, e} \omega}\left(\mu_{v, e}+\frac{4}{3} \mu_{e}\right), \\
F=\frac{i \kappa_{e}}{\omega\left\langle\rho C_{v}\right\rangle_{e}}
\end{gathered}
$$




$$
G=\frac{\left\langle\rho C_{v} \beta^{-1}(\gamma-1)\right\rangle_{e, e}\left\langle\rho(\gamma-1) C_{v} \beta^{-1}\right\rangle_{m, e}}{T \rho_{m, e} \omega^{2}\left\langle\rho C_{v}\right\rangle_{e}} .
$$

Equation (40) can be written in the form

$$
\left(k_{c e}^{-2} \nabla^{2}+1\right)\left(k_{t e}^{-2} \nabla^{2}+1\right) \Phi=0,
$$

so that $\Phi=\Phi_{c}+\Phi_{\imath}$ with

$$
\begin{aligned}
& \left(\nabla^{2}+k_{c e}^{2}\right) \Phi_{c}=0, \\
& \left(\nabla^{2}+k_{t e}^{2}\right) \Phi_{1}=0 .
\end{aligned}
$$

The effective wavenumbers for the compressional and thermal waves are given by, respectively,

$$
\begin{aligned}
& k_{c c}^{-2}=\frac{1}{2}(E-F+G)+\frac{1}{2}\left\{(E-F+G)^{2}+4 E F\right\}^{1 / 2} . \\
& k_{t e}^{-2}=\frac{1}{2}(E-F+G)-\frac{1}{2}\left\{(E-F+G)^{2}+4 E F\right\}^{1 / 2} .
\end{aligned}
$$

As mentioned earlier the compressional wavenumber is the most important one as far as the acoustic wave propagation of the plane wave is concerned. The imaginary part of $k_{c e}$ gives the attenuation while $\omega$ divided by the real part of $k_{c e}$ gives the phase speed.

For future reference we note that the expression (39) for $\left\langle T^{\prime}\right\rangle$ now can be written as

with

$$
\left\langle T^{\prime}\right\rangle=b_{c e} \Phi_{c}+b_{t e} \Phi_{i}
$$

$$
b_{c e}=T\left[-\mathrm{i} \omega \rho_{m, e}+\left\{\frac{i}{\omega}\left\langle c^{2} \rho \gamma^{-1}\right\rangle_{e}+\left(\mu_{v, e}+{ }_{3}^{4} \mu_{e}\right)\right\} k_{c e}^{2}\right] /\left\langle\rho(\gamma-1) C_{v} \beta^{-1}\right\rangle_{m, e} .
$$

The expression for $b_{t e}$ is similar with $k_{c e}$ in the above replaced by $k_{t e}$.

\subsection{An effective-medium model}

To determine the attenuation and phase speed we must now estimate various effective properties of the suspensions. This requires determining five coefficients: $\lambda_{\rho}, \lambda_{p}, \lambda_{T}, \hat{\lambda}_{d}$, and $\lambda_{h}$. Let us begin with the evaluation of $\lambda_{p}$ which represents the ratio of average dilatation amplitude inside the particles to that in the suspension. This is defined by (11), which is equivalent to

$$
\phi \lambda_{p} \nabla \cdot\langle u\rangle(x)=\int_{x-x_{1} \mid \leqslant a}\left\langle\nabla \cdot u_{p}\right\rangle\left(x \mid x_{1}\right) P\left(x_{1}\right) \mathrm{d} V\left(x_{1}\right) .
$$

Here, we have introduced a conditionally averaged field. Thus, $\langle\boldsymbol{u}\rangle\left(\boldsymbol{x} \mid \boldsymbol{x}_{1}\right)$ is the ensemble-averaged velocity amplitude at point $\boldsymbol{x}$ given a particle centred at $\boldsymbol{x}_{1}$. $P\left(x_{1}\right)$ is the probability density for finding a particle with its centre in the vicinity of $x_{1}$. For uniform, monodisperse suspensions $P\left(x_{1}\right)=n=3 \phi /\left(4 \pi a^{3}\right), n$ being the number density of the particles and $\phi$ the particle volume fraction.

We shall use an effective-medium approximation for determining the conditionally averaged fields, and hence, the integrals such as the one appearing on the right-hand side of (51). All effective-medium approximations must satisfy the criterion that far from the test particle, i.e. for $\left|\boldsymbol{x}-\boldsymbol{x}_{1}\right| \rightarrow \infty$, the conditionally averaged fields such as $\langle\boldsymbol{u}\rangle\left(\boldsymbol{x} \mid \boldsymbol{x}_{1}\right)$ must approach the corresponding unconditionally averaged fields such as $\langle\boldsymbol{u}\rangle(\boldsymbol{x})$. On the other hand, for $\left|\boldsymbol{x}-\boldsymbol{x}_{1}\right| \leqslant a$, i.e. for a point inside the test particle, the conditionally averaged fields must satisfy the equations governing the particle 
phase. The simplest kind of effective medium approximation then assumes that the conditionally averaged equation satisfies the suspending fluid equations for $a \leqslant r \leqslant R$ and the unconditionally averaged equations for the suspension for $r \geqslant R$. Here, $r \equiv\left|\boldsymbol{x}-\boldsymbol{x}_{1}\right|$ is the distance from the centre of the particle. Different effective-medium approximations differ in their choice of $R$. Some investigators choose $R=a$ which eliminates the fluid region altogether. This makes the subsequent analysis very simple but, unfortunately, the estimates obtained with $R=a$ are typically inferior, and in some cases unphysical. For example, it may yield negative effective properties at high volume fractions. Other investigators choose $R=a \phi^{-1 / 3}$ with the incorrect reasoning that the volumes occupied by the particle and fluid for $r \leqslant R$ Inust be proportional to the volume fractions of the two phases. In the present study we shall choose $R$ to be given by

$$
\frac{R}{a}=\left(\begin{array}{c}
1-S(0) \\
\phi
\end{array}\right)^{1 / 3},
$$

with $S(\mathbf{0})$ the zero-wavenumber limit of the suspension structure factor defined by

$$
S(\mathbf{0})=\int[P(\boldsymbol{r} \mid \mathbf{0})-P(\mathbf{0})] \mathrm{d} \boldsymbol{r},
$$

where $P(\boldsymbol{r} \mid \mathbf{0})$ is the probability density for finding a particle with its centre near $\boldsymbol{r}$ given that there is a particle with its centre at origin. Note that $P(\boldsymbol{r} \mid \mathbf{0})=\delta(\boldsymbol{r})$ for $r<2 a$. The above choice of $R$ is such that

$$
\int_{r \geqslant 2 a}[P(\boldsymbol{r} \mid \mathbf{0})-P(\mathbf{0})] \mathrm{d} \boldsymbol{r}=\int_{R \leqslant r \leqslant 2 a} P(\mathbf{0}) \mathrm{d} \boldsymbol{r} .
$$

In other words, the excess particle density outside the exclusion region in a suspension is distributed over a distance $r$ ranging from $R$ to $2 a$ in the effective medium.

The structure factor of the suspension can be determined experimentally by a lightscattering technique but in the absence of such information one may choose $S(\mathbf{0})$ to correspond to that of a hard-sphere molecular system for which the well-known Carnahan-Starling approximation yields quite accurate estimates of the structure factor as a function of the volume fraction:

$$
S(\mathbf{0})=\frac{(1-\phi)^{4}}{1+4 \phi+4 \phi^{2}-4 \phi^{3}+\phi^{4}} .
$$

The effective-medium radius $R$ based on $S(0)$ was first introduced by Dodd et al. (1995) who compared the results of rigorous multiparticle interactions for determining the short-time self- and gradient-diffusivity of proteins in bilipid membranes with those obtained by the effective-medium approximation and found a very good agreement between the two. In the problems concerned with determining the collective mobility or the sedimentation velocity, where each particle is acted upon with a constant force, it was shown in Mo \& Sangani (1994) that the conditionally averaged velocity has the correct leading-order behaviour at large $r$ only when $R$ is chosen according to (52).

For small volume fractions, $S(0)$ given by $(55)$ behaves as $1-8 \phi+O\left(\phi^{2}\right)$, and $R \rightarrow 2 a$. Thus, in 'well-stirred' dilute random suspensions the effective medium begins at $r=2 a$ according to (52) and the fluid region $a<r<2 a$ corresponds to the excluded-volume region. Note that the more usual choice $R=a \phi^{-1 / 3}$ would, on the other hand, suggest that the effective medium begins at a very large distance from the test particle in a dilute suspension, which is unphysical except for the situations such 
as dilute periodic or 'well-separated' random suspensions defined by Jeffrey (1973) (For such arrays $S(\mathbf{0})$ is small when $\phi$ is small and (52) also gives $R / a=O\left(\phi^{-1 / 3}\right)$.) Thus it is not surprising that $R$ based on (52) will give better estimates of the effective properites at small to moderate volume fractions compared to those obtained with $R=a \phi^{-1 / 3}$. Indeed, Sangani \& Mo (1997) have shown that the coefficients of $O\left(\phi^{2}\right)$ corrections to the effective conductivity and elasticity obtained using (52) are much closer to the rigorous results for these coefficients obtained by detailed pair interaction calculations than those obtained with $R=a \phi^{-1 / 3}$.

Before we close this brief review of effective-medium approximations, we should perhaps note here one more class of effective-medium approximations made in the literature. These involve immersing a pair of particles in the effective medium. Examples are the calculations by Kim \& Russel (1985) who estimated the permeability of a fixed bed of particles and Ju \& Chen (1994)'s calculations for the effective viscosity and elasticity of suspensions with a hard-sphere spatial distribution. These calculations generally require far greater effort - comparable to direct multiparticle calculations - and do not necessarily yield superior estimates compared with the simple approximations based on a single particle. On the other hand, the single-particle approximations will be inadequate for the suspension problems in which the changes in microstructure due to imposed flow and their effects in turn on the suspension properties must be addressed.

Returning now to the problem of estimating the coefficients $\lambda_{\rho}$ etc. using the effective-medium model consisting of a particle fluid assembly of radius $R$ immersed in a medium with the effective properties of the suspension, we write the velocity inside the test particle in terms of scalar and vector potentials as in the previous subsection. For the plane wave travelling along the $x_{1}$-axis with $\langle\boldsymbol{u}\rangle(\boldsymbol{x})=-\mathrm{i} \boldsymbol{k}_{c e} \exp \left(\mathrm{i} \boldsymbol{k}_{c e} \cdot \boldsymbol{x}\right)$ we have, for $\left|\boldsymbol{x}-\boldsymbol{x}_{1}\right| \leqslant a$,

$$
\begin{aligned}
& \Phi_{c p}\left(\boldsymbol{x} \mid \boldsymbol{x}_{1}\right)=\exp \left(\mathrm{i} \boldsymbol{k}_{c e} \cdot \boldsymbol{x}_{1}\right) \sum_{n=0}^{\infty} \mathrm{i}^{n}(2 n+1) A_{p n} P_{n}(\mu) j_{n}\left(k_{c p} r\right), \\
& \Phi_{t p}\left(\boldsymbol{x} \mid \boldsymbol{x}_{1}\right)=\exp \left(\mathrm{i} \boldsymbol{k}_{c e} \cdot \boldsymbol{x}_{1}\right) \sum_{n=0}^{\infty} \mathrm{i}^{n}(2 n+1) B_{p n} P_{n}(\mu) j_{n}\left(k_{t p} r\right), \\
& A_{p}\left(\boldsymbol{x} \mid \boldsymbol{x}_{1}\right)=\exp \left(\mathrm{i} \boldsymbol{k}_{c e} \cdot \boldsymbol{x}_{1}\right) \sum_{n=0}^{\infty} \mathrm{i}^{n}(2 n+1) C_{p n} P_{n}^{1}(\mu) j_{n}\left(k_{s p} r\right),
\end{aligned}
$$

where $r=\left|\boldsymbol{x}-\boldsymbol{x}_{1}\right|, \mu=\cos \theta, \theta$ being the angle between $\boldsymbol{x}-\boldsymbol{x}_{1}$ and $\boldsymbol{k}_{c e}, j_{n}$ is the spherical Bessel function of both the first kind (regular at $r=0$ ), $P_{n}$ is the Legendre polynomial of degree $n$, and $P_{n}^{1}$ is the associated Legendre polynomial of degree $n$ and order 1. $A_{p}$ is the only non-zero (azimuthal) component of $\boldsymbol{A}$.

Similar expressions can be written for $a<r<R$ for which the relevant wavenumbers in the expressions for $\Phi_{c l}, \Phi_{t l}, A_{l}$ are, respectively, $k_{c l}, k_{t l}$, and $k_{s l}$. The spherical harmonics of both the first kind and second kind (corresponding to waves emanating from $r=0$ ) must be included in the expression. This leads to a set of six unknowns for each mode $n$ describing the motion in the liquid shell. Finally, for $r>R$, the potentials consist of the plane wave corresponding to the unconditional motion plus outgoing waves with wavenumbers $k_{c e}, k_{t e}$, and $k_{s e}$. Thus, a total of 12 unknowns are needed in describing the motion for each mode $n$. These are determined from the boundary conditions of continuity of velocity, traction, temperature, and heat flux amplitudes at $r=a$ and $r=R$. Note that the conditional density and temperature amplitudes can 
be determined from the expressions for $\Phi_{c}, \Phi_{l}$, and $A$ using the expressions given in the previous subsection. We keep a total of $N$ modes (typical calculation used $N=5$ ) and solve the resulting $12 N$ equations numerically.

We return now to the calculation of $\lambda_{\rho}$. We use $\nabla \cdot u_{p}=-\nabla^{2} \Phi_{p}=k_{c p}^{2} \Phi_{c p}+k_{t p}^{2} \Phi_{t p}$ to convert the integral in (51) to integrals over $\Phi_{p}$. Let us introducc a cocfficient $\eta_{c}$ given by

$$
\phi \eta_{c} \Phi_{c}(\boldsymbol{x}) \equiv \int_{\left|x-x_{1}\right| \leqslant a} \Phi_{c p}\left(\boldsymbol{x} \mid \boldsymbol{x}_{1}\right) P\left(\boldsymbol{x}_{1}\right) \mathrm{d} V\left(\boldsymbol{x}_{1}\right) .
$$

Similarly, a coefficient $\eta_{t}$ is introduced with $\Phi_{c p}\left(x \mid x_{1}\right)$ in the above replaced by $\Phi_{t p}\left(\boldsymbol{x} \mid \boldsymbol{x}_{1}\right)$. The coefficient $\lambda_{\rho}$ is related to these two coefficients by

$$
z_{c e}^{2} \lambda_{p}=z_{c p}^{2} \eta_{c}+z_{t p}^{2} \eta_{p}
$$

The integration in (59) must be carried out over all $\boldsymbol{x}_{1}$ such that $\left|\boldsymbol{x}-\boldsymbol{x}_{1}\right| \leqslant a$. To convert this to an integration over $\boldsymbol{r}$ we use the identity

$$
\begin{aligned}
\exp \left[\boldsymbol{i}_{c e} \cdot \boldsymbol{x}_{1}\right] & =\exp \left[\mathrm{i} \boldsymbol{k}_{c e} \cdot \boldsymbol{x}\right] \exp \left[-\mathrm{i} r k_{c e} \mu\right] \\
& =\exp \left[\mathrm{i} \boldsymbol{k}_{c e} \cdot \boldsymbol{x}\right] \sum_{m=0}^{\infty} \mathrm{i}^{m}(-1)^{m}(2 m+1) j_{m}\left(k_{c e} r\right) P_{m}(\mu)
\end{aligned}
$$

Now using $P\left(x_{1}\right)=n$, substituting for $\Phi_{c p}$ from (56) into (59), making use of the above identity, and carrying out the integration, we obtain

$$
\eta_{c}=\frac{3}{z_{c e}^{2}-z_{c p}^{2}} \sum_{n=0}^{\infty}(2 n+1) A_{p n}\left[z_{c p} j_{n-1}\left(z_{c p}\right) j_{n}\left(z_{c e}\right)-z_{c e} j_{n}\left(z_{c p}\right) j_{n-1}\left(z_{c e}\right)\right],
$$

where $z_{c p}=k_{c p} a$ and $z_{c e}=k_{c e} a$. In the above expression $j_{1}$ should be taken to be $\cos (z) / z$. In deriving the above expression use has been made of the identity (Gradshteyn \& Ryzhik 1994 - note that there is a sign error in their 5.54(1))

$$
\begin{aligned}
\int r^{2} j_{n}(\alpha r) j_{n}(\beta r) \mathrm{d} r & =\frac{\pi}{2(\alpha \beta)^{1 / 2}} \int r J_{n+1 / 2}(\alpha r) J_{n+1 / 2}(\beta r) \mathrm{d} r \\
& =\frac{\beta r^{2} j_{n}(\alpha r) j_{n-1}(\beta r)-\alpha r^{2} j_{n-1}(\alpha r) j_{n}(\beta r)}{\alpha^{2}-\beta^{2}}
\end{aligned}
$$

(recall that $\left.j_{n}(z)=(\pi / 2 z)^{1 / 2} J_{n+1 / 2}(z)\right)$.

The expression for $\eta_{r}$ is similar to (61) with $A_{p n}$ in that expression replaced by $B_{p n}$ and $z_{c p}$ by $z_{t p}$. Now $\lambda_{\rho}$ can be evaluated by substituting for $\eta_{c}$ and $\eta_{t}$ in (60).

The coefficient $\lambda_{T}$, which represents the ratio of average temperature amplitude inside the test particle to that in the suspension, is also related to $\eta_{c}$ and $\eta_{t}$. Inside the particle the temperature amplitude is a linear combination of the potentials as given by $\left\langle T_{p}^{\prime}\right\rangle\left(\boldsymbol{x} \mid \boldsymbol{x}_{1}\right)=b_{c p} \Phi_{c p}+b_{t p} \Phi_{t p}$, where $b_{c p}$ and $b_{t p}$ are given by expressions similar to that for $b_{c e}$ given earlier (cf. (50)). Now, since the unconditionally averaged thermal potential, $\Phi_{t}(\boldsymbol{x})$, is zero, the average temperature amplitude is given by $\left\langle T^{\prime}\right\rangle(\boldsymbol{x})=b_{c e} \Phi_{c}$, and therefore

$$
\lambda_{T}=\left(b_{c p} / b_{c e}\right) \eta_{c}+\left(b_{t p} / b_{c e}\right) \eta_{t}
$$

The other $\lambda$ coefficients can be evaluated in a similar manner and are interconnected. To determine $\lambda_{r}$, we need to calculate the average of the $x_{1}$-component of the velocity amplitude inside the test particle at $\boldsymbol{x}_{1}$. Decomposing this velocity into 
three parts corresponding to contributions from the three potentials, we write

$$
\hat{\lambda}_{v}=\lambda_{v}^{\Phi_{c}}+\lambda_{v}^{\Phi_{t}}+\lambda_{v}^{A},
$$

where $\lambda_{v}^{\Phi}$ and $\lambda_{v}^{A}$ are the irrotational and rotational field contributions, respectively. It can be shown that

$$
\lambda_{v}^{\Phi_{t}}=\eta_{c}+\frac{3}{z_{c e}} \sum_{n=0}^{\infty} A_{p n} j_{n}\left(z_{c p}\right)\left[n j_{n-1}\left(z_{c e}\right)-(n+1) j_{n+1}\left(z_{c p}\right)\right] .
$$

The expression for $\hat{\lambda}_{v}^{\Phi_{i}}$ is similar with $\eta_{c}, z_{c p}$, and $A_{p n}$ in the above expression replaced by, respectively, $\eta_{t}, z_{t p}$, and $B_{p n}$. In deriving (66) use has been made of (61) and

$$
\exp \left[-\mathrm{i} \boldsymbol{k}_{c e} \cdot \boldsymbol{r}\right] \nabla_{\boldsymbol{r}} \Phi(\boldsymbol{r})=\nabla_{\boldsymbol{r}}\left\{\exp \left[-\mathrm{i} \boldsymbol{k}_{c e} \cdot \boldsymbol{r}\right] \Phi(\boldsymbol{r})\right\}+\mathrm{i} \boldsymbol{k}_{c e} \exp \left[-\mathrm{i} \boldsymbol{k}_{c e} \cdot \boldsymbol{r}\right] \Phi(\boldsymbol{r}),
$$

with $\boldsymbol{r}=\boldsymbol{x}-\boldsymbol{x}_{1}$. The divergence theorem is used to evaluate the integral of the first term on the right-hand side of (67); the second term on the right-hand side is related to $\eta_{c}$ in (66).

To evaluate the rotational contribution to $\lambda_{t}$ we use the identity

$$
\exp \left[-\mathrm{i} \boldsymbol{k}_{c e} \cdot \boldsymbol{r}\right] \nabla_{\boldsymbol{r}} \times \boldsymbol{A}(\boldsymbol{r})=\nabla_{\boldsymbol{r}} \times\left\{\exp \left[-\mathrm{i} \boldsymbol{k}_{c e} \cdot \mathbf{r}\right] \boldsymbol{A}(\boldsymbol{r})\right\}+\exp \left[-\mathrm{i} \boldsymbol{k}_{c e} \cdot \boldsymbol{r}\right] \mathrm{i} \boldsymbol{k}_{c e} \times \boldsymbol{A}(\boldsymbol{r}) \text {. }
$$

The last term on the right-hand side of the above expression does not contribute to the $x_{1}$-component of the velocity, and the contribution from the first term can be readily evaluated to give

$$
\lambda_{v}^{A}=\frac{3}{z_{c e}} \sum_{n=1}^{\infty} n(n+1) C_{p n} j_{n}\left(z_{s p}\right)\left[j_{n+1}\left(z_{c e}\right)+j_{n-1}\left(z_{c e}\right)\right] .
$$

The result for $\lambda_{v}$ can be used to determine other $\lambda$ coefficients as well. Thus, it can be shown that

$$
\lambda_{\kappa}=\left(b_{c p} / b_{c e}\right) \lambda_{v}^{\Phi_{c}}+\left(b_{t p} / b_{c e}\right) \lambda_{v}^{\Phi_{t}} .
$$

Finally, $\lambda_{d}$, defined by (27), is written as

$$
\lambda_{d}=\lambda_{v}+\lambda_{d}^{\phi_{c}}+\lambda_{d}^{\Phi_{t}}+\lambda_{d}^{A},
$$

where we again made use of (67), with $\Phi$ replaced by $\boldsymbol{u}$. The result for $\lambda_{d}^{\phi_{c}}$ is

$$
\begin{aligned}
i_{d}^{\Phi_{c}}= & \frac{3}{z_{c e}^{2}} \sum_{n=0}^{\infty} A_{p n}\left[\{ z _ { c p } j _ { n } ^ { \prime } ( z _ { c p } ) + ( n + 1 ) j _ { n } ( z _ { c p } ) \} \left\{\left(\frac{(n+1)^{2}}{2 n+3}+\frac{n^{2}}{2 n-1}\right) j_{n}\left(z_{c e}\right)\right.\right. \\
& \left.-\frac{(n+1)(n+2)}{2 n+3} j_{n+2}\left(z_{c e}\right)-\frac{n(n-1)}{2 n-1} j_{n-2}\left(z_{c e}\right)\right\} \\
& \left.+\frac{(n+1)(2 n+1)}{2 n+3} j_{n}\left(z_{c p}\right)\left\{(n+2) j_{n+2}\left(z_{c e}\right)-(n+1) j_{n}\left(z_{c e}\right)\right\}\right] .
\end{aligned}
$$

An expression for $\lambda_{d}^{\Phi_{i}}$ is obtained from $\lambda_{d}^{\Phi_{i}}$ by replacing $A_{p n}$ by $B_{p n}$ and $z_{c p}$ by $z_{t p}$. The contribution from $\boldsymbol{A}$ is given by

$$
\lambda_{d}^{A}=\frac{3}{z_{c e}^{2}} \sum_{n=1}^{\infty}(n+1) C_{p n}\left[-\frac{n(n+2)}{2 n+3} X_{n, n+2}-\frac{n(2 n+1)}{(2 n+3)(2 n-1)} X_{n, n}+\frac{n(n-1)}{2 n-1} X_{n, n-2}\right]
$$




\begin{tabular}{|c|c|c|c|c|}
\hline & Polystyrene & Glass & Water & Glycerol/water \\
\hline Density $\left(\mathrm{g} \mathrm{cm}^{-3}\right)$ & 1.055 & 2.3 & 1.0 & 1.08 \\
\hline Thermal conductivity $\left(\mathrm{J} \mathrm{K}^{-1} \mathrm{~m} \mathrm{~s}\right)$ & $1.15 \times 10^{-1}$ & $9.6 \times 10^{-1}$ & $5.87 \times 10^{-1}$ & $4.5 \times 10^{-1}$ \\
\hline Specific heat $\left(\mathrm{J} \mathrm{g}^{-1} \mathrm{~K}\right)$ & 1.19 & 0.836 & 4.19 & 4.19 \\
\hline Thermal expansion coefficient $\left(\mathrm{K}^{-1}\right)$ & $2.04 \times 10^{-4}$ & $3.2 \times 10^{-6}$ & $2.04 \times 10^{-4}$ & $3.22 \times 10^{-4}$ \\
\hline Sound speed $\left(\mathrm{cm} \mathrm{s}^{-1}\right)$ & $2.3 \times 10^{5}$ & $5.2 \times 10^{5}$ & $1.48 \times 10^{5}$ & $1.6 \times 10^{5}$ \\
\hline Shear viscosity $\left(\mathrm{g} \mathrm{cm}^{-1} \mathrm{~s}^{2}\right)$ & $\ldots$ & - & $1.01 \times 10^{-2}$ & $3 \times 2 \times 10^{-2}$ \\
\hline Bulk viscosity $\left(\mathrm{g} \mathrm{cm}^{-1} \mathrm{~s}^{2}\right)$ & $\ldots$ & - & $3 \times 10^{-2}$ & $9.6 \times 10^{-2}$ \\
\hline Shear rigidity $\left(\mathrm{g} \mathrm{cm}^{-1} \mathrm{~s}^{2}\right)$ & $1.27 \times 10^{10}$ & $2.8 \times 10^{11}$ & - & - \\
\hline
\end{tabular}

TABLE 1 . The values of the physical properties used in the present study.

with the short-hand notation

$$
X_{n, m} \equiv-\frac{3}{2} j_{n}\left(z_{s p}\right) j_{m}\left(z_{c e}\right)-\frac{1}{2}\left[z_{s p} j_{n}^{\prime}\left(z_{s p}\right) j_{m}\left(z_{c e}\right)+z_{c e} j_{n}\left(z_{s p}\right) j_{m}^{\prime}\left(z_{c e}\right)\right] .
$$

The scheme for estimating various effective properties and attenuation is as follows: (i) Assume initially that the effective properties of the suspension are the same as that of pure liquid. (ii) Determine the coefficients $A_{p n}, B_{p n}$, etc. by solving the twelve equations resulting from the application of boundary conditions at $r=a$ and $r=R$ for each mode $n$ up to $n=5$. (iii) Estimate $\lambda_{\rho}, \lambda_{k}, \lambda_{v}$, etc. using the expressions given in this section. (iv) Estimate the effective properties of the suspension. (v) Repeat steps (ii)-(iv) until all the effective properties have converged to within a specified limit. The attenuation of the wave is given by the imaginary part of $k_{c e}$.

\section{Comparison with known analytical results}

We shall assess the effective-medium approximation in two steps. In the first, we consider various limiting situations where we expect some of the effective properties to be dominated by multiparticle interactions in Stokes or Laplace fields for which rigorous results have been obtained in recent years through direct numerical solution of the multiparticle system with hard-sphere spatial configurations. The second step will be to compare the theory with the experimental data available in the literature and some new data generated in our laboratory. This will be done in $\S 5$.

As we have seen the acoustic problem has many variables. This makes it meaningless to present results in terms of one or two non-dimensional numbers. We shall instead choose a particular solid-liquid system and then vary either the radius of the particle or the frequency. The relevant physical properties for glass-water and polystyrenewater systems to be considered in the present study are given in table 1. In some calculations we shall vary the thermal conductivity or density of the particles without varying other physical properties to explore the effect of these properties. In some limiting cases it may be possible to solve a simplified set of equations instead of the $12 \mathrm{~N}$ set of equations required by our scheme. However, since our primary purpose is to assess the effective-medium approximation and the computer program written for this purpose we use the same program in all the comparisons shown here.

The effective viscosity of the suspension will be in general complex with the imaginary part multiplied by frequency being the elasticity of the suspension. Results of rigorous multiparticle computations are available in the literature for the case when inertia is negligible (Stokes flow) and a uniform strain rate is applied to suspensions of rigid particles in an incompressible, Newtonian fluid. The spatial distribution 


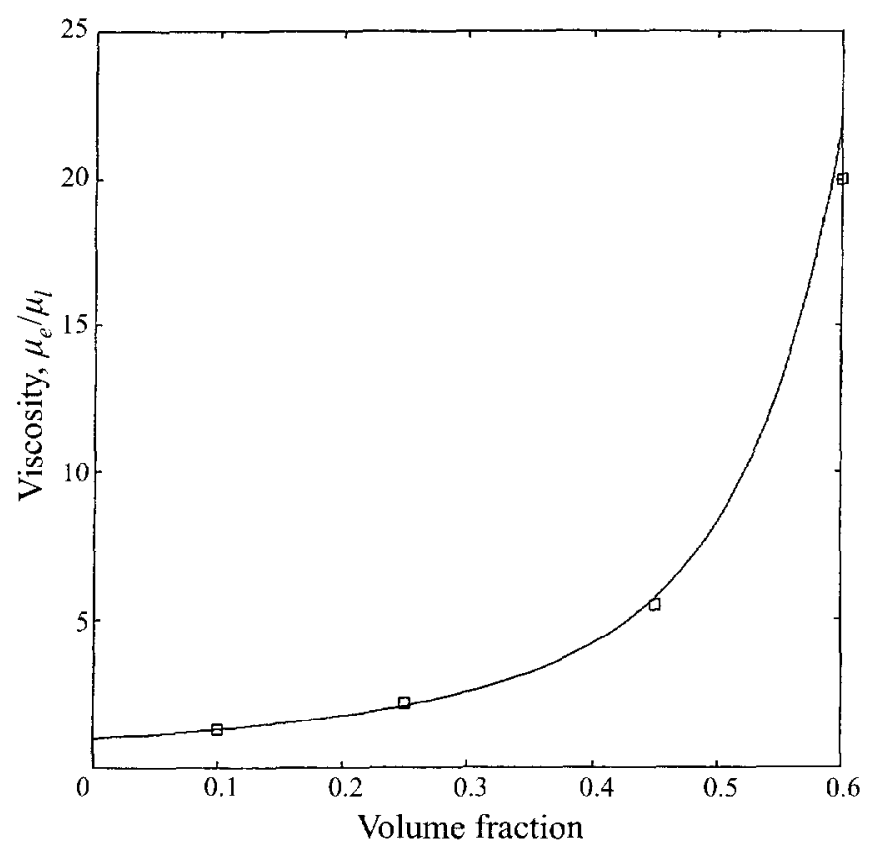

FIGURE 1. Ratio of effective viscosity to liquid viscosity as a function of volume fraction. Limiting values for wavelengths large compared to particle size and viscous boundary layers much larger than the particles. Solid line is theory; squares are numerical simulation results from Sangani \& Mo (1997).

of the particles corresponded to the hard-sphere molecular systems for which $S(\mathbf{0})$ given by (55) applies. Ladd (1990) obtained the results with volume fractions $(\phi)$ in the range 0-0.45. Mo \& Sangani (1994) and Sangani \& Mo (1997) repeated and confirmed his results and also obtained an additional result for $\phi=0.6$. Their results are shown in figure 1 . To see how well the effective-medium model developed in the present study approximates these values we must pick frequencies for which the quasi-steady Stokes flow approximation will be expected. The ratio of unsteady to viscous terms in the momentum equation for the liquid is $\rho_{l} \omega a^{2} / \mu_{l}$. For $a=10^{-5} \mathrm{~cm}$ and $f=\omega / 2 \pi=10^{6} \mathrm{~Hz}$ this number equals $6 \times 10^{-3}$ (we have taken water as the suspending liquid but multiplied the viscosity by 10). The wave nature of the governing equations depends on the ratio $k_{c l} a$ which equals $2 \pi$ times the ratio of particle radius to the wavelength in pure liquid. When this number is small the liquid may be treated as essentially incompressible. For $a$ and $f$ listed above, $k_{c l} a$ for water equals $4.2 \times 10^{-4}$. Finally, our calculations account for small deformations of the particles. For particles to be treated as rigid, their shear modulus $\tilde{\mu}$ divided by the frequency must be much larger than the viscosity of the water. At $\omega=10^{6} / 2 \pi \mathrm{s}^{-1}$, the ratio $\tilde{\mu} /\left(\omega \mu_{l}\right)$ equals $2 \times 10^{7}$ and therefore the glass particles may be treated as rigid.

The solid curve in figure 1 represents the estimates of the effective viscosity obtained by the effective medium model for the aforementioned conditions. The ratio $\operatorname{Re}\left(\mu_{e}\right) / \mu_{l}$ varies from unity to about 20 as $\phi$ is varied from 0 to 0.6 . At high volume fractions significant viscous dissipation occurs in the narrow gap regions between the pairs of particles in close proximity and this dominates the effective viscosity behaviour at high $\phi$. This phenomenon cannot be expected to be modelled accurately by the 


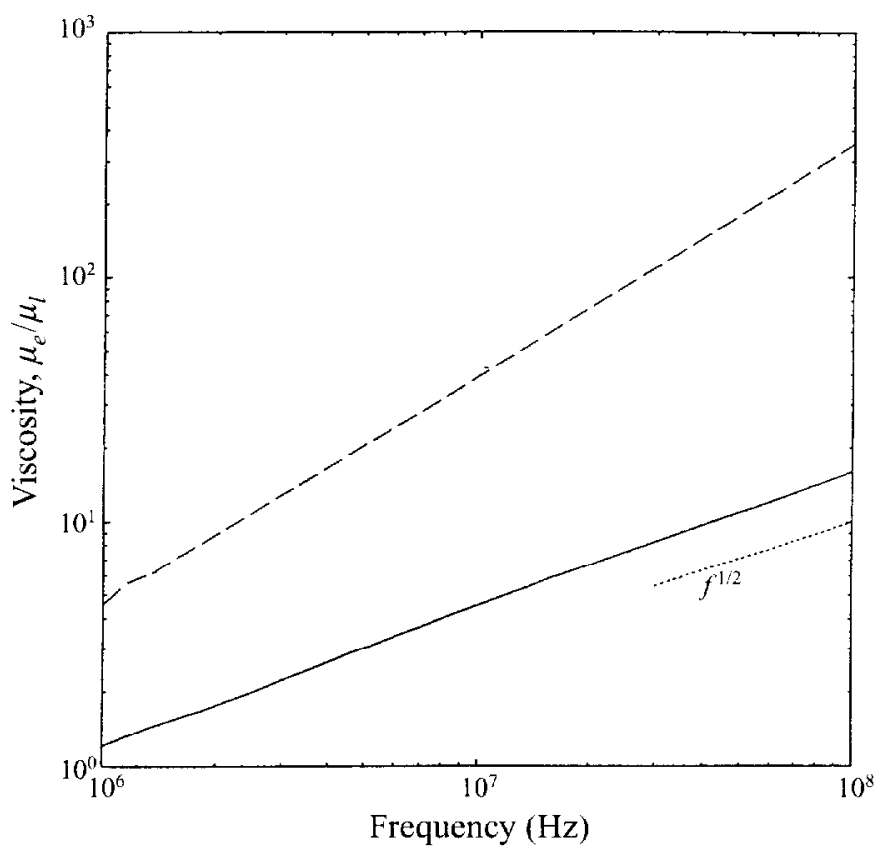

Figure 2. Real part minus its limiting value at large wavelengths $(-)$ and minus imaginary part $\left.(-)^{-}\right)$of the ratio of effective viscosity to liquid viscosity as a function of frequency. Particle volume fraction is 0.3 . The viscous boundary layers at the lowest frequencies are small compared to the particle size (at $1 \mathrm{MHz},\left(\mu_{l} /\left(\rho_{l}\left(\omega^{2} a^{2}\right)\right)^{1 / 2}=0.01\right)$.

single-particle approximation used here and therefore the excellent agreement found here for $\phi=0.45$ and 0.6 may be regarded as fortuitous. It should also be noted that the particles in highly concentrated suspensions may begin to be supported by the other particles through the formation of a continuous network such that the suspension behaves like a fluid-filled porous medium. The present analysis should not be applied to such suspensions.

Figure 2 shows that the real and imaginary parts of the effective viscosity increase with frequency in the range where the unsteady term begins to become comparable to the viscous term in the liquid momentum equation. The results for the real part of the effective viscosity may be rationalized as follows. At relatively large frequencies we expect the viscous effects to be confined to small Stokes layers of thickness $\delta=O\left(\left(\mu_{l} / \rho_{l} \omega\right)^{1 / 2}\right)$ surrounding each particle. The effective viscosity is the rate of energy dissipation per unit volume of the suspension divided by the square of mean velocity gradient $\dot{\gamma}=O\left(k_{c e}\langle\boldsymbol{u}\rangle\right)$. At high frequencies the dominant contribution to dissipation arises from the Stokes layers whose volume per unit suspension volume is $O\left(\delta a^{2} n\right), n$ being the number density of particles, and the velocity gradient in these layers is $O(\dot{\gamma} a / \delta)$. The effective viscosity must therefore roughly scale as $a / \delta$ or $a\left(\omega \rho_{l} / \mu_{l}\right)^{1 / 2}$ for frequencies at which $\delta$ is small compared with $a$. The observation that the real part of effective viscosity should increase with frequency as $\omega^{1 / 2}$ is consistent with the results of figure 2. The ratio $\left(\mu_{l} / \rho_{l}\left(\omega a^{2}\right)^{1 / 2}\right.$ is about 0.01 for $f=1 \mathrm{MHz}$ indicating that indeed the Stokes layers are thin at these frequencies (note that we have replaced $\mu_{l}$ by its value for water divided by 1000 ).

The imaginary part of the effective viscosity is also seen to increase with increasing 
frequency in figure 2. This elastic nature of the suspension is expected at higher frequencics.

We now compare the effective-medium results for $\lambda_{v}$ with the known results. Recall that $\lambda_{v}$ represents the ratio of the velocity amplitude in the particle phase to that in the suspension. At lower frequencies for which $k_{c e} a$ is small and the scattering losses are small, the attenuation will be dominated by the imaginary part of $\lambda_{v}$ as suggested by Sangani et al. (1991) who evaluated the real and imaginary parts of $\lambda_{v}$ for a special case when the frequency is large enough for the Stokes layers to be small compared with the particle radius but small enough for $k_{c a} a$ to be small, i.e. for the suspension to be essentially incompressible. We shall compare the results of effective-medium approximation with their results next.

Sangani et al. gave their results in terms of added mass, Basset, and viscous drag coefficients. The force balance on a particle in the suspension was written as

$$
\langle\boldsymbol{F}(t)\rangle=\rho_{l} \mathscr{V}\langle\dot{\boldsymbol{u}}\rangle+\frac{1}{2} \rho_{l} \mathscr{V} C_{a}\langle\dot{\boldsymbol{u}}-\dot{\boldsymbol{v}}\rangle+6 a^{2} \sqrt{\pi \rho_{l} \mu_{l}} C_{b} \int_{-\infty}^{t}\langle\boldsymbol{u}-\dot{\boldsymbol{v}}\rangle(\tau) \frac{\mathrm{d} \tau}{\sqrt{t-\tau}}+6 \pi a \mu_{l} C_{d}\langle\boldsymbol{u}-\boldsymbol{v}\rangle
$$

where $\boldsymbol{F}$ is the force on the particle, $\mathbf{v}$ is the velocity of the particle, $C_{a}, C_{b}$ and $C_{d}$ are the added mass, Basset and viscous drag coefficients and $\mathscr{T}$ the volume of a particle. Dots above variables denote time derivatives. Noting that $\boldsymbol{F}(t)=\rho_{p} \mathscr{V} \boldsymbol{v}$ and $\langle\boldsymbol{v}\rangle=\hat{\lambda}_{v}\langle\boldsymbol{u}\rangle$, and taking the time-dependence of variables to be $\mathrm{e}^{-\boldsymbol{i} \cdot \boldsymbol{m}}$, the force balance (75) gives

$$
C_{a}+9 \Omega C_{d}+9 \Omega^{2} C_{d}=\frac{2\left(\rho^{*} \hat{\lambda}_{v}-1\right)}{1-\lambda_{v}}
$$

with $\Omega \equiv\left(i \mu_{l} /\left(\rho_{l} \omega a^{2}\right)^{1 / 2}\right.$ and $\rho^{*} \equiv \rho_{p} / \rho_{l}$. Sangani et al.'s analysis is valid when the magnitude of $\Omega$ is small compared with unity, and the terms of $O\left(\Omega^{3}\right)$ or smaller are neglected in (76). For small $\Omega, \lambda_{v}$ can be expanded in a series $\lambda_{v}=\lambda_{v}^{(0)}+\Omega \lambda_{v}^{(1)}+\cdots$ to yield the relations

$$
C_{a}=\frac{2\left(\rho^{*} \lambda_{v^{\prime}}^{(0)}-1\right)}{1-\lambda_{v}^{(0)}}, \quad C_{b}=\frac{2 \lambda_{t}^{(1)}\left(\rho^{*}-1\right)}{9\left(1-\lambda_{i}^{(0)}\right)^{2}} .
$$

The coefficients $\lambda_{v}^{(0)}$ and $\lambda_{v}^{(1)}$ were evaluated from the effective-medium theory results for $\lambda_{v}$ at small $k_{c l} a$ and small $|\Omega|$ by extrapolating to $\Omega=0$ and numerically differentiating the results with respect to $\Omega$, respectively. Figures 3 and 4 show a comparison with the rigorous multiparticle calculations of Sangani et al. who determined $C_{a}, C_{b}$, and $C_{d}$ as a function of $\phi$ and $\rho^{*}$ for periodic as well as random arrays of spheres. The results for $C_{a}$ for the body-centred cubic and random arrays were very close to each other while that for the simple cubic arrays differed by about $12 \%$ at $\phi=0.5$. It was also found that the dependence on $\rho^{*}$ was rather weak, typically variations within $5 \%$ occurred as $\rho^{*}$ was varied from zero to infinity. The results of Sangani et al. shown in figures 3 and 4 correspond to $\rho^{*}=0$ while the effective-medium results correspond to glass particles in water with $\rho^{*}=2.55$. We see an excellent agreement between the added mass coefficient obtained by the effective-medium approximation and for random or body-centred cubic arrays. The effective-medium approximation for $C_{b}$ deviates systematically from the random arrays result with the maximum deviation of about $20 \%$ at $\phi=0.5$. The theory in this case is much closer to the results for the simple cubic arrays.

Next we consider the case when $\Omega$ is very large, i.e. frequencies at which the 


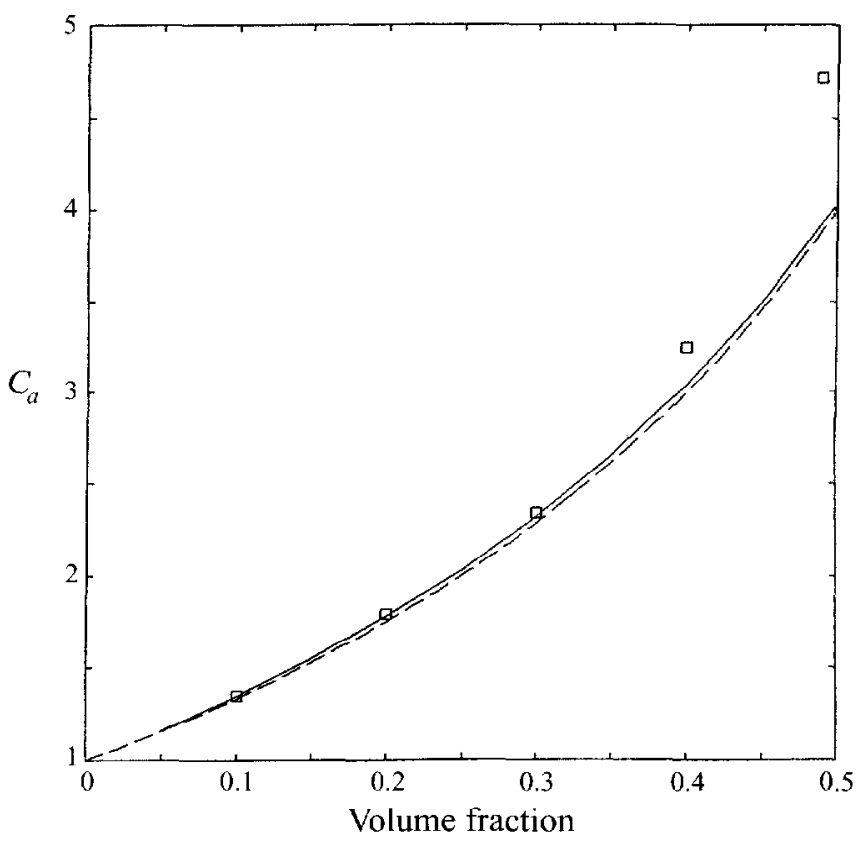

FIGURE 3. Added mass coefficient $C_{a}$ as a function of volume fraction. Solid line is the theoretical result for wavelengths and viscous boundary layers much larger than particle size. Broken line and squares are the random array and simple cubic array results of Sangani et al. (1991). Particle to liquid density ratio is 2.55 .

viscous drag coefficient makes the leading contribution to $\lambda_{v}$. The results in this case can be compared with the results of multiparticlc Stokes flow calculations by Ladd (1990) and Mo \& Sangani (1994). Two kinds of results are available in the Stokes flow literaturc. The first is the hindrance factor in sedimentation in which the average velocity of the particles is determined for the case when the forces acting on all the particles are the same. The second is the calculation of the permeability of a fixed bed of particles. There the average force on the particles is calculated for particles that all have the same (zero) velocity, different from the mean velocity of the suspension. Neither situation applies to oscillatory flows but one expects that the results for the fixed bed resistivity would be most applicable for large $\rho^{*}$ and those of the hindrance factor for very small $\rho^{*}$. Figure 5 compares the results of Ladd and Mo \& Sangani for these two quantities with the results obtained using the effective-medium approximation with $\rho^{*}=10$. These results were obtained with $|\Omega|=22$ and $k_{c} a=0.001$. The results for the sedimentation-hindrance factor were obtained only up to $\phi=0.45$ in the present investigation while Mo \& Sangani had obtained an additional value for the fixed bed resistivity at $\phi=0.6$. Their result for $\phi=0.6$ was in excellent agreement with the well-known Carman-Kozney correlation. We see that at least up to $\phi=0.45$, the hindrance factor and the fixed-bed resistivity are not too different from each other, and that the effective-medium results are in excellent agreement for the entire range of $\phi$.

Next, we compare the results for the effective conductivity. When $k_{c \mid} a$ is small the viscous and thermal effects contribute most to the total attenuation. When the density ratio is close to unity the translational oscillations and hence viscous attenuation are small and the thermal effects become the primary source of attenuation. The effective 


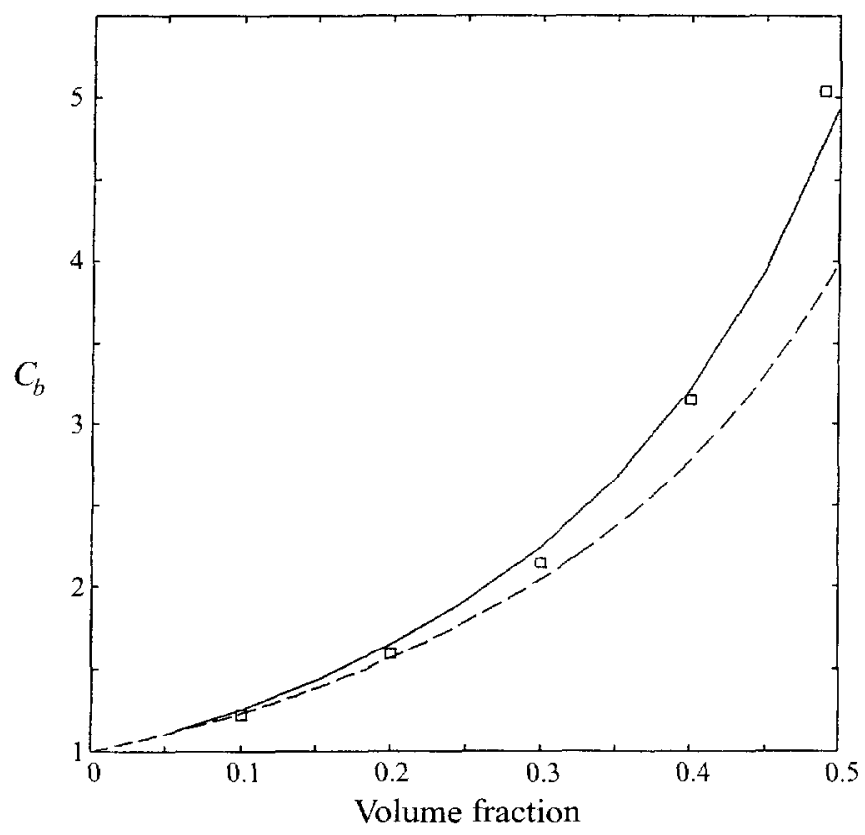

FigurE 4. Basset coefficient $C_{b}$ as a function of volume fraction. Solid line is the theoretical result for wavelengths and viscous boundary layers much larger than particle size. Broken line and squares are the random array and simple cubic array results of Sangani et al. (1991). Particle to liquid density ratio is 2.55 .

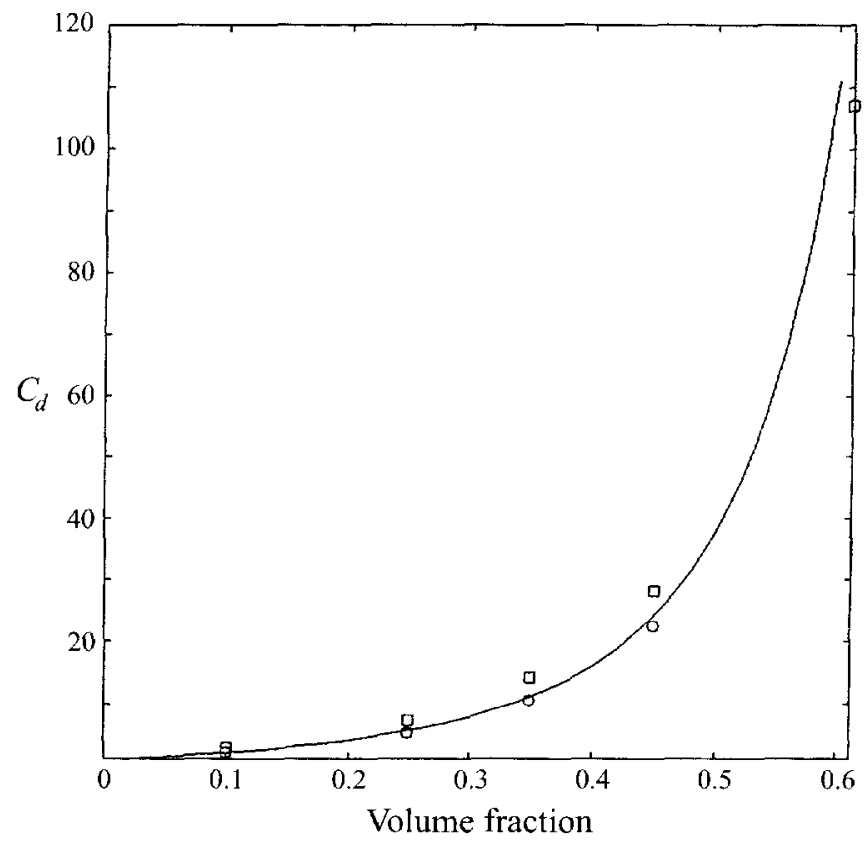

FIGURE 5. $C_{d}$ as a function of volume fraction. Lines are theoretical results obtained for wavelengths much larger and viscous boundary layers much smaller than particle size. Squares are numerical simulation results for the fixed-bed resistivity by Mo \& Sangani (1994), circles are numerical simulation results for the hindrance factor by Ladd (1990). 


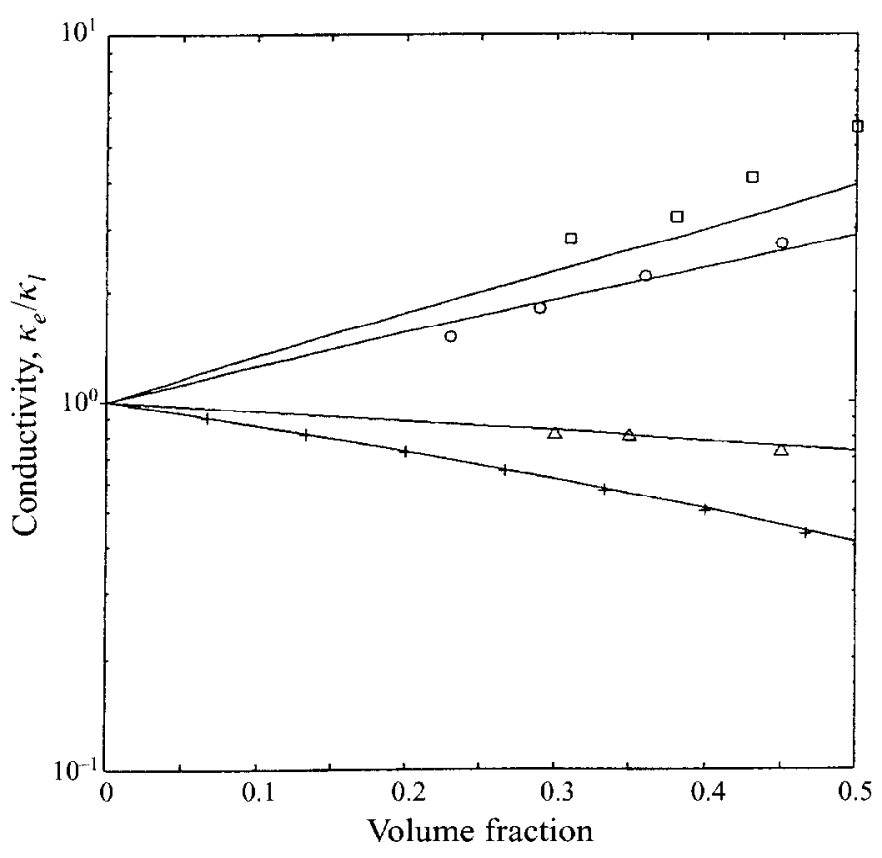

FIGURE 6. Ratio of effective conductivity to liquid conductivity as a function of volume fraction. Lines are theory for wavelengths large compared to particle size, symbols are experimental data from Turner (1976). Results are shown for $\kappa_{p} / \kappa_{l}=0.01(+), 0.51(\triangle), 10.8(\bigcirc)$ and $160(\square)$.

conductivity as a function of $\phi$ and the conductivity ratio $\kappa_{p} / \kappa_{l}$ was determined experimentally by Turner (1976) who used liquid fluidized beds of nearly monodisperse spheres. Sangani \& Yao (1988) and Bonnecaze \& Brady (1991) have carried out multiparticle calculations for the same cases and found generally good agreement between the simulations results and the experimental data of Turner. Figure 6 shows the comparison between the effective-medium approximation and the data of Turner. Calculations were made with the polystyrene-water system with $f=1 \mathrm{MHz}$ for which $k_{c} a$ equals $4.2 \times 10^{-4}$ and the ratio of unsteady term to the steady conduction term $\rho_{l} C_{p, l} \omega a^{2} / k_{l}$ equals 0.05 . The thermal conductivity of the particles was varied keeping other parameters fixed to determine the effect of conductivity ratio. Agreement is generally very good except for the highest particle-to-liquid conductivity ratio of 160 and $\phi=0.5$ for which the effective-medium approximation underpredicts the effective conductivity by about $30 \%$. At such high conductivity ratios the narrow gap regions between pairs of particles in dense suspensions contribute siginificantly to the overall heat flux and this is not captured accurately by the effective-medium approximation. The spatial distribution of the particles could also affect significantly the results at high $\phi$. For low-conductivity particles we see an excellent agreement between the experiments and the effective-medium approximation. It may be noted that the well-known Maxwell relation

$$
\frac{\kappa_{e}}{\kappa_{l}}=\frac{1+2 \alpha \phi}{1-\alpha \phi}
$$

with $\alpha=\left(\kappa_{p}-\kappa_{l}\right) /\left(\kappa_{p}+2 \kappa_{l}\right)$ also gives accurate estimates of the effective conductivity for $\kappa_{p} / \kappa_{l}-0$.

Figure 7 shows the results for the real and imaginary parts of the effective con- 


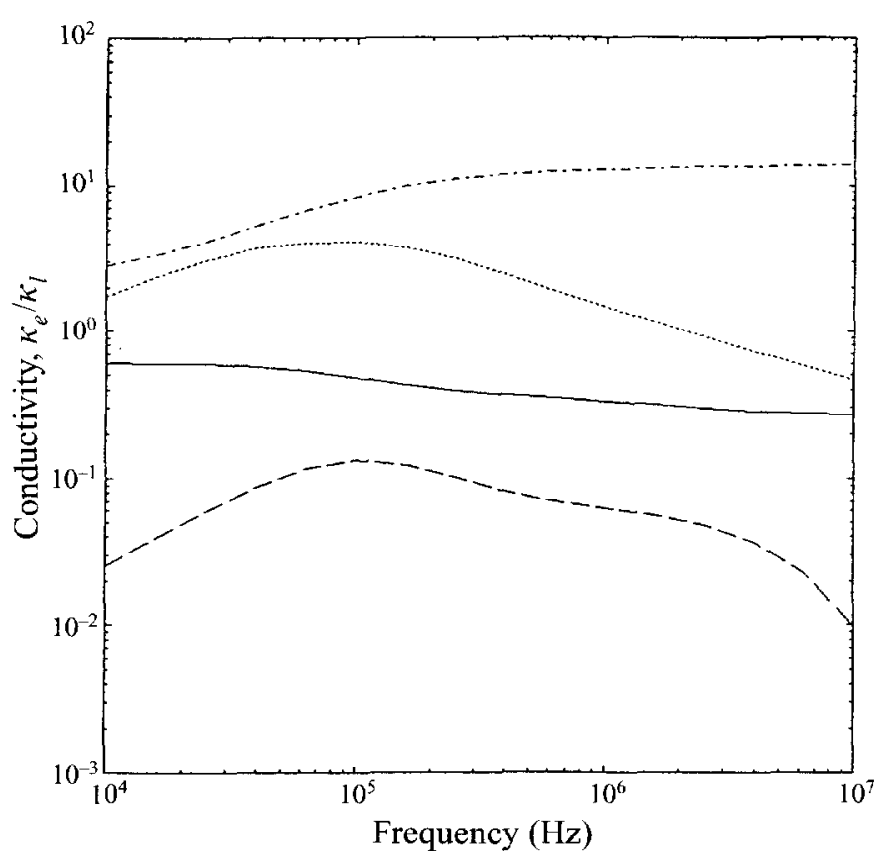

Figure 7. Real and imaginary part of the ratio of effective conductivity to liquid conductivity as functions of frequency. Particle volume fraction is $0.3 ; k_{c l} a=4 \times 10^{-2}$ at $10 \mathrm{MHz}$ for all cases. Solid line (real part) and dashed line (imaginary part), $\kappa_{p} / \kappa_{l}=2 \times 10^{-3}$; dashed-dotted line (real part) and dotted line (imaginary part), $\kappa_{p} / \kappa_{t}=20$.

ductivity as a function of frequency for two particle-to-liquid conductivity ratios. For $\kappa_{p} / \kappa_{l}>1$ the real part of the conductivity is seen to increase with the frequency. This result is similar to the one discussed for the effective viscosity (cf. figure 2). The opposite is true for the particles whose conductivity is smaller than the fluid conducitivity. The imaginary part of the conductivity is seen to reach a maximum at frequencies for which the thermal layer thickness is comparable to particle radius.

In summary, we have shown in this section that the effective-medium approximation yields very accurate estimates of the coefficients $\lambda_{d}$ (effective viscosity), $\lambda_{v}$ (added mass, Basset force, and viscous drag), and $\lambda_{\kappa}$ (conductivity) for the monodisperse, random suspensions in the limits in which the results of exact multiparticle interactions are available.

The two coefficients for which no exact results are available are $\lambda_{\rho}$ and $\lambda_{T}$ but the computed results for these coefficients show expected trends. For example, figure 8 shows results for the real and imaginary parts of $\hat{\lambda}_{T}$ which represents the ratio of average temperature amplitude inside the particles to that in the suspension. The results are shown for polystyrene-water mixture with $a=0.11 \mu \mathrm{m}$, a system which was studied by Allegra \& Hawley (1972). When the thermal diffusion length, $\left(\kappa_{p} / \omega \rho_{p} C_{v, p}\right)^{1 / 2}$, becomes much larger than the particle radius, the temperature inside the particle will be the same as the suspension temperature and $\lambda_{T}$ will approach unity. This is the situation for frequencies less than $1 \mathrm{MHz}$. At frequencies that are large enough so that the thermal layer inside the particles is thin compared with the radius but small enough to keep the wavelength large compared with the radius, we expect the particle temperature amplitude to be governed by the temparature variations in the adiabatic case. A simple calculation shows that in this limit $\lambda_{T}$ and 


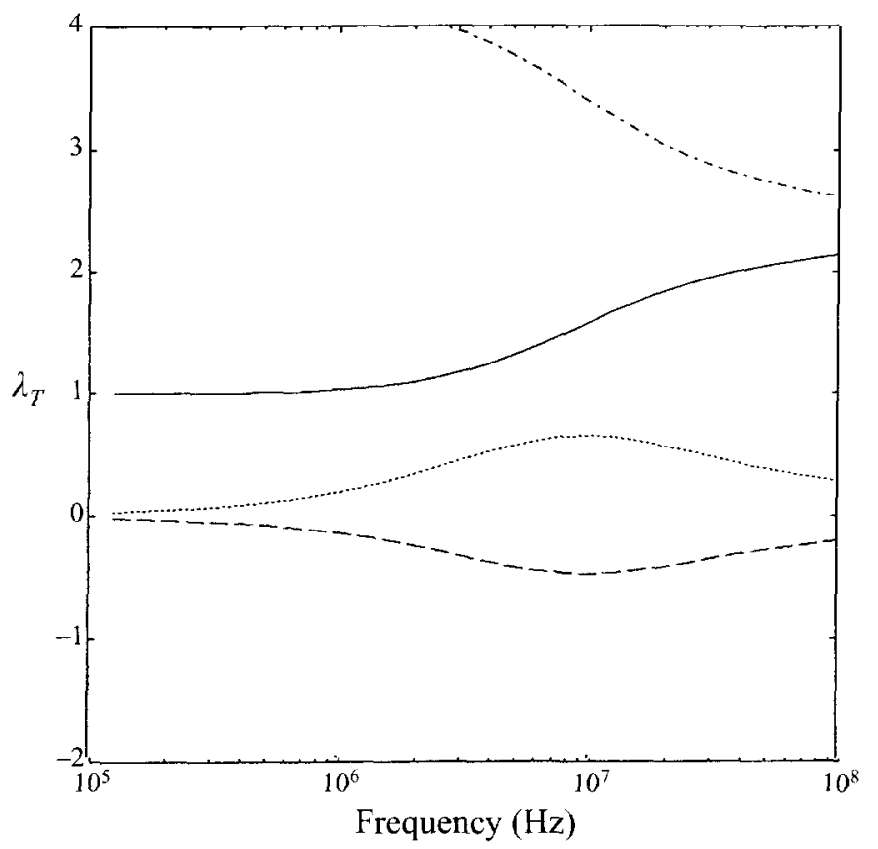

FIGLRE 8. Real and imaginary parts of $\lambda_{T}$ as a function of frequency for polystyrene particles $(0.11 \mu \mathrm{m}$ radius) in water at 0.3 volume fraction. Solid line (real part) and dashed line (imaginary part) are the full results; the dashed-dotted line (real part) and the dotted line (imaginary part) are the adiabatic result (79).

$\lambda_{\rho}$ are related by

$$
\lambda_{T}=\frac{\left(\gamma_{p}-1\right) \lambda_{\rho} k_{c e}^{2}}{i \omega b_{c e} \beta_{p}} .
$$

The dot-and-dashed curve in figure 8 is obtained by first computing $\lambda_{\rho}$ using the effective-medium approximation and then using (79) to estimate $\lambda_{T}$. We see that at high frequencies the result for $\lambda_{T}$ obtained in this manner approaches that obtained from the direct evaluation using the effective-medium approximation. The imaginary part of $\lambda_{T}$ is seen to vanish in the limits of high and low frequencies as one approaches, respectively, the adiabatic and isothermal limits.

All the results discussed in this section correspond to the limit of small $k_{c l} a$ for which the scattering losses are insignificant. Since all indications suggest that the effective-medium approximation is very accurate, we expect the theory to predict the thermal and viscous attenuations for small $k_{c e} a$ very accurately. Rigorous calculations are not available for $k_{c^{\prime}} a=O(1)$ and we shall mostly depend on the experimental data to assess the effective-medium theory in this regime.

\section{Experimental set-up}

The experimental set-up for measuring attenuation is shown in figure 9. The suspension is hand-stirred in a vessel with transmitting and receiving transducers mounted flush with the inner walls. The distance between the transducers in a typical vessel was $5 \mathrm{~cm}$, the width and the height of the vessel being 8 and $13 \mathrm{~cm}$, respectively. In dense suspensions for which greater attenuation is expected, the experiments were carried out with smaller vessels with the acoustic path lengths as small as $1.3 \mathrm{~cm}$. 


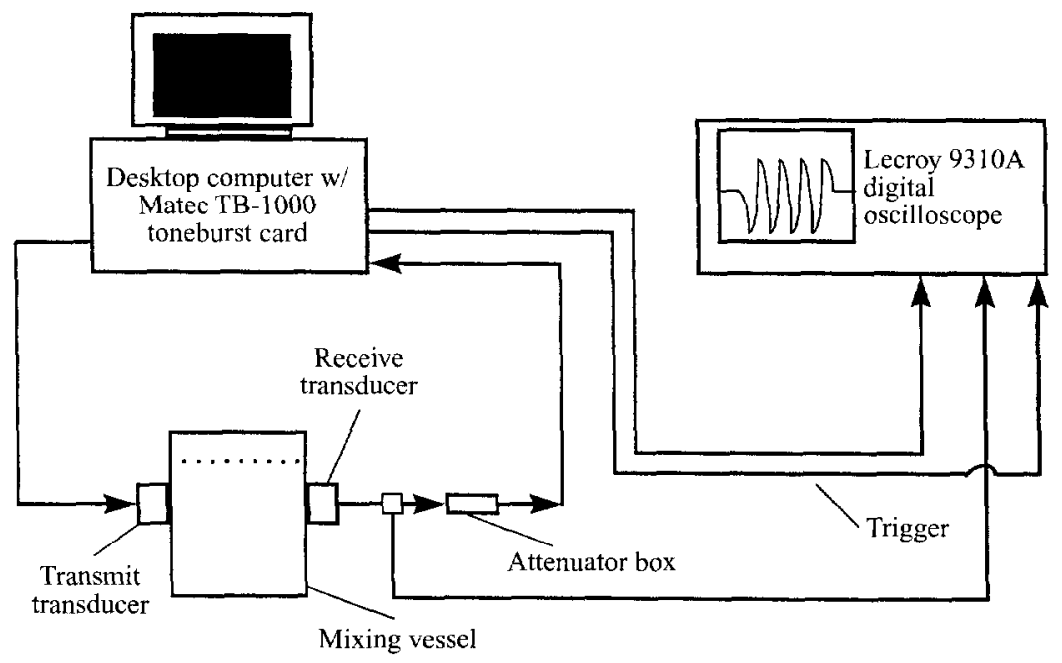

FIGURE 9. The schematic of the experimental set-up.

The transducers were of piezoelectric videoscan immersion type manufactured by Panametrics Inc. To cover a relatively broad range of frequencies, we used transducers with centre frequencics of $1.0,2.25,5.0,7.5$ and $10.0 \mathrm{MHz}$. The first two were $1.3 \mathrm{~cm}$ in radius while the other two were $1 \mathrm{~cm}$ in radius.

A Matec TB-1000 digital synthesizer card installed in a desk-top computer was used to generate monochromatic tonebursts that propagated through the suspension and were received by the receiving transducer. The signal was then sent to a LeCroy Model 9310A digital oscilloscope where its amplitude was measured. Attenuation data were obtained for six to eight frequencies for each transducer pair. Thus, the measurements were typically carried out at frequencies between 1 and $12 \mathrm{MHz}$. The suspension was hand-stirred before each measurement. To calculate the excess attenuation caused by the presence of particles, we also measured the amplitude of the signal received by the transducer for the pure liquid case. The excess attenuation for a given particle concentration is then determined using

$$
\alpha=-\frac{1}{L} \log \left(\frac{V_{\text {mix }}}{V_{l}}\right),
$$

where $V_{\text {mix }}$ and $V_{l}$ are the voltage amplitudes of the received signals in the mixture and pure liquid, respectively, and $L$ is the distance between the transducers.

Further details about the experimental set-up can be found in Norato (1999).

\section{Comparison with experiments}

Several experimental results have been presented for dense slurries in the literature. In this section we shall compare with these data as well as with results obtained in our laboratory. Allegra \& Hawley (1972) measured attenuation for nearly monodisperse polystyrene particles of radius $0.11 \mu \mathrm{m}$ in water. The acoustic frequency range used by these investigators was roughly $5-50 \mathrm{MHz}$. This corresponds to the non-dimensional wavenumbers $k_{c l} a$ in the range of $0.002-0.02$. At such small wavenumbers the scattering losses are negligible, and since the density of polystyrene $\left(1.07 \mathrm{~g} \mathrm{~cm}^{-3}\right)$ is close to that of water, the translational oscillations of the particles and henec the vis- 
cous attenuation are negligible. Thus, the thermal effects dominate the attenuation in Allegra \& Hawley's experiments. The difference in thermal expansion coefficients between the particles and the suspending liquid causes the temperature amplitude inside the particles to differ from that in the liquid. This causes a heat flux through the surface of the particles that is out of phase with the sound wave and leads to thermal attenuation.

Allegra \& Hawley showed that when the thermal boundary layers as well as the wavelength are much greater than the particle radius and the suspension is dilute (i.e. when $\kappa_{l} /\left(\rho_{l} C_{p, l} \omega a^{2}\right) \gg 1, k_{c \mid} a \ll 1$, and $\left.\phi \ll 1\right)$, the attenuation is given by

$$
\alpha=\frac{1}{6} \phi \omega^{2} a^{2} c_{l} T \rho_{l} \rho_{p}^{2} C_{p, p}^{2}\left(\begin{array}{cc}
\beta_{l} & \beta_{p} \\
\rho_{l} C_{p, l} & \rho_{p} C_{p, p}
\end{array}\right)^{2} 1\left[\begin{array}{l}
1 \\
\kappa_{p}
\end{array}+\begin{array}{c}
\kappa_{p} \\
\kappa_{l}
\end{array}\right] .
$$

The attenuation increases as $f^{2}$ in this limit. On the other hand, when the boundary layers are much smaller than the particles while $k_{c t} a$ is still small, their analysis predicts that the attenuation will increase with frequency as $f^{1 / 2}$.

Allegra \& Hawley (1972) compared their data with a theory for dilute suspensions and found good agreement between the two for dilute suspensions. Since the effectivemedium theory reduces to their theory for dilute suspensions as $\phi \rightarrow 0$, we also expect a very good agreement at small volume fractions. Allegra \& Hawley compared the two in several of their figures but did not specify the volume fraction of the particles used in obtaining the data except for one in which they show the attenuation as a function of $\phi$ at several frequencies. We show their data for the lowest volume fraction, $\phi=0.058$, in figure 10 . The asymptotic cxpression (81) is also shown in the figure; it is seen that the experiments were carried out at frequencies for which the thermal laycrs arc comparable to particle radius.

As noted by Allegra \& Hawley, the attenuation is sensitive to the thermal properties of polystyrenc particles. If we take these properties to be the same as given by these investigators and reported in table 1 , we find that the predicted attenuation is slightly greater than the experimental values as indicated by the solid line in figure 10 . However, there is some uncertainty about the values of the physical properties as given by Allegra \& Hawley. In their paper they show that their results depend quite strongly on the equilibrium temperature - because the physical properties do - and that there is a significant discrepancy between the theory and experiments in this temperature dependence. Especially, the attenuation at temperatures $\leqslant 20^{\circ} \mathrm{C}$ is overpredicted. Allegra \& Hawley mentioned that the factor $\beta_{p} /\left(\rho_{p} C_{p, p}\right.$ ) (cf. (81)) introduces the uncertainty. To be able to have a fair comparison between the dense slurry data and the effective-medium theory we have therefore changed the value of $\beta$ for polystyrene somewhat (decreased by $11 \%$ ) to get the best fit at low volume fractions, which is seen to be excellent.

Figure 11 compares the effective-medium approximations with the attenuation data as a function of volume fraction of the particles at different frequencies. We see an excellent agreement at all volume fractions. (Slight differences seen are within the error introduced in reading the data from Allegra \& Hawley's figures or due to small temperature variations that could occur during the experiments.) Note that simply using the dilute theory of Allegra \& Hawley (1972) would have overpredicted the attenuations at 0.5 volume fraction by as much as $50 \%$.

The scattering attenuation was small in the experiments by Allegra \& Hawley (1972) since $k_{c^{\prime}} a$ for their experiments was much less than unity. To extend the range of $k_{c l} a$ over which the theory can be tested against experiments we have conducted 


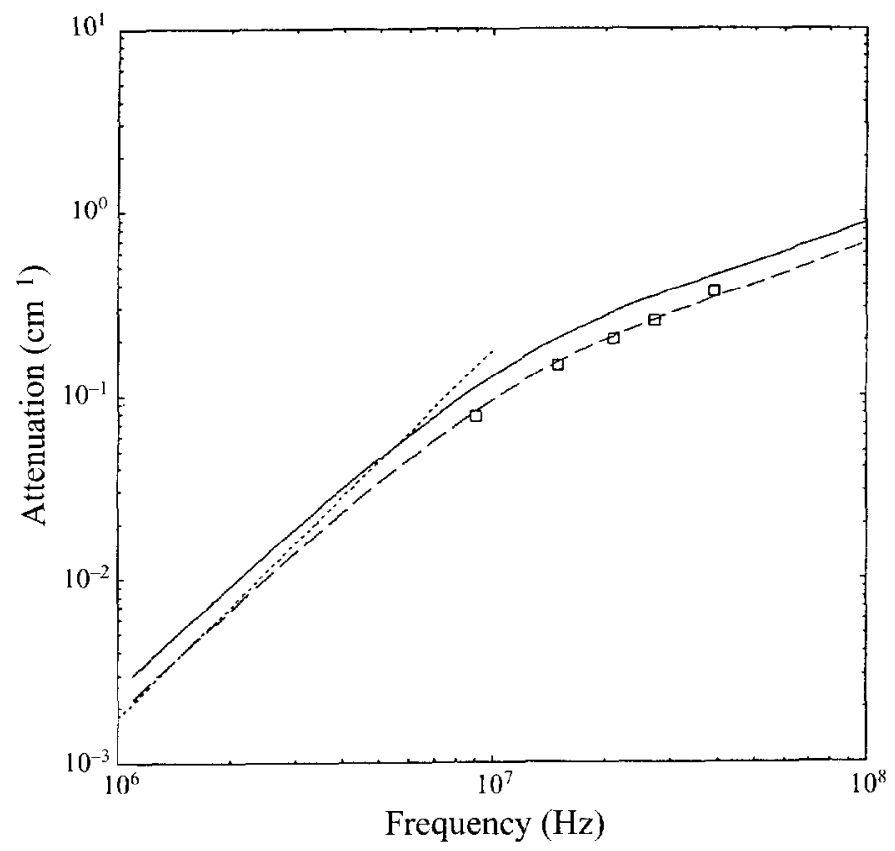

FIGLRE 10. Comparison with experimental dilute-slurry results by Allegra \& Hawley (1972) for the attenuation in a mixture of polystyrene particles of $0.11 \mu \mathrm{m}$ radius in water at 0.05 volume fraction. Squares are experiments, solid line is the theoretical result. The broken line is the theoretical result when the thermal expansion coefficient is changed from $2.04 \times 10^{-4}$ to $1.82 \times 10^{-4} \mathrm{~K}^{-1}$. The dotted line is the asymptotic result (81).

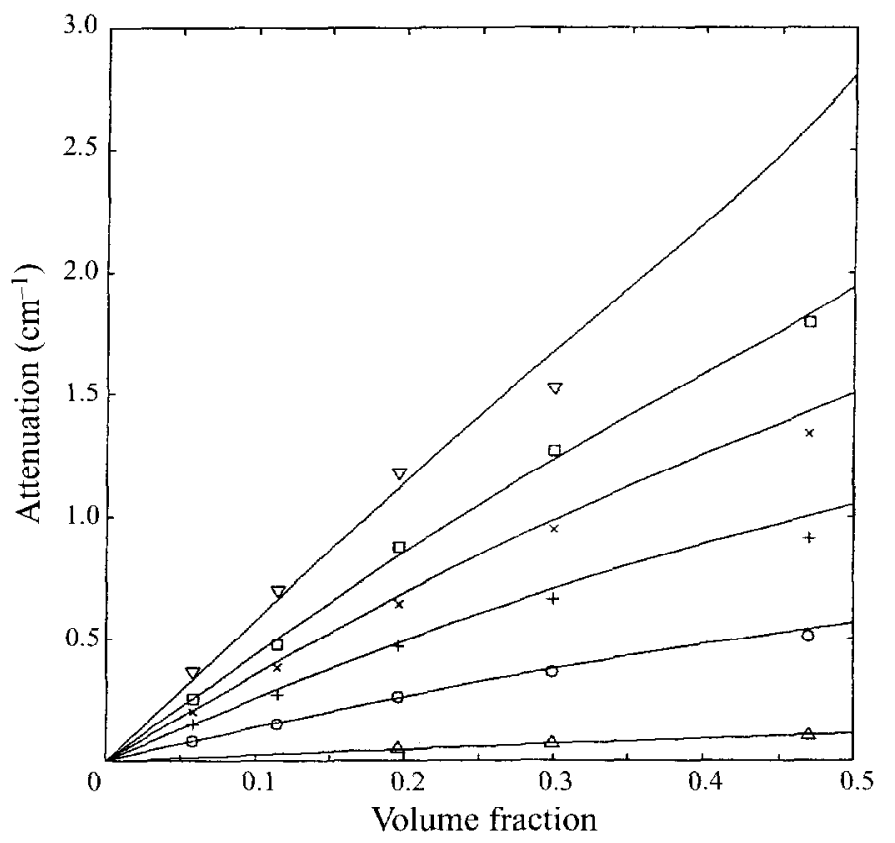

FIGURE 11. Comparison with experimental dense-slurry results by Allegra \& Hawley (1972) for the attenuation for polystyrene particles of $0.11 \mu \mathrm{m}$ at different frequencies. $\triangle, 3 \mathrm{MHz} ; \mathrm{O}, 9 \mathrm{MHz}$; ,$+ 15 \mathrm{MHz} ; \times, 21 \mathrm{MHz} ; \square, 27 \mathrm{MHz}$ and $\nabla, 39 \mathrm{MHz}$. 


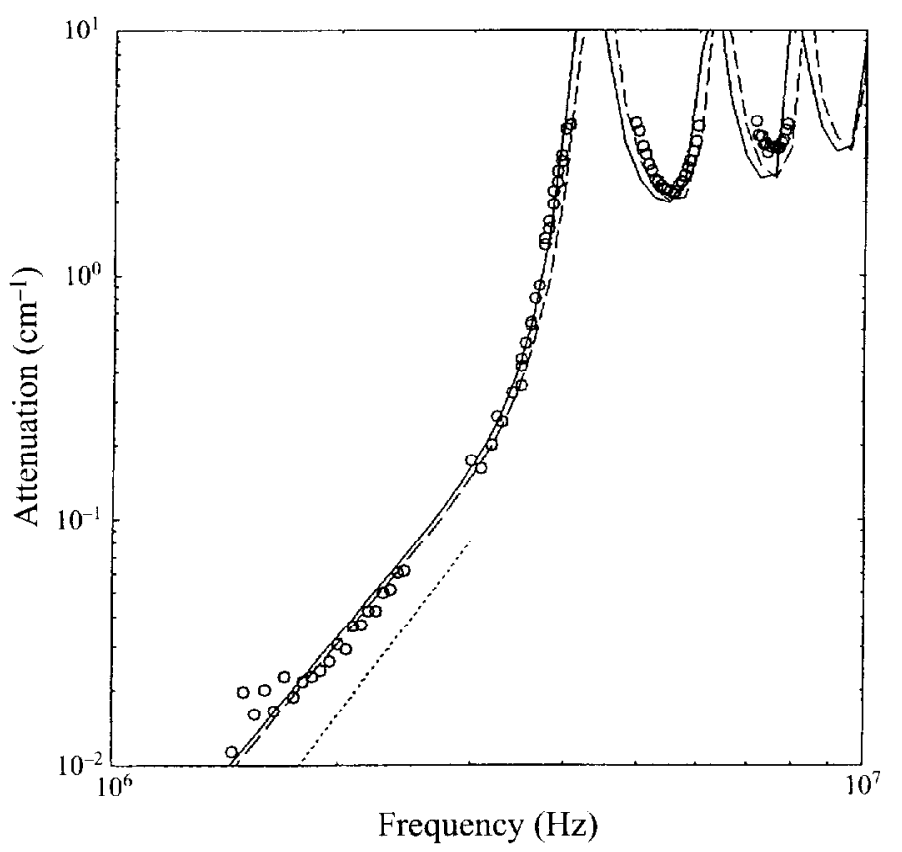

FIGURE 12. Experimental and theoretical results for the attenuation in a mixture of polystyrene particles (mean radius $79 \pm 3 \mu \mathrm{m}$ and $1.8 \mu \mathrm{m}$ standard deviation) in water at 0.05 volume fraction. Circles are experiments, solid and broken lines are the theory for monodisperse particles of $79 \mu \mathrm{m}$ and $77 \mu \mathrm{m}$ radius, respectively.

experiments for much larger polystyrene particles. The particles were specified by the manufacturer to have a mean radius of $79 \pm 3 \mu \mathrm{m}$ with a standard deviation of $1.8 \mu \mathrm{m} ; k_{c l} a$ in our experiments varied between 0.5 and 2.6. The comparison between the theory and experiments is shown in figure 12. At small frequencies (or small $k_{c l} a$ ) the attenuation due to scattering is expected to increase in proportion to $f^{4}$. This behaviour is observed roughly for $k_{c / a}<1.3$. At higher frequencies the resonance effects due to various shape deformations of the particles become important as discussed in more detail by Spelt et al. (1999) who examined the problem of determining size distributions for dilute suspensions. The first three peaks seen in figure 12 correspond to the resonances in $n=2,3$, and 4 modes (cf. (56)).

As we can see from figure 12 , the agreement between the theory and the experiments is very good. A possible explanation for the slight differences observed near the resonance peaks is the uncertainty in the mean particle size as specified by the manufacturcr. Changing the size of the particles from 79 to $77 \mu \mathrm{m}$ radius (which is within the specifications) is seen in figure 12 to improve the comparison. Alternatively, an excellent agreement can also be observed by accounting for the size distribution of particles.

Most of the data shown in figure 12 were taken for a suspension with $\phi=0.05$. High attenuation near the resonance peaks is not measurable and this explains the gaps seen in the data near those frequencies. We repeated some experiments with $\phi=0.025$ and with smaller vessels which decreased the acoustic path length between the two transducers and obtained a few data points near the resonances but additional measurements with very low volume fractions appeared unnecessary.

Since the volume fraction used in this measurement is rather small $(\phi=0.05)$, an 
excellent comparison between the theory and experiments should not be regarded as a true test of the effective-medium approximation. Rather, it shows that the data taken in our laboratory are reliable and that our analysis and the computer program for the effective-medium approximation gives correct results over a wide range of frequencies. To test the effective-medium theory higher volume fractions must be used but we encounter two problems. First, the monodisperse polystyrene particles in this size range are extremely expensive and secondly the range of frequencies for which the attenuation at higher volume fractions would be measurable will be rather narrow to provide a good test of the effective-medium approximation.

Experiments on dense slurries in the frequency range that is dominated by scattering effects before the resonance peaks were done by Atkinson (1991) and Atkinson \& Kytömaa (1992). We have compared their data for the dilute suspensions with the present theory and found that, although the agreement at the lower half of their frequency range is reasonable, at higher frequencies the experimental results for the attenuation were consistently lower than the theoretical results (at 0.045 volume fraction and $0.7 \mathrm{MHz}$ frequency the difference was a factor two). It was found that the differences could not be resolved by changing the physical properties, the size of the particles or by allowing for a size distribution of the particles. Since we do not see any reason for the theory to be inapplicable at such low volume fractions, we did not pursue further comparison at higher volume fractions. Instead, we shall compare the theory with the experiments we have conducted for the glass-water system.

Since the large glass particles are difficult to keep suspended in water, we added glycerol to increase the viscosity and density of the suspending medium. Soda-lime glass particles were used. The volume fraction size distribution was measured using a light scattering instrument and gave a mean radius of $63 \mu \mathrm{m}$ and a standard deviation of $8.5 \mu \mathrm{m}$ (the volume fraction distribution is related to the size distribution $P(a)$ by $\left.\phi(a)=(4 / 3) \pi a^{3} P(a)\right)$. The distribution is shown in figure 13 together with a fit used in calculations discussed below (a lognormal size distribution for $P(a)$ was used). The instrument could measure the particle radius up to about $240 \mu \mathrm{m}$. It was estimated that about $1.5 \%$ of the particles by volume had radius that exceeded this value.

We first discuss results for a dilute suspension. Figure 14 shows the attenuation as a function of frequency at $\phi=0.05$. At the frequency of $1 \mathrm{MHz}$, the non-dimensional wavenumber $k_{c l} a$ based on mean radius is about 0.25 . Thus, throughout the frequency range we expect the scattering losses to be the most significant. At low frequencies, the attenuation is approximately proportional to $f^{4}$. Note that at very low frequencies the viscous attenuation will become more significant, and if the Stokes layers are small compared with the particle radius, then the attenuation will be proportional to $f^{1 / 2}$. At higher frequencies the attenuation appears to level off, unlike the case of polystyrene particles which exhibited distinct resonance peaks. This qualitative difference arises due to different shear moduli of glass and polystyrene (Spelt et al 1999).

The solid curve in figure 14 is obtained by using the size distribution shown in figure 13 which ignores the particles larger than $240 \mu \mathrm{m}$. We see that the agreement between the theory and the experiments is very good at frequencies above $2 \mathrm{MHz}$. Significant discrepancy exists, however, at lower frequencies. This may be due to the presence of larger particles. If we assume that, in addition to the size distribution shown in figure 14 we had $1.5 \%$ by volume of particles with a radius of $540 \mu \mathrm{m}$, then we obtain the dashed curve shown in figure 14. These larger particles contribute most to the attenuation for smaller frequencies. Alternatively, it is possible that some of the assumed physical properties of the water-glycerol system (cf. table 1) may 


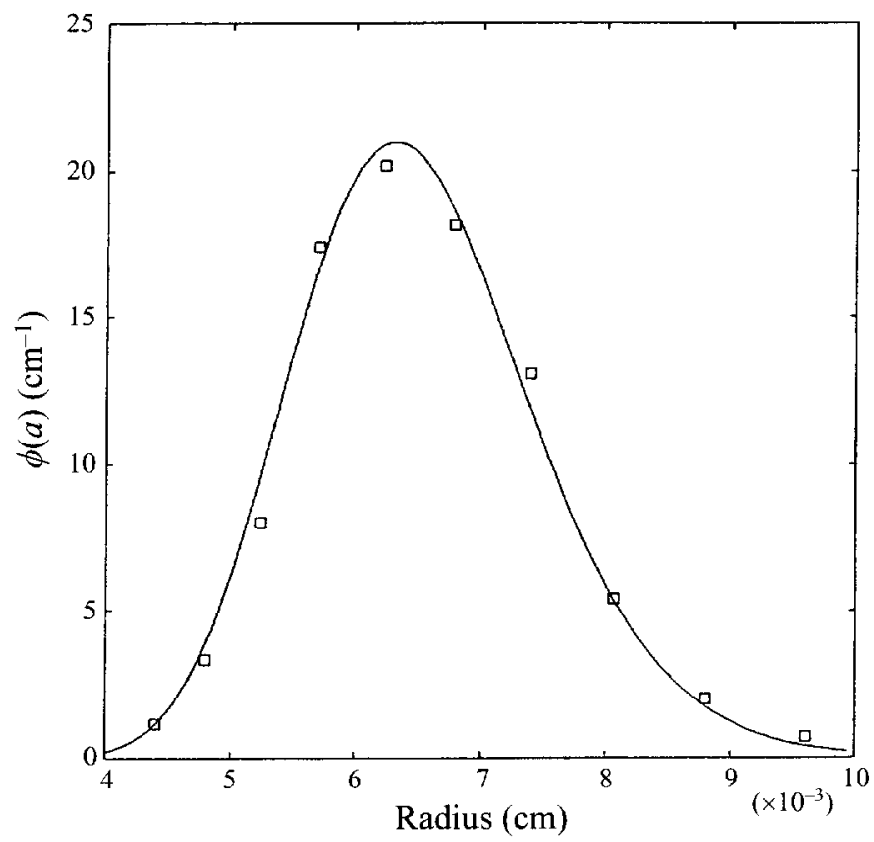

FIGURE 13. Volume-fraction distribution of the glass particles used in figure 14. Circles are measurements, the solid line is a fit using a log-normal size distribution.

be inaccurate and this may lead to the observed discrepancy at lower frequencies. The density and viscosity were measured in our laboratory but the other properties (sound speed and attenuation) were estimated using a volume-average mixture rule. In view of these uncertainties we shall only compare the experimental data for dense suspensions for frequencies greater than $2 \mathrm{MHz}$ where the agreement for dilute suspensions is good.

Since the size distribution is somewhat broad, we must modify the effective-medium theory to account for polydispersity. The coefficients $\lambda_{\rho}$, etc. to be used in determining the effective properties of the suspension are now replaced by $\sum_{n=0}^{M} \phi\left(a_{i}\right) \lambda_{p}\left(a_{i}\right)$, etc. where $M$ is the number of particle size bins. Here, $\lambda_{\rho}\left(a_{i}\right)$ represents the ratio of average dilatation amplitude inside the particle of radius $a_{i}$ to that in the suspension. To estimate such coefficients we assume that the particle of radius $a_{i}$ is surrounded by the liquid up to $r=R_{i}$ and the effective medium for $r>R_{i}$. We take $R_{i} / a_{i}$ to be the same for all particle sizes and given by the same expression as in the case of a monodisperse suspension (cf. (52)). This is probably not a good estimate of $R_{i} / a_{i}$ since one would expect $R_{i} / a_{i}$ for larger particles to be smaller than for monodisperse suspensions as the volume exclusion for larger particles is smaller when smaller particles are present in the suspension. However, since there are no known analytical, rigorous solutions for polydisperse suspensions, a more complicated scheme for estimating $R_{i} / a_{i}$ would be difficult to justify.

The dense-slurry data for the glass-water/glycerol suspensions are shown in figure 15. The frequency range is $2.5-5 \mathrm{MHz}$ for which the comparison at $\phi=0.05$ shown in figure 14 was good. The agreement is seen to be very good up to $\phi=0.3$. At higher volume fractions, however, wc obscrve significant discrepancy. The theory predicts the attenuation to be a monotonically increasing function of $\phi$ while the experiments exhibit maxima near $\phi=0.3$. The measurements were made two or 


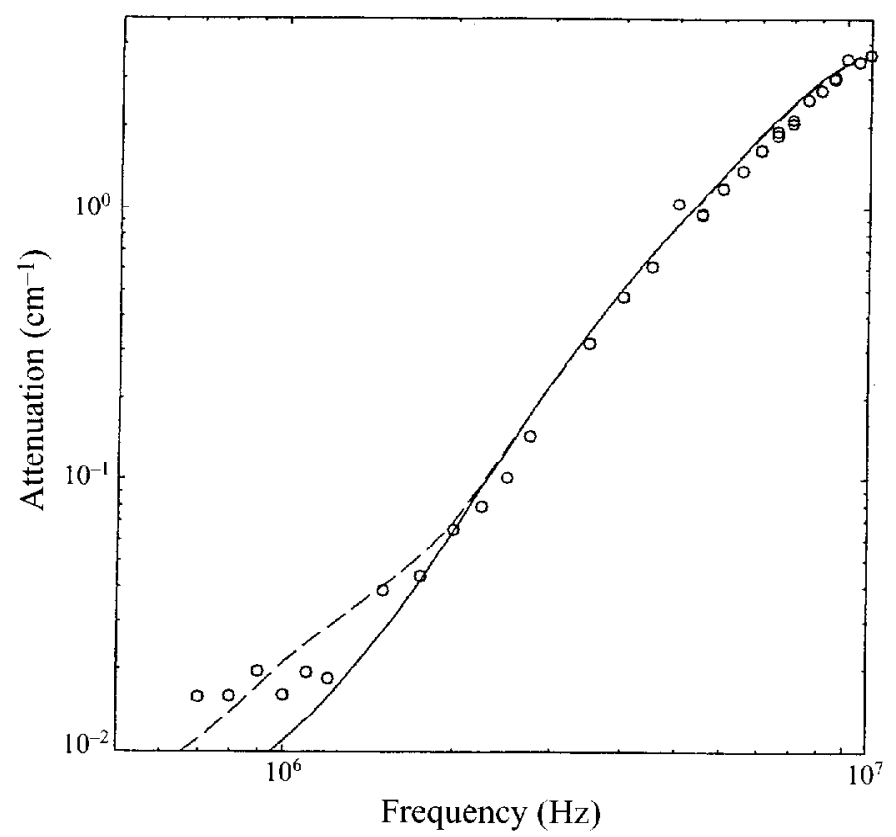

FIGURE 14. Experimental and theoretical results for the attenuation in a mixture of glass particles (mean radius $63 \mu \mathrm{m}$ and $8.5 \mu \mathrm{m}$ standard deviation) in glycerol at 0.05 volume fraction. Circles are experiments, solid and broken lines are the theoretical predictions.

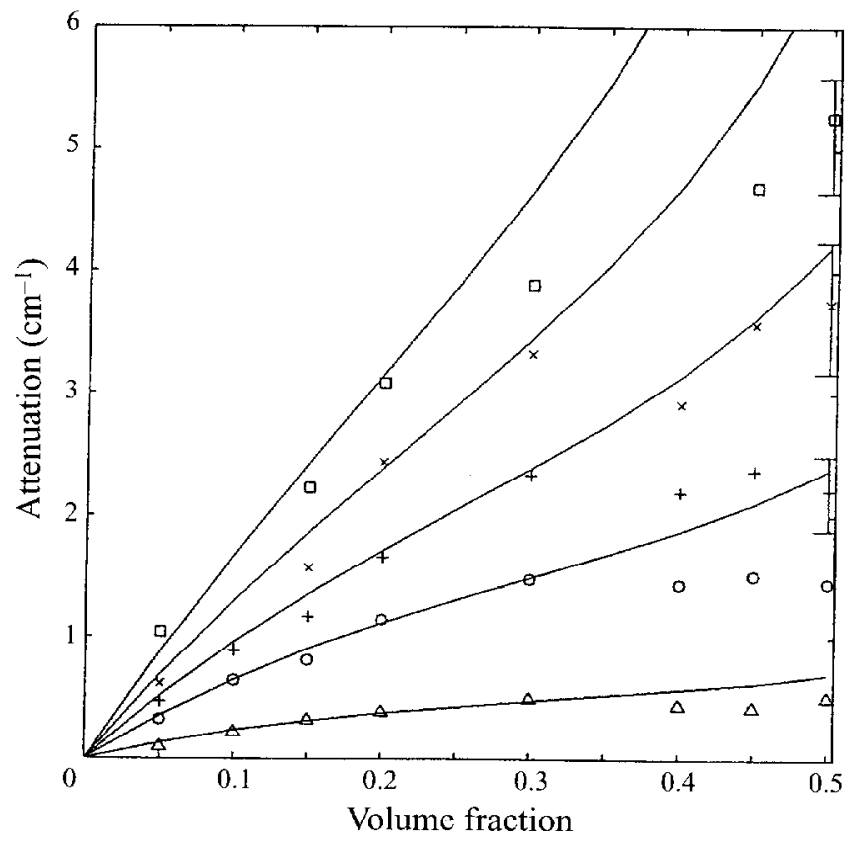

FIGURE 15. Experimental and theoretical results for the attenuation as a function of volume fraction for different frequencies, using the same glass particles and glycerol as in figure 11. Symbols are experiments, solid lines theory for monodisperse particles and broken lines theory for polysdisperse particles. $\triangle, 2.5 \mathrm{MHz} ; 0,3.5 \mathrm{MHz} ;+, 4 \mathrm{MHz} ; \times, 4.5 \mathrm{MHz} ; \square, 5 \mathrm{MHz}$. 


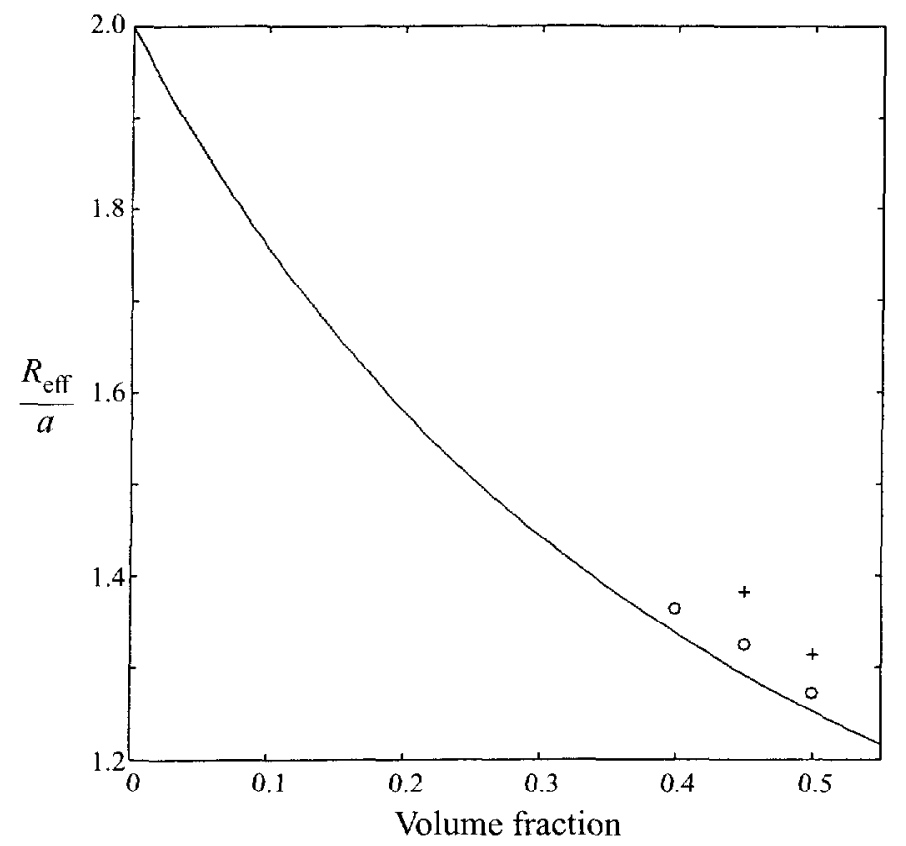

FIGURE 16. Liquid-shell to particle radius ratio as a function of volume fraction. Solid line is (52). Symbols are the values that would have to be used to get very good agreement with the experimental data shown in figure 15 at high volume fractions. $0,2.5 \mathrm{MHz}$ data;,$+ 5 \mathrm{MHz}$.

three times for each $f$ at $\phi=0.3$ and 0.5. At smaller volume fractions the data were quite reproducible and the error bars were typically smaller than the size of the symbols shown in figure 15. However, larger variations were observed at higher volume fractions as exemplified by the vertical bars around the data points. Although these error bars are quite significant, we see that the theory consistently overpredicts the attenuation for $\phi>0.3$.

As mentioned earlier, there is some concern about the proper choice of $R_{i} / a_{i}$ to be used in the effective-medium approximations for polydisperse suspensions. To see how the choice of $R_{i} / a_{i}$ affects the results, we calculated the values of $R_{i} / a_{i}$ (assumed to be independent of the particle radius) at which the theory and experiments would coincide for $\phi \geqslant 0.3$ at 2.5 and $5 \mathrm{MHz}$. The results are shown in figure 16 . The solid line in that figure corresponds to the value used in the results presented in figure 15. We see that only slight changes in $R / a$ are needed to make the theory predictions coincide with the experimental data. In other words, the results for the attenuation are very sensitive to the choice of $R / a$ in very dense suspensions. Finally, the fact that the scatter in the attenuation data is significant at higher volume fractions suggests that the attenuation might be quite sensitive to the manner in which the suspension is stirred. As noted earlier we used hand-stirring just before taking the attenuation measurement. Perhaps using a fluidized bed would have produced different attenuation data at high volume fractions.

The comparisons shown so far were dominated by the thermal and scaltering effects. Experiments in which the viscous losses are significant were carried out by Hampton (1967), but those were for clay particles which are highly non-spherical. To assess the theory for the viscous regime, we have measured attenuation for a suspension of small glass particles in water. The size distribution for these particles is shown in figure 


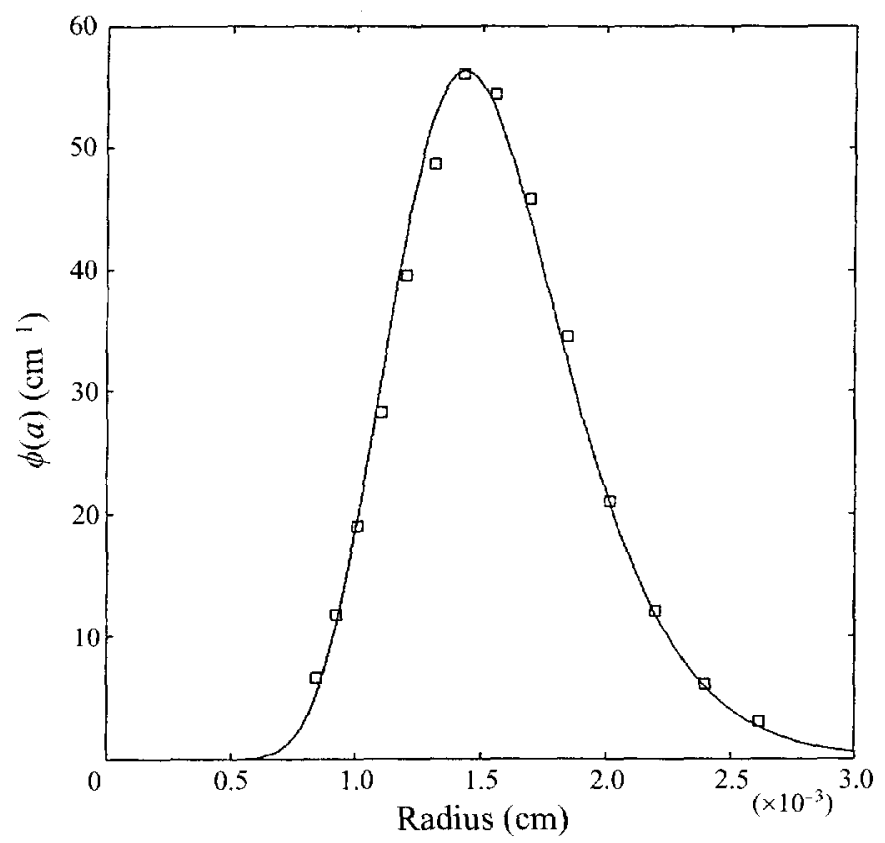

Figure 17. Volume-fraction distribution of the glass particles used in figurc 18. Circles are measurements, the solid line is a fit using a log-normal size distribution.

17 together with the fit used in the calculations. The mean radius is $15 \mu \mathrm{m}$ and the standard deviation is $3.5 \mu \mathrm{m}$. These particles have a very small terminal velocity and it is not necessary to add glycerol to keep them suspended. For the frequency range over which we could measure attenuation, i.e. for $0.7-10 \mathrm{MIIz}$, the non-dimensional wavenumber $k_{c l} a$ varies from 0.03 to 0.5 . The particle-to-liquid (pure water) density ratio in this case is 2.55 , and the viscous attenuation dominates the lower part of the frequency range, while the scattering attenuation becomes important at higher frequencies.

The results for volume fractions $0.05,0.2,0.3$, and 0.4 are shown in figure 18 . We see that the measured attenuation is proportional to $f^{1 / 2}$ in the viscous range, which is to be expected for the case when the Stokes layers are thin compared with the particle radius (see, e.g., Allegra \& Hawley 1972). We see an excellent agreement between the theory and experiments. It may be noted that the attenuation does not vary linearly with the volume fraction, indicating that the effective-medium approximation represents a significant improvement over the dilute theory. We also note that, unlike the case of larger particles, the attenuation increases monotonically with the volume fraction for the entire range of frequencies over which the measurements are made.

\section{Phase speed}

While the physics of acoustics is very interesting, it appears that the determination of the particle volume fraction from acoustic measurements will be, in general, difficult because of the sensitive dependence of the acoustics on physical properties of the particles and liquid and the particle size distribution. Since the phase speed is relatively less sensitive to the particle size, it might be more advantageous to measure the phase speed. The scattering regime can lead to large attenuation and resonance 


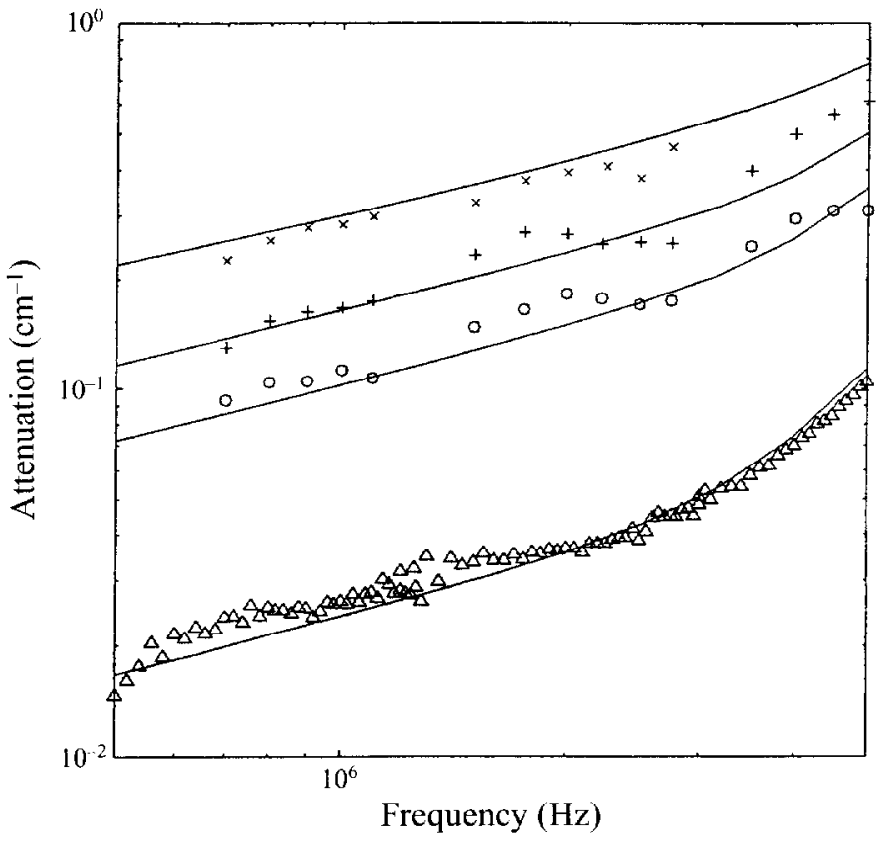

FIGURE 18. Experimental and theoretical results for the attenuation as a function of frequency at different volume fractions, using glass particles in water. Symbols are experiments, solid lines are theory. Volume fractions are $\triangle, 0.05 ; 0,0.2 ;+, 0.3$ and $\times, 0.4$.

behaviour sensitive to the mechanical properties of particles. The phase speed near the resonance frequencies can vary significantly. Thus, it is desirable to carry out measurements at low frequencies where the scattering effects will be insignificant.

When $k_{c l} a$ is small the phase speed can be measured for cases for which the Stokes layers are much smaller than the particle radius. In this limit the speed is nearly independent of the particle radius. Figure 19 shows the phase speed as a function of volume fraction in this limit for a glass-water system with two different sizes. Note that the speed is essentially the same for both particle sizes. The attenuation under these conditions would be proportional to $a^{-1}$ (Allegra \& Hawley 1972). Figure 19 also shows results for the case when the Stokes layers are much thicker than the particle radius. Once again, in this limit the phase speed is nearly independent of the particle radius while the attenuation would vary significantly with the particle radius as $a^{2}$. Note that the phase speed as a function of volume fraction goes through a minimum in the low-frequency limit. The monotonic increase at high frequency might be more suitable for determining the volume fraction. Thus, the ideal frequency for measuring the phase speed corresponds to the one for which the Stokes layers are thin compared with the particle radius and $k_{c} a$ is small.

\section{Summary}

We have derived equations for describing small-amplitude acoustic wave propagation through a suspension. The equations are similar to those for a single-phase medium but require closures for estimating the effective properties of the suspension. We used an effective-medium model to solve for the conditionally averaged temperature, density, and velocity fields inside a test particle, and estimated thereby the 


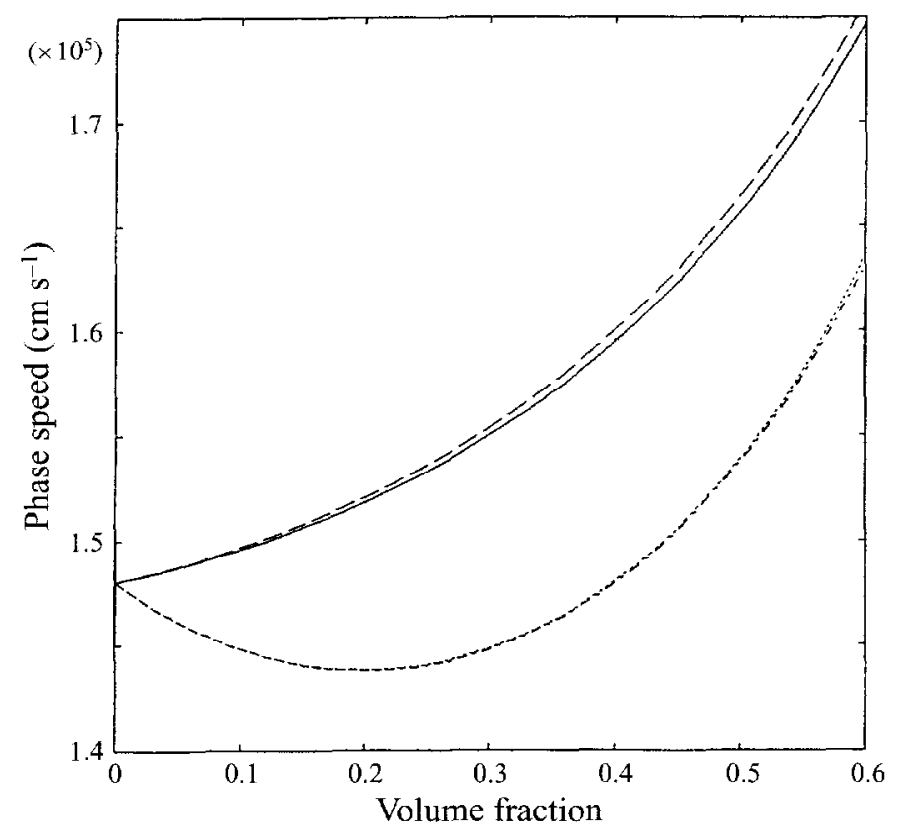

Figtre 19. Wave speed as a function of volume fraction for two limiting cases. Solid line, $a^{2} \omega p_{l} / \mu_{i}=3 \times 10^{3}$ and $k_{c l} a=8 \times 10^{-4}$; long-dashed line the same, but with the particle radius increased by a factor of 5; dashed-dotted line, $a^{2} \omega \rho_{l} / \mu_{i}=3 \times 10^{-4}$ and $k_{c l} a=8 \times 10^{-4}$; short-dashed linc the same but with the particle radius increased by a factor of 5 .

effective properties such as the density, heat capacity, conductivity, viscoelasticity, and compressibility in a self-consistent manner. When the wavelength is large compared with the particle radius the multiparticle interactions in the suspension can be approximated by Stokes or Laplace equations for which a number of effective properties have been determined in recent years through rigorous multiparticle calculations. We show that the estimates obtained using the effective-medium approximation for various properties are in excellent agreement with these rigorous calculations. The theory is also shown to be excellent agreement with the experimental data for the polystyrene-water system by Allegra \& Hawley (1972). The ratio of particle radius to wavelength was small in these experiments. To test the theory for larger particles we have conducted experiments both for polystyrene particles and glass particles in water. The agreement with the data for the polystyrene-water system which exhibits several resonances due to shape oscillations is excellent. However, the comparison was limited to dilute suspensions because of the unavailability of concentrated monodisperse suspensions in the particle size range of interest. The glass-water system had significant polydispersity but covered a broad range of volume fractions. The agreement between the theory and experiments for small particles in which the viscous attenuation dominates is excellent while for large particles for which the scattering losses dominate the agreement is good only up to $\phi=0.3$. At higher volume fractions the attenuation measured in our laboratory decreased, in contrast with the theory prediction.

In view of the remarkable success of the effective-medium approximation in predicting the attenuation in solid-liquid systems, it seems that the procedure used here may also find applications in other acoustic problems, e.g. in the electroacoustics of 
colloidal suspensions (O'Brien 1990) and in acoustics of fluid-saturated porous media (Burridge \& Keller 1981).

This work was supported by the Department of Energy, Environmental Science Management Program, under Grant DE-FG07-96ER14729. The computations were performed using the resources of NCSA at University of Illinois. AS also acknowledges the support provided by the National Science Foundation under Grant CTS9632227.

\section{REFERENCES}

Allegra, J. R. \& Hawlfy, S. A. 1972 Attenuation of sound in suspensions and emulsions: theory and experiments. J. Acoust. Soc. Am. 51, 1545-1563.

AtKinson, C. M. 1991 Acoustic wave propagation and non-intrusive velocity measurements in highly concentrated suspensions. PhD Thesis, MIT.

Atknson, C. M. \& KrtömaA, H. K. 1992 Acoustic wave speed and attenuation in suspensions. Intl J. Multiphase Flow 18, 577-592.

BonneCAZE, R. T. \& BRADY, J. F. 1991 The effective conductivity of random suspension of spherical particles. Proc. R. Soc. Lond. A 432, 455-465.

Burridge, R. \& Keller, J. B. 1981 Poroelasticity equations derived from microstructure. J. Acoust. Soc. Am. 70, 1140-1146.

Carstensen, E. L. \& Foldy, L. L. 1947 Propagation of sound through a liquid containing bubbles. J. Acoust. Soc. Am. 19, 481-501.

Dond, T. L., Hammer, D. A., Sangani, A. S. \& Kuch, D. L. 1995 Numerical simulations of the effect of hydrodynamic interactions on diffusivities of integral membrane proteins. $J$. Fluid Mech. 293, 147-180.

Duraiswami, R., Prabhukumar, S. \& Chahine, G. L. 1998 Bubble counting using an inverse acoustic scattering method. J. Acoust. Soc. Am. 104, 2699-2717.

Epstein, P. S. \& CarharT, R. R. 1953 The absorption of sound in suspensions and emulsions. I. Water fog in air. J. Acoust. Soc. Am. 25, 553-565.

Gradshiteyn, I. S. \& Ryzhik, I. M. 1994 Table of Integrals, Series, and Products, 5th Edn. Academic.

Hampton, L. D. 1967 Acoustic properties of sediments. J. Acoust. Soc. Am. 42, 882-890.

JefrReY, D. J. 1973 Conduction through a random suspension of spheres. Proc. R. Soc. Lond. A 335, 355-367.

JU, J. W. \& CHEN, T. M. 1994 Effective elastic moduli of two-phase composites containing randomly dispersed spherical inhomogeneities. Acta Mech. 103, 123-144.

KIM, S. \& Russel, W. B. 1985 Modelling of porous media by renormalization of the Stokes equations. J. Fluid Mech. 154, 269-286.

LADD, A. J. C. 1990 Hydrodynamic transport coefficients of random dispersions of hard spheres. J. Chem. Phys. 93, 3484-3494.

Landau, L. D. \& Lifshitz, E. M. 1986 Theory of Elasticity, 3rd Edn. Pergamon.

Mo, G. \& SANGani, A. S. 1994 A method for computing Stokes flow interactions among spherical objects and its application to suspension of drops and porous particles. Phys. Fluids 6, 16371652.

Norato, M. A. 1999 Acoustic probe for the characterization of solid-gas-liquid slurries. PhD Thesis, Syracuse University.

O'BRIEN, R. W. 1990 The clectroacoustic equations for a colloidal suspension. J. Fluid Mech. 212, 81-93.

Prosperetti, A. 1984 Bubble phenomena in sound fields: part one. Ultrasonics 22, 69-77.

SANGANI, A. S. \& Mo, G. 1997 Elastic interactions in particulate composites with perfect as well as imperfect interfaces. J. Mech. Phys. Solids 45, 2001-2031.

SANGANI, A. S. \& YAO, C. 1988 Bulk thermal conductivity of composites with spherical inclusions. J. Appl. Phys. 63, 1334-1341.

Sangani, A. S., Zhang, D. Z. \& Prosperetri, A. 1991 The added mass, Basset, and viscous drag coefficients in nondilute bubbly liquids undergoing small-amplitude oscillatory motion. Phys. Fluids A 3, 2955-2970. 
Spelt, P. D. M., Norato, M. A., Sangani, A. S. \& Tavlarides, L. L. 1999 Determination of particle size distributions from acoustic wave propagation measurements. Phys. Fluids 11, $1065-1080$.

TURnER, J. C. R. 1976 Two-phase conductivity, the electrical conductance of liquid-fluidized beds of spheres. Chem. Engng Sci. 31, 487-492. 


\title{
Determination of particle size distributions from acoustic wave propagation measurements
}

\author{
Peter D. M. Spelt, Michael A. Norato, Ashok S. Sangani, and Lawrence L. Tavlarides \\ Department of Chemical Engineering and Materials Science, Syracuse University, Syracuse, \\ New York 13244
}

(Received 4 August 1998; accepted 20 January 1999)

\begin{abstract}
The wave equations for the interior and exterior of the particles are ensemble averaged and combined with an analysis by Allegra and Hawley [J. Acoust. Soc. Am. 51, 1545 (1972)] for the interaction of a single particle with the incident wave to determine the phase speed and attenuation of sound waves propagating through dilute slurries. The theory is shown to compare very well with the measured attenuation. The inverse problem, i.e., the problem of determining the particle size distribution given the attenuation as a function of frequency, is examined using regularization techniques that have been successful for bubbly liquids. It is shown that, unlike the bubbly liquids, the success of solving the inverse problem is limited since it depends strongly on the nature of particles and the frequency range used in inverse calculations. (c) 1999 American Institute of Physics. [S1070-6631(99)01405-1]
\end{abstract}

\section{INTRODUCTION}

Determining the particle size distribution of a solidliquid mixture is of great practical interest. It has been suggested in the literature that this distribution may be determined by measuring the attenuation of a sound wave propagating through the mixture as a function of the frequency of the wave. The main premise is that the attenuation caused by a particle as a function of frequency depends on its size and therefore the attenuation measurements can be inverted to determine the particle size distribution-at least when the total volume fraction of the solids is small enough so that the particle interactions and detailed microstructure of the slurry play an insignificant role in determining the acoustic response of the slurry. Indeed, this general principle has been exploited successfully to determine the size distribution of bubbles in bubbly liquids. ${ }^{1-3}$ Commercial "particle sizers" based on acoustic response are in the process of being developed/marketed for characterizing solid-liquid mixtures. ${ }^{4}$ The main objective of this paper is to investigate under what circumstances such a problem can be solved for solid-liquid systems. It will be shown that the success of the acoustic method for determining detailed particle size distributions is limited, depending on the nature of the particles and the frequency range over which input data (attenuation) are available.

The problem of determining the acoustic response of a slurry given its particle size distribution is referred to as the forward problem. When the total volume fraction of the particles is small, the problem is relatively simple since then one only needs to understand the interaction between a single particle and the incident sound wave. This has been examined by a number of investigators in the past with notable contributions from Allegra and Hawley ${ }^{5}$ and Epstein and Carhart ${ }^{6}$ who considered suspensions of particles as well as drops. The former investigators also reported experimental results verifying the theory for relatively small particles for which the acoustic wavelength is large compared with the particle radius. The theory developed by these investigators is quite general and accounts for attenuation by thermal, viscous, and scattering effects as described in more detail in Secs. II and III. The case of monodisperse nondilute suspensions has been examined by Varadan et al. ${ }^{7}$ who used an effective medium approximation to account for particle interactions, but their analysis was concerned only with the attenuation due to scattering. In Sec. II we present the theory for the forward problem with the main aim of reviewing the important physical effects causing the attenuation. The derivation for the attenuation proceeds along different lines than that followed by Epstein and Carhart or Allegra and Hawley in the way the one particle analysis is used to predict the attenuation of the suspension. These investigators calculated the energy dissipation per one wavelength to estimate the attenuation while we use the method of ensemble averages to determine both the phase speed and attenuation of waves. The method of ensemble averages will be more convenient for developing a suitable expression for attenuation in nondilute suspensions, if desired, using either an appropriate effective-medium approximation or direct numerical simulations.

In Sec. III we present new experimental data for nearly monodisperse polystyrene particles whose radii are comparable to the wavelength and validate the theory described in Sec. II over a nondimensional frequency range much broader than examined by previous investigators. We also summarize in that section the different physical mechanisms that cause attenuation in suspensions. The attenuation as a function of frequency is shown to undergo several peaks owing to the resonances in shape oscillations in agreement with the theory prediction. It also gives some indication of the range of frequency and attenuation measurable with our acoustic device.

In Sec. IV we consider the inverse problem, i.e., the 
problem of determining the particle size distribution given the total attenuation as a function of frequency and the physical properties of the particles and the suspending liquid. At small particle volume fractions, this amounts to solving a linear integral equation for the unknown size distribution. This is an ill-posed problem: small changes/errors in the attenuation data can cause large changes in the size distribution. Thus, for example, several very different particle distributions could give rise to essentially the same attenuationfrequency curve. This, of course, is a rather well-known difficulty in most inverse problems which involve solving a Fredholm integral equation of the first kind with a smooth kernel. Techniques have been developed to "regularize" the problem. We use the well-known Tikhonov regularization techniques, ${ }^{8}$ which replaces the ill-posed original problem with another well-posed problem involving an integrodifferential equation whose solution minimizes the fluctuations in the particle size distribution. Minimizing of the fluctuations is rationalized on the grounds that in most practical situations the particle size distribution is smooth. This regularization technique has been shown to work very well for the inverse problem in bubbly liquids. ${ }^{2}$

We apply the above technique to suspensions of polystyrene and glass particles. We find that the technique works well for the polystyrene particles but not for all glass particles. We also find that for polystyrene particles the technique works only when the attenuation is given over an appropriate frequency range-a frequency range that is too narrow or too broad may give erroneous estimates of the distribution. An alternative inverse technique based on linear programing also failed to produce the correct particle size distribution for the cases for which the Tikhonov scheme failed. This suggests that the prospects for determining the detailed particle size distribution from acoustic mcasurcments are somewhat limited. (In situations where more might bc known about the nature of particle size distribution, e.g., unimodal with a Gaussian or log-normal distribution, one might be ablc to detcrmine the size distribution through appropriate curve fitting as has been done, for example, by McClements and Coupland, ${ }^{9}$ but this is not addressed here.)

The reasons why the size distributions for some particle suspensions are not recovered by the inverse techniques while the same techniques were found to be quite successful for bubble suspensions can be given in terms of differing resonance nature of these suspensions. In the case of bubbles in most typical applications, the resonance occurs at frequencies for which the wavelength is relatively large compared with the bubble radius. This resonance is due to volume oscillations; the shape-dependent resonances are unimportant and, as a consequence, there is effectively one resonance frequency for each bubble size. Thus, the peaks in the attenuation-frequency curve give a reasonable indication of the bubble sizes. The situation with the particles is different as their resonance behavior is governed by shape oscillations. For polystyrene particles, several resonance peaks corresponding to different shape oscillations arise even for monodisperse particles, and, as a result, it is difficult to determine whether a given resonance peak arises from a different shape oscillation mode of the same particle or from a particle of different size. For glass particles, on the other hand, there are no significant resonance peaks even for monodisperse particles, and the attenuation behavior for different sizes is not significantly different to allow accurate results for the size distribution.

\section{THE FORWARD PROBLEM}

The wave equations for both the interior and exterior of particles have been derived by Epstein and Carhart. ${ }^{6}$ They were interested in the attenuation of sound waves in fog and therefore their analysis was concerned with drops instead of particles. The stress tensor for a viscous fluid used by them for the interior of the drops was subsequently replaced by Allegra and Hawley ${ }^{5}$ by that of an elastic solid to determine the attenuation of sound waves in a solid-liquid suspension. In this section we shall ensemble average a resulting wave equation to obtain the effective wave number of the suspension at arbitrary volume fraction, the real and imaginary parts of which give the wave speed and attenuation. Thus, the attenuation is not calculated by means of an energy dissipation argument, ${ }^{5,6}$ but directly from averaging the relevant wave equation. The result contains certain coefficients that remain to be evaluated for a given microstructure. In the present study, since we are primarily concerned with determining the size distribution, we shall evaluate the coefficients in the limit of small volume fractions. In a separate study, where we shall present experimental results for nondilute suspensions, we shall extend the theory to treat nondilute suspensions.

\section{A. Theory}

Epstein and Carhart ${ }^{6}$ first linearized the conservation equations for mass, momentum, and energy. The pressure and internal energy were then eliminated by introducing the linearized equations of state to yield equations in terms of density, velocity, and temperature. Next, the time dependence of all quantities were expressed by the factor $\exp (-\iota \omega t)$-which is henceforth suppressed--and the velocity was expressed as

$$
\mathbf{v}=-\nabla \Phi+\nabla \times \mathbf{A}
$$

with $\nabla \cdot \mathbf{A}=0$. With this form of $\mathbf{v}$ it is possible to eliminate the temperature and density from the governing equations to yield a fourth-order partial differential equation for $\Phi$ and a second-order equation in $\mathbf{A}$. The former, in turn, can be split into two second-order wave equations upon a substitution $\Phi=\phi_{c}+\phi_{T}$ to finally yield three wave equations:

$$
\begin{aligned}
& \left(\nabla^{2}+k_{c}^{2}\right) \phi_{c}=0, \\
& \left(\nabla^{2}+k_{T}^{2}\right) \phi_{T}=0, \\
& \left(\nabla^{2}+k_{s}^{2}\right) \mathbf{A}=\mathbf{0} .
\end{aligned}
$$

The wave numbers in the above equations are given by

$$
\begin{aligned}
\frac{1}{k_{c}^{2}}= & \frac{c^{2}}{2 \omega^{2}}\left[1-\iota(e+\gamma f)+\left((1-\iota(e+\gamma f))^{2}\right.\right. \\
& \left.+4 f(\iota+\gamma e))^{1 / 2}\right],
\end{aligned}
$$




$$
\begin{aligned}
\frac{1}{k_{T}^{2}}= & \frac{c^{2}}{2 \omega^{2}}\left[1-\iota(e+\gamma f)-\left((1-\iota(e+\gamma f))^{2}\right.\right. \\
& \left.+4 f(\iota+\gamma e))^{1 / 2}\right], \\
k_{s}= & (1+\iota)(\omega \rho / 2 \mu)^{1 / 2},
\end{aligned}
$$

with

$$
e \equiv(4 \mu / 3+\kappa) \omega /\left(\rho c^{2}\right) ; \quad f \equiv \sigma \omega / c^{2} .
$$

Here, $c$ is the phase speed in pure liquid, $\rho$ is the density, $\kappa$ and $\mu$ are, respectively, the compressional and dynamic viscosity, $\gamma=C_{p} / C_{v}$ is the ratio of specific heats at constant pressure and volume, $\tau$ is the thermal conductivity, and $\sigma=\tau / \rho C_{p}$ is the thermal diffusivity.

Inside the particles similar equations hold with the dynamic viscosity replaced by $\tilde{\mu} /(-\iota \omega)$ and the wave speed by $((\tilde{\Lambda}+2 \tilde{\mu} / 3) / \tilde{\rho})^{1 / 2}$, where $\tilde{\mu}$ and $\tilde{\lambda}$ are the Lamé constants, while the compressional viscosity is left out. Henceforth a tilde refers to the interior of particles.

At small values of $e$ and $f$ (such as in water), the above expressions for $k_{c}$ and $k_{T}$ simplify to

$$
\begin{aligned}
& k_{c}=\omega / c+\frac{\iota}{2}[(4 \mu / 3+\kappa) / \rho+(\gamma-1) \sigma] \omega^{2} / c^{3}, \\
& k_{T}=(1+\iota)(\omega / 2 \sigma)^{1 / 2} .
\end{aligned}
$$

Equation (1) and its counterpart inside the particles describe the sound wave propagation through the suspension. Note that the wave number has an imaginary part; sound waves in pure fluid are attenuated by viscous and thermal energy dissipation $;^{10}$ the term inside the square brackets in (8) is commonly referred to as the "diffusivity of sound." The total attenuation coefficient in both liquid and in the solid particle will henceforth be treated as additional physical properties. The other two wave equations describe waves that arise from thermal conduction and finite viscosity: we note that the modulus of $k_{T}$ in Eq. (8) is inversely proportional to the thermal penetration depth $\sqrt{\sigma / \omega}$ and that of $k_{s}$ to the viscous penetration depth $\sqrt{\mu / \rho \omega}$. The thermal $\left(\phi_{T}\right)$ and shear (A) waves have generally very high attenuation and are unimportant in acoustic applications.

We now proceed to ensemble average the wave equation (1) to find an expression for the effective wave number of a wave propagating through a solid-liquid suspension. Introducing an indicator function $g$, defined to be unity inside the particles and 0 outside, the ensemble-averaged value of $\phi_{c}$ is

$$
\left\langle\phi_{c}\right\rangle=\left\langle g \widetilde{\phi}_{c}+(1-g) \phi_{c}\right\rangle .
$$

To obtain a wave equation for $\left\langle\phi_{c}\right\rangle$ we first take the gradient of the above equation to yield

$$
\nabla\left\langle\phi_{c}\right\rangle=\left\langle g \nabla \bar{\phi}_{c}+(1-g) \nabla \phi_{c}\right\rangle+\left\langle(\nabla g)\left(\tilde{\phi}_{c}-\phi_{c}\right)\right\rangle .
$$

As argued by Sangani, ${ }^{11}$ upon assuming that the particles' spatial distribution is homogeneous on a macroscale, the last term in (9), being a vector, can only depend on quantities such as $\nabla\left\langle\phi_{c}\right\rangle$ and $\nabla \nabla^{2}\left\langle\phi_{c}\right\rangle$. Anticipating that $\left\langle\phi_{c}\right\rangle$ will satisfy a wave equation we express the last term on the righthand side of the above equation in terms of $\nabla\left\langle\phi_{c}\right\rangle$, i.e., we write

$$
\left\langle(\nabla g)\left(\tilde{\phi}_{c}-\phi_{c}\right)\right\rangle=\lambda_{1} \nabla\left\langle\phi_{c}\right\rangle,
$$

where $\lambda_{1}$ depends on the parameters such as the volume fraction, $k_{c}$, and $\widetilde{k}_{c}$. The divergence of (9) is given by

$$
\begin{aligned}
\nabla^{2}\left\langle\phi_{c}\right\rangle= & \left\langle g \nabla^{2} \tilde{\phi}_{c}+(1-g) \nabla^{2} \phi_{c}\right\rangle+\langle(\nabla g) \\
& \left.\cdot\left(\nabla \bar{\phi}_{c}-\nabla \phi_{c}\right)\right\rangle+\lambda_{1} \nabla^{2}\left\langle\phi_{c}\right\rangle \\
= & -k_{c}^{2}\left\langle\phi_{c}\right\rangle-\left(\widetilde{k}_{c}^{2}-k_{c}^{2}\right)\left\langle g \widetilde{\phi}_{c}\right\rangle+\langle(\nabla g) \\
& \left.\cdot\left(\nabla \widetilde{\phi}_{c}-\nabla \phi_{c}\right)\right\rangle+\lambda_{1} \nabla^{2}\left\langle\phi_{c}\right\rangle .
\end{aligned}
$$

Writing

$$
\left\langle(\nabla g) \cdot\left(\nabla \bar{\phi}_{c}-\nabla \phi_{c}\right)\right\rangle=\lambda_{2} \bar{k}_{c}^{2}\left\langle\phi_{c}\right\rangle, \quad\left\langle g \bar{\phi}_{c}\right\rangle=\lambda_{3}\left\langle\phi_{c}\right\rangle,
$$

we find that $\left\langle\phi_{c}\right\rangle$ satisfies a wave equation

$$
\left(\nabla^{2}+k_{\text {eff }}^{2}\right)\left\langle\phi_{c}\right\rangle=0
$$

with the effective wave number given by

$$
k_{\mathrm{eff}}^{2}=\frac{k_{c}^{2}+\lambda_{3}\left(\widetilde{k}_{c}^{2}-k_{c}^{2}\right)-\lambda_{2} \widetilde{k}_{c}^{2}}{1-\lambda_{1}} .
$$

The real part of the effective wave number is the frequency divided by the phase speed in the mixture and the imaginary part the attenuation.

Up to this point the analysis is rigorous and without any assumption. Applying the boundary conditions of continuity of temperature, flux, velocity, and traction at the surface of the particles, and solving the resulting boundary value problem numerically, it is possible, in principle, to determine the phase speed and attenuation at arbitrary volume fraction using the above formulation. Special simplifications can be made when the wavelength is large compared with the particles and when the viscous and thermal depths are small compared with the particle radius for which numerical computations using the multipole expansions developed in recent years (see, e.g., Ref. 12) can be readily used for determining the attenuation at arbitrary volume fractions. Alternatively, one may use a suitable effective-medium approximation to account for the particle interactions in nondilute suspensions using the above framework. We shall pursue this further in a separate study ${ }^{13}$ devoted to nondilute suspensions where we shall also present experimental data. Since our interest in the present study is in determining size distributions, it is necessary to consider only the simplest case of dilute suspensions.

In dilute suspensions the particle interactions can be neglected, and the coefficients $\lambda_{1-3}$ can be evaluated from the solution for $\phi_{c}$ for a single particle given by Allegra and Hawley. ${ }^{5}$ Accordingly, the conditionally averaged $\left\langle\phi_{c}\right\rangle\left(\mathbf{x} \mid \mathbf{x}_{1}\right)$ given a particle centered at $\mathbf{x}_{1}$ is given by

$$
\begin{aligned}
\left\langle\phi_{c}\right\rangle\left(\mathbf{x} \mid \mathbf{x}_{1}\right)= & \exp \left(\iota \mathbf{k}_{c} \cdot \mathbf{x}\right)+\exp \left(\iota \mathbf{k}_{c} \cdot \mathbf{x}_{1}\right) \\
& \times \sum_{n=0}^{\infty} \iota^{n}(2 n+1) A_{n} h_{n}\left(k_{c} r\right) P_{n}(\mu),
\end{aligned}
$$


where $r=\left|\mathbf{x}-\mathbf{x}_{1}\right|, \mu=\cos \theta, \theta$ being the angle between $\mathbf{x}$ $-\mathbf{x}_{1}$ and $\mathbf{k}_{c}, h_{n}$ is the spherical Bessel function of the third kind (corresponding to an outgoing scattered wave), and $P_{n}$ is the Legendre polynomial of degree $n$. The first term on the right-hand side of the above expression is the unconditionally averaged $\left\langle\phi_{c}\right\rangle(\mathbf{x})$ whose amplitude is taken to be unity with no loss of generality.

Inside the particle centered at $\mathbf{x}_{1}$ wc have

$$
\begin{aligned}
\left\langle\bar{\phi}_{c}\right\rangle=\left(\mathbf{x} \mid \mathbf{x}_{1}\right)= & \exp \left(\iota \mathbf{k}_{c} \cdot \mathbf{x}_{1}\right) \sum_{n=0}^{\infty} \iota^{n}(2 n+1) \\
& \times \tilde{A}_{n} j_{n}\left(\tilde{k}_{c} r\right) P_{n}(\mu),
\end{aligned}
$$

where $j_{n}$ is the spherical Bessel function of the first kind. Similar expressions are written for the conditionally averaged $\phi_{T}$ and $\mathbf{A}$. This results in expressions with a set of six unknowns for each mode $n$. Application of the aforementioned boundary conditions of continuity of velocity, traction, temperature, and heat flux yield six equations in these six unknowns for each $n$. There were some typographical errors in the equations given by Epstein and Carhart ${ }^{6}$ and Allegra and Hawley; ${ }^{5}$ the correct equations are given in the Appendix. Although it is possible to solve for the unknowns analytically in certain limiting cases, it is best to solve them numerically since we are interested in covering a wide frequency range for inverse calculations.

We now return to the calculations of the coefficients $\lambda_{1-3}$. Upon using the identity

$$
\nabla g=-\mathbf{n} \delta\left(\mathbf{x}-\mathbf{x}_{i}\right),
$$

with $\mathbf{x}_{i}$ being a point on solid-liquid interface and $\mathbf{n}$ the unit normal vector at the point, $\lambda_{1}$ is given by

$$
\begin{aligned}
\lambda_{1} \nabla\left\langle\phi_{c}\right\rangle(\mathbf{x})= & -\int_{\left|\mathbf{x}-\mathbf{x}_{1}\right|=a} \mathbf{n}\left[\left\langle\bar{\phi}_{c}\right\rangle\left(\mathbf{x} \mid \mathbf{x}_{1}\right)-\left\langle\phi_{c}\right\rangle\left(\mathbf{x} \mid \mathbf{x}_{1}\right)\right] \\
& \times P\left(\mathbf{x}_{1}\right) d A_{1} .
\end{aligned}
$$

Here, $P\left(x_{1}\right)$ is the probability density for finding a particle in the vicinity of $\mathbf{x}_{1}$. Similarly, we have for $\lambda_{2}$ and $\lambda_{3}$

$$
\begin{aligned}
\lambda_{2} \widetilde{k}_{c}^{2}\left\langle\phi_{c}\right\rangle(\mathbf{x})= & -\int_{\left|\mathbf{x}-\mathbf{x}_{1}\right|=a} \mathbf{n} \cdot \nabla\left[\left\langle\widetilde{\phi}_{c}\right\rangle\left(\mathbf{x} \mid \mathbf{x}_{1}\right)\right. \\
& \left.-\left\langle\phi_{c}\right\rangle\left(\mathbf{x} \mid \mathbf{x}_{1}\right)\right] P\left(\mathbf{x}_{1}\right) d A_{1},
\end{aligned}
$$

and

$$
\lambda_{3}\left\langle\phi_{c}\right\rangle(\mathbf{x})=\int g(\mathbf{x})\left\langle\bar{\phi}_{c}\right\rangle\left(\mathbf{x} \mid \mathbf{x}_{1}\right) P\left(\mathbf{x}_{1}\right) d V_{1} .
$$

The above integrals must be evaluated while keeping in mind that the integration variable is $\mathbf{x}_{1}$. Thus, for example, in (15) and (16) we must consider all particles whose surfaces pass through the point $\mathbf{x}$. To carry out these integrals we use the identity

$$
\begin{aligned}
e^{\iota \mathbf{k}_{c} \cdot \mathbf{x}_{1}} & =e^{\iota \mathbf{k}_{c} \cdot \mathbf{x}^{-\imath r k_{\mathrm{c}} \mu}} \\
& =e^{\iota \mathbf{k}_{c} \cdot \mathbf{x}} \sum_{m=0}^{\infty} \iota^{m}(-1)^{m}(2 m+1) j_{m}\left(k_{c} r\right) P_{m}(\mu)
\end{aligned}
$$

and the orthogonality of the Legendre polynomials over spherical surfaces. The resulting expressions are

$$
\begin{aligned}
\lambda_{1}= & \frac{3 \phi}{z} \sum_{n=0}^{\infty}\left[(n+1) j_{n+1}(z)-n j_{n-1}(z)\right] \\
& \times\left[\tilde{A}_{n} j_{n}(\tilde{z})-j_{n}(z)-A_{n} h_{n}(z)\right], \\
\lambda_{2}= & -\frac{3 \phi}{\tilde{z}} \sum_{n=0}^{\infty}(2 n+1) j_{n}(z) \\
& \times\left[\tilde{A}_{n} j_{n}^{\prime}(\tilde{z})-\frac{z}{\tilde{z}} j_{n}^{\prime}(z)-A_{n} \frac{z}{\tilde{z}} h_{n}^{\prime}(z)\right], \\
\lambda_{3}= & \frac{3 \phi}{2 z \tilde{z}} \tilde{A}_{0}\left(\frac{\sin (\tilde{z}-z)}{\tilde{z}-z}-\frac{\sin (\tilde{z}+z)}{\tilde{z}+z}\right)+\frac{3 \phi}{z^{2}-\widetilde{z}^{2}} \sum_{n=1}^{\infty}(2 n+1) \\
& \times \tilde{A}_{n}\left[\tilde{z} j_{n-1}(\tilde{z}) j_{n}(z)-z j_{n}(\tilde{z}) j_{n-1}(\tilde{z})\right],
\end{aligned}
$$

where in the expression for $\lambda_{1}$ the $j_{n-1}$-term in the $n=0$ contribution is to be left out. Here, $\phi$ is the volume fraction of the solids, $z \equiv k_{c} a$ and $\tilde{z} \equiv \widetilde{k}_{c} a$ are the nondimensional wavenumbers, and primes denote derivatives. Expressions (19)-(21), together with the cxpression for the effective wave number (12), complete the description of a solid-liquid mixture at low volume fractions.

In the above we have derived expressions for the attenuation and wave speed by calculating the effective wave number directly. An alternative derivation of the attenuation coefficient is to calculate the energy dissipation per wavelength in the mixture and divide the result by the energy per wavelength. The result for the attenuation per unit length is then ${ }^{5,6}$

$$
\alpha=-\frac{3 \phi}{2 z^{2} a} \sum_{n=0}^{\infty}(2 n+1) \operatorname{Re} A_{n} .
$$

It can be shown that the two methods give essentially the same result for the attenuation in the limit $\phi \rightarrow 0$ with $z^{-2} \operatorname{Re} A_{n}$ in the above replaced by $\operatorname{Re}\left(A_{n} / z\right) / \operatorname{Re}(z)$ in the ensemble-averaging method presented here.

The above analysis may be extended to account for the effect of finite volume fraction through a suitable effectivemedium approximation. Sangani ${ }^{11}$ showed that the first correction of $O\left(\phi^{3 / 2}\right)$ to the dilute $O(\phi)$ approximation for bubbly liquids can be simply derived through an effectivemedium approximation. This correction is most significant near the resonance frequency of bubbles, and to correctly capture the behavior near resonance it is important to replace the pure liquid wave number ( $k_{c}$ in the above analysis) by the effective wave number. Thus, in the present context, $z \equiv k_{c} a$ in (19)-(21) for $\lambda_{1-3}$, is replaced by $z_{\mathrm{eff}} \equiv k_{\mathrm{eff}} a$, while the wave number in pure liquid in the expression for $k_{\text {eff }}$, (12), has to be retained. The latter expression is then iterated to obtain a converged solution for $k_{\text {eff }}$. The effective-medium approximations have been found to be quite useful in the related study of light scattering by suspensions (see, e.g., Ref. 14). For very high volume fractions the other physical properties of the so-called effective medium must also be modified. In a separate study, ${ }^{13}$ where we shall 
report experimental data for dense slurries, we shall examine several different versions of effective-medium approximations in more detail.

Finally, the above analysis can be extended in a straightforward manner to account for the particle size distribution when the total volume fraction of the particles is small. Let us write the attenuation by particles of radius between $a$ and $a+d a$ as an attenuation density $\hat{\alpha}(f, a)$ [where $f$ is the frequency of the wave, $f=\omega /(2 \pi)]$ times the volume fraction of those particles $\phi(a) d a$; we shall refer to $\phi(a)$ as the volume fraction distribution. At low volume fractions these contributions can be "summed" over all particle sizes to give for the total attenuation $\alpha_{\mathrm{tot}}(f)$ :

$$
\alpha_{\mathrm{tot}}(f)=\int_{a=0}^{\infty} \hat{\alpha}(f, a) \phi(a) d a .
$$

It is customary to express the particle size distribution in terms of its number density distribution $P(a)$. The volume fraction distribution is related to $P(a)$ by $\phi(a)$ $=\left(4 \pi a^{3} / 3\right) P(a)$.

The effective-medium approach described earlier can also be readily extended to account for the particle size distribution. The coefficients $\lambda_{1-3}$ are first determined as functions of $a$ for an assumed value of the effective wave number and these are integrated after multiplying by $\phi(a) d a$ to yield estimates for the average values of $\lambda_{1-3}$ for the suspension. These are substituted in (12) to determine $k_{\text {eff }}$. If this estimate of $k_{\text {eff }}$ is different from the the assumed value, then $\lambda_{1-3}$ are estimated for the new value of $k_{\text {cff }}$, and the process is repeated until the assumed and estimated values of the effective wave numbers agree with each other.

\section{DISCUSSION AND COMPARISON WITH EXPERIMENTAL DATA}

Figures 1 and 2 show the predictions for the attenuation and wave speed as a function of frequency $f$ for $79 \mu \mathrm{m}$ radius polystyrene particles at a volume fraction of 0.05 . The frequency $f$ in $\mathrm{Hz}$ is related to $\omega$ by $\omega=2 \pi f$. The physical properties used in the computations are given in Table I. ${ }^{15}$ We note that the wave speed only changes if the frequency becomes very large and that these changes coincide with strong changes in the attenuation as well. Hence we expect that the measurement of the phase speed will not provide significantly new information over that obtained from the attenuation measurements alone as far as the problem of determining the size distribution is concerned. On the other hand, since the phase speed at low frequencies is nearly independent of the frequency or $k_{\mathrm{c}} a$, it might be possible to use the low frequency speed data to determine the total volume fraction of the particles regardless of its size distribution. We shall focus in the present study on the results for attenuation as they are the most sensitive to the particle size distribution.

The attenuation of sound waves in a suspension is different from that in pure liquid because of four effects. First, the attenuation of sound in pure solid is different from that in pure liquid, and hence simply the presence of the particles changes the attenuation from that of pure liquid. Second,

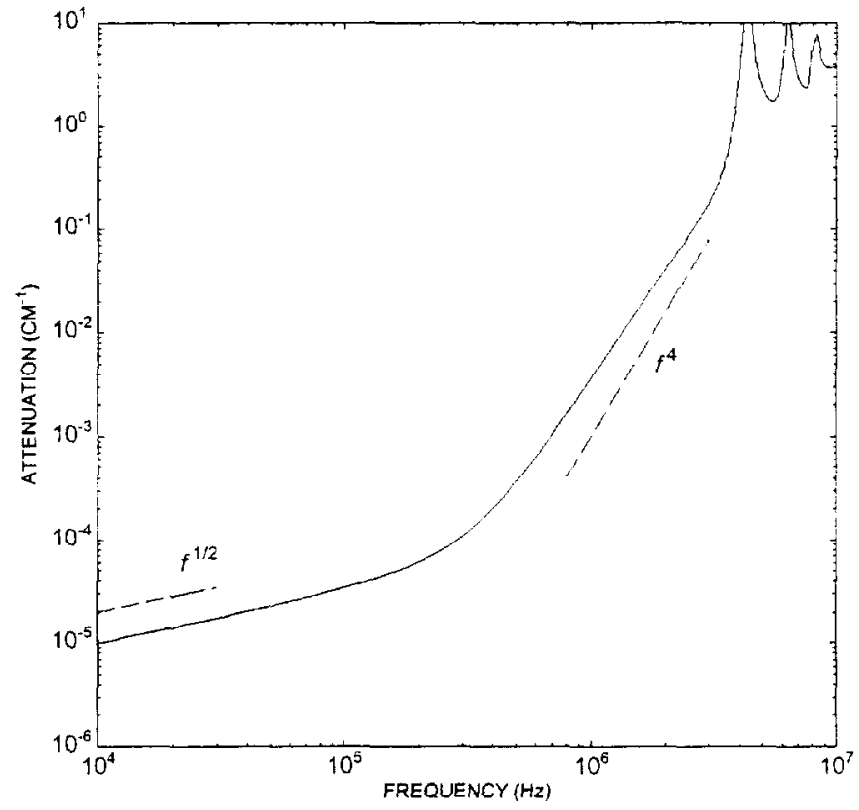

FIG. 1. Example of the dependence of attenuation on frequency $f$ for a mixture of monodispersed polystyrene particles in water. Dashed lines are asymptotic slopes of the attenuation for small and large frequencies.

changes in temperature are different in a solid than in a liquid, and this causes a heat flux through the surface of the particles. This heat flux is out of phase with the sound wave passage and this leads to attenuation referred to as the thermal attenuation. Third is the viscous energy dissipation caused due to the motion of the boundary of the suspended particles. Finally, the fourth effect is the attenuation due to scattering.

Allegra and Hawley ${ }^{5}$ showed that when the particle size is much smaller than the wavelength and much greater than the thermal and viscous penetration depths $(\sigma / \omega)^{1 / 2}$ and

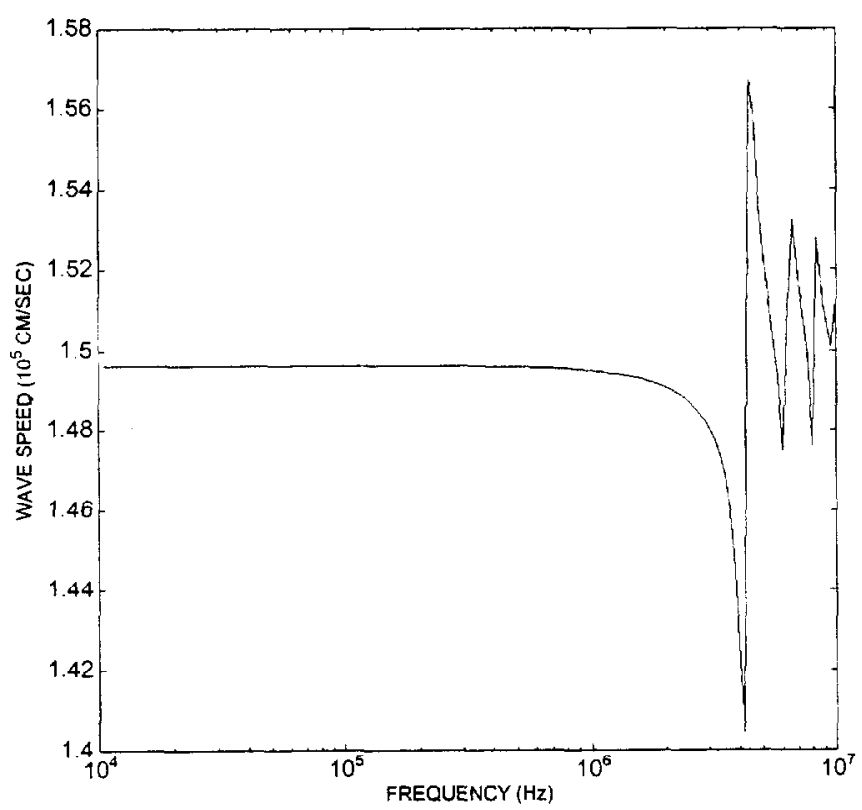

FIG. 2. Example of the dependence of wave speed on frequency $f$ for a mixture of monodispersed polystyrene particles in water. 
TABLE I. The values of the physical properties that are used in this paper. The properties of water and polystyrene were taken from Ref. 6; the properties of glass from various sources, most nolably Ref. 15.

\begin{tabular}{|c|c|c|c|}
\hline & Polystyrene & Glass & Water \\
\hline density $\left(\mathrm{g} / \mathrm{cm}^{3}\right)$ & 1.055 & 2.3 & 1.0 \\
\hline thermal conductivity $(\mathrm{J} / \mathrm{K} \cdot \mathrm{cm} \cdot \mathrm{sec})$ & $1.15 \times 10^{-3}$ & $9.6 \times 10^{-3}$ & $5.87 \times 10^{-3}$ \\
\hline specific heat $(\mathrm{J} / \mathrm{g} \cdot \mathrm{K})$ & 1.19 & 0.836 & 4.19 \\
\hline thernal expansion coefficient $(1 / \mathrm{K})$ & $2.04 \times 10^{-4}$ & $3.2 \times 10^{-6}$ & $2.04 \times 10^{-4}$ \\
\hline attenuation coefficient per freq $\left(\mathrm{sec}^{2} / \mathrm{cm}\right)$ & $10^{-15}$ & $10^{-15}$ & $2.5 \times 10^{-16}$ \\
\hline sound speed $(\mathrm{cm} / \mathrm{sec})$ & $2.3 \times 10^{5}$ & $5.2 \times 10^{5}$ & $1.48 \times 10^{5}$ \\
\hline shear viscosity $\left(\mathrm{g} / \mathrm{cm} \cdot \mathrm{sec}^{2}\right)$ & & & $1.01 \times 10^{-2}$ \\
\hline shear rigidity $\left(\mathrm{g} / \mathrm{cm} \cdot \sec ^{2}\right)$ & $1.27 \times 10^{10}$ & $2.8 \times 10^{11}$ & $\ldots$ \\
\hline
\end{tabular}

$(\nu / \omega)^{1 / 2}$, the resulting viscous and thermal attenuations increase as $f^{1 / 2}$. On the other hand, when the penetration depths are much greater than the particles, both attenuation contributions increase as $f^{2}$. This transition occurs at very low frequencies-about $2 \mathrm{~Hz}$ for $100 \mu$ radius particles in water-and will not be considered here. Attenuation due to scattering becomes important when the nondimensional wave number $z=k_{c} a$ becomes comparable to unity. For small but finite $z$ the scattering losses increase as $f^{4}$. Thus, one expects that the change in the attenuation behavior from $f^{1 / 2}$ at low frequencies to $f^{4}$ at high frequencies will provide an important indication of the particle size. These asymptotic ranges are indicated in Fig. 1. We see that the transition to the $f^{4}$ behavior does not fully occur for the particles considered here. As the frequency is increased particles undergo several resonances as described in more detail below, and this is responsible for the several peaks seen in Fig. 1.

Figure 3 shows the contributions to the total attenuation from each $P_{n}$ mode. The $n=0$ mode corresponds to radial (volume) oscillations of the particles, $n=1$ to the translational oscillations, $n=2$ to the ellipsoidal $P_{2}$-shape deforma-

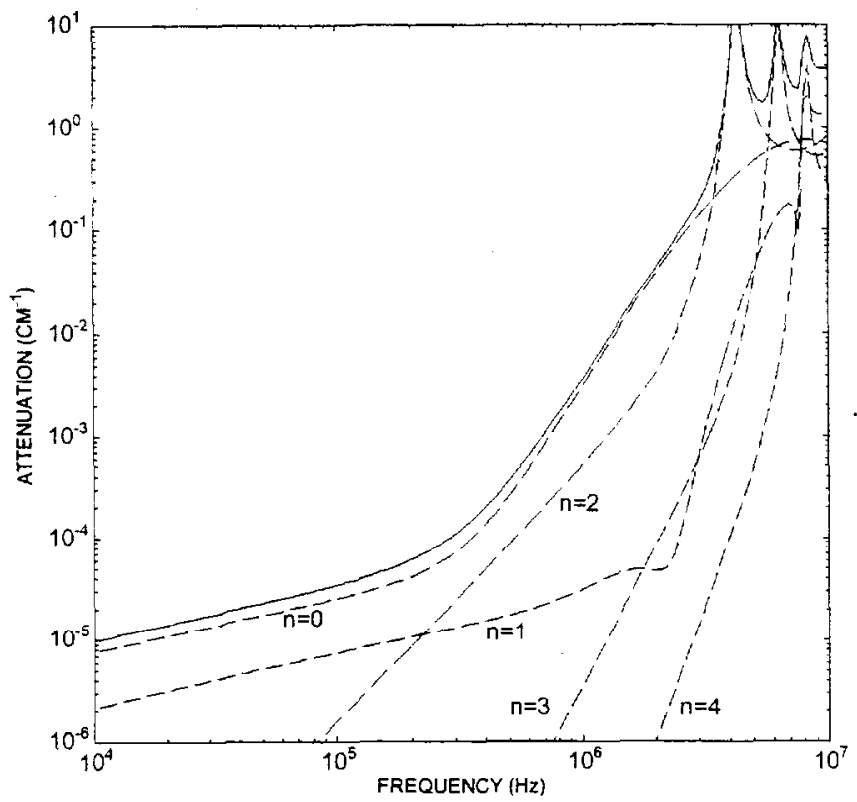

FIG. 3. Contributions from the first five modes $n$ in (19)-(21) to the total attenuation [the imaginary part of $k_{\text {err }}$, which is given by (12)]. Polystyrene particles in water. tion oscillations, and so on. The density of polystyrene particles is essentially the same as that of water, hence the particles' translational oscillations are very small. As a consequence, the viscous attenuation is small and the small frequency behavior is governed by the thermal attenuation of the $n=0$ mode. At higher frequencies the $n=0$ mode begins to increase first as $f^{4}$ due to scattering losses but the contribution from the $n=2$ mode soon becomes important as it undergoes a resonance at about $3 \mathrm{MHz}$ frequency. The $n=3$ and $n=1$ modes undergo resonances next, and so on. We see that the $n=0$ mode undergoes a broad maximum around $9 \mathrm{MHz}$. Although not shown here, it too undergoes a resonance with a sharp downward peak at about $21 \mathrm{MHz}$. Thus, we see that the attenuation varies with frequency in a rather complicated manner at high frequencies owing to various resonances. We should note here that the behavior of this kind for polystyrene particles has also been reported by other investigators in the past. For example, Anson and Chivers ${ }^{16}$ and Ma, Varadan, and Varadan, ${ }^{14}$ who restricted their analysis to scattering losses only, found essentially the same behavior, and in earlier investigations ${ }^{17,18}$ mainly focusing on the determination of waves reflected by immersed objects, high-amplitude reflected waves were found at certain resonance frequencies.

Figure 4 shows attenuation as a function of nondimensional wave number $k_{c} a$ for particles of radii 50 and 79 microns. We see that the curves for these two radii are essentially the same, indicating that, at least for polystyrene particles, the thermal or viscous effects have negligible influence on the resonance frequency. The first resonance corresponding to $n=2$ appears at $k_{c} a \simeq 1.4$.

Allegra and Hawley ${ }^{5}$ tested (22) extensively against their experiments and found very good agreement. However, their particles were always smaller than $1 \mu \mathrm{m}$ radius, so that the wavelength was always much greater than the particle size. No resonance behavior was observed in their experiments. Although the above-mentioned paper by Ma, Varadan, and Varadan ${ }^{14}$ presents experimental data on light scattering in the small-wavelength regime, no data on attenuation of sound waves by particles were presented. To test how well the theory works for larger particle sizes, we carried out an experiment that will be described in detail (along with more experiments on concentrated slurries) elsewhere. ${ }^{13}$ In this experiment the attenuation of sound waves was measured in a frequency range of $1-10 \mathrm{MHz}$ in a solid-liquid mixture of 


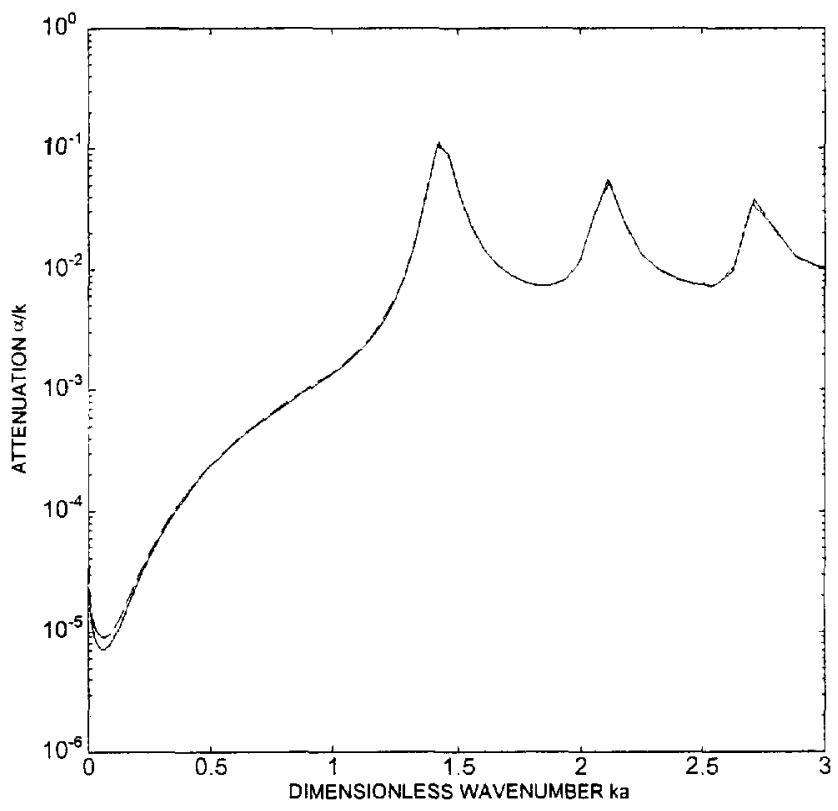

FIG. 4. Attenuation divided by wave number as a function of the wave number times the particle radius in the scattering regime for monodispersed polystyrene particles $(-, a=79 \mu \mathrm{m} ;--, a=50 \mu \mathrm{m})$. The volume fractions of the particles in both cases are the same and equal to 0.05 .

polystyrene particles with $79 \pm 3 \mu$ mean radius and $1.8 \mu$ standard deviation at 0.05 volume fraction. Monochromatic tonebursts, at incremental frequencies, were transmitted by a transducer on one side of a small vessel in which the mixture was being stirred; a second transducer received the signal and sent it to a LeCroy 9310A digital oscilloscope. The amplitude of the signal for pure water was measured, as was that for the solid-liquid mixture. The excess attenuation was determined by

$$
\alpha=-\frac{1}{d} \log \left(\frac{V_{\text {mix }}}{V_{\mathrm{H}_{2} \mathrm{O}}}\right),
$$

where $d$ is the distance between the transducers and $V_{\text {mix }}$ and $V_{\mathrm{H}_{2} \mathrm{O}}$ are the voltage amplitudes of the received signals in the mixture and pure water, respectively. The distance between the transducers was $2 \mathrm{in}$. at low frequencies and $1 \mathrm{in}$. at higher frequencies; this was necessary because the attenuation at higher frequencies was too large to produce significant signal-to-noise ratio in the larger vessel.

Figure 5 shows the comparison between theory and experiment. At the two gaps in the frequency domain (where the theory predicts very high peaks) the attenuation became again so large that the signal-to-noise ratio vanished even in the smallest vessel. Good agreement is found between experiments and the theory except near resonance frequencies where small differences are seen. There are two possible reasons for these small differences. The first is concerned with the finite volume-fraction effect. To investigate this we have also plotted in Fig. 5 a result from an effective-medium approach described in the previous section. The resulting attenuation changes, but in the wrong direction. The second reason is that the particles were not exactly monodispersed. Using the method described in the previous section, a log-

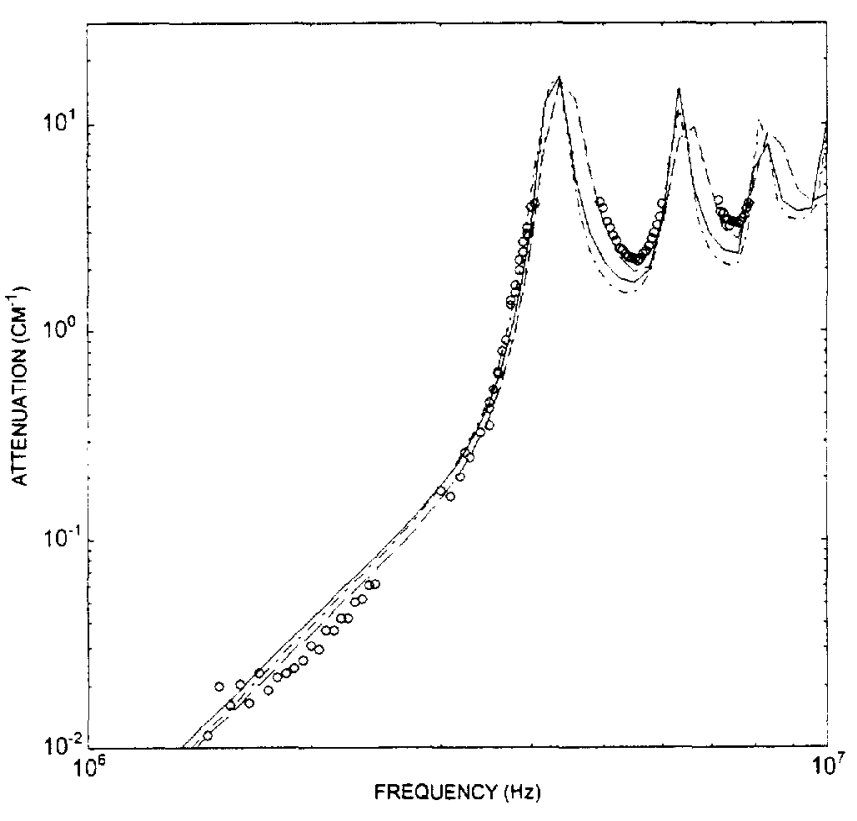

FIG. 5. Comparison with experimental data for the attenuation as a function of frequency. Polystyrene particles of radius $a=79 \mu \mathrm{m}$ and 0.05 volume fraction. $O$, experiments; —_ t t t -..-., theory for monodispersed particles with effective medium correction for finite volume fraction effects; ---, theoretical result with a particle size distribution with a mean particle radius of $77 \mu \mathrm{m}$ and standard deviation of $2.5 \mu \mathrm{m}$ (this is within the range specified by the manufacturer).

normal particle size distribution was introduced with a mean radius of 77 and $2.5 \mu \mathrm{m}$ standard deviation, which lies within the manufacturers' specifications. The result for the attenuation, the dashed curve in Fig. 5, shows close agreement with the data. Thus, we conclude that the agreement between the theory and experiment is excellent, and that the small observed differences are due to small polydispersity of the suspension.

The attenuation behavior displayed by polystyrene particles is not generic, as can be seen from Fig. 6 which shows the attenuation behavior for glass particles. Since the density of the glass particles is significantly different from that of water, the glass particles execute significant translational oscillations. As a consequence, the low-frequency behavior is completely governed by the viscous effects and the $n=1$ mode. Note that the small frequency attenuation is about two orders of magnitude greater for glass particles than for the polystyrene particles. Also we see a considerably different behavior at higher frequencies. The attenuation does not seem to peak at several frequencies. Rather, for each $n$ we see broad "hills" separated by narrow "valleys." The total attenuation does not appear to go through several resonances. The difference in the behavior for the glass and polystyrene particles at these high frequencies seems to arise mainly from the different elastic properties of the two materials.

\section{THE INVERSE PROBLEM}

We now consider the inverse problem: given the total altenuation $\alpha_{\text {tot }}$ as a function of $f$ we wish to determine $\phi(a)$ using (23). The straightforward method of solving the inte- 




FIG. 6. Attenuation by monodispersed glass particles (of $79 \mu \mathrm{m}$ radius) in water as a function of frequency and the contributions from the first three modes $n$ in (19)-(21) to the total attenuation [the imaginary part of $k_{\text {eff }}$, which is given by (12)].

gral equations, i.e., discretizing the integral domain into a number of elements and converting the integral equation into a system of linear equations in unknowns $\phi\left(a_{k}\right)$ at a selected number of points $a_{k}$ in the domain, cannot be used since the resulting equations will be ill conditioned. Figure 7 illustrates the ill-posed nature of the problem. Figure 7(a) shows two very different particle distributions whose attenuation versus frequency curves are seen in Fig. 7 (b) to be essentially the same. These curves were obtained by starting with a smooth, log-normal particle size distribution [the dashed curve in Fig. 7(a)] and generating the attenuation versus frequency data using the forward theory [the circles in Fig. 7(b)]. A $1 \%$ random noise was then added to the data and (25) with $\epsilon=0$, which is equivalent to the integral equation (23), was subsequently solved to yield the particle size distribution indicated by the solid line in Fig. 7(a). The pluses in Fig. 7(b) correspond to the attenuation determined from the forward theory using the new particle distribution. Note that the attenuation is evaluated with a smaller frequency increment than the one used for the original distribution. We see that the attenuation from the two distributions agree with each other to within $1 \%$ for the frequencies marked by circles. The highly oscillatory particle distribution does show an oscillatory behavior in between the frequency increments, particularly at $10 \mathrm{MHz}$, but these oscillations occur only for a very narrow frequency range and would have been missed altogether had the attenuation been determined only at the input frequencies.

\section{A. Method}

Since the true particle distribution is expected to be smooth, we must only allow solutions that are reasonably smooth. There are several ways of accomplishing this. In the
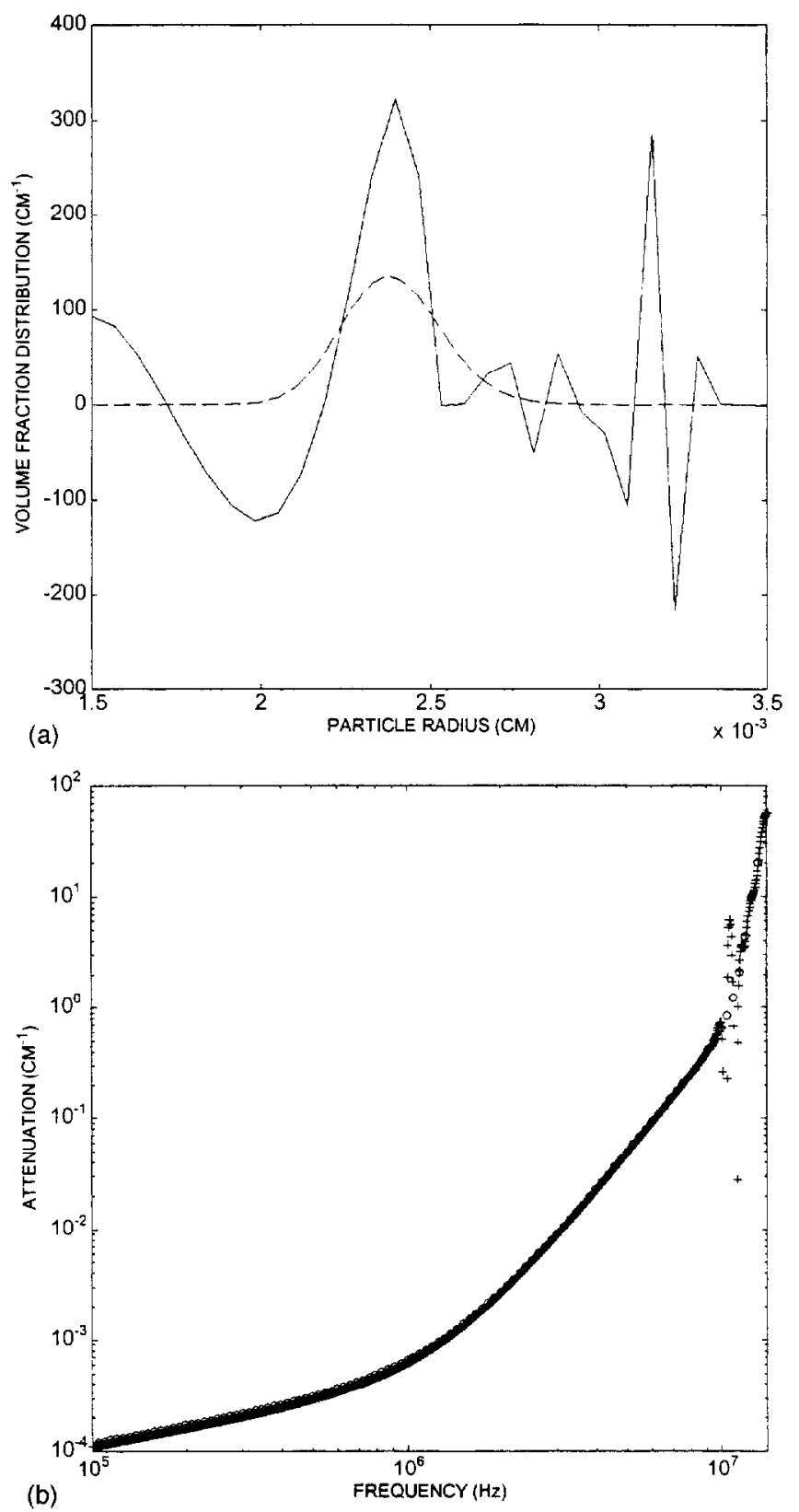

FIG. 7. Influence of fluctuations superimposed on the volume fraction distributions (a) on attenuation data (b). In (b) the circles correspond to the result when using the dashed distribution of (a) and the pluses when using the solid line in (a).

present study, we shall use primarily a regularization technique due to Tikhonov ${ }^{8}$ which was successfully used for bubbly liquids by Duraiswami. ${ }^{2}$ An alternative method is presented at the end of this section. Accordingly, we multiply (23) with $\hat{\alpha}(f, a) d f$ and integrate over the frequency range to obtain a simpler integral equation in which the right-hand side is only a function of $a$ :

$$
\begin{gathered}
\int_{f_{\min }}^{f_{\max }} \int_{a_{\min }}^{a_{\max }} \hat{\alpha}(f, a) \hat{\alpha}\left(f, a^{\prime}\right) \phi\left(a^{\prime}\right) d a^{\prime} d f \\
=b(a) \equiv \int_{f_{\min }}^{f_{\max }} \alpha_{\mathrm{tot}}(f) \hat{\alpha}(f, a) d f,
\end{gathered}
$$

where $\left(a_{\min }, a_{\max }\right)$ and $\left(f_{\min }, f_{\max }\right)$ are the radius and frequency ranges. The above integral equation is now regular- 
ized as explained below by adding a small term $\epsilon(\phi$ $-l^{2} \phi^{\prime \prime}$ ) (where primes denote derivatives) to its left-hand side. Thus, we obtain

$\epsilon\left[\phi(a)-l^{2} \phi^{\prime \prime}(a)\right]+\int_{a_{\min }}^{a_{\max }} K\left(a, a^{\prime}\right) \phi\left(a^{\prime}\right) d a^{\prime}=b(a)$,

where $l$ is a suitably chosen lengthscale and $K\left(a, a^{\prime}\right)$ is a kernel defined by

$$
K\left(a, a^{\prime}\right)=\int_{f_{\min }}^{f_{\max }} \hat{\alpha}(f, a) \hat{\alpha}\left(f, a^{\prime}\right) d f .
$$

Equation (25) is an integro-differential equation and needs two boundary conditions. Usual practice is to take the derivative of $\phi(a)$ to be zero at the two end points:

$$
\phi^{\prime}\left(a_{\min }\right)=\phi^{\prime}\left(a_{\max }\right)=0 .
$$

Note that $a_{\min }$ and $a_{\max }$ are not known $a$ priori in general. One expects $\phi$ to be zero also at the two end points. Thus, the range $\left(a_{\min }-a_{\max }\right)$ must be determined by trial and error so that both $\phi$ and its derivatives are approximately zero at the extreme values of $a$.

Now it can be shown that the solution of (25) subject to the boundary conditions given by (27) minimizes

$$
E+\epsilon \int_{a_{\min }}^{a_{\max }}\left[\{\phi(a)\}^{2}+l^{2}\left\{\phi^{\prime}(a)\right\}^{2}\right] d a,
$$

where $E$ is the measure of error between the actual attenuation and the computed attenuation:

$$
E=\int_{f_{\min }}^{f_{\max }}\left|\int_{a_{\min }}^{a_{\max }} \hat{\alpha}(f, a) \phi(a) d a-\alpha_{\mathrm{tot}}(f)\right|^{2} d f .
$$

Since both $E$ and the second term in (28), i.e., the integral, are non-negative, minimization of (28) ensures that the solution of (25) will be free from large oscillations in $\phi$. In other words, highly oscillatory distributions such as the one shown in Fig. 7(a) are rendered inadmissible when (25) is solved with finite, positive $\epsilon$ in place of the original integral equation (24). Thus, we have regularized the problem of determining $\phi$.

If we choose a large $\epsilon$, then we decrease the oscillations in $\phi$ but increase the error in $\phi(a)$ since then the equation solved is significantly different from the original integral equation. Small $\epsilon$, on the other hand, yields unrealistic $\phi(a)$ having large oscillations when the data $\alpha_{\text {tot }}(f)$ are not exact. An optimum choice of $\epsilon$ then depends on the magnitude of uncertainty/error in the attenuation-frequency data. In the calculations we shall present here the exact $\alpha_{\text {tot }}(f)$ is first determined using the forward theory for a given $\phi(a)$ and a small random noise of about $1 \%$ magnitude is added to it before the inverse calculations are made (the effect of noise magnitude is discussed below). Thus, we have an estimate of the error in the data, but in general this estimate may not be known reasonably accurately. To determine the optimum $\epsilon$, we solve (25) for several different $\epsilon$ 's and plot $E$ versus $\epsilon$ to find a minimum in $E$. This, however, may lead to distributions in which $\phi(a)$ may have unphysical negative values for some $a$. The constraint $\phi(a) \geqslant 0$ for all $a$ is satisfied $a$ posteriori by setting $\phi(a)=0$ for all $a$ 's for which the solu- tion of (25) gave negative values of $\phi$. The computed value of $E$ for a given $\epsilon$ is then based on $\phi(a) \geqslant 0$.

The integro-differential equation (25) was solved as follows. After discretizing the domain $\left(a_{\min }-a_{\max }\right)$ into $N-1$ equal segments and the frequency domain into $M-1$ logarithmically equal segments we first evaluate the kernel $K\left(a_{i}, a_{j}\right)$ for $i, j=1,2, \ldots, N$ [cf. (26)] using a trapezoidal rule for the integration over the frequency range. As pointed out by Duraiswami, ${ }^{2}$ it is essential to calculate the integral over particle radius very accurately. We assume that $\phi(a)$ varied in a piecewise linear manner in each segment and use a 12 point Gauss-Legendre quadrature to evaluate the integral in (25). A second-order central difference formula was used to evaluate $\phi^{\prime \prime}(a)$ at all points except the end points $a_{\min }$ and $a_{\max }$. The boundary conditions $\phi^{\prime}\left(a_{\min }\right)=0$ and $\phi^{\prime}\left(a_{\max }\right)$ $=0$ were approximated using, respectivcly, second-order forward and backward difference formulas. Application of (25) at all the discretization points together with the boundary conditions can be expressed with the above scheme as a system of linear equations:

$$
\sum_{j=1}^{N} A_{i j} \phi_{j}=b_{i}, \quad i=1,2, \ldots, N
$$

where $\phi_{j}=\phi\left(a_{j}\right)$ and $b_{i}=b\left(a_{i}\right)$. The above set of equations was normalized by dividing all the equations with the greatest element of the kernel $K\left(a_{i}, a_{j}\right), K_{m}$ for all $i, j$, times the segment length $\Delta a=\left(a_{\max }-a_{\min }\right) /(N-1)$. This set of equations was subsequently solved using a standard IMSL subroutine for linear equations.

Once $\phi_{j}$ are determined for a selected value of $\epsilon$, we satisfy the constraint $\phi_{j} \geqslant 0$ by setting, as mentioned earlier, $\phi_{j}=0$ for all negative $\phi_{j}$. The error $E$ as given by (29) was subsequently evaluated using a trapezoidal rule for integration over the frequency range. The optimum value of $\epsilon$ was determined by stepping logarithmically through several values of $\epsilon$ and plotting $E$ versus $\epsilon$.

A typical result $\left(N=30, M=112, f_{\min }=0.1 \mathrm{MHz}, f_{\max }\right.$ $=17 \mathrm{MHz}, a_{\min }=15 \mu \mathrm{m}$ and $a_{\max }=35 \mu \mathrm{m}$ ) for the error $E$ in the resulting attenuation as a function of $\epsilon$ is shown in Fig. 8. Note that $\epsilon$ here is the actual $\epsilon$ divided by $K_{m} \Delta a$. We see a clearly defined optimum value of $\epsilon$. Computations were also made with larger $M$ to confirm that the resulting volume fraction distribution was not affected by the further refinement in the integration over the frequency range. A remark should also be made of the choice for the length $l$ in (25). We may regard both $\epsilon$ and $l$ as parameters to be chosen so as to minimize the error $E$. Taking $l=\left(a_{\max }-a_{\min }\right) / n$ we computed $E$ by varying both $\epsilon$ and $n$ with $n$ varied from 1 to $N$. The three-dimensional plot of $E$ versus $n$ and $\epsilon$ showed that $E$ was much more sensitive to the choice of $\epsilon$ than it was to $n$. In general, the results with $n$ close to $N$ were slightly better than with those near $n=1$. Based on this observation we chose $n=30$. For larger values of $N(N>40)$ we found that chousing $n=N$ led to more oscillatory behavior for $\phi_{j}$. This is to be expected since choosing larger $n$, and, hence, smaller $l$, permits larger values of $\phi^{\prime}(a)$. 


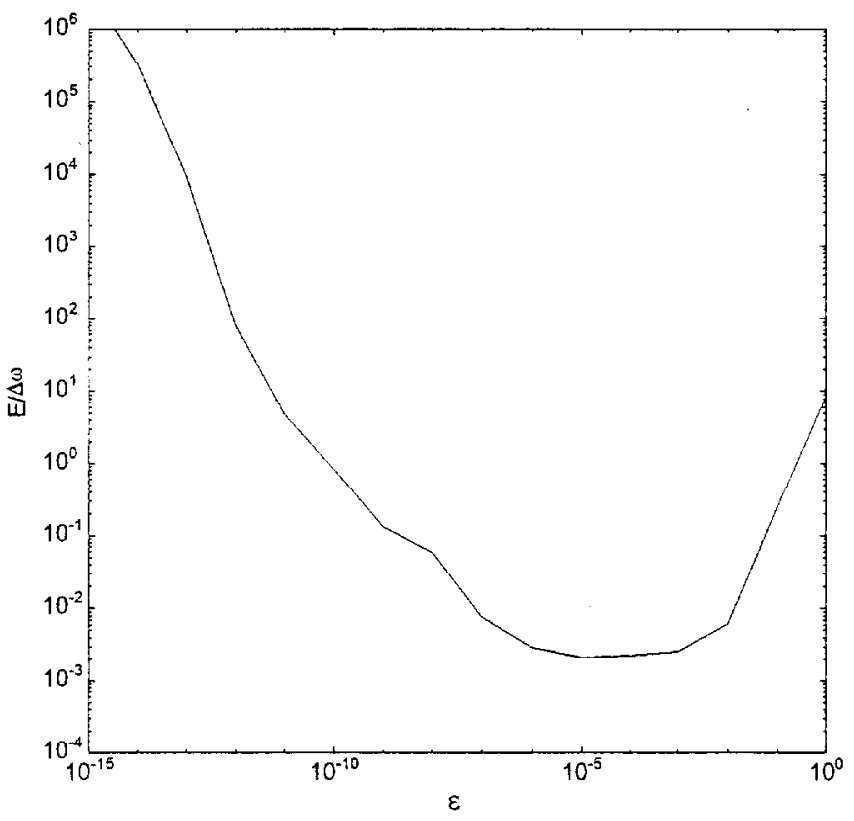

FIG. 8. Typical dependence of the error in the attenuation for the solved volume fraction distribution as a function of the regularization parameter $\epsilon$. The (small) parameter $\epsilon$ should be chosen such that this error is minimized. The minimum was always found to be well-defined

\section{B. Results and discussion}

We now present results for the volume fraction distribution obtained using the above technique. As mentioned earlier, we used the forward theory to generate attenuation data for an assumed volume fraction distribution. Small random noise can be added to the data thus generated to mimic possible errors arising in the attenuation measurement. This is satisfactory since we are primarily interested in assessing the procedure for solving the inverse problem. If the procedure gives erroneous results even for this case, it will certainly break down in practice using the experimentally generated data.

The frequency range over which the attenuation measurements are carried out in our laboratory is $0.1-15 \mathrm{MHz}$. We shall choose here the same range to investigate the success and limitations of the above technique to solve the inverse problem although we shall also consider cases with a larger frequency range to inquire if better estimates of $\phi(a)$ could be achieved if the attenuation data at higher frequencies were to be made available. This is important since the acoustic instruments operating up to $150 \mathrm{MHz}$ are available.

We consider first particle sizes that are of the same order of magnitude as the wavelength somewhere in this frequency range, which is the case for particles of about $10-100 \mu$ radius (for larger particles observed behavior of the attenuation is shifted to lower frequencies). A particle size distribution that is often used is a log-normal distribution, which results in volume fraction distributions such as the smooth one shown in Fig. 7(a). We altempt therefore to recover that distribution from the corresponding attenuation data. As in the forward problem, we shall investigate polystyrene particles and glass particles in water, as the first are almost neutrally buoyant and deformable whilc the latter are very

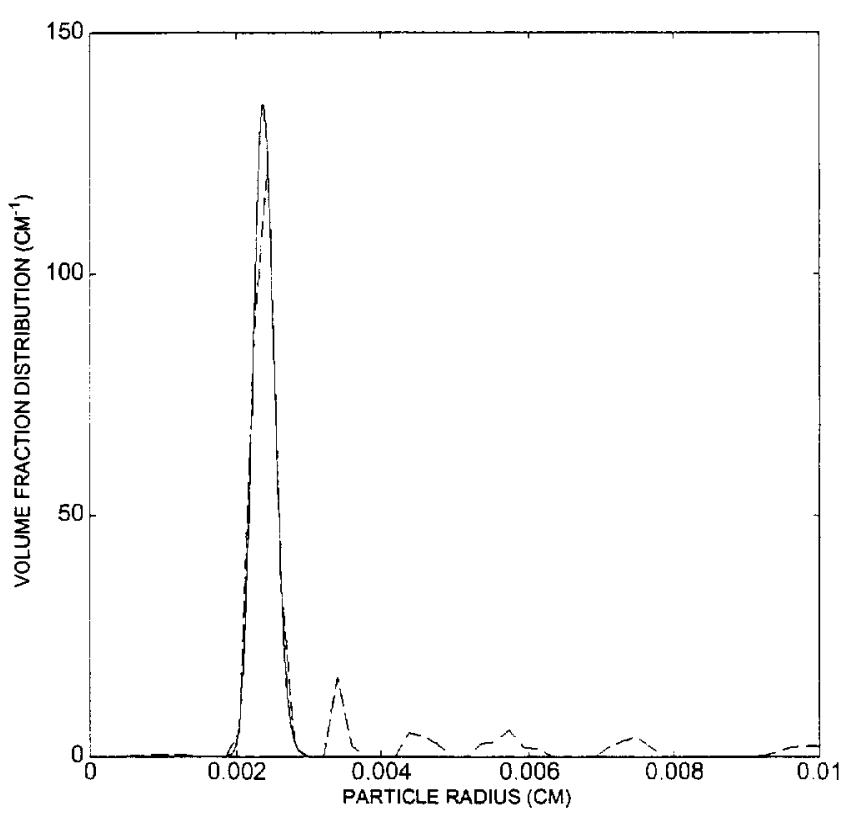

FIG. 9. Solving the inverse problem for polystyrene particles. The solid line is the volume fraction distribution used to generate attenuation data [shown in Fig. 12(a), with $f_{\max }$ as indicated by a square]; the dashed line is the solution of the inverse problem when taking the particle radius range to be $1-100 \mu \mathrm{m}$ and using 50 "bins" of particle sizes.

rigid and much heavier than water; the physical properties used in the present calculations are listed in Table I.

We begin with the results for polystyrene particles with a narrow size distribution in the range of $20-30 \mu \mathrm{m}$. The particle size range for the inverse calculations is first taken to be much greater-5-100 $\mu \mathrm{m}$; the frequency range was 0.1-17 MHz. Figure 9 shows that the volume fraction distribution as evaluated from the inverse technique is in very good agreement with the input distribution. The result for the size distribution can be improved further by making the particle size range smaller (a close-up of the improved result is shown in Fig. 11).

In Fig. 10 we consider a more complicated, bimodal size distribution in the range of $20-45 \mu \mathrm{m}$ with peaks at about 25 and $38 \mu \mathrm{m}$. The attenuation as a function of frequency for this distribution is shown in Fig. 10a. The maximum frequency used for inverse calculations is indicated by a square; it is seen that the frequency range includes the first two resonance peaks of the attenuation curve. From Fig. 10(b) we see once again that the inverse procedure recovers this distribution very well.

One of the difficulties in solving an ill-posed problem is that small errors in the input (attenuation) data can cause large changes in the solution. Of course, errors are always present in the experimentally obtained attenuation data. The calculations presented so far were made with no added noise. To mimic the practical situation, we added random noise of 5\% standard deviation to the input data; this is about the same as the order of magnitude of the errors present in the experimental results shown in Fig. 5. The resulting volume fraction distribution, shown in Fig. 11, does confirm that small fluctuations in the input data only cause small deviations in the output. When the calculations were repeated with 

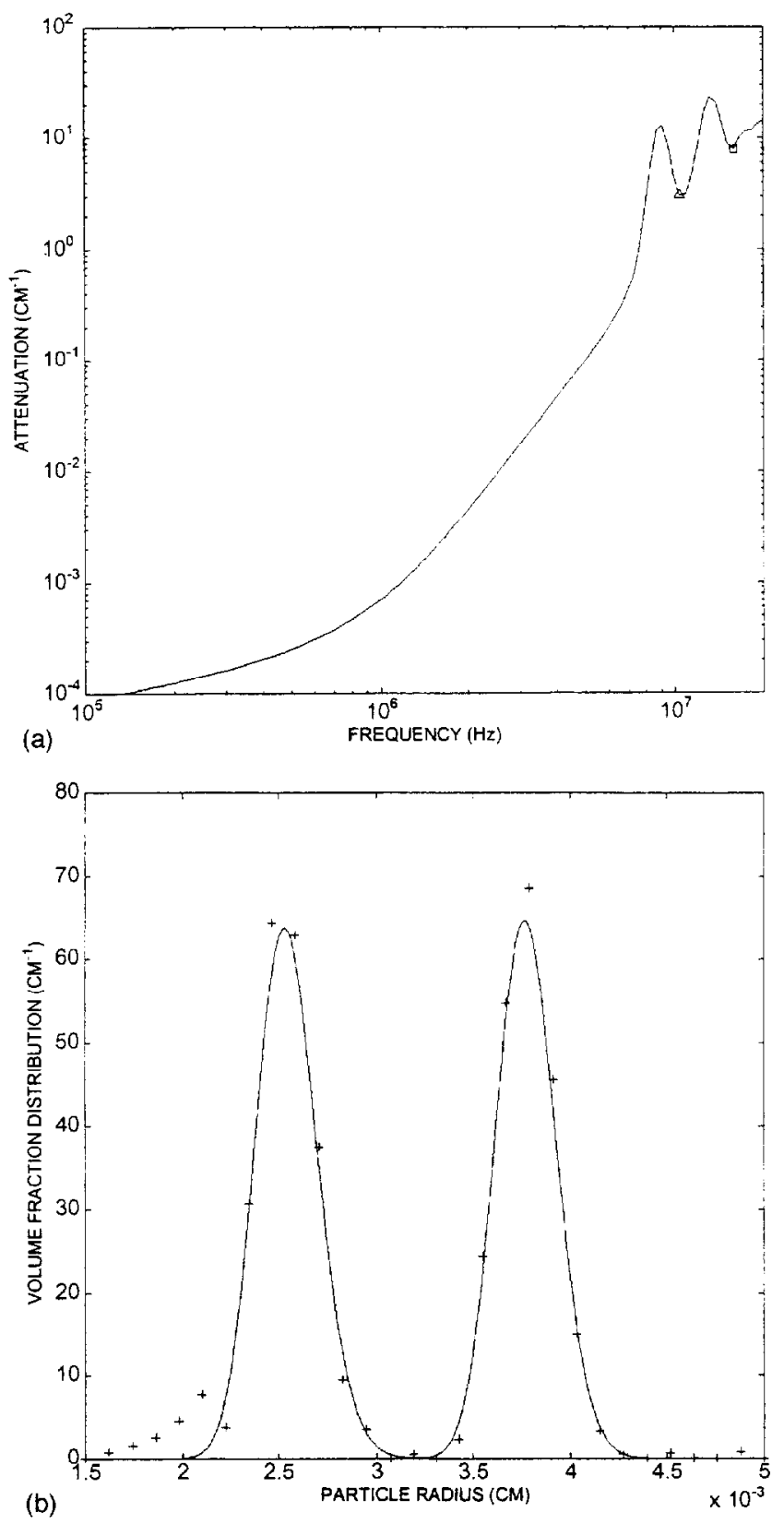

FIG. 10. Attenuation (a) and the solution of the inverse problem (b) for a bimodal distribution of polystyrene particles, using 30 particle size bins. In (b), the solid line is the exact result, markers represent the inverse problem solution when using for $f_{\max }$ the value indicated by a square in (a). Results when cutting of the frequency range at the point marked by a triangle are discussed along with Fig. 14

a noise of $10 \%$ standard deviation, the computed particle size distribution was found to be considerably different from the input distribution, although the main features of the size distribution were preserved by the inverse computations.

The results discussed so far suggest that the inverse problem can be solved with reasonable success. We now illustrate some limitations. The inverse method gave erroneous particle size distributions for smaller particles when the same frequency range as the above was used. Of course, in order that the size of the particles be determined there must be at least one transition-from the thermal attenuation dominated regime to the scattering dominated regime which

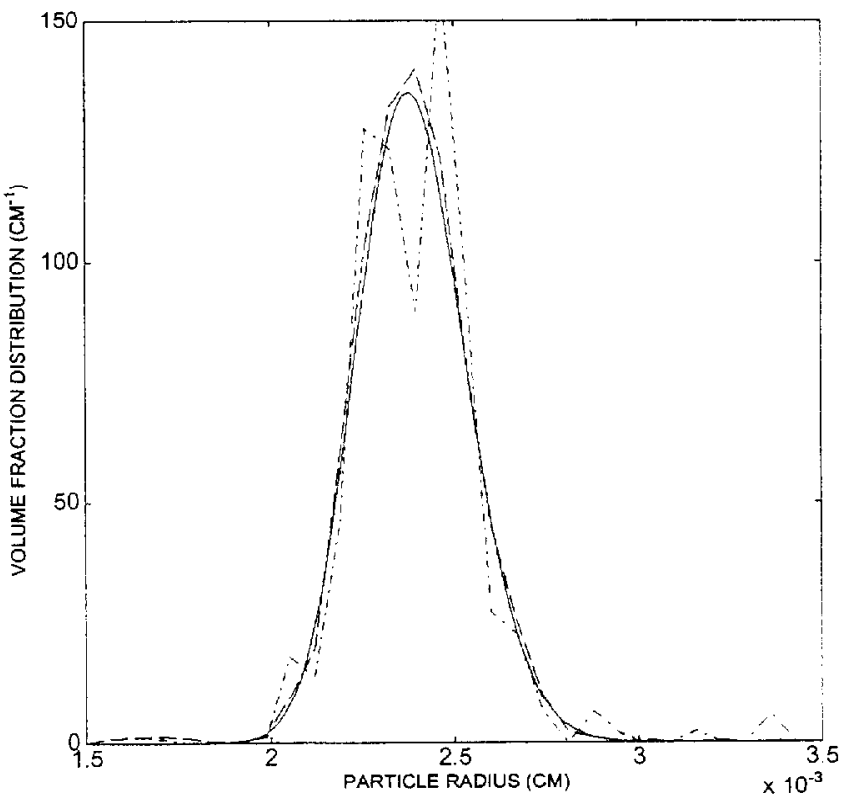

FIG. 11. Solution of the inverse problem when random noise of $5 \%$ standard deviation is introduced in the attenuation (input) data. Solid line is the exact result; the broken line is the result when no noise is introduced (already shown in Fig. 8); and the dash-dotted curve is the result after introduction of the noise. Polystyrene particles in water.

occurs roughly speaking at $k_{c} a=O(1)$. If the particles are very small, then this transition may not occur over a fixed frequency range. However, as we shall presently see, the results are very sensitive to the frequency range chosen for computations even when this transition is included in the range.

Figure 12 shows the effect of varying $f_{\max }$ on the computed distribution. As seen in the figure the resonance in the shape oscillations of the (polystyrene) particles leads to a change in the slope of the curve just before the first resonance. This transition occurs just beyond the point marked by a circle in Fig. 12(a). We see a marked improvement in the results in Fig. 12(b) when $f_{\max }$ is chosen corresponding to a point marked plus in Fig. 12(a) over those obtained with a point corresponding to the circle which does not include the second change in slope. The point marked plus corresponds to a frequency greater than the frequency at which the second change in slope occurs for larger particles but smaller than that for smaller particles. This seems to give rise to an inverse solution which is reasonably accurate for larger particles but not for smaller particles. Also shown in Fig. 12(b) are the results when $f_{\max }$ is chosen to coincide with the end of first peak, the point marked square in Fig. 12(a). This is seen to yield very accurate results for the size distribution.

One might suppose that covering a broad enough frequency range will alleviate the difficulties seen above. This, unfortunately, is not the case. Figure 13 shows the results for three different $f_{\max }$. The dashed curve corresponds to cutting off the frequency range at the end of first peak as in Fig. 12, the dashed-dotted line to the end of three peaks, and the dotted line to $10^{9} \mathrm{~Hz}$, a frequency about 50 times greater than the first resonance frequency. We see that the rcsults of 

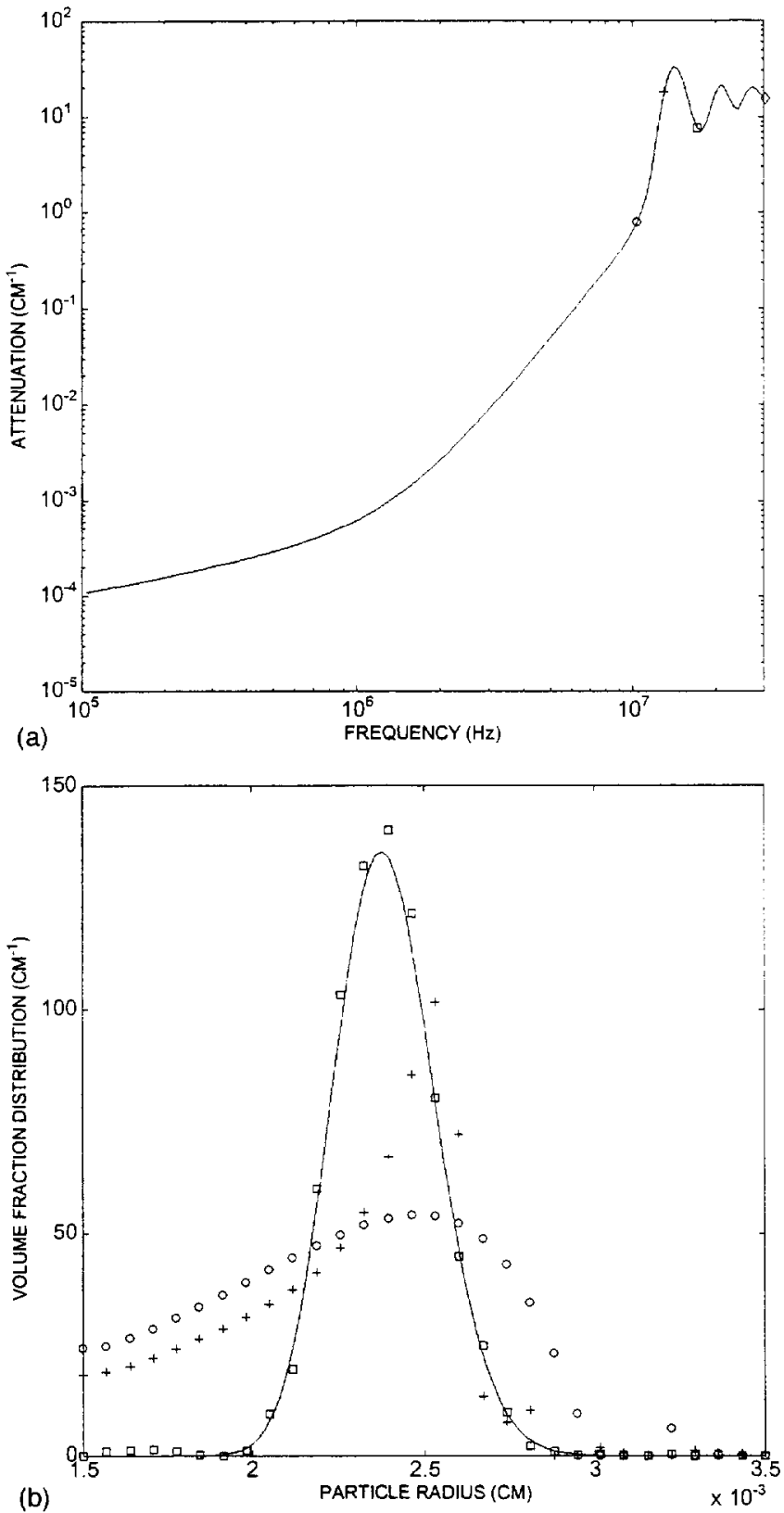

FIG. 12. Influence of the size of the frequency range over which attenuation is specified on the solution of the inverse problem. Polystyrene particles. (a) Input-attenuation data and four different upper bounds on the frequency. (h) Results from the inverse problem from these different ranges, using the same marker type. The solid line is the exact result; $\square$, result when cutting off the frequency range just at the end of the first peak in the attenuation; + , result when cutting of the frequency range after the second change in slope of the attenuation; and $O$, result when cutting off before the second change in slope. Cutting off the frequency range at the point marked " $\diamond$ " is discussed along with Fig. 13.

inverse calculations actually deteriorate if a much larger range of frequency is employed, notwithstanding the fact that measurements over such a broad frequency range could itself be a very challenging task. One may rationalize this result as follows. As seen in Fig. 1 a monodisperse suspension will exhibit several resonance frequencies corresponding to various shape oscillation $P_{n}(n=2,3, \ldots)$ modes. Thus, a second peak in the attenuation-frequency curve for polystyrene particles may correspond either to say, a $P_{3}$ mode of a larger

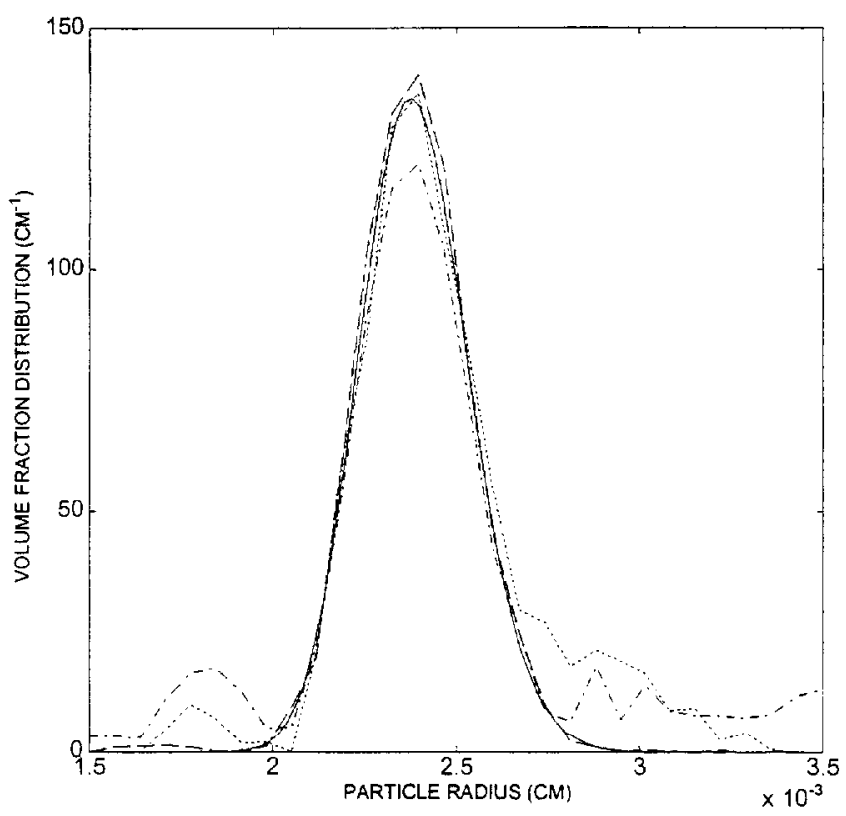

FIG. 13. Too big a frequency range over which the attenuation is available for polystyrene particles also detcriorates the result: the solid line is the exact result; the dashed line is the inverse problem result when using attenuation data of Fig. 12(a) below the point marked by " $\square$," the dashed-dotted line represents the result when this end point is shifted to the point marked by " $\diamond$ " and the dotted line is the result when this end point is shifted to $1000 \mathrm{MHz}$.

particle, or may correspond to a $P_{2}$ mode of a smaller particle. In our calculations we used only up to the first six modes $(n \leqslant 5)$, but in practice the acoustic response may be further complicated by the higher-order modes for frequencies of order $10^{9} \mathrm{~Hz}$ considered here.

Since including a wide frequency range with several resonance peaks seems to adversely affect the inverse calculation, one may consider cutting off the attenuation data beyond first peak. This, however, may not work if the distribution is truly bimodal as was the case considered earlier in Fig. 10. If we omit the second resonance peak from the attenuation data by considering a maximum frequency that is less than the point marked square in Fig. 10(a), say, that marked by the circle, we get a poor inversion as shown in Fig. 14. The inverse technique computes accurately the volume fraction distribution of larger particles whose resonance was included in the data but fails to predict that for smaller particles.

Figure 15 shows results for a broad, unimodal distribution. The resonance peaks of different particles overlap in this case resulting in the absence of peaks in the attenuationfrequency curve [Fig. 15(a)]. Figure 15(b) shows the results of inversion for three different cut-off frequencies. The largest frequency, marked by a square in Fig. 15(a), is larger than the second transition frequency of small as well as large particles, and this seems to produce excellent inverse results.

In most of the inverse calculations shown so far which yielded poor results, we note that the failure is particularly severe for smaller particles. One may rationalize this by observing that the total error $E$ will be dominated by the errors at large frequencies since the attenuation there is very large. 


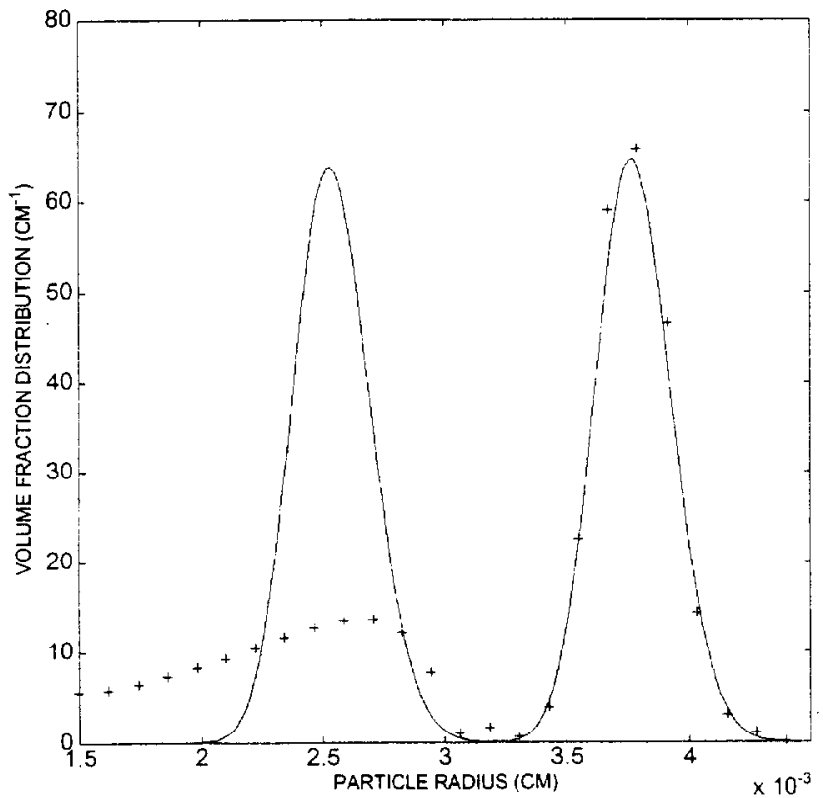

FIG. 14. As in Fig. 10(b), but after cutting off the frequency range over which the attenuation was given between the first and second (attenuation) peak, indicated by a triangle in Fig. 10(a).

When $k_{c} a_{\min }<1$ in the frequency domain that is considered, the small particles' volume fraction is seen from Figs. 12(b) and 14 to be underestimated, while the large particles' volume fraction is overestimated. To decrease the relative importance of the attenuation at high frequencies, we solved a slightly different inverse problem in which both the attenuation and $\hat{\alpha}$ were divided by $f^{2}$. However, only small improvements were found by modifying the attenuation data this way. The inverse-problem result shown in Fig. 14 was in fact obtained in this way.

Some insight into why the choice of $f_{\max }$ drastically affects the results may be gained from Fig. 16, which shows the three-dimensional plots for the kernel $K\left(a_{i}, a_{j}\right)$ for the same values of $f_{\max }$ as considered in Fig. 12. We see that when $f_{\max }=10.4 \mathrm{MHz}$, corresponding to the circle in Fig. 12(a), the kernel has a maximum for $a_{i}=a_{j}=a_{\max }$. The kernel for smaller particles is very small and, as a consequence, the inverse technique could determine the larger particle size volume fraction correctly but failed for smaller particles. In contrast to this the kernel for $f_{\max }=17.1 \mathrm{MHz}$, corresponding to the end of first peak, shows significant variations for a wide range of values of $a_{i}$ and $a_{j}$, and this apparently leads to a much better inverse solution. Finally, the kernel for $f_{\max }=30.4 \mathrm{MHz}$, corresponding to the end of the third resonance peak, shows a less pronounced structure.

It is also instructive to examine the kernel and the results of inverse calculations for the problem of determining bubble-size distribution in bubbly liquids examined by Duraiswami. ${ }^{2}$ The inverse procedure works very well for bubbly liquids as can be seen from Fig. 17(a) which shows the input and computed bubble size distributions to be in excellent agreement. The kernel for this case has smooth variations over a wide range of bubble radii as seen in Figure 17(b). The attenuation as a function of frequency is shown in
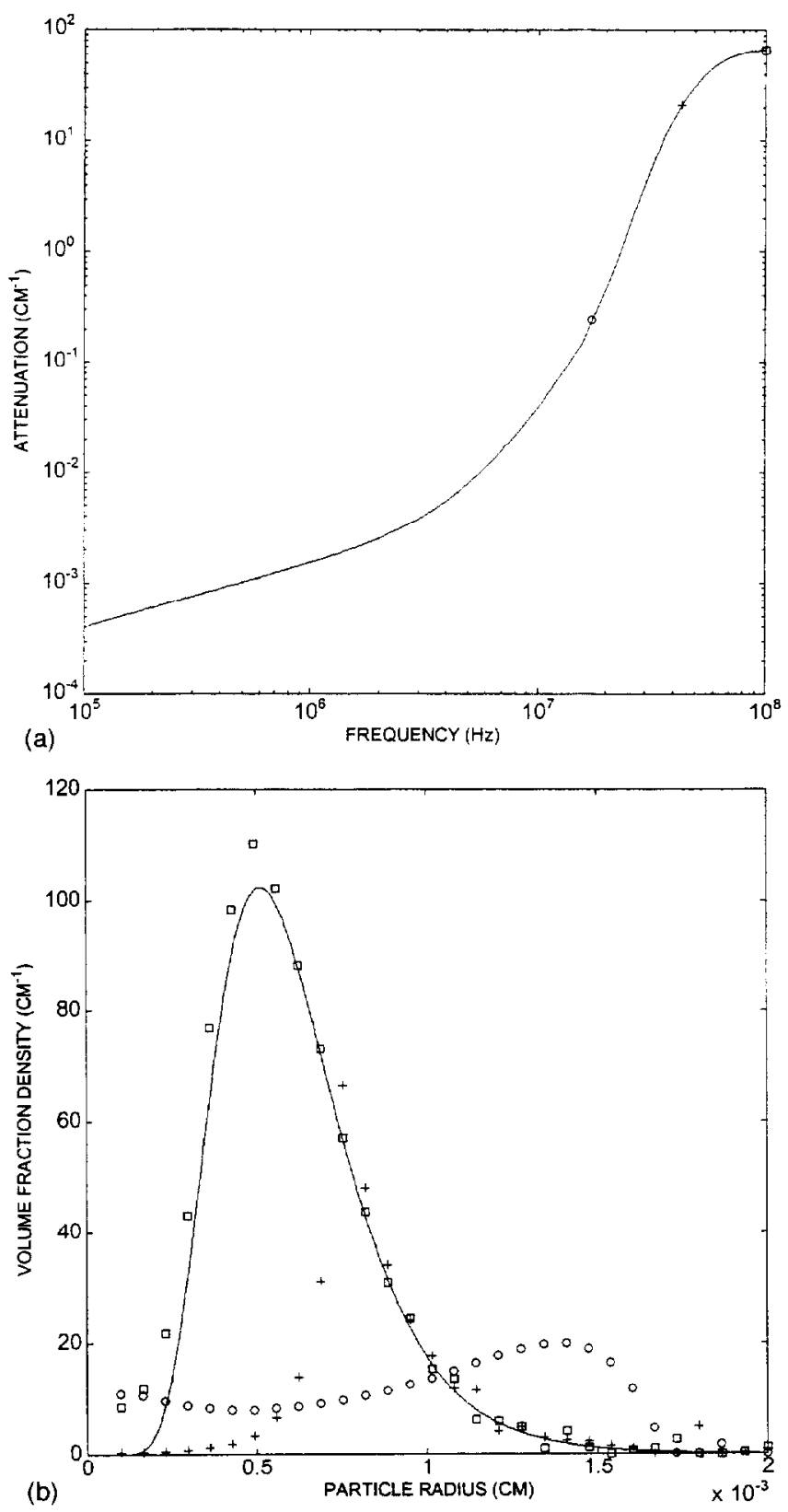

FIG. 15. As Fig. 12, but with a broader size distribution.

Fig. 17(c). The main reason for the success of the inverse technique for bubbly liquids seems to be that there is one resonance frequency for bubbles of each size. As long as the frequency range is broad enough to cover the resonance frequency of all the bubbles, it is possible to determine the size distribution.

The results presented so far were for polystyrene particles. We have also carried out inverse calculations for glass particles. As indicated earlier (cf. Fig. 6) there is no clear, sharp resonance frequency peak for glass particles. As a consequence, the inverse calculations for the glass particles did not show, in general, good agreement with the input size distribution.

The results presented so far show that the success of Tikhonov regularization to solve the inverse problem is limited. Although we have given plausible reasons for why the 


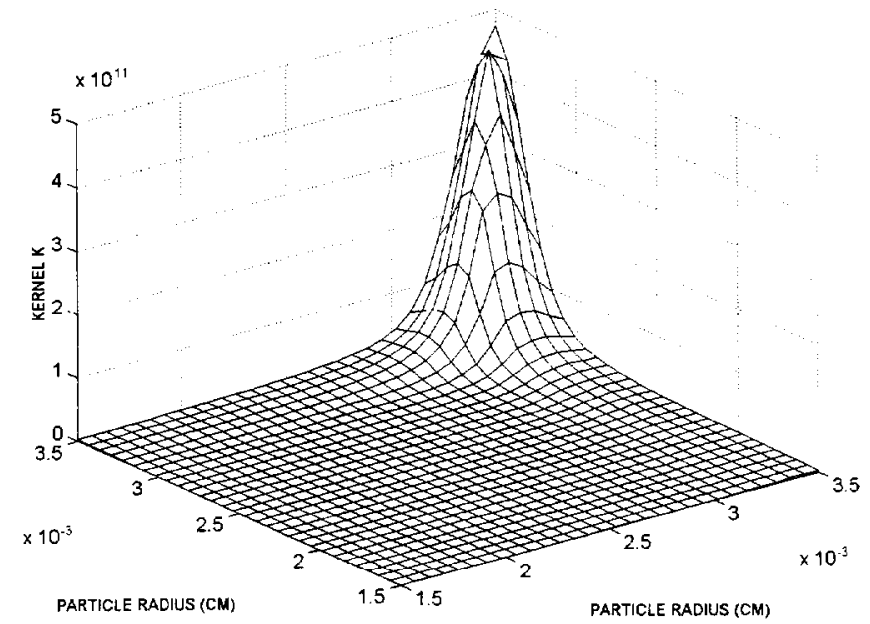

(a)

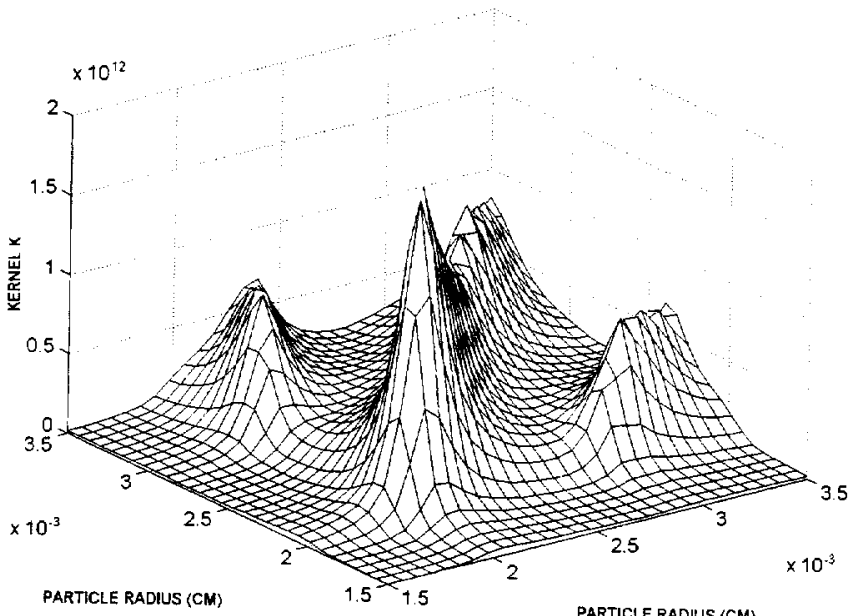

(b)

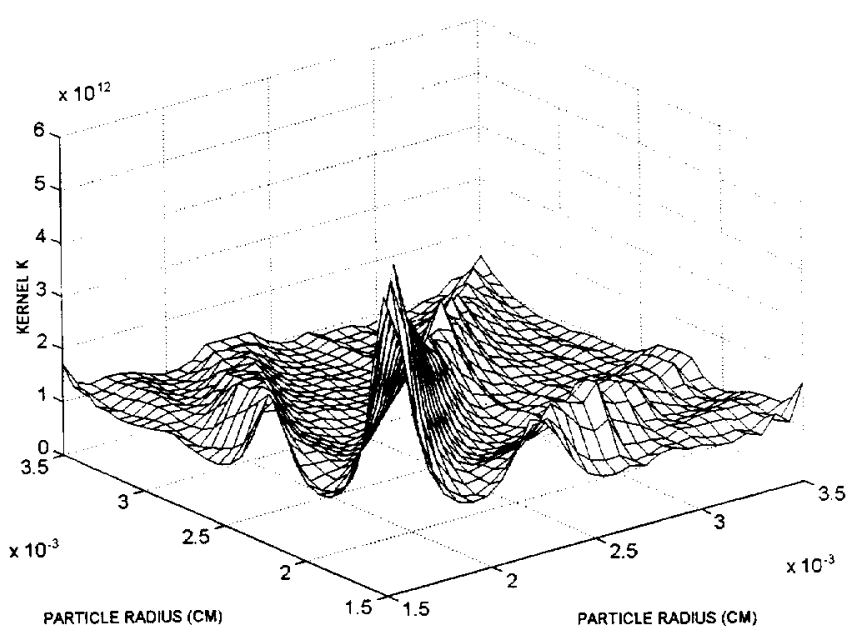

(c)

PARTICLE RADIUS ICM

FIG. 16. The kernel $K\left(a_{i}, a_{j}\right)$ for polystyrene particles when using for $f_{\max }$ the value indicated in Fig. 12(a) by a $O$ (a), + (b), and $\square$ (c).

method works well for bubbles but not for all particles, it is possible that other techniques for solving the inverse problem may be more successful. For that reason we have attempted an alternative method ${ }^{2,3,19}$ based on linear programing that we shall briefly describe here.

The constraint $\phi(a) \geqslant 0$ for all $a$ was satisfied only $a$ posteriori in the Tikhonov scheme. To ensure that the error is minimized while satisfying this constraint, we reformulate the original inverse problem as an optimization problem. The simplest scheme is to minimize the error

$$
\int_{f_{\min }}^{f_{\max }}\left|\int_{a_{\min }}^{a_{\max }} \hat{\alpha}(f, a) \phi(a) d a-\alpha_{\mathrm{tot}}(f)\right| d f
$$

instead of the integral of the square of the quantity enclosed by two vertical bars at each frequency. Constraints on the solution are used $a$ priori in optimization via linear programming; here we use that $\phi(a) \geqslant 0$. Imposing an upper bound on the total volume fraction (maximum packing) can also be incorporated but is not essential. After discretizing the frequency range by $M$ and $\phi(a)$ in $N$ discrete values we write $\sum_{j=1}^{N} B_{i j} \phi\left(a_{j}\right)-\alpha_{\mathrm{tot}}\left(f_{i}\right)=u_{i}-v_{i}, u_{i}, v_{i} \geqslant 0, i=1,2, \ldots, M$.

Here, $B_{i j}$ is the discretized form of the integral operator in (31) and $u_{i}$ and $v_{i}$ are, as yet, unknown, non-negative variables. Now, it can be shown ${ }^{19}$ that minimizing the absolute value of (32) is equivalent to minimizing

$$
\sum_{i=1}^{M}\left(u_{i}+v_{i}\right)
$$

with (32) as a constraint together with the constrains $u_{i}, v_{i}$ $\geqslant 0(i=1, \ldots, M)$ and $\phi\left(a_{i}\right) \geqslant 0(i=1, \ldots, N)$. Essential here is the notion that at the optimum $u_{i} v_{i}=0$ for each $i$, which makes the solutions of the two minimization problems (31) and (33) identical.

The above scheme was applied to a number of cases that were also examined using the Tikhonov method. It was found that, in general, the linear programing scheme produced inferior results. A typical example is shown in Fig. 18 where the Tikhonov method is seen to yield far better results for the size distributions. This technique also did not yield good inverse results for the case of glass particles.

\section{CONCLUSION}

A theory for the attenuation and wave speed of solidliquid suspensions at low particle volume fractions is described. The theory is shown to be in excellent agreement with the experimental data measured in our laboratory. Tikhonov regularization and linear programing techniques are employed to solve the inverse problem of determining the particle size distribution from the attenuation-frequency data. Although these techniques are successful in solving the inverse problem in several cases, we have also shown that the results are very sensitive to the choice of frequency range, the physical properties of the particles, and the nature of particle size distribution (unimodal, bimodal, etc.). Since the same techniques worked very well for bubbly liquids, we attribute the failure in solving the inverse problem satisfactorily to the complex resonance behavior of slurries. We conclude therefore that the prospects of using acoustic probes for on-line monitoring of particle size distribution of slurries are somewhat limited unless some additional information on the particle size distribution (e.g., unimodal) is available. 

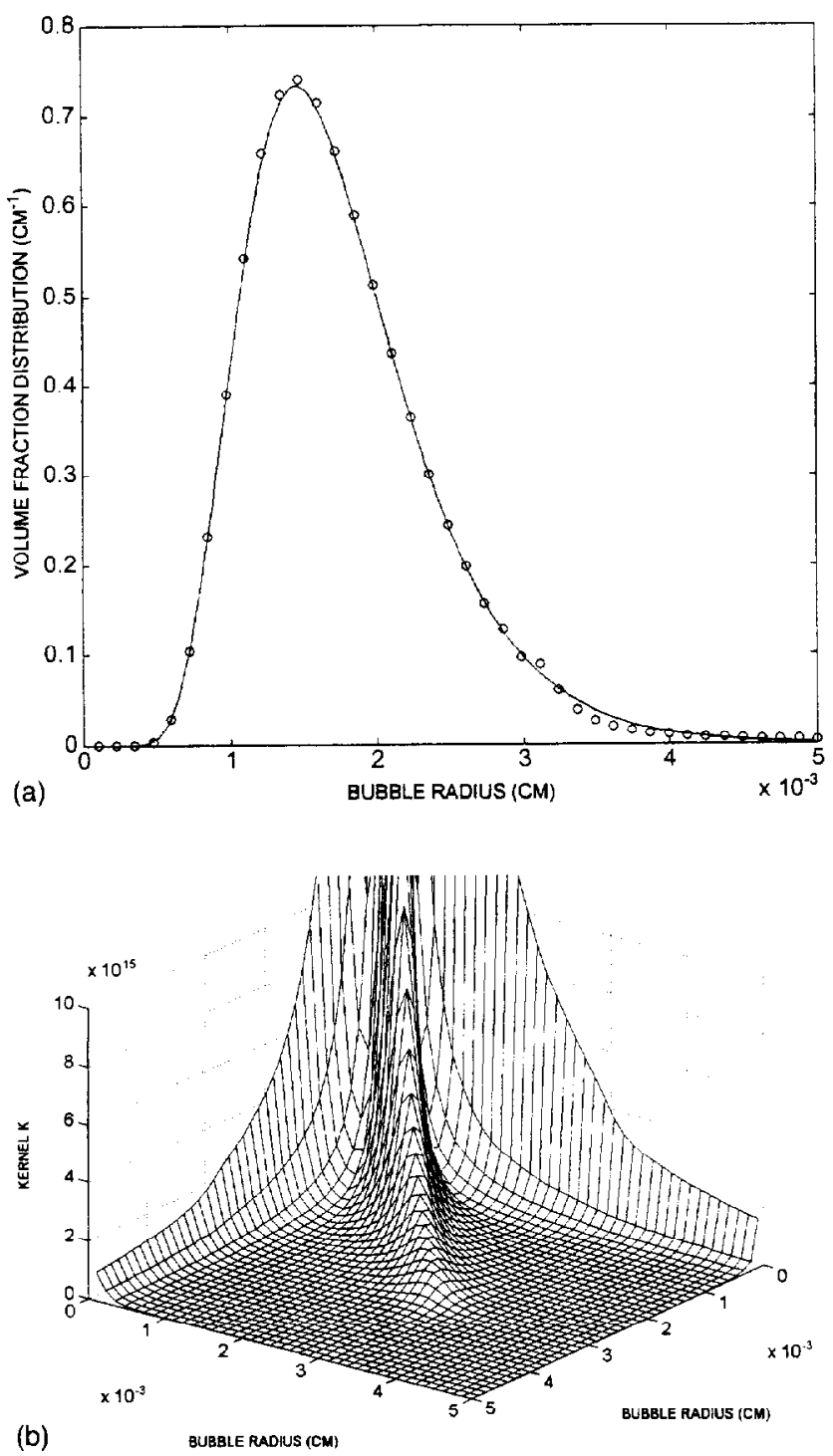

\section{ACKNOWLEDGMENTS}

Financial support for this work was provided by U.S. Department of Energy, Environmental Management Science Program, under Grant No. DE-FG07-96ER14729. Computations were made using the facilities of the Cornell Theory Center and the National Center of Supercomputing Applications at the University of Illinois at Urbana-Champaign.

\section{APPENDIX: EQUATIONS FOR $\boldsymbol{A}_{n}$}

In this appendix we give the set of linear equations for unknowns that include the coefficients $A_{n}$ required to calculate the attenuation from (12) and (19)-(21) or (22). These equations are derived from the boundary conditions on the surface of a test particle. In addition to the coefficients $A_{n}$, $\bar{A}_{n}$ of the solution of (1) outside and inside the particle, respectively, similar coefficients arise due to the solution of (2) and (3), denoted by $B_{n}$ and $C_{n}$. Note that (3) is an equation for the vector $\mathbf{A}$ rather than a scalar velocity potential, but only the azimuthal component of $\mathbf{A}$ is nonzero, hence only a scalar coefficient $C_{n}$. In the following, we use the notation $z_{c}=k_{c} a, z_{T}=k_{T} a$, and $z_{s}=k_{s} a$ :

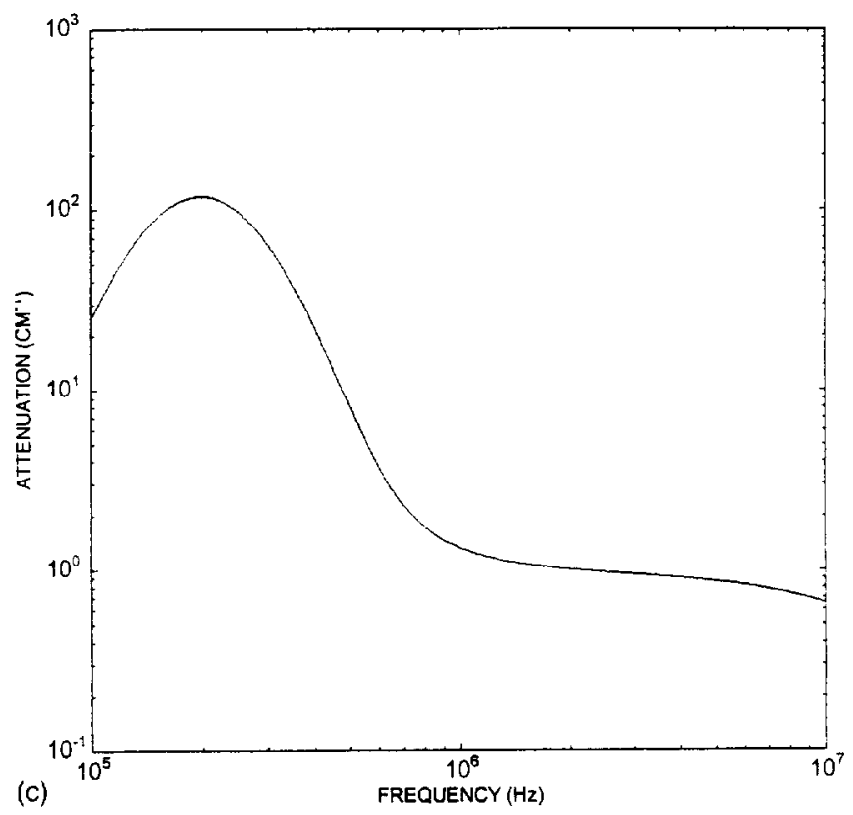

FIG. 17. Results for air bubbles in water. (a) Inverse problem result with a total volume fraction equal to 0.004 , together with the the kernel $K\left(a_{i}, a_{j}\right)$ (b) and the attenuation as a function of frequency (c).

$$
\begin{aligned}
& z_{c} j_{n}^{\prime}\left(z_{c}\right)+A_{n} z_{c} h_{n}^{\prime}\left(z_{c}\right)+B_{n} z_{T} h_{n}^{\prime}\left(z_{T}\right)-C_{n} n(n+1) h_{n}\left(z_{s}\right) \\
& =\tilde{A}_{n} \tilde{z}_{c} j_{n}^{\prime}\left(\tilde{z}_{c}\right)+\widetilde{B}_{n} \tilde{z}_{T} j_{n}^{\prime}\left(\tilde{z}_{T}\right)-\widetilde{C}_{n} n(n+1) j_{n}\left(\tilde{z}_{s}\right), \\
& j_{n}\left(z_{c}\right)+A_{n} h_{n}\left(z_{c}\right)+B_{n} h_{n}\left(z_{T}\right)-C_{n}\left(h_{n}\left(z_{s}\right)+z_{s} h_{n}^{\prime}\left(z_{s}\right)\right) \\
& =\tilde{A}_{n} j_{n}\left(\tilde{z}_{c}\right)+\widetilde{\boldsymbol{B}}_{n} j_{n}\left(\tilde{z}_{T}\right)-\tilde{C}_{n}\left(j_{n}\left(\tilde{z}_{s}\right)+\tilde{z}_{s} j_{n}^{\prime}\left(\tilde{z}_{s}\right)\right) \text {, } \\
& b_{c}\left[j_{n}\left(z_{c}\right)+A_{n} h_{n}\left(z_{c}\right)\right]+B_{n} b_{T} h_{n}\left(z_{T}\right) \\
& =\tilde{A}_{n} \tilde{b}_{c} j_{n}\left(\tilde{z}_{c}\right)+\widetilde{B}_{n} \widetilde{b}_{T} j_{n}\left(\tilde{z}_{T}\right), \\
& \tau\left(z_{c} b_{c}\left[j_{n}^{\prime}\left(z_{c}\right)+A_{n} h_{n}^{\prime}\left(z_{c}\right)\right]+B_{n} b_{T} z_{T} h_{n}^{\prime}\left(z_{T}\right)\right) \\
& =\tilde{\tau}\left(\tilde{A}_{n} \widetilde{b}_{c} \tilde{z}_{c} j_{n}^{\prime}\left(\tilde{z}_{c}\right)+\widetilde{B}_{n} \tilde{b}_{T} \tilde{z}_{T} j_{n}^{\prime}\left(\tilde{z}_{T}\right)\right), \\
& (-\iota \omega \mu)\left(\left[\left(z_{s}^{2}-2 z_{c}^{2}\right) j_{n}\left(z_{c}\right)-2 z_{c}^{2} j_{n}^{\prime \prime}\left(z_{c}\right)\right]+A_{n}\left[\left(z_{s}^{2}\right.\right.\right. \\
& \left.\left.-2 z_{c}^{2}\right) h_{n}\left(z_{c}\right)-2 z_{c}^{2} h_{n}^{\prime \prime}\left(z_{c}\right)\right]+B_{n}\left[\left(z_{s}^{2}-2 z_{T}^{2}\right) h_{n}\left(z_{T}\right)\right. \\
& \left.\left.-2 z_{T}^{2} h_{n}^{\prime \prime}\left(z_{T}\right)\right]+C_{n} 2 n(n+1)\left[z_{s} h_{n}^{\prime}\left(z_{s}\right)-h_{n}\left(z_{s}\right)\right]\right) \\
& =\tilde{A}_{n}\left[\left(\omega^{2} \tilde{\rho} a^{2}-2 \tilde{\mu} \tilde{z}_{c}^{2}\right) j_{n}\left(\tilde{z}_{c}\right)-2 \tilde{\mu} \tilde{z}_{c}^{2} j_{n}^{\prime \prime}\left(\tilde{z}_{c}\right)\right] \\
& +\widetilde{B}_{n}\left[\left(\omega^{2} \tilde{\mu} a^{2}-2 \tilde{\mu} \tilde{z}_{T}^{2}\right) j_{n}\left(\tilde{z}_{T}\right)-2 \tilde{\mu} \tilde{z}_{T}^{2} j_{n}^{\prime \prime}\left(\tilde{z}_{T}\right)\right] \\
& +\tilde{C}_{n} 2 \tilde{\mu} n(n+1)\left[\tilde{z}_{s} j_{n}^{\prime}\left(\tilde{z}_{s}\right)-j_{n}\left(\tilde{z}_{s}\right)\right],
\end{aligned}
$$




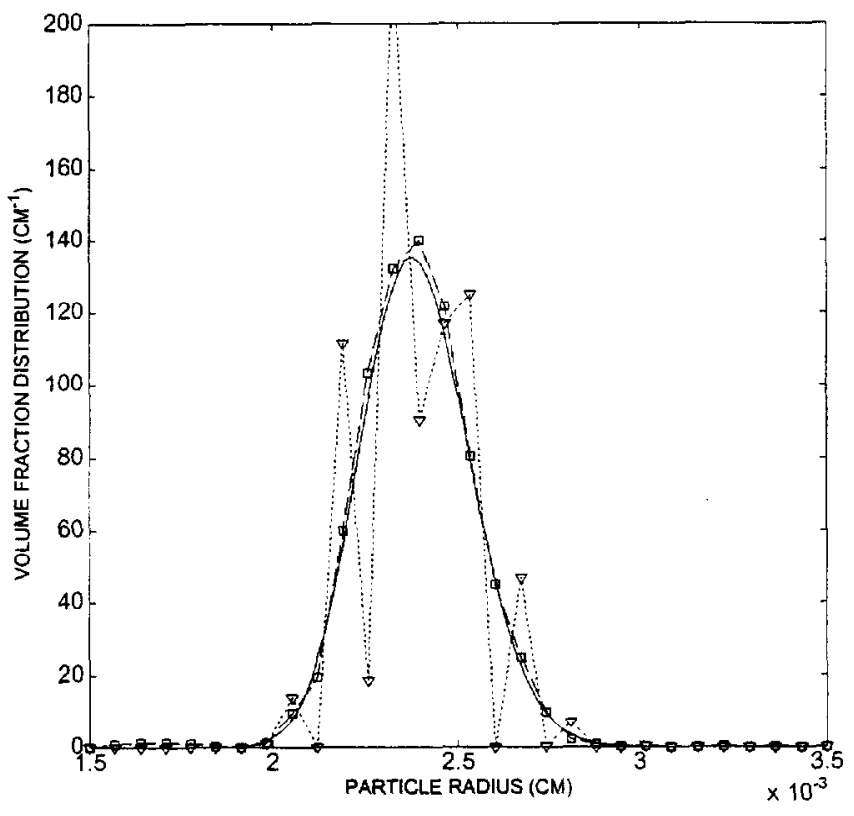

FIG. 18. Comparison of the results for the inverse problem of polystyrene particles (exact solution is the solid line) using the linear programming method (.. . .) and the Tikhonov method (-- $\square$-.). In both cases the attenuation was cut off at the same frequency, indicated by the square in Fig. 12(a).

$$
\begin{aligned}
(-\iota & \omega \mu)\left(z_{c} j_{n}^{\prime}\left(z_{c}\right)-j_{n}\left(z_{c}\right)+A_{n}\left[z_{c} h_{n}^{\prime}\left(z_{c}\right)-h_{n}\left(z_{c}\right)\right]\right. \\
& +B_{n}\left[z_{T} h_{n}^{\prime}\left(z_{T}\right)-h_{n}\left(z_{T}\right)\right]-\left(C_{n} / 2\right)\left[z_{s}^{2} h_{n}^{\prime \prime}\left(z_{s}\right)\right. \\
& \left.\left.+\left(n^{2}+n-2\right) h_{n}\left(z_{s}\right)\right]\right) \\
& =\tilde{\mu}\left(\tilde{A}_{n}\left[\tilde{z}_{c} j_{n}^{\prime}\left(\tilde{z}_{c}\right)-j_{n}\left(\tilde{z}_{c}\right)\right]+\tilde{B}_{n}\left[\tilde{z}_{T} j_{n}^{\prime}\left(\tilde{z}_{T}\right)-j_{n}\left(\tilde{z}_{T}\right)\right]\right. \\
& \left.-\left(\tilde{C}_{n} / 2\right)\left[\tilde{z}_{s}^{2} j_{n}^{\prime \prime}\left(\tilde{z}_{s}\right)+\left(n^{2}+n-2\right) j_{n}\left(\tilde{z}_{s}\right)\right]\right) .
\end{aligned}
$$

Here, $b_{c}$ and $b_{T}$ are given by

$$
b_{c}=\frac{(1-\gamma) \omega^{2}}{\beta c^{2}}, \quad b_{T}=-\frac{\gamma}{c_{1}^{2} \beta}\left[\omega^{2}-\left(\frac{c_{1}^{2}}{\gamma}-\frac{4 \iota \omega \mu}{3 \rho}\right) k_{T}^{2}\right]
$$

with $\beta$ the thermal expension coefficient and $c_{1}$ the liquidequivalent of the speed of sound for spherical compressional waves in an elastic isotropic solid $\tilde{c}_{1}=\sqrt{(\tilde{\lambda}+2 \tilde{\mu} / 3) / \tilde{\rho}}$. The Lamé constant $\tilde{\lambda}$ is not really needed when the speed of sound $(c)$ of longitudinal compressional waves is specified, as we can also write $c_{1}^{2}=c^{2}\left(1-4 \tilde{\mu} /\left(3 \rho c^{2}\right)\right)$. The above equations have also been given by Epstein and Carhart ${ }^{5}$ and Allegra and Hawley. ${ }^{6}$ However, in both there are typographical errors: in Ref. 5 , the last $j_{n}^{\prime}\left(\widetilde{z}_{s}\right)$ in (A2) is erroneously replaced by $h_{n}^{\prime}\left(\tilde{z}_{s}\right)$; in Ref. 6 the signs of both $\left(n^{2}+n-2\right)$-terms are wrong, while the last $h_{n}\left(z_{s}\right)$ on the left-hand side of (A6) has the argument $\tilde{z}$ instead and the first $z_{s}$ after $C_{n}$ is replaced by $\tilde{z}_{s}$. Not correcting the typographical errors in Ref. 6 would have altered the results significantly.

${ }^{1} \mathrm{~K}$, W. Commander and R. J. McDonald, "Finite-element solution of the inverse problem in bubble swarm acoustics," J. Acoust. Soc. Am. 89, 592 (1991).

${ }^{2} \mathrm{R}$. Duraiswami, "Bubble density measurement using an inverse acoustic scattering technique," in ASME Cavitation and Multiphase Forum, Washington DC, edited by O. Furuya (ASME, New York, 1993), Vol. 153, p. 67.

${ }^{3}$ R. Duraiswami, S. Prabhukumar, and G. L. Chahine, "Bubble counting using an inverse acoustic scattering method," J. Acoust. Soc. Am. 104, 2699 (1998).

${ }^{4} \mathrm{~T}$. Oja and F. Alba, "Acoustic attenuation spectroscopy for particle sizing of high concentration dispersions," presentation at the NIST International Workshop on Ultrasonic and Dielectric Characterization Techniques for Suspended Particulates Gaithersburg, MD (1997).

5. R. Allegra and S. A. Hawley, "Attenuation of sound in suspensions and emulsions: Theory and experiments," J. Acoust. Soc. Am. 51, 1545 (1972).

"P. S. Epstein and R. R. Carhart, "The absorption of sound in suspensions and emulsions. I. Water fog in air," J. Acoust. Soc. Am. 25, 553 (1953).

${ }^{7}$ V. K. Varadan, V. N. Bringi, V. V. Varadan, and Y. Ma, "Coherent attenuation of acoustic waves by pair-correlated random distribution of scatterers with uniform and Gaussian size distributions," J. Acoust. Soc. Am. 73, 1941 (1983).

${ }^{8}$ R. Kress, Linear Integral Equations (Springer, Berlin, 1989).

${ }^{9}$ D. J. McClements and J. N. Coupland, "Theory of droplet size distribution measurements in emulsions using ultrasonic spectroscopy," Colloids Surf., A 117, 161 (1996).

${ }^{10} \mathrm{M}$. J. Lighthill, "Viscosity effects in sound waves of finite amplitude," in Surveys in Mechanics, edited by G. K. Batchelor and R. M. Davies (Cambridge U.P., Cambridge. England. 1956).

${ }^{11}$ A. S. Sangani, "A pairwise interaction theory for determining the linear acoustic properties of dilute bubbly liquids," J. Fluid Mech. 232, 221 (1991)

${ }^{12}$ A. S. Sangani, D. Z. Zhang, and A. Prosperetti, "The added mass, Basset, and viscous drag coefficients in nondilute bubbly liquids undergoing small-amplitude oscillatory motion,"' Phys. Fluids A 3, 2955 (1991).

${ }^{13}$ P. D. M. Spelt, M. A. Norato, A. S. Sangani, M. S. Greenwood, and L. L. Tavlarides, "Attenuation of sound in concentrated suspensions: Theory and experiments," submitted to J. Fluid Mech.

${ }^{14}$ Y. Ma, V. K. Varadan, and V. V. Varadan, "Comments on "Ultrasonic propagation in suspensions," " J. Acoust. Soc. Am. 87, 2779 (1990).

${ }^{15}$ R. E. Bolz, CRC Handbook of Tables for Applied Engineering Science (CRC, Boca Raton, FL, US $\Lambda$, 1973).

${ }^{16} \mathrm{~L}$. W. Anson and R. C. Chivers, "Ultrasonic propagation in suspensions-A comparison of a multiple scattering and an effective medium approach," J. Acoust. Soc. Am. 85, 535 (1989).

${ }^{17} \mathrm{~J}$. J. Faran, "Sound scattering by solid cylinders and spheres," J. Acoust. Soc. Am. 23, 405 (1951).

${ }^{18} \mathrm{R}$. C. Chivers and L. W. Anson, "Calculations of the backscattering and radiation force functions of spherical targets for use in ultrasonic beam assessment," Ultrasonics 20, 25 (1982).

${ }^{19}$ L. M. Delves and J. L. Mohamed, Computational Methods for Integral Equations (Cambridge U.P., Cambridge, England, 1985). 


\title{
Ultrasonic Characterization of Low Volume Fraction Solid-Liquid Slurries
}

by

\author{
Mark Jefferson Hedges
}

Abstract of Thesis

January 2001

The United States is encumbered with a huge amount of liquid radioactive waste as a result of the nuclear weapons program. The waste is stored in underground tanks, all of which need to be emptied and many of which are leaking. In order to process the waste it must be transported by pipeline to the treatment centers. The waste contains suspended solids so plugging of the pipeline is a major concern. There is a need to characterize the waste during transfer in order to reduce the risk of plugging.

Ultrasound is an attractive choice for this application. It has the ability to penetrate optically dense liquids and its non-intrusive nature protects operators from the harmful effects of radiation.

Previous work on using ultrasound to characterize slurries examined relatively ideal systems of monodispersed particles in water. No work was done on systems with volume fractions less than 0.05 . Theory is available which accurately predicted attenuation at low volume fractions but it was not known if reliable experimental data could be collected under these conditions. 
Attenuation measurements are performed on systems of soda-lime glass beads (radius $=16 \mu \mathrm{m}$ ) in water, clay in water, and a nuclear waste simulant consisting of precipitated salts in saturated supernate. Solids volume fraction studied range from 0.004 to 0.05 . Both a Pulse/FFT and a Toneburst method are used to measure attenuation. The Pulse/FFT method is found to be more accurate. As expected the relationship between attenuation and volume fraction is found to be linear, and it is found that reliable attenuation measurements can be made in all three systems even at solids volume fraction of under 0.01 .

Sound speed is measured in systems of soda-lime glass beads (radius $16 \mu \mathrm{m}$ ) and Potter's beads (radius $60 \mu \mathrm{m}$ ). Volume fraction ranged from 0 to 0.4 . Experiments indicate that particle size has little effect on sound speed. Experimental data of sound speed in soda-lime glass slurries agrees well with theory. Experiments performed at low volume fraction showed that with very precise monitoring of temperature it is possible to use speed to characterize slurries, however, practical considerations weigh against this.

Future work involves automating the procedure to measure attenuation and creating an on-line monitor on a spoolpiece that can be installed in a flow loop. The system will monitor slurry and the suspending liquid simultaneously and give data in real time. 


\title{
Ultrasonic Characterization of Low Volume Fraction Solid-Liquid Slurries
}

\author{
by \\ Mark Jefferson Hedges \\ B.S. Ch.E., Syracuse University, 1998 \\ Thesis \\ Submitted in partial fulfillment of the requirements for the \\ degree of Master of Science in Chemical Engineering \\ in the Graduate School of Syracuse University
}

January 2001

Approved Professor Lawrence L. Tavlarides

Date 


\section{Table of Contents}

List of Tables.......................................... vii

List of Figures........................................ viii

Acknowledgments...................................... xi

1. Introduction........................................... 1

2. Literature review..................................... 4

2.1 Attenuation..................................... 4

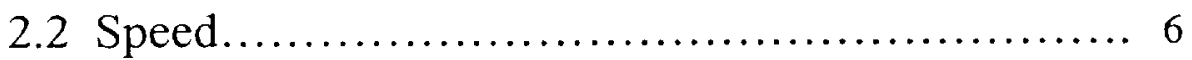

2.3 Conclusions................................... 6

3. Experimental....................................... 7

3.1 Attenuation Background ......................... 7

3.2 Attenuation Experiments....................... 13

3.2.1 Soda-Lime Glass.........................13

3.2 .2 Clay ...................................... 16

3.2.3 Crystallized Salt Solution.................. 17

3.3 Speed Background ............................... 19

3.3.1 Speed Experiments....................... 24

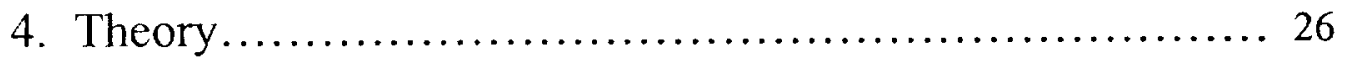

5. Results............................................ 30

5.1 Attenuation in Soda Lime Glass................. 30

5.1.1 Comparison with theory ................... 34

5.2 Attenuation in Clay................................ 39

5.3 Attenuation in Chemical Slurry Surrogate.............39

5.3.1 Comparison with theory .................. 45

5.4 Reproducibility of Data.......................... 46

5.5 Speed Experiments.............................. 50

5.5.1 Comparison with theory $\ldots \ldots \ldots \ldots \ldots \ldots . \ldots 53$ 
6. Conclusions........................................... 53

7. Future Work.......................................... 55

References.............................................. 58

Appendix A: Procedure for synthesizing Crystallized

Salt Solution.................................. 60

Appendix B: Transducer Specifications.................... 62

Appendix C: Speed Measurement Procedure.................. 63 


\section{List of Tables}

Table 3.1: $\quad$ Properties of Soda-lime Glass Beads used in this study

Table 3.2: Composition of Chemical slurry surrogate

Table 5.1: $\quad$ Particle size distribution of chemical slurry surrogate

Table 5.2: Values used for theoretical modeling of chemical slurry surrogate system 


\section{List of Figures}

Figure 3.1: Schematic diagram of Pulse/FFT setup used to measure attenuation. A spike pulse is generated by the pulser/receiver and is transmitted to the transmitting transducer which is in contact with the sample. After traveling through the sample and being acquired by the receiving transducer the pulse is routed through the pulser/receiver to the oscilloscope.

Figurc 3.2: Schematic diagram of toneburst setup used to measure attenuation. $\Lambda$ toneburst card located in a PC generates an electrical signal of constant frequency and transmits that signal to the transmitting transducer which is in contact with the sample. After traveling through the sample and being acquired by the receiving transducer the pulse is routed through the toneburst card to the oscilloscope.

Figure 3.3: Schematic diagram of attenuation test cell. Cell is constructed of Plexiglas with a nominal pathlength of two inches. Transducers with centerline frequencies of $1 \mathrm{MHz}$ to $10 \mathrm{MHz}$ are mounted on each side to complete the cell.

Figure 3.4: Schematic diagram of setup used to measure speed. A spike pulse is generated by the pulser/receiver and is transmitted to the transducer, which is coupled to a Plexiglas $®$ buffer with ultrasound gel. The signal travels through the buffer and some signal is reflected back at the buffer/sample interface. The remaining signal travels through the sample and is reflected off a brass plate mounted at the far end of the cell. The same transducer that emitted the signal picks up both reflections and routes the reflected signals through the Pulser/Receiver to the oscilloscope.

Figure 3.5: Schematic diagram of speed test cell. Cell is constructed of Plexiglas with a brass reflector plate. The reflector plate is interchangeable, with current plates creating pathlengths of $1 / 2$ inch or one inch. A single transducer is mounted on one side, coupled with ultrasound gel. The signal passes through a buffer rod of Plexiglas (thickness: $1 / 4$ inch) before coming in contact with the sample.

Figure 3.6: Typical waveform produced from speed test setup. The first peak, at $t \sim 6 \mu \mathrm{s}$, is reflected at the buffer-rod sample interface. The second peak, at t $-23 \mu \mathrm{s}$, is reflected off the brass plate at the far end of the cell. The smaller peaks at $t$ $\sim 11 \mu \mathrm{s}$ and $\mathrm{t} \sim 28 \mu \mathrm{s}$ are echoes of the larger peaks.

Figure 5.1: Attenuation versus volume fraction for a slurry of soda lime glass particles (radius $=16 \mu \mathrm{m}$ ) in water at three frequencies. The frequencies studied are $(x)$ $12 \mathrm{MHz}$, (o) $10 \mathrm{MHz}$, and (+) $8 \mathrm{MHz}$. 
Figure 5.2: Attenuation versus volume fraction for a slurry of soda lime glass particles (radius $=16 \mu \mathrm{m}$ ) at very low volume fraction. The frequencies studied are $(x)$ $12 \mathrm{MHz},(\mathrm{o}) 10 \mathrm{MHz}$, and (+) $8 \mathrm{MHz}$.

Figure 5.3: Typical attenuation versus frequency behavior for a $0.4 \%$ (volume) soda lime glass slurry. Transducers used are $(\times) 1 \mathrm{MHz},\left(^{*}\right) 2.25 \mathrm{MHz},\left(\right.$ o) $5 \mathrm{MHz},\left(^{+}\right)$ $7.5 \mathrm{MHz},(\Delta) 10 \mathrm{MHz}$.

Figure 5.4: Effect of time between preparation and testing in soda lime glass slurries. The data marked $(x)$ was collected immediately following preparation of the slurry. The data marked (o) was collected after that same slurry was allowed to sit one week.

Figure 5.5: Comparison of pulse/FFT and toneburst methods of measuring attenuation. Pulse/FFT data is shown on top, with toneburst data shown on bottom.

Figure 5.6: Comparison of methods of acquiring baseline signal. The baseline signal for the data shown on top was acquired separately from the sample signal. The baseline signal for the data shown on bottom was acquired by letting the solids settle.

Figure 5.7: Comparison of theoretical prediction and experimental data of attenuation versus frequency for slurries of soda lime glass in water. The points represent experimental data and the lines represent theoretical predictions. The same pair of $10 \mathrm{MHz}$ transducers was used to gather all data. Volume fractions shown are $(0) 0.004,(+) 0.01,(\Delta) 0.02$, and $(x) 0.05$.

Figure 5.8: Attenuation versus volume fraction for slurries of clay ( 2 parts kaolin to one part Bentonite, by weight) in water at three frequencics. The frequencics studied are (o) $12 \mathrm{MHz},(+) 10 \mathrm{MHz}$, and ( $x) 8 \mathrm{MHz}$.

Figure 5.9: Attenuation versus volume fraction for crystallized salt solution system at three frequencies. The frequencies studied are (o) $12 \mathrm{MHz},(\times) 10 \mathrm{MHz}$, and (+) $8 \mathrm{MHz}$.

Figure 5.10: Attenuation versus frequency for crystallized salt solution. Points represent experimental data, the line is a linear fit.

Figure 5.11: Photographs of crystallized salt solution particles. The photograph on top shows the larger particles, at a magnification factor of approximately 50 . The photograph on the bottom shows the smaller particles, at a magnification factor of approximately 130 . 
Figure 5.12: Results of the two particle size model to predict attenuation in the crystallized salt solution. It was assumed that the solids had two particle sizes, a smaller size with radius of $5 \mu \mathrm{m}$ and a larger size with a radius of 50 $\mu \mathrm{m}$. The ratio of the two particle sizes was varied. Volume fraction ratios shown $(5 \mu \mathrm{m}: 50 \mu \mathrm{m})$ are: (o) 30:70, (x) 50:50, and $(\Delta) 70: 30 .(+)$ represents experimental data.

Figure 5.13: Results of expanding the two particle size model to predict attenuation in crystallized salt solution to include actual measured particle size information. Again it was assumed that the solids were of two different types. The first type was still taken to be spheres of radius $5 \mu \mathrm{m}$. The larger particles were of various sizes, as shown in Table 5.2. Volume fraction ratios shown (5 $\mu \mathrm{m}:$ larger particles) are: $(+)$ 30:70, $(\Delta)$ 50:50, and $(\times)$ 60:40. $(+)$ represents experimental data.

Figure 5.14: Reproducibility of data in liquid solid systems. Four experimental runs were performed on each system, with new slurry prepared for each run. All data is at $10 \mathrm{MHz}$. Systems shown are (top) SLG, (middle) BKC, (bottom) CSS.

Figure 5.15: Effect of volume fraction on phase speed in solid-liquid slurries. Particles are $(x)$ Soda lime glass $(r=16 \mu \mathrm{m})$ and $(+)$ Potter's beads $(r=60 \mu \mathrm{m})$. Volume fraction ranges from 0.0 to 0.4 .

Figure 5.16: Phase speed differential versus volume fraction for low volume fraction soda-lime glass beads. The phase speed differential is the difference between the phase speed in slurry and the speed in the suspending liquid (water). Data is represented by points. The line is a linear fit.

Figure 5.17: Comparison of measured phase speed with theoretical phase speed. Experimental data is represented by points, while the line represents theoretical calculations. Frequency is $2 \mathrm{MHz}$, temperature $25^{\circ} \mathrm{C}$.

Figure 7.1: Conceptual Diagram of Acoustic Monitor on Spool Piece. The unit includes the primary acoustic monitor transducer cluster for slurry; and the by-pass loop with slurry pump, cross-flow slurry filter with pressure actuated backflush piston, and secondary acoustic monitor with interrogative cell for supernate. 


\section{Acknowledgments}

I would like to thank the following persons for their invaluable assistance toward the completion of this work:

Professor Lawrence L. Tavlarides, my research advisor, for his leadership and ability. His suggestions, ideas, and criticisms were the driving force behind this study and were essential to my graduate education.

Professor Ashok S. Sangani, for his help in the theoretical aspects of this study.

Dr. Peter Spelt, for his indispensable assistance in performing the theoretical calculations included in this study.

Professor D. Julian McClements, University of Massachusetts, Amherst, for his very generous assistance in helping me use his technique to measure phase speed.

My parents, Daniel C. and Elizabeth Hedges, for their support during my education.

Heather Marie Wilson, for her love and patience. I know it was not easy for her to wait for me but her support helped me complete this work.

Sandie Friske, for making me feel at home even when I was not. 
The assistance of the following people is greatly appreciated:

Technicians: Mr. Dick Chave and Mr. John Banas

Students: Mr. Jun Sig Lee and Mr. Sang Kyun

Administrative Staff: Mrs. Ruth Dewey, Mrs. Linda Lowe, and Ms. Linda Vanderhoof.

The following organizations are recognized for their financial support:

Department of Chemical Engineering and Materials Science, Syracuse University.

The Graduate School of Syracuse University.

US Department of Energy, Environmental Management Science Program

Grant number DE-FG07-96ER 14729. 


\section{Introduction}

The cold war era nuclear arms race has left the United States encumbered with a large amount of radioactive waste. Currently hundreds of millions of gallons of radioactive waste are stored at several sites across the country. The waste, contained in underground tanks, is a non-uniform mixture of sludge, supernate, and salt cake.

Generally, the major components are sodium nitrate and sodium nitrite. Additional ions present include aluminate, hydroxide, and carbonate. The supernates are basic, with $\mathrm{pH}$ ranging from 10 to greater than 14 . Radioactive components in the supernate and dissolved salt cake are materials such as ${ }^{99} \mathrm{Tc},{ }^{137} \mathrm{Cs},{ }^{235} \mathrm{Pu},{ }^{237} \mathrm{~Np}$, and ${ }^{90} \mathrm{Sr}$ (Golcar 2000).

Production of new weapons grade radioactive material in the United Stated has stopped and the cleanup phase has been in progress for a number of years. The fact that many of the storage tanks are leaking only increases the urgency to deal with the problem.

In order to treat the waste it must first be removed from the storage tanks and transported to treatment centers located on site. The tanks are located some distance from the treatment centers, some as far as six miles away (Hylton, 2000). For safety reasons, it is often desirable to transfer the materials by pipeline, rather than tanker truck. Plugging is a major concern when transferring slurries by pipcline. If a pipeline becomes plugged there are major costs in money and time, as a new pipeline has to be laid, and the old pipeline remediated. As of 1996 five of the six cross-site pipelines at the Hanford Site were unusable due to plugging (Hudson 1996). The risk of plugging can be reduced by 
careful monitoring of the slurry properties (suspended solids levels, density, and viscosity).

The treatment of nuclear waste is a complicated process, consisting of many different operations run in series and in parallel. Many of these operations involve handling of liquid/solid systems. For example, one of the methods for treating radioactive tank supernate involves using an adsorbent such as crystalline silica titanate to remove the radioactive cesium ions and then imprisoning the now radioactive absorbent in a glass frit. This disposal method largely involves the handling of solid/liquid systems and some method of characterizing the flowing stream is needed.

Ultrasound has several properties which make it an attractive choice for characterizing radioactive slurry. It has the ability to penetrate optically dense slurries. When used properly it can give accurate information even in the presence of small amounts of gas bubbles. It is nonintrusive, protecting the operator from the effects of radiation. It also is a very sensitive technique and can give accurate readings at very low solid concentrations.

Two different kinds of information that can be obtained from ultrasound are attenuation and speed. Attenuation is the absorbency of sound by the material. It is almost always highly dependent on the frequency of the ultrasonic signal and the size and physical properties of the particles. Attenuation is highly sensitive to the presence of solids particles and gas bubbles, and is somewhat tolerant of changes in temperature. In theoretical terms, attenuation is the imaginary part of the wave number.

The three modes of attenuation are viscous, thermal, and scattering. The nondimensional number $\mathrm{k}_{\mathrm{cl}} \mathrm{a}$, which is the product of particle radius and wavenumber, can 
be used to determine which modes are significant. The viscous energy dissipation results from translational, volume, and shape oscillations of particles. Thermal attenuation arises as a result of non-adiabatic changes in temperature of the particles, as the particles expand and contract due to the passage of a sound wave. Viscous and thermal attenuation are most significant at small values of $\mathrm{k}_{\mathrm{cl}}$ a. Thermal attenuation is particularly significant at low $\mathrm{k}_{\mathrm{cl}} \mathrm{a}$ when the densities of the particles and liquid are similar. Attenuation due to scattering is the major source of attenuation at higher frequencies, i.e. when $\mathrm{k}_{\mathrm{cl}} \mathrm{a}=\mathrm{O}(1)$.

Other kind of information that can be obtained using ultrasound is sound speed. In many materials (such as water) speed is nearly independent of frequency. However, in some systems such as suspensions, speed is dependent on the frequency of the ultrasonic signal. Such systems are termed highly dispersive. Speed is not very dependent on particle size and is not as sensitive to the presence of solid particles as is attenuation, particularly when the speed of sound in the solid phase is close to the speed of sound in the liquid phase. Speed is strongly dependent on temperature.

There are several methods currently being evaluated for the characterization of radioactive slurries. A study was conducted which evaluated 12 in-line or in-tank monitoring systems. Based on this study, two systems were selected for testing with radioactive slurries (Hylton and Bayne, 1999). One method, studied by Hylton (2000) uses Coriolis meters, which measure density of a material. Two meters are used, one measuring the density of the slurry and another the density of the supernate. Solids loading is calculated assuming a constant solids density. The other method given consideration is an ultrasonic probe developed by Argonne National Laboratory (Hylton 
and Bayne, 1999). Using a $1 \mathrm{mHz}$ transducer, the probe simultaneously measures ultrasonic attenuation and speed.

Current slurry characterization techniques leave much to be desired, particularly at low solids concentrations. The Argonne ultrasonic probe has an error of over $50 \%$ at solids concentrations under 5 weight percent (Hylton 1999). This error is caused in large part by the presence of bubbles in the waste stream. The work presented in this study will show that when used properly ultrasound can give reliable data even in the presence of small amounts of gas.

\section{Literature Review}

Many researchers have performed theoretical and experimental work involving

measuring and predicting speed and attenuation in multiphase mixtures. Since the focus of this paper is on experimental studies of multiphase systems literature involving experimental studies of multiphase systems will be discussed.

\subsection{ATTENUATION:}

Allegra and Hawley (1972) studied attenuation as a function of frequency in polystyrene particle-water systems. They studied particles ranging in radius from 0.044 to 0.635 microns, in the regime where thermal attenuation is the predominant phenomenon. In addition to reporting the data on attenuation these investigators also developed a theory.

Atkinson and Kytomaa (1991) measured attenuation and speed in clay-water and silica-water systems. The silica particles studies were of radius $500 \mu \mathrm{m}$, and volume fraction ranged from 0.05 to 0.6 . The effective-medium approximation theory they 
developed agreed well with their experimental data, except when $k_{\mathrm{cl}} \mathrm{a}$ became $\mathrm{O}(1)$ and multiple scattering effects become dominant.

Greenwood et al. (1993) studied attenuation in kaolin clay - water systems. They used a toneburst method where the system is interrogated by an ultrasonic signal of constant frequency. The frequency range studied was 0.5 to $3.0 \mathrm{MHz}$, and the maximum solid concentration was $44 \%$. They found that for their system attenuation appeared to depend linearly on frequency over the range studied. Several different possible explanations are given for this behavior. The two given the most consideration to yield a linear relationship between attenuation and frequency are a combination of viscous and thermal attenuation or a hydrodynamic model which considers the increase in viscosity as the slurry becomes more concentrated.

Norato (1999) developed the experimental techniques used in this study to measure attenuation. Systems studied included polystyrene-water and various types of glass beads in water. Volume fractions studied ranged from $5 \%$ to $45 \%$. Also studied was the effect of the presence of small amounts of gas on attenuation in solid-liquid systems.

Spelt et al. (2000) studied attenuation in polystyrene slurries and concentrated suspensions of glass particles. An effective medium theory was used to predict attenuation. Toneburst and Pulse/Fast Fourier Transform (FFT) methods were used to measure attenuation experimentally (Norato 1999). It was found that the theory matched the experiment well at volume fractions below 0.3 . 


\subsection{SPEED}

McClements and Fairlcy (1990) developed a technique to measure the speed of sound in a liquid. This method uses a dual reflection pulse-echo cell to cancel out any delay in the electronics, thus giving an excellent time-of flight measurement, accurate to $\pm 0.3 \mathrm{~m} / \mathrm{s}$. McClements later (1991) employed a Fourier transform to determine ultrasonic speed as a function of frequency. These investigators used speed to determine (among other things) sugar contents in fruit juices. The method developed by McClements and co-workers was adopted for use in this study.

Tsouris et al. (1995) used a pulse-echo method to determine local volume fraction in liquid-liquid dispersions. A weighed average model, corrected for reflection and refraction, was used to predict volume fraction from time-of-flight measurements. No correction was made for delay in the monitoring equipment.

Cowan et al. (1998) used a method similar to McClements and co-workers to measure phase speed in systems of glass beads of average radius $0.438 \mathrm{~mm}$. They studied the scattering regime with frequencies between 1 and $5 \mathrm{MHz}$ and volume fractions from 0.21 to 0.61 . An effective medium theory was employed which agreed well with the experimental data.

\subsection{CONCLUSIONS}

Considerablc work has been performed studying ultrasonic attenuation in multiphase systems, both experimental and theoretical. Most experimental work studied systems of higher volume fraction particles, and little work has been done examining volume fractions under $5 \%$. The work done by Norato (1999) proved that current theory 
predicts attenuation well at low volume fraction but did not determine if reliable experimental data can be collected at low volume fraction. In addition, the systems studied were fairly ideal (monodisperse and very well characterized), and were not very representative of actual radioactive waste.

Ultrasonic speed work has concentrated on time-of flight measurements, generally ignoring dispersive effects, with the exception of the work done by McClements and coworkers and Cowen et Al. In addition, most speed experiments studied emulsions or other liquid-liquid systems. Little work has been done studying ultrasonic speed in solidliquid systems or in systems with significant dispersive effects.

Accordingly, the purpose of the work in this study is twofold. The usefulness of attenuation in characterizing low volume fraction slurries will be determined. Since speed is more tolerant of changes in particle size than attenuation the usefulness of speed in characterizing low volume fraction slurries will also be determined.

\section{Experimental}

\subsection{ATTENUATION BACKGROUND:}

Attenuation as a function of frequency is measured for three systems: (a) sodalime glass beads in water (referred to as the SLG system), (b) a physical radioactive waste slurry surrogate of Bentonite and kaolin clay in water (referred to as the BKC system), and (c) a chemical radioactive slurry surrogate consisting of a crystallized salt solution in supernate (referred to as the CSS system). The volume fraction range studied is approximately 0.005 to 0.1 . Multiple replicates are performed to ensure reproducibility of data, and determine the error in the results. 
The term 'attenuation" that has been used throughout this discussion is more appropriately called excess attenuation - that is, it is the additional attenuation of sound due to the presence of solids in the suspending liquid. Excess attenuation is determined by measuring the amplitude of the ultrasonic signal in the slurry (hereafter referred to as the sample reading) and comparing that to the amplitude of the ultrasonic signal in the pure liquid (referred to as the baseline reading). Since electrical transducers are used to create and measure the ultrasonic signal the amplitude is proportional to the voltage created by the transducer. Thus, for a given frequency and transducers $d$ distance apart the attenuation is determined by:

$$
\alpha=-\frac{1}{d} \ln \left(\frac{v_{\text {slurry }}}{v_{\text {liquid }}}\right)
$$

where $v_{\text {slurry }}$ is the amplitude of the electrical signal produced by the transducers interrogating the slurry system and $v_{\text {liquid }}$ is the amplitude of the electrical signal produced by the transducers interrogating the suspending liquid alone, with no solids present.

Two different methods are used to obtain voltage information at a specific frequency. The first method employs a spike pulse waveform followed by an FFT analysis. The other method uses a waveform of only one frequency (a toneburst). The voltages can then be read directly off of an oscilloscope and compared.

The spike pulse / FFT method as depicted in Figure 3.1 is by far the easier and more accurate way to measure attenuation. An ultrasonic pulse generator (Panametrics 5052PR) generates an electric pulse and sends it to the emitting transducer. The actuated 


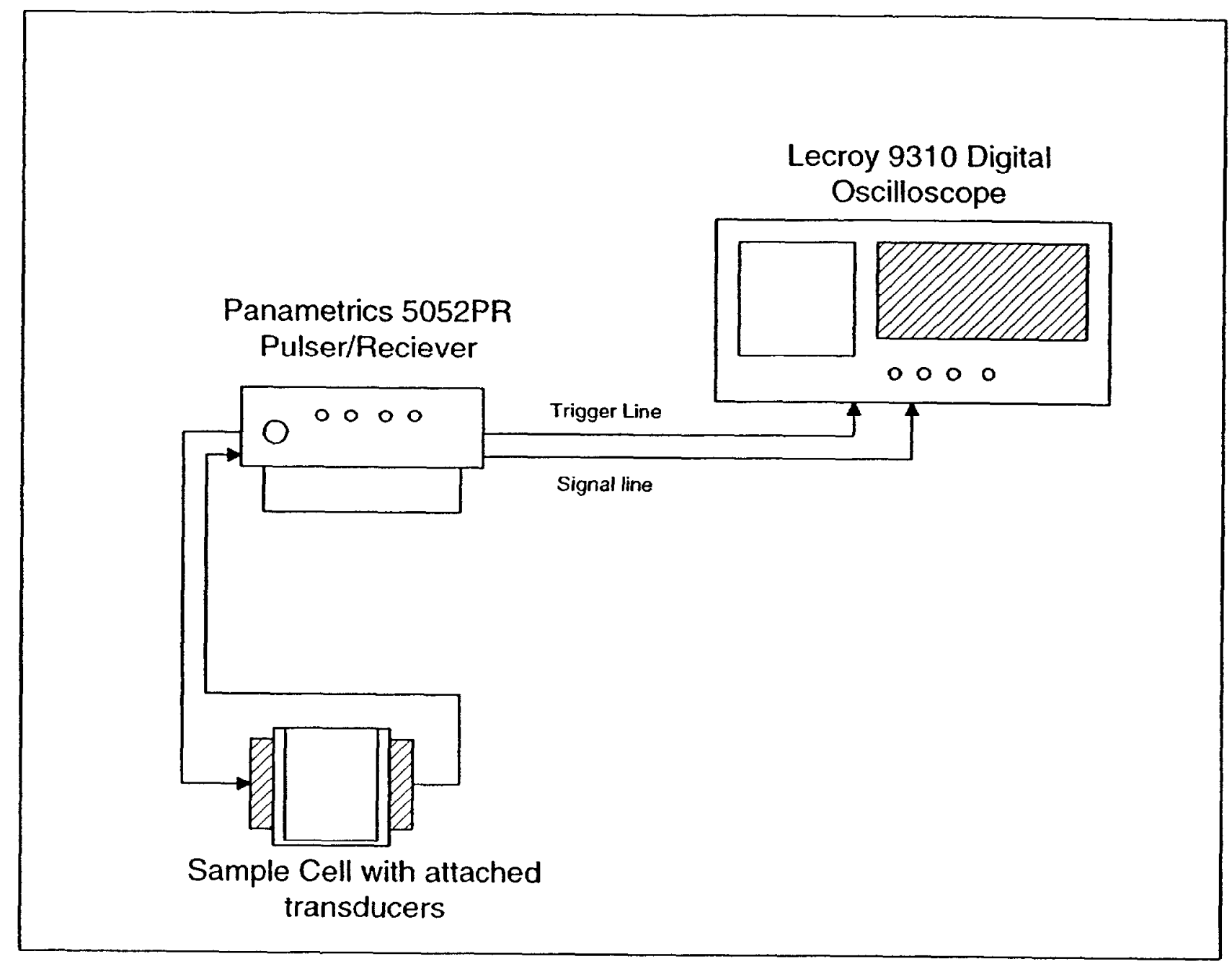

Figure 3.1: Schematic diagram of Pulse/FFT setup used to measure attenuation. A spike pulse is generated by the pulser/receiver and is transmitted to the transmitting transducer which is in contact with the sample. After traveling through the sample and being acquired by the receiving transducer the pulse is routed through the pulser/receiver to the oscilloscope. 
emitting transducer transmits an acoustic pulse through the sample actuating the receiving transducer, where the signal is transmitted to and read by an oscilloscope (Lecroy 9310A). An FFT analysis of the amplitude is performed on the spike pulse, outputting voltage as a function of frequency for all of the component frequencies of the pulse. This procedure is performed both on the suspending liquid and the solid-liquid slurry. The voltages are used in eqn. 1 to calculate the attenuation.

The toneburst method as depicted in Figure 3.2 is somewhat simpler in that no Fourier transforms are necessary, but is more tedious because an experiment needs to be nun for each specific frequency for which a measurement is made. A computer controlled toneburst card (Matec TB1000) is used to generate a signal of a constant, specific frequency, and transmit that signal to the send transducer. Again, the signal actuates the emitting transducer, propagates through the sample, and actuates the receive transducer, where it is read by the oscilloscope. The peak-to-peak voltage of the signal is calculated and these values are used in eqn. 1 to calculate attenuation.

It is instructive to describe the method of data capture by the oscilloscope. In order to minimize error due to abnormal waveforms, and random noise, the oscilloscope actually takes an average of a certain designated number of spike pulses or toneburst sweeps before the FFT or amplitude calculation is made. The number of sweeps used to calculate the average is important because it determines the sampling time. If the sampling time is too long significant settling of the suspended solids in the test cell may occur, causing error due to appreciable variation of the actual suspended solids concentration in the test cell. If the number of sweeps taken is too small then random signal noise may be significant. This may be less of a problem in pipe slurry 

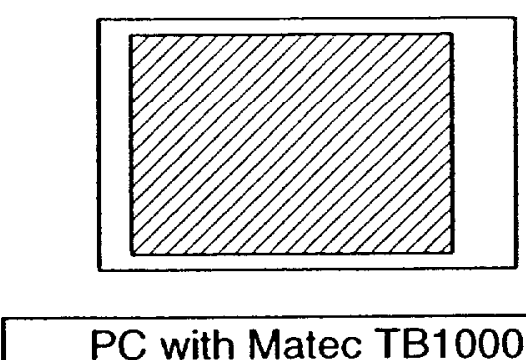

Toneburst Card

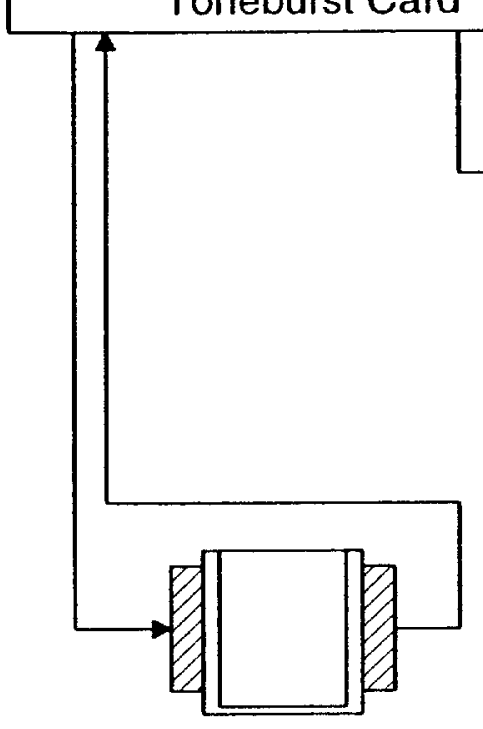

Sample Cell with attached transducers

Figure 3.2: Schematic diagram of toneburst setup used to measure attenuation. A toneburst card located in a PC generates an electrical signal of constant frequency and transmits that signal to the transmitting transducer which is in contact with the sample. After traveling through the sample and being acquired by the receiving transducer the pulse is routed through the toneburst card to the oscilloscope. 
measurements. It was found that 50 sweeps produced an acceptable average waveform with little noise, and had a short enough sampling time ( 2 scconds) that settling of the slurry was insignificant. This finding is reinforced using Stokes law - under average experimental conditions terminal settling velocity is $3.4 \times 10^{-4} \mathrm{~m} / \mathrm{s}$, giving a $6.7 \times 10^{-2} \mathrm{~cm}$ drop during the two second sampling period.

As mentioned above, the spike pulse / FFT was found to be the preferred method for measuring attenuation. It was found to be much more accurate and consistent. The data it produces have much less noise than data resulting from the toneburst method. This is mainly due to the fact that only 2 experiments need to be performed using the pulse/FFT method (sample and reference) per pair of transducers, while up to 60 experiments per pair of transducers would be needed using the toneburst method to cover the same range and resolution. Nevertheless, both methods were employed to prove the consistency of the spike pulse/FFT.

The transducers used were immersion transducers made by Panametrics. Like a speaker, a specific transducer can only create/read ultrasonic signals in a specific frequency range. In order to cover the entire range desired $(0.5$ to $13 \mathrm{mHz})$, different transducers with different center-line frequencies must be employed. Five different pairs of transducers were used in this study, with centerline frequencies of $1 \mathrm{mHz}, 2.25 \mathrm{mHz}, 5$ $\mathrm{mHz}, 7.5 \mathrm{mII}$, and $10 \mathrm{mHz}$. See Appendix III for the frequency range, model number and serial number of all transducers used.

The test cell used for all attenuation experiments was a Plexiglas vessel with a nominal pathlength of two inches. The two inch pathlength was chosen because 2 inches is the diameter of the pipe used to transfer slurries at Hanford. The cell used can 
accommodate transducer pairs with centerline frequencies of $1 \mathrm{MHz}$ to $10 \mathrm{MHz}$. See

Figure 3.3 for the schematic of the cell.

\subsection{ATTENUATION EXPERIMENTS}

\subsubsection{SODA LIME GLASS}

The first system studied is soda-lime glass beads in distilled water. Volume fraction ranged from 0.004 to 0.05 . The particles, supplied by Cataphote® , Inc., have an average radius of $32 \mu \mathrm{m}$, and standard deviation of $7.6 \mu \mathrm{m}$. Particle density is 2.54 $\mathrm{g} / \mathrm{cm}^{3}$. See Table 3.1 for further information on physical properties.

\begin{tabular}{|c|c|c|}
\hline & Soda-lime Glass & Water \\
\hline Density $\left(\mathrm{g} / \mathrm{cm}^{3}\right)$ & 2.54 & 1.0 \\
\hline $\begin{array}{l}\text { Thermal Conductivity } \\
(\mathrm{J} / \mathrm{K} \cdot \mathrm{cm} \cdot \mathrm{s})\end{array}$ & $9.6 \times 10^{-3}$ & $5.87 \times 10^{-3}$ \\
\hline $\begin{array}{l}\text { Specific Heat } \\
(\mathrm{J} / \mathrm{g} \cdot \mathrm{K})\end{array}$ & 0.836 & 4.19 \\
\hline$\left(\mathrm{K}^{-1}\right)$ Thermal Expansion Coefficient & $3.2 \times 10^{-6}$ & $2.04 \times 10^{-4}$ \\
\hline $\begin{array}{l}\text { Attenuation Coefficient per } \mathrm{f}^{2} \\
\left(\mathrm{~s}^{2} / \mathrm{cm}\right)\end{array}$ & $1.0 \times 10^{-15}$ & $2.5 \times 10^{-16}$ \\
\hline $\begin{array}{l}\text { Sound Speed } \\
(\mathrm{cm} / \mathrm{s})\end{array}$ & $5.2 \times 10^{5}$ & $1.48 \times 10^{5}$ \\
\hline $\begin{array}{l}\text { Shear Viscosity } \\
\left(\mathrm{g} / \mathrm{cm} \cdot \mathrm{s}^{2}\right)\end{array}$ & - & $1.01 \times 10^{-2}$ \\
\hline $\begin{array}{l}\text { Shear Rigidity } \\
\left(\mathrm{g} / \mathrm{cm} \cdot \mathrm{s}^{2}\right)\end{array}$ & $2.8 \times 10^{11}$ & - \\
\hline \multicolumn{3}{|c|}{$\begin{array}{l}\text { Table 3.1: Physical properties of Soda-lime glass / water system. Properties are from } \\
\text { Kinsler et al. (1982) and Bolz (1973) }\end{array}$} \\
\hline \multicolumn{3}{|c|}{ Slurries were prepared by weighing a calculated amount of solids into a } \\
\hline
\end{tabular}




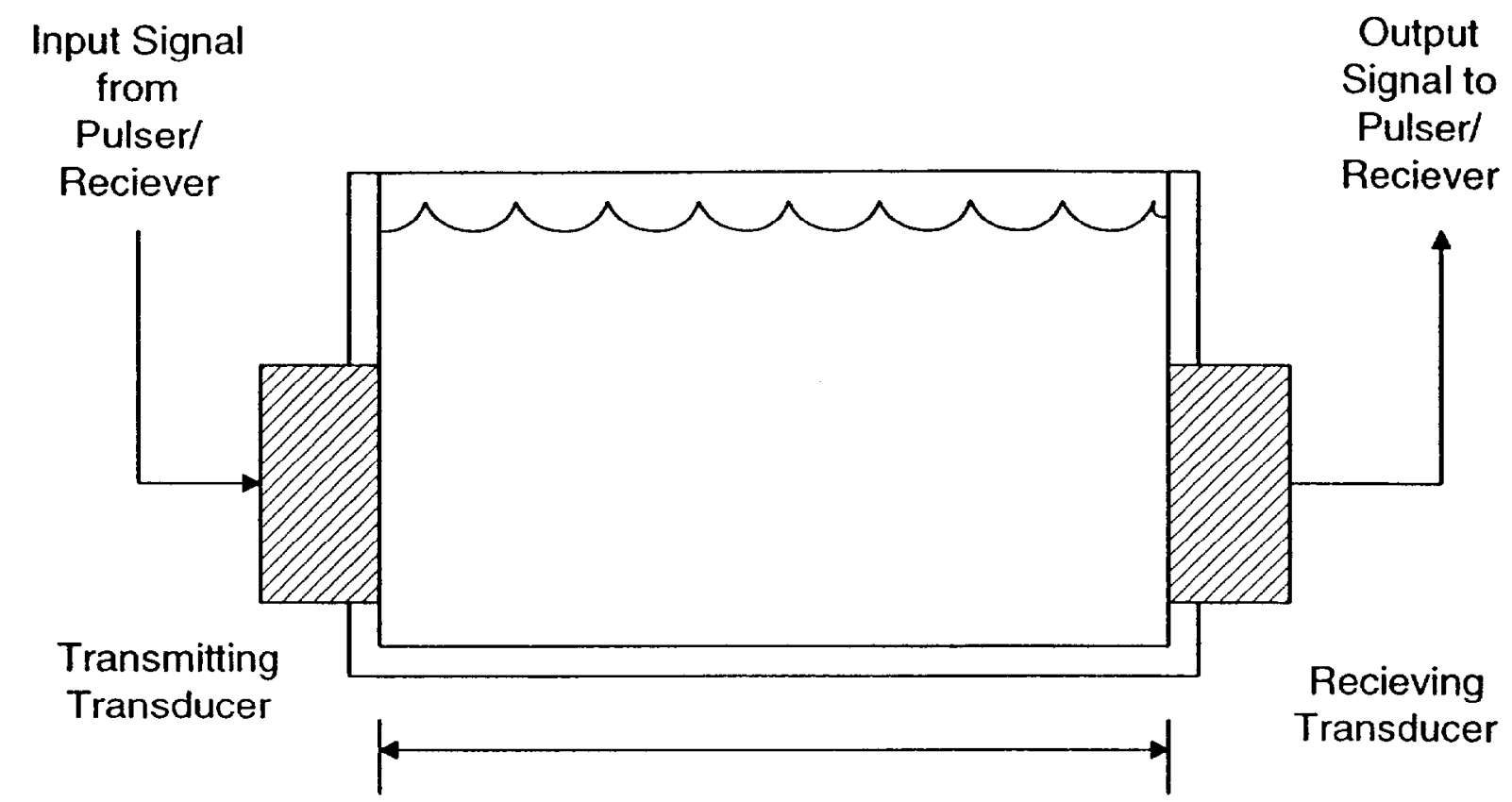

Two Inch Pathlength

Figure 3.3: Schematic diagram of attenuation test cell. Cell is constructed of Plexiglas with a nominal pathlength of two inches. Transducers with centerline frequencies of $1 \mathrm{MHz}$ to $10 \mathrm{MHz}$ are mounted on each side to complete the cell. 


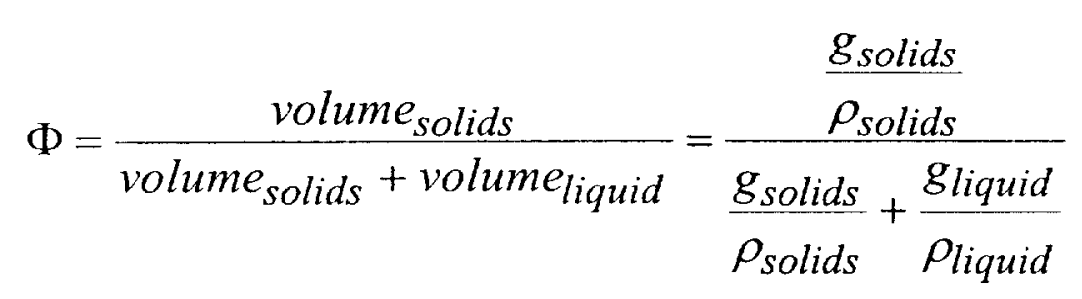

where

$$
\begin{aligned}
& \mathrm{g}_{\text {solids }}=\text { mass of solid particles added }(\mathrm{g}) \\
& \mathrm{g}_{\text {liquid }}=\text { mass of suspending liquid added }(\mathrm{g}) \\
& \rho_{\text {solid }}-\text { density of solid particles }\left(\mathrm{g} / \mathrm{cm}^{3}\right) \\
& \rho_{\text {liquid }}=\text { density of suspending liquid }\left(\mathrm{g} / \mathrm{cm}^{3}\right)
\end{aligned}
$$

Slurries were prepared immediately prior to testing. The test cell was prepared with the appropriate transduccrs, and the pathlength measured using a dial caliper. The sample was hand stirred with a stainless steel spatula and a reading was taken.

In order to determine the best frequency range for studying low $\Phi$ slurries, a sample was interrogated with all 5 pairs of transducers. In addition, both the pulse/FFT and toneburst methods were employed.

The standard method of obtaining a baseline reading had been to fill the cell with water, take a reading, and empty and dry the cell. Slurry would then be added to the cell to collect the sample reading. This method had several disadvantages - it was time consuming and it introduced more possibilities for error. For example, the handling of the cell necessary to empty, dry, and refill it could cause the pathlength to change slightly. In addition, the time lapse between gathering the two signals could lead to slight changes in the waveform produced by the pulser/receiver or toneburst card. The attenuation calculation is based on the assumption that the slurry and the suspending liquid are both subjected to the same waveform. Any change in that waveform could lead to error. 
Without stirring, the soda-lime glass beads settle to the bottom of the vessel quickly. This behavior was exploited to improve the method of determining the water signal. Since only a small amount of solids was used for these experiments, after they settled the space between the transducers contained only distilled water. Therefore, the completely settled system was used to acquire the baseline reading. Immediately after the baseline reading was taken, the system was stirred until all solids were resuspended and the sample reading was taken.

\subsubsection{CLAY SURROGATE}

After preliminary work with soda lime glass at low $\Phi$ it was decided to attempt to use ultrasound to characterize a system more representative of the nuclear waste. A number of surrogate slurries have been developed to simulate nuclear waste (Golcar 2000 ), one being a primarily rhealogical surrogate composed of bentonite and kaolin clays. The slurry selected for these experiments consisted of two parts (dry weight) kaolin to one part bentonite.

Kaolin clay is composed mostly of silica and alumina arranged in alternating layers. A particle is generally square shaped, with sides less then $1-2 \mu \mathrm{m}$ in length. The particles, held together by strong hydrogen bonds, can be 70 to 100 layers thick. (Holtz and Kovacs, 1981) Bentonite is actually a mixture of several different types of minerals, with smectitc as the major component (Grim 1968).

Since Bentonite particles swell when added to water it is important to prepare the

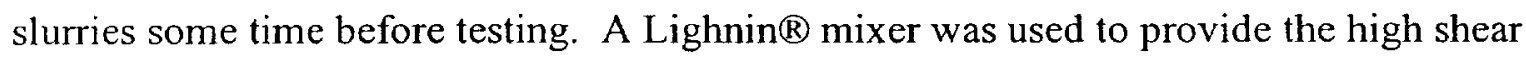
that is needed to properly disperse clay. Kaolin was added to water and the mixture was 
stirred until the clay was dispersed (usually about 15 minutes). Then bentonite was added and the mixture was stirred again. The slurries were allowed to sit overnight and then mixed again before testing.

The test procedure was similar to that performed on the soda lime glass. Only one set of transducers $(10 \mathrm{MHz})$ was used. The pulse/FFT method was the only testing method employed. Since lay does not settle readily, a baseline reading was acquired in distilled water. Since the density of the clay particles was not known slurries were prepared on a weight fraction basis, with weight fractions ranging from one to five percent.

\subsubsection{CRYSTALLIZED SALT SOLUTION}

The third system studied is a crystallized salt solution proposed by researchers at the Hanford site to represent the chemical and physical behavior of an average composition of Hanford supernate nuclear waste containing suspended saltcake particles, with the exception of the radioactive properties. All of the major components in the waste tanks are present in the chemical slurry surrogate, and they are in the correct proportions. See Table 3.2 for the chemical species present, and the concentration of each species:

\begin{tabular}{lccc}
\hline $\begin{array}{l}\text { Chemical } \\
\text { Species }\end{array}$ & $\begin{array}{c}\text { Feed Solution } \\
(M)\end{array}$ & $\begin{array}{c}\text { Concentrated Solution } \\
(M)\end{array}$ & Weight Percent \\
\hline $\mathrm{NaOH}$ & 1.61 & 2.3 & 5.9 \\
$\mathrm{NaAl}(\mathrm{OH})_{4}$ & 1.54 & 2.2 & 16.3 \\
$\mathrm{NaNO}_{3}$ & 2.59 & 3.7 & 20.3 \\
$\mathrm{NaNO}_{2}$ & 2.24 & 3.2 & 14.3 \\
$\mathrm{Na}_{2} \mathrm{CO}_{3}$ & 0.42 & 0.6 & 4.1 \\
$\mathrm{H}_{2} \mathrm{O}$ & & & 31.7 \\
\hline
\end{tabular}

Table 3.2: Crystallized Salt Solution components 
In brief, the solution is prepared by dissolving all of the solids in water and then evaporating the solution down to $70 \%$ of the original volume to form a precipitate. The complete procedure is described in Appendix I. The mixture is centrifuged to separate the solids (precipitate) from the liquid (supernate). The solids are stored wet to prevent caking and the density of the supernate is measured.

Again, the procedure used to determine the attenuation of the surrogate slurry was similar to that used on the soda lime glass beads, except that only the $10 \mathrm{MHz}$ transducers and the pulse/FFT method were employed. The baseline reading is acquired in supernate. Solids are added gradually with intermediate testing to get attenuation data at different weight fractions.

The actual volume (or weight) fraction studied is not known, since it is difficult to measure the density of the solids, and the solids are not dried out completely. Accordingly, the following procedure is employed to estimate the weight percent solids in the slurry. Assuming a packing efficiency of $60 \%$ and a solid density of $2.2 \mathrm{gm} / \mathrm{cm}^{3}$ (typical of sodium salts, according to Weast, 1972) an approximate dry weight percent of the concentrated solids feedstock is given by:

$$
W_{d r y}=W_{w e t} \times \frac{1 \mathrm{~cm}^{3}}{1.91 \mathrm{~g}} \times 0.6 \times \frac{2.2 \mathrm{~g}}{\mathrm{~cm}^{3}}
$$

where:

$0.6=$ Assumed packing efficiency (volume fraction of concentrated solids)

$2.2 \mathrm{~g} / \mathrm{cm} 3=$ Assumed dry solids density

$1.91 \mathrm{~g} / \mathrm{cm} 3=$ Assumed density of wet solids 


\subsection{SPEED BACKGROUND}

Previous work (Norato 1999) characterizing slurries using ultrasound had dealt with measuring attenuation exclusively. Theory was developed (Spelt et al., 2000) that predicted ultrasonic speed. In order to test the theory and evaluate the utility of speed information in characterizing slurries it was deemed desirable to develop a method to measure ultrasonic speed as a function of frequency in liquid slurries. The technique developed by McClements and co-workers (1991) was selected because it is proven successful and is similar to the method already in use to measure attenuation

The technique uses a dual reflectance method, along with an FFT, to measure sound speed. The experimental setup, shown in Figure 3.4, is very similar to the Spike Pulse/FFT setup used to measure attenuation. The test cell, as depicted in Figure 3.5, is similar to that used to measure attenuation, but only one transducer is used. Instead of being in direct contact with the fluid, the transducer is in contact with a Plexiglas buffer. Ultrasound gel (Parker Laboratories Scan gel) is used to couple the transduccr to the Plexiglas. The sample is on the other side of the Plexiglas buffer. A brass reflector plate is mounted on the rear of the cell. The reflector plate is constructed of brass due to its high impedance and its resistance to corrosion. The reflector is removable and the pathlength can be altered by installing a thicker or thinner reflector plate. For these experiments two reflector plates were used, a thinner one that gives a pathlength of one inch, and a thicker one that gives a pathlength of $1 / 2$ inch.

A spike pulse waveform is used to energize the transducer. As the sound travels through the cell it is actually reflected twice - once at the Plexiglas/sample interface and 


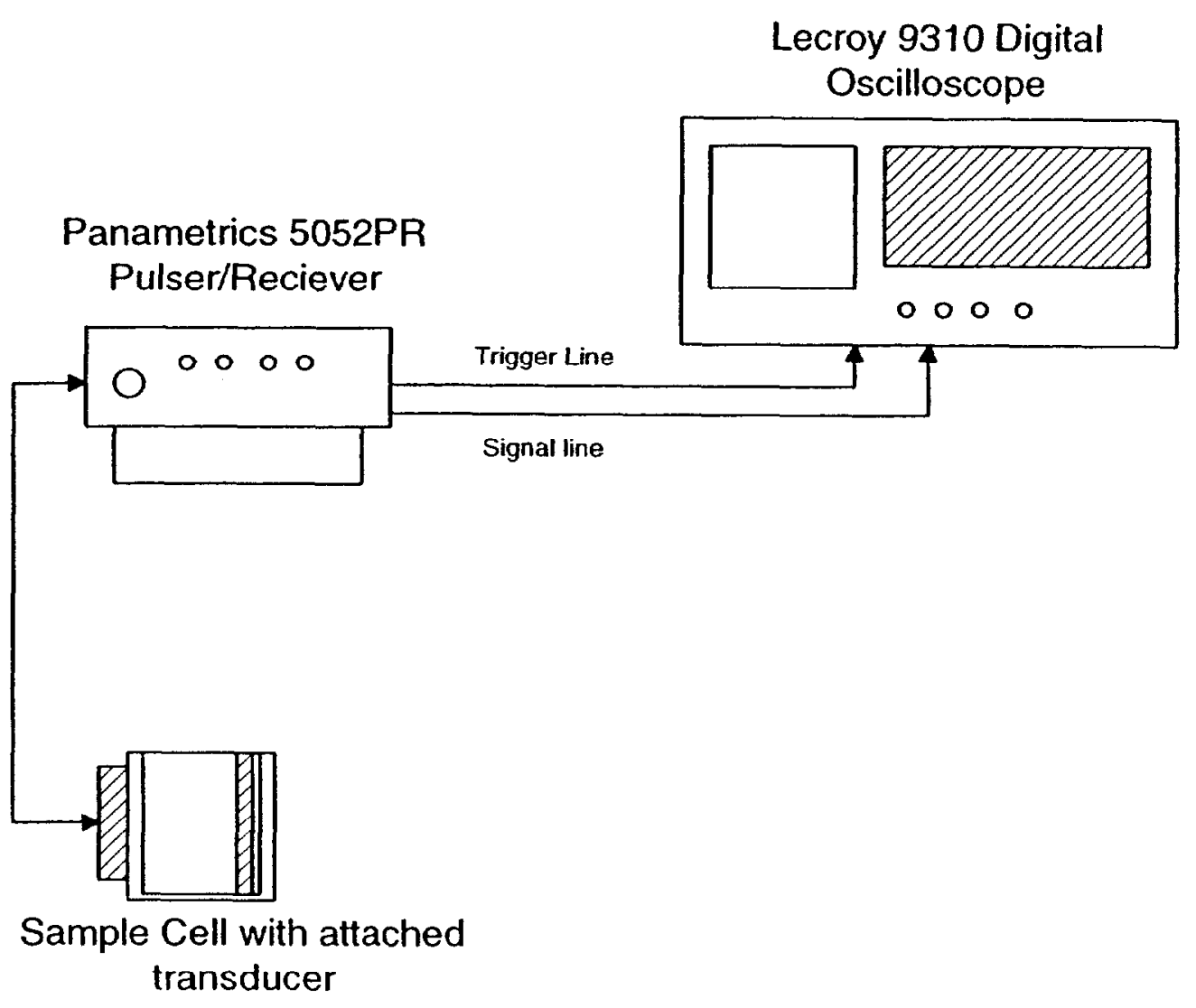

Figure 3.4: Schematic diagram of setup used to measure speed. A spike pulse is generated by the pulser/receiver and is transmitted to the transducer, which is coupled to a Plexiglas $($ buffer with ultrasound gel. The signal travels through the buffer and some signal is reflected back at the buffer/sample interface. The remaining signal travels through the sample and is reflected off a brass plate mounted at the far end of the cell. The same transducer that emitted the signal picks up both reflections and routes the reflected signals through the Pulser/Receiver to the oscilloscope. 
Signal $\ln /$

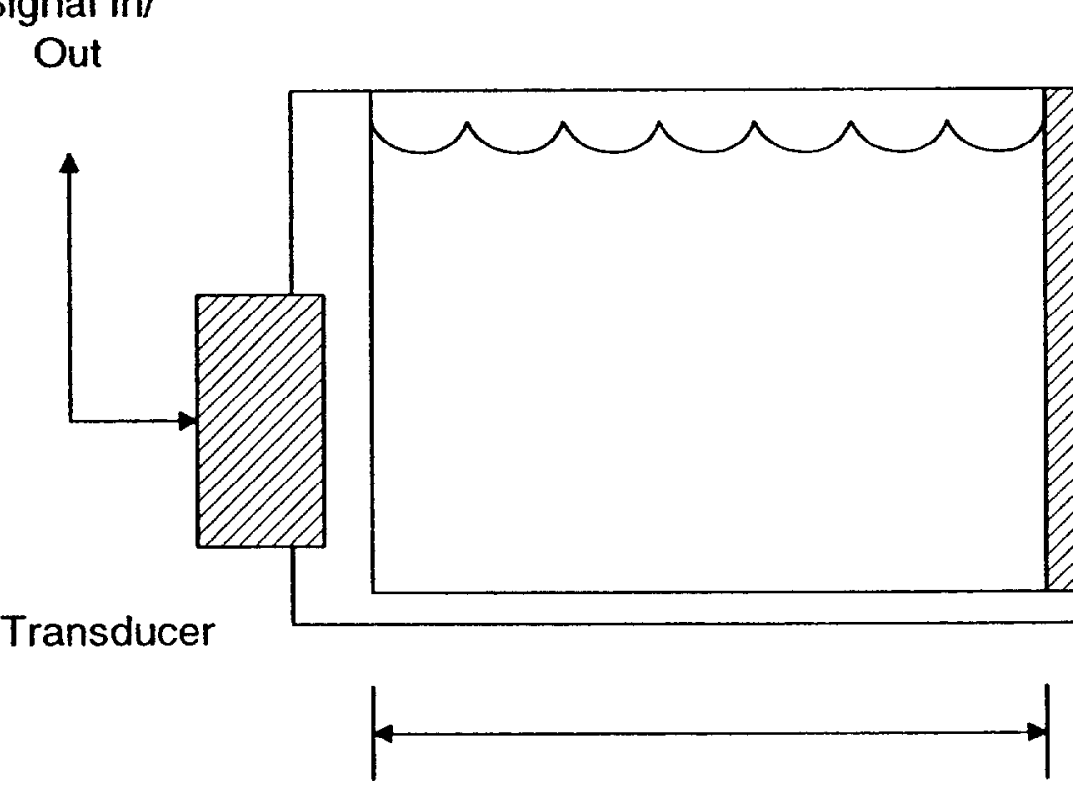

1" or $1 / 2$ " Pathlength

Figure 3.5: Schematic diagram of speed test cell. Cell is constructed of Plexiglas with a brass reflcctor plate. The reflector plate is interchangeable, with current plates creating pathlengths of $1 / 2$ inch or one inch. A single transducer is mounted on one side, coupled with ultrasound gel. The signal passes through a buffer rod of Plexiglas (thickness: $1 / 4$ inch) before coming in contact with the sample. 
again off the brass reflector at the far end of the cell. See Figure 3.6 for a typical waveform produced by this sctup. Since travel time through the wires, transducers, Plexiglas buffer, and other electrical components is common to both signals the exact travel time through the sample can be calculated by subtracting the time of the first peak from the time of the second peak. As shown below, by using an FFT to calculate the phase difference between the two reflected signals the speed as a function of frequency can be measured.

The test cell must be designed properly in order to produce acceptable data. The Plexiglas buffer must be thick enough such that the transducer stops ringing before the first reflection reaches it. Plexiglas, like all materials, absorbs sound, and so if the buffer is too thick the signal will be attenuated and signal will be lost unnecessarily. In addition, the buffer thickness and sample pathlength must be designed so that signals which reflect internally in the buffer rod do not interfere with the second echo returning from the brass reflector.

The phase speed is calculated using the following equation (McClements, 1991):

$$
c=\text { pathlength } \times \frac{360 \cdot f}{\left(n \cdot 360+\delta \theta-\theta_{d}-180\right)}
$$

where

$$
\mathrm{c}=\text { phase speed }
$$

$\mathrm{f}=$ frequency

$\delta \theta=$ phase difference from FFT

$\theta_{\mathrm{d}}=$ phase correction due do diffraction (usually negligible)

$\mathrm{n}=$ number of waves $\sim \Delta \mathrm{t}^{*} \mathrm{f}$

and $\Delta t($ above $)=$ time difference between main peaks of the two reflections 


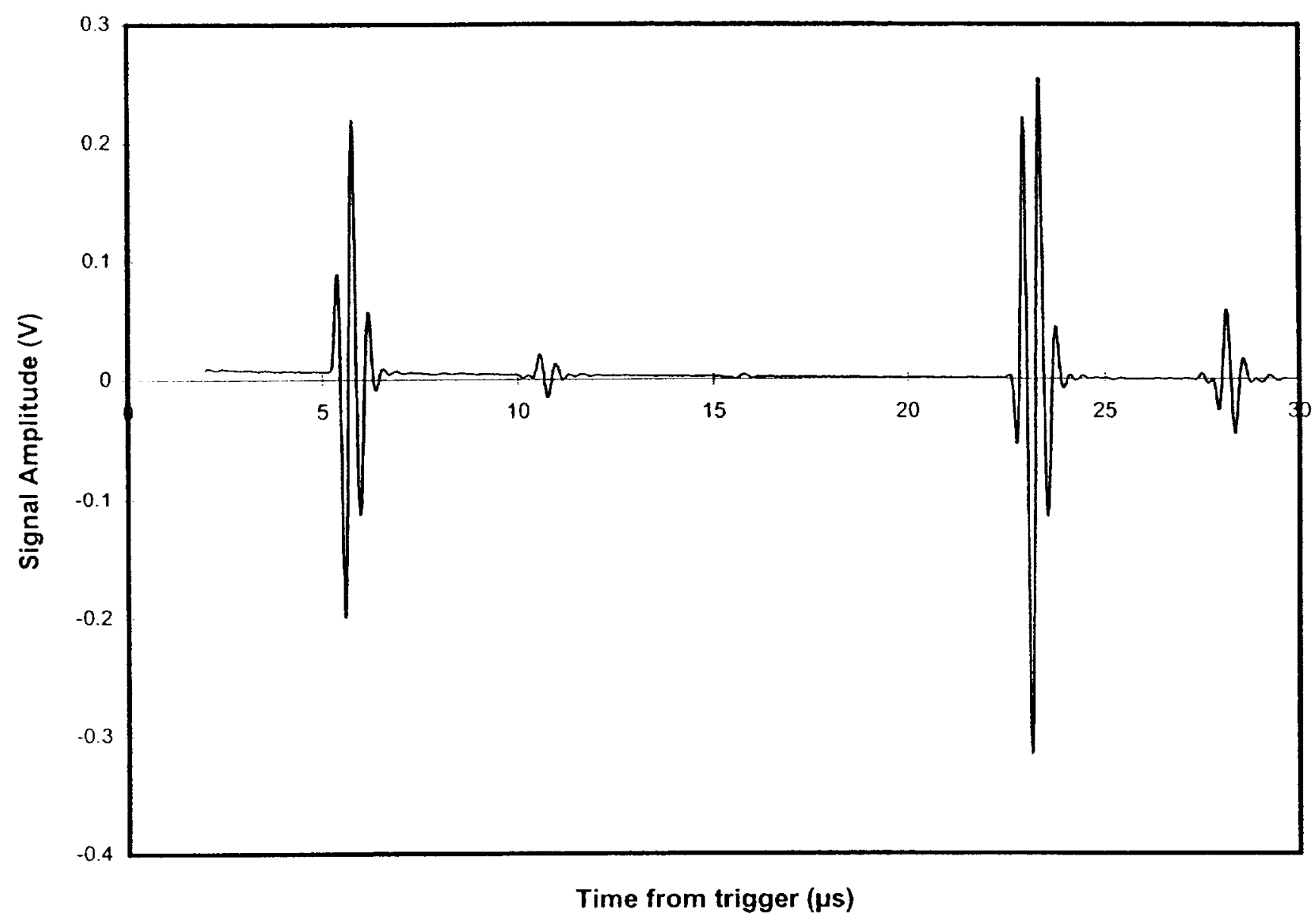

Figure 3.6: Typical waveform produced from speed test setup. The first peak, at $t \sim 6 \mu \mathrm{s}$, is reflected at the buffer-rod sample interface. The second peak, at $\mathrm{t}-23 \mu \mathrm{s}$, is reflected off the brass plate at the far end of the cell. The smaller peaks at $t$ $\sim 11 \mu \mathrm{s}$ and $\mathrm{t} \sim 28 \mu \mathrm{s}$ are echoes of the larger peaks. 
The main peaks of the two waveforms are superimposed and a phase FFT operation is performed on both. The difference in phase between the first and second echo as a function of frequency is determined. The 180 degree correction is required because the signal is inverted as it reflects off the brass plate. In practice, it was easier to superimpose the waveforms if the second echo is inverted employing software available with the oscilloscope. Accordingly, the 180 degree correction was not necessary.

The speed of sound in water is strongly dependent on temperature. At room temperature the speed of sound increases approximately $3 \mathrm{~m} / \mathrm{s}$ for every degree Celsius the temperature is increased. Although this change is small compared to the speed of sound in water at room temperature $\left(\sim 1500 \mathrm{~m} / \mathrm{s}\right.$ at $\left.23^{\circ} \mathrm{C}\right)$ it has a significant effect when trying to correctly discern the volume fraction of suspended particles. In order to provide better temperature control the test cell (with attached immersion transducer) is placed in a water bath (Neslab GP-300). Slurry temperature was maintained at $25^{\circ} \mathrm{C} \pm 0.1^{\circ}$, verified by a digital thermometer (Cole-Parmer Thermistor Model 8502-12).

\subsubsection{SPEED EXPERIMENTS}

Speed measurements are made in a system consisting of the same soda-lime glass beads described above that were used for attenuation. In order to gauge the effect of particle size on speed larger particles, manufactured by Potter's Industries (P-0060, lot 0422(98)) are also tested. These particles are larger than those previously studied, with a mean diameter of $125 \mu \mathrm{m}$ and a standard deviation of $19 \mu \mathrm{m}$. Although larger than the soda-lime glass beads the Potter's beads are composed of the same material (silica) so 
physical properties for the Potter's beads were taken to be the same as those for soda-lime glass.

In order to accurately measure the speed of sound the pathlength must be known very precisely. For a $1 / 2$ inch pathlength, a measurement error of $1 / 1000$ of an inch results in an error of $3 \mathrm{~m} / \mathrm{s}$ under typical conditions. In order to be able to measure the absolute sound speed with as little error as possible it is necessary to measure the pathlength to the $1 / 10,000$ of an inch. Since it is impossible to measure this accurately in a direct fashion, the cell is calibrated using distilled water. The cell is filled with water and the transit time was measured at different temperatures. The speed of sound in water is very accurately known (Lynnworth) so with careful monitoring of temperature the pathlength can be calculated to the desired accuracy of $1 / 10,000$ of an inch.

The speed of sound is measured in slurries of both the soda-lime glass particles and Potters' beads suspended in distilled water. Volume fraction ranges from $5 \%$ to $45 \%$. Initially all 5 transducers were used, to cover the entire available frequency range, but for most experiments the $2.25 \mathrm{MHz}$ transducer provided all the data necessary.

Experiments are also conducted at low volume fraction. Speed is measured in soda-lime glass slurries, at volume fractions of $0.004,0.01,0.03$, and 0.05 . For these experiments a "settled solids" approach similar to that used to measure attenuation is used. Speed is measured in distilled water, with the solids settled. Then the system is stirred to resuspend the solids, and the speed in the slurry is measured. The difference between the two velocities is calculated. 


\section{Theory}

The theory used to predict attenuation in solid-liquid two phase systems is given in Spelt et $\Lambda 1$. (2000). It uses an effective medium approach to predict viscous, thermal, and scattering losses. In this approach the particle is surrounded by a spherical shell of fluid. This particle-cell combination is immersed in a uniform suspension (the effective medium). The properties of the effective medium are determined by solving the governing equations for the particle-cell combination so they are consistent with the averaged equations for the suspension.

The theory in its entirety is very complcx and will not be discussed here. $\Lambda$ brief summary valid for dilute slurries, where one only has to consider the affects of a sound wave on a single particle will be given.

Epstein and Carhart (1953) first derived the wave equations for the interior and exterior of solid particles. They linearized the equations conserving mass, momentum, and energy, and eliminated pressure and internal energy using the linearized equations of slate to give relationships in terms of speed, density, and temperaturc. The specd vector $v$ can be expressed in terms of the vector potential $A$ and a scalar potential $\phi$ as shown:

$$
v=-\nabla \phi+\nabla \times A
$$

with $\nabla \cdot A=0$. It is then possible to eliminate temperature and density to give a forthorder partial differential equation for $\phi$ and a second order equation in $\mathrm{A}$. The $\phi$ expression can be split into two second order wave equations by substituting 
$\phi=\phi_{c}+\phi_{T}$, with $\phi_{c}$ and $\phi_{T}$ being the scalar potentials of the compressional and thermal waves. The result is three wave equations:

$$
\begin{aligned}
& \left(\nabla^{2}+k_{c}^{2}\right) \phi_{c}=0 \\
& \left(\nabla^{2}+k_{T}^{2}\right) \phi_{T}=0 \\
& \left(\nabla^{2}+k_{s}^{2}\right) A=0
\end{aligned}
$$

The wavenumbers in the above equations are given by

$$
\begin{gathered}
\frac{1}{k_{c}^{2}}=\frac{c^{2}}{2 \omega^{2}}\left[1-i(e+\not f)+\left\{(1-i(e+\not f))^{2}+4 f(i+\gamma e)\right\}^{1 / 2}\right] \\
\frac{1}{k_{T}^{2}}=\frac{c^{2}}{2 \omega^{2}}\left[1-i(e+\gamma f)-\left\{(1-i(e+\not f))^{2}+4 f(i+\gamma e)\right\}^{1 / 2}\right] \\
k_{s}=(1+i)\left(\frac{\omega \rho}{2 \mu}\right)^{1 / 2}
\end{gathered}
$$

with

$$
e \equiv\left(\frac{4 \mu}{3}+\kappa\right) \frac{\omega}{\rho c^{2}} ; f \equiv \frac{\sigma \omega}{c^{2}}
$$

In these expressions $c$ is the phase speed in the pure liquid, $\rho$ is density, $\kappa$ and $\mu$ the compressional and dynamic viscosities, $\gamma$ the ratio of specific heats $\left(\mathrm{C}_{\mathrm{p}} / \mathrm{C}_{\mathrm{v}}\right), \tau$ the thermal conductivity, and $\sigma=\tau / \rho C_{p}$ the thermal diffusivity. 
Similar equations are valid inside the particles, with the dynamic viscosity

replaced by $\mu /(-i \varpi)$ and the wave speed by $((\tilde{\lambda}+2 \tilde{\mu} / 3) / \widetilde{\rho})^{1 / 2}$, where $\tilde{\lambda}$ and $\tilde{\mu}$ are the Lamé constants. The compressional viscosity of the solid is set to zero. The tilde notation refers to quantities inside the particles.

At small values of $e$ and $f$ (as in water) the above expressions for $k_{c}$ and $k_{T}$ simplify to

$$
k_{c}=\frac{\varpi}{c}+\frac{i}{2}\left[\left(\frac{4}{3} \mu+\kappa\right) \frac{1}{\rho}+(\gamma-1) \sigma\right] \frac{\varpi^{2}}{c^{3}}
$$

and

$$
k_{T}=(1+i)(\varpi / 2 \sigma)^{1 / 2}
$$

Equation (2) and its inside-particle counterpart represent the sound propagation through the slurry. The imaginary part of the wavenumber equals the attenuation. The bracketed term in equation. (9) is referred to as the 'diffusivity of sound'. The total attenuation coefficients in the liquid and in the solid particle will be treated as additional physical propertics. The other two wave equations ( 3 and 4) describe waves due to thermal conduction and finite viscosity. Note that the modulus $\mathrm{k}_{\mathrm{T}}$ in $(10)$ is inversely proportional to the thermal penetration depth $\sqrt{\sigma / \varpi}$ and $\mathrm{k}_{\mathrm{s}}$ is inversely proportional to the viscous penetration depth $\sqrt{\mu / \rho \pi}$. Thermal $\left(\phi_{\mathrm{T}}\right)$ and shear (A) waves usually have very high attenuation and are unimportant.

It is possible in principle to determine the phase speed and attenuation at arbitrary volume fractions using the above equations. The result can be determined by applying 
boundary conditions of continuity to temperature, speed, heat flux and traction and solving the problem numerically.

The potential $\phi_{\mathrm{c}}$ outside a particle located at $\mathrm{x}_{1}$ can be expressed as:

$$
\phi_{c}(x)=\exp \left(i k_{c} \bullet x\right)+\sum_{n=0}^{\infty} i^{n}(2 n+1) A_{n} h_{n}\left(k_{c} r\right) P_{n}(\mu)
$$

where $r=\left|x-x_{1}\right|, \mu=\cos \theta, \theta$ as the anglc between $x-x_{1}$ and $k_{c}$. The spherical Bessel function of the third kind (or Hankel function) $h_{n}$ corresponds to an outgoing scattered wave and $P_{n}$ is the Legendre polynomial of degree $n$.

Inside the particle centered at $x_{1}$ we have

$$
\left\langle\widetilde{\phi}_{c}\right\rangle=\exp \left(i k_{c}\right) \sum_{n=0}^{\infty} i^{n}(2 n+1) \widetilde{A}_{n} j_{n}\left(\widetilde{k}_{c} r\right) P_{n}(\mu)
$$

where $\mathrm{j}_{\mathrm{n}}$ is the spherical Bessel function of the first kind. Similar expressions are written for $\phi_{\mathrm{T}}$ and A. Expressions with a set of six unknowns for each mode $\mathrm{n}$ result. Application of the boundary conditions of continuity of speed, traction, temperature, and heat flux give six equation with six unknowns for each $\mathrm{n}$. In a few limiting cases it is possible to solve for the unknowns analytically. However, it is best to solve the equations numerically since it is desired to cover a large range of frequencies.

After the coefficients are determined, the attenuation can be calculated per unit length using the result given by Allegra and Hawley (1972) as:

$$
\alpha=-\frac{3 \phi}{2 z^{2} a} \sum_{n=0}^{\infty}(2 n+1) \operatorname{Re} A_{n}
$$


When the total volume fraction is small the above method can be extended to account for a non-uniform particle size distribution by summing up the attenuation caused by each individual particle size present:

$$
\alpha_{\text {tot }}(f)=-\sum_{a=0}^{\infty} \hat{\alpha}(f, a) \phi(a) d a
$$

where $\hat{\alpha}(f, a)$ is the attenuation density and $\phi(a) d a$ is the volume fraction of a particular size range between $a$ and $a+d a$. Equation (14) is the basis of the computer program provided by Dr. Peter Spelt (2000) used with his assistance to calculate theoretical attenuation in solid-liquid slurries.

\section{Results}

Experimental results for the three systems studied follow. Included are comparisons of expcrimental results with theory and a study evaluating the reproducibility of the experimental data.

\subsection{ATTENUATION IN DILUTE SODA-LIME GLASS BEADS}

Figure 5.1 shows attenuation versus volume fraction at three different frequencies - $8 \mathrm{MHz}, 10 \mathrm{MHz}$, and $12 \mathrm{MHz}$, for dilute soda-lime glass beads in water. Volume fraction ranged from $0.04 \%$ to $5 \%$. As expected the relationship between attenuation and volume fraction is linear, with $\mathrm{R}^{2}$ ranging from 0.9853 to 0.9986 . Note that attenuation is significant (and measurable) even at very low volume fractions. Figure 5.2 shows the data at extremely low volume fraction (less than $2 \%$ ).

Figure 5.3 shows a typical attenuation versus frequency curve. Note the unexpected maximum at low frequencies. It is suspected that the particles are slightly 


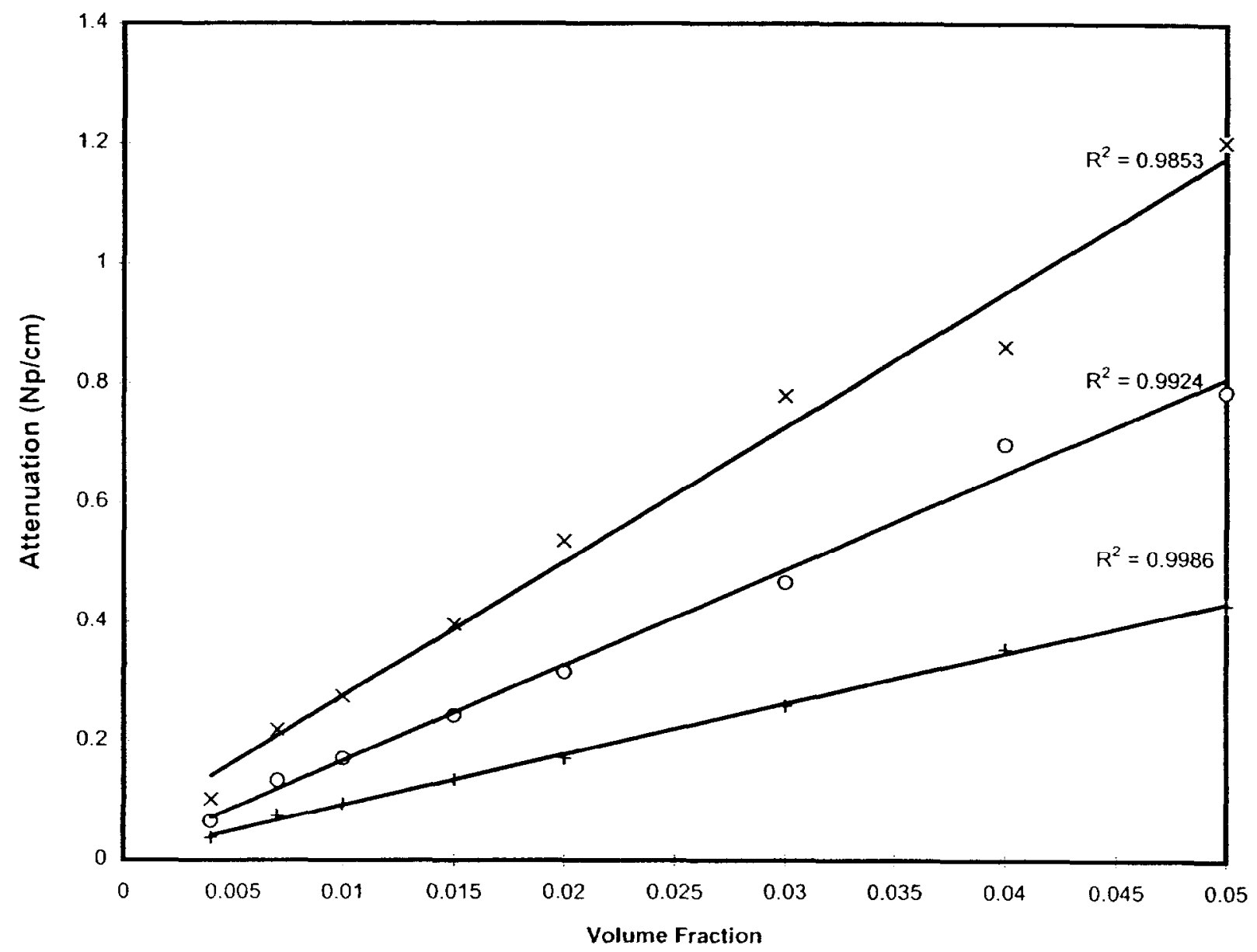

Figure 5.1: Attenuation versus volume fraction for a slurry of soda lime glass particles (radius $=16 \mu \mathrm{m}$ ) in water at three frequencies. The frequencies studied are $(x)$ $12 \mathrm{MHz}$, (o) $10 \mathrm{MHz}$, and (t) $8 \mathrm{MHz}$. 


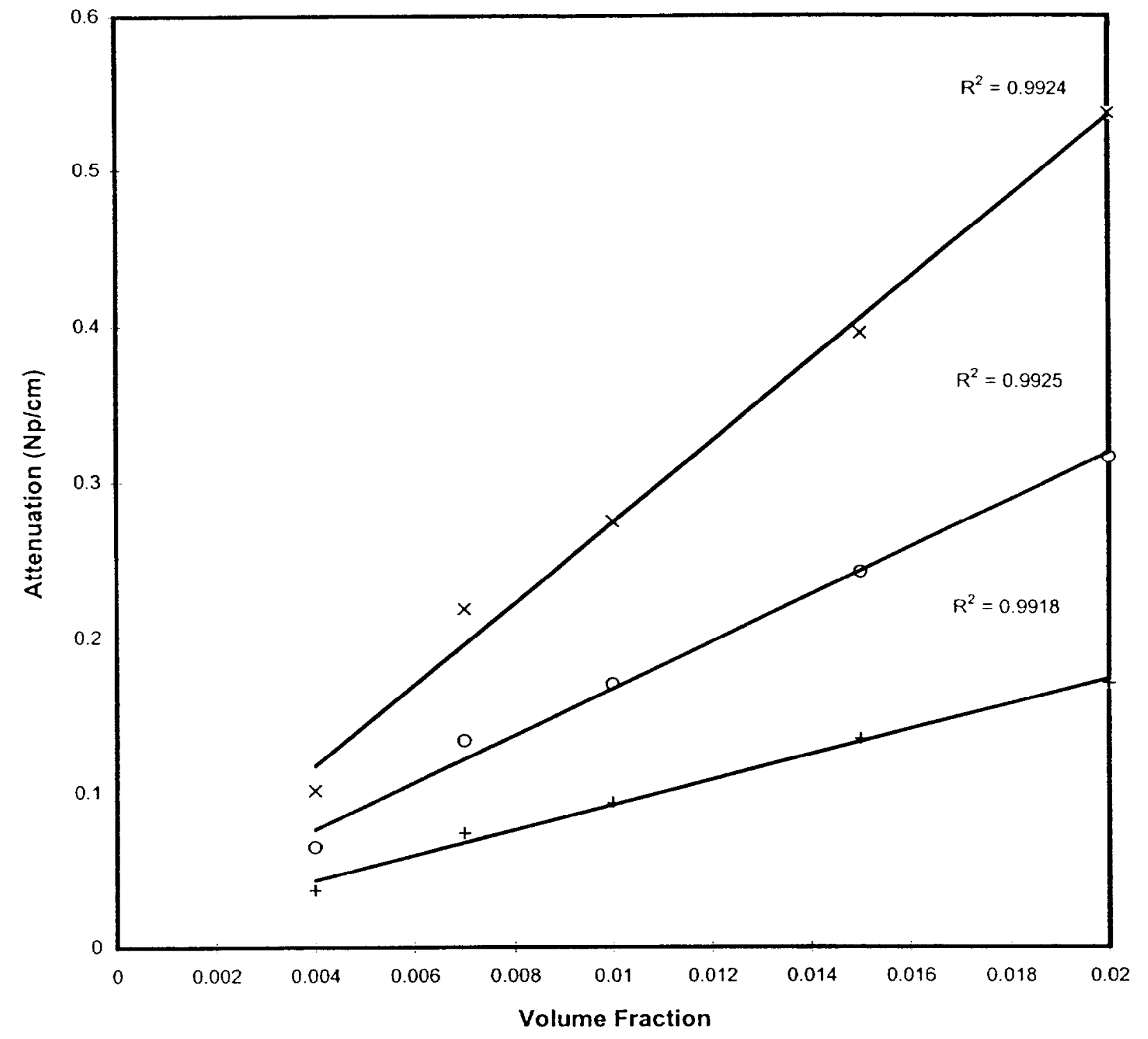

Figure 5.2: Attenuation versus volume fraction for a slurry of soda lime glass particles (radius $=16 \mu \mathrm{m}$ ) at very low volume fraction. The frequencies studied are $(x)$ $12 \mathrm{MHz},(\mathrm{o}) 10 \mathrm{MHz}$, and (+) $8 \mathrm{MHz}$. 


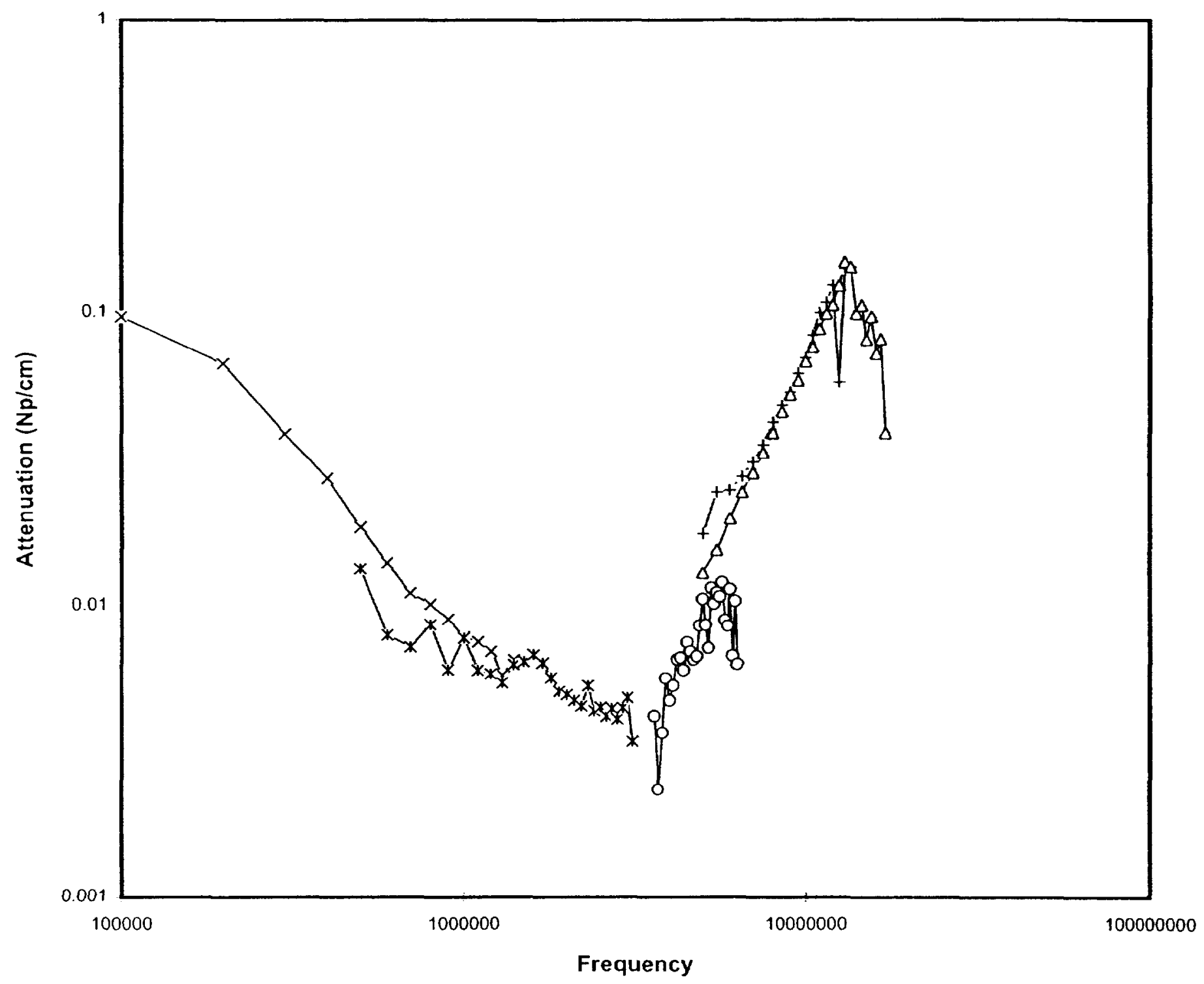

Figure 5.3: Typical attenuation versus frequency behavior for a $0.4 \%$ (volume) soda lime glass slurry. Transducers used are (×) $1 \mathrm{MHz},\left(^{*}\right) 2.25 \mathrm{MHz},(0) 5 \mathrm{MHz},\left(^{+}\right)$ $7.5 \mathrm{MHz},(\Delta) 10 \mathrm{MHz}$. 
porous, and this increase in attenuation is due to air entrained in the particles, as the behavior is similar to that observed when air bubbles were present in slurry (Norato 1999). In these experiments the slurry was tested immediately after preparation so there is no time for entrained air to diffuse out of the particles. In a subsequent experiment the slurry is prepared a week prior to testing, to allow time for any entrapped gas to diffuse out from within the porous particles and disengage. As shown in Figure 5.4, the low frequency attenuation decreased significantly. It was also noted that the attenuation at higher frequencies was essentially unchanged. Gas bubbles resonate at a specific frequency which is a function of the size of the bubble. At frequencies much higher than the resonance frequency the presence of gas bubbles has little effect on attenuation As mentioned previously, several different methods are attempted in order to determine the best way to measure attenuation at low volume fraction. Figure 5.5 shows a comparison between the pulse/FFT method and the toneburst method. The pulse/FFT data are less noisy and are more consistent from transducer pair to transducer pair.

Figure 5.6 shows the difference between acquiring the baseline signal in the settled slurry and acquiring the baseline signal separately. The signal acquired using the pulse / FFT method is significantly less noisy.

\subsubsection{COMPARISON WITH THEORY}

As shown in Figure 5.7, the effective medium theory prcdicts the bchavior of the system well. Attenuation varied linearly with volume fraction. The slight deviation present at lower frequencies may be caused in part by entrained air, as discussed previously. 


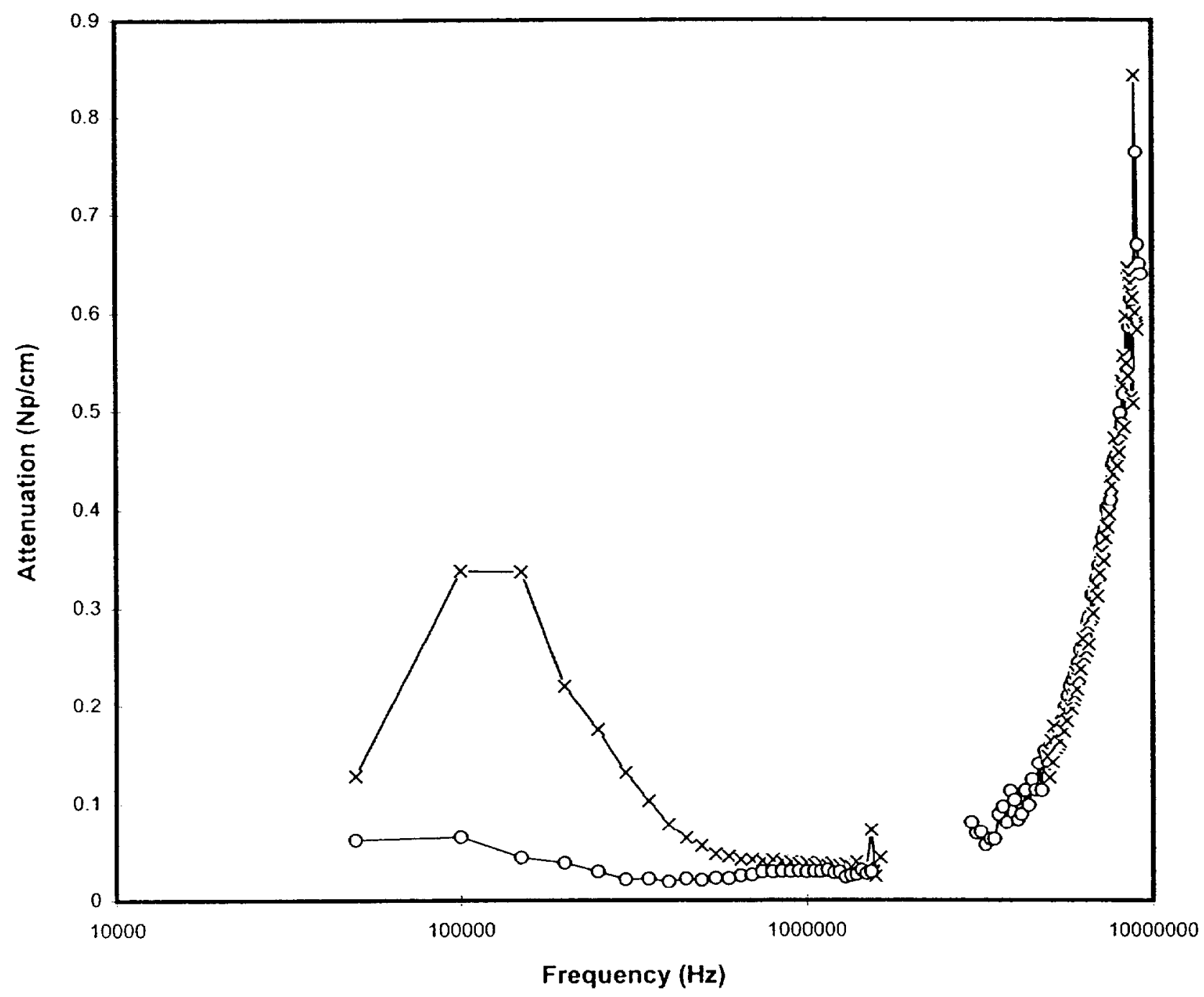

Figure 5.4: Effect of time between preparation and testing in soda lime glass slurries. The data marked $(x)$ was collected immediately following preparation of the slurry. The data marked (o) was collected after that same slurry was allowed to sit one week. 

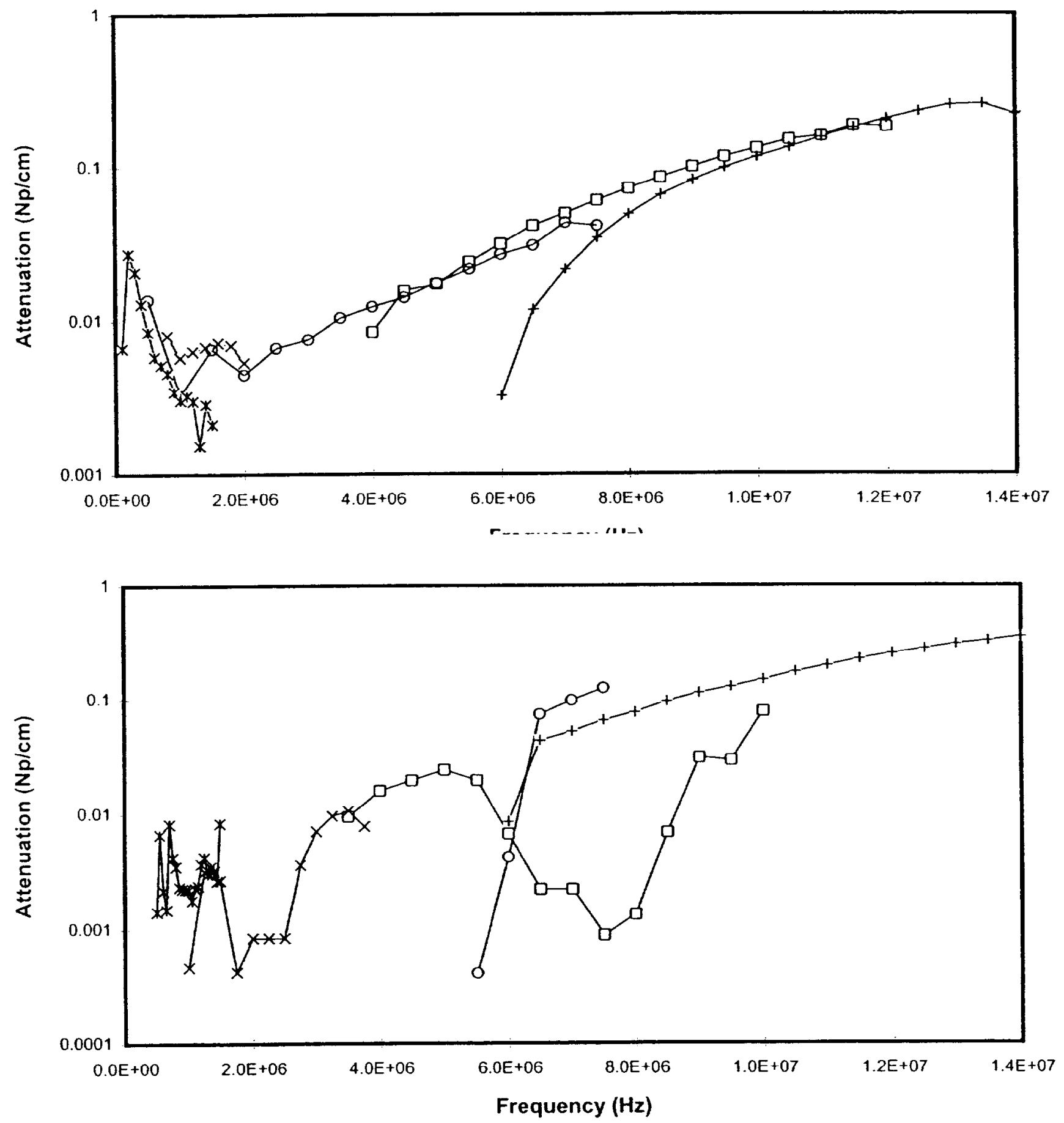

Figure 5.5: Comparison of pulse/FFT and toneburst methods of measuring attenuation. Pulsc/FFT data is shown on top, with toneburst data shown on bottom. 

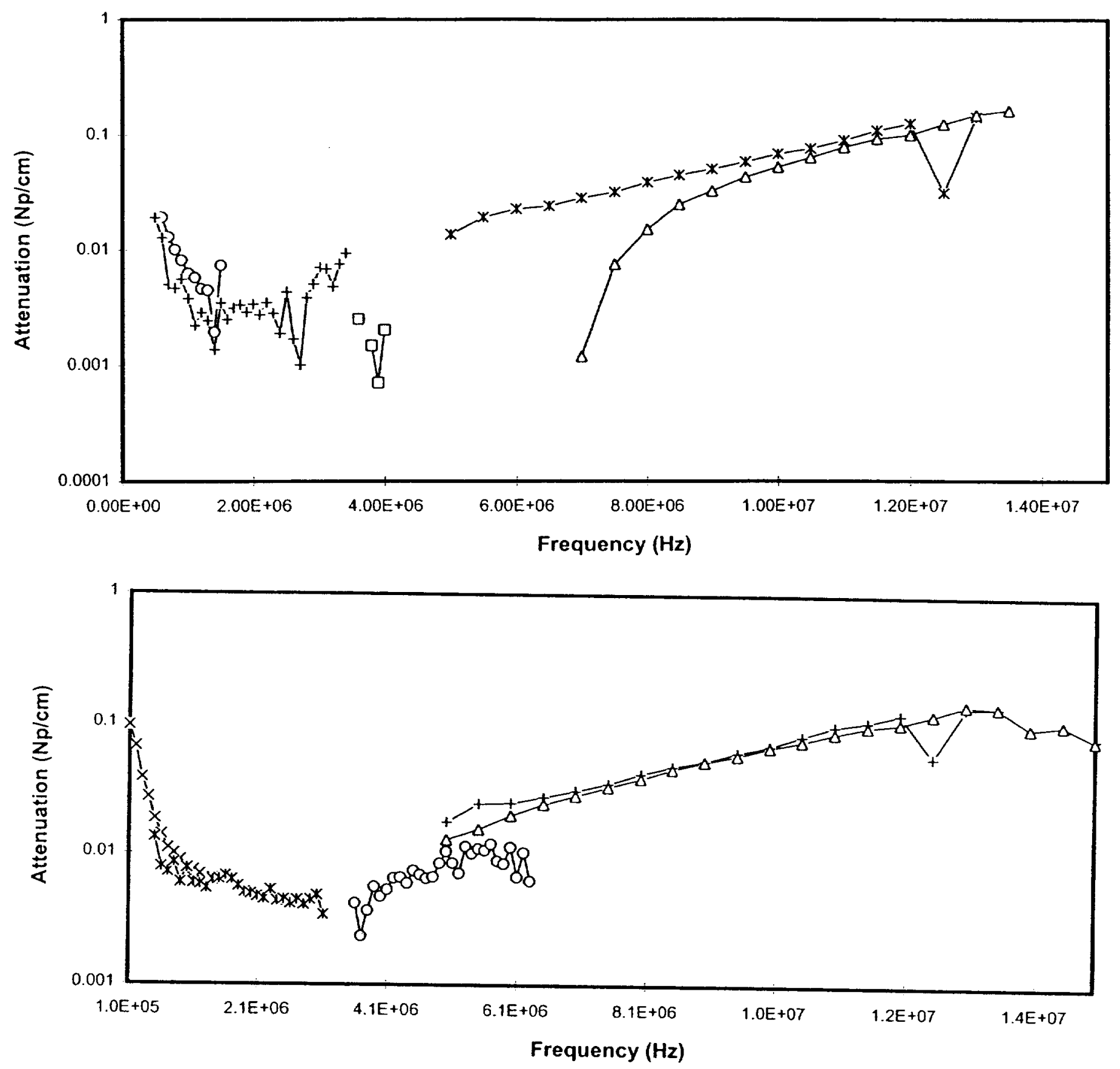

Figure 5.6: Comparison of methods of acquiring baseline signal. The baseline signal for the data shown on top was acquired separately from the sample signal. The baseline signal for the data shown on bottom was acquired by letting the solids settle. 


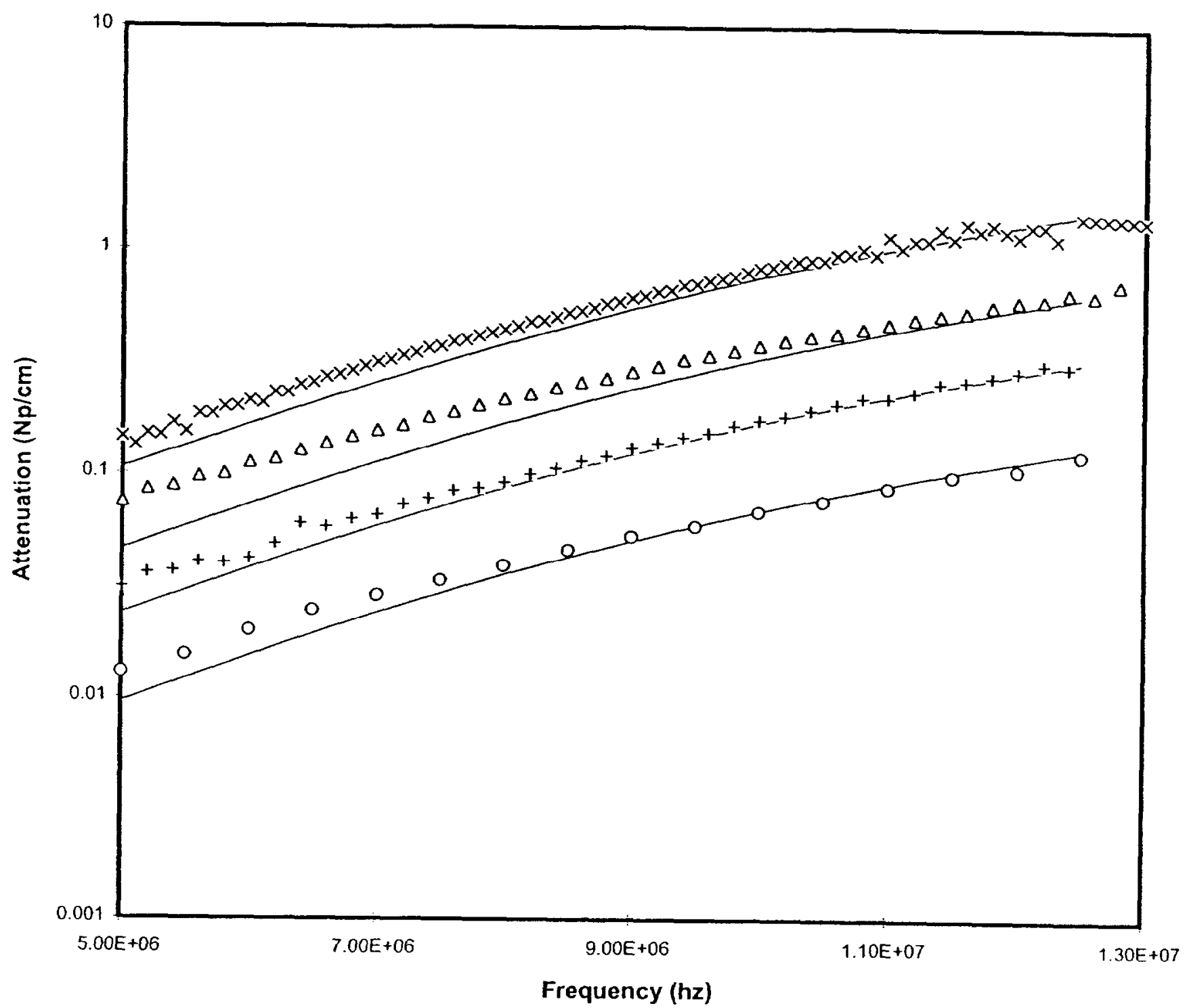

Figure 5.7: Comparison of theoretical prediction and experimental data of attenuation versus frequency for slurries of soda lime glass in watcr. The points represent experimental data and the lines represent theoretical predictions. The same pair of $10 \mathrm{MHz}$ transducers was used to gather all data. Volume fractions shown are $(0) 0.004,(+) 0.01,(\Delta) 0.02$, and $(x) 0.05$. 


\subsection{ATTENUATION IN CLAY MIXTURES}

Figure 5.8 displays attenuation versus volume fraction in the clay mixture system. Results are similar to those found in soda-lime glass. Attenuation is a linear function of volume fraction at constant frequency $\left(\mathrm{R}^{2}\right.$ ranges from 0.9997 to 0.9999$)$, and increases with increasing frequency. There is less scatter with the BKC system than with the SLG system. This is probably due to the fact that clay does not settle easily and variations due to nonhomogeneity are much less.

Suprisingly, the linear fit does not pass through the origin, whereas the linear fit for the SLG system does. As mentioned before, clay particles swell in water and are not as rigid as solid particles such as soda-lime glass beads. The swelling may cause unexpected behavior such as that observed.

\subsection{ATTENUATION IN CHEMICAL SURROGATE}

Figure 5.9 displays attenuation as a function of weight fraction in the CSS system. The relationship is linear as expected. Also of interest is Figure 5.10, which displays attenuation as a function of frequency for $\sim 1 \%$ (weight) chemical slurry surrogate. The relationship between attenuation and frequency also appears linear. The relationship between attenuation and frequency in monodisperse systems is usually nonlinear- scaling as $\mathrm{f}^{1 / 2}$ at low frequencies and as $\mathrm{f}^{4}$ at high frequencies.

There are two possible explanations for this phenomena. The system could be in an intermediate state between the viscous and scattering regions where attenuation scales roughly linearly with frequency. Although possible, this explanation is unlikely because any such behavior would likely be confined to a small range of frequencies. The 


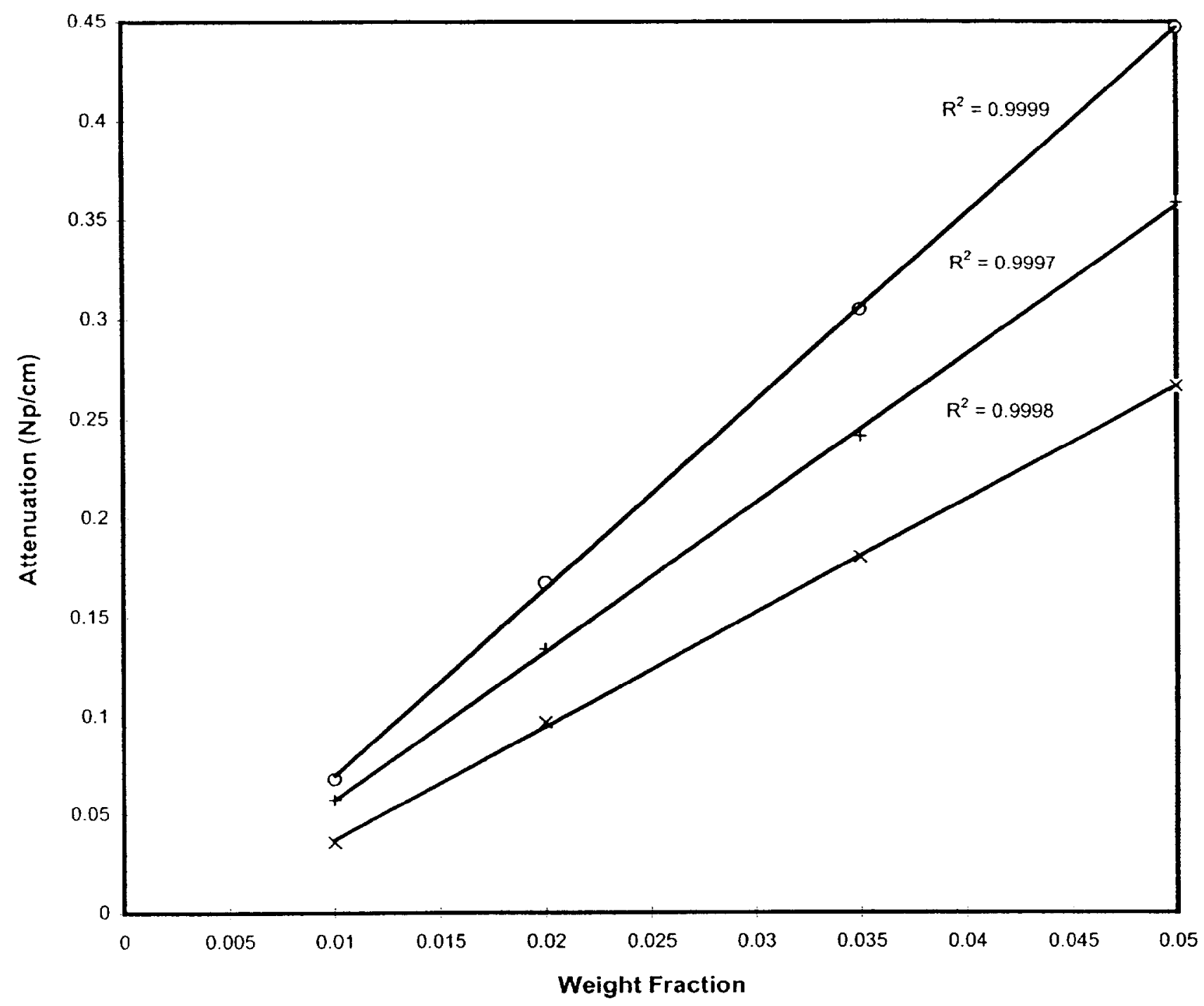

Figure 5.8: Attenuation versus volume fraction for slurries of clay (2 parts kaolin to one part Bentonite, by weight) in water at three frequencies. The frequencies studied are (o) $12 \mathrm{MHz},(+) 10 \mathrm{MHz}$, and (x) $8 \mathrm{MHz}$. 


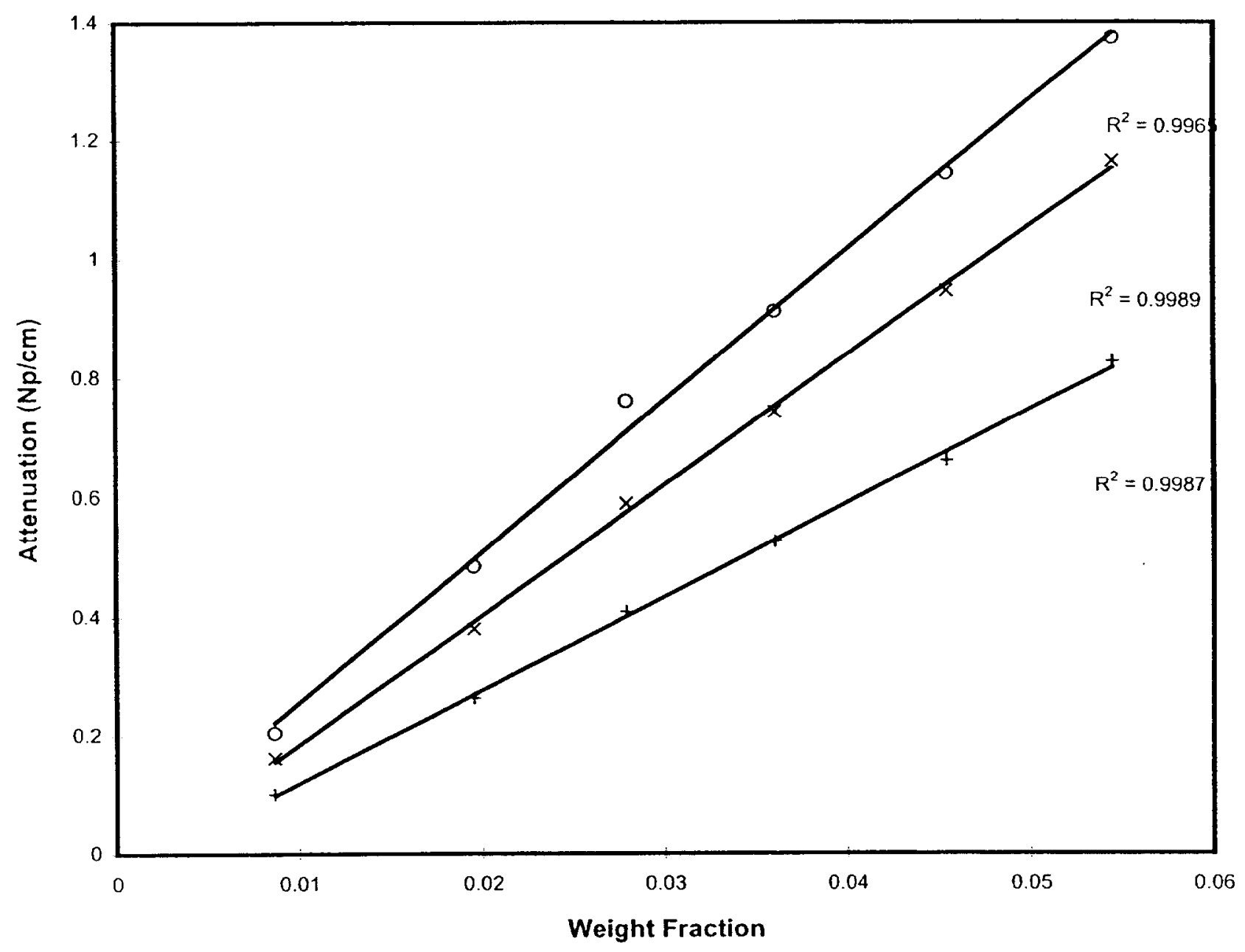

Figure 5.9: Attenuation versus volume fraction for crystallized salt solution system at three frequencies. The frequencies studied are (o) $12 \mathrm{MHz},(\times) 10 \mathrm{MHz}$, and (+) $8 \mathrm{MHz}$. 


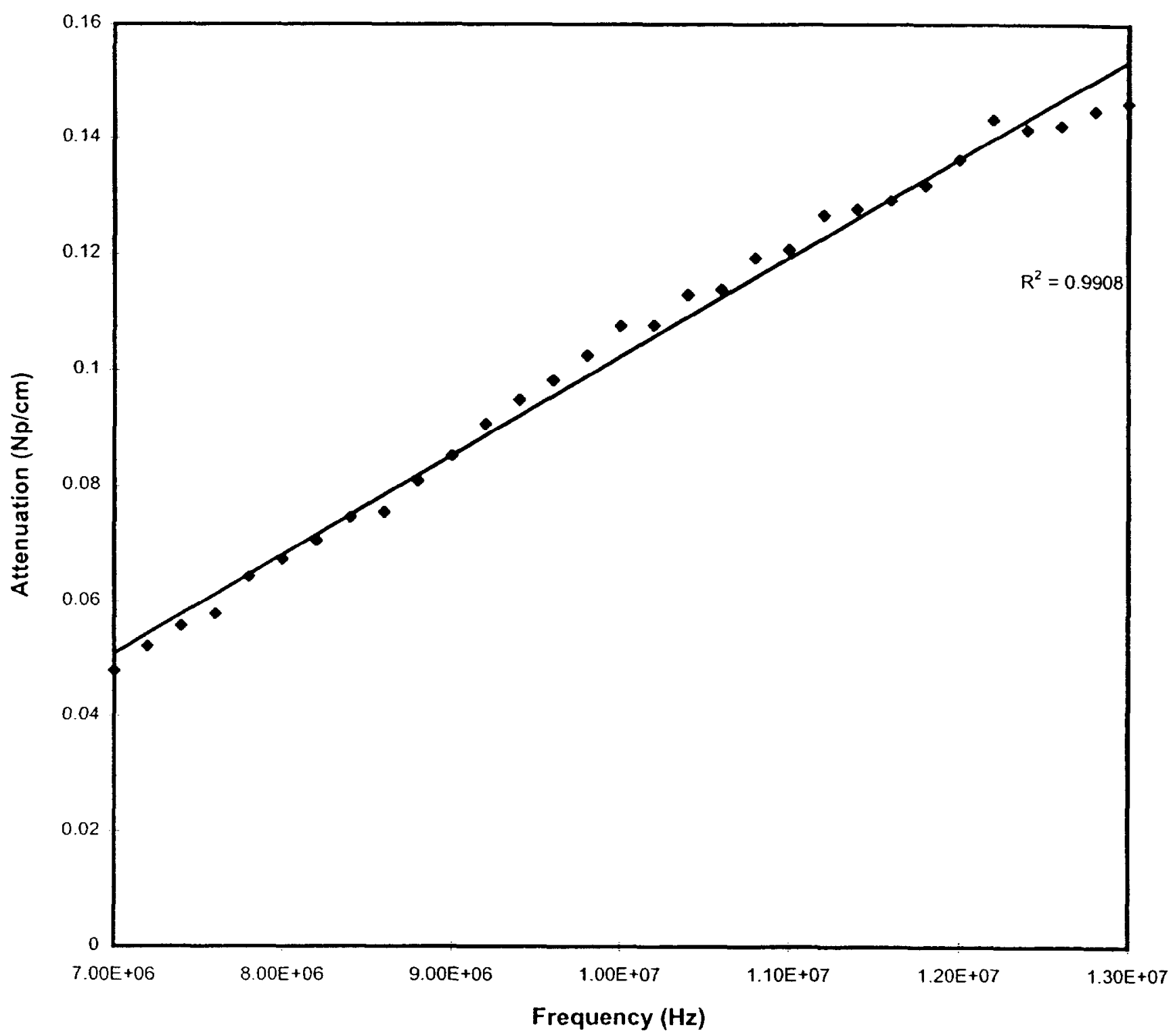

Figure 5.10: Attenuation versus frequency for crystallized salt solution. Points represent experimental data, the line is a linear fit. 
attenuation behavior was linear across a relatively broad frequency band $(7-13 \mathrm{MHz})$. It is unlikely that a system would be in a transition region over this range.

A more likely explanation is that the system is highly polydisperse. If the solids are composed of small particles with a few much larger particles then an approximately linear relationship could result. Small particles would have a small $\mathrm{k}_{\mathrm{cl}} \mathrm{a}$ and be in the viscous or thermal attenuation range. I arger particles would have $a \mathrm{k}_{\mathrm{cl}} \mathrm{a}$ closer to unity where scattering effects are more dominant. The combination of the two could produce a pseudo-linear relationship.

To explore the issue of a polydispersed system, an effort was made to estimate the particle size distribution using a microscopic particle size analysis. A sample of diluted slurry is dried on a slide and examined under a microscope. It is observed that there are two types of solids - small $(\sim 10 \mu \mathrm{m}$ in length $)$ needlc-like particles and larger $(\sim 100 \mu \mathrm{m}$ diameter) cubic crystals. Particle size distribution, shown in Table 5.1, is calculated from photographs of the system. See Figure 5.11 for photographs of the large particles and the small particles. The system is calibrated by taking photographs of a precision gradicule.

\begin{tabular}{ll}
\hline $\begin{array}{l}\text { Particle Size Range } \\
(\text { Diameter, } \mu \mathrm{m})\end{array}$ & $\begin{array}{l}\text { Volume Fraction } \\
(\mathrm{n}=161 \text { particles })\end{array}$ \\
\hline $0-38$ & 0 \\
$38-77$ & 0.04 \\
$77-115$ & 0.28 \\
$115-154$ & 0.33 \\
$154-192$ & 0.19 \\
$192-230$ & 0.10 \\
$230-270$ & 0.03 \\
$270+$ & 0.04 \\
\hline
\end{tabular}

Table 5.1: PSD of CSS solids 

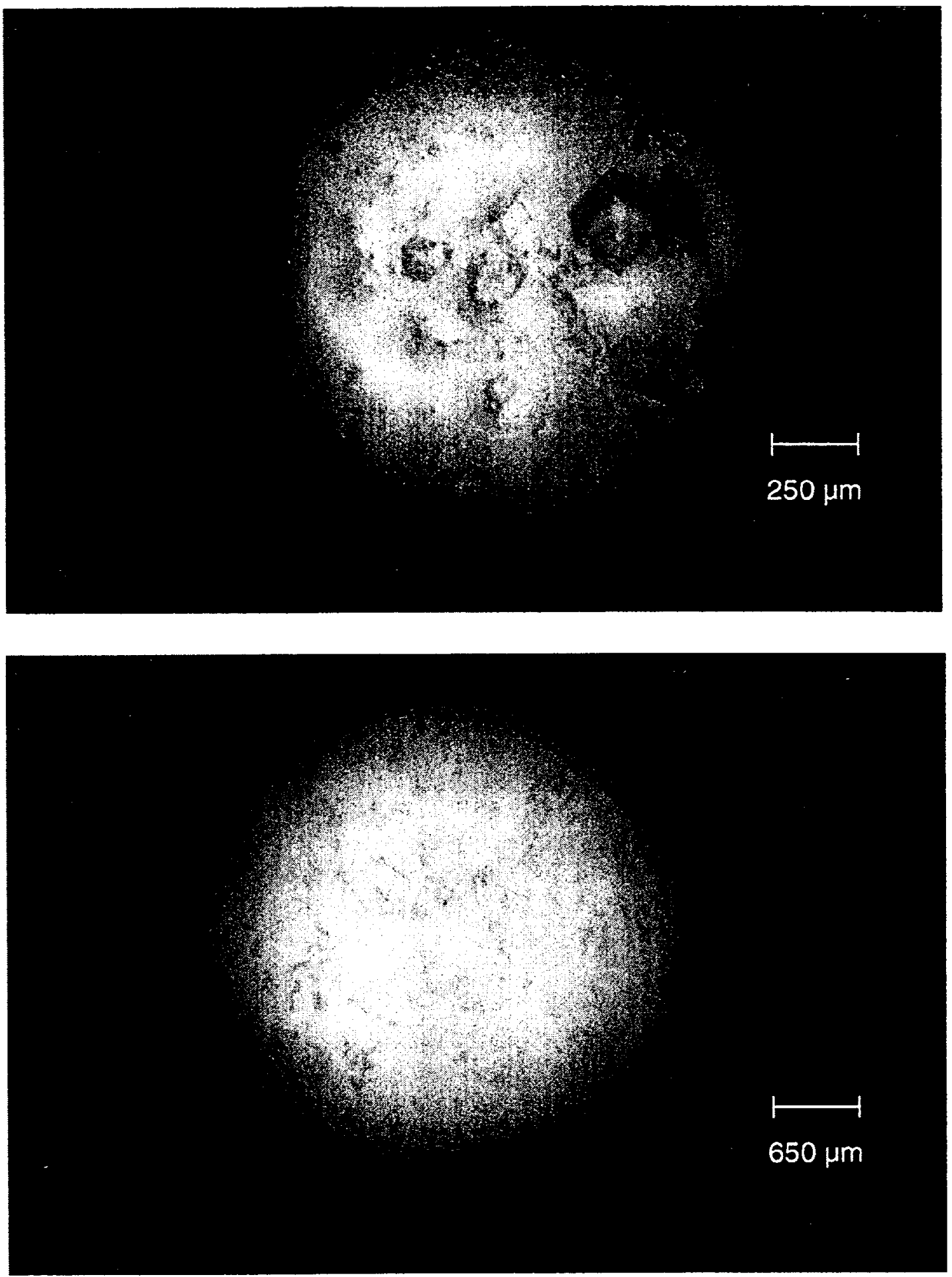

Figure 5.11: Photographs of crystallized salt solution particles. The photograph on top shows the larger particles, at a magnification factor of approximately 50 . The photograph on the bottom shows the smaller particles, at a magnification factor of approximately 130 . 
No particle size distribution is available for the needlelike particles as they are too small to accurately measure (particularly their diameter) with the apparatus available.

The approximate average length appear to be $10 \mu \mathrm{m}$.

\subsubsection{COMPARISON WITH THEORY}

The program used to estimate attenuation for the soda-lime glass system is for monodisperse particles, and cannot be used with the surrogate. Instead, a program that considers different particle sizes is used. Since the surrogate system is significantly different physically from the water / glass bead system a number of physical constants have to be changed. The values used are shown in Table 5.2:

\begin{tabular}{lcc}
\hline & Solid & Supernate \\
\hline $\begin{array}{l}\text { Density }\left(\mathrm{g} / \mathrm{cm}^{3}\right) \\
\text { Thermal Conductivity }\end{array}$ & $2.2(\mathrm{a})$ & $1.48(\mathrm{~b})$ \\
$(\mathrm{J} / \mathrm{K} \cdot \mathrm{cm} \cdot \mathrm{s})$ & & \\
Specific Heat & $0.836(\mathrm{c})$ & $4.19(\mathrm{c})$ \\
$(\mathrm{J} / \mathrm{g} \cdot \mathrm{K})$ & & \\
Thermal Expansion Coefficient & $3.2 \times 10^{-6}(\mathrm{c})$ & $2.04 \times 10^{-4}(\mathrm{c})$ \\
$\left(\mathrm{K}^{-1}\right)$ & & $5.87 \times 10^{-3}(\mathrm{c})$ \\
$\begin{array}{l}\text { Attenuation Coefficient per } \mathrm{f}^{2} \\
\left(\mathrm{~s}^{2} / \mathrm{cm}\right)\end{array}$ & $1.0 \times 10^{15}(\mathrm{c})$ & $2.5 \times 10^{16}(\mathrm{c})$ \\
$\begin{array}{l}\text { Sound Speed } \\
(\mathrm{cm} / \mathrm{s})\end{array}$ & $5.2 \times 10^{5}(\mathrm{c})$ & $2.14 \times 10^{5}(\mathrm{~b})$ \\
$\begin{array}{l}\text { Shear Viscosity } \\
\left(\mathrm{g} / \mathrm{cm} \cdot \mathrm{s}^{2}\right)\end{array}$ & - & $1.48 \times 10^{-1}(\mathrm{~b})$ \\
$\begin{array}{l}\text { Shear Rigidity } \\
\left(\mathrm{g} / \mathrm{cm} \cdot \mathrm{s}^{2}\right)\end{array}$ & & \\
\hline
\end{tabular}

Table 5.2: Physical constants used for theoretical calculations for C.SS system.

a: estimated (Weast, 1972)

b: measured

c: Taken to be the same as that for soda-lime glass beads

Note that due to the nature of the surrogate system several parameters could not be measurcd. They are instead estimated using average values of sodium salts given in Weast (1972) or taken to be the same as those of the soda-lime glass / water system. 
Attenuation is a linear function of volume fraction at low volume fraction so only one volume fraction needs to be used for comparison. All computer simulations are run with a total solid volume fraction of one percent.

As a first approximation the system is taken to have two particle sizes - small ( 5 $\mu \mathrm{m}$ radius) and large (50 $\mu \mathrm{m}$ radius). The parameter varied is the ratio of the volume fractions of the two particles. As shown in Figure 5.12, a ratio of $70 \%$ (volume) smaller particles to $30 \%$ (volume) larger particles is consistent with experimental data.

Efforts to more accurately characterize the surrogate system using the measured particle size distribution met with mixed success. The two particle model explained above was modified so that the smaller particles were still taken to be spheres of $5 \mu \mathrm{m}$ radius, while the larger particles were of several particle sizes as shown in Table 5.1. As shown in Figure 5.13, the predicted behavior is not as linear as that found using the model which assumed two particle sizes. This could be due to several factors. As mentioned previously, not all physical parameters of the slurry system can be measured. Some have to be estimated (see Table 5.2). In addition, it is not possible to get detailed information on the smaller particles size distribution. Finally, it is not possible to determine experimentally the ratio between the small and large particles.

\subsection{REPRODUCIBILITY OF DATA}

Several replicate runs were performed in order to gauge the reproducibility of the data for attenuation versus volume fraction plots. Four replicate experiments are performed on each of the three systems: SLG, BKC, and CSS. Fresh slurries were prepared for each experiment. No slurries were reused. Reproducibility of all three systems was acceptable, as shown in Figure 5.14. The SLG and CSS systems showed 


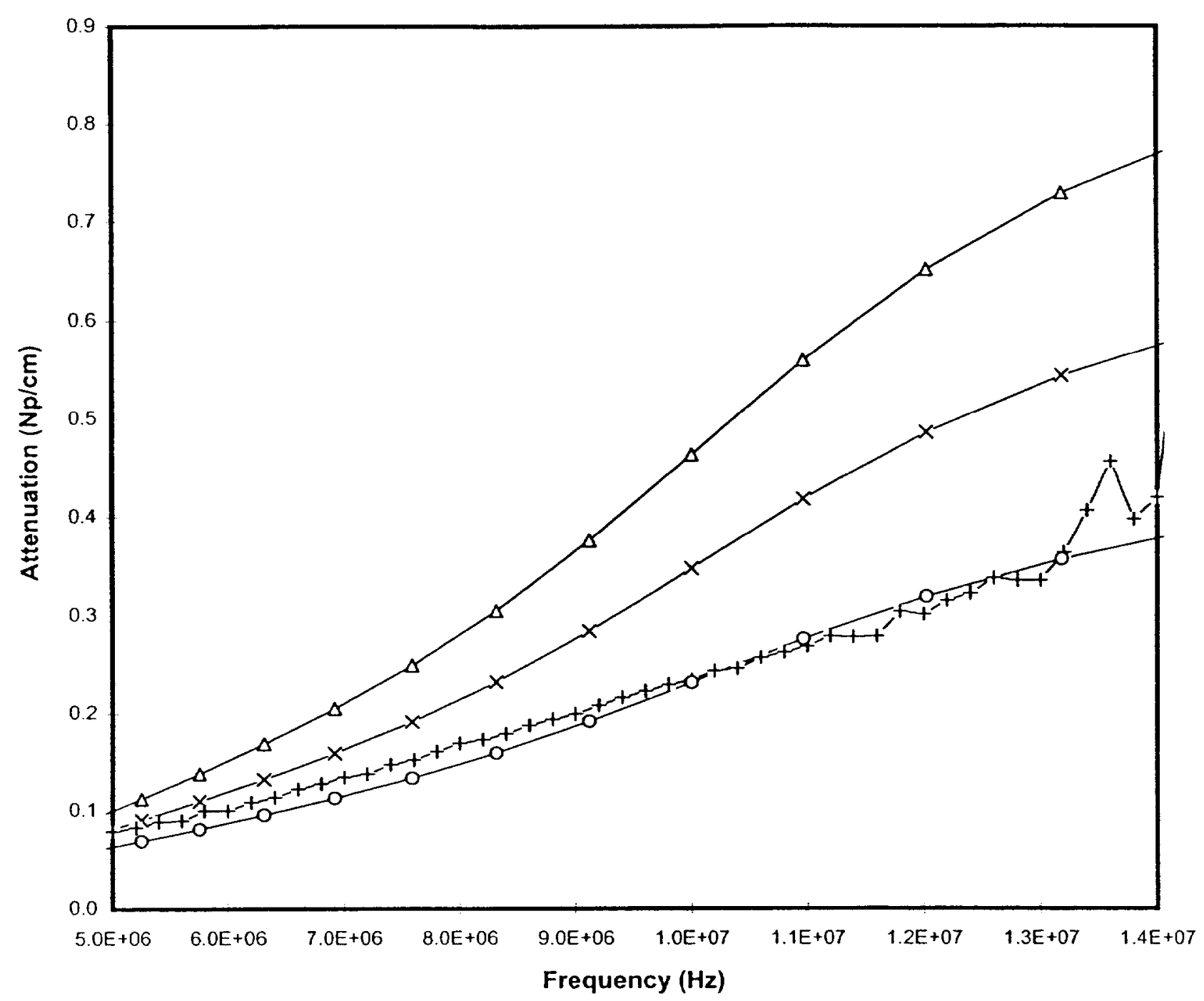

Figure 5.12: Results of the two particle size model to predict attenuation in the crystallized salt solution. It was assumed that the solids had two particle sizes, a smaller size with radius of $5 \mu \mathrm{m}$ and a larger size with a radius of 50 $\mu \mathrm{m}$. The ratio of the two particle sizes was varied. Volume fraction ratios shown $(5 \mu \mathrm{m}: 50 \mu \mathrm{m})$ are: (o) 30:70, (x) 50:50, and $(\Delta) 70: 30$. (+) represents experimental data. 


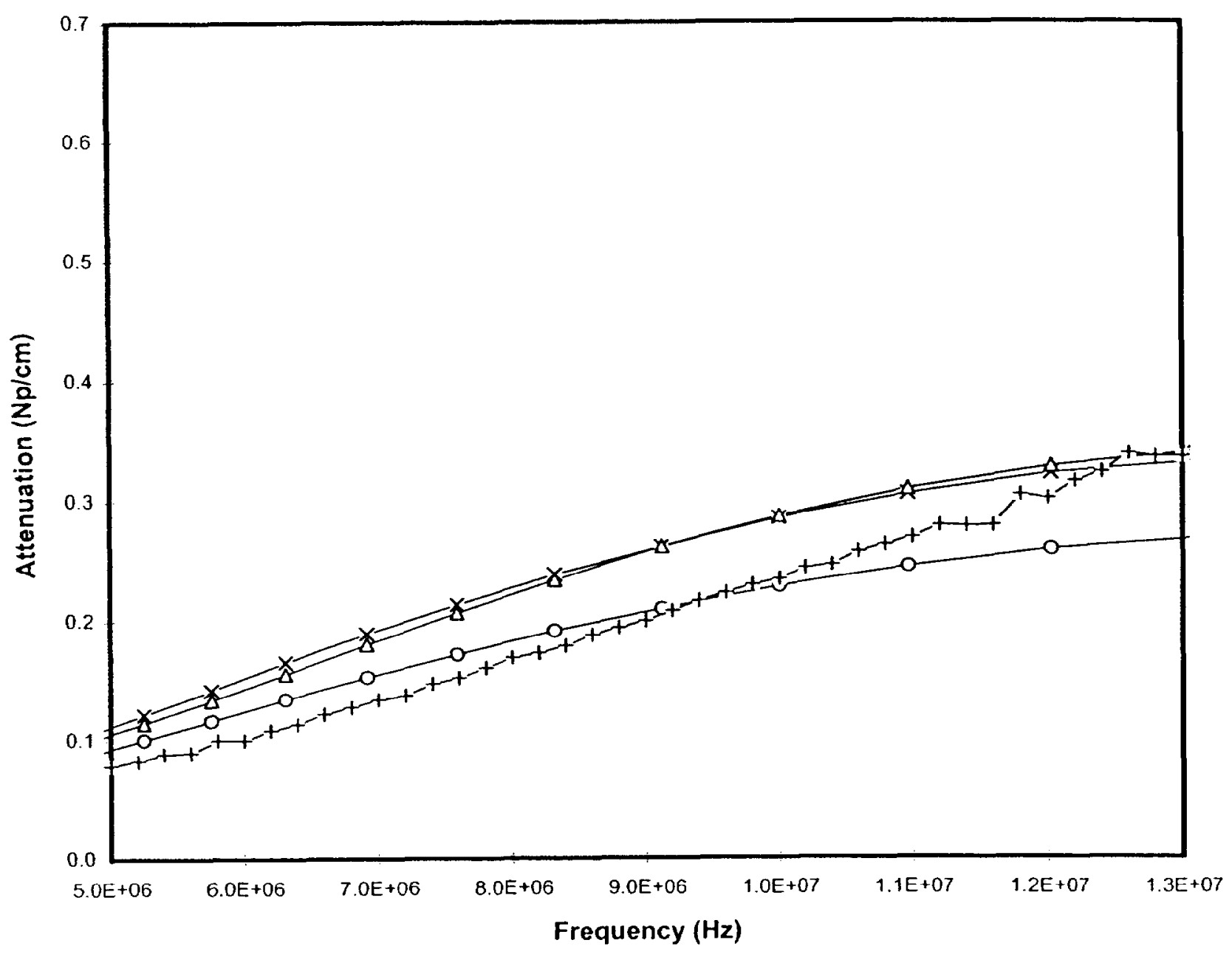

Figure 5.13: Results of expanding the two particle size model to predict attenuation in crystallized salt solution to include actual measured particle size information. Again it was assumed that the solids were of two different types. The first type was still taken to be spheres of radius $5 \mu \mathrm{m}$. The larger particles were of various sizes, as shown in Table 5.2. Volume fraction ratios shown $(5$ $\mu \mathrm{m}$ :larger particles) are: (+) 30:70, ( $\triangle)$ 50:50, and $(\times) 60: 40 .(+)$ represents experimental data. 

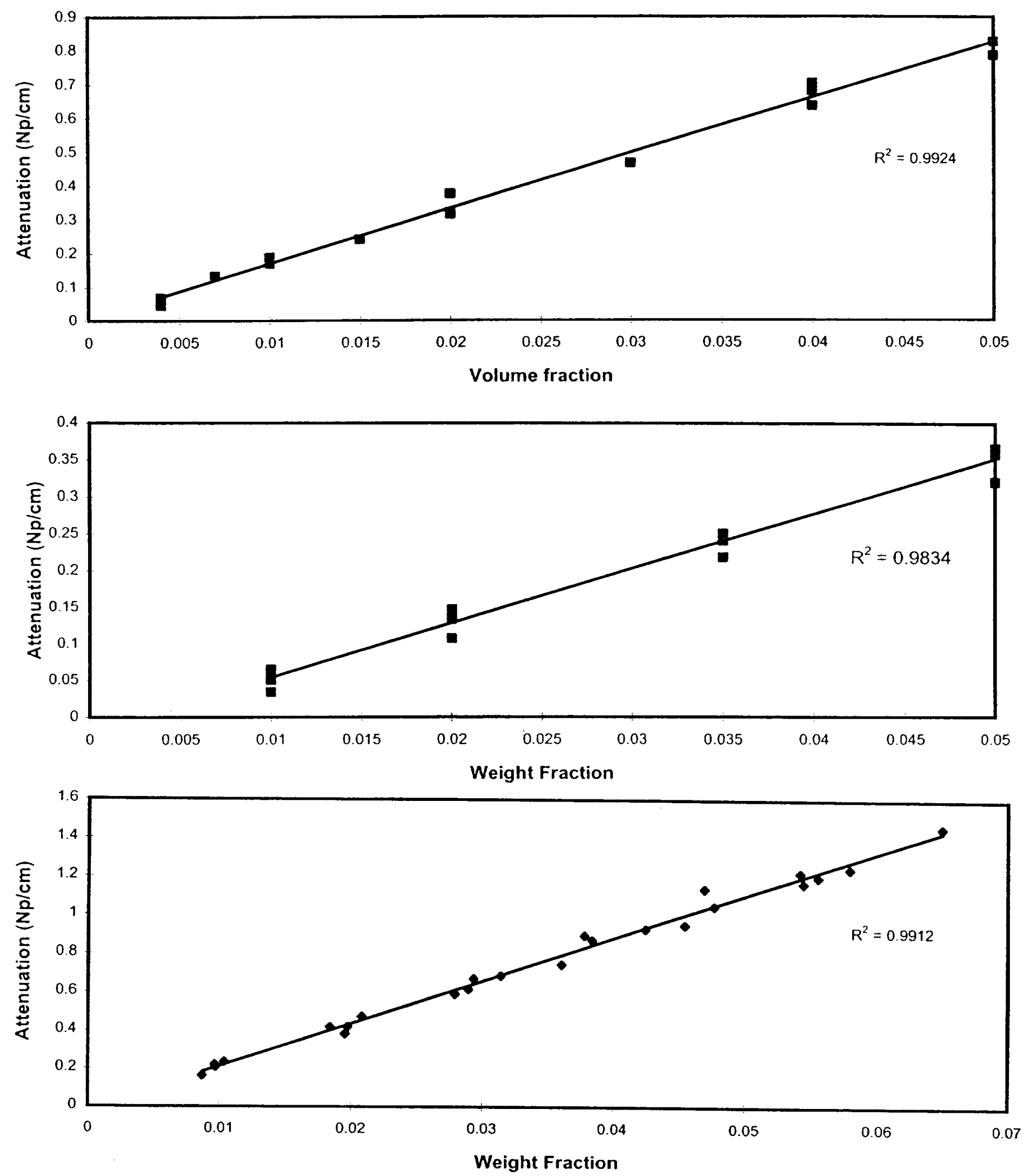

Figure 5.14: Reproducibility of data in liquid solid systems. Four experimental runs were performed on each system, with new slurry prepared for each run. All data is at $10 \mathrm{MHz}$. Systems shown are (top) SLG, (middle) BKC, (bottom) CSS 
similar amounts of scatter, having $\mathrm{R}^{2}$ values of 0.992 . The BKC system displayed slightly more scatter, with an $\mathrm{R}^{2}$ value of 0.983 .

\subsection{SPEED EXPERIMENTS}

Figure 5.15 shows the effect of volume fraction on ultrasonic speed in the sodalime glass system and the Potter's bead system at a frequency of $2.25 \mathrm{MHz}$ over a broader range of volume fractions ( 0.0 to 0.4$)$. The behavior is nonlinear as expected. The speeds measured in the two systems are roughly similar. The greatest deviation is present when volume fractions are low, less than $20 \%$. The speed in the Potter's beads is less than the speed in the soda-lime glass beads. This is likely due to experimental error. Large particles settle much faster than small particles, particularly at low volume fractions, and it is very difficult to keep the slurry homogenous. The effective volume fraction seen by the transducers could be lower than the actual volume fraction, causing the measured speed to be lower than expected.

Figure 5.16 shows the results of the low volume fraction speed experiments. The linear fit is acceptable but practical considerations decided against further consideration of using phase speed to characterize low VF slurries. At low volume fractions the change in speed due to the solids is very small (less than $10 \mathrm{~m} / \mathrm{s}$, or $0.7 \%$ of the total speed) and any change in temperature or any non-uniformity of the slurry is significant.

No speed experiments were performed with the CSS system. In order to calculate the theoretical attenuation in the CSS system the ultrasonic speed in the supernate is measured. The value is found to be quite high $-\sim 2200 \mathrm{~m} / \mathrm{s}$, and rather close to the expected speed in the salt $(\sim 2500 \mathrm{~m} / \mathrm{s})$. Thus any change in speed due to the presence of 


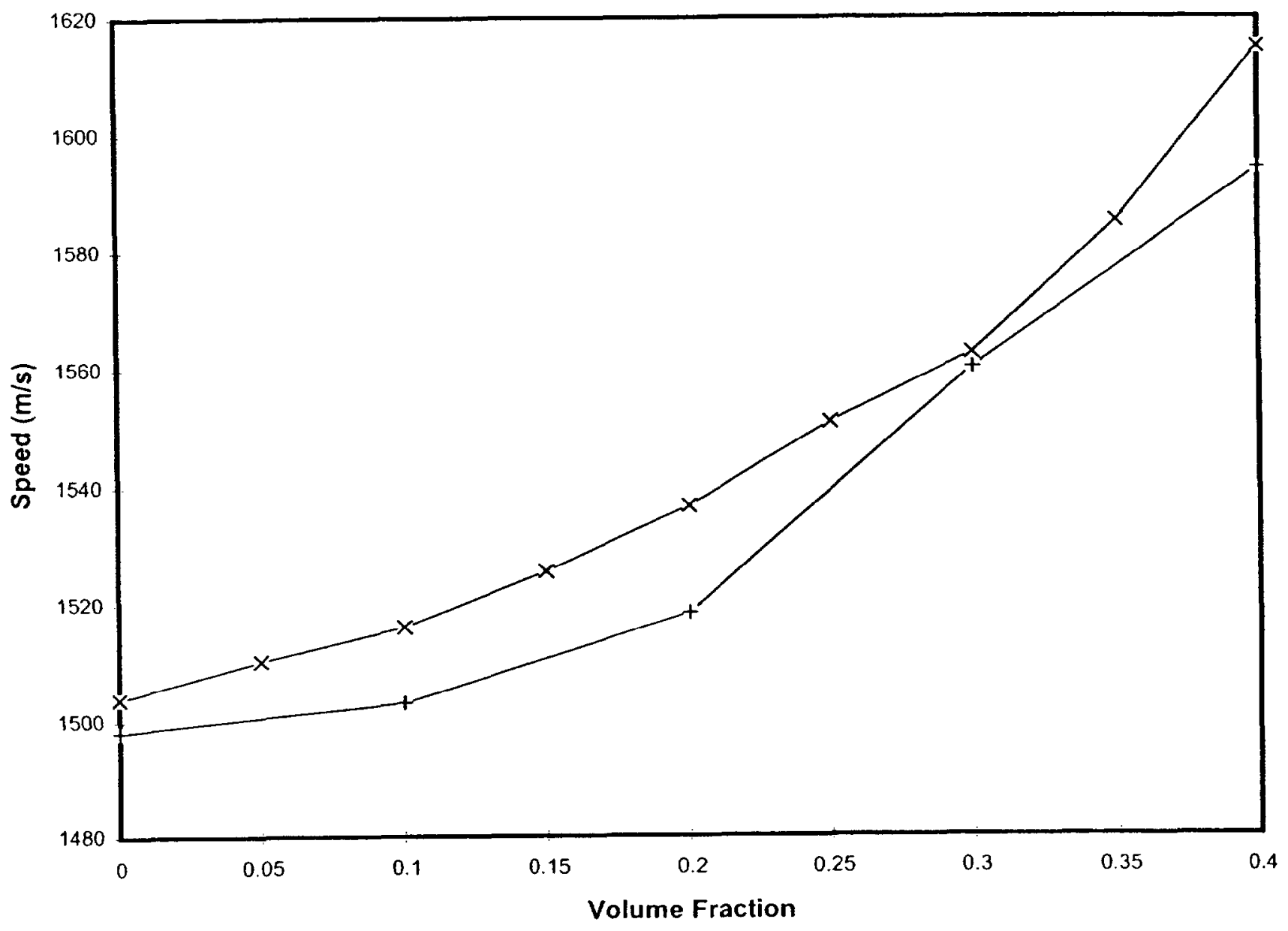

Figure 5.15: Effect of volume fraction on phase speed in solid-liquid slurries. Particles are $(x)$ Soda lime glass $(r=16 \mu \mathrm{m})$ and $(+)$ Potter's beads $(r=60 \mu \mathrm{m})$. Volume fraction ranges from 0.0 to 0.4 . 


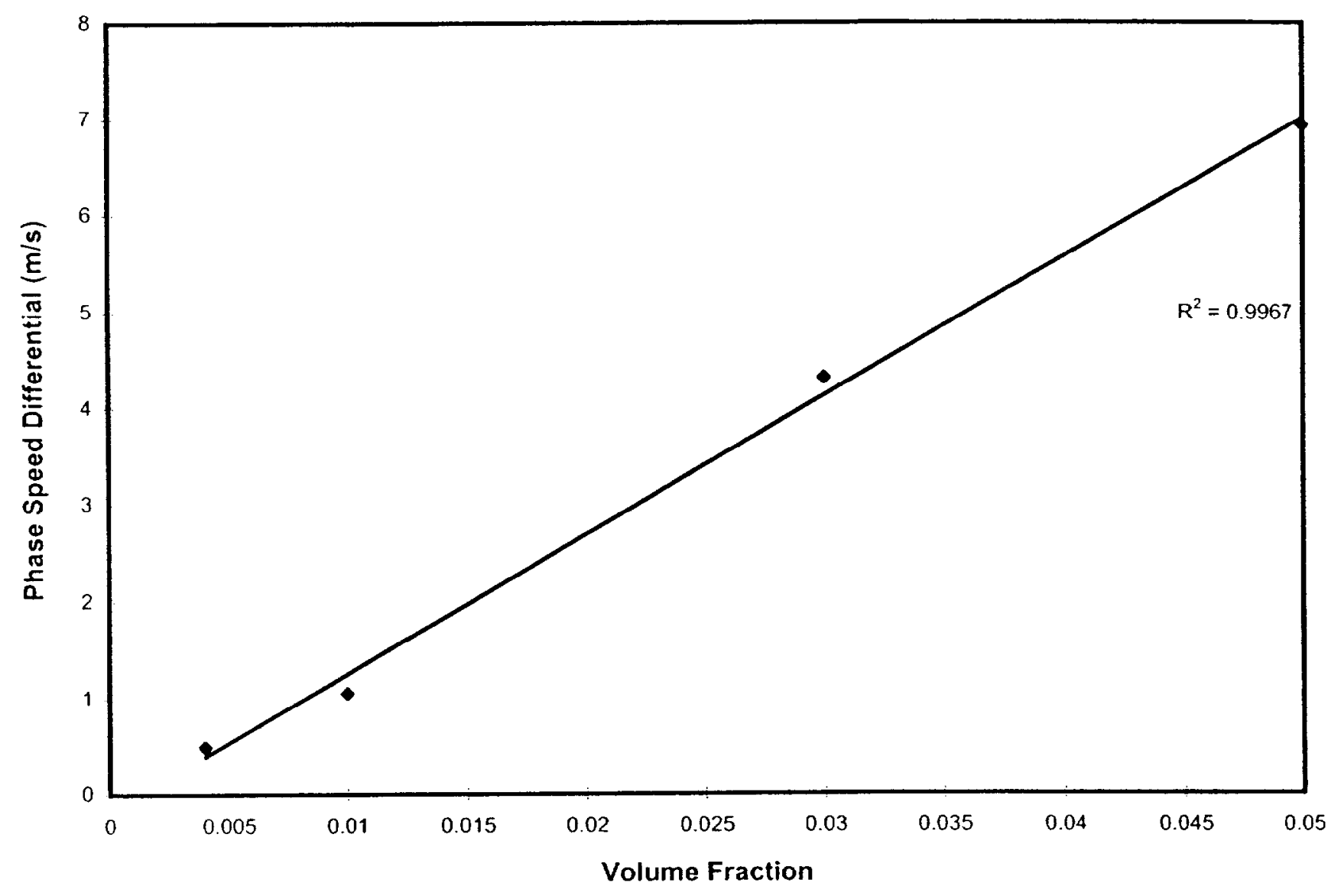

Figure 5.16: Phase speed differential versus volume fraction for low volume fraction soda-lime glass beads. The phase speed differential is the difference between the phase speed in slurry and the speed in the suspending liquid (water). Data is represented by points. The line is a linear fit. 
salt would be very small and difficult to measure, particularly at low volume fractions. Accordingly, these measurements were not made.

\subsubsection{COMPARISON WITH THEORY}

Figure 5.17 compares the speed measured in soda-lime glass beads with the speed predicted by the computer simulations. The simulation agrees very well with the experiment. These results demonstrate that the basic premises of the theory hold for this system over a wide range of volume fractions.

\section{Conclusions}

The following conclusions can be drawn from this study.

The acoustic monitor is shown capable to accurately measure volume fraction of solid-liquid slurries in low concentration ranges of 0.4 to 10 volume percent by analysis of the attenuation of the transmitted wave. A linear relationship exists in this region between attenuation and volume fraction for three slurry systems studied: a) soda-lime glass beads in water (SLG), (b) a physical radioactive waste slurry surrogate of Bentonite and kaolin clay in water (BKC), and (c) a chemical radioactive slurry surrogate consisting of a crystallized salt solution in supernate (CSS). This relationship exists in the presence of small amounts of gas bubbles when the frequencies of interrogation are much greater than the resonance frequency of the gas bubbles. The frequency ranges studied are 8 $\mathrm{MHz}$ to $12 \mathrm{MHz}$ for all three systems. Replicate experiments conducted for the three systems demonstrate linear behavior with regression coefficients of 0.983 to 0.992 .

The theory developed by Spelt et al. (2000) describes well the attenuationfrequency behavior for the well characterized SLG system for 0.4 to 5 volume percent 


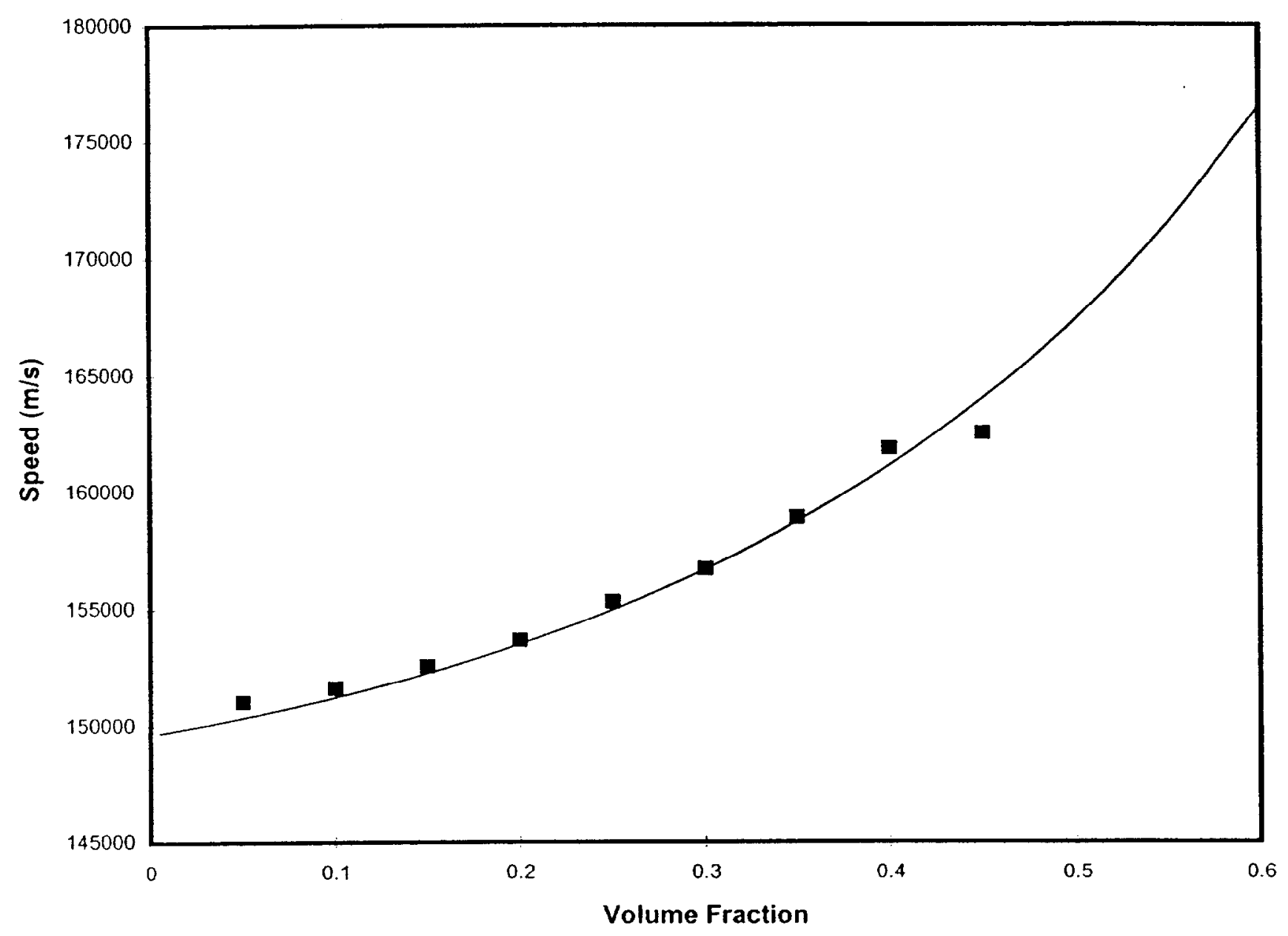

Figure 5.17: Comparison of measured phase speed with theoretical phase speed. Experimental data is represented by points, while the line represents theoretical calculations. Frequency is $2 \mathrm{MHz}$, temperature $25^{\circ} \mathrm{C}$. 
slurries. Attempts to apply the theory to the CSS system met with limited success due to the complexity of the salt crystals morphologies (at least two different crystal structures exist, each with broad particle size distributions), and unknown physical properties of these crystals are estimated.

The sender-receiver mode experimental setup using pairs of transducers mounted in a slurry sample cell is employed with fast Fourier transform analysis to obtain the attenuation measurements. The technique is the same as that employed earlier by Norato (1999) and provides accurate results.

Acoustic speed through the well characterized SLG slurries can be accurately measured using the reflectance method adapted from McClements (1991) for a broad range of volume fractions of 5.0 to 40 percent. With proper control of temperature, speed can be measured to $\pm 0.3 \mathrm{~m} / \mathrm{s}$. The theory of Spelt et al. (2000) is in close agreement with these data. For low volume fractions (less than 10 volume percent) accurate data can be produced with very careful monitoring of temperature. These results show that the reflectance method and theory can be a valuable tool to characterizing solids content if solid-liquid slurries at large volume fractions.

\section{Future Work}

Future work involves automating the procedure to measure attenuation and manufacturing an on-line monitor on spoolpiece to characterize flowing slurries. The basic experimental setup will be similar to that currently employed, except the procedure will be automatic, controlled by a computer. A computer controlled pulser/receiver will be used to generate the spike pulse. The pulse will then be routed through a high voltage, 
high bandwidth computer controlled multiplexer to the appropriate transducers. The multiplexer will act as a switchbox, enabling the use of several different transducers without having to manually change the signal wires. The signal will be read on a digital oscilloscope card installed in a PC and attenuation will be calculated automatically.

The baseline signal will be acquired continuously, using the same system used to acquire the sample signal. $A$ Mott $B$ filter will be used to separate the solids from the liquid stream. This stream will pass through a transducer bank and be interrogated by the appropriate transducer pairs before being returned to the slurry line. See Figure 7.1 for a schematic of the wiring setup and the spoolpiece. 


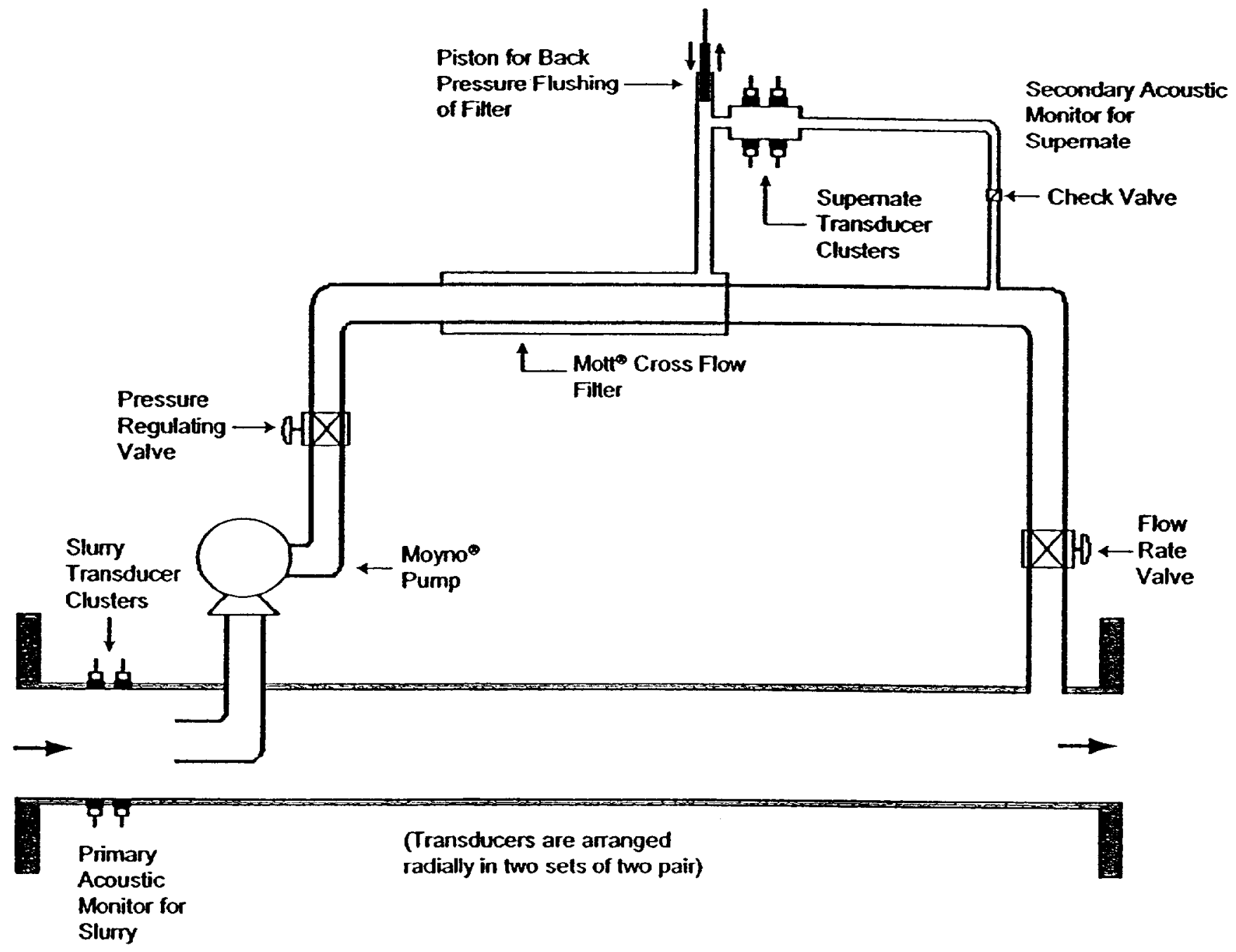

Figure 7.1: Conceptual Diagram of Acoustic Monitor on Spool Piece. The unit includes the primary acoustic monitor transducer cluster for slurry; and the by-pass loop with slurry pump, cross-flow slurry filter with pressure actuated backflush piston, and secondary acoustic monitor with interrogative cell for supernate. 


\section{References}

Allegra, J.R. and Hawley, S.A., "Attenuation of sound in suspensions and Emulsions: Theory and experiments," J. Acoust. Soc. Am. 51, 1545 (1972).

Atkinson, C.M. and Kytomaa, H.K., "Acoustic wave speed and attenuation in suspensions," Int. J. Multiphase Flow. 18, 577 (1993).

Bolz, R.E., CRC Handbook of tables for Applied Engineering Science (CRC Press, Boca Raton, FL, USA, 1973).

Cowan, M.L., Beaty, K., Page, J.H., Liu, Z., and Sheng, P, "Group Velocity of Acoustic Waves in Strongly Scattering Media: Dependence on the Volume Fraction of Scatterers," Physical Review E. 58, 6626 (1998)

Epstien, P.S. and Carhart, R.R., "The absorption of sound in Suspensions and Emulsions. I. Water Fog in Air," J. Acoust. Soc. Am. 25, 553 (1953)

Golcar, G.R., Colton, N.G., Darab, J.G., and Smith, H.D., "Hanford Tank Waste Simulants Specifications and Their Applicability for the Retrieval, Pretreatment, and Vitrification Processes," PNWD-2455/BNFL-RPT-012 Rev. 0, 2000.

Grim, R.E., Clay Minerology (McGraw-Hill Book Company, New York NY, USA, 1968, pg. 567.

Greenwood, M.S., Mai, J.L., and Good, M.S., “Attenuation Measurements of Ultrasound in a Kaolin-Water Slurry: A Linear Dependence Upon Frequency, " $J$. Acoust. Soc. Am. 94, 908 (1993).

Holtz, R.D., and Kovacs, W.D., An Introduction to Geotechnical Engineering (Prentice-Hall, Inc., Englewood Cliffs, NJ, USA, 1981, pp. 81-82

Hudson, J.D., "Defining Waste Acceptance Criteria for the Hanford Replacement Cross-Site Transfer System," PNNL-11146/UC-2030, National Technical Information Service, 1996

Hylton, T.D., and Bayne, C.K., "Testing of In-Line Slurry Monitors and Pulsair Mixers with Radioactive Slurries," ORNL/TM-1999/111, National Technical Information Service, 1999

Hylton, T.D., "An Evaluation of a Dual Coriolis Mcter System for In-line Monitoring of Suspended Solids Concentrations in Radioactive Slurries," ORNL/TM-2000/184, National Technical Information Service, 2000. 
Kinsler, L.E., Frey, A.R., Coppens, A.B., and Sanders, J.V., Fundamentals of Acoustics (John Wiley \& Sons, Inc., New York, NY, USA, 1982).

McClements, D.J., "Characterisation of Emulsions Using a Frequency Scanning Ultrasonic Pulse Echo Reflectometer," Proc. Inst. Acoust., 13, 71 (1991).

Norato, M.A., "Acoustic Probe for the Characterization of Solid-Gas-Liquid Slurries," Ph.D. Thesis, Syracuse University (1999).

Spelt, P.D.M., Norato, M.A., Sangani, A.S., Greenwood, M.S., and Tavlarides, L.L., "Attenuation of Sound in Concentrated Suspensions: Theory and Experiments", J. Fluid Mechanics., 420, 1 (2000)

Spelt, P.D.M, Impcrial College, London, England. Private Correspondence. JulySeptember 2000.

Tsouris, C., Norato, M.A., and Tavlarides, L.L., "A Pulse-Echo Ultrasonic Probe for Local Volume Fraction Measurements in Liquid-Liquid Dispersions," I \& EC Res. 34, 3154 (1995).

Weast, R.C., CRC Handbook of Chemistry and Physics (The Chemical Rubber Co., Cleveland, OH, USA, 1972). 


\section{Appendix A: Procedure for synthesizing Crystallized Salt Solution}

The procedure used to synthesis the surrogate slurry follows. Source is Golcar (2000), although a few changes were necessary due to equipment limitations.

Simulant ID: SY1-SIM-91A

The simulant is prepared by evaporating (under reduced pressure) excess water from a dilute feedstock solution.

Feed Solution Preparation:

1. Weigh out the following:

\begin{tabular}{ll}
\hline Compound & g needed for $1-\mathrm{L}$ of feedstock \\
\hline $\mathrm{NaOH}$ & 64.4 \\
$\mathrm{NaAl}(\mathrm{OH}) 4$ & 126.23 \\
$\mathrm{NaNO} 2$ & 154.56 \\
$\mathrm{NaNO} 3$ & 220.12 \\
$\mathrm{Na} 2 \mathrm{CO} 3$ & 45.15
\end{tabular}

2. To make $1 \mathrm{~L}$ of solution use a $2 \mathrm{~L}$ beaker, starting with about $500 \mathrm{ml}$ water. Use a round 'leflon stir bar for agitation.

3. Heat water to about $90 \mathrm{C}$ and add sodium aluminate. Stir and heat until the solution is almost clear; waiting too long results in the precipitation of aluminum hydroxide.

4. Turn off heat and slowly add $\mathrm{NaOH}$ pellets.

5. Add sodium carbonate and stir until dissolved. Some cooling may be necessary to get all the carbonate into solution.

6. Turn heat back on and add sodium nitrite followed by sodium nitrate

7. Add water when necessary to dissolve solids, trying to have a total of $900 \mathrm{ml}$ solution when finished.

Slurry Preparation:

The solution prepared above is evaporated down in a RotoVap until the final volume is $\sim 700 \mathrm{ml}$.

1. Assemble RotoVap instrument with accessory hot water bath. Connect vacuum line to house vacuum. Connect condenser water line to house cold water. 
2. Fill RotoVap vessel with $700 \mathrm{ml}$ water, mount in RotoVap, and mark water line on outside of vessel with a permanent marker. The purpose of this line is to gauge when to stop the RotoVap process. When the slurry level comes even with the marked line then the process is over, as the desired volume of $700 \mathrm{ml}$ is reached.

3. Fill water bath so that water level is $1 \mathrm{~cm}$ from the inside lip, with the RotoVap vessel immersed. Set water bath to $90^{\circ} \mathrm{C}$.

4. Begin evaporation. Set rotation level at the $9: 30$ position $(\sim 65 \mathrm{rpm})$.

5. Stop evaporation once slurry level reaches the mark placed in step 2 .

6. Allow to cool with stirring overnight.

\section{Slurry Separation:}

The slurry is separated in a centrifuge (Fisher Marathon $21000 \mathrm{CF}$ ). Slurry is placed in centrifuge beakers and spun until all solids have settled. The supernate is then carefully drawn of. The solids are collected and centrifuged again.

1. Fill four $200 \mathrm{ml}$ centrifuge beakers with slurry. Using balance verify that the mass of all four vessels is within one gram of each other.

2. Centrifuge 20 minutes at 2000 RPM.

3. Carefully pipette solid-free supernate into storage vessel. Leave approximately $1 \mathrm{~cm}$ of supernate on top of settled solids.

4. Combine all solids and centrifuge again (2000 RMP, 10 minutes), using a beaker filled with water as a counterweight.

5. Carefully pipette solid-free supernate into storage vessel. Stop placing the supernate into the vessel when it appears that solids are being drawn into the pipette with the supernate.

6. Remove all remaining supernate and discard. Transfer wet solids into storage container. 


\section{Appendix B: Transducer Specifications}

All transducers are produced by Panametrics, Inc. (Waltham, Ma) and are Videoscan immersion transducers.

\begin{tabular}{llllll}
\hline $\begin{array}{l}\text { Model } \\
\text { Number }\end{array}$ & $\begin{array}{l}\text { Serial } \\
\text { Number }\end{array}$ & $\begin{array}{l}\text { Element } \\
\text { Diameter }(\mathrm{cm})\end{array}$ & $\begin{array}{l}\text { Center } \\
\text { Frequency } \\
(\mathrm{MHz})\end{array}$ & $\begin{array}{l}\text { Bandwidth } \\
(-6 \mathrm{~dB}) \\
(\%)\end{array}$ & $\begin{array}{l}\text { Operating Range } \\
\text { (MHz) }\end{array}$ \\
\hline V302 & 211353 & 2.54 & 0.935 & 65.24 & 0.6 to 1.4 \\
V302 & 211354 & 2.54 & 0.950 & 65.26 & 0.6 to 1.4 \\
V304 & 217679 & 2.54 & 2.11 & 63.51 & 1.4 to 3.0 \\
V304 & 217680 & 2.54 & 2.08 & 63.46 & 1.4 to 3.0 \\
V308 & 219594 & 1.90 & 4.88 & 56.41 & 3.0 to 7.0 \\
V308 & 237702 & 1.90 & 4.65 & 60.22 & 3.0 to 7.0 \\
V320 & 222752 & 1.27 & 7.95 & 64.15 & 5.0 to 11.0 \\
V320 & 222753 & 1.27 & 8.05 & 55.90 & 5.0 to 11.0 \\
V311 & 221617 & 1.27 & 9.90 & 64.65 & 6.0 to 12.0 \\
V311 & 266829 & 1.27 & 10.4 & 48.80 & 6.0 to 12.0 \\
\hline
\end{tabular}




\section{Appendix C: \\ Speed Measurement Procedure}

\section{B.1 Slurry Speed Measurement Procedure:}

For a detailed explanation of the pulse echo technique see $\mathrm{McClements}$ and co-workers $(1990,1991)$. The following proccdurc describes all steps necessary to measure sound speed in liquids.

\section{Data Acquisition:}

1. Turn on cooling water and water bath. Set water bath temperature to $26^{\circ} \mathrm{C}$. The test

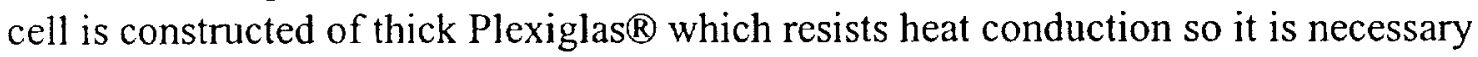
to have the water bath at a slightly higher temperature than that desired for the slurry.

2. Assemble test cell. Choose pathlength desired (1/2" or 1") and install appropriate reflector plate to back of test cell. Choose transducer required and place in appropriate mount, taking care that the transducer surface is flush with the mount. Tighten set screw when using 7.5 or $10 \mathrm{MHz}$ transducer. Apply a small amount of ultrasound gel to transducer well in test cell. Clamp mounted transducer to test cell using supplied screws, taking care to tighten each side gradually.

3. Attach co-axial wire to transducer and 5052 PR Pulser Receiver.

4. Fill cell with slurry, place in water bath and allow to come to desired temperature $\left(25^{\circ} \mathrm{C}\right)$. Monitor sample temperature with digital thermometer.

5. Turn on oscilloscope. Refer to Norato (1999) for instructions on operating oscilloscope.

6. Verify that 5052PR is set to pulse-echo mode. Switch labeled " $1 / 2$ " should be set to "1".

7. Check fixed settings of 5052PR. Rep rate should be set to zero (minimum setting but not on "EXT"). Gain should be set to $40 \mathrm{~dB}$. H.P. filter should be set to "out".

8. Set variable settings of 5052PR. Energy, attenuation, and damping are varied depending on transducer used and experimental conditions: Refer to Table C.1 below for a starting setting for each transducer: 


\begin{tabular}{cccc}
\hline Transducer & Energy $^{*}$ & Attenuation & Damping \\
\hline $1 \mathrm{MHz}$ & 4 & 46 & 0 \\
$2.25 \mathrm{MHz}$ & 4 & 46 & 4 \\
$5 \mathrm{MHz}$ & 3 & 48 & 10 \\
$7.5 \mathrm{MHz}$ & 1 & 36 & 10 \\
$10 \mathrm{MHz}$ & 1 & 4 & 10 \\
\hline
\end{tabular}

Table C.1: Suggested settings of Pulser/Reciever.

* Energy should not be set above 3 for transducers of centerline frequency $5 \mathrm{MHz}$ or greater.

9. Fine tune variable settings of $5052 \mathrm{PR}$. The goal is to create a spike pulse with as high an amplitude as possible, that is not squared off when viewed on the oscilloscope. Additionally, damping should be set such that the baseline voltage is near zero before the first echo is received by the oscilloscope. Once damping is set properly vary attenuation until either the first or second echo waveform begins to square off. Then increase attenuation by four.

10. Check math settings of oscilloscope. Correct settings are:

Trace (A) - Summed average of Channel 1, 50 sweeps

Trace (B) - Functions, Negation of M1

Trace (C) - Phase FFT of (A)

Trace (D) - Phase FFT of (B)

11. Gather waveforms. Center second ccho in oscilloscope. Have Volts/Division set such that the entire waveform takes up about $1 / 3$ of the window, but take care that any echoing waveforms are not displayed. Activate Trace A and Clear Sweeps to take new reading. Then go to Waveform Store and store A to M1.

12. Center first echo in oscilloscope, with all settings equal to those used in the previous step. Activate Trace A and Clear Sweeps to take reading.

13. Perform FFT Operation. Turn off Channel 1 and turn on Trace (A) and (B). Superimpose waveforms on the main peak of both waveforms. It is helpful to zoom in when doing this. Using cursors, determine the time of the main peak of both waveforms and record. Keeping the waveforms superimposed, zoom out and activate Trace (C) and Trace (D).

14. Save FFT data. Turn off Trace (A) and Trace (B). Insert floppy disk in drive. Go to Waveform Store and set "to FLPY" (floppy). Set Data Format to ASCII, MathCad Format. Store "All Displayed" to "FLPY" and press "Do Store". For more information on storing waveforms to floppy see Norato (1999). 


\section{Calculations:}

Calculations are performed in MS Excel. There is a template file for performing phase speed calculations named PSPEED.XLS, located in the C:IPSPEED directory.

1. Open PSPEED.XLS in MS Excel. Immediately save file as different filename. Do not make changes to PSPEED.XLS before saving it as a different filename.

2. Follow instructions in spreadsheet. Fill in experimental conditions and peak times recorded earlier. Open FFT data files and cut and paste data.

3. In order to correctly subtract the phase information given by the FFT procedure all phase angles must be positive. Therefore make corrections of $+360^{\circ}$ to all negative phase angles.

4. Phase speed is now automatically calculated and displayed in column labeled "Phase Speed". 


\title{
Acoustic Probe for the Characterization of Solid-Gas-Liquid Slurries
}

\author{
by \\ Michael Anthony Norato \\ B.S. Ch.E., Syracuse University, 1991 \\ M.S. Ch.E., Syracuse University, 1994
}

\section{DISSERTATION}

Submitted in partial fulfillment of the requirements for the degree of Doctor of Philosophy in Chemical Engineering in the Graduate School of Syracuse University

May 1999

Approved

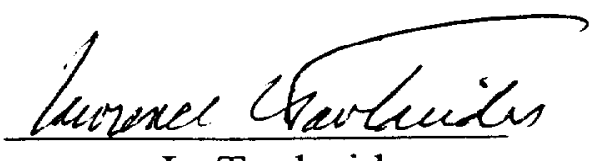

Professor Lawrence L. Tavlarides

Date

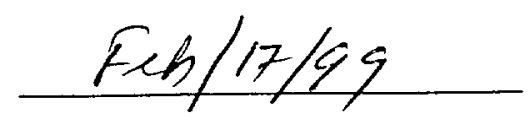


(C) Copyright 1999

Michael Anthony Norato 


\title{
Acoustic Probe for the Characterization of Solid-Gas-Liquid Slurries
}

by

\author{
Michael Anthony Norato
}

Abstract of Dissertation

May 1999

The development of nuclear weapons technology during the Cold War Era has left a legacy of large quantities of radioactive waste which are stored throughout the US Department of Energy (US DOE) Nuclear Weapons Complex. During the proposed remediation stages of processing, it will be necessary to characterize and monitor these waste slurries by remote methods.

Acoustic probes have shown promise because of their non-intrusive nature and ability to penetrate optically opaque slurries.

A forward theory for the acoustic response in both dilute and concentrated solidliquid slurries is developed. It is based on ensemble averaging of the equations of motion in the solid and liquid phases to obtain expressions for the "effective properties" of the slurry mixture in terms of coefficients which appear in the equations of motion for the solid particle. The attenuations predicted from the theory are in generally good agreement with the experimental data obtained by Toneburst and Pulse/FFT data acquisition methods for solid-liquid slurries of soda-lime glass particles of $14.9 \mu \mathrm{m}$ and 
$65 \mu \mathrm{m}$ mean radius and polystyrene particles of $79 \mu \mathrm{m}$ radius at concentrations ranging from $5 \%$ to $50 \%$ solids by volume.

The forward theory is readily extended to systems containing more than one dispersed phase, such as particles and gas bubbles, and the theory predictions are observed to be in good agreement with preliminary attenuation data obtained in solid-gasliquid slurries of soda-lime glass particles of $14.9 \mu \mathrm{m}$ mean radius at $5 \%$ and $10 \%$ by volume and gas bubbles ranging from approximately $25 \mu \mathrm{m}$ to $150 \mu \mathrm{m}$ radius at volume fractions on the order of $10^{-5}$.

An inverse theory is also developed to determine the concentration and solids volume fraction distribution in a solid-liquid slurry given its experimentally obtained acoustic response. A Tikhonov scheme is employed to regularize the ill-posed integrodifferential equations and solve them as a linear programming problem. Solution of the inverse problem is found to be successful in several cases, but the results are observed to be sensitive to the choice of frequency range, the physical properties of the particles, and the nature of the particle volume fraction distribution. 


\section{Table of Contents}

List of Tables..................................................... ix

List of Figures.................................................. $\quad x$

Acknowledgments.......................................... $\quad x v i$

Chapter 1: Introduction.........................................

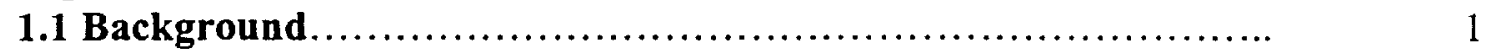

1.2 Objective of Research....................................... 4

1.3 Organization of Dissertation................................. 4

Chapter 2: Literature Survey.................................. 6

2.0 Introduction to Literature Review........................... 6

2.1 Ultrasonic Experimental Techniques.......................... 6

2.1.1 Measurement Techniques in Solid-Liquid Systems........... 6

2.1.2 Measurement Techniques in Liquid-Liquid Dispersions and Emulsions.............................................. 8

2.1.3 Measurement Techniques in Three-Phase Systems.......... 10

2.2 Bubble Generation Techniques in Acoustic Studies................ 11

2.3 Photomicrographic Imaging Techniques....................... 13

Chapter 3: Experimental Studies................................ 16

3.1 Experimental Equipment................................. 16

3.1.1 Mixing Vessels and Test Cells........................... 16

3.1.2 Ultrasonic and Accompanying Electronic Equipment...... 17

3.1.3 Photomicrographic Imaging System..................... 26

3.2 Experimental Procedure-Solid-Liquid Slurries................ 30

3.2.1 Dilute Soda-Lime Glass Slurries...................... 30

3.2.2 Dilute Polystyrene Slurries.............................. 33

3.2.3 Concentrated Soda-Lime Glass Slurries................. 34

3.3 Experimental Procedure-Bubble Generation................. 35

3.4 Experimental Procedure—Solid-Gas-Liquid Slurries........... 39

3.5 Quality of Experimental Results............................. 40

3.5.1 Comparison of Attenuation Measurement Techniques..... 40

3.5.2 Reproducibility of Experimental Results................. 42 
Chapter 4: Theory and Experiments for Dilute Solid-Liquid

Suspensions.

4.1 Theory for the Attenuation of Sound in Dilute Solid-Liquid

Suspensions.................................................... $\quad 50$

4.2 Attenuation Measurements in Dilute Solid-Liquid Systems.......

4.2.1 Attenuation in Soda-Lime Glass Bead Slurries............... 55

4.2.2 Attenuation in Polystyrene Bead Slurries................... 57

Chapter 5: Inverse Problem for Determination of Particle Size

Distribution ............................................ 65

5.1 Inverse Problem Theory.................................... 65

5.2 Inverse Problem Calculation Results..........................

Chapter 6: Effective Medium Theory and Experiments for Concentrated

Slurries................................................... 107

6.1 Effective Medium Theory.......................................... 107

6.1.1 Linearized Equations............................... 110

6.1.2 Ensemble Averaged Linearized Equations for Suspensions.. $\quad 112$

6.1.3 Wave Equations for the Suspension........................ 121

6.1.4 An Effective-Medium Model................................ 124

6.2 Attenuation Measurements in Concentrated Solid-Liquid Systems. 134

Chapter 7: Theory and Experiments for Solid-Gas-Liquid Slurries........ 142

7.1 Theory for the Effective Wavenumber in Solid-Gas-Liquid Slurries. $\quad 142$

7.2 Attenuation Measurements in Solid-Gas-Liquid Slurries............ 142

Chapter 8: Conclusions and Future Work .................................. 153

8.1 Conclusions..................................................... 153

8.2 Recommendations for Future Work .............................. 154

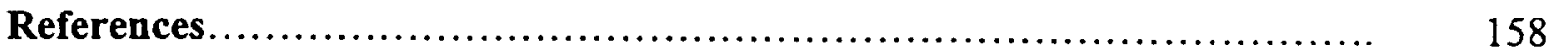

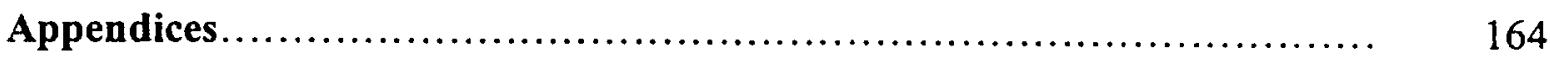

Appendix A: Boundary Conditions from Epstein and Carhart (1953)

and Allegra and Hawley (1972).......................... 164

Appendix B: Experimental Procedures.............................. 168

B.1 Slurry Attenuation Measurement Procedures....................... 168

B.1.1 General Considerations................................... 168

B.1.2Operation of the Matec TB-1000 Toneburst Generator and

Panametrics 5052PR Pulser/Receiver....................... 169

B.1.2.1 Matec TB-1000 Toneburst Generator................. 170

B.1.2.2 Panametrics 5052PR Pulser/Receiver................ 171

B.1.3Operation of the LeCroy 9310A Dual $400 \mathrm{MHz}$ (Digital) 
Oscilloscope.

B.1.3.1 Saving Waveforms to Floppy Diskette.

B.1.3.2 Oscilloscope Set up for Toneburst Measurements...... 174

B.1.3.3 Oscilloscope Set up for Pulse/FFT Measurements....... 174

B.1.4Operation of the Photomicrographic Imaging System....... 176

Appendix C: MATLAB ${ }^{\text {TM }}$ Routines.................................. 186

Appendix D: Experimental Parameter Series Coding.................... 206

Appendix E: Discussion of Transducer Bandwidth..................... 209

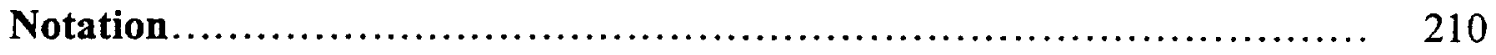

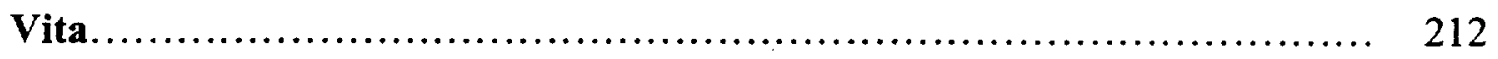




\section{List of Tables}

Table 3.1: Specifications for the Panametrics, Inc. (Waltham, MA) Videoscan immersion transducer employed in this study .......................

Table 3.2: Values of physical properties used in theoretical calculations. The values for polystyrene are from Epstein and Carhart (1953). The properties of soda-lime glass are from Kinsler et al.(1982) and Bolz (1973). The properties of glycerin are from Perry's Chemical Engineer's Handbook (1984) and the CRC Handbook of Chemistry and Physics (1984).

Table D.1: Transducer combinations employed to obtain attenuation data in the various slurries investigated in this study

Table D.2: Experimental method combinations employed to obtain attenuation data in the various slurries investigated in this study.

Table D.3: Experimental mixing vessel/test cell combinations employed to obtain attenuation data in the various slurries investigated in this study

Table D.4: Various slurries investigated in this study 


\section{List of Figures}

Figure 3.1: Schematic diagram representing five different Plexiglas test cells employed in the solid-liquid and solid-gas-liquid

experimentation.

Figure 3.2: Schematic diagram of the experimental set-up used in the Toneburst

Technique.

Figure 3.3: Oscilloscope traces for a 1.0 MHz toneburst transmitted through distilled water and through a $5 \%$ by volume soda-lime glass slurry.......... 20

Figure 3.4: Schematic diagram of the experimental set-up used in the Pulse/FFT Technique

Figure 3.5: Oscilloscope trace and FFT Spectrum of received signal through water for a $1.0 \mathrm{MHz}$ transducer with negative spike pulse input signal......

Figure 3.6: Schematic diagram of the experimental set-up for the photomicrographic video imaging system.

Figure 3.7: Comparison of attenuation spectra results obtained in a $5 \%$ soda-lime glass slurry by both the Toneburst and the Pulse/FFT Techniques. - E1301.. 41

Figure 3.8: Mean attenuation versus frequency curve with error bars representing the average difference between individual attenuation values and the mean value. These data are for a $5 \%$ (by volume) soda-lime glass slurry in water. - E1 101

Figure 3.9: Mean attenuation versus frequency curve with error bars representing the average difference between individual attenuation values and the mean value. These data are for a $10 \%$ (by volume) Potter's beads slurry in a mixture of glycerin/water. - E5182

Figure 3.10: Mean attenuation versus frequency curve with error bars representing the average difference between individual attenuation values and the mean value. These data are for a $30 \%$ (by volume) Potter's beads slurry in a mixture of glycerin/water. - E6192

Figure 3.11: Mean attenuation versus frequency curve with error bars representing the average difference between individual attenuation values and the mean 
value. These data are for a $50 \%$ (by volume) Potter's beads slurry in a mixture of glycerin/water. - E6112 ...................................

Figure 4.1: Comparison between experimental results and forward problem theory predictions for the attenuation versus frequency curves for soda-lime glass slurries at $5 \%$ (lower curve) and $10 \%$ solids by volume. The experimental solids size distribution has a mean radius of $14.9 \mu \mathrm{m}$ with standard deviation of $3.56 \mu \mathrm{m}$. The forward theory predictions are based on a log-normal distribution with a mean radius of $14 \mu \mathrm{m}$ and standard deviation of $7 \mu \mathrm{m}$. - E1101

Figure 4.2: Comparison of forward theory with experimental data for the attenuation as a function of frequency. These data are for polystyrene particles of radius $a$ $=79 \mu \mathrm{m}$, at $5 \%$ by volume. O, experimental data; - theory for monodispersed particles; -..--, theory for monodispersed particles with effective medium correction for finite volume fraction effects; :- - , theoretical result with a particle size distribution with a mean radius of 79 $\mu \mathrm{m}$ and standard deviation of $2.5 \mu \mathrm{m}$ (this is the particle size range specified by the particle manufacturer). - E2363............................... 58

Figure 4.3: Contributions of the first five modes of resonance ( $n=1$ through 5$)$ to the total attenuation (the imaginary part of $k_{\text {eff }}$ ) for $79 \mu \mathrm{m}$ radius polystyrene particles in water

Figure 4.4: Example of the dependence of attenuation on frequency, $f$, for a slurry of polystyrene particles in water. Dashed lines are asymptotic slopes of the attenuation for low and high frequencies............................ 62

Figure 4.5: Contributions of the first three modes of resonance ( $n=1$ through 3$)$ to the total attenuation (the imaginary part of $k_{e f f}$ ) for $79 \mu \mathrm{m}$ radius monodispersed glass particles in water.

Figure 5.1a: Two significantly different particle volume fraction distributions which yield similar attenuation spectra.

Figure 5.1b: Attenuation spectra obtained from the volume fraction distributions in Figure 5.1a. The circles correspond to the results obtained when using the distribution shown by a dashed line in Figure 5.1 a, and the crosses correspond to the result obtained when using the distribution shown by the solid line in Figure 5.1a......................................... 68

Figure 5.2: Error in the attenuation as a function of $\varepsilon$ 
Figure 5.3: Inverse problem solution for polystyrene particles in water. The solid curve is the volume fraction distribution used to generate the attenuation curve shown in Figure 5.7a (with $f_{\max }$ indicated by a square); and the dashed curve is the solution to the inverse problem for particle radius range of 1 to $100 \mu \mathrm{m}$ and using 50 'bins' of particle sizes..............

Figure 5.4: Solution of the inverse problem when a random noise of $5 \%$ standard deviation is introduced in the attenuation (input) data. The solid line is the exact result; the dashed curve is the result when no noise is introduced; the dash-dotted curve is the result after the introduction of the noise. These calculations are for polystyrene in water............................

Figure 5.5: Solution of the inverse problem for a bimodal distribution of polystyrene particles, using 30 particle size bins. The solid curve is the exact result, and the markers represent the inverse problem solution when using a value of $f_{\max }$ indicated by a square in Figure $5.6 \ldots \ldots \ldots \ldots \ldots \ldots \ldots \ldots . . . \ldots$

Figure 5.6: Attenuation versus frequency curve for polystyrene particle volume

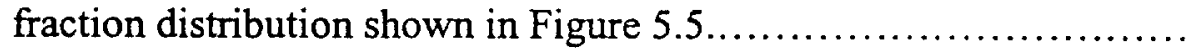

Figure 5.7a: Input attenuation data for four different upper bounds on frequency to determine the influence of the frequency range over which the attenuation is specified on the solution of the inverse problem. This attenuation curve is for polystyrene in water. The solid curve is the exact result; $\square$, cutting off the frequency range after the first attenuation peak; + , cutting off the frequency range just after the second change in slope in the attenuation curve; $O$, cutting off the frequency range just before the second change in slope in the attenuation curve; and $\diamond$, cutting off the frequency range just after the third attenuation peak

Figure 5.7b: Results of the inverse problem solution for four different frequency

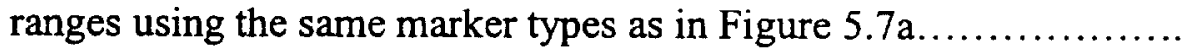

Figure 5.8: Using too large of a frequency range over which the attenuation data are available for polystyrene particles in water can deteriorate the results. The solid curve is the exact result; the dashed curve is the inverse problem result when using the data of Figure $5.7 \mathrm{a}$ below the point marked by a ' $\square$ ' the dash-dotted curve represents the results when this endpoint is shifted to the point marked by ' $O$ '; and the dotted curve is the result

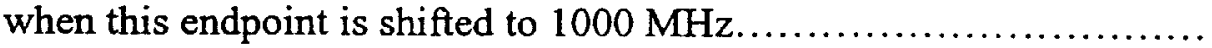

Figure 5.9: Inverse problem solution for a bimodal distribution of polystyrene particles (same distribution as in Figure 5.5) when cutting off the frequency range 
over which the attenuation was given between the first and second

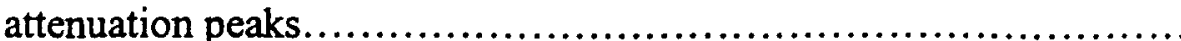

Figure 5.10a: Attenuation versus frequency curve for the same inverse calculation for polystyrene particles in water whose results are shown in Figures 5.7a $\&$ b, but with a broader size distribution....................... $\quad 90$

Figure5.10b: Resulting volume fraction distribution for the broader distribution attenuation data shown in Figure 5.10a...................... 91

Figure 5.11a: The kernel, $K\left(a_{i}, a_{j}\right)$ for polystyrene particles in water when using for $f_{\max }$ the value of frequency indicated by a ' $\mathrm{O}$ ' in Figure $5.7 \mathrm{a} . . . . .93$

Figure 5.11b: The kernel, $K\left(a_{i}, a_{j}\right)$, for polystyrene particles in water when using for $f_{\max }$ the value of frequency indicated by a ' + ' in Figure $5.7 \mathrm{a} . . . . .994$

Figure 5.12c: The kernel, $K\left(a_{i}, a_{j}\right)$, for polystyrene particles in water when using for $f_{\max }$ the value of frequency indicated by a ' $\square$ ' in Figure $5.7 \mathrm{a} \ldots \ldots .95$

Figure5.12a: Results of the inverse problem solution for air bubbles in water. Bubble volume fraction distribution for a total gas phase volume fraction equal to 0.004

Figure 5.12b: The kernel, $K\left(a_{i}, a_{j}\right)$, for air bubbles in water at a total gas phase volume fraction of 0.004

Figure 5.12c: The attenuation spectrum for air bubbles in water at a total gas phase

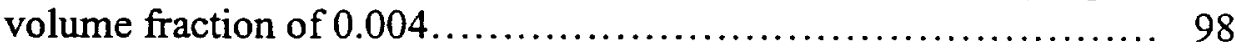

Figure 5.13a: An inverse problem result for small glass particles.............. 100

Figure 5.13b: An inverse problem result for lager glass particles............... 101

Figure 5.13c: The kernel, $K\left(a_{i}, a_{j}\right)$, for the larger glass particles whose results are shown in Figure 5.13b........................................... 102

Figure 5.14a: Attenuation spectrum for polystyrene particles in water where the data are used in the linear programming results shown in Figure 5.14b..... 105

Figure 5.14b: Inverse problem results using the linear programming method. The linear programming results (.. ...) are compared with results of the Tikhonov method (-- $\square--)$ and the exact solution (solid curve)......

Figure 6.1: Attenuation versus frequency behavior for a slurry of Potter's beads in a 
mixture of glycerin/water at $5 \%$ by volume-forward theory and experimental data. - E2162 ..........................................

Figure 6.2: Attenuation as a function of solids volume fraction at various frequencies in slurries of Potter's beads in glycerin/water. Data are shown for $1.5 \mathrm{MHz}$ $(\Delta) ; 1.75 \mathrm{MHz}(0) ; 2.0 \mathrm{MHz}(+) ; 2.25 \mathrm{MHz}(\times) ; 2.5 \mathrm{MHz}(\square)$; and 2.75 $\mathrm{MHz}(\nabla)$. The solid curves represent the results of the effective medium calculations using a particle size distribution with $65 \mu \mathrm{m}$ mean radius and standard deviation of $11 \mu \mathrm{m}$. - E4172 ......................... 137

Figure 6.3: Attenuation as a function of solids volume fraction at various frequencies in slurries of Potter's beads in glycerin/water. Data are shown for $3.0 \mathrm{MHz}$ $(\Delta) ; 3.5 \mathrm{MHz}(\mathrm{O}) ; 4.0 \mathrm{MHz}(+) ; 4.5 \mathrm{MHz}(x)$; and $5.0 \mathrm{MHz}(\square)$ The solid curves represent the results of the effective medium calculations using a particle size distribution with $65 \mu \mathrm{m}$ mean radius and standard deviation of $11 \mu \mathrm{m}$. - E6173........................... 139

Figure 6.4: Attenuation as a function of solids volume fraction for the data of Allegra and Hawley (1972). The symbols represent experimental data at $3 \mathrm{MHz}(\Delta)$; $9 \mathrm{MHz}(0) ; 15 \mathrm{MHz}(+) ; 21 \mathrm{MHz}(\times) ; 27 \mathrm{MHz}(\square)$; and $39 \mathrm{MHz}(\nabla)$.The solid curves represent the results of the effective medium approach used in this study

Figure 7.1: Attenuation versus frequency data for solid-gas-liquid systems of sodalime glass beads in water at $5 \%$ (lower curve) and $10 \%$ solids by volume with bubbles generated by a limewood aerator with air flow rate of 150 $\mathrm{ml} / \mathrm{hr}$. The solid-gas-liquid data $(+)$ are shown with solid-liquid data $(\bullet)$ at the same solids concentrations. The solids size distribution has a mean radius of $14.9 \mu \mathrm{m}$ with standard deviation of $3.56 \mu \mathrm{m}$. - E2231 ........ 144

Figure 7.2: Attenuation versus frequency data for solid-gas-liquid systems of sodalime glass beads in water at $5 \%$ (lower curve) and $10 \%$ solids by volume with bubbles generated by an electrolyzer at $40 \mathrm{~V} ; 4$ to $8 \mathrm{~mA} ;<1 \mathrm{~W}$. The solid-gas-liquid data $(+)$ are shown with solid-liquid data $(\bullet)$ at the same solids concentrations. The solids size distribution has a mean radius of $14.9 \mu \mathrm{m}$ with standard deviation of $3.56 \mu \mathrm{m}$. - E2231 ............. 146

Figure 7.3: Comparison between experimental solid-gas-liquid attenuation data $(+)$ at $5 \%$ (lower curve) and $10 \%$ solids (by volume) and forward problem theory predictions (solid curves) employing the same solids concentrations and a log-normal bubble size distribution with $23 \mu \mathrm{m}$ mean radius and $4.3 \mu \mathrm{m}$ standard deviation. The bubble volume fraction used in the theory predictions is $1.5 \times 10^{-5}$. - E2231 
Figure 7.4: Results of predicting the attenuation due to the presence of bubbles in a solid-gas-liquid slurry and simply subtracting that attenuation from the total attenuation. The symbols represent experimental data for the solidliquid $(\bullet)$ and solid-gas-liquid $(+)$ slurries, and the curves represent the forward theory predictions for those slurries and the difference after subtracting the bubble attenuation. - E2231 ...................... 150

Figure B.1: Photograph of the 0.005 " diameter circle on the reticle used in calibrating the photomicrographic video imaging system......................

Figure B.2: Photograph of a electrolytically generated "bubble stream". The bubbles are are generated at $40 \mathrm{~V}, 6$ to $8 \mathrm{~mA},<1 \mathrm{~W}$.

Figure B.3: Calibration plot relating measured particle diameters to the NIST certified diameters of polystyrene particle standards....

Figure B.4: Bubble size distribution determined by the microphotographic video imaging system and the log-normal size distribution fitted to the data.. 185 


\section{Acknowledgments}

I would like recognize and thank the following individuals for their indispensable contributions to the completion of this work:

Professor Lawrence L. Tavlarides, my research advisor, for his suggestions, criticism, and guidance, both personal and professional, throughout the course of this work, and indeed, my entire undergraduate and graduate education experience. I am very grateful for all he has done for me over the years.

Professor Ashok S. Sangani, my co-advisor on my doctoral research, for his assistance, guidance, and patience in the theoretical aspects of this work.

Drs. Margaret Greenwood and Stephen Doctor, and all the staff of the NonDestructive Evaluation Section at the Battelle Pacific Northwest National Laboratory in Richland, WA, for the instruction, guidance, and friendship they provided during my four months at PNNL and throughout the course of this work.

Dr. Costas Tsouris, and all the staff of the Chemical Technology Division of the Oak Ridge National Laboratory, for the friendship, support, and assistance provided periodically throughout the course of this work. I would also like to thank his wife and colleague, Professor Sotira Yiacoumi, and her students at Georgia Institute of Technology for making some the early bubble size measurements used in this work. 
Dr. Peter D.M. Spelt, for contributing his considerable talent in performing the theoretical portions of this work. I would also like to thank him for his advice, criticism, and friendship.

My parents, Dr. and Mrs. Joseph F. Norato, for their support and criticism throughout the years of my education.

Ella-Marie A. LaPlante, for her patience, understanding, love, and support. I know I did not always make it easy for her, but she stayed on through the final and most difficult year of my work...and for that, I am eternally grateful.

The technical and administrative assistance of the following people on various occasions is appreciated:

Technicians: Mr. John Banas, Mr. John Chabot, Mr. Dick Chave, Mr. Ronnie Judd, and Mr. John Kotlarz.

Students: $\quad$ Mr. Daniel Wright, Mr. Richard Henry, and Mr. Ted Hromadka (PNNL). Administrative Staff: Mrs. Ruth Dewey, Mrs. Mickey Hunter, Mrs. Linda Lowe, and Ms. Linda Vanderhoof.

The financial support provided by the following organizations is greatly appreciated: Department of Chemical Engineering and Materials Science, Syracuse University. 
The Graduate School of Syracuse University.

Associated Western Universities.

US Department of Energy, Environmental Management Science Program

Grant \# DE-FG07-96ER14729. 


\section{Chapter 1: Introduction}

\subsection{Background}

For nearly five decades, the US government has been building and stockpiling nuclear weapons without a well defined plan for the safe and permanent disposal of the highly toxic byproducts routinely created by the continual refining and manufacturing of plutonium and uranium (Garr 1992). The development and application of nuclear weapons technology during the Cold War Era has left a legacy of large quantities of radioactive waste which are stored throughout the fifteen facilities which comprise the US Department of Energy (US DOE) Nuclear Weapons Complex. The production of weapons grade nuclear materials began in the early 1940's and continued until the late 1980's. Nuclear weapons production efforts have generated 100,000 kilograms of plutonium. The PUREX (Plutonium Uranium Extraction) process creates more than 340 gallons of high-level radioactive waste and more than 55,000 gallons of low- to intermediate-level radioactive waste for every kilogram of plutonium produced (Garr 1992). Much of these wastes was stored with little or no documentation (Levi 1992), making characterization of the wastes an integral part of any potential remediation scheme.

It is estimated by the DOE that cleanup of the entire nuclear weapons complex will take thirty years and require about $\$ 100$ billion, while some outside estimates predict that it will cost at least twice that much (Garr 1992). There are hundreds of waste storage 
tanks across the US DOE Nuclear Weapons Complex that will require remediation in the next several decades. During the proposed retrieval, pretreatment, immobilization, and closure stages of the waste processing it will be necessary to characterize and monitor various properties of the resulting waste slurries by remote, non-intrusive methods. Real time monitoring of the size and volume fraction of solid particles in these slurries by acoustic probes has shown promise because of their (i) non-intrusive nature; (ii) simplicity of operation (unlike NMR and X-Ray tomography); and (iii) ability to penetrate concentrated and optically opaque slurries (unlike laser Doppler anemometry which requires refractive index matching).

Although acoustic probes have been commonly used for monitoring flows of single-phase fluids (McLeod 1967), their application to monitor multiple phase mixtures has not yet fully reached its potential. A number of investigators in recent years have been involved in developing probes for measuring the volume fractions in solid-liquid suspensions (Atkinson and Kytomaa 1993; Greenwood et al. 1993; Martin et al. 1995) and liquid-liquid dispersions (Bonnet and Tavlarides 1987; Tavlarides and Bonnet 1988; Yi and Tavlarides 1990; Tsouris and Tavlarides 1993; Tsouris et al. 1995). In particular, Atkinson and Kytomaa (1993) suggest that the acoustic technique can be used to determine both the velocity and the volume fraction of solids while Martin et al. (1995) claim that the acoustic probe can be used to obtain information on the size distribution of the particles. The acoustic probe is also used and commercially available (Malvern Instruments, Pen Kem, Inc.) for at-line (i.e., using samples or slip-streams drawn from a process line as opposed to on-line, implying simply being immersed in a mixing tank or 
installed on a process pipeline) particle size measurements in solid-liquid slurries. Finally, an acoustic technique is also used for determining bubble size distributions in bubbly liquids (Duraiswami et al. 1998).

The work of the investigators just cited has established the potential of the acoustic probe for characterizing/monitoring two-phase flows in relatively ideal, wellcharacterized suspensions. One major obstacle to its wide-spread use in slurry processing, however, arises from the interference caused by the presence of small amounts of gas. The presence of a low volume fraction of gas bubbles formed, for example, by radiolytic degradation processes, cavitation from slurry pumps, or vapor entrainment could significantly interfere with the transmitted acoustic signal preventing straightforward application of a probe. Since the compressibility of the gas is typically several orders of magnitude greater than the compressibility of liquids and solids, the acoustic wave propagation is significantly affected even when the gas is present in relatively small amounts.

It may be possible, however, to overcome the interference introduced by the gas phase by carefully selecting the frequency range for interrogation and by making use of the theory of acoustic wave propagation in bubbly liquids in analyzing the acoustic response of the suspension. Since the gas bubbles are formed by a variety of different mechanisms and their size distribution in actual flows can vary considerably, an important problem to be solved is to determine the noise introduced by the bubbles and to remove it from the acoustic signal obtained from the suspension to yield the underlying information about the suspended solid phase. 


\subsection{Objective of Research:}

The objective of this study is to investigate the potential applicability of the acoustic probe to characterize solid-liquid and solid-gas-liquid slurries. In order to achieve this objective it is necessary develop a forward theory to predict the acoustic response of a solid-liquid slurry, given an a priori knowledge of its microstructure, which can then be compared with experimental results. To be practical, the theory must include consideration of both dilute and concentrated slurries. Further, it is desired to develop an inverse theory to determine the solids concentration and volume fraction distribution in a solid-liquid slurry from its experimentally obtained acoustic response. Lastly, it is desired to extend the forward theory for solid-liquid systems to solid-gas-liquid slurries and compare theory predictions with experimental results for these systems.

\subsection{Organization of Dissertation:}

The work presented in this study consists of four main areas of investigation which are discussed separately in individual chapters. The experimental data in each area of study are acquired by slight variations of two main experimental techniques. Chapter 2 gives a literature survey of experimental techniques which are especially relevant to this study. Chapter 3 describes the experimental equipment, instrumentation, and procedures employed in this study. Chapter 4 presents theory and a comparison with experimental data of the attenuation of acoustic waves in dilute solid-liquid suspensions. Chapter 5 examines the inverse problem to determine the solids concentration and particle size distribution in solid-liquid slurries. In Chapter 6 a theory is presented for predicting the 
attenuation of acoustic waves in concentrated slurries, and the predictions of the theory are compared with experiments. Chapter 7 gives the preliminary results of attenuation measurements in solid-gas-liquid slurries. Finally, conclusions and recommendations for future work are given in Chapter 8. 


\section{Chapter 2: Literature Survey}

\subsection{Introduction to Literature Review:}

It is the goal of this chapter to present a literature review of experimental techniques which are relevant to the work performed in this investigation. Only literature pertaining to the experimental studies is presented here. The literature surveys related to the various theories are presented in the respective chapters in which they are relevant.

\subsection{Ultrasonic Experimental Techniques:}

\subsubsection{Measurements Techniques in Solid-Liquid Systems:}

Experimentation using ultrasound techniques has been going on for quite some time. Urick (1947 and 1948), for example, measured the velocity and attenuation of ultrasound in solid-liquid suspensions in two different experimental studies using a fairly simple ultrasound interferometer which consisted of a "bare" quartz (piezoelectric) crystal and relatively simple electronics operated in a pulse-echo mode. Since that time, many other investigators have made attenuation and/or phase speed measurements in solid-liquid suspensions using more sophisticated instrumentation as technology, especially in the field of electronics, has advanced.

Allegra and Hawley (1972) used a semiautomatic version of the conventional pulse technique employed by Kessler et al. (1970) to measure attenuation as a function of frequency in suspensions of polystyrene spheres in water as well as in cmulsions of 
toluene and hexane in water. They observed that the attenuation data obtained from the liquid-liquid emulsions by their technique yielded good agreement with predictions made by the theory of Epstein and Carhart (1953). They also extended the Epstein and Carhart theory to solid-liquid suspensions and observed good agreement between the theory predictions and experimental results.

Atkinson (1991) used a commercially available $1.0 \mathrm{MHz}$ immersion transducer (Panametrics Model V302) as a transmitter and a custom made polyvinylidene fluoromer (PVDF) receiver to perform phase speed and attenuation measurements in packed beds and fluidized beds of $1.0 \mathrm{~mm}$ glass beads in water. Atkinson (1991) reported that the advantage of using the piezoelectric polymer, PVDF, as the acoustic receiver was that it could be custom made into virtually any size, and that PVDF hydrophones have fairly flat acoustic response in the range of DC (implying zero frequency) to several Megahertz.

Alba (1992) patented an at-line (next to the process line) device which measures concentration and size distribution of particles in suspensions. It employed piezoelectric transducers mounted in tubes in a vessel wall allowing for variable distance between transducers through a sliding contact. The input signal to the transmitter transducer was a continuous wave transmitted for a few milliseconds at a time. Attenuation measurements were then made at two or more separation distances. This device is reported to be applicable to solid-liquid slurries containing particles ranging in size from 0.01 to 1,000 $\mu \mathrm{m}$ at concentrations from $0.1 \%$ to $70 \%$ by volume. The particle size distribution is determined by fitting the resulting measured attenuation spectrum to one calculated from an a priori assumed distribution, such as log-normal or Gaussian. The main 
disadvantages of this device are that it requires a knowledge of the materials which constitute the slurry and the assumption that the particle population can be described by a commonly known type of size distribution.

Greenwood et al.(1993) employed two piezoelectric transducers of $2.25 \mathrm{MHz}$ centerline frequency separated by four inches in a tank to measure the attenuation as a function of frequency from 0.5 to $3.0 \mathrm{MHz}$ in a kaolin/water slurry for solids volume fractions up to 0.24 . The transmitter transducer was driven by signals from a toneburst swept frequency generator. Measurements were made by photographing the voltage versus frequency of the signal received which was displayed on an oscilloscope screen for each slurry concentration studied and comparing the voltage values at six individual frequencies for each concentration with the corresponding values in water. The attenuation was then calculated from ratios of the slurry voltages at each frequency to those in water at the same frequency. It was observed that the attenuation is proportional to the frequency and solids volume fraction within the investigated ranges of these parameters. These results further demonstrated that it is feasible to use attenuation measurements to determine the solids concentration in slurries.

\subsubsection{Measurement Techniques in Liquid-Liquid Dispersion and}

\section{Emulsions:}

Much work has been done in the monitoring and characterization of liquid-liquid dispersions using an ultrasonic technique. Bonnet and Tavlarides (1987) developed an

ultrasonic technique which used the time-averaged model of Kuster and Toksoz (1974) to 
predict the dispersed phase volume fraction in liquid-liquid dispersions and suspensions based on the difference in travel time of the ultrasound in the pure materials and in the dispersion. This technique was later improved to account for effects of the dispersed phase (such as the sphericity and polydispersity of the drops) on the travel time by modifications to the time-averaged model (Yi and Tavlarides 1990; Tsouris and Tavlarides 1990; Tsouris and Tavlarides 1993). These modifications allowed the technique to be employed under conditions of large dispersed phase volume fraction and in situations where the velocity of sound was greater in the organic phase than in the aqueous phase. These improvements in the ultrasonic technique eventually led to the development of a miniaturized, in-situ pulse-echo ultrasonic probe to measure local dispersed phase volume fraction in liquid-liquid dispersions (Tsouris et al.1995), which was also shown to have the capability to detect phase inversion in these dispersions.

McClements (1992) measured ultrasound velocity and attenuation coefficients in $10 \mathrm{wt} \%$ emulsions of $n$-hexadecane in water using a device called a Frequency Scanning Ultrasonic Pulse-Echo Reflectometer (FSUPER). This device measured the amplitude and phase differences, through Fourier analysis, between signals transmitted through a Perspex buffer rod in the sample cell and the buffer rod plus emulsion sample. McClements (1992) observed good agreement between the data obtained in that study and multiple scattering theory for dilute emulsions of dispersed phase volume fraction less than 0.13 . However, there was some deviation from the multiple scattering theory for more concentrated emulsions, especially at low frequencies and small drop sizes. McClements (1992) suggested that this deviation between theory and experiment could 
be due to interaction between the drops, which is not included in the multiple scattering theory. Another suggested source of discrepancy was interference with the measurement by the incoherent part of the scattered signal.

\subsubsection{Measurement Techniques in Three-phase Systems:}

Okamura et al. (1989) and Uchida et al. (1989), in two related works, used the degree of phase difference between acoustic signals obtained in liquid, solid-liquid, and three phase systems to empirically determine the concentration of solids in three phase reactors. The main premise of their work was that the presence of the gas phase did not affect the phase shift of the signal received which was induced due to the presence of the solid particles alone. Therefore, one could determine the solids concentration in a three phase slurry simply by measuring the phase lead in the signal received. This technique is successful because the transmitted signal never actually encounters any of the gas phase. When the transmitted signal does encounter gas bubbles, it is completely blocked. So the signals upon which the measurements are made are only signals acquired when no gas bubbles are encountered. Therefore, this technique is essentially measuring the solids hold up in a two phase system.

Soong et al. (1995) used a technique based on that of Okamura et al. (1989) to perform ultrasonic measurements of the solids concentration in a three phase slurry consisting of water, nitrogen bubbles, and glass beads. Particular attention was placed on the effects of the gas flow. The bubbles were estimated to be of the order of 3 to $5 \mathrm{~mm}$ in size. The authors concluded that the attenuation is slightly affected by the gas flow rate, 
and the transit time of the ultrasonic signal is not affected by the gas flow rate. Both the amplitude ratio and the travel time were affected by the solids concentration.

Soong et al. (1996) performed similar measurements in three phase slurries of molten paraffin wax, nitrogen bubbles, and glass beads. In this study, the bubble size was also about $5 \mathrm{~mm}$. This study concluded that both the amplitude and the transit time of an ultrasonic signal were influenced by the variation of solids concentration in paraffin wax. It was, again, found that the variation of nitrogen flow had very little effect on the observed transit times. The gas probably did not have a great effect on the measurements because of the bubble size. Bubbles in the size range of 3 to $5 \mathrm{~mm}$ have extremely low resonance frequencies (about $1 \mathrm{kHz}$ ). Therefore, the measurements were made at frequencies well beyond the bubble resonance frequency. Bubbles of this size would only affect the transmitted signal if they were present in a large enough concentration to completely block the signal. These techniques are successful because the experimental conditions are such that the gas phase is not really relevant to the measurements.

\subsection{Bubble Generation Techniques in Acoustic Studies:}

Carstensen and Foldy (1947) conducted measurements of the transmission, scattering and reflection of sound by screens of bubbles. The bubbles were generated by a novel device called a microdisperser. This apparatus consisted of two concentric glass tubes. The inner tube was of capillary dimension $(0.01 \mathrm{~cm}$ i.d.), and it carried air at approximately 0.5 to $2.5 \mathrm{psi}$ above the hydrostatic pressure at its terminus. The outer tube directed a flow of water over the inner capillary tube. The effect of the water flow 
was to strip air bubbles away from the capillary tube end as soon as they formed. Coarse control of the bubble size was provided by varying the water flow rate, and bubbles were generated in the size range of approximately 100 to $400 \mu \mathrm{m}$.

Silberman (1957) measured the attenuation and phase speed of sound in bubbly liquids by establishing standing waves in steel pipes filled with a gas-water mixture. The attenuation was then determined from pressure measurements. The bubbles in this study were produced by pumping gas at extremely small flow rates through hypodermic needles and other small diameter tubing. This procedure consistently produced bubbles with diameters of 1 to $3 \mathrm{~mm}$ at approximately $1 \%$ by volume. The Silberman (1957) study is considered significant because of its superior control of the bubble size and the accuracy of the data which generally agreed well with the theory, except in the immediate neighborhood of bubble resonance.

Kol'tsova et al. (1979) measured the excess attenuation in liquids containing between $0.025 \%$ and $0.02 \%$ (by volume) of small hydrogen bubbles. The bubbles were created by electrolysis. The cathode of the electrolyzer consisted of $0.02 \mathrm{~mm}$ diameter copper wire wound around rectangular Bakelite plates at a spacing of 1 to $3 \mathrm{~mm}$. Several plates were stacked together with the wire turns interconnected on one side. The wire turns on the opposite side were polished such that the wires were severed to form stubs from which the bubbles emanated. The anode was a thin platinum wire stretched above the cathode. A $3 \% \mathrm{NaCl}$ solution was hydrolyzed to create bubbles with radii in the range of 7 to $45 \mu \mathrm{m}$. These bubble sizes are much smaller than those described in 
previous investigations, and they are the approximate bubble sizes which are of interest in the present study.

\subsection{Photomicrographic Imaging Techniques:}

Photomicrographic techniques of various types have been employed rather widely in the past to measure particle, bubble, and drop sizes in a vast array of dispersed phase systems. For example, Kirou et al. (1988) employed a photomicrographic technique, which was a modification of that developed by Bapat (1982), to measure droplet size in an Oldshue-Rushton extraction column. This method consisted of a microscope tube interfaced with a $35 \mathrm{~mm}$ camera. The microscope objective viewed the droplets through a special window port in the wall of the extraction column which was designed to prevent droplets from passing between the objective lens and those droplets in the objective's focal plane. Backlighting was provided by a rigid fiber optic light conduit which also passed through the port and was bent to face the photographic window. The light conduit was coupled to a microflash unit which was synchronized to the camera shutter. Droplets were photographed as they passed between the fiber optic conduit and the window, and their sizes were measured using a semi-automatic technique.

Kol'tsova et al. (1979) employed a photographic technique to measure the size distributions of microbubbles in their study of the attenuation of ultrasound in bubbly liquids. Although not many details of the bubble size measurements are provided, it seems that the bubbles were either photographed or viewed visually through optical glass windows in the walls sample test cell with the aid of a long distance microscope. 
Pacek et al. (1994) employed a video technique to study the dynamics of phase inversion in liquid-liquid dispersions. Their technique allowed for the collection of data on the transient mean drop size and the transient drop-size distribution during phase inversion. The system of Pacek et al. (1994) consisted of a stereo microscope with a very shallow depth of field attached to a high speed video camera. Sharp images of dispersed phase drops were captured by using a strobotach synchronized with the video camera at its framing rate. Pacek et al. (1994) were able to photograph dispersed phase droplets of sizes of $40 \mu \mathrm{m}$ and larger for accurate measurements at dispersed phase concentrations of up to $70 \%$ by volume. The photographs were analyzed semiautomatically via computer to yield cumulative and frequency drop size distributions to any mean size.

Tsouris et al. (1995) employed a high speed imaging system to visualize drops produced at the tip of the nozzle of an electrostatic spraying apparatus. This system consisted of a Kodak Ektapro Intensified Imager which had a maximum frame speed of 12,000 frames per second. The imager was connected to a video recorder, a monitor, and a printer. This system was employed to measure large drops (approximately 0.5 to 2.5 mm diameter) of TCE (trichloroethylene) electrostatically sprayed into water.

Burns et al. (1997) used a video based digital imaging system to measure sizes of bubbles generated by various techniques such as electrolysis (electroflotation), electrostatic spraying, and dispersed air flotation (DAF). An image analysis system was employed to measure the equivalent circular diameter of the bubbles produced. The hardware employed depended upon the size of the bubbles produced. For the techniques which produced relatively large bubbles (diameters larger than about $100 \mu \mathrm{m}$ in diameter), the bubbles were 
photographed by a high speed video camera fitted with a magnifying lens. Smaller bubbles (less than approximately $100 \mu \mathrm{m}$ in diameter) were photographed with the video camera interfaced with a long distance microscope capable of magnification of up to 230 to 250 times. The camera in each set up was interfaced to a video recorder, a monitor, and a printer. A light source was set up behind the test cell, shining toward the camera so that the bubbles appeared as dark images on a light background. The image analysis system consisted of a control computer and software which measured the area of individual bubbles and converted it to an equivalent circular diameter. The calibration factor for the bubble size measurements was determined by videotaping and measuring a wire of known diameter. The identical imaging system was used by Shin et al. (1997) to measure sizes of bubbles produced in experiments involving the electrostatic spraying of air into water. 


\section{Chapter 3: Experimental Studies}

\subsection{Experimental Equipment:}

It should be noted that a number of combinations of transducers, mixing vessels and test cells, and experimental techniques were employed to obtain the attenuation data presented in this study. Therefore, in the caption of each figure which presents attenuation data obtained in this study, there is an "experimental parameter number". This number begins with a capital letter " $E$ " (indicating experimental parameter series) and is followed by four numbers. Each of the four numbers refers to a particular set of parameters used in the experiments to yield that data set. The key to extracting the specific experimental parameters from the experimental series number is given in Appendix D.

\subsubsection{Mixing Vessels and Test Cells:}

Initial Experiments in solid-liquid systems in this study have been conducted in a cylindrical polyvinyl chloride (PVC) vessel of approximately $3.9 \mathrm{~L}$ in volume with 15.5 cm I.D. and $20.5 \mathrm{~cm}$ height. Four transducer ports spaced at $90^{\circ}$ allow for the employment of two transducer pairs in each experimental "run". A "false bottom" can be inserted into the vessel to allow for a smaller sample volume of $1.6 \mathrm{~L}$.

Also, various other vessels are employed depending upon the nature of the system to be interrogated. In instances where the materials under consideration are quite 
expensive (such as polystyrene spheres) allowing only small slurry volumes, or the volume fraction of solids is high making suspension difficult, Plexiglas test cells of rectangular "box" geometry and various nominal transducer separation distances are employed. There are four different test cells with nominal transducer separation distances of $1.27 \mathrm{~cm}, 2.54 \mathrm{~cm}, 5.08 \mathrm{~cm}$, and $10.2 \mathrm{~cm}$. A schematic diagram of a Plexiglas test cell is shown in Figure 3.1. These cells are designed to accommodate only a small sample volume while still being large enough to avoid the effects of signal reflection off the cell walls due to spreading of the transmitted ultrasound beam as described in Kinsler et al. (1982). When these test cells are used, the suspension of the slurry is provided through manual agitation with a glass stirring rod.

\subsubsection{Ultrasonic and Accompanying Electronic Equipment:}

In regard to electronic equipment, there are two distinct experimental set-ups which are employed. The first setup is shown in Figure 3.2, and it is based on an experimental system employed by the Non-Destructive Evaluation Section of the Energy Division at the Battelle Pacific Northwest National Laboratory. This system consists of a desktop computer with a Matec TB-1000 digital synthesizer card capable of generating monochromatic tonebursts from 0.05 to $20 \mathrm{MHz}$ in frequency. An example of a $1 \mathrm{MHz}$ toneburst in both water and a $5 \%$ soda lime glass slurry is shown in Figure 3.3. A toneburst of a given frequency is sent to the transmitter piezoelectric transducer (Panametrics Videoscan Immersion Transducers Models V391, V302, V304, V308, V320, and V311), through the medium (either water or slurry), to the receiver transducer. 


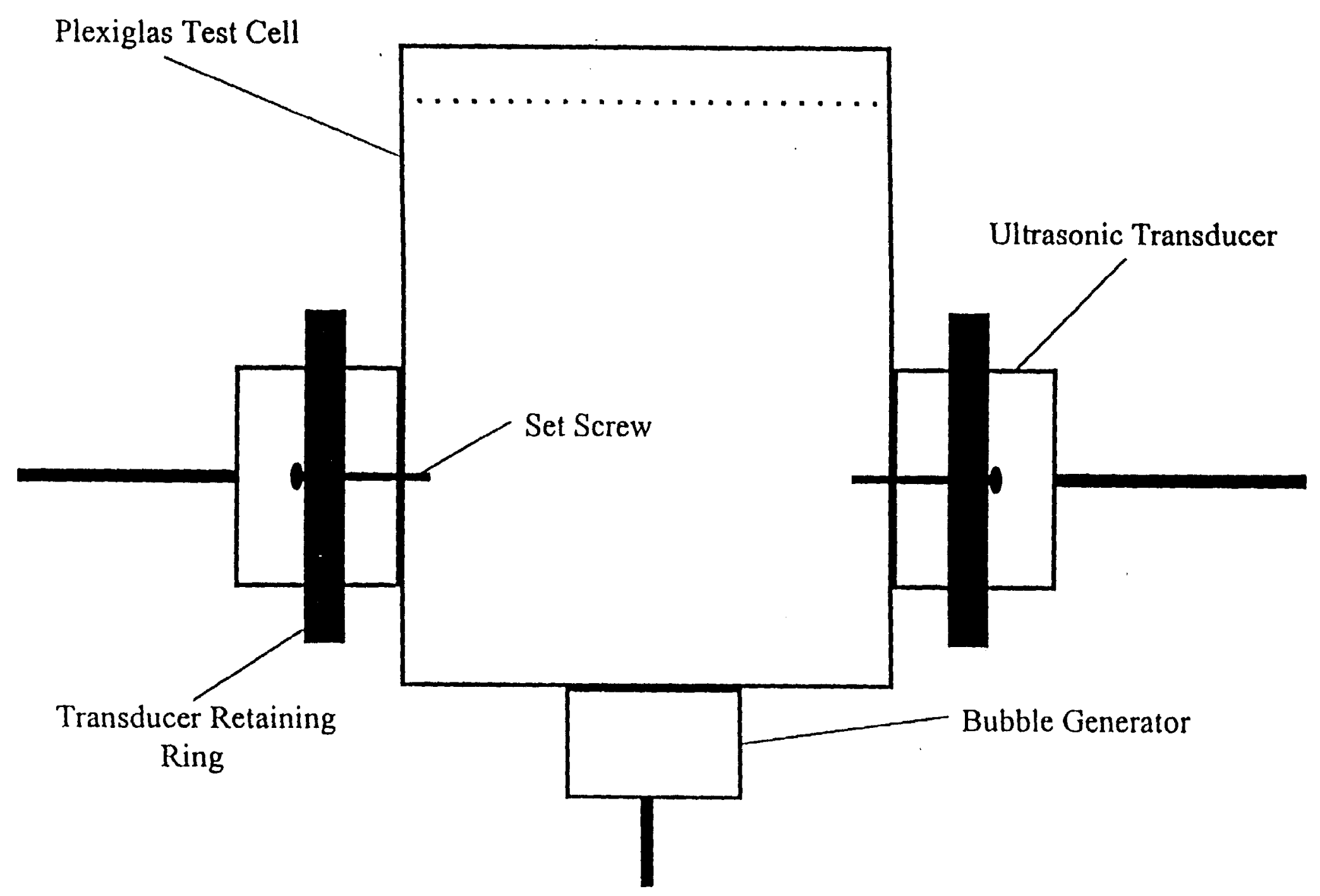

Eigure 3.1: Schematic diagram representing five different Plexiglas test cells employed in the solid-liquid and solid-gas-liquid experimentation. 


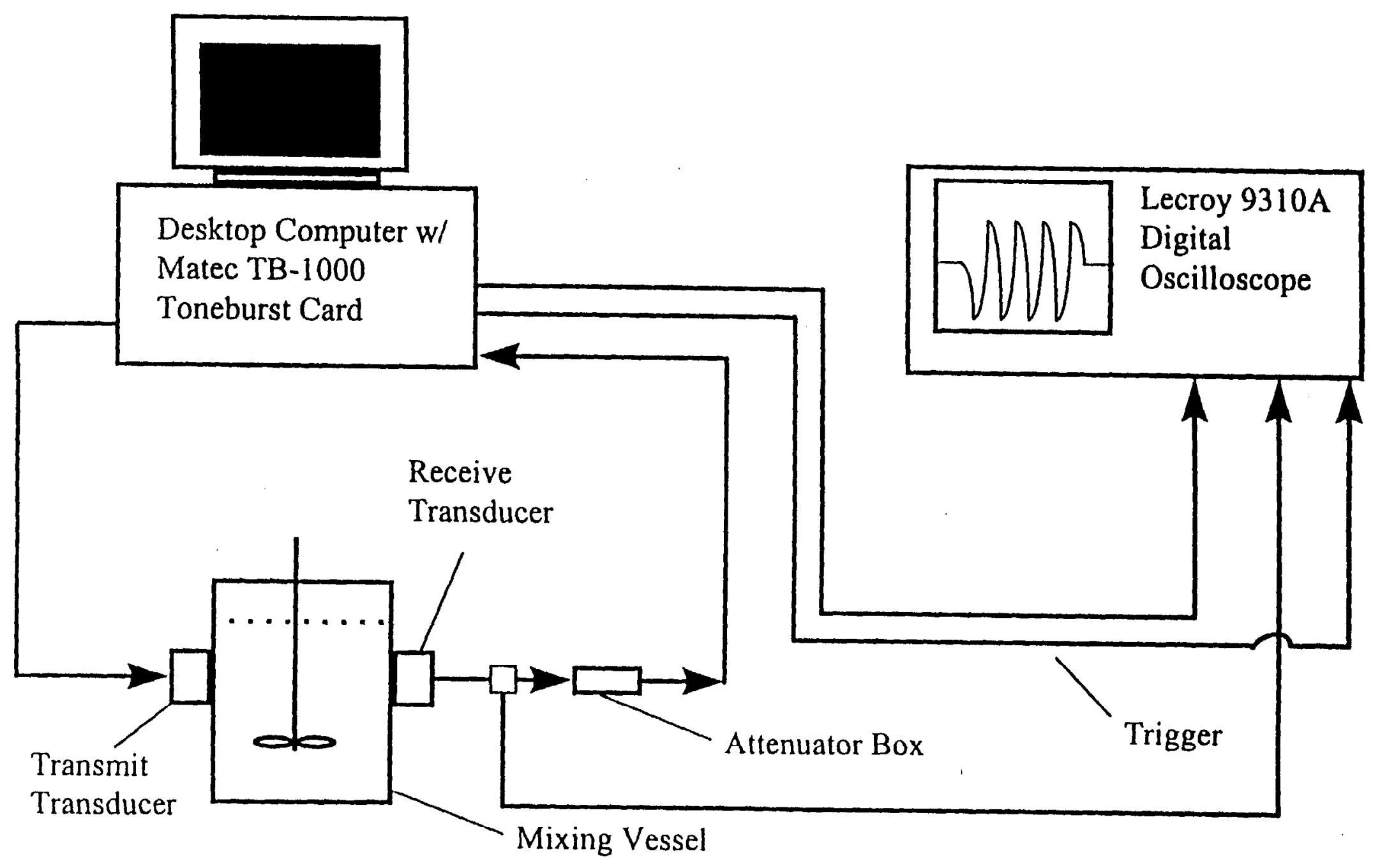

Eigure 3.2: Schematic diagram of the experimental set-up used in the Toneburst Technique. 


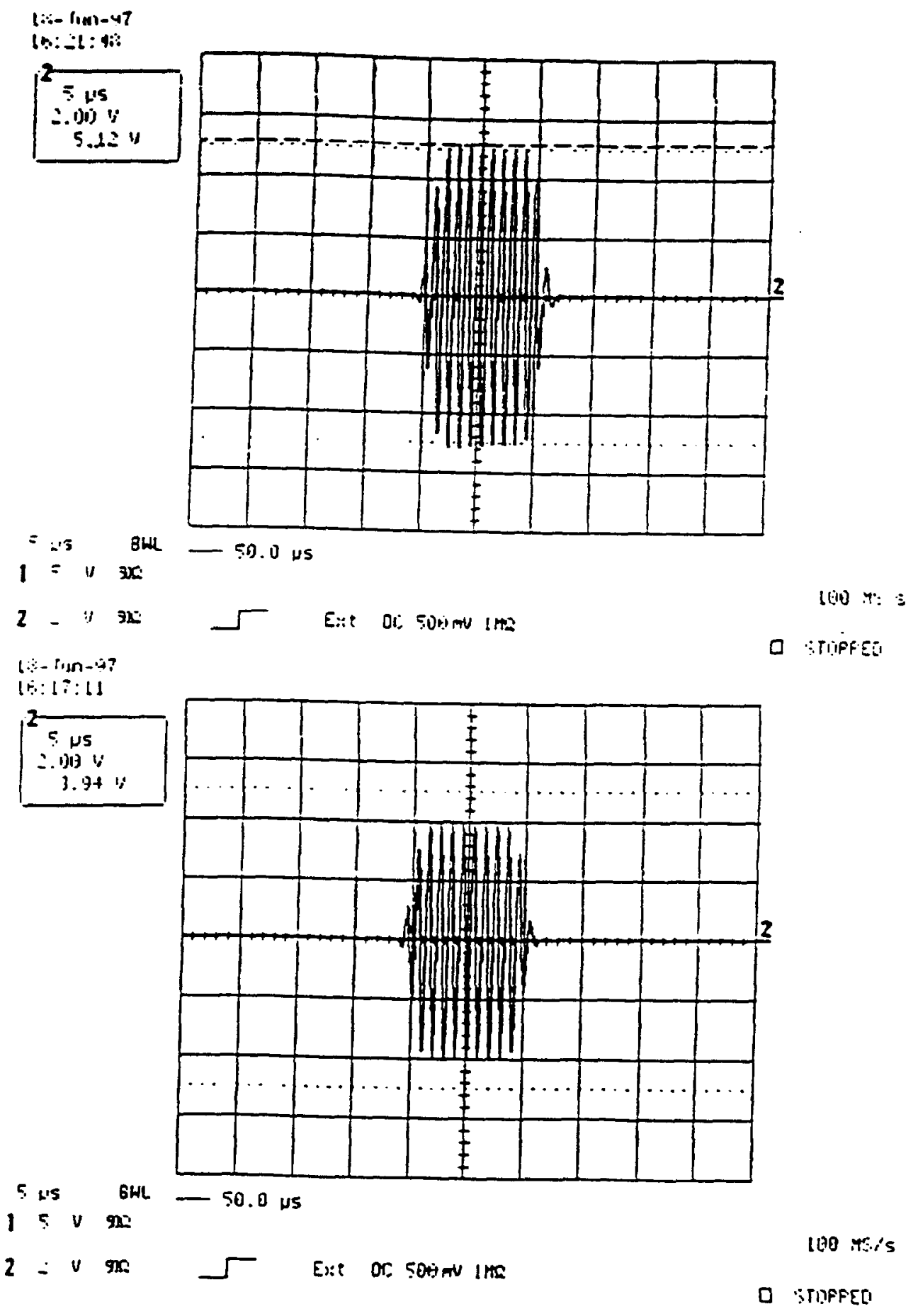

Figure 3.3: Oscilloscope traces for a $1.0 \mathrm{MHz}$ toneburst transmitted through distilled water and through a $5 \%$ by volume soda-lime glass slurry. 
The technical specifications associated with each transducer used in this study, including transducer bandwidth, are given in Table 3.1. A discussion of the determination of transducer bandwidth is given in Appendix E. The received signal can be either sent through the built in amplifier (capable of amplification from 0 to $70 \mathrm{~dB}$ ) on the toneburst card (via a Pasternak Enterprises No. PE7008-1 50 Ohm attenuator box, if necessary) to a LeCroy Model 9310A digital oscilloscope, or directly to the oscilloscope where the amplitude of the received toneburst is measured. The amplitude data are then entered into computer data files which are then loaded into a MATLAB ${ }^{\mathrm{TM}}$ code which, after correcting for attenuator and amplifier settings, calculates the attenuation, $\alpha$ (in $\mathrm{Np} / \mathrm{cm}$ ), according to the formula:

$$
\alpha=-\frac{1}{d} \log \left(\frac{V_{\mathrm{mix}}}{V_{\mathrm{H}_{2} \mathrm{O}}}\right),
$$

where $d$ is the distance (in $\mathrm{cm}$ ) between the transducers, and $V_{\text {mix }}$ and $V_{\mathrm{H} 2 \mathrm{O}}$ are the voltage amplitudes of the signals received in the mixture and pure water, respectively. It should be noted that the term "log" in equation (3.1) refers to the natural logarithm. Various MATLAB $^{\text {TM }}$ codes written to manipulate the experimental data are shown in Appendix C.

The second experimental setup is similar to the first, but in this case the Matec TB-1000 card and computer are replaced by a Panametrics Model 5052 PR Pulser/Receiver (cf. Figure 3.4). The pulser/receiver generates a negative spike pulse signal (instead of a toneburst) of 270 Volts which is sent to the transmitter transducer, propagates through the medium under investigation, and is received by the receiver transducer. The signal received then goes through the receiver which has an amplifier 


\begin{tabular}{|l|l|l|l|l|l|}
\hline Model \# & Serial \# & $\begin{array}{l}\text { Element } \\
\text { Diameter } \\
(\mathrm{cm})\end{array}$ & $\begin{array}{l}\text { Center } \\
\text { Frequency } \\
(\mathrm{MHz})\end{array}$ & $\begin{array}{c}\text { Bandwidth } \\
(-6 \mathrm{~dB}) \\
(\%)\end{array}$ & $\begin{array}{l}\text { Operating } \\
\text { Range } \\
(\mathrm{MHz})\end{array}$ \\
\hline V391 & 226750 & 2.86 & 0.457 & 68.85 & 0.3 to 0.7 \\
\hline V391 & 228834 & 2.86 & 0.475 & 69.47 & 0.3 to 0.7 \\
\hline V302 & 211353 & 2.54 & 0.935 & 65.24 & 0.6 to 1.4 \\
\hline V302 & 211354 & 2.54 & 0.950 & 65.26 & 0.6 to 1.4 \\
\hline V304 & 217679 & 2.54 & 2.11 & 63.51 & 1.4 to 3.0 \\
\hline V304 & 217680 & 2.54 & 2.08 & 63.46 & 1.4 to 3.0 \\
\hline V308 & 219586 & 1.90 & 5.03 & 56.72 & 3.0 to 7.0 \\
\hline V308 & 219594 & 1.90 & 4.88 & 56.41 & 3.0 to 7.0 \\
\hline V320 & 222752 & 1.27 & 7.95 & 64.15 & 5.0 to 11.0 \\
\hline V320 & 222753 & 1.27 & 8.05 & 55.90 & 5.0 to 11.0 \\
\hline V311 & 221617 & 1.27 & 9.90 & 64.65 & 6.0 to 12.0 \\
\hline V311 & 221619 & 1.27 & 10.1 & 56.72 & 6.0 to 12.0 \\
\hline
\end{tabular}

Table 3.1: Specifications for the Panametrics, Inc. (Waltham, MA) Videoscan immersion transducers employed in this study. 


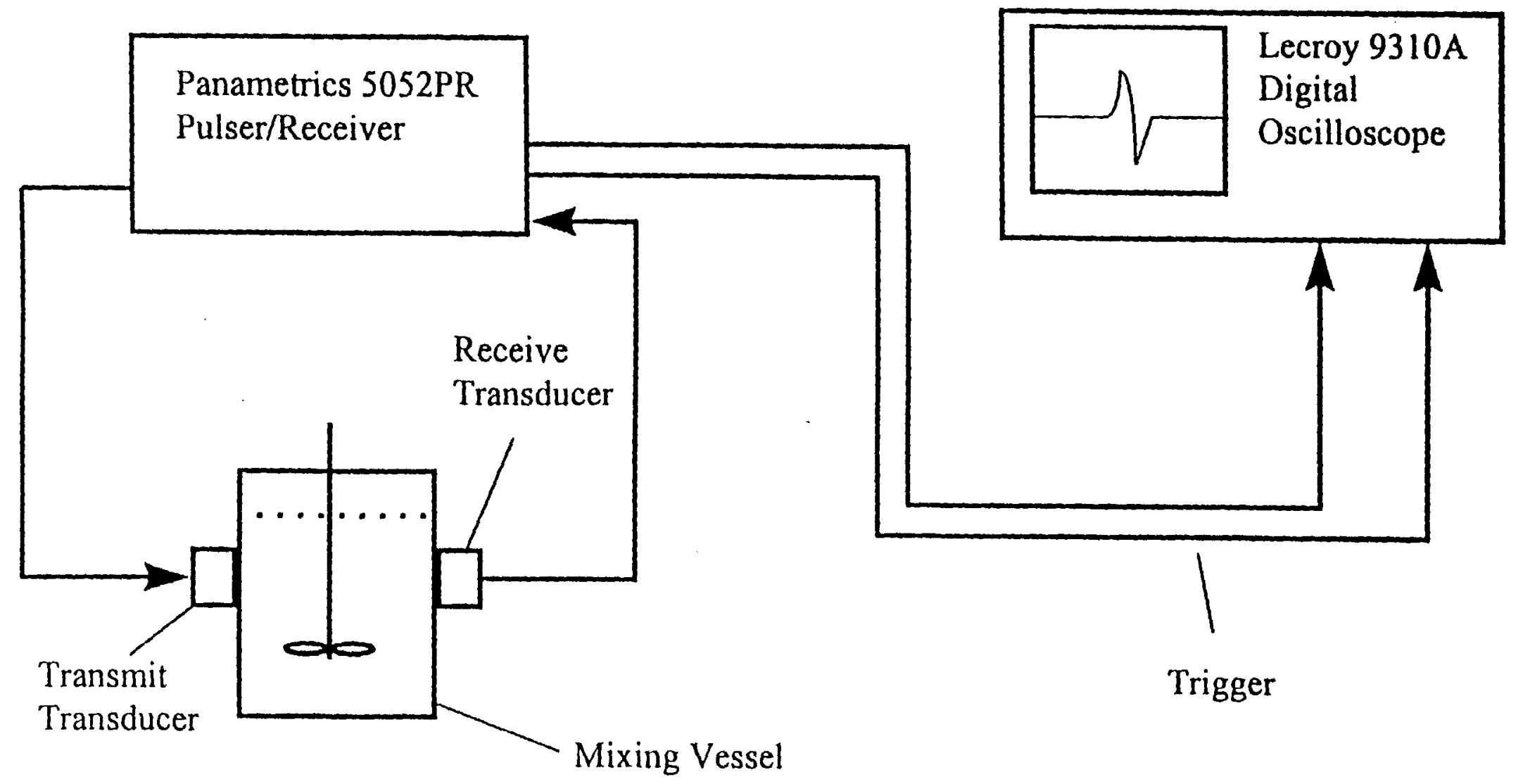

Eigure 3.4: Schematic diagram of the experimental set-up used in the Pulse/FFT Technique. 
capable of either 20 or $40 \mathrm{~dB}$ of gain and an attenuator capable of 0 to $58 \mathrm{~dB}$ of attenuation. The received pulse is then sent to the oscilloscope where the signal is averaged, and a Fast Fourier Transform (FFT) is performed on the averaged waveform. The average is usually calculated over 50 to 100 sweeps of the time domain duration of the signal of interest. The number of sweeps used in the averaging is dependent upon how quickly conditions in the sample are changing. If the particles or bubbles are large and tend to separate from the mixture quickly, fewer sweeps are used in the averaging of the waveform received. Through trial and error it has been determined that 50 sweeps allows for smoothing (averaging) of the received signal before the phases of a given slurry can separate. An example of a received pulse and its FFT magnitude spectrum (used by Panametrics a calibration for a $1.0 \mathrm{MHz}$ transducer) is shown in Figure 3.5. The magnitude spectrum generated by the FFT is then saved on a floppy disk as an ASCII data file and loaded into a MATLAB ${ }^{\mathrm{TM}}$ code where the attenuation is calculated in a manner identical to that described previously in this section.

The advantage to the Pulse/FFT technique over that of the Toneburst technique is that the entire operating frequency range of a given pair of transducers can be covered in a single measurement rather than requiring measurements at incremental frequencies over the transducer operating range, as is the case of the toneburst measurements. A pulse is comprised of the sum of many sinusoidal waves of various frequencies (Ramirez 1985), and thus, the FFT of a received pulse of a given amplitude will exhibit magnitude values over the entire operational frequency range of a given pair of transducers. The Pulse/FFT 


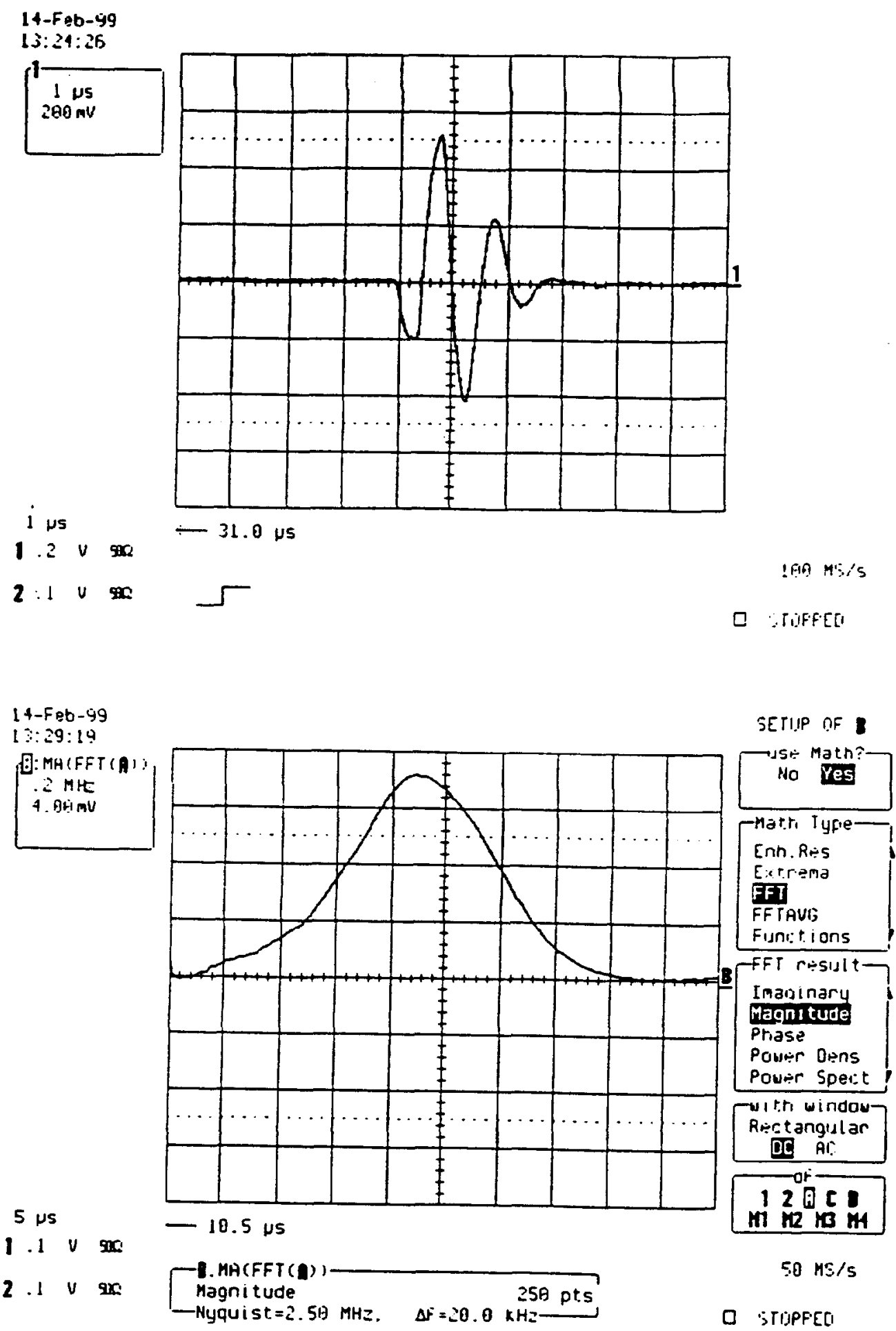

Figure 3.5: Oscilloscope trace and FFT Spectrum of received signal through water for a $1.0 \mathrm{MHz}$ transducer with negative spike pulse input signal. 
technique greatly increases the speed and efficiency of attenuation data acquisition. A comparison between results of the two data acquisition techniques is made in Section 3.5

\subsubsection{Photomicrogrphic Video Imaging System:}

In the present study bubble sizes in solid-gas-liquid slurries are estimated through the use of a digital photomicrographic video imaging system. This imaging system consists of a Company 7 "Questar" QM-100 long distance microscope (identical to that used by Burns et al. (1996) and Shin et al. (1997)) providing magnification of approximately 152 times at a working distance of $17.8 \mathrm{~cm}$ (7'). The microscope is coupled to a Cohu Model 4810 2/3" format monochrome CCD video camera. The CCD video camera is synchronized to a strobe light via a Global Specialty Corporation 4001 Pulse Generator and interfaced in parallel with an Apple Power Macintosh G3 desktop computer equipped with a Scion VG-5 PCI frame-grabber board and an Apple ColorSync 20 "high resolution" monitor. The strobe light provides backlighting so that the bubbles appear as dark images on a light background, and the strobe is synchronized with the camera so that the moving bubbles appear as though "frozen" in space. The imaging system setup is shown schematically in Figure 3.6.

Images obtained by the $\mathrm{CCD}$ video camera are sent to the computer where image analysis is performed. Initially, the image analysis was performed using the Scion Image software package provided with the frame-grabber board. However, that software package has been replaced by a more advanced, privately distributed, software package called Image SXM, version 1.61-7 (Barrett 1998). 


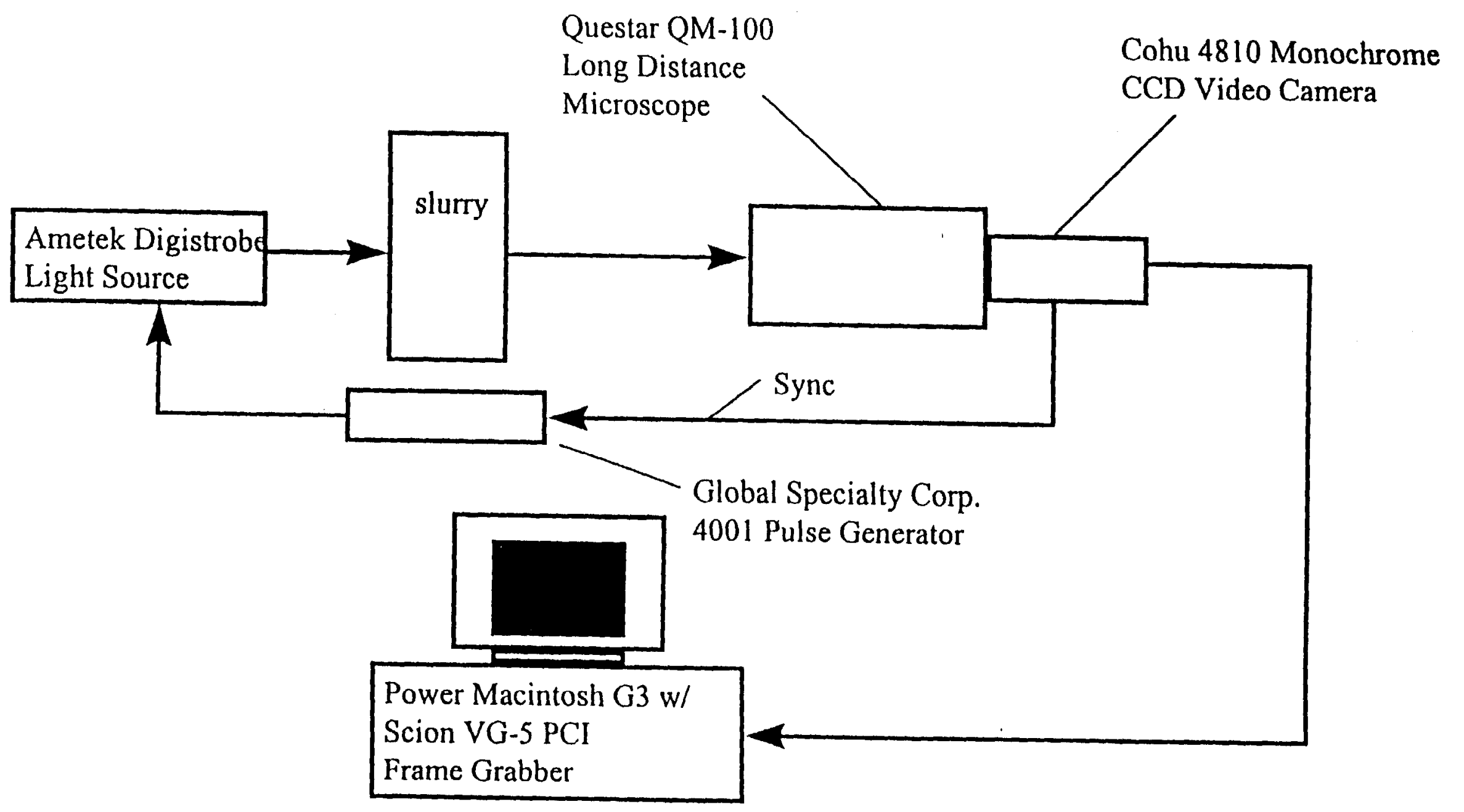

Eigure 3.6: Schematic diagram of the experimental set-up for the photomicrographic video imaging system. 
The most difficult consideration involved with the imaging system is that of the proper lighting to be used. As was discussed by Bongiovanni et al. (1997), the error in photographing spherical particles can be considerable depending upon the relative positions of the backlighting, the object, and the objective lens. However, bright field backlighting is the most desirable lighting technique (as opposed to, say, dark field lighting) in the consideration of possible three phase particle and bubble size measurements. In dark field illumination, it is difficult to distinguish between solid particles and bubbles. Also, an illumination configuration such as that employed by Kirou et al. (1988) is not feasible as the test cell size is too small to accommodate the fiber optic apparatus; and, according to Bongiovanni et al. (1997), the relative size and close proximity of the light source to the focal distance of the particles or bubbles will introduce large errors in the size measurements. Therefore, since the experimental measurements and the optical apparati involved are similar, bubble measurements are made by the method employed by Burns et al. (1997) and Shin et al. (1997), and a calibration is performed using particles of known sizes to determine how much, if any, error is present in the size measurements by this method. Back lighting is provided by a strobotach placed about $61 \mathrm{~cm} \mathrm{(24")} \mathrm{behind} \mathrm{the} \mathrm{test} \mathrm{cell.} \mathrm{The} \mathrm{microscope} \mathrm{is} \mathrm{placed} \mathrm{at} \mathrm{a}$ working distance of approximately $17.8 \mathrm{~cm}\left(7^{\prime \prime}\right)$ from the center of the test cell. As the strobotach has a lighting element that is only 4" in diameter, the error in the size measurements should be relatively small. The $17.8 \mathrm{~cm}$ working distance is chosen because it is a working distance which provides for reasonable magnification of the 
smallest calibration particles while still allowing the largest particles to be photographed at the same working distance.

The calibration is begun by setting the scaling factor for the conversion from pixel count to size measurement. This task is performed by photographing a reticle marked with circles of various sizes. Diameter measurement is performed on the smallest circle on the reticle, which is $0.005 "(127 \pm 2 \mu \mathrm{m})$ in diameter. This information is then used to make the pixel to length conversion. This calibration is performed before each series of measurements or whenever the focus of the microscope is adjusted. Further calibration is performed to determine the overall measurement error by photographing NIST traceable polystyrene spheres (Duke Scientific Corp., Palo Alto, CA) of certified diameter at lighting conditions identical to those to be used in actual bubble measurements. Four different sphere diameters are used in the calibration. The sizes (diameters) are, respectively, $25.02 \pm 0.12 \mu \mathrm{m}, 50.4 \pm 1.0 \mu \mathrm{m}, 103 \pm 1.8 \mu \mathrm{m}$, and $158 \pm 3.2 \mu \mathrm{m}$. A series of photographs is obtained for each particle diameter and an average of 50 diameter measurements are made from these photographs at each particle size. The diameter measurements for each particle size are averaged, and the average particle diameter is plotted versus the known (certified) particle diameter. A best fit least squares line is then fitted to these data using the "polyfit" routine in MATLAB" ${ }^{\text {TM }}$. The least squares fit yields the following relationship between the measured and true particle diameters:

$$
d_{\text {meas }}=1.25 d_{\text {true }}-10.4
$$

where $d_{\text {meas }}$ and $d_{\text {true }}$ are the measured and true (known) particle diameters, respectively. The calibration curve is shown in Figure B.3 in Appendix B. 


\subsection{Experimental Procedure-Solid-Liquid Slurries:}

\subsubsection{Dilute Soda-Lime Glass Slurries:}

The initial solid-liquid attenuation experiments are performed in slurries of sodalime glass beads in distilled water at various solids volume fraction. The soda-lime glass beads have been purchased from Cataphote, Inc. and have a density of $2.54 \mathrm{~g} / \mathrm{cm}^{3}$. The physical properties of these particles are shown in Table 3.2. Particle size distribution measurements have been performed on these beads at the Battelle Pacific Northwest National Laboratory (PNNL), and it has been determined that the batch of beads used in these experiments has a particle mean diameter of $32 \mu \mathrm{m}$ and standard deviation of 7.6 $\mu \mathrm{m}$ (Greenwood, private communication 1998). Solids volume fractions of the various slurries is confirmed by making mass measurements of known volumes of suspended slurry upon completion of experimental runs. The solids volume fraction is determined from the following equation:

$$
\phi_{s}=\frac{\rho_{\text {slurry }}-\rho_{\mathrm{H}_{2} \mathrm{O}}}{\rho_{\text {solid }}-\rho_{\mathrm{H}_{2} \mathrm{O}}},
$$

where $\phi_{s}$ is the solids volume fraction in the slurry, and $\rho_{\text {slurry, }} \rho_{\text {solid }}$, and $\rho_{H 2 O}$ are, respectively, the slurry, solid, and water densities.

Two pairs of transducers are put in place for each experimental series performed in the $1.6 \mathrm{~L} \mathrm{PVC} \mathrm{mixing} \mathrm{vessel.} \mathrm{Before} \mathrm{performing} \mathrm{each} \mathrm{experiment,} \mathrm{the} \mathrm{separation}$ distance between each pair of transducers is measured and recorded, and the vessel is then filled with distilled water. The voltage amplitude of monochromatic tonebursts over incremental frequencies is measured over the operational range of each pair of tranducers 


\begin{tabular}{|c|c|c|c|c|}
\hline & Polystyrene & $\begin{array}{c}\text { Soda-lime } \\
\text { Glass }\end{array}$ & Water & $\begin{array}{c}\text { Glycerin/ } \\
\text { Water } \\
\text { Mixture }\end{array}$ \\
\hline Density $\left(\mathrm{g} / \mathrm{cm}^{3}\right)$ & 1.055 & 2.54 & 1.0 & 1.08 \\
\hline Thermal Conductivity & $1.15 \times 10^{-3}$ & $9.6 \times 10^{-3}$ & $5.87 \times 10^{-3}$ & $4.5 \times 10^{-3}$ \\
\hline Specific Heat $(\mathrm{J} / \mathrm{g} \cdot \mathrm{K})$ & 1.19 & 0.836 & 4.19 & 4.19 \\
\hline $\begin{array}{c}\text { Thermal Expansion } \\
\text { Coefficient }(1 / \mathrm{K})\end{array}$ & $2.04 \times 10^{-4}$ & $3.2 \times 10^{-6}$ & $2.04 \times 10^{-4}$ & $3.22 \times 10^{-4}$ \\
\hline Attenuation Coefficient & $1.0 \times 10^{-15}$ & $1.0 \times 10^{-15}$ & $2.5 \times 10^{-16}$ & - \\
per $f^{2}\left(\mathrm{~s}^{2} / \mathrm{cm}\right)$ & & & & \\
\hline Sound Speed $(\mathrm{cm} / \mathrm{s})$ & $2.3 \times 10^{5}$ & $5.2 \times 10^{5}$ & $1.48 \times 10^{5}$ & $1.6 \times 10^{5}$ \\
\hline Shear Viscosity $\left(\mathrm{g} / \mathrm{cm} \cdot \mathrm{s}^{2}\right)$ & - & - & $1.01 \times 10^{-2}$ & $3.2 \times 10^{-2}$ \\
\hline Shear Rigidity $\left(\mathrm{g} / \mathrm{cm} \cdot \mathrm{s}^{2}\right)$ & $1.27 \times 10^{10}$ & $2.8 \times 10^{11}$ & - & - \\
\hline
\end{tabular}

Table 3.2: Values of physical properties used in theoretical calculations. The values for polystyrene are from Epstein and Carhart (1953). The properties of soda-lime glass are from Kinsler et al. (1982) and Bolz (1973). The properties of glycerin are from Perry's Chemical Engineer's Handbook (1984) and the CRC Handbook of Chemistry and Physics (1984). 
in the toneburst technique, or an FFT is performed on the received waveform of a negative spike pulse input signal for each pair of transducers in the Pulse/FFT technique. In each case it is important to record the pertinent operating parameters of each particular pulse generating apparatus. In the case of the toneburst technique, where the Matec TB1000 digital synthesizer is used, the recorded parameters consist of the frequency, the amplifier gain, and attenuator box settings (if necessary). In the case of the Pulse/FFT technique the recorded parameters are the pulse repetition rate, the pulse energy, the receiver attenuation, the high pass filter setting, the damping, and the receiver gain. The digital oscilloscope is set to parameters which are appropriate for the given experimental run, and these parameters are either recorded manually or shown in printed "hardcopies" of oscilloscope waveform traces.

After voltages are measured by either technique for the distilled water, the water is drained from the vessel and replaced with the slurry to be interrogated. The slurry is suspended via agitation by a Lightnin' Labmaster II ${ }^{\mathrm{M}}$ Model TS 2510 portable mixer fitted with a $5.08 \mathrm{~cm}$ diameter marine propeller type impeller. It is important to make certain that the impeller and shaft are positioned off center in the mixing vessel so that they are not in the travel path of the acoustic signal. The agitation speed is varied as the agitation speed employed in each case is the highest speed achievable such that the solids are suspended, but there is no vortex "tube" which extends into the acoustic travel path. These conditions are usually satisfied by agitation speeds ranging from 300 to $600 \mathrm{rpm}$. Voltage measurements are then made for the slurries in the same fashion as those made for the distilled water. 
The voltage data for both the distilled water and the slurries are then entered into ASCII data files which are loaded into MATLAB ${ }^{\text {TM }}$ codes to calculate the attenuation by using equation (3.1) after correcting for the receiver gain and receiver attenuation.

\subsubsection{Dilute Polystyrene Slurries:}

Attenuation measurements are also made in dilute suspensions of polystyrene beads in water. The polystyrene beads are manufactured by Duke Scientific Corp., Palo Alto, CA and are composed of polystyrene with $2 \%$ divinylbenzene (considered pure polystyrene in this study). The physical properties of these particles are also given in Table 3.2. They are purchased as nominally "monodispersed" $158 \mu \mathrm{m}$ diameter polystyrene spheres, but the calibration information provided by the manufacturer shows that spheres actually have a rather narrow size distribution with mean diameter of $158 \pm 5$ $\mu \mathrm{m}$ and standard deviation of $3.6 \mu \mathrm{m}$. Due to the very narrow size distribution, the spheres are somewhat expensive, and only a small quantity has been purchased which come from the manufacturer in a $10 \%$ (by wt.) suspension in water. Since the particles have a density of $1.05 \mathrm{~g} / \mathrm{cm}^{3}$, weight percent is approximately equal to volume percent. The purchased quantity is diluted to a $5 \%$ (by volume) suspension, and there is sufficient volume of slurry to perform attenuation measurements in the $5.08 \mathrm{~cm}$ test cell.

Attenuation measurements are performed in this slurry using both the toneburst and Pulse/FFT techniques described previously in Section 3.1.2. 


\subsubsection{Concentrated Soda-Lime Glass Slurries:}

Attenuation measurements are also performed in relatively concentrated slurries of soda-lime glass beads. These beads are soda-lime glass beads manufactured by Potter's Industries, Inc. (Brownwood, TX) with a specified density of $2.54 \mathrm{~g} / \mathrm{cm}^{3}$. The physical properties of these particles are shown in Table 3.2. These particles have also been subjected to particle size measurements at the Battelle Pacific Northwest National Laboratory, and it has been determined that the particles have a mean diameter of $125 \mu \mathrm{m}$ with standard deviation of $19 \mu \mathrm{m}$.

Bccause these particles are rather large, even concentrated slurries are difficult to suspend in a pure water continuous phase. Therefore, the suspending liquid which is employed is a mixture of approximately $29 \%$ (by wt.) of glycerin (approximately $99 \%$ reagent grade, Sigma Chemical Company) in distilled water. The addition of the glycerin increases the liquid phase density to approximately $1.08 \mathrm{~g} / \mathrm{cm}^{3}$, and the liquid phase viscosity by approximately a factor of three. The liquid phase viscosities of these solutions range from $0.0259 \mathrm{~g} / \mathrm{cm} \cdot \mathrm{s}$ to $0.0308 \mathrm{~g} / \mathrm{cm} \cdot \mathrm{s}$. Thus, the solid particles settle much more slowly, and attenuation measurements can be made. It should be noted that even with the addition of glycerin, the particles still settle sufficiently quickly that the suspension of large volumes of slurry is difficult. Therefore, only small volumes of the concentrated slurries are prepared, and all measurements are performed in the various "box" geometry test cells.

Slurries are prepared at $5 \%, 10 \%, 15 \%, 20 \%, 30 \%, 40 \%, 45 \%$, and $50 \%$ solids by volume. These slurries are then subjected to attenuation measurements in the 
various test cells by both the toneburst and Pulse/FFT techniques described previously. It should also be noted here that the "pure liquid" reference voltages in these experiments are not made in pure distilled water, but rather in the individual glycerin/water mixtures in which the solids of each slurry are suspended. Each time a slurry is prepared, a portion of the suspending liquid mixture is put aside and saved to be used as the "reference liquid" for the attenuation measurements in that slurry. The average physical properties for the glycerin/water mixtures are given in Table 3.2 .

\subsection{Experimental Procedure-Bubble Generation:}

Several different methods have been employed to generate bubbles for attenuation measurements in bubbly liquids and solid-gas-liquid systems. Each method has its particular advantages and disadvantages with respect to reproducible bubble generation rate, bubble size distribution, compatibility with the ultrasound measurement equipment and slurry solids, etc.

The first bubble generation method which has been employed is an electrolysis method based on that used by Kol'tsova et al. (1979). Electrolysis has the advantage of producing relatively small bubbles $(<100 \mu \mathrm{m}$ diameter), but it can be difficult to control when operated in a continuous mode as the electrolysis causes the electrical properties of the water to change with time. These changes cause variations with time in the current provided to the electrolyzer which makes reproducible bubble production difficult. Also, continuous operation of the electrolyzer over an extended period of time causes significant increases in the temperature of the medium under interrogation. As the 
density of most media changes with temperature, and the attenuation of acoustic energy in materials is strongly dependent upon the material density, these temperature changes are most undesirable. The possibility of using the electrolytic bubble generation method in a non-continuous fashion has also been explored and will be discussed in more detail later in this section. Another significant disadvantage to using the electrolytic generation technique is that the ultrasonic transducers are grounded. Because of this grounding, the safety controls in the DC power supply (Sigma-Aldrich Model PS 4010-1) cause it to shut off due to a "ground leak error" fault whenever the transducers are in direct contact with the sample under investigation.

The second bubble generation technique investigated is an electrostatic spraying technique based on that used by Tsouris et al. (1995). This technique produces bubbles which are approximately the same size as those produced by electrolysis, however, this technique has also proven to be difficult to employ reproducibly. Electrostatic spraying of air into water requires the use of de-ionized water. As the process is operated, ions begin to accumulate in the water, and the technique becomes less effective with time. This situation is further exacerbated by the addition of solids, such as soda-lime glass beads, which have a fairly high content of ions which will migrate from the solids into the liquid phase. The aforementioned factors, coupled with the solids also tending to clog the capillary spray nozzle make the electrostatic spraying technique difficult to control, and therefore, undesirable as a bubble generation technique.

The third bubble generation technique which has been explored is the use of air pumped through an aquarium aerator stone. The particular aerator used is manufactured 
by Coralife (Energy Savers Unlimited, Inc., Harbor City, CA) and is made from limewood. Limewood has the unique property that it contains a number of small straight pores which allow for the production of relatively small bubbles. The aerator is operated by interfacing it with a Cole-Parmer Model 74900 syringe pump and pumping air at various metered flow rates. This method of bubble generation is quite successful in that bubbles are generated fairly consistently over long periods of time even with solids present. This technique is the one employed in the initial "proof of principle" solid-gasliquid attenuation measurement experiments. A notable characteristic of this method is that the bubbles produced are of fairly large diameter and wide size distribution relative to those produced by the other methods, such as electrolysis. The bubbles produced from the limewood aerator, when operated in water at an air flow rate of $150 \mathrm{ml} / \mathrm{hr}$, are photographed and measured using the photomicrographic imaging system described in Section 3.1.3, and the bubble population is determined to have a mean diameter of 141 $\mu \mathrm{m}$ with standard deviation of $67 \mu \mathrm{m}$. The bubble volume fraction is estimated to be approximately 0.02 . These bubble size characteristics are somewhat undesirable as bubbles of these sizes have rather low resonance frequencies; it is, therefore, difficult to examine the effects of bubble resonance with bubbles of these sizes.

As was previously mentioned, the possibility of producing bubbles by electrolysis in a non-continuous manner has been explored. It has been determined, by trial and error, that if bubbles are generated electrolytically only for a time period which is long enough to establish bubble flow, mix the vessel contents, and make an acoustic measurement, fairly reproducible electrolyzer performance can be maintained. The other drawback to 
the electrolytic technique is the recurrence of "ground leak error" faults when the transducers are in place which would cause the DC power supply to shut off. This problem is rectified by covering the wear plates of the transducers with Parafilm ${ }^{\top M}$, a paraffin laboratory covering wrap, and coupling the Parafilm ${ }^{T M}$ to the transducers with ultrasound gel. Also, the transducers have to be disconnected from the pulse generator until bubble flow is established. Incorporation of these improvements allows for the use of electrolysis as a method of bubble generation in the attenuation experiments. The electrolytic set up in the test cells is rather simple. The cathode (negatively charged electrode) consists of an approximately $30 \mathrm{~cm}$ length of $1.3 \mathrm{~mm}$ diameter copper electrical wire with the insulation stripped away from approximately $1 / 4$ " of the wire end and the individual wire strands separated, run vertically through an $1 / 8$ " NPT to Swagelok fitting in the bottom of the cell so that it sticks upright perpendicular to the flat bottom of the cell. Care is taken to avoid the cathode wire protruding into the acoustic path between transducers. The anode (positively charged electrode) is an approximately $4 \mathrm{~cm}$ length of $1 / 8$ " diameter stainless steel rod positioned against the test cell wall with approximately one centimeter of its length immersed below the liquid surface. When the DC power supply is turned on, bubbles are generated at the cathode and rise upward toward the anode. Bubbles are generated in all experiments at $40 \mathrm{~V} ; 4$ to $8 \mathrm{~mA}$; and $<1$ W. The bubbles generated in water at these conditions are photographed and measured using the photomicrographic imaging system described in Section 3.1.3. These bubbles are found to have a mean diameter of $51 \mu \mathrm{m}$ with standard deviation of $26 \mu \mathrm{m}$. The bubble volume fraction is estimated to be approximately 0.002 . 


\subsection{Experimental Procedure-Solid-Gas-Liquid Slurries:}

The attenuation measurement experiments in solid-gas-liquid slurries are very similar in nature and procedure to those performed in solid-liquid slurries. In fact, the experimental procedure for the solid-gas-liquid systems actually includes a step where the attenuation is measured in a solid-liquid slurry without the presence of gas bubbles. Solid-liquid slurries are prepared at $5 \%$ and $10 \%$ solids (by volume) using the same soda-lime glass beads which are described in Section 3.2.1. Attenuation measurements are performed in these slurries using the Pulse/FFT technique described previously in Section 3.1.2. Once attenuation measurements are made in the solid-liquid slurry of interest in the particular experiment, bubbles are generated using either electrolysis to generate relatively small, narrowly distributed bubbles (approximately 25 to $76 \mu \mathrm{m}$ in diameter) or air pumped at $150 \mathrm{ml} / \mathrm{hr}$ through the limewood aerator stone to generate larger, more widely distributed bubbles (approximately 74 to $210 \mu \mathrm{m}$ in diameter). In the case where the bubbles are generated electrolytically, the DC power supply is operated at $40 \mathrm{~V} ; 4$ to $8 \mathrm{~mA}$; and $<1 \mathrm{~W}$. When the LCD display on the power supply shows that it is operating at the specified conditions, the (now solid-gas-liquid) slurry is mixed thoroughly with a glass or plastic stirring rod and a measurement is made. The data are recorded as per the procedure described in Section 3.1.2, and "hardcopies" of the oscilloscope traces and FFT spectra are entered into the laboratory notebooks. All computer files are stored both on the hard drive of the data acquisition computer (Gateway 2000 P5-66) and on 3.5" floppy diskette. Also in these experiments, bubbles are generated by both methods in the water used to make the reference measurements 
after the reference measurements are made. Pulse/FFT measurements are made in these bubbly liquids, and the data are recorded in order to calculate bubbly liquid attenuations for later comparison with solid-gas-liquid attenuations in an attempt to obtain information about any effects the solids may have on the bubble size.

\subsection{Quality of Experimental Results:}

\subsubsection{Comparison of Attenuation Measurement Techniques:}

Upon development of the Pulse/FFT technique for obtaining attenuation data in the various slurries, one of the most important issues to be addressed is how well the attenuation data obtained by this technique compare with those obtained by the Toneburst measurement technique. A comparison of attenuation data obtained by both techniques in a $5 \%$ (by vol.) slurry of soda-lime glass beads in water is shown in Figure 3.7. From this figure is clear that the two methods of obtaining data are equivalent under most conditions. It is found, however, that in dilute slurries the Pulse/FFT technique yields better results at lower frequencies than the toneburst technique. The results are better for the Pulse/FFT technique because the toneburst measurements are made by manually moving the horizontal oscilloscope cursors to correspond with the wave peaks and troughs in the received toneburst signal. At lower frequencies, the attenuation is small, and the error in the measurements becomes large relative to the magnitude of the measurements, themselves (on the order of $20 \%$ relative error). In the case of the Pulse/FFT technique, the amplitude as a function of frequency is calculated from the received pulse waveform by the oscilloscope as part of the FFT algorithm. Since 


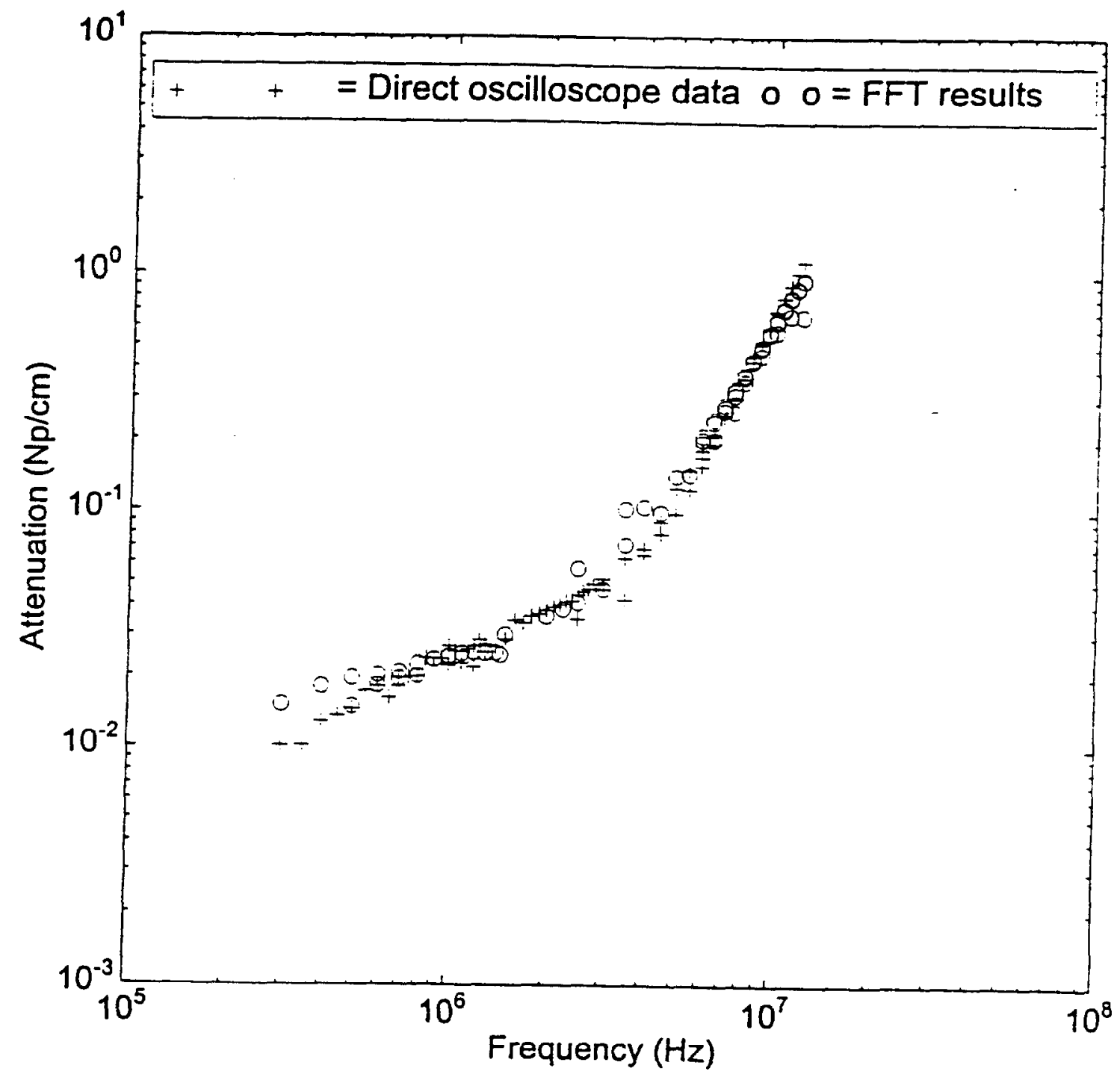

Figure 3.7: Comparison of attenuation spectra results obtained in a $5 \%$ soda-lime glass slurry by both the Toneburst and the Pulse/FFT Techniques. - E1301. 
Pulse/FFT method is an automated measurement technique, the measurement error at lower frequencies is smaller than that observed in the manually performed toneburst measurements.

\subsubsection{Reproducibility of Experimental Results:}

In order to examine the reproducibility of experimental results, complete repeat experiments have been performed at various times throughout the course of this study. These duplicate experiments are performed in addition to collecting data by more than one technique (as discussed in the previous section) in order to establish an overall sense of the reproducibility of the experimental results. As the acoustic attenuation behavior of these various slurries is quite complicated, and the data for these specific systems (e.g. particle type and size, and gas phase concentration) are unique, it is difficult to perform a rigorous error analysis to determine the absolute accuracy of the results. It is, however, possible to investigate the quality of the data with regard to the reproducibility of results and their agreement with theoretical predictions.

Experiments are repeated (total of three experiments) in the attenuation measurements made in the soda-lime glass slurries at $5 \%$ solids by volume using the Toneburst technique. Also, repeated experiments are performed in the concentrated slurry experiments with the Potter's beads slurries in glycerin/water. Duplicate experiments are performed at $50 \%$ (two duplicates), $30 \%$, and $10 \%$ solids by volume.

Figure 3.8 shows the average (solid line) attenuation versus frequency curve, with error bars, for three experiments using slurries of $5 \%$ (by volume) soda-lime glass beads 
in water. The error in the measurements is taken to be the mean difference between the experimentally determined attenuation value and the mean attenuation value at each frequency. It can be seen from Figure 3.8 that the data, except at the lowest frequencies where the attenuations are rather small, fall within $5 \%$ (relative error) of the average value. Even at the lowest frequencies, the measurement error still permits results which are within $20 \%$ of the mean value of the attenuation. These results demonstrate that the experimental measurements in these slurries are rather reproducible.

Similarly, Figures $3.9,3.10$ and 3.11 show the average attenuation versus frequency curves, with error bars, for $10 \%, 30 \%$, and $50 \%$ (by volume) slurries, respectively, of Potter's Beads in a mixture of glycerin and water. In Figure 3.9, which shows the error in the $10 \%$ Potter's beads slurry measurements, the error in the attenuation measurements is relatively small up to, and including, that at $3.0 \mathrm{MHz}$. At 3.0 $\mathrm{MHz}$ the relative error is $1.7 \%$, while at $3.5 \mathrm{MHz}$, the error jumps to $18 \%$. The error in the attenuation measurements at $3.5 \mathrm{MHz}$ is more than 10 times that at $3.0 \mathrm{MHz}$. The error continues to increase with increasing frequency up to $32 \%$ at $5.0 \mathrm{MHz}$. The error is considerably larger at frequencies above $3.0 \mathrm{MHz}$ because these are the frequencies at which the operating ranges of the various transducers used in the measurements overlap. There often tend to be slight differences in measurements between the individual transducer pairs at overlapping frequencies, even though the results should ideally match. These differences are usually small enough so as not to present a problem from a reproducibility standpoint. However, because of the large particle size and density difference between the phases, the Potter's beads slurries are particularly difficult to 


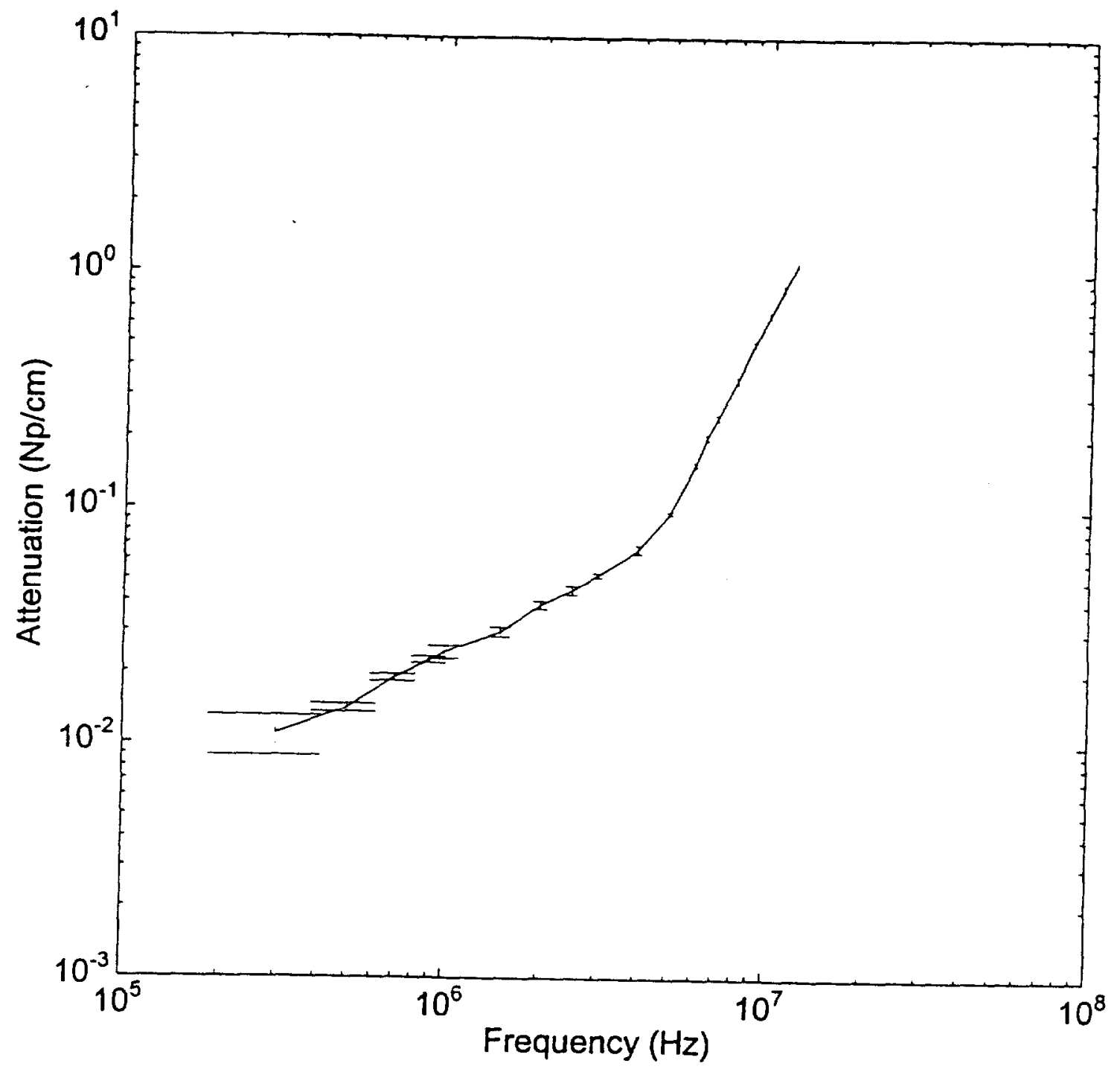

Figure 3.8: Mean attenuation versus frequency curve with error bars representing the average difference between individual attenuation values and the mean value. These data are for a $5 \%$ (by volume) soda-lime glass slurry in water. - E1101. 


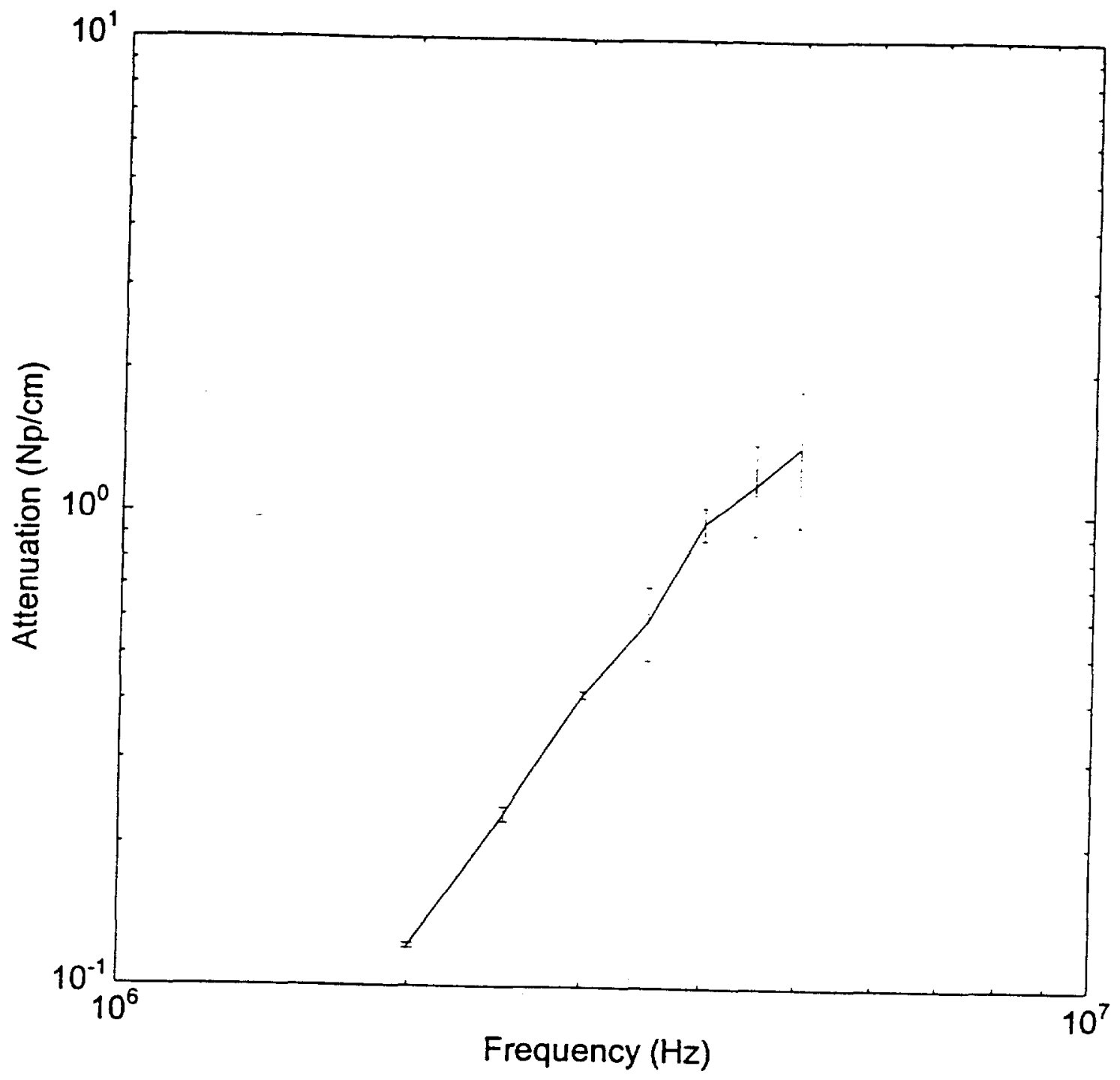

Figure 3.9: Mean attenuation versus frequency curve with error bars representing the average difference between individual attenuation values and the mean value. These data are for a $10 \%$ (by volume) Potter's beads slurry in a mixture of glycerin/water. - E5182. 
suspend uniformly. Therefore, any discrepancies between measurements will be amplified in the data for these slurries because of differences in the uniformity of the suspension.

A similar situation is seen for the $30 \%$ Potter's beads slurry data shown in Figure 3.10. Here the error in the attenuation measurements becomes relatively large at 2.0 $\mathrm{MHz}$, and at 3.0 MHz and 3.5 MHz. These frequencies also overlap in the operating ranges of the transducers used in these experiments. Therefore, as seen previously in Figure 3.9, there is an increased error at these frequencies because of differences in the results obtained by the different transducers.

Figure 3.11 shows the error in the attenuation measurements in a $50 \%$ (by volume) Potter's beads slurry. The error in the measurements in the $50 \%$ slurry appears to be more uniform relative to that in the $10 \%$ and $30 \%$ slurries in that there are no data points where the error band is exceptionally large. It is not clear why the error is more uniform for this slurry. One possible explanation could be that the data for the $50 \%$ Potter's beads slurry are obtained in the test cell with the shortest acoustic path length $(2.54 \mathrm{~cm})$. It is possible that the slurry volume between the transducers in this test cell is small enough that there is more consistency in the slurry mixing conditions and solids concentration which are interrogated by the different transducer pairs.

In general it can be said that the experimental error is larger in the Potter's Beads slurries than in the soda-lime glass slurries. The largest relative error in the soda-lime glass beads slurries data is $19 \%$ at $0.3 \mathrm{MHz}$ where the attenuation is extremely small; while the $30 \%$ Potter's Beads slurry measurements display an error as high as $32 \%$ at 


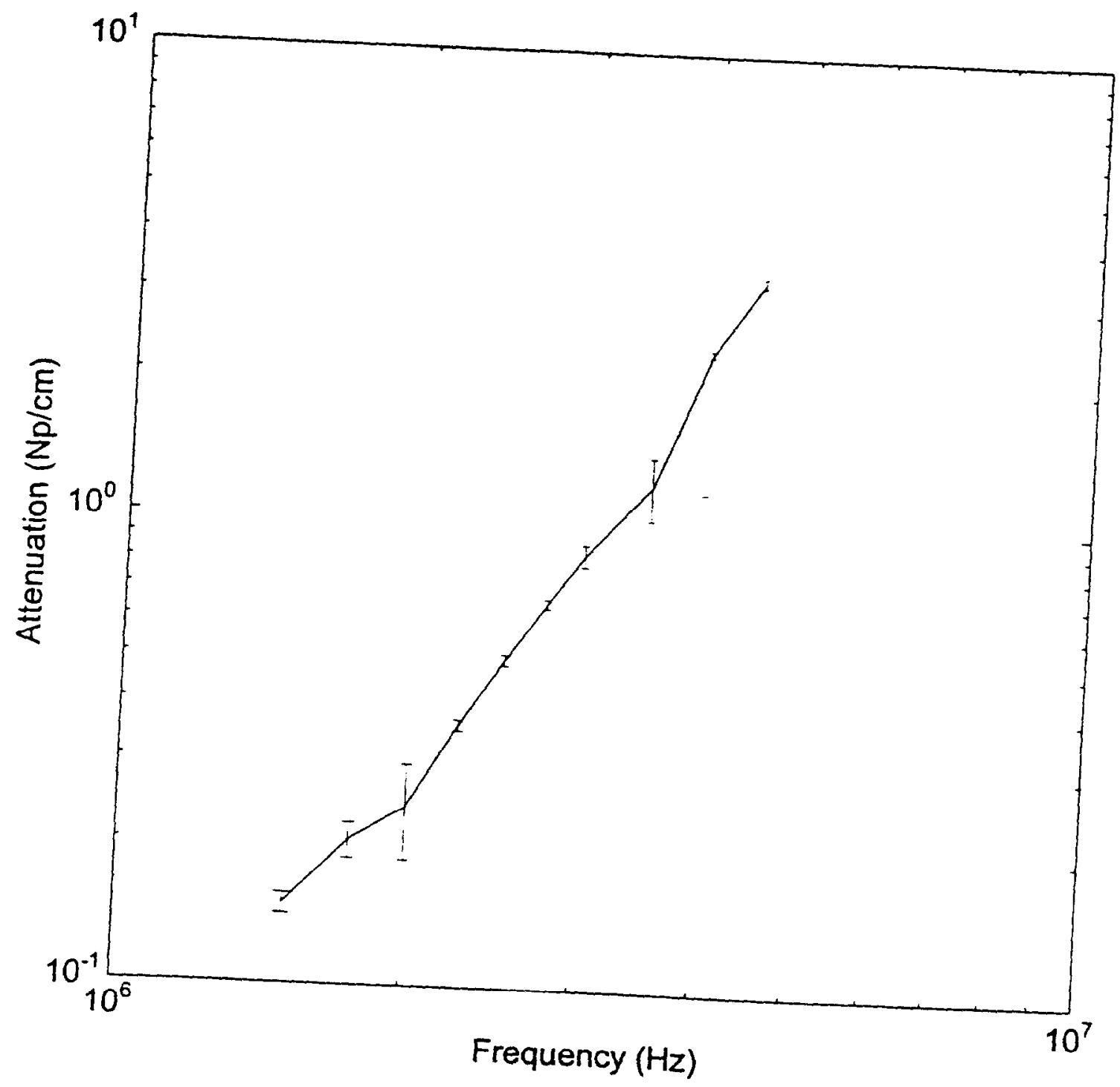

Figure 3.10: Mean attenuation versus frequency curve with error bars representing the value. These data between individual attenuation values and the mean a mixture of glycerin/water. 


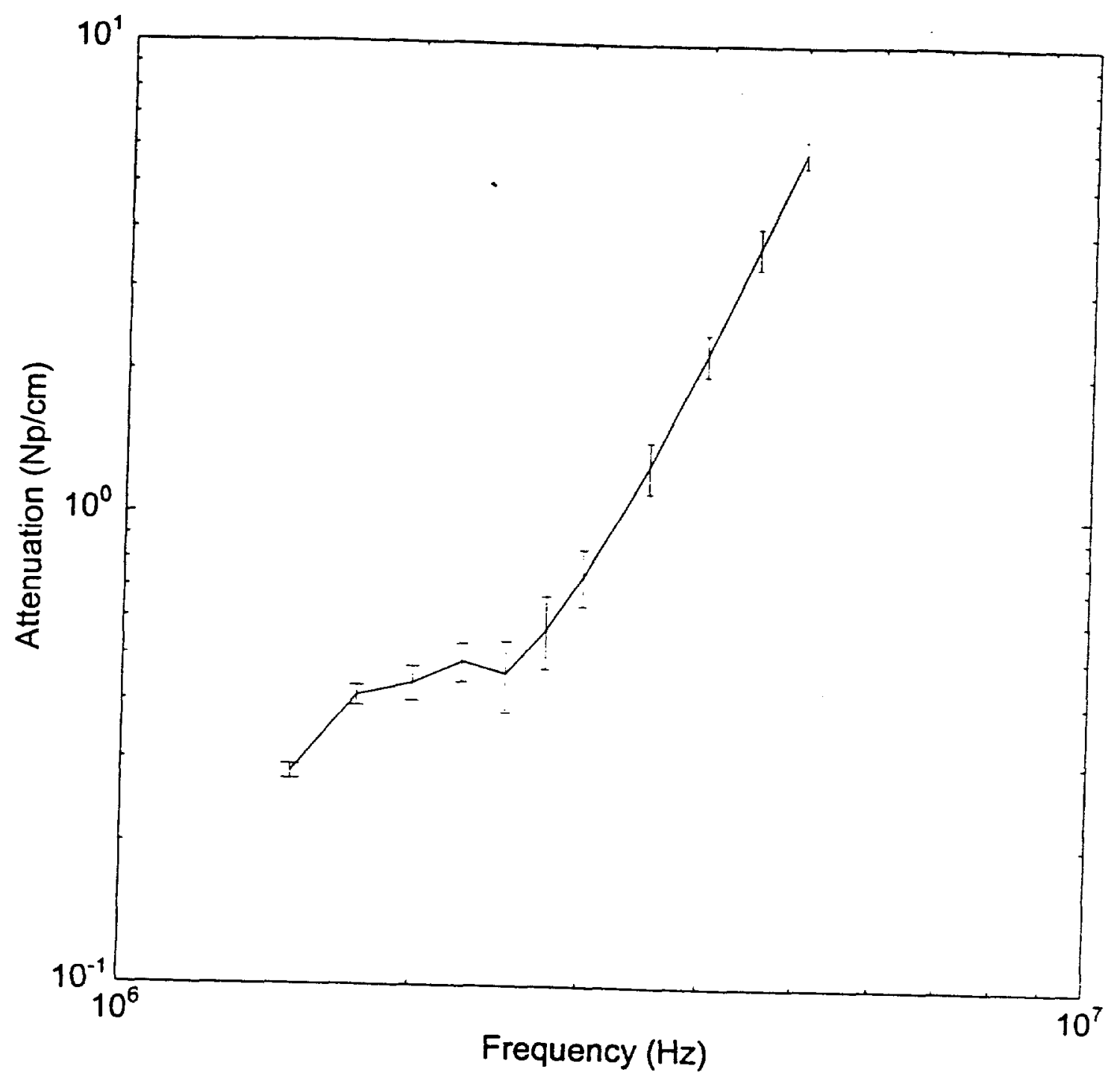

Figure 3.11: Mean attenuation versus frequency curve with error bars representing the average difference between individual attenuation values and the mean value. These data are for a $50 \%$ (by volume) Potter's beads slurry in a mixture of glycerin/water. - E6112. 
5.0 MHz. This larger error is, again, most likely due to variations in the mixing conditions in the Potter's Beads slurries caused by the combination of relatively large particle size and the density difference between the solid and liquid phases. 


\section{Chapter 4: Theory and Experiments for Dilute Solid-Liquid Suspensions}

\subsection{Theory for the Attenuation of Sound in Dilute Solid-Liquid Suspensions:}

The determination of the acoustic response of a slurry given a priori details of its microstructure is referred to as the forward problem. When the total volume fraction of the solids is small, the problem is relatively simple since one only needs to understand the interactions between a single particle and an incident sound wave. This phenomenon has been studied by a number of investigators in the past with notable contributions from Epstein and Carhart (1953) and Allegra and Hawley (1972), who considered suspensions of particles as well as drops. Allegra and Hawley (1972) also reported experimental results verifying the theory for relatively small particles for which the acoustic wavelength is large compared with the particle radius. The theory developed by these investigators is quite general and accounts for attenuation by thermal, viscous, and scattering effects. This theory is briefly described in this chapter, and its predictions are compared with experimental data for dilute systems of polystyrene particles in water and soda-lime glass beads in water. A more complete description of the governing equations is given in Chapter 6, where a theory is developed for concentrated suspensions.

The wave equations for both the interior and exterior of the solid particles were initially derived by Epstein and Carhart (1953). These investigators first linearized the conservation equations for mass, momentum, and energy. The pressure and internal 
energy are eliminated by introducing the linearized equations of state to yield equations in terms of density, velocity, and temperature. Next, the time dependence of all quantities

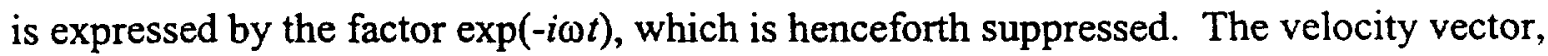
$\mathbf{v}$, can always be represented in terms of a scalar potential, $\Phi$, and a vector potential, $\mathbf{A}$, as

$$
\mathbf{v}=-\nabla \Phi+\nabla \times \mathbf{A}
$$

with $\nabla \cdot \mathbf{A}=0$. With this form of $\mathbf{v}$ it is possible to eliminate the temperature and density from the governing equations to yield a fourth-order partial differential equation for $\Phi$ and a second-order equation in $\mathbf{A}$. The former can be split in two second-order wave equations upon substitution of $\Phi=\phi_{C}+\phi_{T}$ (where $\phi_{C}$ and $\phi_{T}$ represent the scalar potentials of the compressional and thermal waves, respectively) to finally yield three wave equations:

$$
\begin{aligned}
& \left(\nabla^{2}+k_{c}^{2}\right) \phi_{c}=0 \\
& \left(\nabla^{2}+k_{T}^{2}\right) \phi_{T}=0 \\
& \left(\nabla^{2}+k_{s}^{2}\right) \mathbf{A}=\mathbf{0},
\end{aligned}
$$

The wavenumbers in the above equations are given by

$$
\begin{aligned}
& 1 / k_{c}^{2}=\frac{c^{2}}{2 \omega^{2}}\left[1-i(e+\not f)+\left((1-i(e+\not f))^{2}+4 f(i+\gamma e)\right)^{1 / 2}\right] \\
& 1 / k_{\mathrm{T}}^{2}=\frac{c^{2}}{2 \omega^{2}}\left[1-i(e+\not f)-\left((1-i(e+\not f))^{2}+4 f(i+\gamma e)\right)^{1 / 2}\right]
\end{aligned}
$$




$$
k_{s}=(1+i)(\omega \rho / 2 \mu)^{1 / 2} \text {, }
$$

with

$$
e \equiv(4 \mu / 3+\kappa) \omega /\left(\rho c^{2}\right) ; \quad f \equiv \sigma \omega / c^{2}
$$

Here, $c$ is the phase speed in the pure liquid; $\rho$ is the density; $\kappa$ and $\mu$ are, respectively, the compressional and dynamic viscosities; $\gamma=C_{p} / C_{\nu}$ is the ratio of specific heats at constant pressure and volume; $\tau$ is the thermal conductivity; and $\sigma=\tau / \rho C_{p}$ is the thermal diffusivity.

Inside the particles similar equations hold with the dynamic viscosity replaced by $\tilde{\mu} /(-i \omega)$ and the wave speed by $((\tilde{\lambda}+2 \tilde{\mu} / 3) / \tilde{\rho})^{1 / 2}$, where $\tilde{\mu}$ and $\tilde{\lambda}$ are the Lamè constants, and the compressional viscosity is left out. Henceforth, a tilde refcrs to the inside of the particles.

At small values of $e$ and $f$ (such as in water), the above expressions for $k_{C}$ and $k_{\mathrm{T}}$ simplify to

$$
\begin{gathered}
k_{c}=\omega / c+\frac{i}{2}\left[\left(\frac{4}{3} \mu+\kappa\right) / \rho+(\gamma-1) \sigma\right] \omega^{2} / c^{3} \\
k_{T}=(1+i)(\omega / 2 \sigma)^{1 / 2} .
\end{gathered}
$$

Equation (4.2) and its counterpart inside the particles describe the sound wave propagation through the suspension. Note that the wavenumber has an imaginary part; sound waves in pure fluid are attenuated by viscous and thermal energy dissipation (Lighthill 1956); the term inside the square brackets in equation (4.9) is commonly 
referred to as the 'diffusivity of sound'. The total attenuation coefficient in both the liquid and in the solid particle will henceforth be treated as additional physical properties. The other two wave equations describe waves that arise from thermal conduction and finite viscosity: we note that the modulus, $k_{T}$ in equation (4.10), is inversely proportional to the thermal penetration depth, $\sqrt{\sigma / \omega}$, and that of $k_{S}$ to the viscous penetration depth, $\sqrt{\mu / \rho \omega}$. The thermal $\left(\phi_{T}\right)$ and shear $(\mathbf{A})$ waves have generally very high attenuation and are unimportant in acoustic applications.

Applying the boundary conditions of continuity of temperature, heat flux, velocity, and traction at the surface of the particles, and solving the resulting boundary problem numerically, it is possible to, in principle, determine the phase speed and attenuation at arbitrary volume fraction using the above formulation.

The potential, $\phi_{c}$, outside a particle at $\mathbf{x}_{1}$ can be expressed as

$$
\left\langle\phi_{c}\right\rangle(\mathbf{x})=\exp \left(i \mathbf{k}_{c} \cdot \mathbf{x}\right)+\exp \left(i \mathbf{k}_{c} \cdot \mathbf{x}_{1}\right) \sum_{n=0}^{\infty} i^{n}(2 n+1) A_{n} h_{n}\left(k_{c} r\right) P_{n}(\mu)
$$

where $r=\left|\mathbf{x}-\mathbf{x}_{1}\right|, \mu=\cos \theta, \theta$ being the angle between $\mathbf{x}-\mathbf{x}_{1}$ and $\mathbf{k}_{C}, h_{n}$ is the spherical Bessel function of the third kind (or Hankel function) corresponding to an outgoing scattered wave, and $P_{n}$ is the Legendre polynomial of degree of $n$.

Inside the particle centered at $\mathrm{x}_{1}$ we have

$$
\left\langle\widetilde{\phi}_{c}\right\rangle\left(\mathbf{x} \mid \mathbf{x}_{1}\right)=\exp \left(i \mathbf{k}_{c} \bullet \mathbf{x}_{1}\right) \sum_{n=0}^{\infty} i^{n}(2 n+1) \widetilde{A}_{n} j_{n}\left(\widetilde{k}_{c} r\right) P_{n}(\mu)
$$


where $j_{n}$ is the is the spherical Bessel function of the first kind. Similar expressions are written for $\phi T$ and A. This results in expressions with a set of six unknowns for each mode $n$. Application of the boundary conditions of continuity of velocity, traction, temperature, and heat flux yield six equations in six unknowns for each $n$. There were some typographical errors in the equations given by Epstein and Carhart (1953) and Allegra and Hawley (1972); the correct equations are given in Appendix A. Although it is possible to solve for the unknowns analytically in certain limiting cases, it is best to solve them numerically since we are interested in covering a wide frequency range for later inverse calculations.

Once the coefficients are determined, the attenuation can be calculated using the result for the attenuation per unit length given by Allegra and Hawley (1972) as

$$
\alpha=-\frac{3 \phi}{2 z^{2} a} \sum_{n=0}^{\infty}(2 n+1) \Re e A_{n} .
$$

The above analysis can be extended in a straightforward manner to account for the particle size distribution when the total volume fraction of the particles is small. One may write the attenuation by the particles of radius between $a$ and $a+d a$ as an attenuation density $\hat{\alpha}(f, a)$ (where $f$ is the frequency of the wave, $f=\omega /(2 \pi)$ ) times the volume fraction of those particles, $\phi(a) d a$. Here $\phi(a)$ is the particle volume fraction distribution. At low volume fractions these contributions can be "summed" over all particle sizes to give the total attenuation $\alpha_{\text {tot }}(f)$ : 


$$
\alpha_{\text {tot }}(f)=\int_{a=0}^{\infty} \hat{\alpha}(f, a) \phi(a) d a
$$

It should be noted that it is customary to express the particle size distribution in terms of its number density distribution $P(a)$. The volume fraction distribution is related to $P(a)$

by $\phi(a)=\left(4 \pi a^{3} / 3\right) P(a)$

\subsection{Attenuation Measurements in Dilute Solid-Liquid Systems:}

\subsubsection{Attenuation in Soda-Lime Glass Bead Slurries:}

Attenuation data plotted as a function of frequency for soda-lime glass beads at 5 $\%$ and $10 \%$ by volume are shown in Figure 4.1 . These data are collected in the $1.6 \mathrm{~L}$ PVC vessel by the Toneburst measurement technique. These experimental results are plotted along with results of the forward problem calculation based on the theory of Allegra and Hawley (1972) for log-normal distribution of particle sizes. The forward problem calculation used a particle radius of $14 \mu \mathrm{m}$ with a standard deviation of $7 \mu \mathrm{m}$. The actual solids radii range from $0.5 \mu \mathrm{m}$ to $18 \mu \mathrm{m}$, with mean radius at $9 \mu \mathrm{m}$. It is clear from this figure that the agreement between theory and experiment is excellent.

It is interesting to note in this figure the pronounced change in the slope of the attenuation curve. This change in slope occurs where the dominant attenuation regime changes from the inertial regime (Kytomaa 1995) to the geometric scattering regime. The theory predicts that the slope of the attenuation curve should be $1 / 2$, before the transition and four afterwards. That is, the attenuation should scale as $f^{1 / 2}$ before the 


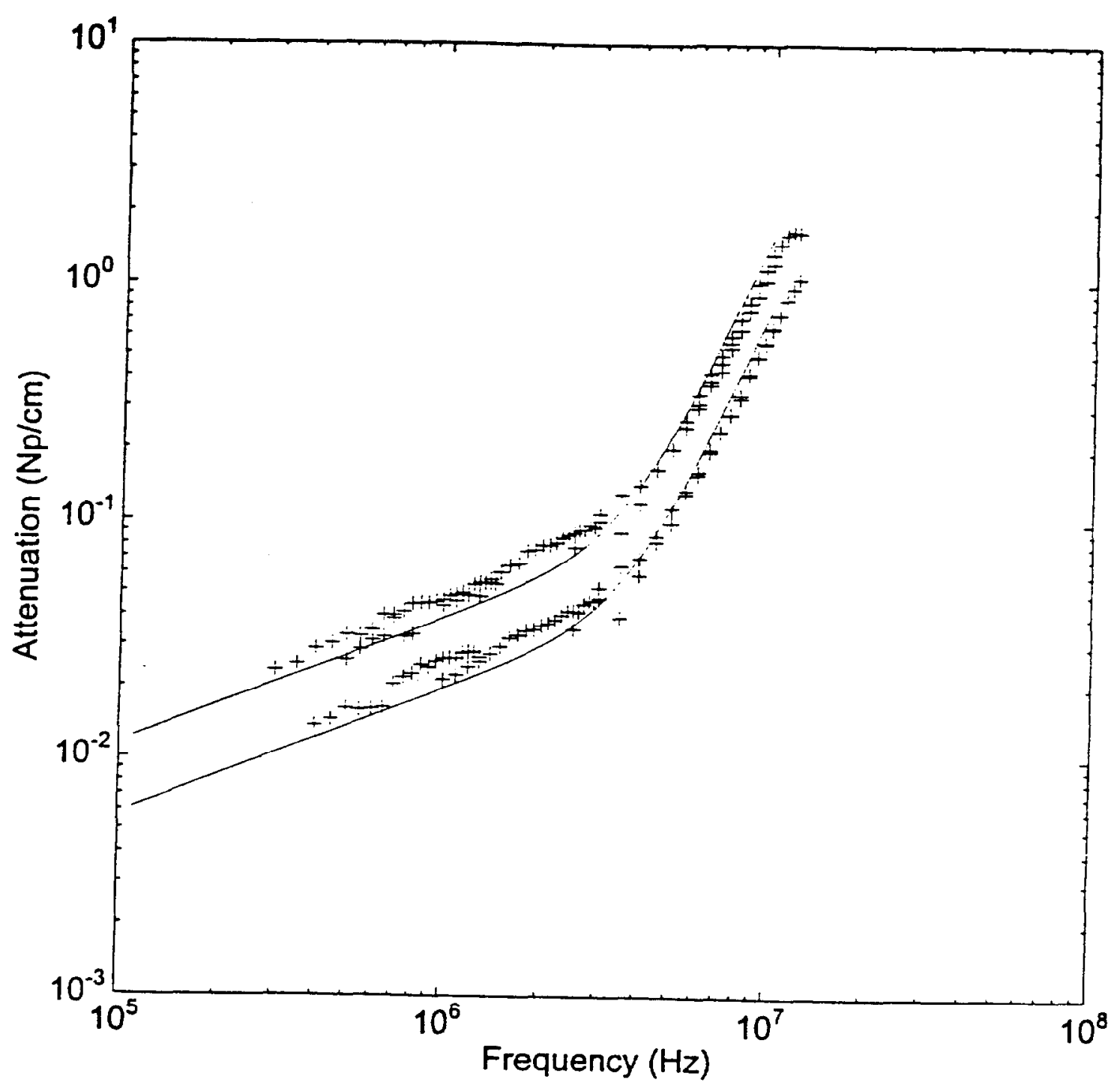

Figure 4.1: Comparison between experimental results and forward problem theory predictions for the attenuation versus frequency curves for soda-lime glass slurries at $5 \%$ (lower curve) and $10 \%$ solids by volume. The experimental solids size distribution has a mean radius of $14.9 \mu \mathrm{m}$ with standard deviation of $3.56 \mu \mathrm{m}$. The forward theory predictions are based on a log-normal distribution with a mean radius of $14 \mu \mathrm{m}$ and standard deviation of $7 \mu \mathrm{m}$. - E1101. 
transition and $f^{4}$ afterwards. However, if one examines the experimental data it can be seen that the data in the inertial regime do scale as $f^{1 / 2}$, but the data in the scattering regime scale with frequency more like $f^{3}$. At first it was thought that this difference occurred because the soda-lime glass particles are polydispersed, but this behavior is seen in theoretical calculations even for monodispersed particles. This behavior is actually due to contributions from more than one mode of resonance, which shall be discussed in more detail in the next section.

\subsubsection{Attenuation in Polystyrene Bead Slurries:}

Figure 4.2 shows the attenuation as a function of frequency for $79 \mu \mathrm{m}$ radius "monodispersed" polystyrene beads. These data are obtained in the $2.54 \mathrm{~cm}$ nominal path length Plexiglas test cell using both the Toneburst and the Pulse/FFT measurement techniques. These data are also compared with several different results of the forward problem theory using different input particle size distributions. Good agreement is seen between experiments and the theory except near the resonance frequencies where small differences appear. The differences were initially thought to possibly be due to finite volume fraction effects, but when the attenuation is calculated using an effective medium approach, shown by a dash-dot line in Figure 4.2, the attenuation actually shifts in a direction which increases the differences. Therefore, the differences probably occur because the particles are not exactly monodispersed. The theory for a monodispersed dispersion, shown by the solid line in Figure 4.2, also shows a difference from experimental data. However, when the theory calculations are performed using a particle 


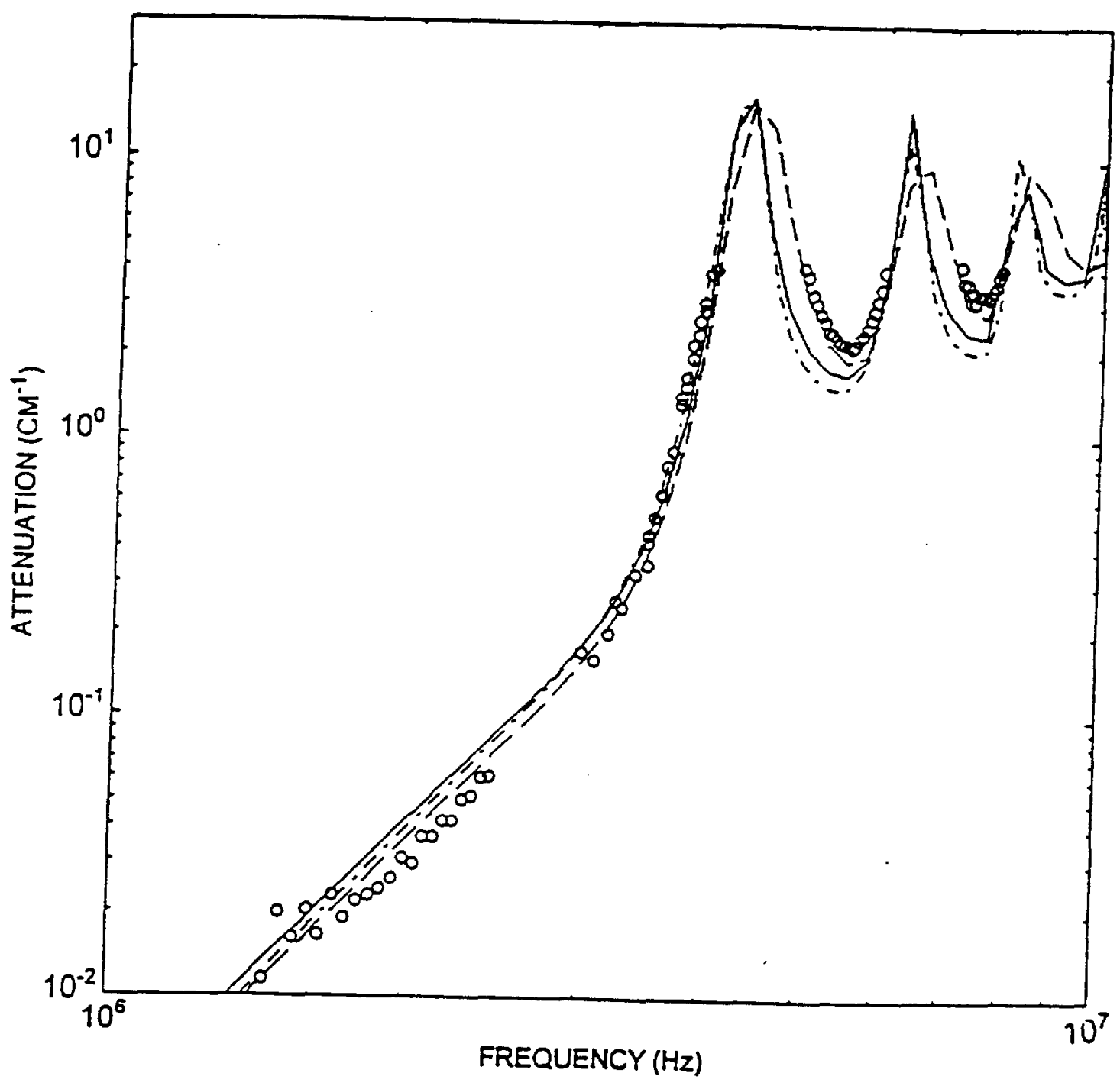

Figure 4.2: Comparison of forward theory with experimental data for the attenuation as a function of frequency. These data are for polystyrene particles of radius $a$ $=79 \mu \mathrm{m}$, at $5 \%$ by volume. O, experimental data; - theory for monodispersed particles; -..-., theory for monodispersed particles with effective medium correction for finite volume fraction effects; - - -, theoretical result with a particle size distribution with a mean radius of 79 $\mu \mathrm{m}$ and standard deviation of $2.5 \mu \mathrm{m}$ (this is the particle size range specified by the particle manufacturer). - E2363. 
size distribution with a mean diameter of $154 \mu \mathrm{m}$ with a standard deviation of $5 \mu \mathrm{m}$, (which lies within the manufacturer's specifications) the result for the attenuation, which is shown as a dashed line in Figure 4.2, shows excellent agreement with the experimental data. Thus, it can be concluded that the small observed differences are due to the polydispersity of the dispersion.

It is important to note the several peaks and troughs in the plot at frequencies above approximately $3 \mathrm{MHz}$. These peaks and troughs are caused by the various modes of resonance which the particles undergo. Each peak corresponds to-dominance by a different mode of resonance.

These transitions to dominance by the different modes of resonance are also responsible for the slope in the scattering regime never truly achieving an $f^{4}$ dependence. The Lengendre polynomials in the equations for the potential describe the contributions to the attenuation from the various modes of oscillation which the particles can undergo. The $n=0$ mode corresponds to radial (volume) oscillations. The $n=1$ mode corresponds to translational oscillations. The $n=2$ mode corresponds to ellipsoidal $P_{2}$-shape oscillations, and so on. Figure 4.3 shows the contributions to the total attenuation as calculated for the polystyrene particles used in the experiments from the forward problem theory. The density of polystyrene particles is essentially the same as that of water, and so the translational oscillations of the particles are small. Therefore, the viscous attenuation is small, and the low frequency behavior is governed by the thermal attenuation of the $n=0$ mode (volume oscillations). At higher frequencies the $n=0$ mode increases first as $f^{4}$ due to scattering losses, but the contribution from the $n=2$ 


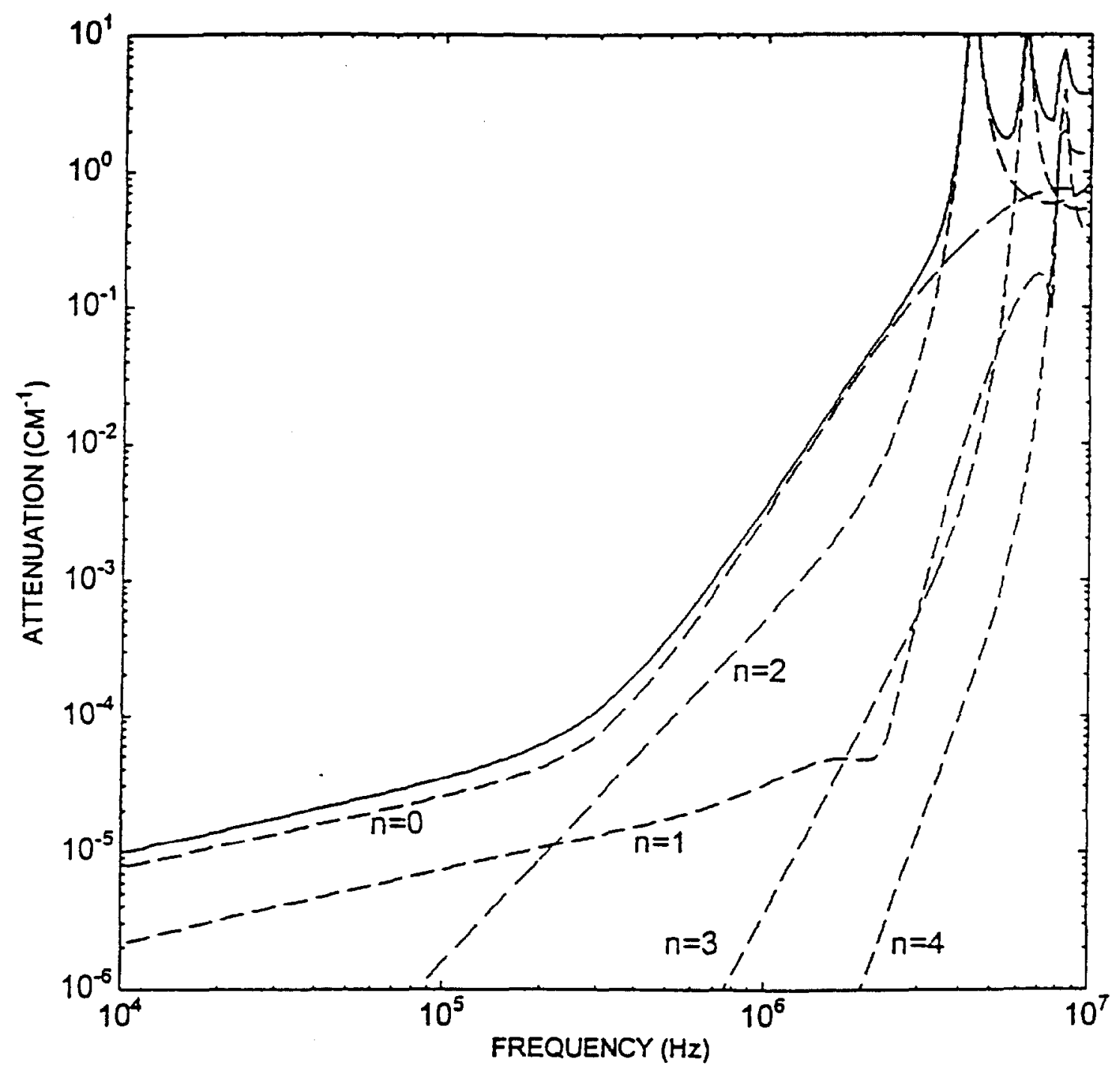

Figure 4.3: Contributions of the first five modes of resonance ( $n=1$ through 5) to the total attenuation (the imaginary part of $k_{e f f}$ ) for $79 \mu \mathrm{m}$ radius polystyrene particles in water. 
mode (ellipsoidal oscillations) soon becomes important as it undergoes resonance at about $3 \mathrm{MHz}$. In the figure we see that the $n=3$ and $n=1$ modes undergo resonance next, etc. Therefore, the attenuation never really achieves the $f^{4}$ dependence that would be predicted by the scattering theory; but rather, it displays the combined behavior shown in Figure 4.4. Thus, it can be said that the resonance frequencies of the various modes of oscillations cause the high frequency behavior of the polystyrene particles to become quite complicated.

It should be noted that the reason why the peaks appear truncated, in the region where the theory predicts very high peaks in the attenuation, is because in those regions the attenuation increases dramatically to levels which are beyond the measuring capabilities of the instrumentation used in this study.

In contrast, these peaks and troughs do not appear in the attenuation behavior predicted by the theory of Allegra and Hawley (1972) for soda-lime glass particles. This behavior is shown in Figure 4.5. Unlike the polystyrene data, the attenuation data for the soda-lime glass particles do not peak at several different frequencies. Rather, for each mode $n$ we see broad "hills" separated by narrow "valleys". The total attenuation does not appear to go through several resonances, but instead has one broad "overall" peak which is a mean contribution, of sorts, of all the individual resonance behaviors. The difference between the behavior for the polystyrene and soda-lime glass particles at the higher frequencies seems to arise mainly from the different elastic properties of the two materials. Also, the density of the soda-lime glass particles is significantly different from that of water. The soda-lime glass particles exhibit significant translational oscillations. 


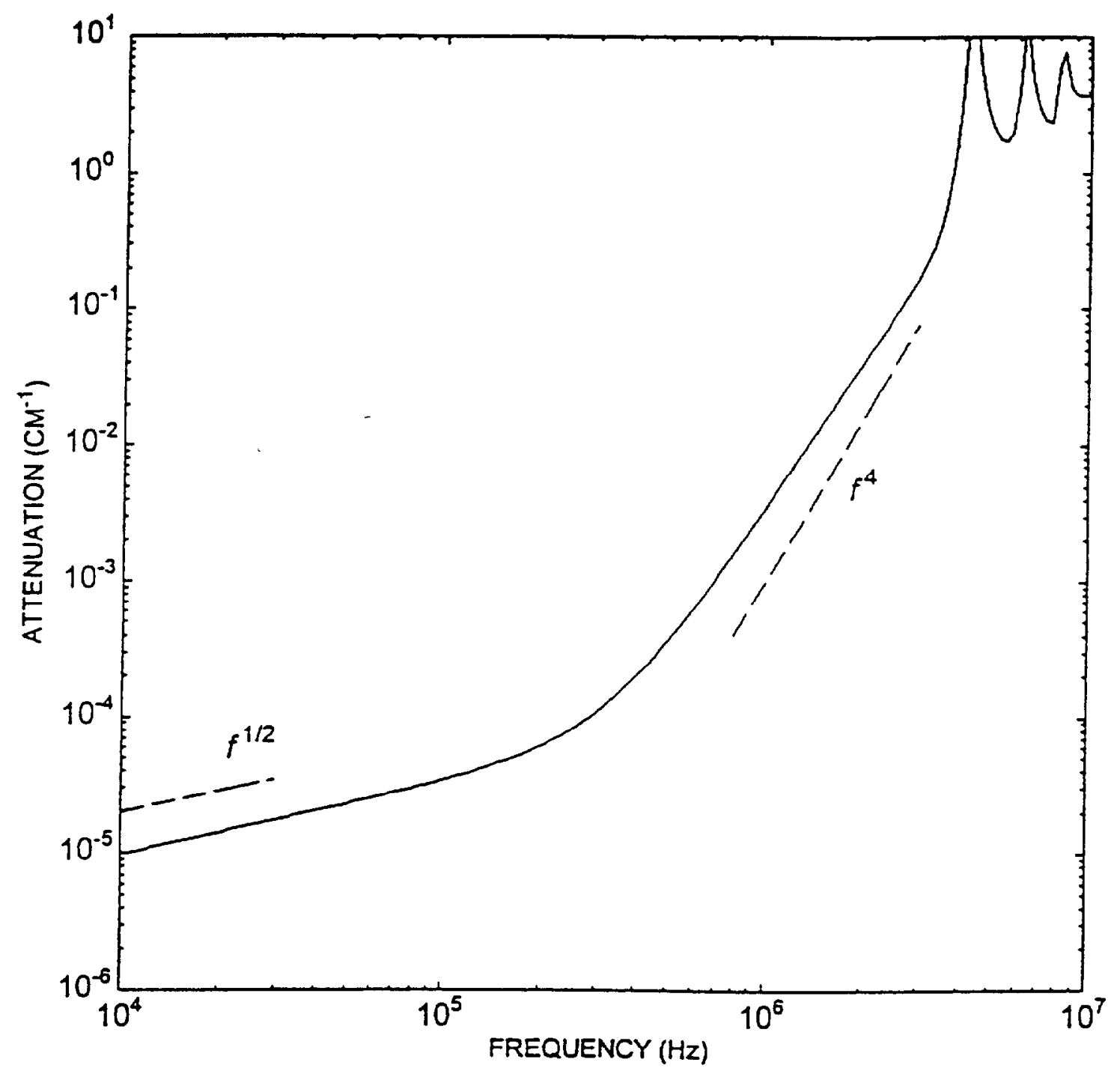

Figure 4.4: Example of the dependence of attenuation on frequency, $f$, for a slurry of polystyrene particles in water. Dashed lines are asymptotic slopes of the attenuation for low and high frequencies. 


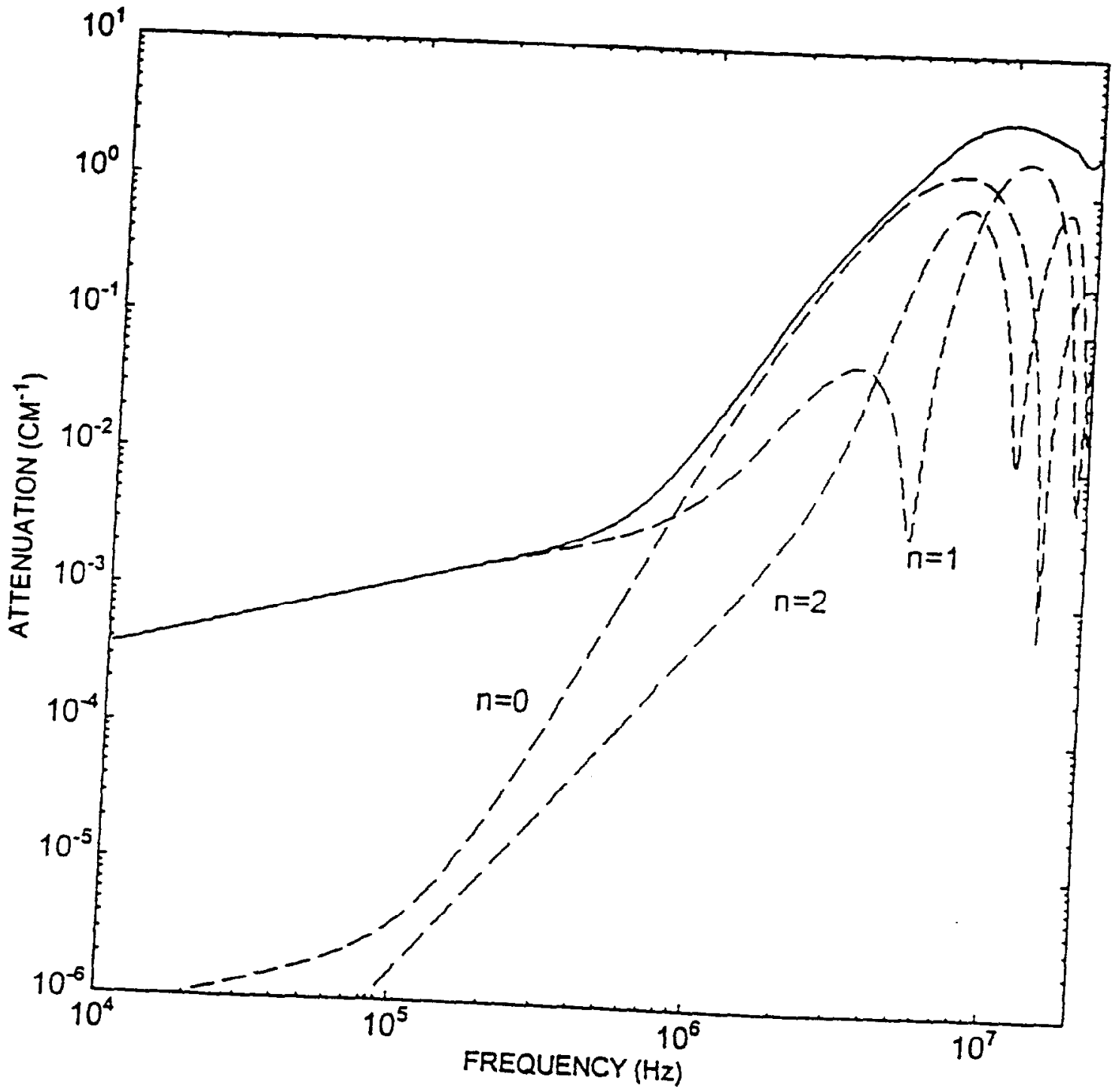

Figure 4.5: Contributions of the first three modes of resonance ( $n=1$ through 3 ) to the total attenuation (the imaginary part of $k_{\text {eff }}$ ) for $79 \mu \mathrm{m}$ radius monodispersed glass particles in water. 
As a result, the low frequency behavior is completely governed by the viscous effects and the $n=1$ mode. It should be noted that small frequency attenuation is about two orders of magnitude greater for the soda-lime glass particles than for the polystyrene particles. 


\section{Chapter 5: Inverse Problem for Determination of Particle Size Distributions}

\subsection{Inverse Problem Theory:}

Determining the particle size distribution of a solid-liquid suspension is of great practical interest. It has been suggested in the literature that this distribution may be determined by measuring the attenuation of a sound wave propagating through the suspension as a function of the frequency of the wave. The main premise is that the attenuation caused by a particle as a function of frequency depends on its size; and, therefore, the attenuation measurements can be inverted to determine the particle size distribution -- at least when the total volume fraction of the solids is small enough such that particle interactions and detailed microstructure of the suspension play an insignificant role in determining the acoustic response of the suspension. Indeed, this general principle has been exploited successfully to determine the size distribution of bubbles in bubbly liquids (Commander and McDonald (1991); Duraiswami (1993); and Duraiswami et al. (1998)). Commercial "particle sizers" based on acoustic response are in the process of being developed/marketed for characterizing solid-liquid mixtures (Oja and Alba (1997)). The main objective of this chapter is to investigate under which circumstances such a problem can be solved for solid-liquid systems. The attenuation is predicted in the calculations from the linear theory of Allegra and Hawley (1972). It will be shown that the success of the acoustic method for determining detailed particle size 
distributions is limited, depending on the nature of the particles and the frequency range over which the input (attenuation) data are available.

We now consider the inverse problem. That is, given only the total attenuation $\alpha_{\text {to }}$ as a function of $f$, it is desirable to determine $\phi(a)$ using equation (4.13). Inverse techniques have been explored by many investigators with regard to the acoustic evaluation of dispersed phase systems.

Duraiswami (1993), after exploring a number of possible regularization strategies such as truncated singular value expansion, moment collocation techniques, and Tikhonov regularization and optimization, employed a Tikhonov regularization scheme and optimization to solve the inverse problem of determining the size distribution of bubbles in bubbly liquids from measurements of attenuation and change in phase speed. The basic idea behind the Tikhonov regularization technique is that the problem is formulated in such a way that the norm of the solution and the norm of the residual error are sought to be minimized. This technique also takes advantage of the fact that the bubble size distribution function is generally a smooth function. Further details of this technique will be discussed later in this chapter.

This type of straightforward method of solving the integral equations, i.e., discretizing the integral domain into a number of elements and converting the integral equation into a system of linear equations in unknowns $\phi\left(a_{k}\right)$ at a selected number of points $a_{k}$ in the domain, cannot be used in the solid-liquid slurry inverse problem since the resulting equations will be ill-posed. Figures $5.1 \mathrm{a} \& \mathrm{~b}$ illustrates the ill-posed nature of the problem. Figure 5.1a shows two very different particles size distributions 


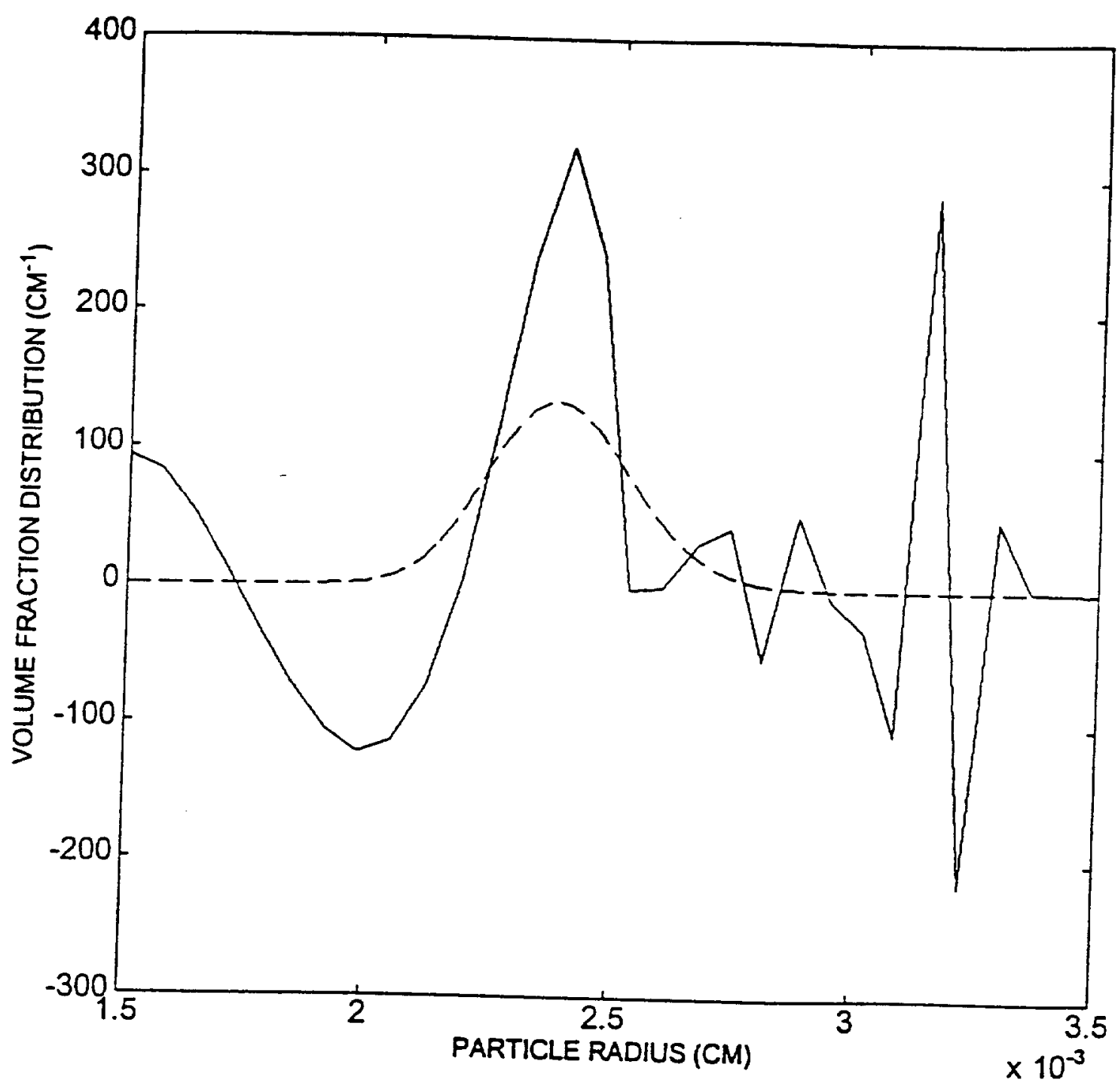

Eigure 5.1a: Two significantly different particle volume fraction distributions which yield similar attenuation spectra. 


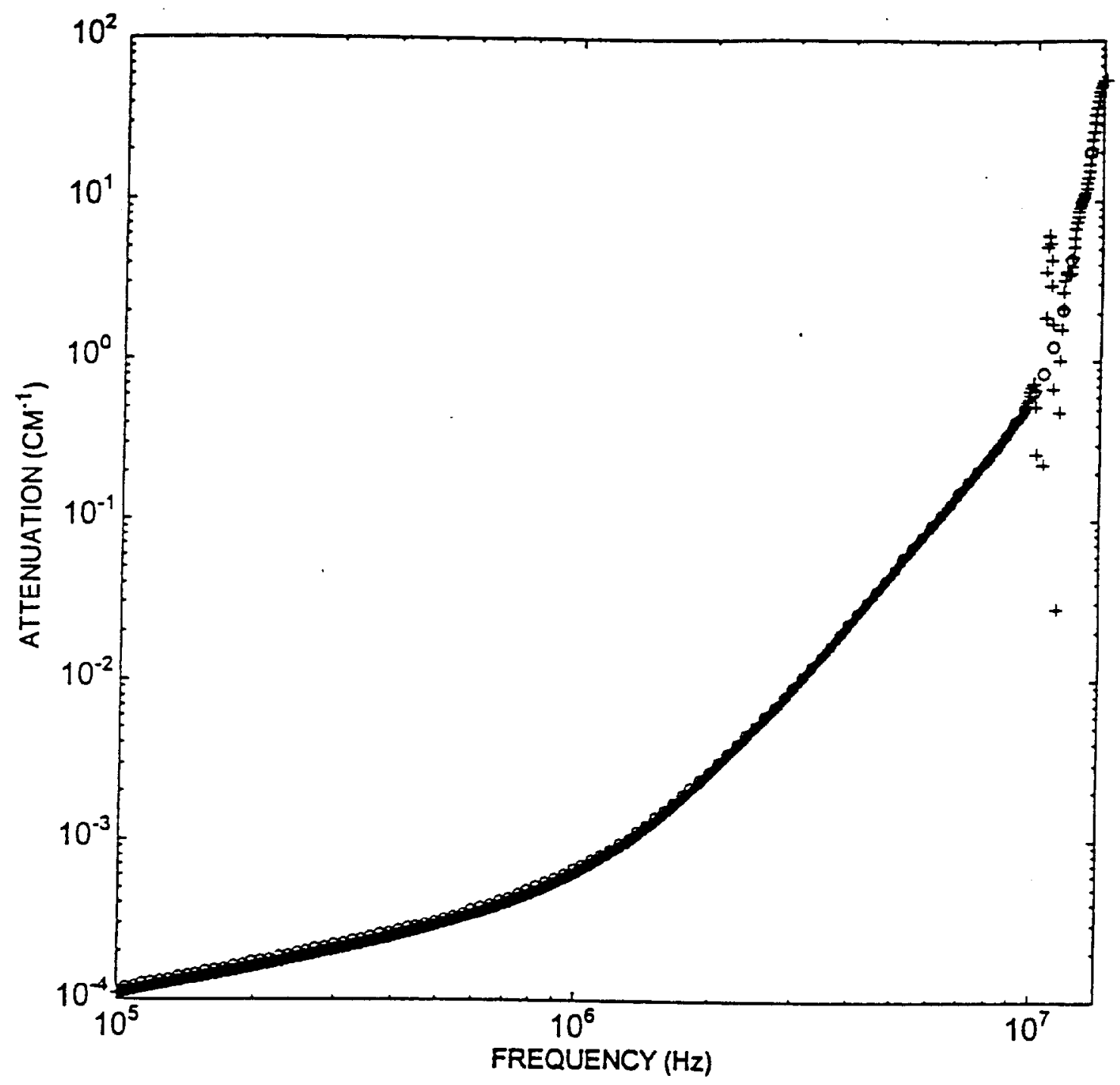

Figure 5.1b: Attenuation spectra obtained from the volume fraction distributions in Figure 5.1a. The circles correspond to the results obtained when using the distribution shown by a dashed line in Figure 5.1a, and the crosses correspond to the result obtained when using the distribution shown by the solid line in Figure 5.1a. 
whose attenuation spectra, shown in Figure 5.1b, are seen to be essentially the same. These two curves were obtained by starting with a smooth, log-normal particles size distribution (dashed curve in Figure 5.1a) and generating the attenuation versus frequency data using the forward theory (circles in Figure 5.1b). A random noise of $1 \%$ was then added to the data, and equation (5.2) with $\varepsilon=0$ (which is equivalent to integral equation (4.13)) was subsequently solved to yield the particle size distribution indicated by the solid line in Figure 5.1a. The crosses in Figure 5.1b correspond to the attenuation spectrum determined from the forward theory using the new particle size distribution. It should be noted that the attenuation is evaluated with a smaller frequency increment than the one used for the original distribution. It is seen that the attenuation from the two distributions agree with each other to within $1 \%$ for the frequencies marked by the circles. The attenuation spectrum for the latter particle size distribution does exhibit an oscillatory behavior in between the frequency increments, particularly at $10 \mathrm{MHz}$, but these oscillations occur only for a very narrow frequency range and would have been missed altogether had the attenuation been determined at only the input frequencies.

Since the true particle size distribution is expected to be smooth, only solutions which are reasonably smooth can be permitted. This restriction may be imposed in several ways. In the present study, the primarily used regularization technique is that due to Tikhonov (Kress 1989) which was successfully used for bubbly liquids by Duraiswami (1993). An alternative method is presented at the end of this section. Accordingly, equation (4.13), which is based on the theory of Allegra and Hawley (1972), is multiplied 
with $\hat{\alpha}(f, a) d f$ and integrated over the frequency range to obtain a simpler integral equation in which the right-hand side is only a function of $a$ :

$\int_{f_{\min }}^{f_{\max }} \int_{a_{\min }}^{a_{\max }} \hat{\alpha}(f, a) \hat{\alpha}\left(f, a^{\prime}\right) \phi\left(a^{\prime}\right) d a^{\prime} d f=b(a) \equiv \int_{f_{\min }}^{f_{\max }} \alpha_{\text {tot }}(f) \hat{\alpha}(f, a) d f$,

where $\left(a_{\min }, a_{\max }\right)$ and $\left(f_{\min }, f_{\max }\right)$ are the radius and frequency ranges. The above integral equation is now regularized as explained below by adding a small term $\varepsilon\left(\phi-l^{2} \phi^{\prime \prime}\right)$ (where primes denote derivatives) to its left-hand side. Thus,

$$
\varepsilon\left[\phi(a)-l^{2} \phi^{\prime \prime}(a)\right]+\int_{a_{\min }}^{a_{\max }} K\left(a, a^{\prime}\right) \phi\left(a^{\prime}\right) d a^{\prime}=b(a)
$$

is obtained, where $l$ is a suitably chosen length scale and $K\left(a, a^{\prime}\right)$ is a kernel defined by

$$
K\left(a, a^{\prime}\right)=\int_{f_{\min }}^{f_{\max }} \hat{\alpha}(f, a) \hat{\alpha}\left(f, a^{\prime}\right) d f
$$

Equation (5.2) is an integro-differential equation and needs two boundary conditions.

Usual practice is to take the derivative of $\phi(a)$ to be zero at the two end points:

$$
\phi^{\prime}\left(a_{\min }\right)=\phi^{\prime}\left(a_{\max }\right)=0
$$

It should be noted that $a_{\min }$ and $a_{\max }$ are not, in general, known a priori. One expects $\phi$ to also be zero at the two end points. Thus, the range $\left(a_{\min }-a_{\max }\right)$ must be determined by trial 
and error so that both $\phi$ and its derivatives are approximately zero at the extreme values of

a.

Now it can be shown that the solution of equation (5.2) subject to the boundary conditions given by equation (5.4) minimizes

$$
E+\varepsilon \int_{a_{\min }}^{a_{\max }}\left[\{\phi(a)\}^{2}+l^{2}\left\{\phi^{\prime}(a)\right\}^{2}\right] d a
$$

where $E$ is the measure of error between the actual attenuation and the computed attenuation:

$$
E=\int_{f_{\min }}^{f_{\max }}\left|\int_{a_{\min }}^{a_{\min }} \hat{\alpha}(f, a) \phi(a) d a-\alpha_{\text {tot }}(f)\right|^{2} d f
$$

Since both $E$ and the second term in equation (5.5), i.e., the integral, are non-negative, minimization of equation (5.5) ensures that the solution of equation (5.2) will be free from large oscillations in $\phi$. In other words, highly oscillatory distributions such as the one shown in Figure 5.1a. are rendered inadmissible when equation (5.2) is solved with finite, positive $\varepsilon$ in place of the original integral equation (5.1). Thus, we have regularized the problem of determining $\phi$.

If a large $\varepsilon$ is chosen, then the oscillations in $\phi$ decrease, but increase the error in $\phi(a)$ increases since then the equation solved is significantly different from the original integral equation. Small $\varepsilon$, on the other hand, yields unrealistic $\phi(a)$ having large oscillations when the data $\alpha_{\text {tot }}(f)$ are not exact. An optimum choice of $\varepsilon$, therefore, depends on the magnitude of the uncertainty or error in the measured attenuation versus frequency 
data.

\subsection{Inverse Problem Calculation Results:}

The theoretical calculations which accompany the experimental results in this dissertation are performed by Dr. Peter D.M. Spelt and Professor Ashok S. Sangani at Syracuse University. Both the theoretical study and computations are performed by these individuals as part of the overall Acoustic Probe Development Project being funded by the US DOE Environmental Management Science Program Grant \# DE-FG0796ER 14729. A formal presentation of the theoretical and computational work presented herein may be found in Spelt et al. (1998).

As mentioned in the previous section, an optimum choice of parameter, $\varepsilon$, depends on the magnitude of the uncertainty or error in the attenuation versus frequency data. This concept is tested by first determining the exact $\alpha_{\text {tot }}(f)$ using the forward theory for a given $\phi(a)$ with a small random noise of about $1 \%$ magnitude added to it before the inverse calculations are performed. This random noise is introduced to simulate the error which would be present in experimentally obtained attenuation versus frequency data. To obtain the optimum value of $\varepsilon$, equation (5.2) is solved for several different $\varepsilon$ 's, and the error $E$ is plotted versus $\varepsilon$ to find a minimum value of $E$. This minimum value of $E$, however, may lead to volume fraction distributions in which $\phi(a)$ may have physically unrealizable negative values for some values of $a$. To prevent the realization of this possibility, the constraint $\phi(a) \geq 0$ for all $a$ is satisfied $a$ posteriori by 
setting $\phi(a)=0$ for all $a$ 's for which the solution of equation (5.2) yields negative values of $\phi(a)$. Thus, the computed value of $E$ is based on $\phi(a) \geq 0$.

The integro-differential equation (5.2) was solved in the following manner. After discretizing the domain $\left(a_{\min }\right.$ to $\left.a_{\max }\right)$ into $N-1$ equal segments and the frequency domain into $M-1$ logarithmically equal segments the kernel $K\left(a_{i}, a_{j}\right)$, is first evaluated for $i, j=$ $1,2, \ldots, N$ (cf. equation 5.3) using a trapezoidal rule for the integration over the frequency range. As was indicated by Duraiswami (1993) it is important to calculate the integral over the particle radius rather accurately. Thus, it was assumed that $\phi(a)$ varied in a piecewise continuous manner in each segment, and a 12-point Gauss-Legendre quadrature was used to evaluate the integral in equation (5.2). A second-order central difference formula was used to evaluate $\phi^{\prime \prime}(a)$ at all points except the endpoints $a_{\min }$ and $a_{\max }$. The boundary conditions $\phi^{\prime}\left(a_{\min }\right)=0$ and $\phi^{\prime}\left(a_{\max }\right)=0$ were approximated using, respectively, second-order forward and backward difference formulae. Application of equation (5.2) at all the discretization points together with the boundary conditions can be expressed with the above scheme as a system of linear equations:

$$
\sum_{j=1}^{N} A_{i j} \phi_{j}=b_{i} \quad i=1,2, \ldots, N
$$

where $\phi_{j}=\phi\left(a_{j}\right)$ and $b_{i}=b\left(a_{i}\right)$. The above set of equations was then normalized by dividing all the equations by the greatest element of the kernel, $K\left(a_{i}, a_{j}\right), K_{m}$ for all $i$, $j$, times the segment length, $\Delta a=\left(a_{\max }-a_{\min }\right) /(N-1)$. This set of equations was subsequently solved using a standard IMSL subroutine for linear equations. 
Once the $\phi_{j}$ are determined for a selected value of $\varepsilon$, the constraint $\phi_{j} \geq 0$ is satisfied by setting, as was mentioned previously, all $\phi_{j}=0$ for all negative values of $\phi_{j}$. The error, $E$, as given by equation (5.6), was subsequently evaluated using a trapezoidal rule for integration over the frequency range. The optimum value of $\varepsilon$ was determined by stepping logarithmically through several values of $\varepsilon$ and plotting $E$ versus $\varepsilon$.

A typical result $\left(N=30, M=112, f_{\min }=0.1 \mathrm{MHz}, f_{\max }=17 \mathrm{MHz}, a_{\min }=15 \mu \mathrm{m}\right.$, and $a_{\max }=35 \mu \mathrm{m}$ ) for the error in the resulting attenuation as a function of $\varepsilon$ is shown in Figure 5.2. It should be noted that here $\varepsilon$ is actually the value of $\varepsilon$ divided by $K_{m} \Delta a$. Upon examining Figure 5.2, one can see a clearly defined optimum value of $\varepsilon$. Computations were also made with larger $M$ to confirm that the resulting volume fraction distribution was not affected by the further refinement in the integration over the frequency range.

It should be noted that both $\varepsilon$ and $l$ are parameters which were chosen so as to minimize the error $E$. The value for $l$ was taken as $l=\left(a_{\max }-a_{\min }\right) / n$, and $E$ was computed by varying both $\varepsilon$ and $n$. The value of $n$ was varied from 1 to $N$. It was found that when $E$ was plotted versus $n$ and $\varepsilon, E$ was much more sensitive to $\varepsilon$ than it was to $n$. In general, it was found that the results where $n$ was close to $N$ were slightly better than those where $n$ was close to unity. Therefore, $n$ was chosen to equal 30 . For larger values of $N(N>40)$, it was found that choosing $n=N$ led to more oscillatory behavior for $\phi_{j}$. This behavior is to be expected since choosing a larger value of $n$, and hence, a smaller value of $l$ will permit larger values of $\phi^{\prime}(a)$. 


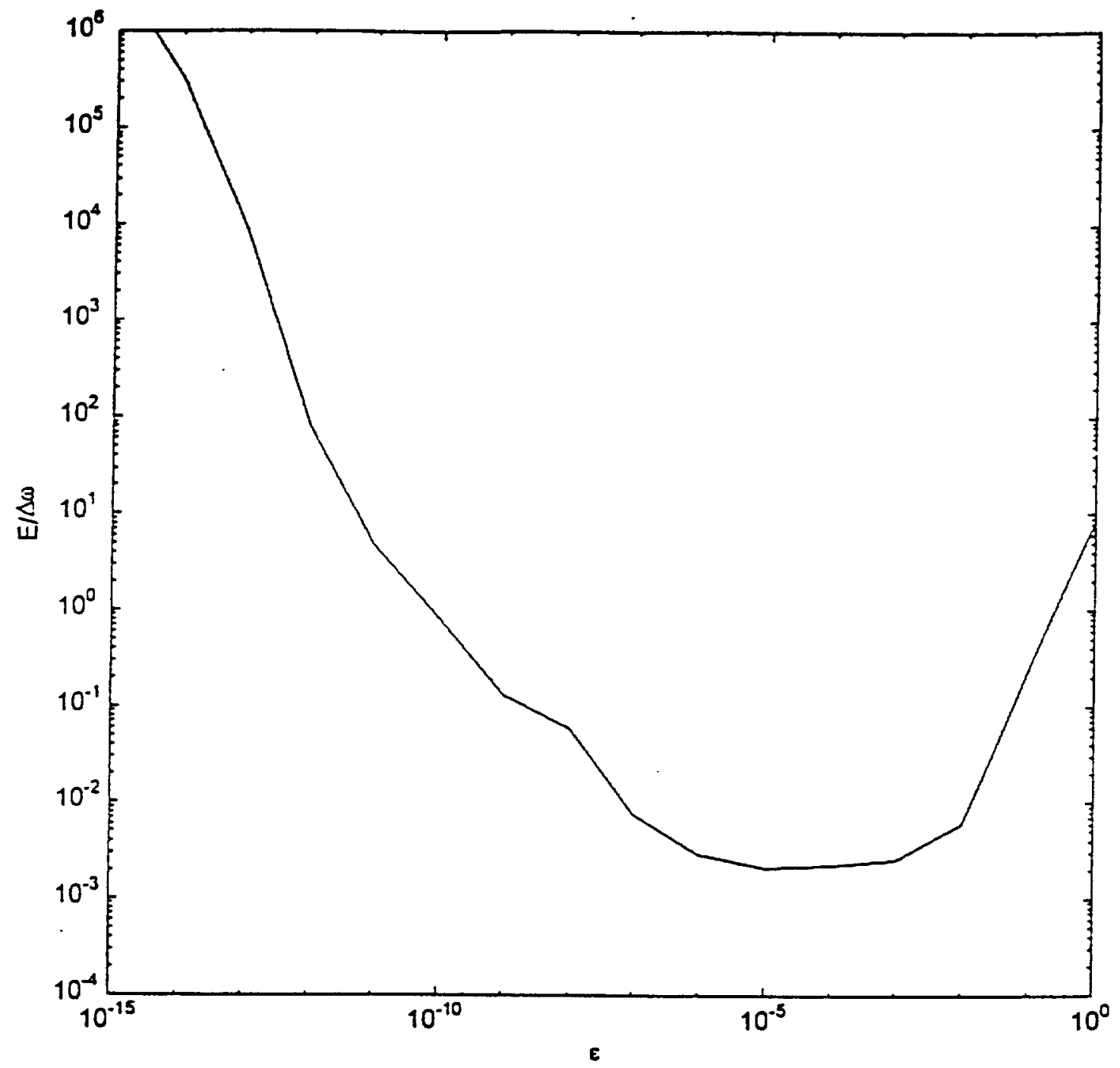

Figure 5.2: Error in the attenuation as a function of $\varepsilon$. 
In order to obtain the results for the volume fraction distribution using the above mentioned regularization technique, the forward theory was used to first generate attenuation data for an assumed volume fraction distribution. Small random noise was added to the generated data to mimic experimental error in measured attenuation data. If the procedure fails for the generated data with small amount of noise added, it would certainly fail for any real experimentally obtained data.

The calculation is initially performed for a frequency range of 0.1 to $15 \mathrm{MHz}$ in order to investigate the success and limitations of the technique. This range roughly coincides with the range of frequency employed in the attenuation measurement experiments. Also considered is a larger range of frequency to determine if better estimates of the particle volume fraction distribution could be achieved if the attenuation data at higher frequencies were available. The examination of higher frequency results is important because there are commercially available acoustic probes which can operate at frequencies up to $150 \mathrm{MHz}$.

The particle sizes first considered are those which are of the same order of magnitude as the wavelength of the sound waves somewhere in the above mentioned frequency range. This requirement is satisfied for particles of size about 10 to $100 \mu \mathrm{m}$ in radius. It should be noted that particles of larger sizes would merely shift the observed behavior to the left in an attenuation versus frequency plot. The smooth particle volume fraction distribution (dashed line) in Figure 5.1 a shows an example of a log-normal particle size distribution which is employed because it is a commonly used and smooth distribution. As in the forward problem calculations, the inverse problem calculations are 
performed for polystyrene and soda-lime glass particles. These two types of particles form a good basis for examination of the capabilities and limitations of the inverse problem technique because the polystyrene particles are almost neutrally buoyant with respect to water and deformable, while the soda-lime glass particles are much more dense than water and quite rigid. The physical properties of these two materials are shown in Table 3.2 in Chapter 3.

The first volume fraction distribution results obtained from the inverse problem are shown in Figure 5.3. These results are for polystyrene particles with a narrow size distribution in the range of 20 to $30 \mu \mathrm{m}$ in radius. However, the particle size range initially employed was somewhat larger, ranging from 5 to $100 \mu \mathrm{m}$ in radius; and the frequency range was 0.1 to $17 \mathrm{MHz}$. The results for this particle size range are shown in Figure 5.3, and it is clear that the volume fraction distribution, evaluated from the inverse technique, is in very good agreement with the original input particle volume fraction distribution. The result for the particle volume fraction distribution can be further improved by making the particle size range smaller (a close-up of the improved result is shown as a dashed line in Figure 5.4).

In Figure 5.5 a more complicated, bimodal, volume fraction distribution is considered for the polystyrene particles. This distribution has a particle radius range of 20 to $45 \mu \mathrm{m}$ with peaks at approximately 25 and $38 \mu \mathrm{m}$. The attenuation as a function of frequency for this volume fraction distribution is shown in Figure 5.6. The maximum frequency used in the inverse calculation is indicated in the figure by a square. It is seen that the frequency range includes the first two resonance peaks of the attenuation curve. 


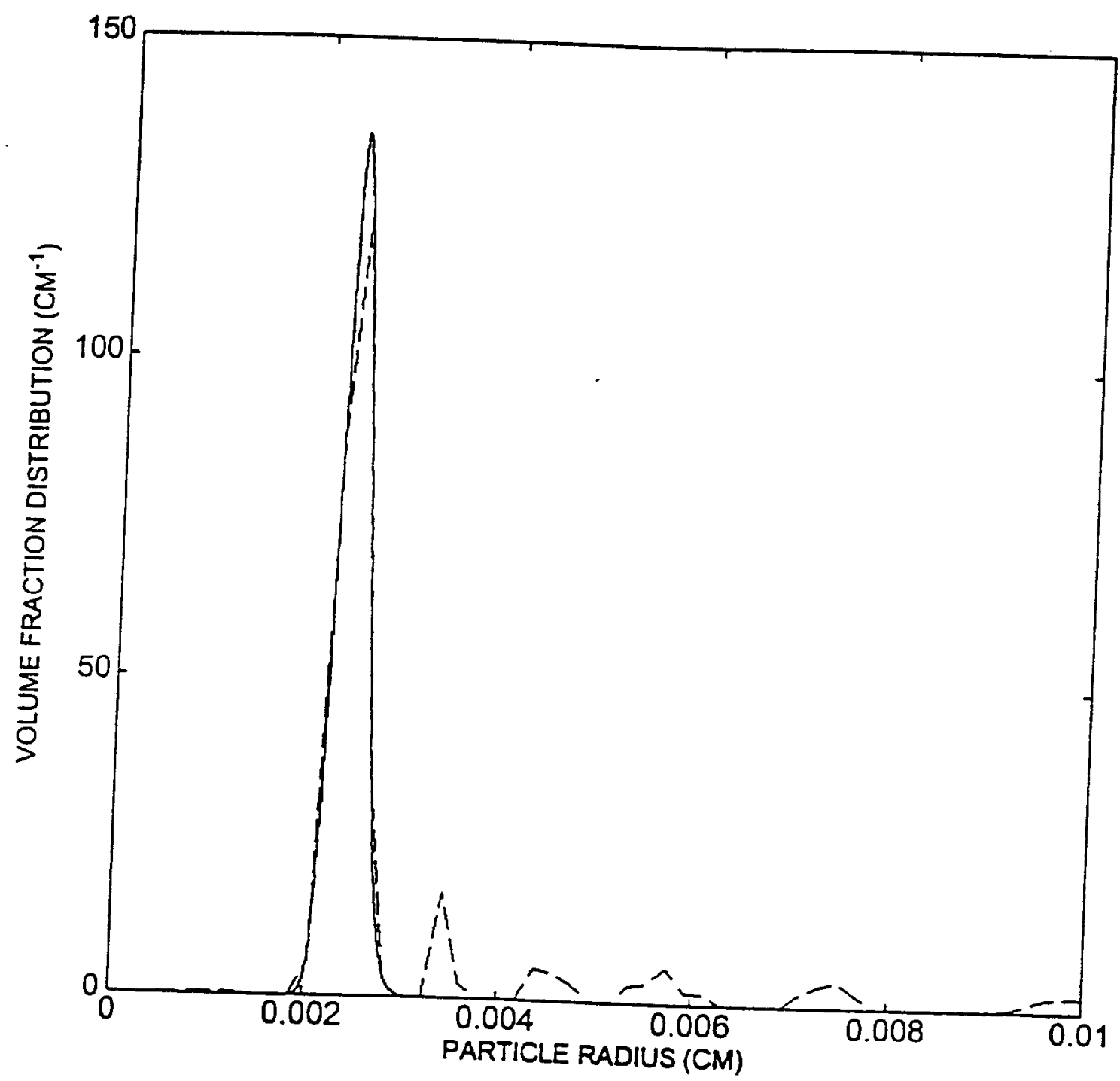

Figure 5.3: Inverse problem solution for polystyrene particles in water. The solid curve is the volume fraction distribution used to generate the attenuation curve shown in Figure 5.7a (with $f_{\max }$ indicated by a square); and the dashed curve is the solution to the inverse problem for particle radius range of 1 to $100 \mu \mathrm{m}$ and using 50 'bins' of particle sizes. 


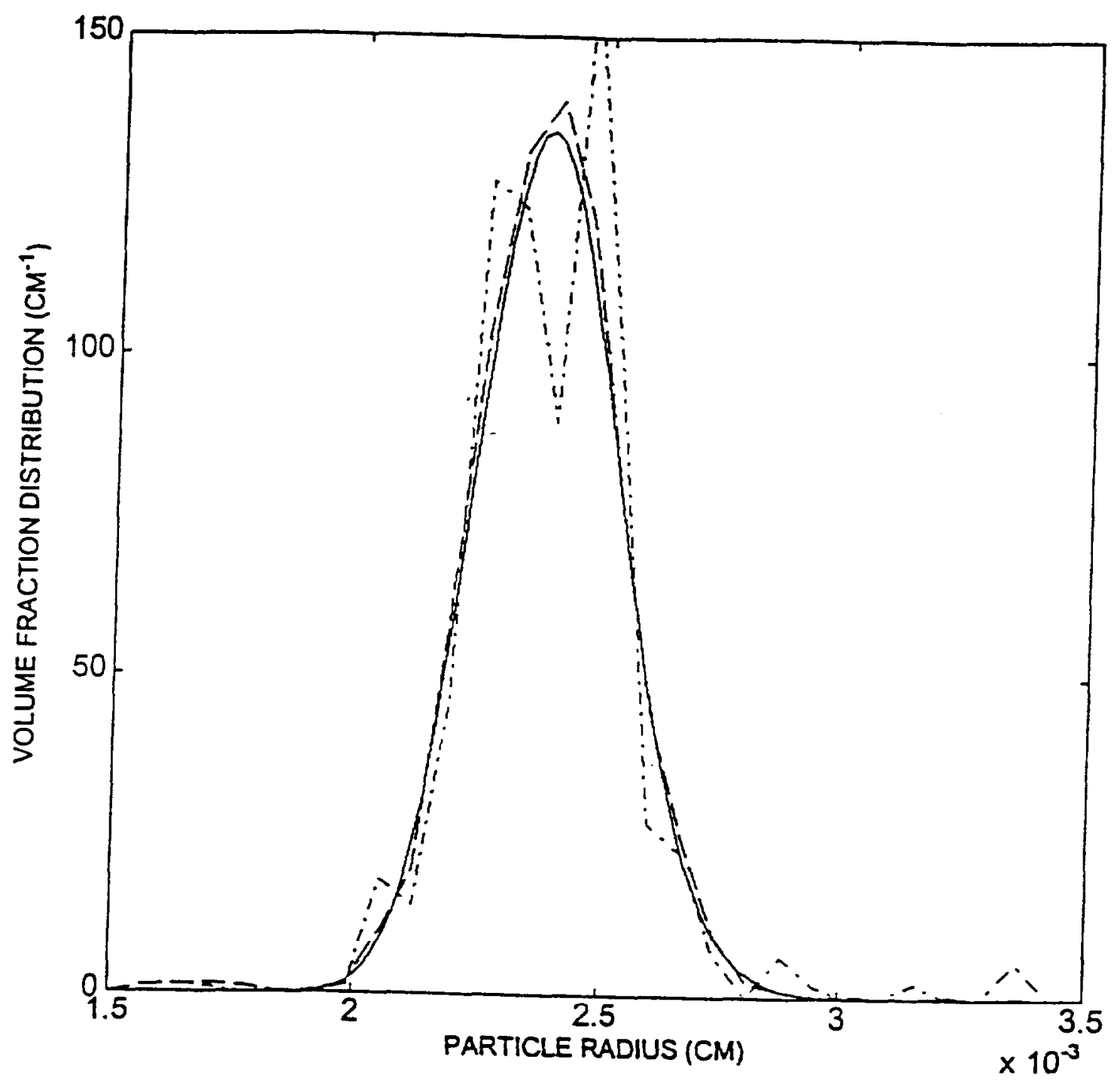

Eigure 5.4: Solution of the inverse problem when a random noise of $5 \%$ standard deviation is introduced in the attenuation (input) data. The solid line is the exact result; the dashed curve is the result when no noise is introduced; the dash-dotted curve is the result after the introduction of the noise. These calculations are for polystyrene in water. 


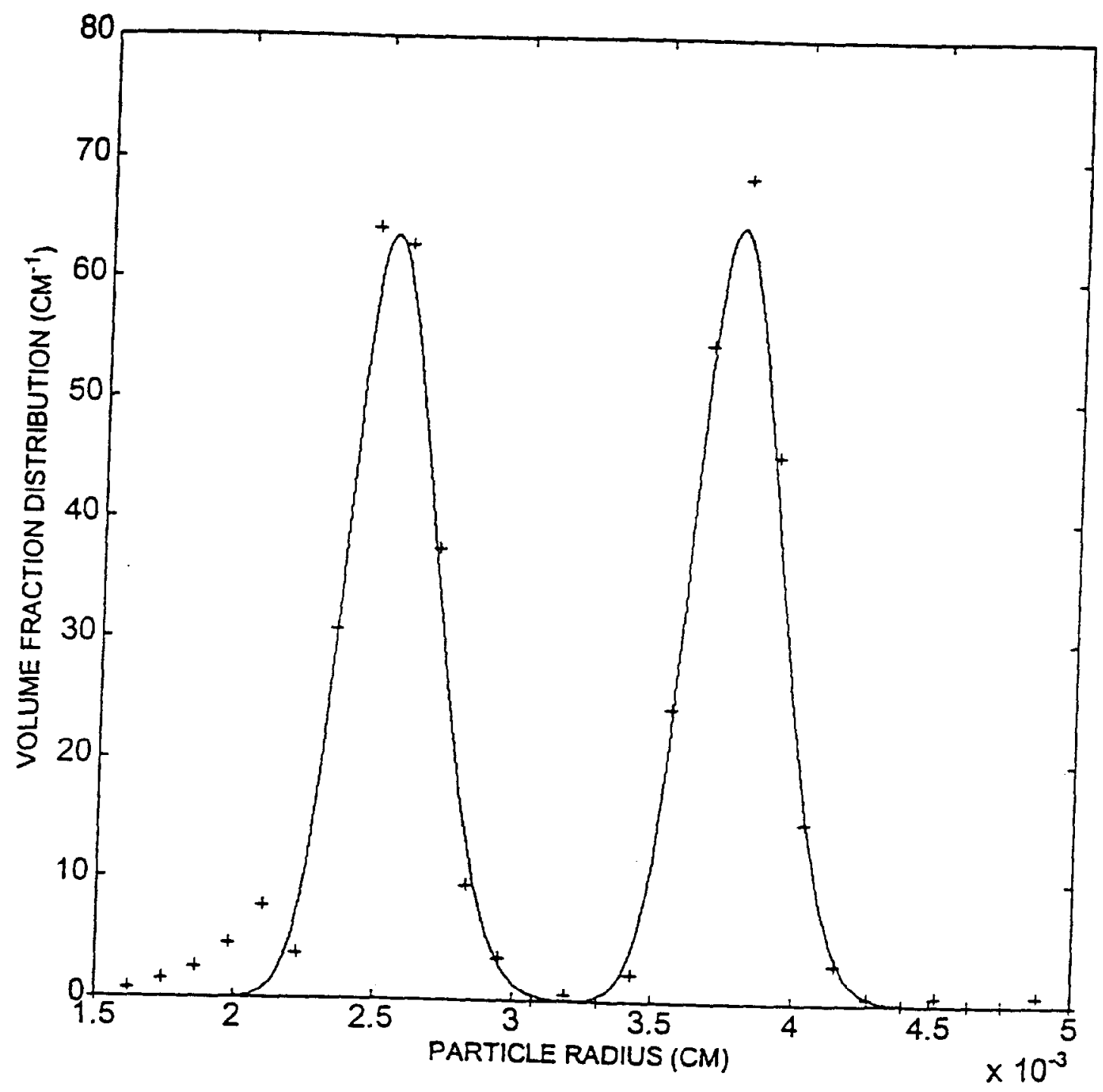

Figure 5.5: Solution of the inverse problem for a bimodal distribution of polystyrene particles, using 30 particle size bins. The solid curve is the exact result, and the markers represent the inverse problem solution when using a value of $f_{\max }$ indicated by a square in Figure 5.6. 


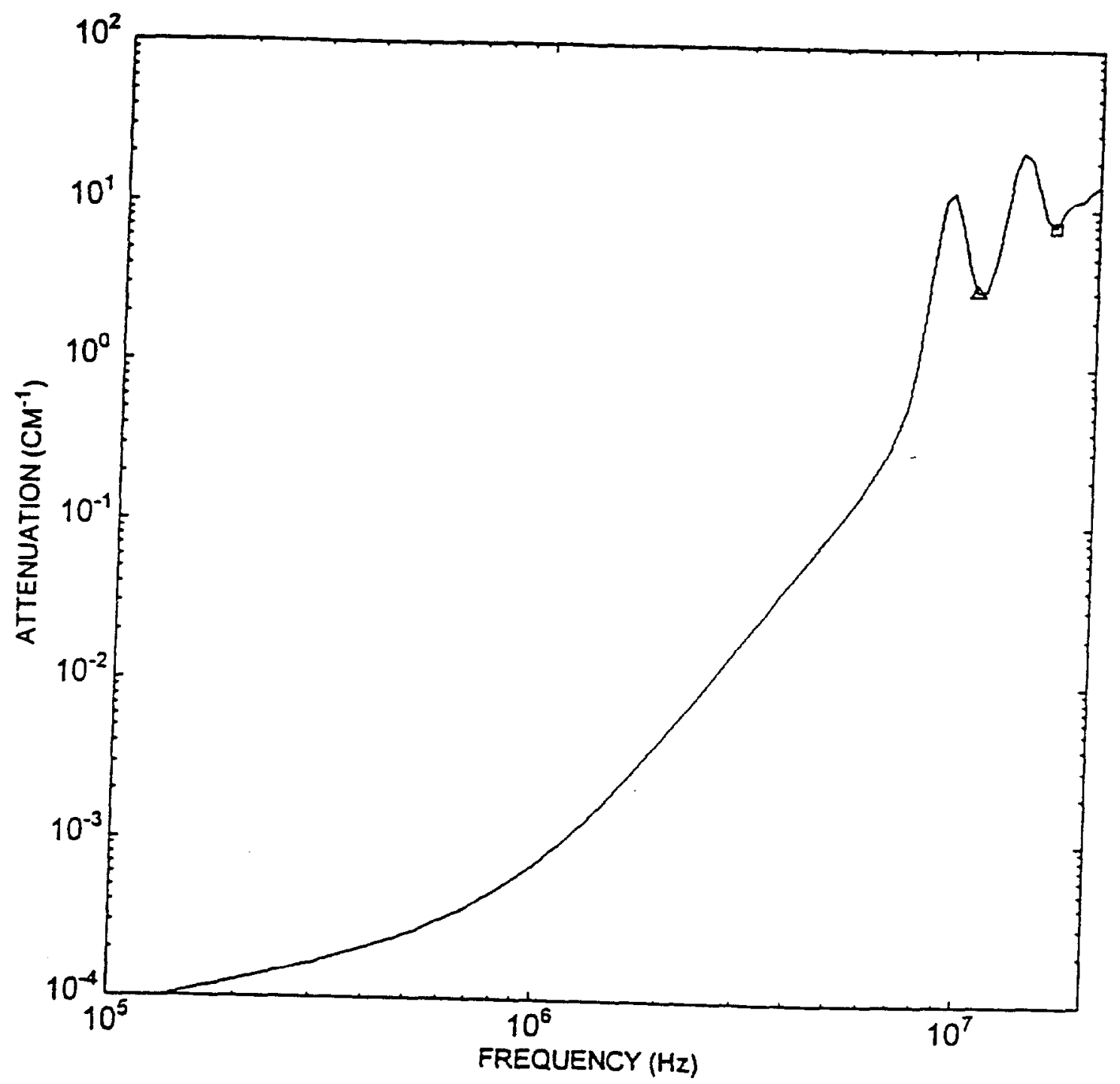

Figure 5.6: Attenuation versus frequency curve for polystyrene particle volume fraction distribution shown in Figure 5.5. 
From the results shown in Figure 5.5 we can see, once again, that the inverse procedure recovers this distribution quite well.

As has been discussed previously, one of the difficulties in solving inverse problems is that they tend to be ill-posed. That is, small errors in the input (attenuation) data can lead to large changes in the solution (volume fraction distribution). Of course, there is always a certain amount of error present in experimentally obtained attenuation data. The inverse problem results presented so far were obtained with no noise added to the input attenuation data. To imitate the practical situation, a random noise of $5 \%$ standard deviation was added to the input data. It turns out this error is approximately of the same order of magnitude as the error in the experimentally obtained attenuation data for polystyrene shown in Figure 4.2. The resulting volume fraction distribution is shown in Figure 5.4 (dash-dot line). The calculations were repeated with a noise of $10 \%$ standard deviation (not shown), and the computed volume fraction distribution was considerably different form the input distribution, but the main features of the distribution were still preserved by the inverse computations.

The results which have so far been presented would suggest that the inverse technique can be employed with reasonable success. There are, however, limitations. The inverse problem technique yielded erroneous volume fraction distributions for smaller particles when the above mentioned frequency range $(0.1 \mathrm{MHz}$ to $17 \mathrm{MHz})$ was used in the computations. In order for the size of the particles to be determined there must be at least one transition in the attenuation versus frequency curve, namely the transition from the thermal attenuation dominated regime to the scattering dominated 
regime which occurs when the dimensionless wavenumber becomes approximately $O(1)$. If the particles are very small this transition may not occur over a fixed frequency range. However, it will be shown that the results are quite sensitive to the frequency range which is chosen for the calculations, even when the transition is included in the frequency range.

Figures $5.7 \mathrm{a} \& \mathrm{~b}$ illustrate the effect of varying the maximum frequency, $f_{\max }$, on the computed volume fraction distribution. As seen in Figure 5.7a the resonance in the shape oscillations of the polystyrene particles leads to a change in the slope of the curve just before the first resonance. This transition occurs just before the point marked with a circle in Figure 5.7a. There appears to be a marked improvement in the results shown in Figure $5.7 \mathrm{~b}$ when $f_{\max }$ is chosen corresponding to a point marked by a cross in Figure $5.7 \mathrm{a}$ over those obtained with the point corresponding to a circle, which does not include the second change in slope. The point marked with a cross corresponds to a frequency greater than the frequency at which the second change in slope occurs for larger particles but less than that for smaller particles. This gives rise to a solution of the inverse problem (cf. Figure $5.7 \mathrm{~b}$ ) which is reasonably accurate for larger particles but not as accurate for smaller particles. Also shown in Figure $5.7 \mathrm{~b}$ are the results when $f_{\max }$ is chosen to coincide with the end of the first resonance peak in the attenuation versus frequency plot. This is the point marked with a square in Figure 5.7a. The resulting volume fraction distribution shows that when the first resonance peak is included in the attenuation data, the resulting volume fraction distribution predicted from the inverse problem solution is quite accurate. 


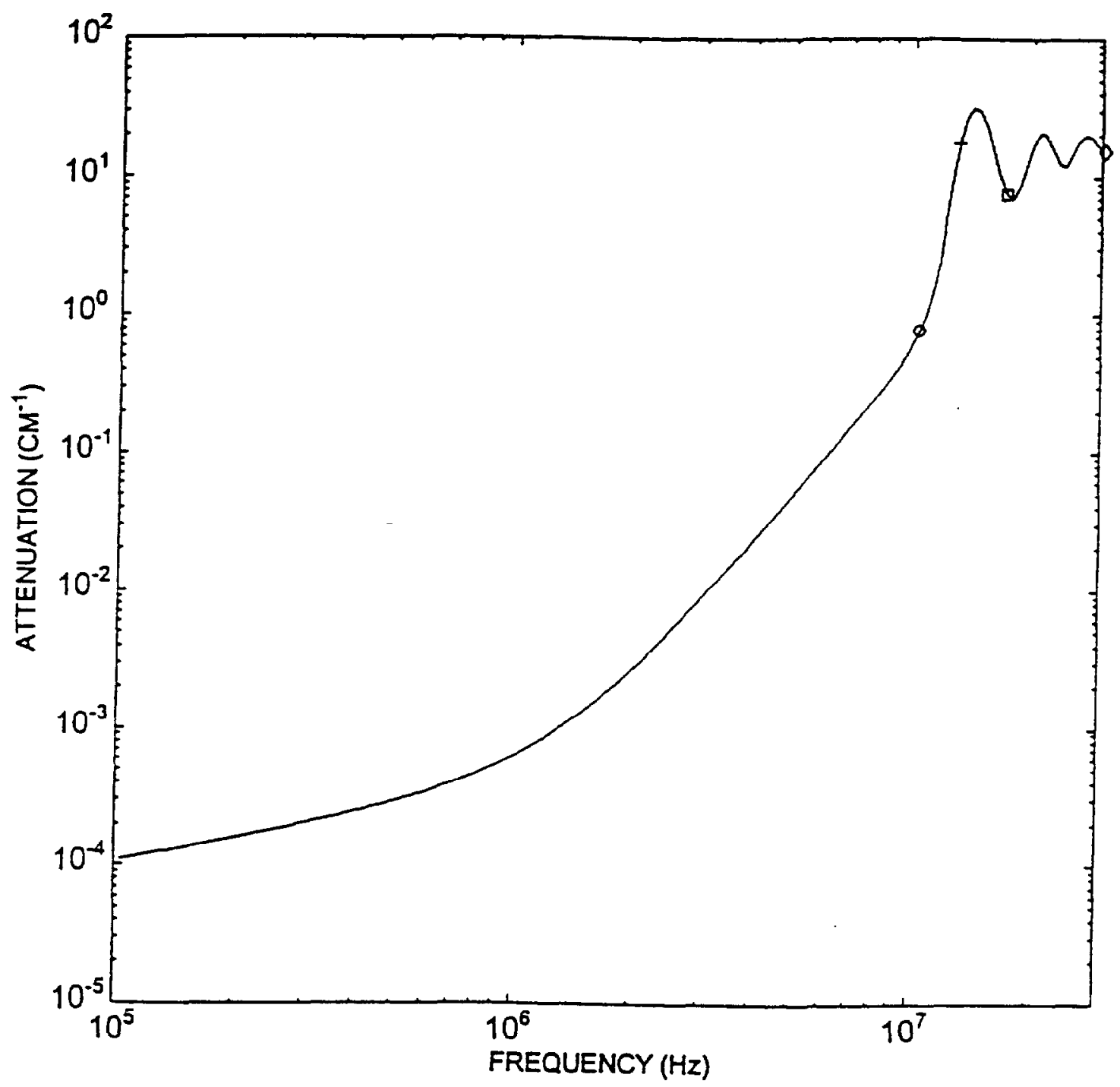

Figure 5.7a: Input attenuation data for four different upper bounds on frequency to determine the influence of the frequency range over which the attenuation is specified on the solution of the inverse problem. This attenuation curve is for polystyrene in water. The solid curve is the exact result; $\square$, cutting off the frequency range after the first attenuation peak; +, cutting off the frequency range just after the second change in slope in the attenuation curve; $\mathrm{O}$, cutting off the frequency range just before the second change in slope in the attenuation curve; and $\diamond$, cutting off the frequency range just after the third attenuation peak. 


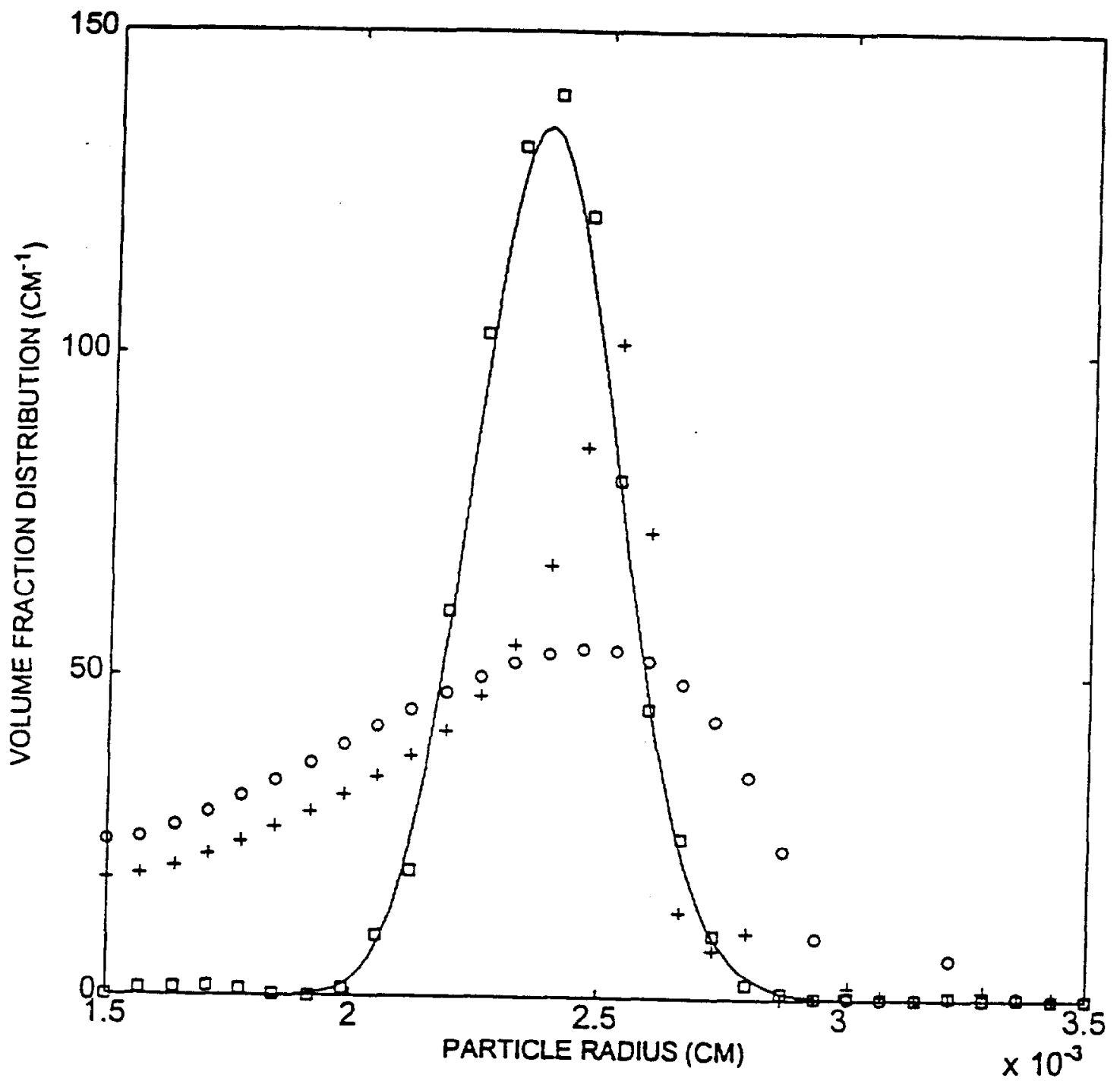

Figure 5.7b: Results of the inverse problem solution for four different frequency ranges using the same marker types as in Figure 5.7a. 
It should, however, be noted that one cannot alleviate all the difficulties mentioned above by merely choosing a wide enough frequency range of interrogation. Figure 5.8 shows results of the inverse problem calculations for three different values of $f_{\max }$. The dashed curve corresponds to terminating the frequency range at the end of the first resonance peak, as in Figure 5.7a. The dash-dot curve corresponds to terminating the frequency range at the end of three resonance peaks; and the dotted curve corresponds to terminating the frequency range at $10^{9} \mathrm{~Hz}$, a frequency which is approximately fifty times greater than the first resonance frequency. Figure 5.8 shows that the results of the inverse calculation actually deteriorate from those calculated after the first resonance peak if a much larger range of frequency is employed. The deterioration of results is due to the different modes of oscillation which may undergo resonance. As is shown in Figure 4.4, a monodisperse suspension will exhibit several resonance frequencies corresponding to various shape oscillation $P_{n}(n=2,3, \ldots)$ modes. Therefore, a second peak in the attenuation versus frequency curve for polystyrene particles could, for example, correspond either to a $P_{3}$ mode for a larger particle, or it could correspond to a $P_{2}$ mode for smaller particle. The inverse calculations performed in this work used only the first six modes ( $n \leq 5)$, but in practice the acoustic response may be further complicated by the higher modes of oscillation for frequencies of the order of $10^{9} \mathrm{~Hz}$ considered here.

Since including an extremely wide frequency range with several resonance peaks seems to adversely affect the inverse calculations, one may consider terminating the attenuation data just beyond the first resonance peak. This strategy may not be successful 


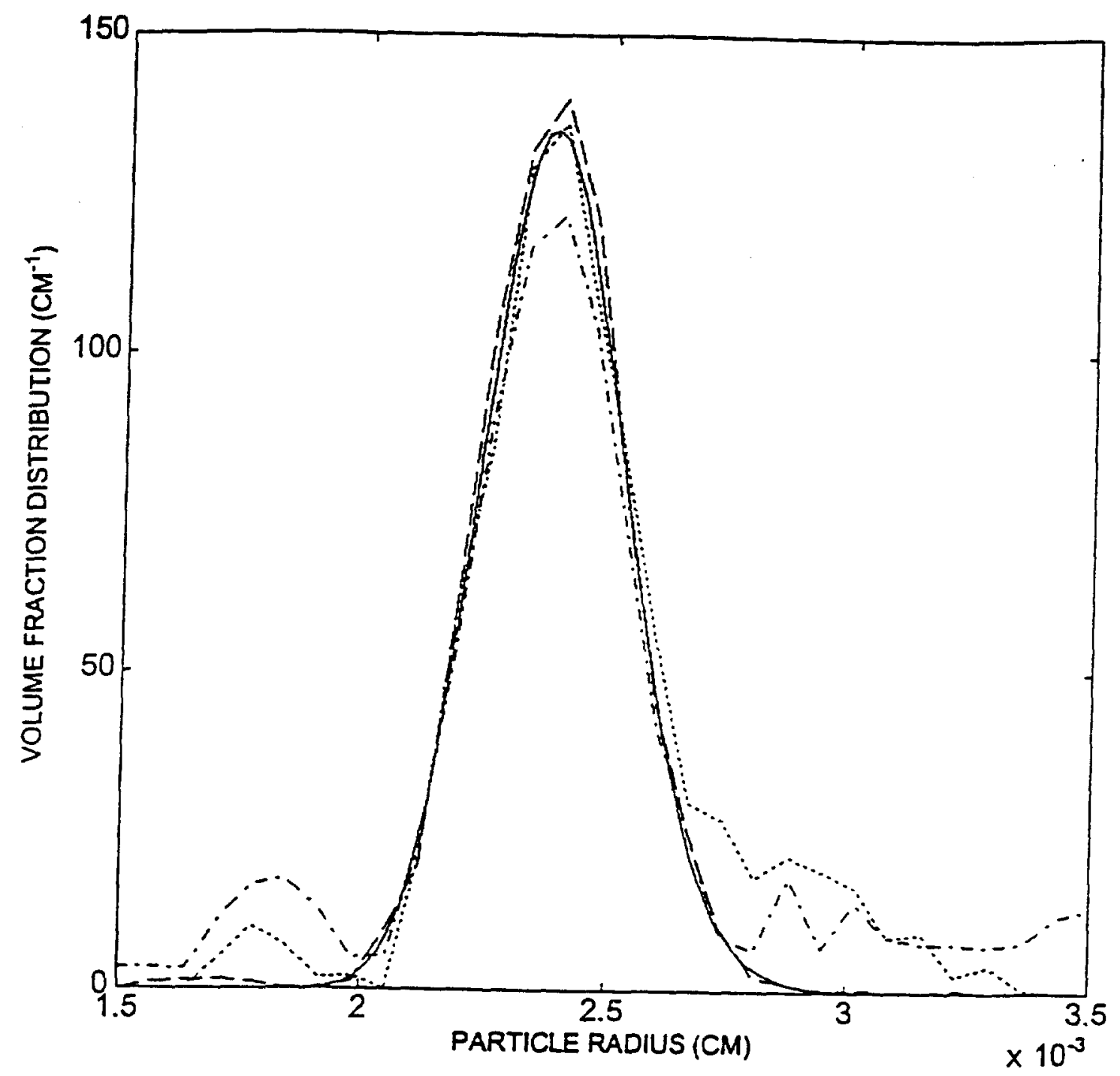

Figure 5.8: Using too large of a frequency range over which the attenuation data are available for polystyrene particles in water can deteriorate the results. The solid curve is the exact result; the dashed curve is the inverse problem result when using the data of Figure 5.7 a below the point marked by a ' $\square$ ' the dash-dotted curve represents the results when this endpoint is shifted to the point marked by ' $O$ '; and the dotted curve is the result when this endpoint is shifted to $1000 \mathrm{MHz}$. 
if the volume fraction distribution is truly bimodal as was seen earlier in Figure 5.5. If the second resonance peak is omitted from the attenuation data by considering a frequency range with a maximum that is less than the frequency marked with a square in Figure 5.6, for example let the maximum be the frequency marked with the triangle; then the inverse calculation yields rather poor results as shown in Figure 5.9. It is interesting to note that the inverse technique computes rather accurately the volume fraction distribution of the larger particles, whose resonance behavior was included in the data; but it fails to accurately predict the volume fraction distribution for the smaller particles, whose resonance behavior is not included in the attenuation data.

Figures $5.10 \mathrm{a} \& \mathrm{~b}$ show the inverse technique results for a very broad unimodal distribution for polystyrene particles. The resonance peaks of the different particles overlap in this case, resulting in the absence of peaks in the attenuation versus frequency curve (cf. Figure 5.10a). Figure 5.10b shows the results of the inverse calculation for three different cut-off frequencies. The largest frequency, marked by a square in Figure $5.10 \mathrm{a}$, is larger than the second transition frequency of small as well as large particles, and this appears to produce excellent results.

We note that in the cases shown so far where the inverse calculations yielded poor results the failure is particularly severe for smaller particles. This phenomenon may be explained when one considers that the total error, $E$, will be dominated by the errors at large frequencies, since the attenuation there is quite large. When $k_{c} a_{\min }<1$ in the frequency domain that is considered, the small particles' volume fraction appears in Figures $5.7 \mathrm{~b}$ and 5.9 to be underestimated, while the large particles' volume fraction is 


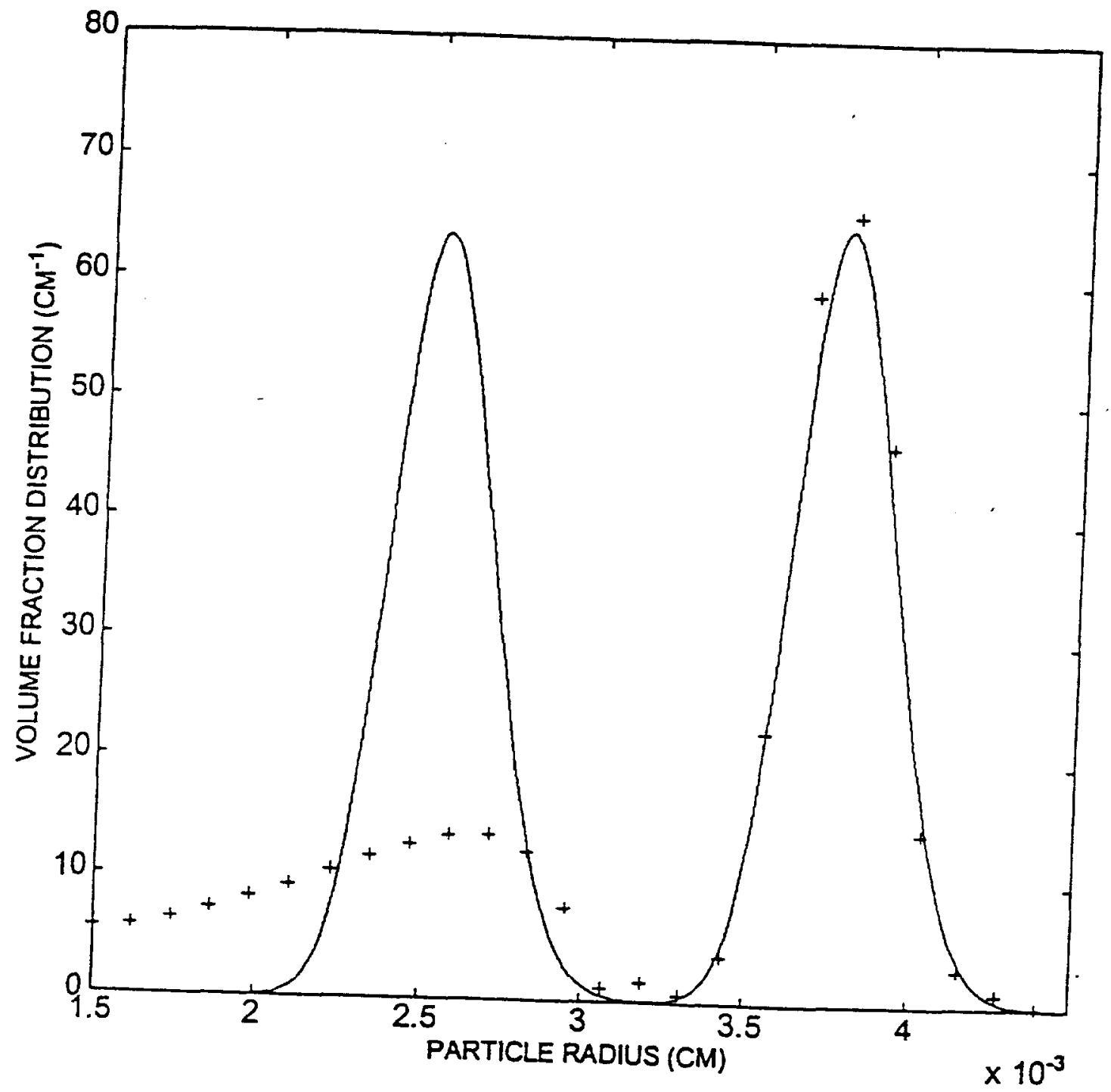

Figure 5.2: Inverse problem solution for a bimodal distribution of polystyrene particles (same distribution as in Figure 5.5) when cutting off the frequency range over which the attenuation was given between the first and second attenuation peaks. 


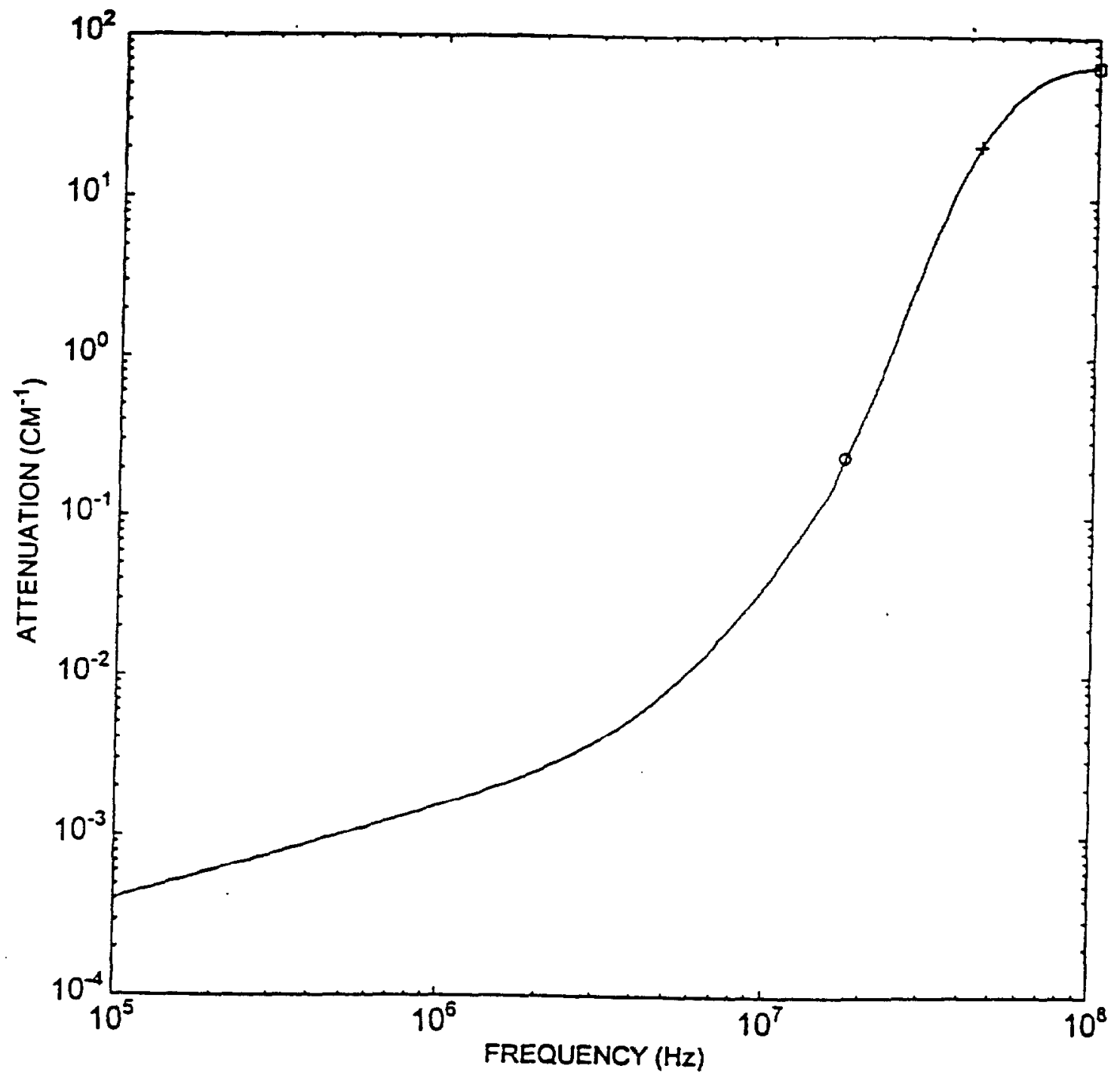

Eigure 5.10a: Attenuation versus frequency curve for the same inverse calculation for polystyrene particles in water whose results are shown in Figures $5.7 \mathrm{a}$ $\&$ b, but with a broader size distribution. 


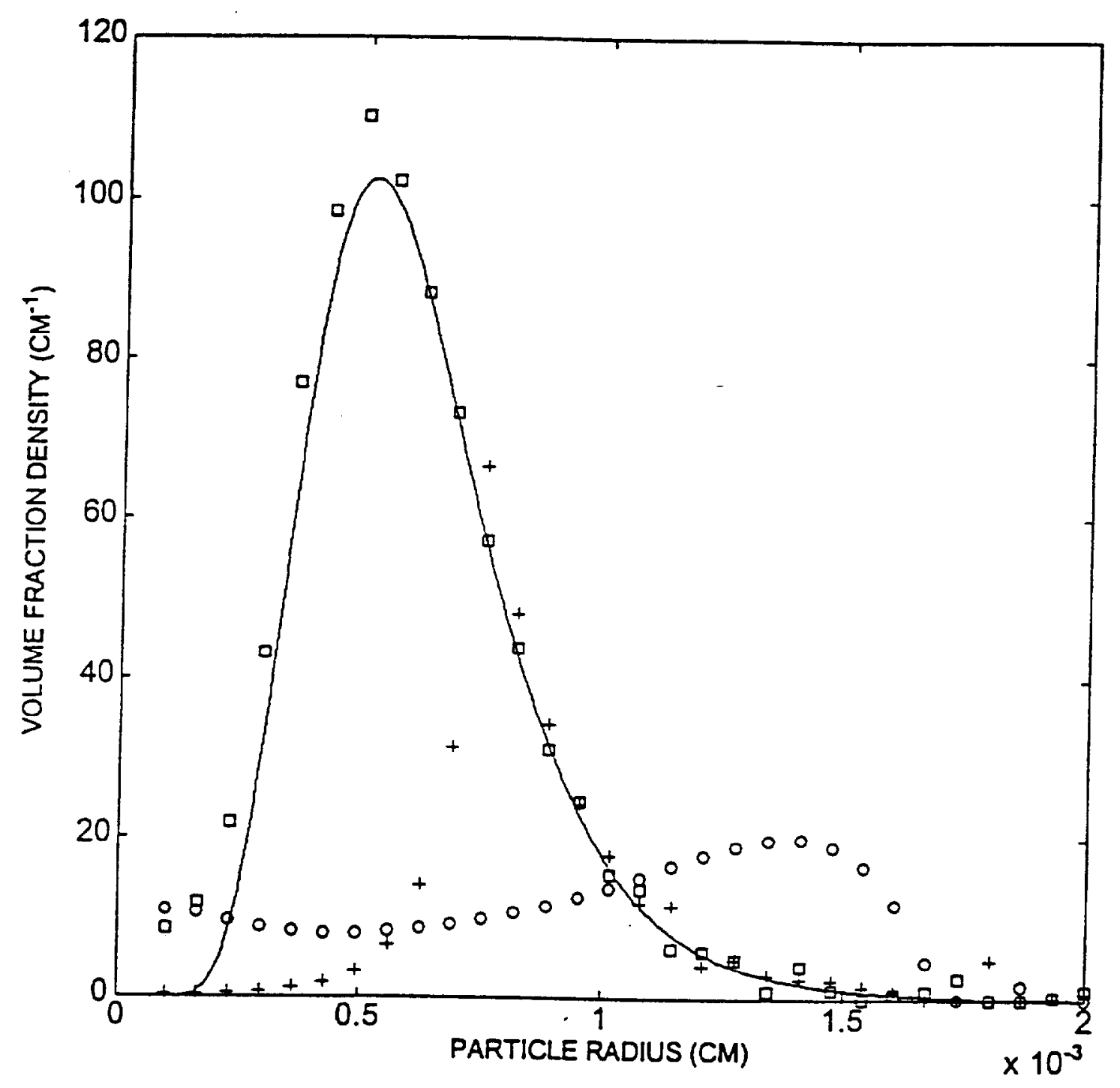

Figure5.10b: Resulting volume fraction distribution for the broader distribution attenuation data shown in Figure 5.10a. 
slightly overestimated. This problem was addressed in some calculations by dividing both the attenuation and $\hat{\alpha}$ by $f^{2}$. However, only small improvements were realized by modifying the attenuation data in this manner. It should be noted that the inverse calculation results presented in Figure 5.9 were obtained in this manner.

Figures $5.11 \mathrm{a}, \mathrm{b}, \& \mathrm{c}$ provide some insight into why the choice of $f_{\max }$ can greatly affect the results of the inverse calculation. These figures show three-dimensional plots of the kernel, $K$ (for the same values of $f_{\max }$ considered in Figures $5.7 \mathrm{a} \& \mathrm{~b}$ ) plotted against particle radius, $a_{i}$ and $a_{j}$. We see that when $f_{\max }=10.4 \mathrm{MHz}$, corresponding to the circle in Figure 5.7a, the kernel has a maximum for $a_{i}=a_{j}=a_{\max }$. The kernel for smaller particles is rather small in comparison; and as a consequence, the inverse technique could determine the larger size particle volume fraction correctly but failed for the smaller particles. In contrast, the kernel for $f_{\max }=17.1 \mathrm{MHz}$, corresponding to the end of the first peak in Figure 5.7a, shows significant variations for a wide range of values of $a_{i}$ and $a_{j}$ (cf. Figure 5.11b). This behavior of the kemel apparently leads to a much more accurate inverse solution. Finally, the kernel for $f_{\max }=30.4 \mathrm{MHz}$, corresponding to the end of the third resonance peak in Figure 5.7a, shows a less pronounced structure (cf. Figure 5.11c) which explains the fact that the inverse results actually deteriorated when using such a wide frequency range.

Inverse problem calculations were also performed to determine the kernel, volume fraction distribution, and attenuation in the bubbly liquids examined by Duraiswami (1993). The inverse procedure worked quite well for bubbly liquids as is shown in Figures 5.12a, b, \& c. Figure 5.12a shows the input and computed bubble volume 


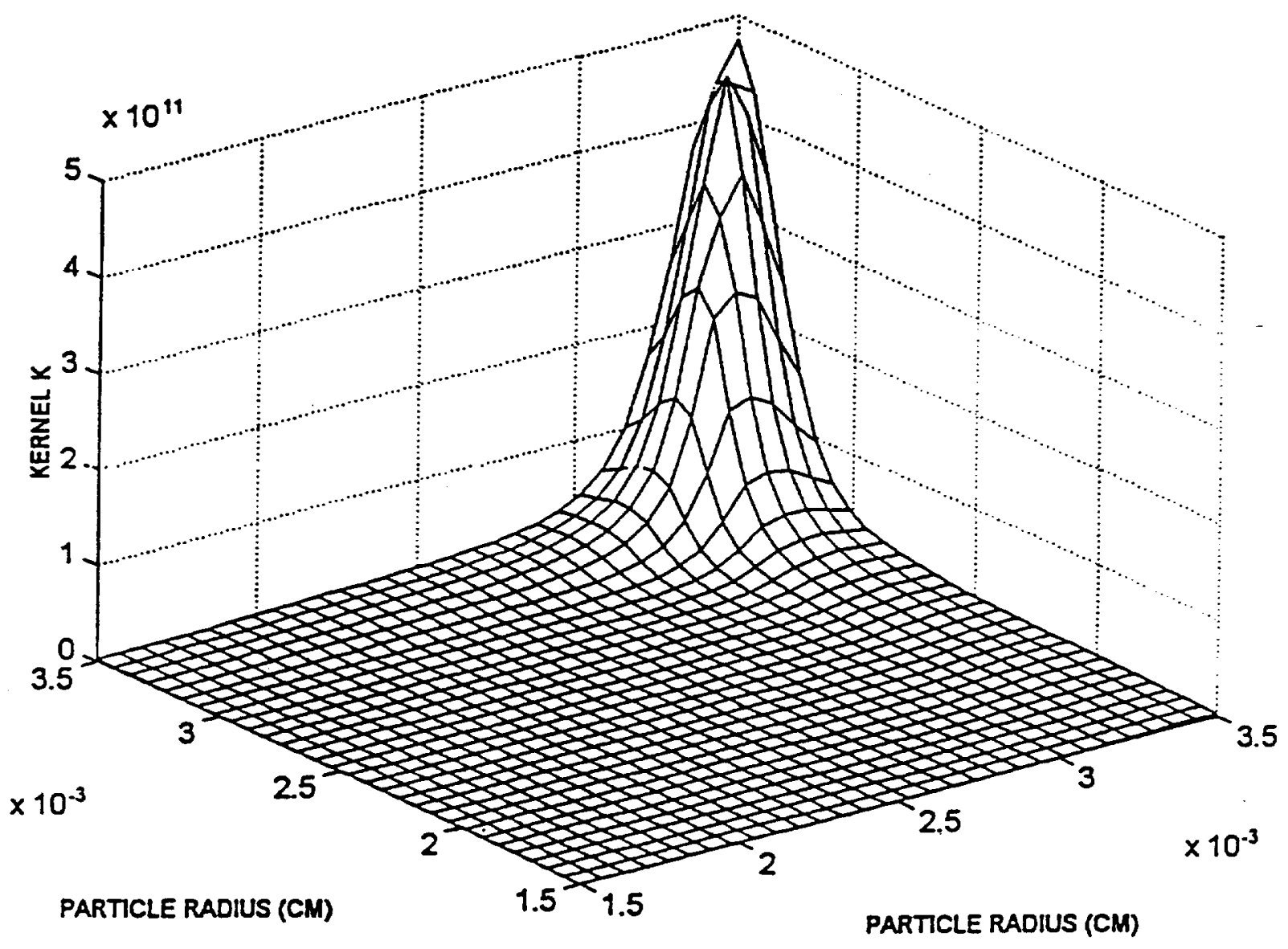

Figure 5.11a: The kernel, $K\left(a_{i}, a_{j}\right)$ for polystyrene particles in water when using for $f_{\max }$ the value of frequency indicated by a ' $\mathrm{O}$ ' in Figure $5.7 \mathrm{a}$. 


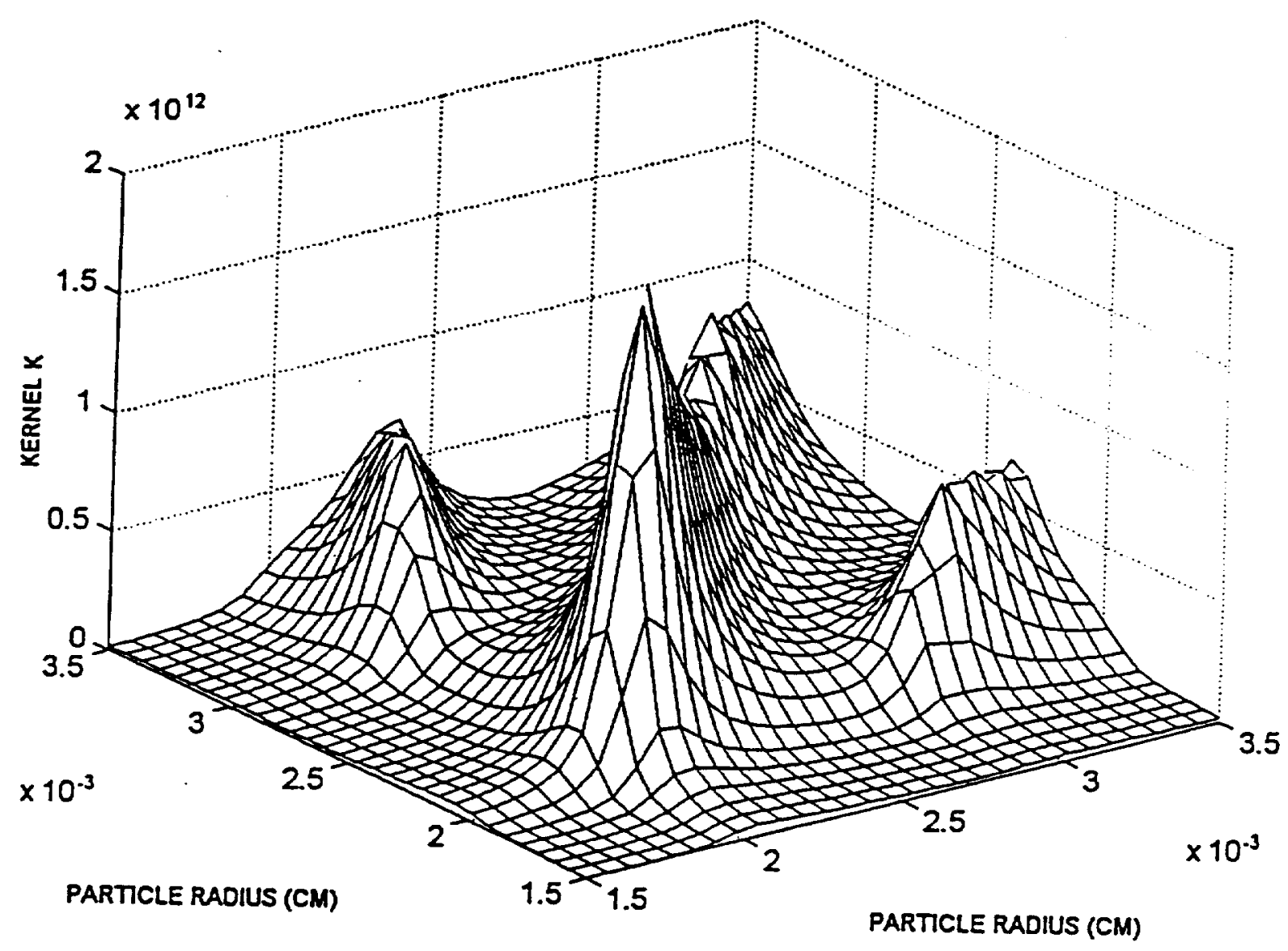

Figure 5.11b: The kemel, $K\left(a_{i}, a_{j}\right)$, for polystyrene particles in water when using for $f_{\max }$ the value of frequency indicated by a ' + ' in Figure $5.7 \mathrm{a}$. 


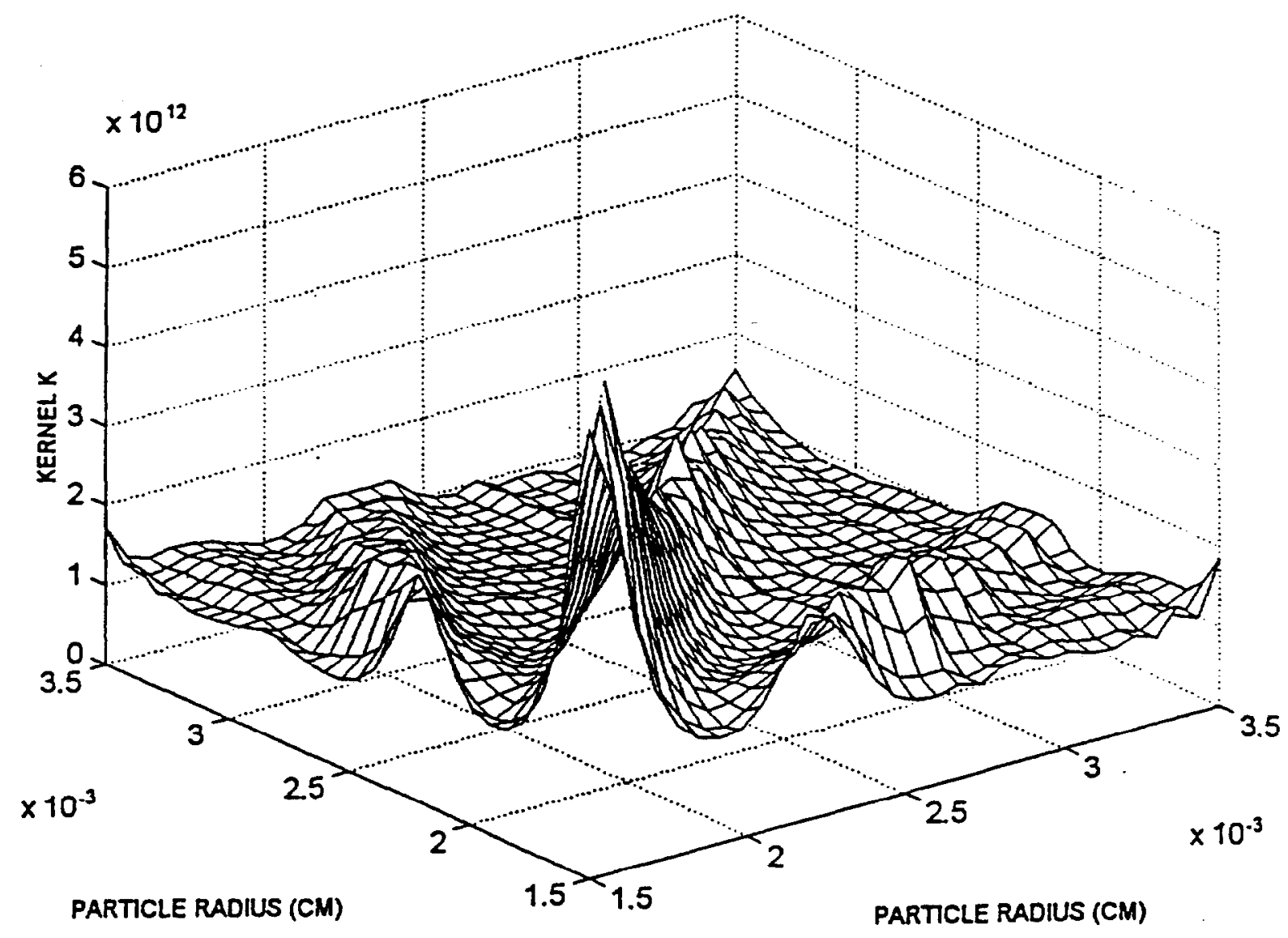

Figure 5.11c: The kernel, $K\left(a_{i}, a_{j}\right)$, for polystyrene particles in water when using for $f_{\max }$ the value of frequency indicated by a ' $\square$ ' in Figure 5.7a. 


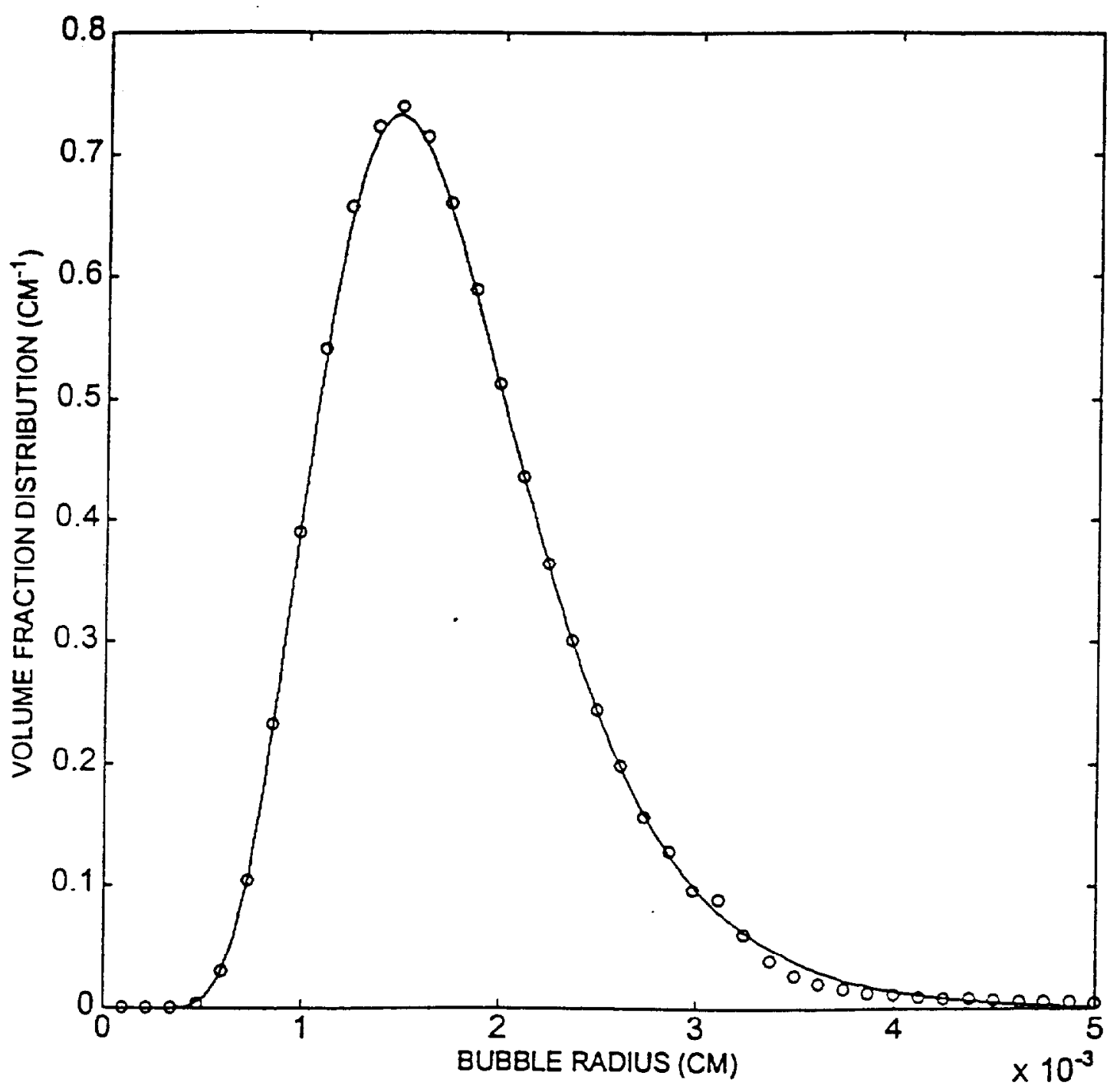

Figure 5.12a: Results of the inverse problem solution for air bubbles in water. Bubble volume fraction distribution for a total gas phase volume fraction equal to 0.004 . 


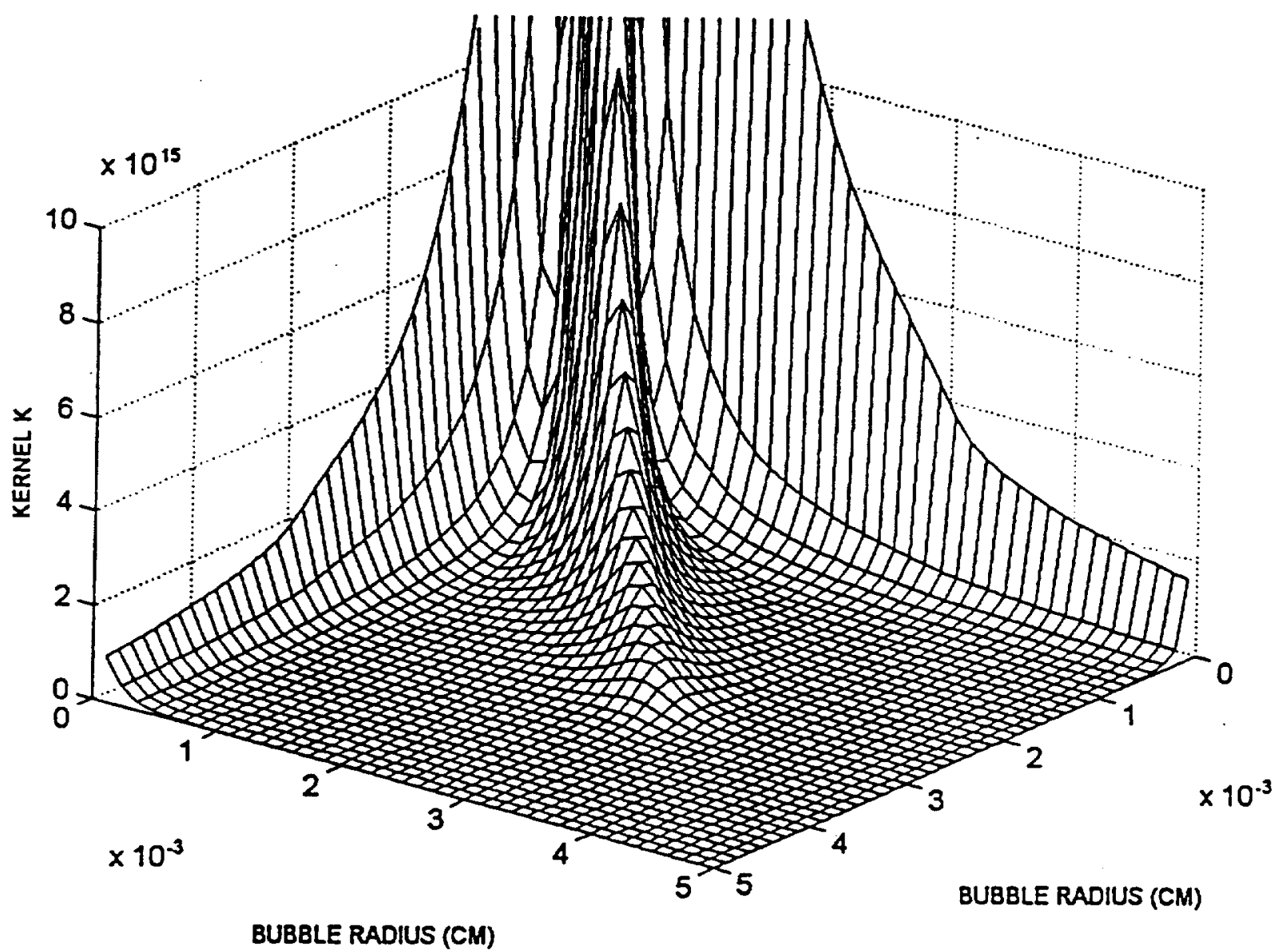

Figure 5.12b: The kernel, $K\left(a_{i}, a_{j}\right)$, for air bubbles in water at a total gas phase volume fraction of 0.004 . 


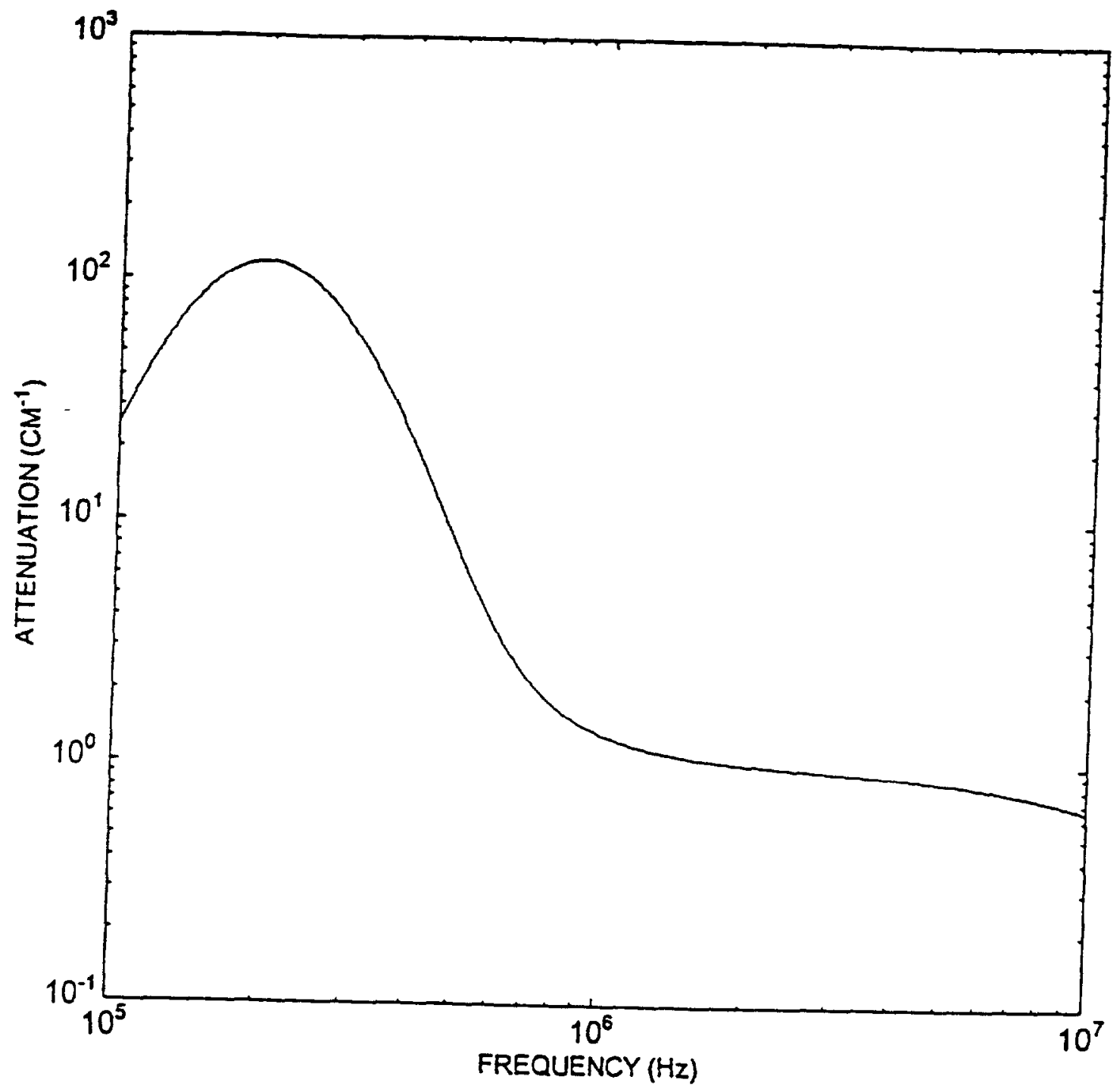

Figure 5.12c: The attenuation spectrum for air bubbles in water at a total gas phase volume fraction of 0.004 . 
fraction distributions to be in excellent agreement. The kernel, as shown in Figure 5.12b, has smooth variations over a wide range of bubble radii. The resulting attenuation as a function of frequency is shown in Figure 5.12c. The main reason why the inverse technique appears to be somewhat more successful for bubbly liquids is that there is only one resonance frequency for bubbles of each size. Therefore, so long as the frequency range is wide enough to cover the resonance frequency of all the bubbles, it is possible to accurately determine the volume fraction distribution.

If one recalls the notable difference between the attenuation data for polystyrene and that for soda-lime glass particles, it was the absence of the multiple resonance peaks for the soda-lime glass particles (cf. Figure 4.5). Along with this fact, it was found that the inverse calculation results for the soda-lime glass particles were generally poor. Figure 5.13a shows an inverse problem result for small soda-lime glass particles which is still reasonable; however, that shown for larger particles in Figure $5.13 \mathrm{~b}$ is quite poor.

The reason for this result lies in the shape of the kernel, which is shown for the latter case in Figure 5.13c. When this kernel is compared with those for polystyrene particles (cf. Figure 5.11b) and for bubbles (cf. Figure 5.12b), it can be seen that the kernel for the soda-lime glass particles is relatively flat. This behavior is indicative of an inverse problem which is rather hard to solve.

Based on the above discussion, it can be said that the success of the Tikhonov regularization in solving the inverse problem is somewhat limited. Although reasons are given as to why this method worked well for bubbly liquids and not as well for slurries, other techniques were examined to investigate whether they might be more generally 


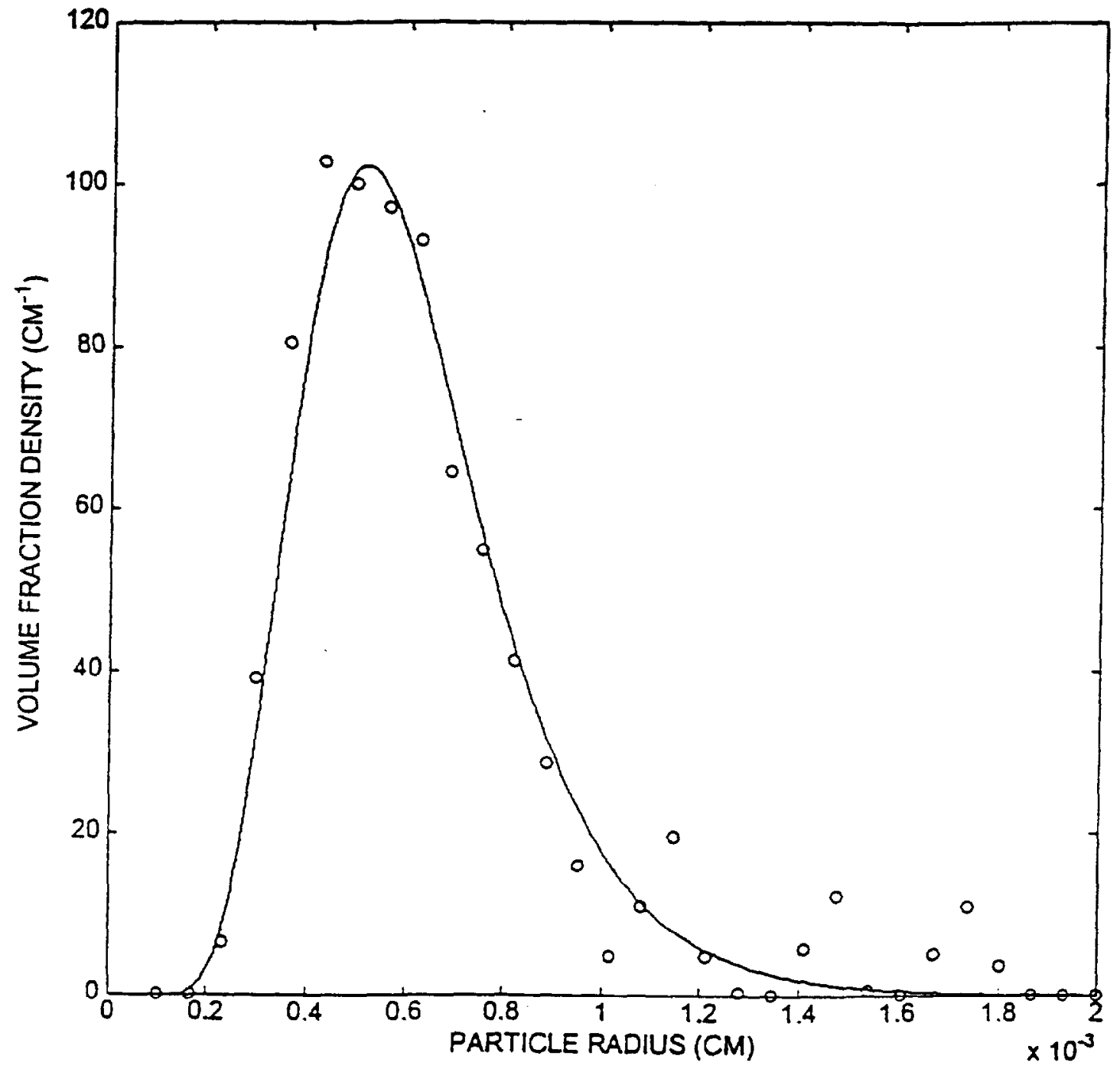

Figure 5.13a: An inverse problem result for small glass particles. 


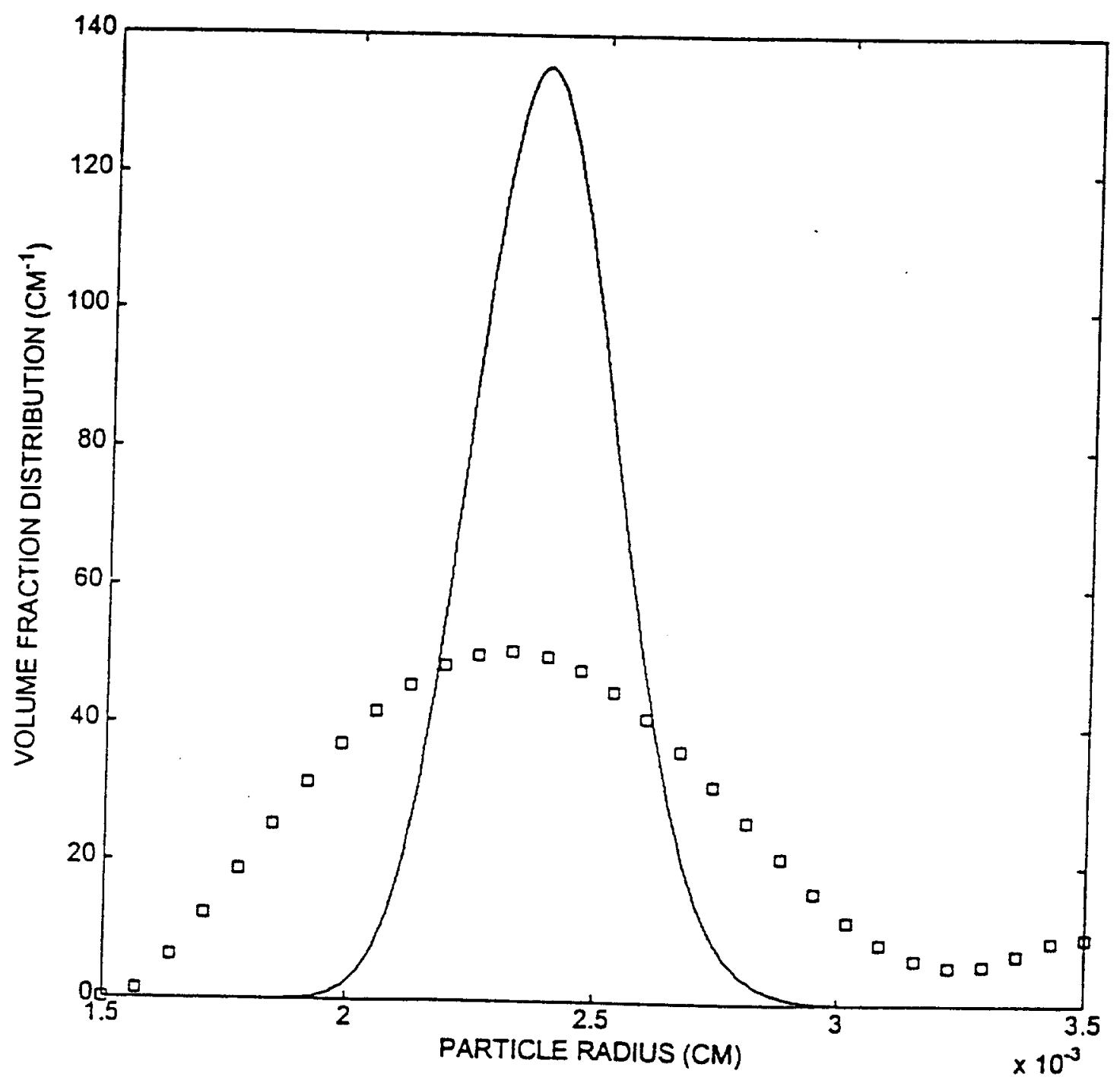

Figure 5.13b: An inverse problem result for larger glass particles. 


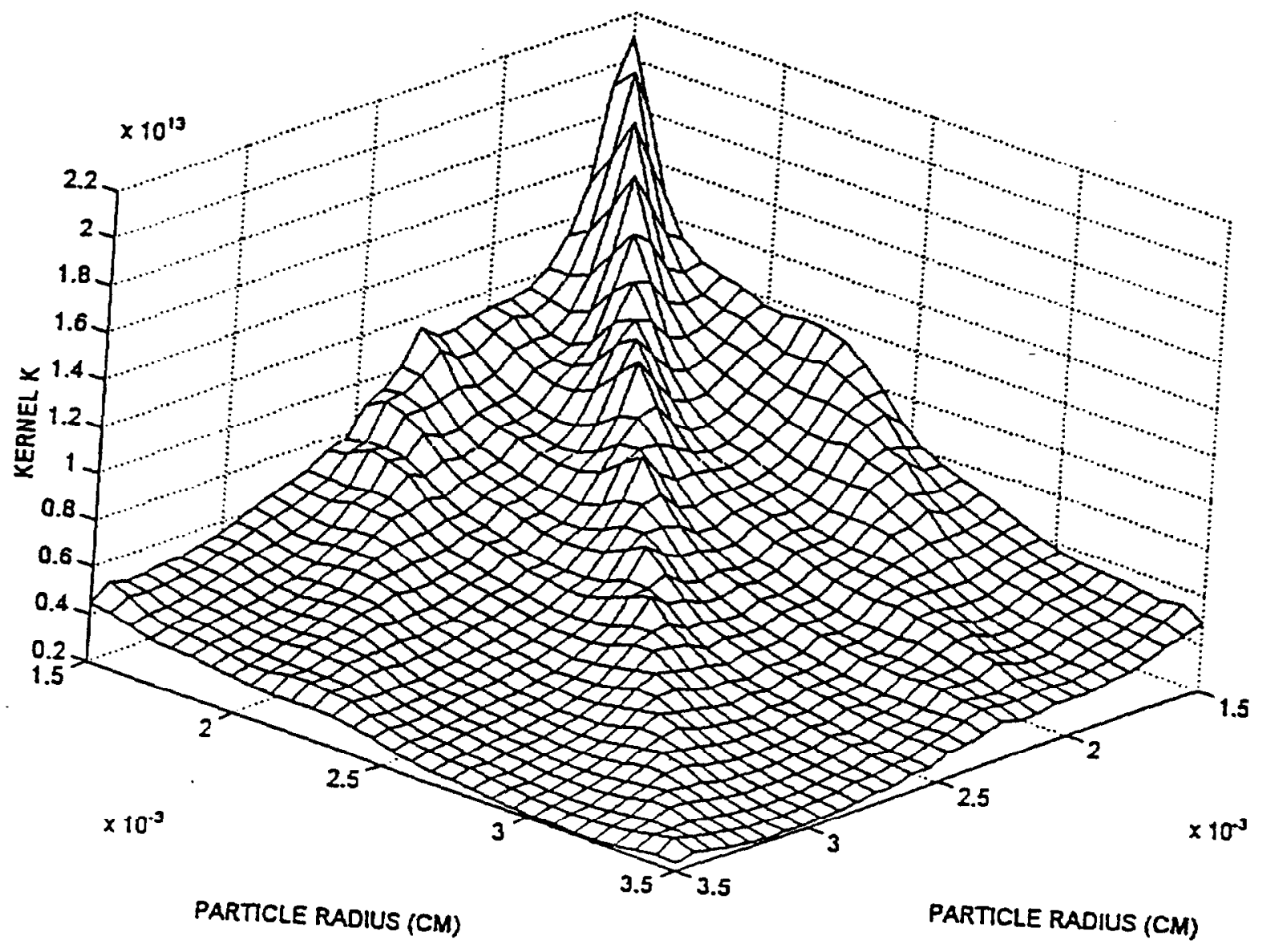

Figure 5.13c: The kemel, $K\left(a_{i}, a_{j}\right)$, for the larger glass particles whose results are shown
in Figure 5.13b. 
successful. With that in mind, an alternative method based on linear programming was attempted.

The constraint $\phi(a) \geq 0$ was satisfied only a posteriori in the Tikhonov scheme. To ensure that the error was minimized while satisfying this constraint, the original inverse problem is reformulated as an optimization problem. The simplest scheme is that to minimize the error

$$
\int_{f_{\min }}^{f \max }\left|\int_{a_{\min }}^{a_{\max }} \hat{\alpha}(f, a) \phi(a) d a-\alpha_{\text {tot }}(f)\right| d f
$$

instead of the integral of the square of the quantity enclosed by the two vertical bars at each frequency. Constraints on the solution are used a priori in optimization via linear programming; here $\phi(a) \geq 0$ is used. Imposing an upper limit on the total volume fraction (maximum packing) can also be incorporated but is not essential. After discretizing the frequency range by $M$ and $\phi(a)$ by $N$ discrete values, one can write

$$
\sum_{j=1}^{N} B_{i j} \phi\left(a_{j}\right)-\alpha_{\text {tot }}\left(f_{i}\right)=u_{i}-v_{i} \quad u_{i}, v_{i} \geq 0 \quad i=1,2, \ldots, M
$$

Here, $B_{i, j}$ is the discretized form of the integral operator in equation (5.8); and $u_{i}$ and $v_{i}$ are, as yet, unknown, non-negative variables. It can be shown (Delves 1985) that minimizing the absolute value of equation (5.9) is equivalent to minimizing

$$
\sum_{i=1}^{M}\left(u_{i}+v_{i}\right)
$$


with equation (5.9) as a constraint together with the constraints $u_{i}, v_{i} \geq 0(i=1, \ldots, M)$ and $\phi\left(a_{i}\right) \geq 0(i=1, \ldots, N)$. It is essential here that at the optimum $u_{i} v_{i}=0$ for each $i$, which makes the solutions of the two minimization problems, equations (5.8) and (5.9), identical.

Although the above linear programming scheme is a completely different method for solving the inverse problem, the results obtained from it were found to be very similar to those obtained from the Tikhonov method. It was shown earlier that the Tikhonov regularization fails when the frequency range over which the attenuation data are provided is made too small. In Figures $5.14 \mathrm{a} \& \mathrm{~b}$ linear programming results are presented for the same problem solved by the Tikhonov method in Figures $5.7 \mathrm{a} \& \mathrm{~b}$. The Tikhonov method results are also presented in Figure 5.14b (open square markers) for comparison. From these figures it is clear that there is no improvement. In fact, there is actually a deterioration of results. Similarly, it was found that increasing the frequency range cut-off beyond the point marked with a square in Figure 5.14a actually made the results worse, as it had in the solution in Figure 5.7a. The other problem where the Tikhonov regularization failed was in the case of relatively large soda-lime glass particles (cf. Figures $5.13 \mathrm{a} \& \mathrm{~b}$ ). The linear programming technique also failed to yield good inverse results for this case 


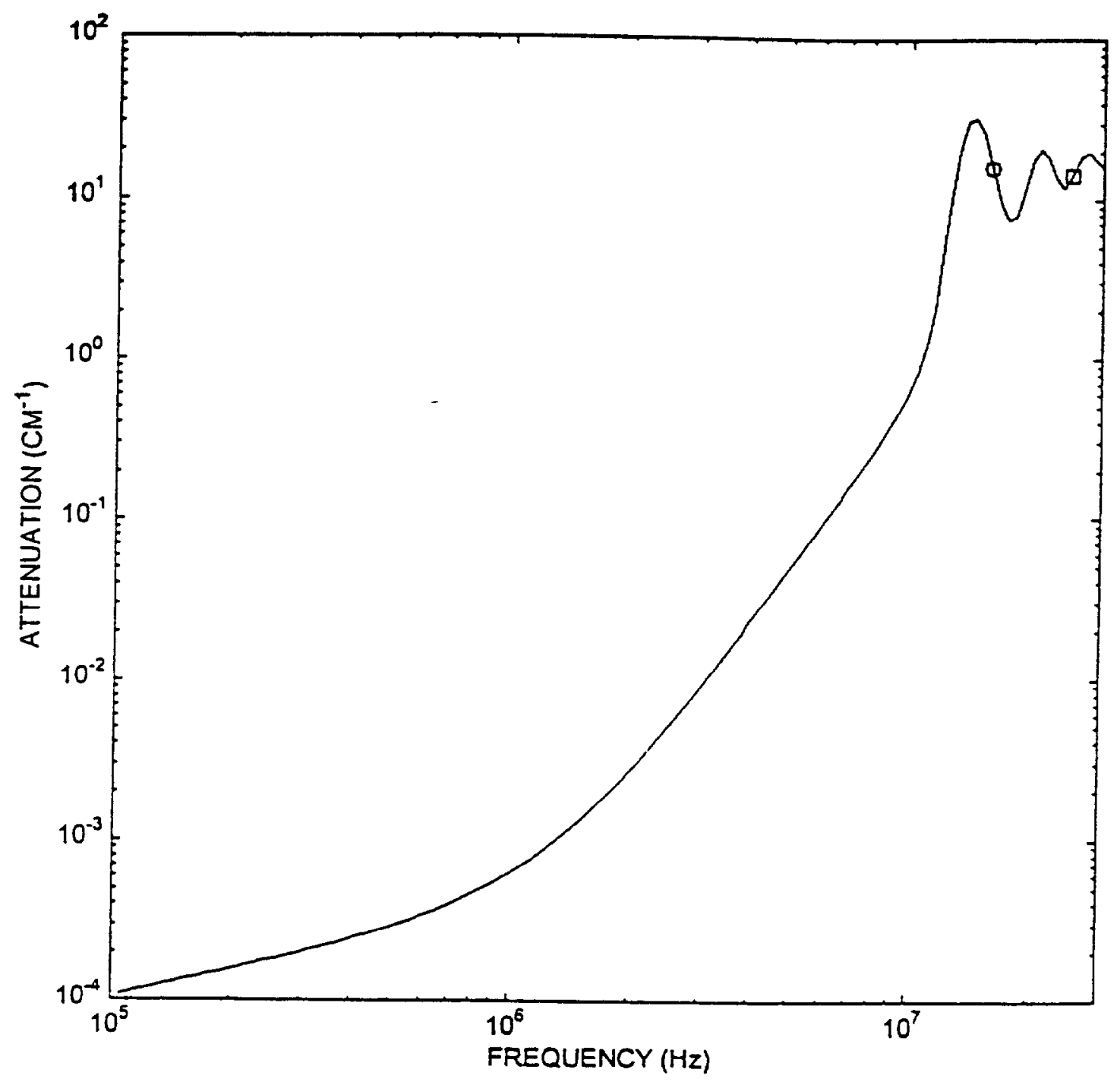

Figure 5.14a: Attenuation spectrum for polystyrene particles in water where the data are used in the linear programming results shown in Figure 5.14b. 


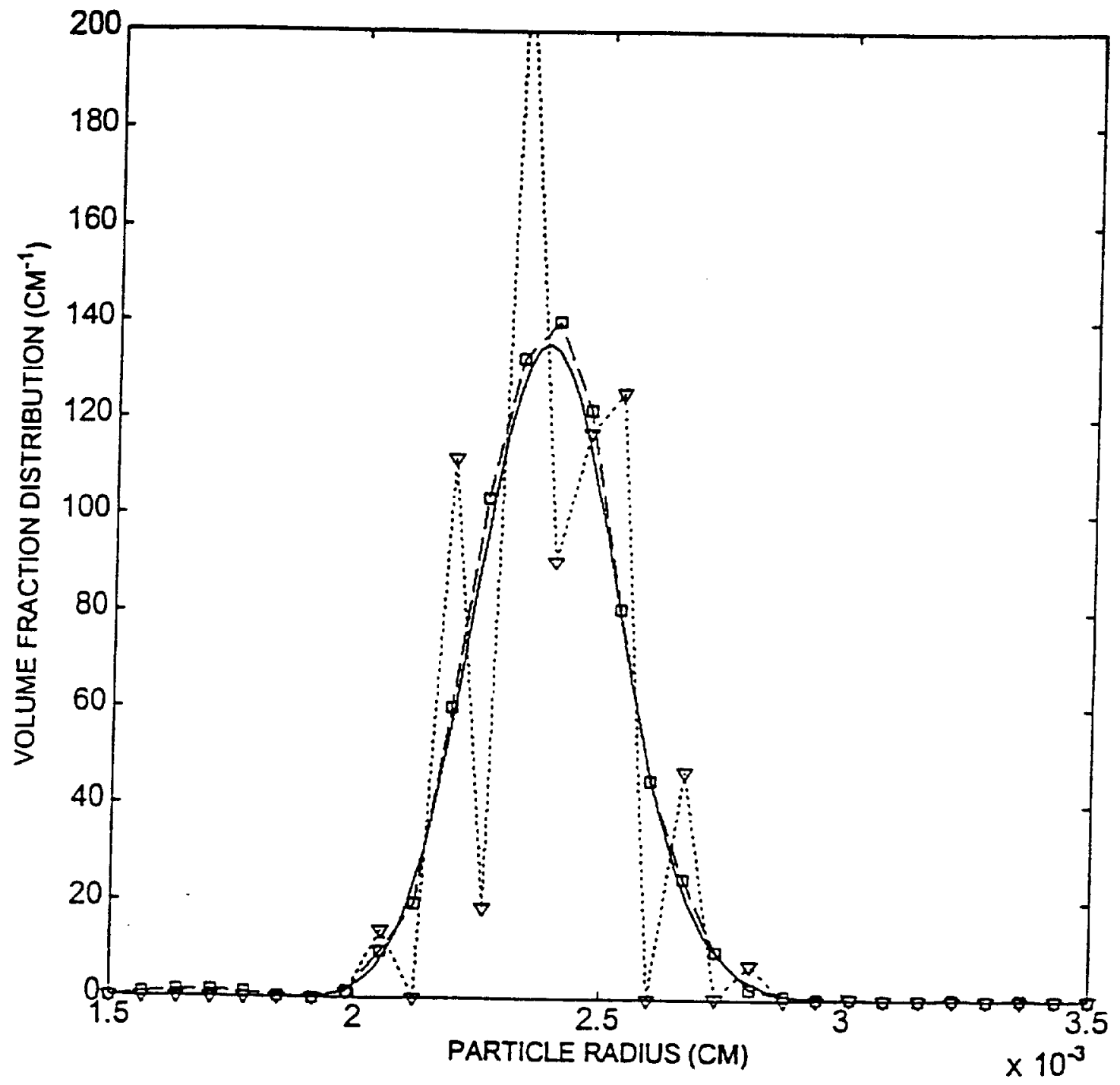

Figure 5.14b: Inverse problem results using the linear programming method. The linear programming results (....$)$ are compared with results of the Tikhonov method (-- $\square--)$ and the exact solution (solid curve). 


\section{Chapter 6: Effective Medium Theory and Experiments for Concentrated Slurries}

\subsection{Effective Medium Theory:}

As mentioned earlier, the measurement of the attenuation of acoustic waves through a suspension of solid or fluid particles can be used to infer the suspension properties.

When the particle volume fraction in the suspension is very small the particle interactions may be neglected, and the attenuation as a function of the sound wave frequency can be determined by examining the interaction of a single particle with the incident wave as has been done by a number of investigators in the past and in Chapter 4 . As mentioned earlier, Foldy (1945) examined the problem of scattering in dilute bubbly liquids. Epstein and Carhart (1953) and Allegra and Hawley (1972) examined, respectively, the case of dilute emulsions and dilute slurries. Since the attenuation behavior is strongly dependent upon particle radius, the attenuation versus frequency data for dilute suspensions may be used to determine particle size distributions, as was shown by Duraiswami (1998) for the case of bubbly liquids. The corresponding problem for dilute slurries has been examined in Chapter 5.

In many processes it is desirable to monitor continuous flow of non-dilute suspensions. The particle interactions can have significant effects on the acoustic behavior of non-dilute suspensions, and at the present, rigorous calculations accounting for their effects are lacking. Direct attack on the problem, i.e. solving the linearized 
energy, momentum, and continuity equations for multiparticle systems, appears daunting even with the development of efficient computers. Thus, it is necessary to develop a suitable approximate theory and to assess its validity by comparison with the experimental data obtained from different kinds of suspensions.

Two approximate theories commonly used for predicting effective properties of non-dilute suspensions are the cell theory and the effective medium theory. The cell theory models particle interaction effects by assuming that each particle is surrounded by a spherical shell of fluid. This particle-cell assembly is assumed to interact with the rest of the suspension in the least possible manner by requiring, for example, that the tangential stress and the heat flux at the boundary between the cell and the surrounding suspension are zero. The cell theory has been used for predicting the average force on a particle in a fixed bed of particles by Happel and Brenner (1973) and for determining the attenuation of sound waves in slurries by Strout (1991). The main criticism of the cell theory is its ad-hoc nature. It is not clear, in general, what conditions must be applied at the cell boundary to ensure the minimal interaction between the cell and the rest of the suspension. Furthermore, it is not even clear that the interaction between the cell and the surrounding suspension must be minimal.

Kuwabara (1959) proposed another model for determining the force on a particle in a fixed bed that assumed that the fluid vorticity, and not the tangential stress, vanishes at the fluid-particle boundary and obtained estimates of the force that are different from those given by Happel and Brenner (1973). 
In the effective medium theory the particle-cell assembly is assumed to be immersed in a uniform suspension with properties which are determined by requiring that the governing equations in the particle-cell assembly are consistent with the averaged equations for the suspension. Semi-theoretical reasoning is used to select proper boundary conditions at the interface between the cell and the effective suspension. This selection of boundary conditions renders the effective medium theories somewhat less $a d$-hoc than the cell theory. As a result, the effective medium theory is the approximate theory of choice whenever rigorous calculations involving multiparticle interactions are not feasible or are to time consuming to be practical.

In recent years, the effective medium theory has proven to yield reasonably accurate estimates in a number of cases for which the detailed multiparticle interaction calculations are carried out with the help of high performance computers. This includes the calculations of the force on a particle in fixed beds, effective viscosity and elasticity of random suspensions (Ladd 1990), the Nusselt number for heat transfer from an array of cylinders (Wang and Sangani 1997), and the diffusivity of proteins in bilipid membranes (Dodd et al. 1995). The effective medium theory is also applied to the light scattering problem, a problem which is quite similar to the acoustic problem of interest here; and its predictions are shown to compare well with experiments on the light scattered by suspensions (Ma et al. (1990)).

In this chapter an effective medium theory is developed to predict the attenuation of sound waves in non-dilute monodispersed suspensions. The accuracy of the theory is 
assessed by comparing its predictions against the experimental data available in the literature and with new data obtained in this study.

\subsubsection{Linearized Equations:}

Consider a small amplitude plane acoustic wave with frequency, $\omega$, propagating through a uniform, monodisperse suspension of solid particles of radius, $a$. Let us write the density as $\rho_{0}+\rho e^{-i \omega t}$, the temperature as $T_{0}+T e^{-i \omega t}$, and the velocity as $\mathbf{u} e^{-i \omega t}$. When the amplitudes of $\rho, \mathbf{u}$, and $T$ are small, the terms involving the products of these quantities can be neglected from the continuity, momentum, and energy equations to obtain the following linearized equations:

$$
\begin{gathered}
\cdot-i \omega \rho+\rho_{0} \nabla \bullet \mathbf{u}=0, \\
-i \omega \rho_{0} u_{i}=\frac{\partial \sigma_{i j}}{\partial x_{j}}, \\
\text { and } \quad-i \omega \rho_{0} C_{v} T=-\frac{\partial q_{j}}{\partial x_{j}}-\rho_{0} C_{v} \beta^{-1}(\gamma-1) \nabla \bullet \mathbf{u} .
\end{gathered}
$$

In writing equation (6.3), we have made use of the linearized equation of state to eliminate the pressure from the usual energy equation. The stress tensor amplitude, $\sigma_{i j}$, for a Newtonian fluid is given by

$$
\sigma_{i j}=\left[\left\{\frac{c^{2} \rho_{0}}{-i \omega \gamma}+\mu_{v}\right\} \nabla \cdot \mathbf{u}-\left\{\frac{\rho_{0}(\gamma-1) C_{v}}{\beta T_{0}}\right\} T\right] \delta_{i j}+d_{i j},
$$


where $d_{i j}$ is the deviatoric component of the rate of strain tensor amplitude

$$
d_{i j}=\mu\left[\frac{\partial u_{i}}{\partial x_{j}}+\frac{\partial u_{j}}{\partial x_{i}}-\frac{2}{3} \delta_{i j} \nabla \cdot \mathbf{u}\right]
$$

In the above equations $C_{v}$ is the constant volume heat capacity and $\gamma=C_{p} / C_{v}$ is the ratio of specific heats, $\mu$ and $\mu_{\nu}$ are the shear and bulk coefficients of viscosity, $c$ is the speed of sound through the fluid, and $\beta$ is the coefficient of thermal expansion. It should be noted that the first and third terms inside the square brackets on the right-hand side of equation (6.4) are related to the thermodynamic pressure amplitude by

$$
p=\left(\frac{\partial p}{\partial \rho}\right)_{T=T_{0}} \rho+\left(\frac{\partial p}{\partial T}\right)_{\rho=\rho_{0}} T=\frac{c^{2}}{\gamma} \rho+\frac{\rho_{0}(\gamma-1) C_{v}}{\beta T_{0}} T
$$

Finally, $q_{j}=-k \partial T / 2 x_{j}$ in equation (6.3) is the heat flux, $k$ being the thermal conductivity.

Inside the solid particles equations similar to equations (6.1) through (6.3) apply with the stress tensor, in this case given by Landau and Lifshitz (1986) as

$$
\sigma_{i j}=\left[\left\{\frac{\tilde{\lambda}+\frac{2}{3} \tilde{\mu}}{-i \omega}\right\} \nabla \cdot \mathbf{u}-\left\{\frac{\rho_{0}(\gamma-1) C_{v}}{\beta T_{0}}\right\} T\right] \delta_{i j}+\tilde{d}_{i j}
$$

where $\tilde{\lambda}$ and $\tilde{\mu}$ are the Lamé constants for the particles, which are assumed to be perfectly elastic. Note that for solids it is customary to write the stress in terms of displacement and not velocity. For small amplitude oscillatory motions the amplitudes of the two are, of course, related by a factor of $1 /(-i \omega)$, and this fact has been used in 
writing the first term on the right-hand side of the above equation. Note also that the factor $\tilde{\lambda}+(2 / 3) \tilde{\mu}$ is the bulk modulus of the solid. Thus, the isotropic part of the stress tensor represented by the terms inside the square brackets in the above equation arises from the density and temperature changes in the solid. The deviatoric stress tensor, $\widetilde{d}_{i j}$, is defined in manner similar to equation (6.5), but with the fluid viscosity replaced by $\mu_{P}=\widetilde{\mu} /(-i \omega)$. This term may be thought of as a "particle viscosity". It should be noted that the Lamè constant, $\tilde{\mu}$, is sometimes referred to as the shear modulus.

The above equations must be solved subject to the boundary conditions of continuity of velocity, temperature, heat flux, and traction $\left(\sigma_{i j} n_{j}, n_{j}\right.$ being the unit outward normal at the particle surface) at the interface between the particles and the fluid. In concentrated suspensions particle interactions are significant and the rigorous evaluation of sound wave speed and attenuation through the suspension would require a very difficult task of solving the above set of equations in a domain containing many particles. We shall introduce suitable approximations to convert this multiparticle problem to a single particle problem in Section 6.1.4. But first, we shall derive ensemble-averaged equations for the suspension and show how their solutions can be related to the overall speed and attenuation of waves through the suspension.

\subsubsection{Ensemble Averaged Linearized Equations for Suspensions:}

In this section we ensemble average the equations for the amplitudes of density, velocity, and temperature in the fluid and solid phases, and obtain, thereby, the linearized 
continuity, momentum, and energy equations for the suspension. It will be shown that the resulting equations have a form similar to the equations for a single phase, provided that the suspension is assigned suitable properties, which we refer to as the effective properties of the suspension. An important outcome of the averaging process will be that it will yield rigorous expressions for various effective properties of the suspension.

Let $g(\mathbf{x})$ be the particle indicator function defined to be unity when the point, $\mathbf{x}$, is inside any of the particles and zero when $\mathbf{x}$ is in the fluid. The properties of the liquid and particles are denoted by the subscripts, $L$ and $P$, respectively. The ensemble averaged variables are denoted by angular brackets.

Multiplying the continuity equation for the liquid by the liquid indicator function, (1-g); and that for the solid particle by $g$; adding the two; and averaging the resulting equation yields the continuity equation for the suspension

$$
-i \omega\langle\rho\rangle+\rho_{0 L}\left\langle(1-g) \nabla \bullet \mathbf{u}_{L}\right\rangle+\rho_{0 P}\left\langle g \nabla \bullet \mathbf{u}_{P}\right\rangle=0 .
$$

The last two terms on the left-hand side of equation (6.8) must now be expressed in terms of the divergence of the average velocity, i.e. $\langle\nabla \bullet \mathbf{u}\rangle$, so that the resulting equation resembles the continuity equation of a single phase medium (cf. equation (6.1)). We begin with the identity

$$
\begin{aligned}
& \rho_{0 L}\left\langle(1-g) \nabla \bullet \mathbf{u}_{L}\right\rangle+\rho_{0 P}\left\langle g \nabla \bullet \mathbf{u}_{P}\right\rangle \\
& \quad=\rho_{0 L} \nabla \bullet\langle\mathbf{u}\rangle+\left(\rho_{0 P}-\rho_{0 L}\right)\left\langle g \nabla \bullet \mathbf{u}_{P}\right\rangle+\rho_{0 L}\left\langle\left(\mathbf{u}_{L}-\mathbf{u}_{P}\right) \bullet \nabla g\right\rangle
\end{aligned}
$$


The gradient of the indicator function is zero at all points except at the particle-fluid interface, where it is proportional to the Dirac delta function owing to the step jump in $g$ across the particle-fluid interface.

More specifically, $\quad \nabla g=-\mathbf{n} \delta\left(\mathbf{x}-\mathbf{x}_{S}\right)$

where $\mathbf{x}=\mathbf{x} S$ represents the surface of the particles; $\delta$ is the Dirac delta function; and $\mathbf{n}$ is the unit normal vector pointing into the liquid, at the particle surface. At the particleliquid interface it is required that the velocity be continuous. Because the velocity is continuous across the solid-liquid interfaces, the last term in equation (6.9) vanishes. The second term on the right-hand side of equation (6.9) still contains as an unknown the quantity, $\left\langle g \nabla \bullet \mathbf{u}_{P}\right\rangle$. Since the equations of small amplitude acoustics are linear, it is expected that this quantity depends linearly on $\langle\nabla \bullet \mathbf{u}\rangle$. Therefore, one can write this term as

$$
\phi \lambda_{\rho} \nabla \bullet\langle\mathbf{u}\rangle(\mathbf{x})=\left\langle g(\mathbf{x})\left(\nabla \bullet \mathbf{u}_{P}\right)(\mathbf{x})\right\rangle,
$$

where $\phi$ is the volume fraction of the solid particles. Substituting $\left\langle g \nabla \bullet \mathbf{u}_{P}\right\rangle$ from equation (6.11) into equation (6.9) yields the continuity equation for the suspension (equation (6.8)) to be given by

$$
-i \omega\langle\rho\rangle+\left\langle\rho_{0}\right\rangle_{c} \nabla \cdot\langle\mathbf{u}\rangle=0,
$$

with the effective equilibrium density of the suspension to be used in the suspension continuity equation, i.e. $\left\langle\rho_{0}\right\rangle_{c}$ is given by 
Physically, $\lambda_{v}$, represents the ratio of the average velocity amplitude inside the particles to that in the suspension. Once again, this coefficient, and such other coefficients to be introduced in this section will, in general, depend on complex multiparticle interactions; and details of its evaluation will be described later.

The right-hand side of equation (6.14) can be simplified by using the identity

$$
\begin{aligned}
\frac{\partial\left\langle\sigma_{i j}\right\rangle}{\partial x_{j}} & \equiv \frac{\partial}{\partial x_{j}}\left\langle g \sigma_{i j}^{P}+(1-g) \sigma_{i j}^{L}\right\rangle \\
& =\left\langle g \frac{\partial \sigma_{i j}^{P}}{\partial x_{j}}\right\rangle+\left\langle(1-g) \frac{\partial \sigma_{i j}^{L}}{\partial x_{j}}\right\rangle+\left\langle\left(\sigma_{i j}^{P}-\sigma_{i j}^{L}\right) \frac{\partial \mathrm{g}}{\partial x_{j}}\right\rangle
\end{aligned}
$$

The last term in the equation (6.17), is related to the jump in traction across the interface and vanishes owing to the boundary condition that $\sigma_{i j} n_{j}$ be continuous at the particlefluid interface. Thus, it follows that the right-hand side of equation (6.14) simply equals the divergence of the average stress in the suspension, i.e. the momentum equation for the suspension is given by

$$
-i \omega\left\langle\rho_{0}\right\rangle_{m}\left\langle u_{i}\right\rangle=\frac{\partial\left\langle\sigma_{i j}\right\rangle}{\partial x_{j}}
$$

We must now supplement the above momentum equation with an expression for the average stress. The linearity of the equations implies that the stress amplitude will be linear in the gradient of the average velocity amplitude and $\langle T\rangle$. 
Let us first consider the isotropic part of the stress, or equivalently, the stress trace. Multiplying the isotropic part in equation (6.4) by $(1-g)$ and that in equation (6.7) by $g$ and averaging, we obtain

$$
\frac{1}{3}\left\langle\sigma_{k k}\right\rangle=\left\{\frac{\left(c^{2} \rho_{0} / \gamma\right)_{m}}{-i \omega}+\mu_{v, e f f}\right\} \nabla \cdot\langle\mathbf{u}\rangle-\left\langle\frac{\rho_{0}(\gamma-1) C_{v}}{\beta T_{0}}\right\rangle_{m}\langle T\rangle,
$$

with

$$
\begin{gathered}
\left\langle c^{2} \rho_{0} / \gamma\right\rangle_{m}=c_{L}^{2} \rho_{0 L} / \gamma_{L}+\phi \lambda_{\rho}\left(\left(\tilde{\lambda}+\frac{2}{3} \tilde{\mu}\right)+c_{L}^{2} \rho_{0 L} / \gamma_{L}\right), \\
\mu_{v, e f f}=\mu_{\nu}\left(1-\phi \lambda_{\rho}\right),
\end{gathered}
$$

and

$$
\left\langle\frac{\rho_{0}(\gamma-1) C_{v}}{\beta T_{0}}\right\rangle_{m}=\frac{\rho_{0 L}\left(\gamma_{L}-1\right) C_{\nu L}}{\beta_{L} T_{0}}+\phi \lambda_{T}\left(\frac{\rho_{0 P}\left(\gamma_{P}-1\right) C_{\nu P}}{\beta_{P} T_{0}}-\frac{\rho_{0 L}\left(\gamma_{L}-1\right) C_{v L}}{\beta_{L} T_{0}}\right)
$$

The coefficient $\lambda_{\rho}$ was defined earlier (cf. equation (6.11)). The parameter, $\lambda_{T}$, , on the other hand, is a new coefficient which is defined as the ratio of the average temperature amplitude inside the particles to that in the mixture, that is

$$
\phi \lambda_{T}\langle T\rangle(\mathbf{x})=\left\langle g(\mathbf{x}) T_{P}(\mathbf{x})\right\rangle
$$

Both the effective $c^{2} \rho_{0} / \gamma$ and the bulk viscosity of the suspension depend on the coefficient, $\lambda_{p}$. This dependence is not surprising since both depend on the average dilatation amplitude inside the particles. The result that the effective bulk viscosity, 
$\mu_{v, \text { eff }}$, of the suspension depends only on the bulk viscosity of the fluid may seem strange at first sight, but it is really a consequence of the way the isotropic part of the stress is defined for the liquid and solids (cf. equations (6.4) and (6.7)). The stress arising from thermal expansion or, equivalently, temperature fluctuations depends on $\rho_{0}(\gamma-1) / \beta T_{0}$ of the two phases and the relative temperature fluctuations in the two phases.

Since the deviatoric stress amplitudes in the individual phases depend only on the velocity gradient amplitude, it is expected that the average deviatoric stress is linear in the gradient of average velocity amplitude. It must also be traceless. If it is assumed that the suspension is macroscopically isotropic, then the average deviatoric stress is characterized by a single effective (shear) viscosity, $\mu_{\text {eff }}$. Thus, we may write

$$
\left\langle d_{i j}\right\rangle=\mu_{e f f}\left[\frac{\partial\left\langle u_{i}\right\rangle}{\partial x_{j}}+\frac{\partial\left\langle u_{j}\right\rangle}{\partial x_{i}}-\frac{2}{3} \delta_{i j} \nabla \cdot\langle\mathbf{u}\rangle\right] .
$$

To obtain the effective (shear) viscosity, $\mu_{e f f}$, one must evaluate only one component of the average deviatoric stress. We can take, without loss of generality, the mean velocity amplitude to be given by

$$
\langle\mathbf{u}\rangle(\mathbf{x})=e^{i k_{c, e f f} x_{1}} \mathbf{e}_{1}
$$

where $\mathbf{e}_{1}$ is the unit vector along the $\boldsymbol{x}_{1}$-axis, taken to be in the direction of propagation of the plane wave, and $k_{c, e f f}$ is the effective wave number for the compressional wave through the suspension. The 11-component of the deviatoric stress is given by 


$$
\left\langle d_{11}\right\rangle=2\left\langle\mu \frac{\partial u_{1}}{\partial x_{1}}\right\rangle-\frac{2}{3}\left\langle\mu \frac{\partial u_{k}}{\partial x_{k}}\right\rangle
$$

The last term on the right-hand side of equation (6.26), being related to the dilatation amplitudes, can be readily related to the coefficient, $\lambda_{\rho}$, introduced earlier. The first term on the right-hand side can be expressed in terms of a coefficient, $\lambda d$, defined by

$$
\phi \lambda_{d} \frac{\partial\left\langle u_{1}\right\rangle}{\partial x_{1}}=\left\langle g(\mathbf{x}) \frac{\partial u_{1}^{P}}{\partial x_{1}}(\mathbf{x})\right\rangle
$$

With this definition, it is straightforward now to relate $\left\langle d_{11}\right\rangle$ to the gradient in the velocity amplitude:

$$
\left\langle d_{11}\right\rangle=2\left[\mu_{L}+\phi \lambda_{d}\left(\mu_{P}-\mu_{L}\right)\right] \frac{\partial\left\langle u_{1}\right\rangle}{\partial x_{1}}-\frac{2}{3}\left[\mu_{L}+\phi \lambda_{\rho}\left(\mu_{P}-\mu_{L}\right)\right] \frac{\partial\left\langle u_{k}\right\rangle}{\partial x_{k}}
$$

Substituting for $\langle\mathbf{u}\rangle$ from equations (6.25) into (6.28) and also into (6.24) with $i=j=1$ and comparing the resulting expressions yields the following expressions for the effective viscosity:

$$
\mu_{e f f}=\mu_{L}+(1 / 2) \phi\left(\mu_{P}-\mu_{L}\right)\left(3 \lambda_{d}-\lambda_{\rho}\right)
$$

Finally, the energy equation for the suspension, obtained by averaging (1-g) times the energy equation for the liquid, plus $g$ times the energy equation for the solid to yield

$$
-i \omega\left\langle\rho_{0} C_{v}\right\rangle_{e}\langle T\rangle=-\frac{\partial\left\langle q_{j}\right\rangle}{\partial x_{j}}-\left\langle\rho_{0} C_{v} \beta^{-1}(\gamma-1)\right\rangle_{e} \nabla \bullet\langle\mathbf{u}\rangle
$$


Here, an argument similar to the one used for equation (6.17) has been used to simplify the energy flux term (thereby using the boundary condition at the particle surface that the heat flux is continuous). It should be noted that in equation (6.30), the effective heat capacity of the suspension is given by

$$
\left\langle\rho_{0} C_{v}\right\rangle_{e}=\rho_{0 L} C_{v L}+\phi \lambda_{T}\left(\rho_{0 P} C_{v P}-\rho_{0 L} C_{v L}\right)
$$

with $\lambda_{T}$ defined by equation (6.23). The effective property, $\left\langle\rho_{0} C_{v} \beta^{-1}(\gamma-1)\right\rangle_{e}$, appearing in the last term in the right-hand side of equation (6.30) is related to $\lambda_{\rho}$, and the expression for evaluating it is obtained by replacing $\lambda_{T}$ in equation (6.22) by $\lambda_{\rho}$.

The average heat flux is given by

$$
\left\langle q_{j}\right\rangle=-\kappa_{e f f} \frac{\partial\langle T\rangle}{\partial x_{j}},
$$

with the effective conductivity given by

$$
\kappa_{e f f}=\kappa_{L}+\phi \lambda_{\kappa}\left(\kappa_{P}-\kappa_{L}\right)
$$

where the coefficient, $\lambda_{\mathrm{k}}$ is the ratio of the average temperature gradient amplitude inside the particles to that in the suspension, i.e.,

$$
\phi \lambda_{\kappa} \frac{\partial\langle T\rangle}{\partial x_{i}}=\left\langle g(\mathbf{x}) \frac{\partial T_{P}}{\partial x_{i}}(\mathbf{x})\right\rangle
$$

In summary, the continuity, momentum, and energy equations for the suspension are given by equations (6.8), (6.18), and (6.30). The averaged stress tensor is given by equations (6.19) and (6.24). The averaged heat flux is given by equation (6.32). These 
equations resemble the equations for the single phase given in Section 6.1 .1 with suitably defined effective properties of the suspension.

\subsubsection{Wave Equations for the Suspension:}

In order to find an expression for the attenuation of sound in the suspension, it is necessary to derive wave equations for the suspension, as was done by Epstein and Carhart (1953) for a pure liquid. The derivation of those authors is followed closely. As shown by these investigators the acoustics equations permit three waves: a thermal wave, a shear or rotational wave, and a compressional wave. The last one is the most significant one as far as the attenuation of a planes acoustic wave is concerned. The other waves are important in determining the disturbance produced by a test particle in the suspension as, we shall see in the next section.

We decompose the average velocity amplitude in scalar and vector potentials as given by

$$
\langle\mathbf{u}\rangle=-\nabla \Phi+\nabla \times \mathbf{A}
$$

Since the curl of a gradient of any scalar function is zero, A can be specified to within a gradient of an arbitrary scalar function. To remove this arbitrariness, an additional restriction is imposed that $\mathbf{A}$ be divergence free, i.e., $\nabla \bullet \mathbf{A}=0$. It may be noted that the vorticity amplitude equals $-\nabla^{2} \mathbf{A}$

Introducing this decomposition in the momentum equation for the suspension (equation (6.18)) and rearranging yields 


$$
\begin{aligned}
& \nabla\left[\omega^{2} \Phi+\left\{\frac{\left\langle c^{2} \rho_{0} / \gamma\right\rangle_{m}}{\left\langle\rho_{0}\right\rangle_{m}}-\frac{i \omega}{\left\langle\rho_{0}\right\rangle_{m}}\left(\mu_{v, e f f}+\frac{4}{3} \mu_{e f f}\right)\right\} \nabla^{2} \Phi\right. \\
& \left.-\frac{i \omega}{\left\langle\rho_{0}\right\rangle_{m}}\left\langle\frac{\rho_{0}(\gamma-1) C_{v}}{\beta T_{0}}\right\rangle_{m}\langle T\rangle\right]=\nabla \times\left[\omega^{2} \mathbf{A}+\frac{i \omega \mu_{e f f}}{\left\langle\rho_{0}\right\rangle_{m}} \nabla \times(\nabla \times \mathbf{A})\right] .
\end{aligned}
$$

Here, the vector identity, $\nabla^{2} \mathbf{a}=\nabla(\nabla \bullet \mathbf{a})-\nabla \times(\nabla \times \mathbf{a})$, is employed. Similarly, the energy equation (equation (6.30)) becomes

$$
-i \omega\left\langle\rho_{0} C_{\nu}\right\rangle_{e}\langle T\rangle=\kappa_{e f f} \nabla^{2}\langle T\rangle+\left\langle\rho_{0} C_{\nu} \beta^{-1}(\gamma-1)\right\rangle_{e} \nabla^{2} \Phi
$$

In equation (6.36) both sides must vanish separately because a rotational vector field cannot balance an irrotational field. Hence, with the right-hand side being zero, using the previously mentioned vector identity and $\nabla \bullet \mathrm{A}=0$ yields

$$
\nabla^{2} \mathbf{A}+k_{s E M}^{2} \mathbf{A}=0
$$

with $k_{s E M}^{2} \equiv \frac{i \omega\left\langle\rho_{0}\right\rangle_{m}}{\mu_{e f f}}$ being the effective wavenumber for shear waves through the suspension. The subscript, $E M$, refers to the effective medium.

The left-hand side of equation (6.36) being zero gives an expression for $\langle T\rangle$ in terms of the velocity potential: 


$$
\langle T\rangle=\frac{\left[-i \omega\left\langle\rho_{0}\right\rangle_{m} \Phi-\left\{\frac{i}{\omega}\left\langle c^{2} \rho_{0} / \gamma\right\rangle_{m}+\left(\mu_{v, \text { eff }}+\frac{4}{3} \mu_{e f f}\right)\right\} \nabla^{2} \Phi\right]}{\left\langle\frac{\rho_{0}(\gamma-1) C_{v}}{\beta T_{0}}\right\rangle_{m}}
$$

Eliminating $\langle T\rangle$ from the energy equation for the suspension (equation (6.37)) by substituting the above result gives

$$
\Phi+(E-F+G) \nabla^{2} \Phi-E F \nabla^{4} \Phi=0
$$

with

$$
\begin{gathered}
E=\frac{\left\langle c^{2} \rho_{0} / \gamma\right\rangle_{m}}{\left\langle\rho_{0}\right\rangle_{m} \omega^{2}}-\frac{i}{\left\langle\rho_{0}\right\rangle_{m} \omega}\left(\mu_{v, e f f}+\frac{4}{3} \mu_{e f f}\right) \\
F=\frac{i \kappa_{e f f}}{\omega\left\langle\rho_{0} C_{v}\right\rangle_{e}} \\
G=\left\langle\rho_{0} C_{v} \beta^{-1}(\gamma-1)\right\rangle_{e}\left\langle\frac{\rho_{0}(\gamma-1) C_{v}}{\beta T_{0}}\right\rangle_{m} /\left(\left\langle\rho_{0}\right\rangle_{m} \omega^{2}\left\langle\rho_{0} C_{v}\right\rangle_{e}\right)
\end{gathered}
$$

Equation (6.40) can be written in the form

$$
\left(k_{c E M}^{-2} \nabla^{2}+1\right)\left(k_{t E M}^{-2} \nabla^{2}+1\right) \Phi=0
$$

so that $\Phi=\Phi_{c}+\Phi_{t}$ with

$$
\left(\nabla^{2}+k_{c E M}^{2}\right) \Phi_{c}=0
$$




$$
\left(\nabla^{2}+k_{t E M}^{2}\right) \Phi_{t}=0
$$

The effective wavenumbers for the compressional and thermal waves are given by, respectively,

$$
\begin{aligned}
& k_{c E M}^{-2}=\frac{1}{2}(E-F+G)+\frac{1}{2}\left\{(E-F+G)^{2}+4 E F\right\}^{1 / 2}, \\
& k_{t E M}^{-2}=\frac{1}{2}(E-F+G)-\frac{1}{2}\left\{(E-F+G)^{2}+4 E F\right\}^{1 / 2} .
\end{aligned}
$$

As mentioned earlier, the compressional wavenumber is the most important one as far as the propagation of the acoustic plane wave is concerned. The imaginary part of $k_{c E M}$ gives the attenuation while $\omega$ divided by the real part of $k_{c E M}$ gives the phase speed.

\subsubsection{An Effective-Medium Model:}

To determine the attenuation and phase speed, we must now estimate the various effective properties of the suspension. This requires determining the five coefficients: $\lambda_{\rho}$, $\lambda_{v}, \lambda_{T}, \lambda_{d}$, and $\lambda_{\mathrm{x}}$. Let us begin with $\lambda_{\mathrm{\rho}}$, which represents the ratio of the average dilatation amplitude inside the particles to that in the suspension.

The coefficient, $\lambda_{\rho}$, is defined by equation (6.11), which is equivalent to

$$
\phi \lambda_{\rho} \nabla \bullet\langle\mathbf{u}\rangle(\mathbf{x})=\int_{\mathbf{x}-\mathbf{x}_{1} \mid \leq a}\left\langle\nabla \bullet \mathbf{u}_{P}\right\rangle\left(\mathbf{x} \mid \mathbf{x}_{1}\right) d V\left(\mathbf{x}_{1}\right)
$$

Here, we have introduced the conditionally averaged field. Thus $\langle\mathbf{u}\rangle\left(\mathbf{x} \mid \mathbf{x}_{1}\right)$ the ensemble averaged velocity amplitude at point $\mathbf{x}$, given that in all the ensembles used in the averaging a test particle is centered at $\mathbf{x}_{1} . P\left(\mathbf{x}_{1}\right)$ is the probability density for 
finding a particle with its center in the vicinity of $\mathbf{x}_{1}$. For uniform, monodisperse suspensions $P(\mathbf{x})=n=3 \phi /\left(4 \pi a^{3}\right), n$ being the number density of the particles and $\phi$ the particle volume fraction.

We shall use an effective medium approximation for determining the conditionally averaged fields, and, hence, the use of the integrals, such as the one appearing on the right-hand side of equation (6.49). All effective medium approximations must satisfy the criterion that far from the test particle, i.e., for $\left|\mathbf{x}-\mathbf{x}_{1}\right| \rightarrow \infty$, the conditionally averaged fields, such as $\langle\mathbf{u}\rangle\left(\mathbf{x} \mid \mathbf{x}_{1}\right)$ must approach the corresponding unconditionally averaged fields, such as $\langle\mathbf{u}\rangle(\mathbf{x})$. On the other hand, for $\left|\mathbf{x}-\mathbf{x}_{1}\right| \leq a$, i.e., for a point inside the test particle, the conditionally averaged fields must satisfy the equations governing the particle phase. The simplest kind of effective medium approximation, then, assumes that the conditionally averaged equation satisfies the suspending fluid equations for $a \leq r \leq R$ and the unconditionally averaged equations for the suspension for $r \geq R$. Here, $r \equiv\left|\mathbf{x}-\mathbf{x}_{1}\right|$ is the distance from the center of the particle. Different effective medium approximations differ in their choice of $R$. Some investigators choose $R=a$, which eliminates the fluid region altogether. This makes the subsequent analysis very simple; but, unfortunately, the estimates obtained with $R=a$ are typically inferior, and in some cases unphysical. For example, it may yield negative effective properties at high volume fractions. Other investigators choose $R=a \phi^{1 / 3}$ with incorrect reasoning that the volumes occupied by 
the particle and fluid for $r \leq R$ must be proportional to the volume fractions of the two phases. In the present study, we shall choose $R$ to be given by

$$
\frac{R}{a}=\left(\frac{1-S(0)}{\phi}\right)^{1 / 3},
$$

with $S(\mathbf{0})$ the zero-wavenumber limit of the suspension structure factor, defined by

$$
S(\mathbf{0})=n \int[P(\mathbf{r} \mid \mathbf{0})-P(\mathbf{0})] d \mathbf{r},
$$

where $P(\mathbf{r} \mid \mathbf{0})$ is the probability density for finding a particle near $\mathbf{r}$ given that there is a particle with its center at the origin. (Note that $P(\mathbf{r} \mid \mathbf{0})=\delta(\mathbf{r})$ for $r \leq a$.) The structure factor of the suspension can be determined experimentally by a light scattering technique; but in the absence of such information, one may choose $S(0)$ to correspond to that of a hard-sphere molecular system for which the well-known Carnahan-Starling approximation yields quite accurate estimates of the structure factor as a function of the volume fraction,

$$
S(0)=\frac{(1-\phi)^{4}}{1+4 \phi+4 \phi^{2}-4 \phi^{3}+\phi^{4}} .
$$

The effective medium radius, $R$, based on $S(0)$ was first introduced by Dodd $e t$ al. (1995), who compared the results of rigorous multiparticle interactions for determining the short-time self- and gradient diffusivity of proteins in bilipid membranes with those obtained by the effective medium approximation and found very good agreement between the two. In the problems concerned with determining the collective mobility or the sedimentation velocity, where each particle is acted upon with a constant force, it was 
shown in Sangani and Mo (1997) that the conditionally averaged velocity has the correct leading order behavior at large $r$ only when $R$ is chosen according to equation (6.50).

For small volume fractions, $S(0)$ given by equation (6.52) behaves as $1-8 \phi+\phi\left(\phi^{2}\right)$ and $R \rightarrow 2 a$. Thus, in "well-stirred" dilute random suspensions, the effective medium begins at $r=2 a$ according to equation (6.50) and the fluid region $a<r$ $<2 a$ corresponds to the excluded region volume region. Note that the more usual choice of $R=a \phi^{-1 / 3}$ would, on the other hand, suggest that the effective medium at a very large distance from the test particle in a dilute suspension which is unphysical except for the situations such as dilute periodic or "well-separated" random suspensions defined by Jeffrey () (For such arrays $S(0)$ is small when $\phi$ is small and equation (6.50) also gives $R / a=O\left(\phi^{-1 / 3}\right)$. ) Thus it is not surprising that $R$ based on equation (6.50) will give better estimates of the effective properties at small to moderate volume fractions compared to those obtained with $R=a \phi^{-1 / 3}$. Indeed, Sangani and Mo (1997) have shown that the coefficients of $O\left(\phi^{2}\right)$ corrections to the effective conductivity and elasticity obtained using equation (6.50) are much closer to the rigorous results for these coefficients obtained by detailed pair interaction calculations than those obtained with $R=a \phi^{-1 / 3}$

Before closing this brief review of effective medium approximations, we should perhaps note here one more class of effective medium approximations in the literature. 
These approximations involve immersing a pair of particles in the effective medium.

Examples are the calculations by Kim and Russel (1995) who estimated the permeability of a fixed bed of particles, calculations for the effective viscosity of suspensions with hard-sphere spatial distributions, and Ma et al. (1990) for the attenuation due to scattering. These calculations generally require far greater effort, comparable to direct multiparticle calculations, and do not necessarily yield superior estimates compared with the simple approximations based on a single particle. On the other hand, the single particle approximations will be inadequate for the suspension problems in which the changes in microstructure due to imposed flow and their effects, in turn, on the suspension properties must be addressed.

Returning now to the problem of estimating coefficients $\lambda_{\rho}$, etc. using the effective medium model consisting of particle-fluid assembly of radius, $R$, immersed in a medium with the effective properties of the suspension, we write the velocity inside the test particle in terms of scalar and vector potentials as in the previous section. For the plane wave traveling along the $x_{1}$-axis with $\langle\mathbf{u}\rangle(\mathbf{x})=\exp \left(i \mathbf{k}_{c E M} \bullet \mathbf{x}\right) \mathbf{e}_{1}$ we have for $\left|\mathbf{x}-\mathbf{x}_{1}\right|$ $\leq a$,

$$
\begin{aligned}
& \left\langle\Phi_{c P}\right\rangle\left(\mathbf{x} \mid \mathbf{x}_{1}\right)=\exp \left(i \mathbf{k}_{c E M} \bullet \mathbf{x}_{1}\right) \sum_{n=0}^{\infty} i^{n}(2 n+1) A_{P n} P_{n}(\mu) j_{n}\left(k_{c P} r\right), \\
& \left\langle\Phi_{t P}\right\rangle\left(\mathbf{x} \mid \mathbf{x}_{1}\right)=\exp \left(i \mathbf{k}_{c E M} \bullet \mathbf{x}_{1}\right) \sum_{n=0}^{\infty} i^{n}(2 n+1) B_{P_{n}} P_{n}(\mu) j_{n}\left(k_{t P} r\right),
\end{aligned}
$$




$$
\left\langle A_{P}\right\rangle\left(\mathbf{x} \mid \mathbf{x}_{1}\right)=\exp \left(i \mathbf{k}_{c E M} \bullet \mathbf{x}_{1}\right) \sum_{n=0}^{\infty} i^{n}(2 n+1) C_{P n} P_{n}^{1}(\mu) j_{n}\left(k_{s P} r\right)
$$

where $r=\left|\mathbf{x}-\mathbf{x}_{1}\right|, \mu=\cos \theta, \theta$ being the angle between $\mathbf{x}-\mathbf{x}_{1}$ and $\mathbf{k}_{c E M}, j_{n}$ is the spherical Bessel function of the first kind (regular at $r=0$ ), and $P_{n}$ is the Legendre polynomial of degree $n\left(P_{n}^{1}\right.$ is associated Legendre polynomial of degree $\left.n\right) . A P$ is the only nonzero (azimuthal) component of $\mathbf{A}$.

Similar expressions can be written for $a<r<R$ for which the relevant wavenumbers in the expressions for $\Phi_{c L}, \Phi_{t L}$, and $A L$ are, respectively, $\bar{k}_{c L}, k_{t L}$, and $k_{s L}$. Both the spherical harmonics of the first kind and second kind (corresponding to waves emanating from $r=0$ ) must be included in the expression. This leads to a set of six unknowns for each mode $n$ describing the motion in the liquid shell. Finally, for $r>$ $R$, the potentials consist of the plane wave corresponding to the unconditional motion plus the outgoing wave with wavenumbers $k_{c E M}, k_{t E M}$, and $k_{s E M}$. Thus a total of 12 unknowns are needed in describing the motion for each mode $n$. These unknowns are determined from the boundary conditions of continuity of velocity, traction, temperature, and heat flux amplitudes at $r=a$ and $r=R$. Note that the conditional density and temperature amplitudes can be determined from the expressions for $\Phi_{c}, \Phi_{t}$, and $A$ using the expressions given in the previous section. We keep a total of $N$ modes (typical calculation uses $N=5$ ) and solve the resulting $12 N$ equations numerically. 
We now return to the calculation of $\lambda_{p}$. One must first substitute for $\nabla \cdot \mathbf{u}_{P}=-\nabla^{2} \Phi_{P}=k_{c P}^{2} \Phi_{c P}+k_{t P}^{2} \Phi_{t P}$ in equation (6.49). One may now define the coefficient, $\eta_{c}$, as

$$
\phi \eta_{c}\left\langle\Phi_{c}\right\rangle(\mathbf{x}) \equiv n \int_{\left|\mathbf{x}-\mathbf{x}_{1}\right| \leq a}\left\langle\Phi_{c P}\right\rangle\left(\mathbf{x} \mid \mathbf{x}_{1}\right) d V\left(\mathbf{x}_{1}\right)
$$

Similarly a coefficient, $\eta_{t}$, is defined with $\Phi_{c}$ replaced by $\Phi_{t}$. The integration in the above must be carried out over all $\mathbf{x}_{1}$ such that $\left|\mathbf{x}-\mathbf{x}_{1}\right| \leq a$. To convert this to an integration over $\mathbf{r}$, we use the identity

$$
\begin{aligned}
& \exp \left[i \mathbf{k}_{c E M} \bullet \mathbf{x}_{1}\right]=\exp \left[i \mathbf{k}_{c E M} \bullet \mathbf{x}\right] \exp \left[-i r k_{c} \mu\right] \\
&=\exp \left[i \mathbf{k}_{c E M} \bullet \mathbf{x}\right] \sum_{m=0}^{\infty} i^{m}(-1)^{m}(2 m+1) j_{m}\left(k_{c} r\right) P_{m}(\mu)
\end{aligned}
$$

Now substituting $\Phi_{c P}$ form equation (6.53) into (6.56) to give, with $z_{c E M} \equiv k_{c E M} a$,

$$
\begin{gathered}
\eta_{c}=\frac{3}{z_{c E M}^{2}-z_{c P}^{2}} \sum_{n=0}^{\infty}(2 n+1) A_{P n}\left[z_{c P} j_{n-1}\left(z_{c P}\right) j_{n}\left(z_{c E M}\right)\right. \\
\left.-z_{c E M} j_{n}\left(z_{c P}\right) j_{n-1}\left(z_{c E M}\right)\right]
\end{gathered}
$$

In the special case of $n=0, j_{n-1}(z)$ should be replaced by $\cos (z) / z$. Here we have used the solution of equation (6.53); the integral over the radius of the product of two spherical Bessel functions and $r^{2}$ is given by Gradshteyn and Ryzhik (1994). Using this result, one can simply write

$$
\lambda_{\rho}=\left(z_{c P}^{2} / z_{c E M}^{2}\right) \eta_{c}+\left(z_{t P}^{2} / z_{c E M}^{2}\right) \eta_{t}
$$


Equation (6.39) shows that the temperature field is linear in $\Phi_{c}$ and $\Phi_{t}$. Following the notation of Epstein and Carhart (1953) and Allegra and Hawley (1972), where the temperature field is written as $T=b_{c} \Phi_{c}+b_{t} \Phi_{t}$, one finds that $\lambda_{T}$, as defined by equation (6.23), is given by

$$
\lambda_{T}=\left(b_{c P} / b_{c E M}\right) \eta_{c}+\left(b_{t P} / b_{t E M}\right) \eta_{t}
$$

Note that the unconditionally averaged $\langle T\rangle=b_{c E M}\left\langle\Phi_{c}\right\rangle$ because the unconditionally averaged $\left\langle\Phi_{t}\right\rangle=0$

The other $\lambda$ 's are evaluated in a similar manner and are inter-connected. The definition of $\lambda_{v}$ is first written as an integral similar to that in equation (6.49) for the component of the velocity inside the particle in the direction of the wave. The velocity inside the particles can now be split up into three parts (two velocity potentials and a rotational contribution), so that one can write

$$
\lambda_{v}=\lambda_{v}^{\Phi_{c}}+\lambda_{v}^{\Phi_{t}}+\lambda_{v}^{\mathbf{A}}
$$

where $\lambda_{v}^{\Phi}$ and $\lambda_{v}^{\mathbf{A}}$ are the irrotational and rotational field contributions, respectively.

The irrotational contributions to $\lambda_{v}$ are

$$
\lambda_{v}^{\Phi_{c}}=\eta_{c}+\frac{3}{z_{c E M}} \sum_{n=0}^{\infty} A_{P n} j_{n}\left[n j_{n-1}\left(z_{c E M}\right)-(n+1) j_{n+1}\left(z_{c P}\right)\right]
$$

and a similar expression for $\lambda_{v}^{\Phi_{t}}$ (just replace $z_{c P}$ by $z_{t P}$ and $A_{P n}$ by $B_{P_{n}}$ ), where terms that give a spherical Bessel function of negative order are omitted. In deriving equation (6.62) use has been made of equation (6.57) and 


$$
\begin{aligned}
\exp \left[-i \mathbf{k}_{c E M} \bullet \mathbf{s}\right] \nabla_{\mathbf{s}} \Phi(\mathbf{s})= & \nabla_{\mathbf{s}}\left\{\left[-i \mathbf{k}_{c E M} \bullet \mathbf{s}\right] \Phi(\mathbf{s})\right\} \\
& +i \mathbf{k}_{c E M} \exp \left[-i \mathbf{k}_{c E M} \bullet \mathbf{s}\right] \Phi(\mathbf{s})
\end{aligned}
$$

with $\mathbf{s}=\mathbf{x}-\mathbf{x}_{1}$. The divergence theorem is used to evaluate the integral of the first term on the right-hand side of equation (6.63); the second term is seen to simply lead to $\eta_{C}$ in equation (6.62). The rotational contribution to $\lambda_{\nu}$ is

$$
\lambda_{v}^{\mathbf{A}}=\frac{3}{z_{c E M}} \sum_{n=0}^{\infty} n(n+1) C_{P n} j_{n}\left(z_{s P}\right)\left(j_{n+1}\left(z_{c E M}\right)+j_{n-1}\left(z_{c E M}\right)\right)
$$

Here, a similar relation to equation (6.63) is used

$$
\begin{aligned}
\exp \left[-i \mathbf{k}_{c E M} \bullet \mathbf{s}\right] \nabla_{\mathbf{s}} \times \mathbf{A}(\mathbf{s}) & =\nabla_{\mathbf{s}} \times\left\{\left[-i \mathbf{k}_{c E M} \bullet \mathbf{s}\right] \mathbf{A}(\mathbf{s})\right\} \\
& +\exp \left[-i \mathbf{k}_{c E M} \bullet \mathbf{s}\right] i \mathbf{k}_{c E M} \times \mathbf{A}(\mathbf{s})
\end{aligned}
$$

Recognizing that the second term on the right-hand side does not contribute to the component in the wave direction of the velocity inside the particle, this term is left out in the evaluation of $\lambda_{v}^{\mathbf{A}}$.

The result for $\lambda_{v}$ can be used to determine other $\lambda$ 's as well. We notice that

$$
\lambda_{k}=\left(b_{c P} / b_{c E M}\right) \lambda_{v}^{\Phi_{c}}+\left(b_{t P} / b_{c E M}\right) \lambda_{v}^{\Phi_{t}}
$$

Finally, $\lambda_{d}$, defined by equation (6.27), equals

$$
\lambda_{d}=\lambda_{d}+\lambda_{d}^{\Phi_{c}}+\lambda_{d}^{\Phi_{t}}+\lambda_{d}^{\mathbf{A}}
$$

where we have, again, made use of equation (6.63), with $\Phi$ replaced by $\mathbf{u}$. The result for $\lambda_{d}^{\Phi_{c}}$ is 


$$
\begin{aligned}
\lambda_{d}^{\Phi_{c}} & =\frac{3}{z_{c E M}^{2}} \sum_{n=0}^{\infty} A_{P n}\left[\{ z _ { c P } j _ { n } ^ { \prime } ( z _ { c P } ) + ( n + 1 ) j _ { n } ( z _ { c P } ) \} \left\{\left(\frac{(n+1)^{2}}{2 n+3}+\frac{n^{2}}{2 n-1}\right)\right.\right. \\
& \left.\times j_{n}\left(z_{c E M}\right)-\frac{(n+1)(n+2)}{2 n+3} j_{n+2}\left(z_{c E M}\right)-\frac{n(n-1)}{2 n-1} j_{n-2}\left(z_{c E M}\right)\right\} \\
& \left.+\frac{(n+1)(2 n+1)}{2 n+3} j_{n}\left(z_{c P}\right)\left\{(n+2) j_{n+2}\left(z_{c E M}\right)-(n+1) j_{n}\left(z_{c E M}\right)\right\}\right]
\end{aligned}
$$

Again, $\lambda_{d}^{\Phi_{t}}$ is obtained from $\lambda_{v}^{\Phi_{c}}$ by replacing $A_{P n}$ by $B_{P n}$, and $z_{c P}$ by $z_{t P}$. Those terms which would give a spherical Bessel function of negative order at $n \leq 1$ are omitted from this formula. The evaluation of $\lambda_{d}$ is complete with

$$
\begin{aligned}
\lambda_{d}^{\mathrm{A}}=\frac{3}{z_{c E M}^{2}} & \sum_{n=1}^{\infty}(n+1) C_{P n}\left[-\frac{n(n+2)}{2 n+3} X_{n, n+2}\right. \\
& \left.-\frac{n(2 n+1)}{(2 n+3)(2 n-1)} X_{n, n}+\frac{n(n-1)}{2 n-1} X_{n, n-2}\right]
\end{aligned}
$$

with the short hand notation

$$
\begin{aligned}
X_{n, m} \equiv & -\frac{3}{2} j_{n}\left(z_{s P}\right) j_{m}\left(z_{c E M}\right)-\frac{1}{2}\left(z_{s P} j_{n}^{\prime}\left(z_{s P}\right) j_{m}\left(z_{c E M}\right)\right. \\
& \left.+z_{c E M} j_{n}\left(z_{s P}\right) j_{m}^{\prime}\left(z_{c E M}\right)\right) .
\end{aligned}
$$

Again, the terms which give a spherical Bessel function of negative order at $n=1$ are to be omitted.

This completes the calculation of the attenuation. The attenuation is the real part of $k_{C E M}$, given by equation (6.47), the right-hand side of which contains the effective 
medium properties that have been expressed in the unknown parameters, $\lambda_{\rho}$, etc. These parameters are calculated from the above expressions by solving first, at each $n$, for the coefficients, $A_{P n}$, etc., from the boundary conditions at the particle and shell interfaces. In the boundary conditions the effective medium properties show up as well (e.g., the effective viscosity and conductivity), so that this procedure is repeated until the $\lambda$ 's have converged.

\subsection{Attenuation Measurements in Concentrated Solid-Liquid Systems:}

In order to test the effective medium theory for concentrated slurries (solids volume fractions greater than about $10 \%$ by volume), attenuation measurements are performed in slurries of Potter's beads in mixtures of glycerin in distilled water.

The first set of measurements is actually performed on a dilute ( $5 \%$ by volume) slurry over the entire experimental frequency range. Although this is not a concentrated slurry, these data are necessary in order to test the theoretical effective medium calculations. Any effective medium approach to the forward problem theory should reduce to the theory of Allegra and Hawley (1972) for small solids volume fraction. Having data at lower volume fractions, such as $5 \%$, allows for "tuning" the effective medium approach by providing a basis for comparison between the results of the Allegra and Hawley (1972) theory, the effective medium approach, and experimental data. If the effective medium approach is correctly formulated, the attenuation spectra generated in all three cases should be essentially the same. Data are collected using both the 
Toneburst and Pulse/FFT techniques. The experimentally obtained attenuation spectrum is shown, together with results predicted by the theory, in Figure 6.1 .

Attenuation measurements are then made in slurries of $10 \%, 15 \%, 20 \%, 30 \%$, $40 \%, 45 \%$, and $50 \%$ solids by volume. Figures $6.2 \& 6.3$ show attenuation as a function of solids volume fraction in the Potter's Beads slurries for several different frequencies. In this figure, the experimental data (markers) are also compared with results of theoretical calculations (solid curves) employing the effective medium approach using a mean particle diameter of $130 \mu \mathrm{m}$ with standard deviation of $22 \mu \mathrm{m}$. The results for lower frequencies $(1.5,1.75,2.0,2.25$, and $2.75 \mathrm{MHz})$ are shown in Figure 6.2, and the agreement between theory and experiment is rather good up to a solids volume fraction of approximately 0.30 . Agreement is better at frequencies above $2 \mathrm{MHz}$ than for those below. The experimental data at all frequencies appear to go through a maximum at solids volume fraction of approximately 0.30 . It should be noted that similar behavior was seen by Atkinson (1991), who also observed a maximum at a solids volume fraction of approximately 0.30 , in the attenuation versus concentration curves he obtained for $1.0 \mathrm{~mm}$ diameter glass beads in a fluidized bed. The attenuation data from this study then go through a minimum at a volume fraction of approximately 0.4 or 0.5 , and then the attenuation begins to increase again. This behavior is interesting in that the attenuation curves predicted by the effective medium theory show monotonically increasing behavior. There is definitely a change in the apparent slope of the theoretical curves, but there are no local maxima or minima. Nevertheless, it can be said that there is fairly good agreement between the effective medium theory and 


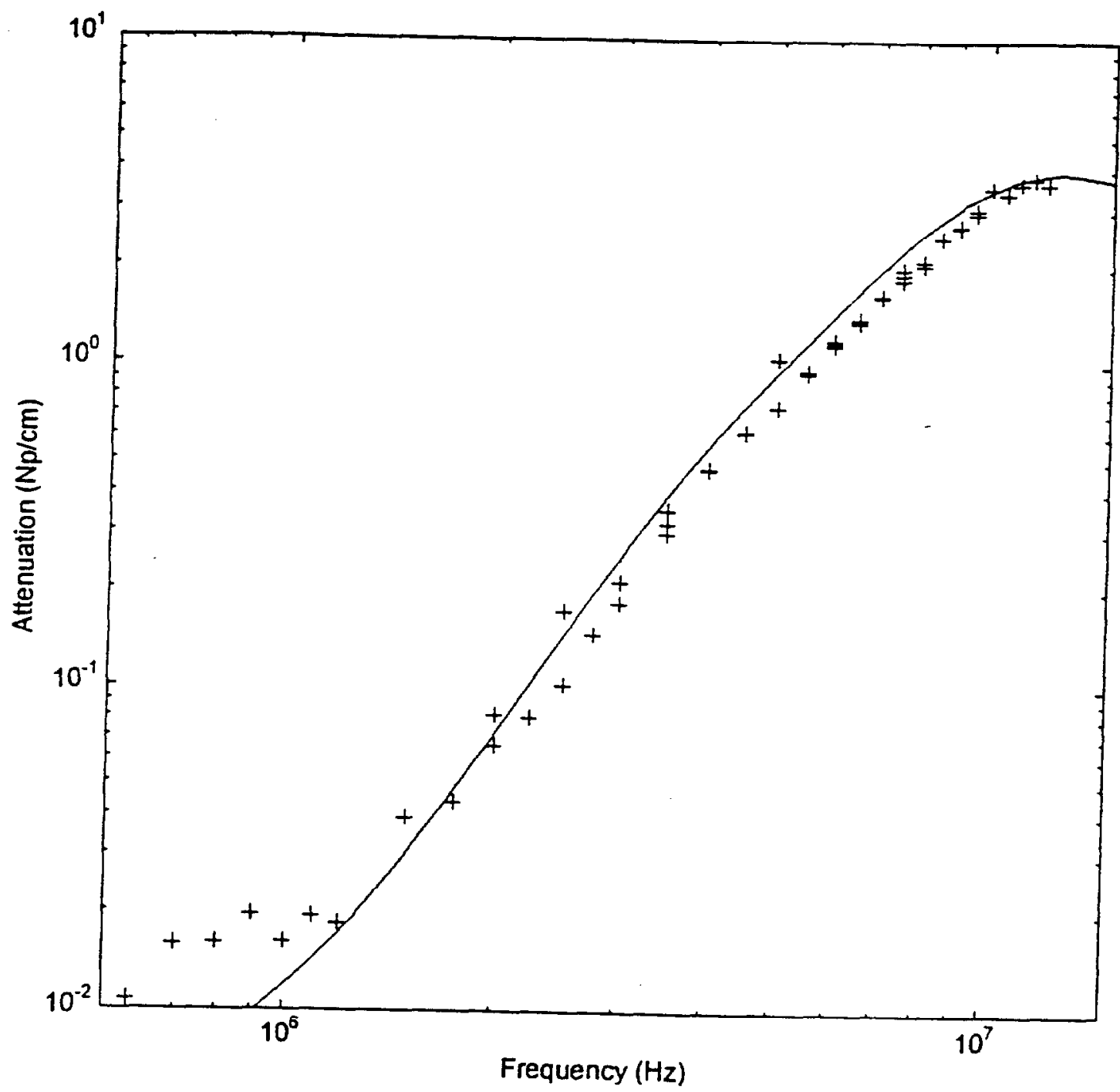

Figure 6.1: Attenuation versus frequency behavior for a slurry of Potter's beads in a mixture of glycerin/water at $5 \%$ by volume-forward theory and
experimental data. - E2162. 


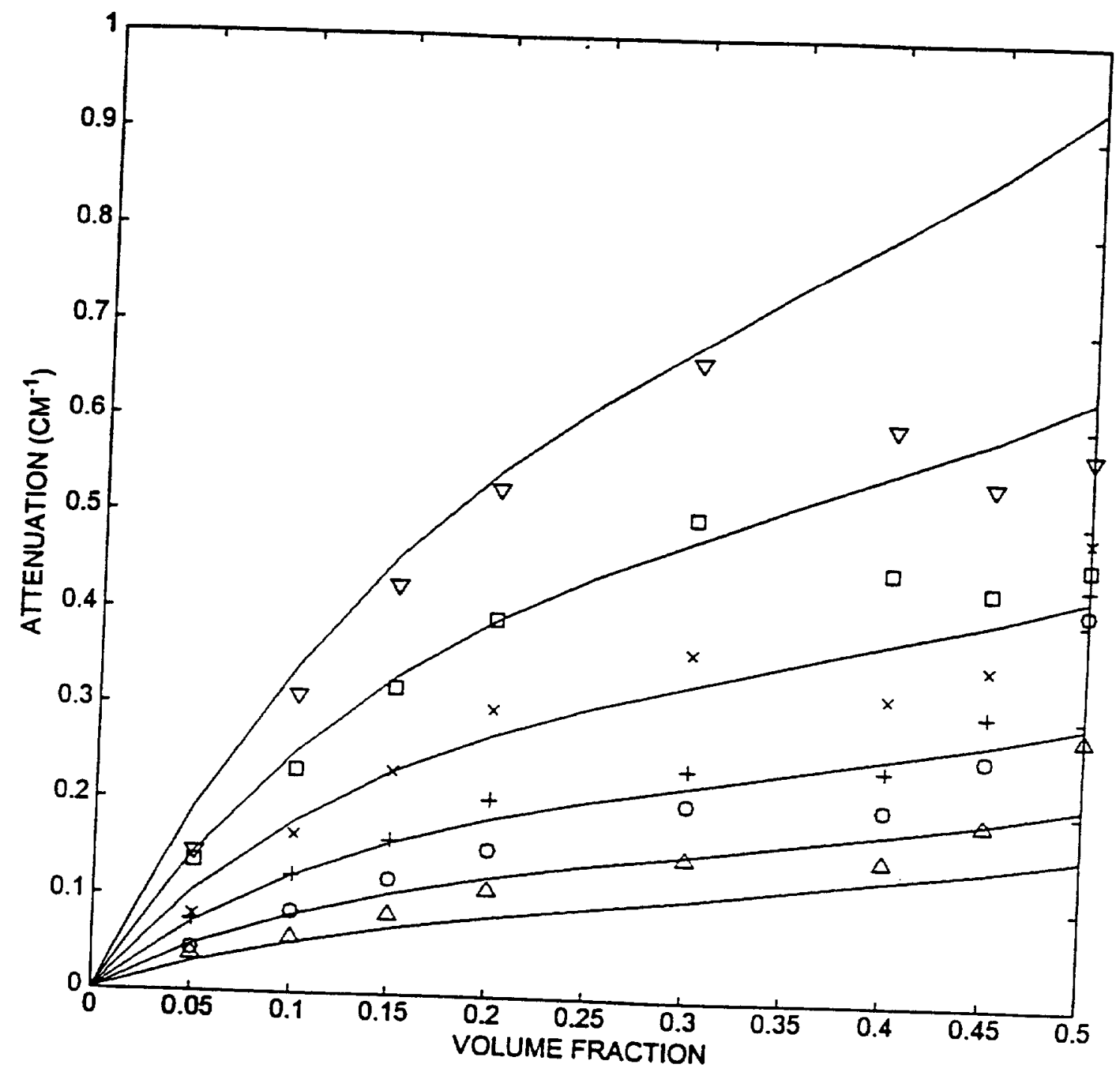

Figure 6.2: Attenuation as a function of solids volume fraction at various frequencies in slurries of Potter's beads in glycerin/water. Data are shown for $1.5 \mathrm{MHz}$ $(\Delta) ; 1.75 \mathrm{MHz}(\mathrm{O}) ; 2.0 \mathrm{MHz}(+) ; 2.25 \mathrm{MHz}(\times) ; 2.5 \mathrm{MHz}(\square)$; and 2.75 calculations using a particle rizesent the results of the effective medium standard deviation of $11 \mu \mathrm{m}$. - E4172. 
experimental data at these lower frequencies for solids volume fractions up to approximately 0.30 .

The theory predictions begin to deviate more from the experimental data as the frequency increases. Figure 6.3 shows the experimental attenuation as a function of solids volume fraction in the Potter's Beads slurries for the relatively higher frequencies $(3,3.5,4,4.5$ and $5 \mathrm{MHz})$, along with the corresponding effective medium theory predicted attenuation curves. The experimental data and theory prediction for $3 \mathrm{MHz}$ show fairly good agreement up to a solids volume fraction of approximately 0.4 . Otherwise for higher frequencies, the deviation between data and theory becomes noticeable at solids volume fractions greater than about 0.2 . It should, again, be noted here that the experimental attenuation data display a local minimum between solids volume fractions of 0.35 and 0.5 . And again, these local minima are not predicted by the effective medium theory. The deviation at the higher volume fraction and higher frequencies could also be attributed to the increased scatter in the attenuation data at the higher solids volume fractions, and the attenuation is especially large (implying small received voltage signals) for the higher frequencies at these higher solids volume fractions.

A more encouraging look into the efficacy of the effective medium approach can be achieved when the theoretical results are compared with other experimental data. For example, Figure 6.4 shows results of the effective medium approach (solid curves) compared with the experimental attenuation data (markers) of Allegra and Hawley (1972), which are for $0.11 \mu \mathrm{m}$ radius polystyrene particles in water at 3, 9, 15, 21, 27, 


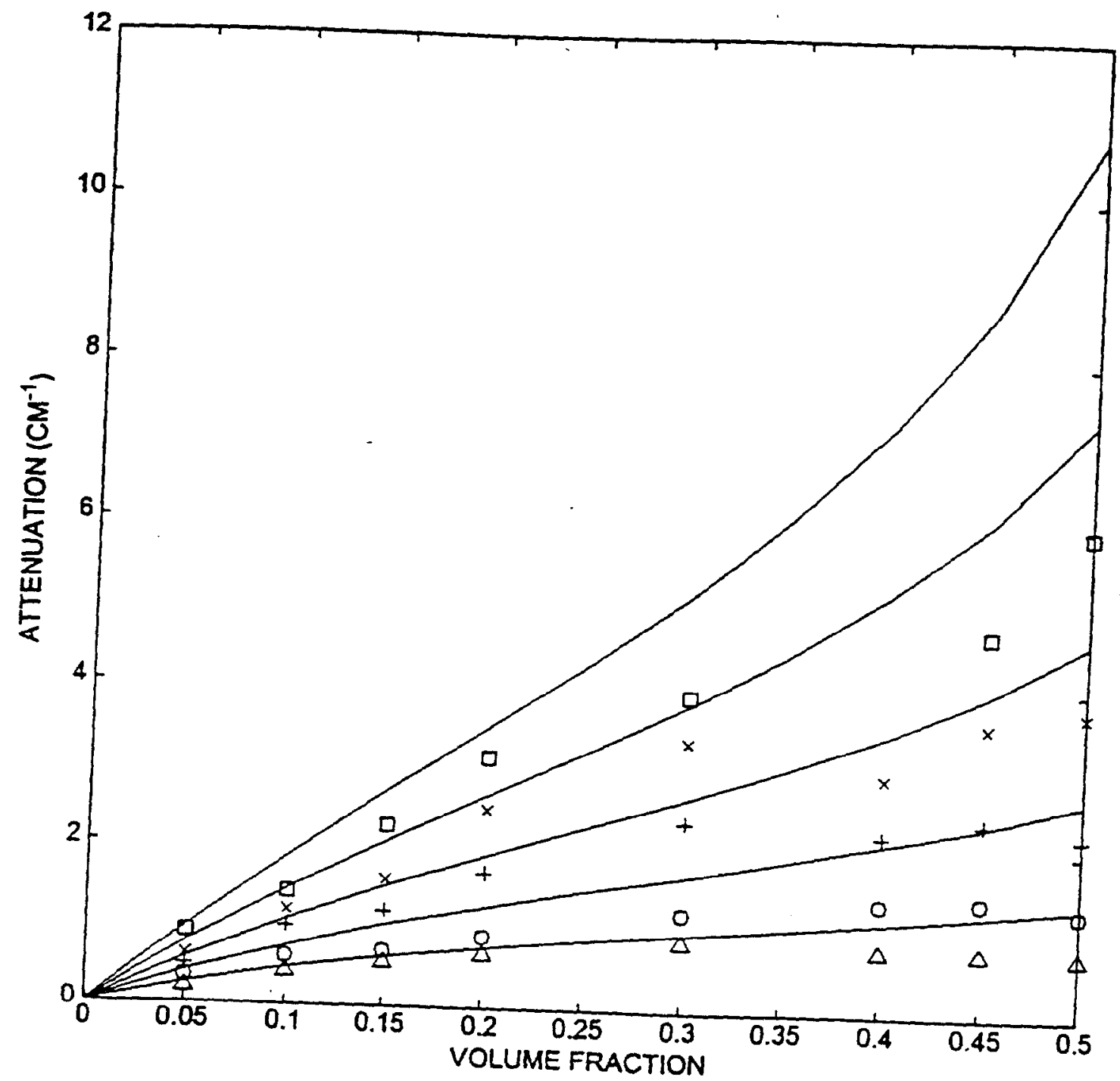

Figure 6.3: Attenuation as a function of solids volume fraction at various frequencies in slurries of Potter's beads in glycerin/water. Data are shown for $3.0 \mathrm{MHz}$ $(\Delta) ; 3.5 \mathrm{MHz}(\mathrm{O}) ; 4.0 \mathrm{MHz}(+) ; 4.5 \mathrm{MHz}(x)$; and 5.0 MHz (口) The solid curves represent the results of the effective medium calculations using a particle size distribution with $65 \mu \mathrm{m}$ mean radius and standard deviation of $11 \mu \mathrm{m}$. - E6173. 


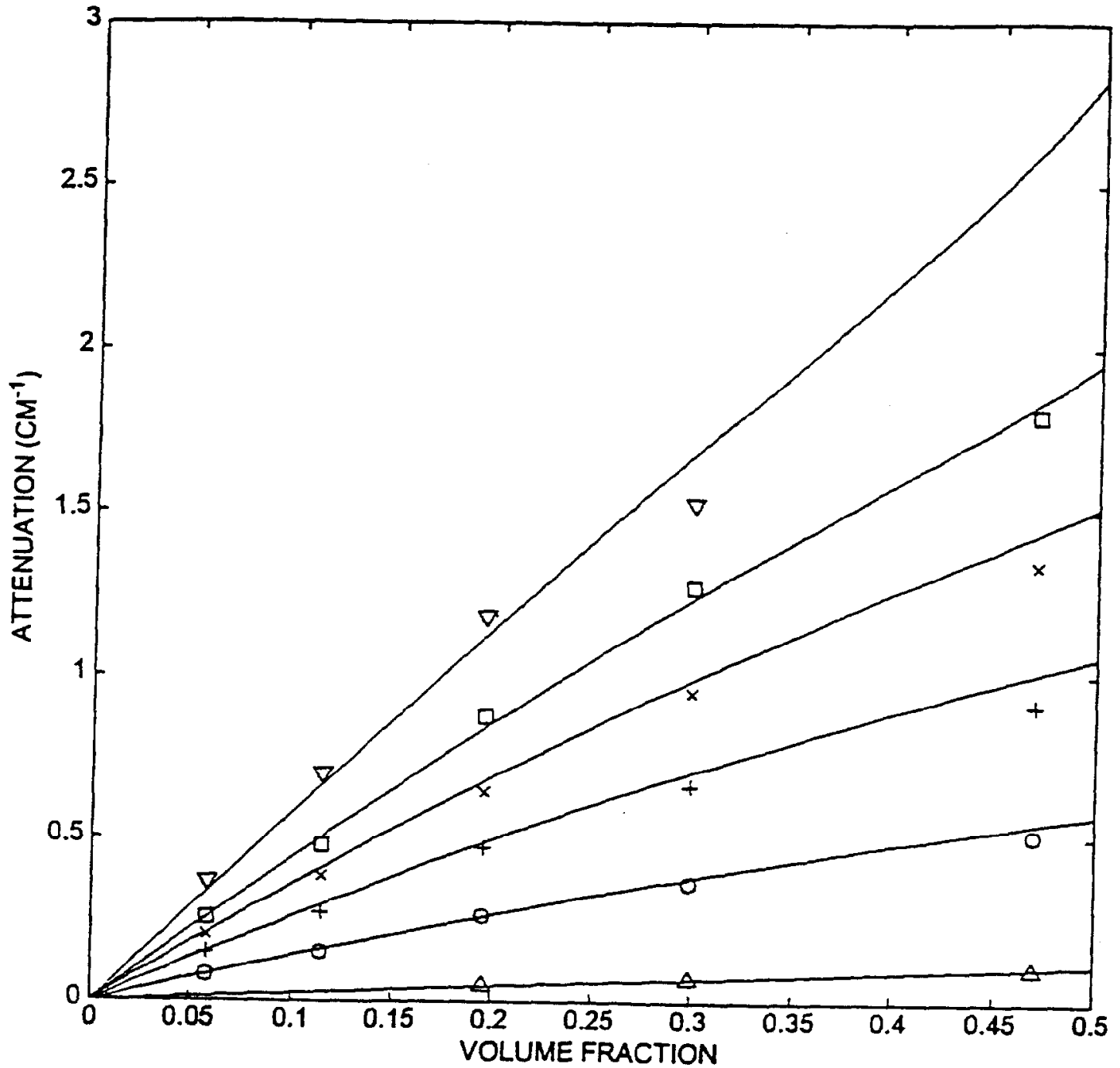

Figure 6.4: Attenuation as a function of solids volume fraction for the data of Allegra and Hawley (1972). The symbols represent experimental data at $3 \mathrm{MHz}(\Delta)$; $9 \mathrm{MHz}(\mathrm{O}) ; 15 \mathrm{MHz}(+) ; 21 \mathrm{MHz}(\times) ; 27 \mathrm{MHz}(\square)$; and $39 \mathrm{MHz}(\nabla)$.The solid curves represent the results of the effective medium approach used in this study. 
and $39 \mathrm{MHz}$. For these systems, the agreement between the experimental data and theory is rather good for all solids volume fractions over the range which attenuation data are available for frequencies up to $27 \mathrm{MHz}$. Even at $39 \mathrm{MHz}$, the agreement between the experimental data and theory is fairly good up to a solids volume fraction of approximately 0.2 .

Based on the results shown in Figure 6.4, the effective medium theory appears to describe the attenuation behavior as a function of solids volume fraction quite well, or at least when the particles are small and are of an elastic material like polystyrene. It is not clear as to why the results for the Potter's Beads slurries were not as good as for the slurry investigated by Allegra and Hawley (1972). It should be noted that the Potter's particles are soda-lime glass and they are, on average almost three orders of magnitude larger than the particles used by Allegra and Hawley (1972). The differences between the results for the two sets of data could be attributed to a behavior dependence based upon the different, particle size governed, attenuation regimes as was seen in the attenuation versus frequency curves. 


\section{Chapter 7: Theory and Experiments for Solid-Gas- Liquid Slurries}

\subsection{Theory for the Effective Wavenumber in Solid-Gas-Liquid Slurries}

The theory developed in the previous chapter can be readily extended to treat suspensions having more than one species, e.g., solid particles and gas bubbles, and particles of different sizes. In this chapter we compare the predictions of the theory to experiments in solid-gas-liquid systems.

\subsection{Attenuation Measurements in Solid-Gas-Liquid Slurries:}

Measurements of attenuation as a function of frequency were performed in solidgas-liquid systems at low gas volume fraction. The first systems investigated were comprised of soda-lime glass spheres (as described in Section 3.2.1) in water at $5 \%$ and $10 \%$ by volume. The gas phase bubbles were produced first by electrolysis and then by sparging air at $150 \mathrm{ml} / \mathrm{hr}$ through a limewood aerator (as described in Section 3.3). The results of these attenuation measurements are shown in Figures 7.1 and 7.2. In these figures, the solid-liquid data are shown on the same plot as the solid-gas-liquid data for comparison. It should be noted that the gas phase volume fraction in the solid-gas-liquid systems is fairly small. In fact, the gas volume fraction is estimated, by use of the photomicrographic imaging system, at approximately 0.02 for the systems where the bubbles were generated by the limewood aerator and at approximately 0.002 for the 
systems where the bubbles were generated by electrolysis (and in both cases are found to be overestimates based on comparison with forward theory predictions "fitted" to the experimental data). Therefore, addition of the gas phase has little effect on the solids volume fraction; and it can assumed that the solids concentration remains essentially constant between the solid-liquid and solid-gas-liquid systems.

Figure 7.1 shows the attenuation versus frequency results for bubbles generated by the limewood aerator operating at a gas flow rate of $150 \mathrm{ml} / \mathrm{hr}$. Comparing the curves for the solid-gas-liquid systems with those of the solid-liquid systems at the same solids concentration, the effect of the gas phase, even at these low concentrations, is quite apparent. The attenuation for the solid-gas-liquid slurries is essentially constant up to about $3 \mathrm{MHz}$, where the total attenuation is dominated by the contribution of the gas bubbles. Then, at higher frequencies, the attenuation begins to increase fairly rapidly as it becomes dominated by the attenuation due to the scattering regime for the solids. In fact, above approximately $6 \mathrm{MHz}$, the attenuation behavior is completely controlled by the attenuation due to the solid particles. This behavior is not surprising as the aerator generated bubbles are estimated to have a mean diameter of approximately $141 \mu \mathrm{m}$, and bubbles of this size resonate at about $0.046 \mathrm{MHz}(46 \mathrm{kHz})$. This resonance frequency is well below the range of frequencies investigated in this experimental study. Therefore, the contribution to the total attenuation by the bubbles is expected to be fairly small, and, the total attenuation in this region should be dominated by the solids. Whether or not the true bubble diameter is actually $141 \mu \mathrm{m}$ is not really relevant to the three phase behavior in this tail region. As long as the bubble size is such that the resonance frequency is 


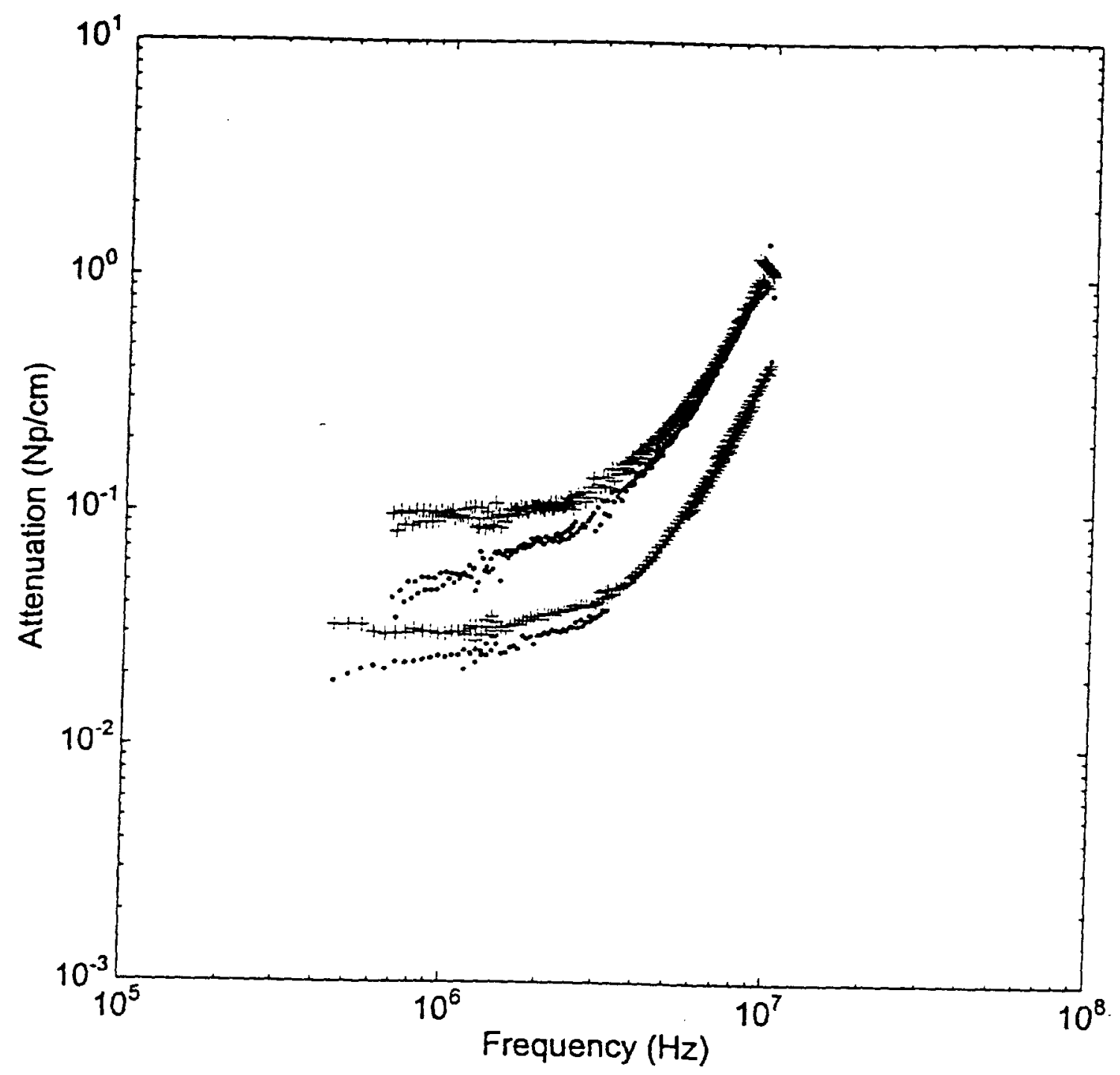

Eigure 2.1: Attenuation versus frequency data for solid-gas-liquid systems of sodalime glass beads in water at $5 \%$ (lower curve) and $10 \%$ solids by volume with bubbles generated by a limewood aerator with air flow rate of 150 $\mathrm{ml} / \mathrm{hr}$. The solid-gas-liquid data $(+)$ are shown with solid-liquid data $(\bullet)$ at the same solids concentrations. The solids size distribution has a mean radius of $14.9 \mu \mathrm{m}$ with standard deviation of $3.56 \mu \mathrm{m}$. - E2231. 
below $0.1 \mathrm{MHz}$, the solid-gas-liquid data should behave in the manner shown in Figure 7.1. A resonance frequency of $0.1 \mathrm{MHz}$ implies a bubble diameter of approximately 65 $\mu \mathrm{m}$, which is less than one half of the reported value from the photomicrographic bubble measurements. The photomicrographic technique does not, however, overestimate the bubble diameter by a factor of two. Therefore, in this context it is adequate to use the reported bubble diameter as a basis to interpret the solid-gas-liquid slurry behavior.

The influence of bubble size on the behavior of the solid-gas-liquid systems can be seen when the results in Figure 7.1 are compared with those shown in Figure 7.2. Figure 7.2 shows the attenuation spectra for the same soda-lime glass slurries as were shown in Figure 7.1. However, in this case, the bubbles for the solid-gas-liquid slurries were generated by electrolysis rather than by the aerator. Bubbles generated by electrolysis tend to have a smaller mean diameter (as described in Section 3.3), and this smaller diameter has an effect on the solid-gas-liquid attenuation results. In Figure 7.2 the attenuation for the solid-gas-liquid slurries is considerably higher in the $0.5 \mathrm{MHz}$ to 1 MHz region than it is for the solid-gas-liquid slurries shown in Figure 7.1. Also, the attenuation for the solid-gas-liquid slurries in Figure 7.2 is never constant as in Figure 7.1. The reason for this difference is the considerably smaller mean diameter of the bubbles in the solid-gas-liquid slurries in Figure 7.2. The electrolytically generated bubbles, with a mean diameter of $51 \mu \mathrm{m}$, have a resonance frequency of approximately $0.13 \mathrm{MHz}$. This frequency is much closer to (but still outside) the frequency range of investigation of these experiments. Therefore, the frequency range is such that the resonance peak has not completely decayed away to the constant attenuation behavior 


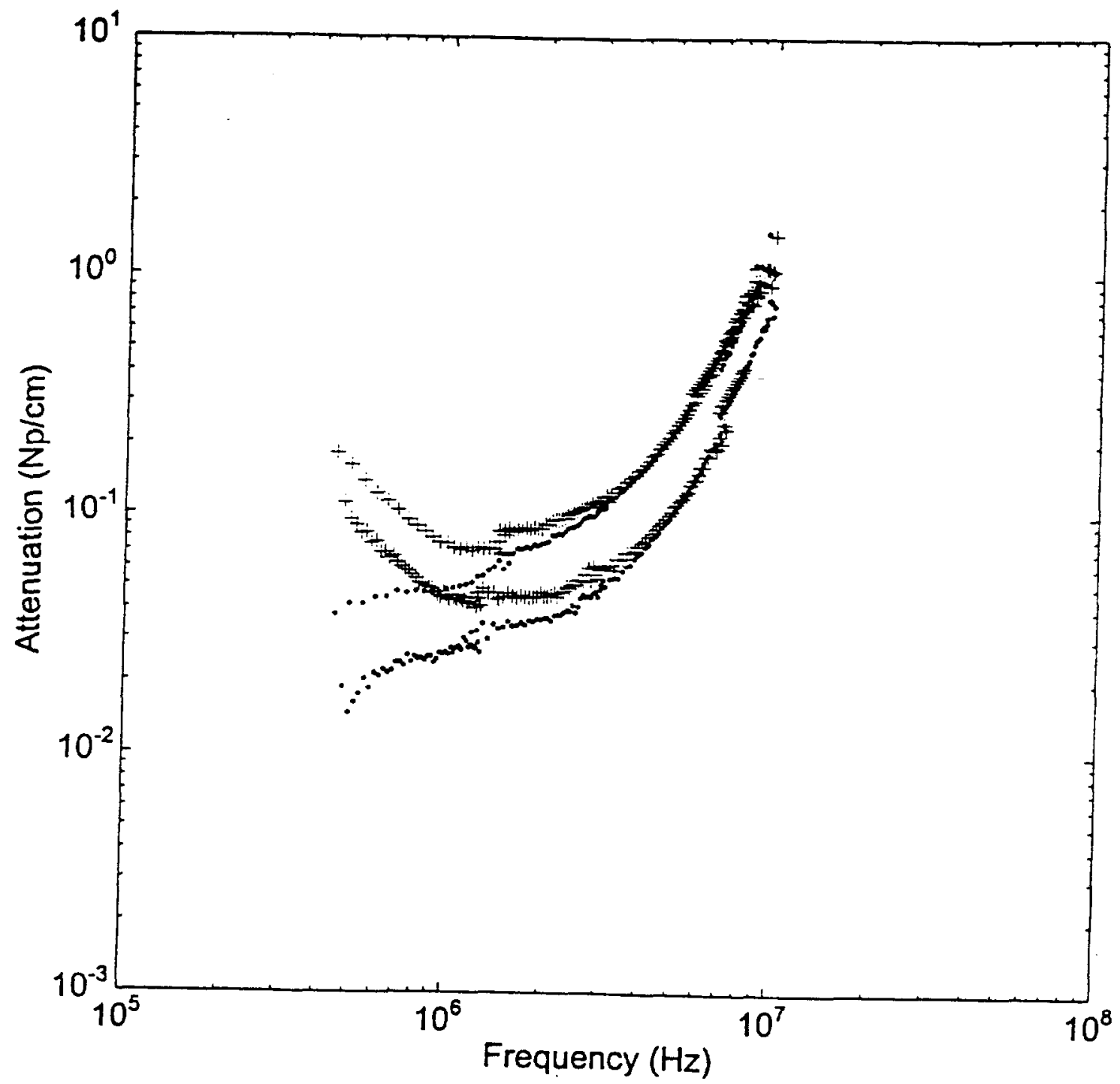

Figure 7.2: Attenuation versus frequency data for solid-gas-liquid systems of sodalime glass beads in water at $5 \%$ (lower curve) and $10 \%$ solids by volume with bubbles generated by an electrolyzer at $40 \mathrm{~V} ; 4$ to $8 \mathrm{~mA} ;<1 \mathrm{~W}$. The solid-gas-liquid data $(+)$ are shown with solid-liquid data $(\bullet)$ at the same solids concentrations. The solids size distribution has a mean radius of $14.9 \mu \mathrm{m}$ with standard deviation of $3.56 \mu \mathrm{m}$. - E2231. 
observed in Figure 7.1. There is enough vestigial evidence in the lower frequency (bubble attenuation dominated) region of the solid-gas-liquid attenuation spectra to suggest the presence of the bubble resonance peak at approximately $1 \mathrm{MHz}$. It should, again, be noted that the solid-gas-liquid attenuation curves in Figure 7.2 are not constant over a significant frequency range as seen in Figure 7.1. Rather, the attenuation curves go through a minimum at approximately 1.5 to $2 \mathrm{MHz}$, at which the attenuation due to the scattering by the solid particles begins to dominate the overall attenuation behavior of the slurry.

The most significant observation which can be made from Figures 7.1 and 7.2 is, as predicted by the theory for sound propagation in bubbly liquids, at sufficiently high frequencies, the attenuation due to the presence of the bubbles in solid-gas-liquid systems becomes so small as to be completely masked by that due to the solids. Therefore, by carefully choosing the frequency range of interrogation, it is possible to create conditions under which the presence of the gas bubbles (at small gas volume fraction) is essentially irrelevant, and the total attenuation is dominated by that of the solids. Also, in the event that some information about the size distribution and concentration of the bubbles is known a priori, it may be possible to predict the attenuation behavior of the bubbles and effectively subtract it from the overall measured attenuation caused by the solid-gasliquid slurry. This approach would then yield the attenuation due to the solid-liquid slurry alone. This point will be addressed in more detail shortly.

Although it is not clear if the presence of the solids changes the gas phase volume fraction in the solid-gas-liquid slurry relative to the bubbly liquid alone, it appears that 
the volume fraction of the solids does not affect the volume fraction of the bubbles in the system once the solids are present. This observation is made from examination of Figure 7.3. The figure shows experimental attenuation data for solid-gas-liquid systems with solids at $5 \%$ and $10 \%$ by volume. Also plotted are the attenuation curves predicted by the forward theory using the same solids volume fractions and size distribution as in the experiments and a log-normal bubble size distribution with a $23 \mu \mathrm{m}$ mean diameter and $4.3 \mu \mathrm{m}$ standard deviation. The bubble size distribution used in the forward theory predictions is determined by fitting the theory results to the experimental data. The bubble volume fraction in each forward theory prediction is held constant at $1.5 \times 10^{-5}$. It can be seen that both theoretical attenuation curves fit their respective experimental data reasonably well. The only difference between the conditions used in generating the two theory curves is in the volume fraction of the solids. When the solids volume fraction in the theory curves is adjusted for the increasing experimental volume fraction from 0.05 to 0.10 , the fit between the theory prediction and experimental data is maintained. Therefore, although the volume fraction of the solids changes by a factor of two, the volume fraction of the gas bubbles remains unchanged. Thus the volume fraction of the solids does not appear to affect the bubble volume fraction in solid-gas-liquid slurries.

It has been previously mentioned that if some information about the bubble size distribution and volume fraction is known, it may be possible to predict the attenuation due to the bubbles in a solid-gas-liquid slurry and effectively subtract that attenuation from the total to yield just that due to the solids. This procedure has been carried out in Figure 7.4. This figure shows the result of subtracting the bubble attenuation from the 


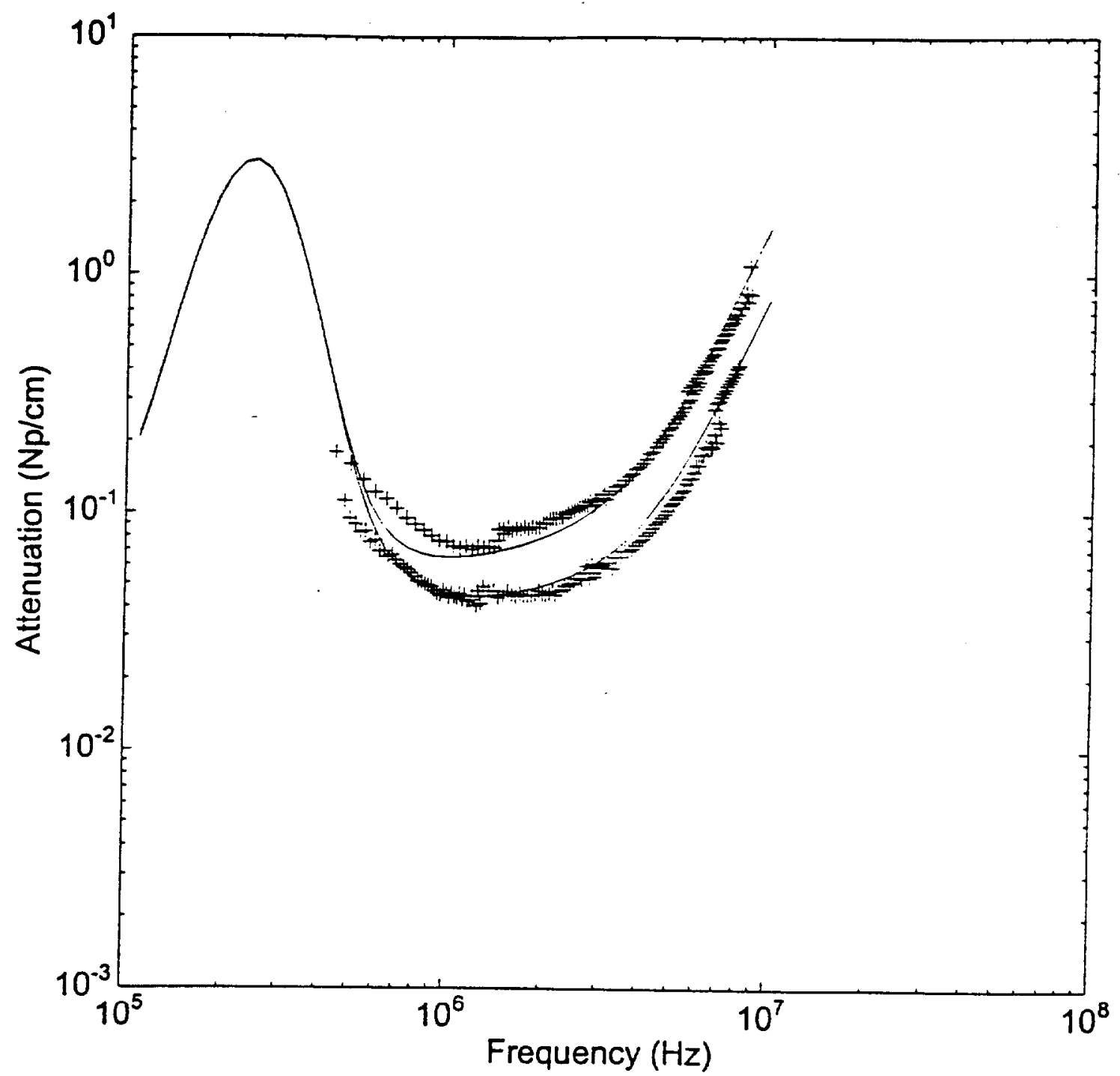

Eigure 73: Comparison between experimental solid-gas-liquid attenuation data $(+)$ at $5 \%$ (lower curve) and $10 \%$ solids (by volume) and forward problem theory predictions (solid curves) employing the same solids concentrations and a log-normal bubble size distribution with $23 \mu \mathrm{m}$ mean radius and $4.3 \mu \mathrm{m}$ standard deviation. The bubble volume fraction used in the theory predictions is $1.5 \times 10^{-5}$. - E2231. 


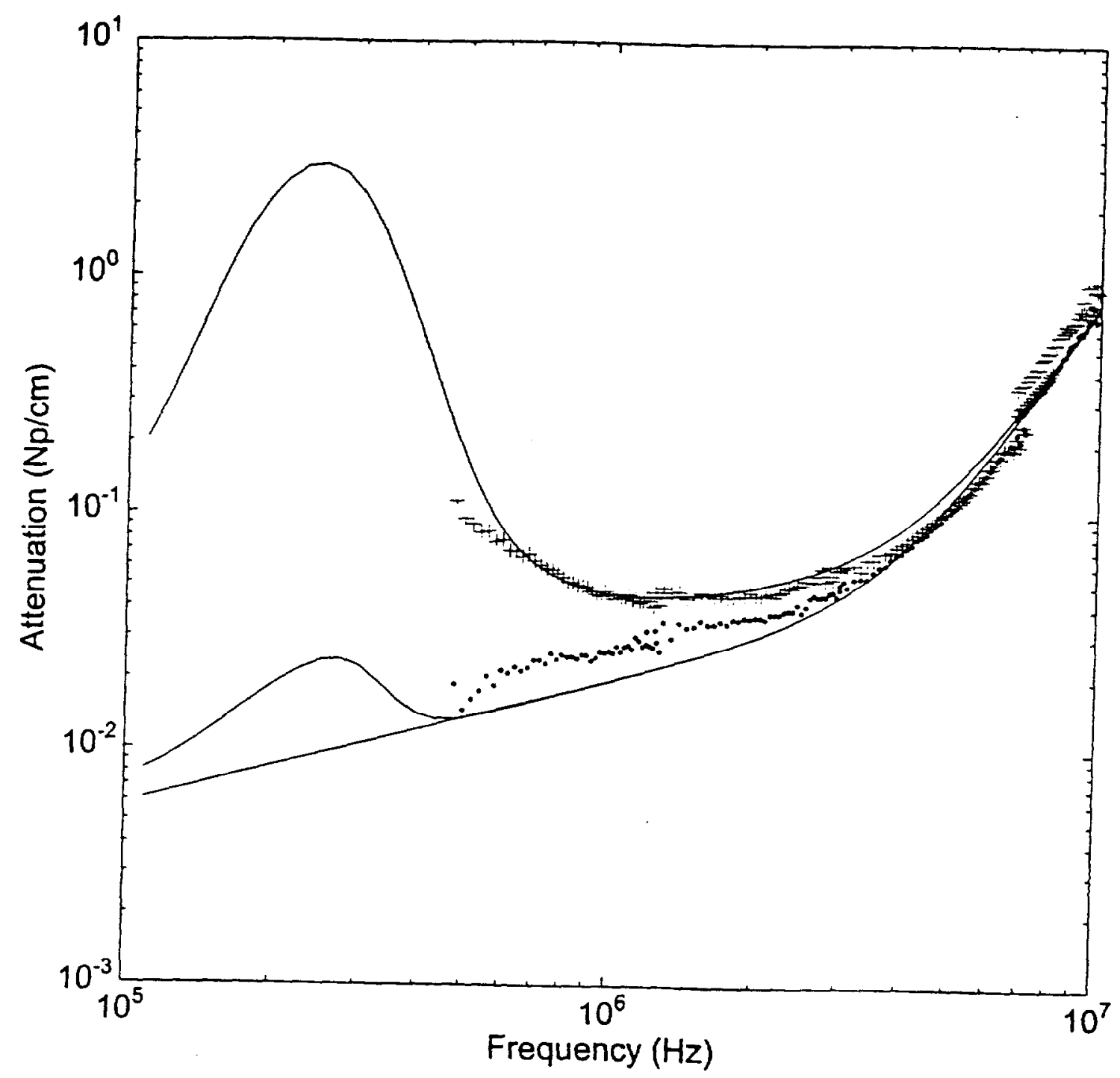

Figure 7.4: Results of predicting the attenuation due to the presence of bubbles in a solid-gas-liquid slurry and simply subtracting that attenuation from the total attenuation. The symbols represent experimental data for the solidliquid $(\bullet)$ and solid-gas-liquid $(+)$ slurries, and the curves represent the forward theory predictions for those slumies and the difference after subtracting the bubble attenuation. - E2231. 
total and compares it with the solid-liquid attenuation at $5 \%$ solids by volume. The solid-gas-liquid forward theory attenuation curve is the same as that shown for the $5 \%$ solids shown in Figure 7.3. It can be seen in this figure that the theory prediction resulting from the subtraction of the bubble attenuation from the total attenuation matches rather well with the solid-liquid data over the frequency range where those data are available. There is still a residual "hump" in the theory curve for the subtraction result at frequencies near the bubble resonance frequency. This "hump" is due to the non-linear volume fraction dependence of the bubble attenuation in this region which prevents simple subtraction from completely removing it from the total attenuation to yield only that due to the solids. However, the bubble resonance region is not the region of interest in this approach. It is the behavior in the "tail' region, well beyond the bubble resonance frequency which is of interest, and the subtraction of the bubble attenuation there yields results which compare fairly closely with the solid-liquid attenuation data.

As with the solid-liquid slurry experiments, it is of interest to investigate what effects, if any, would be contributed to the attenuation behavior of the slurry by a more elastic solid particle material, such as polystyrene. Therefore, further solid-gas-liquid attenuation experiments are attempted in systems containing polystyrene beads (Duke Scientific Corp., Palo Alto, CA) of $136 \mu$ m mean diameter, with standard deviation of $13.5 \mu \mathrm{m}$, in water. However, no data can be collected due to the presence of other physicochemical phenomena which interfere with the attenuation measurements. Attenuation measurements could be made in the solid-liquid only systems within the same experimental restrictions described previously. However, when bubbles are 
introduced into the slurry via the limewood aerator, the polystyrene particles are carried by the gas bubbles to the surface of the liquid phase where a stable foam layer is formed. It is apparent that a dispersed air flotation system has been established. The process of dispersed air flotation is described by Burns et al. (1997). The foam layer is quite stable, and the polystyrene particles were intimately entrained within the surface foam layer. The foam layer does not allow bubbles entering the test cell from the bottom to escape, and bubbles from the surface foam layer are re-entrained when the slurry is mixed. The end result is a continuous increase in the gas phase volume fraction over the course of the attenuation measurement. This increase in the gas volume fraction soon causes the attenuation of the ultrasound in the slurry to become so large as to be unmeasureable.

Attempts have been made to rectify the flotation situation, such as washing the surfactant from the particles and varying the slurry $\mathrm{pH}$ to both the alkaline and acidic extremes. In all cases stable surface foam layers form when the gas is introduced, and there is visible flocculation of the polystyrene particles.

Some information can be gleaned from the polystyrene solid-gas-liquid experiments. The most prominent point is that, in the event that the density difference between the solid and liquid phase is small, gas entrained in the slurry will not be a major consideration in its characterization. The results of this set of experiments indicate that even at relatively small gas phase volume fraction, the gas bubbles will induce flotation of the solids and cause them to be removed from the slurry into a froth. The resulting problem of acoustic characterization of foams with entrained solids is beyond the scope of this work. 


\section{Chapter 8: Conclusions and Future Work}

\subsection{Conclusions:}

The theory of Allegra and Hawley (1972) for dilute suspensions is shown to be in excellent agreement with experimental attenuation data obtained for slurries of soda-lime glass spheres in water and polystyrene spheres in water.

An effective medium approximation for the attenuation in concentrated slurries is employed in which the equations of motion are derived in a manner similar to that used in the dilute theory. However, the effective medium approach considers a solid particle to be centered inside a pure liquid "shell". Results of the effective medium approximation are compared with data obtained in fairly concentrated slurries, of up to $50 \%$ solids by volume, of $125 \mu \mathrm{m}$ mean diameter Potter's Beads (soda-lime glass) in a mixture of glycerin and water. The agreement between the effective medium approximation and the experimentally obtained attenuation data is quite good up to volume fractions of approximately 0.30 . At higher volume fractions, there is significant deviation.

In solid-gas-liquid slurries it is determined that, at sufficiently high frequencies, the total attenuation of ultrasound in the slurry is dominated by the attenuation due to the solid particles. In addition, the bubble volume fraction in the solid-gas-liquid system is not affected by the volume fraction of the solid particles. It is also determined that, if the bubble size distribution and volume fraction are known a priori, the attenuation due to the bubbles in a solid-gas-liquid slurry may be simply subtracted from the total 
attenuation in the slurry to yield only that due to the solids at frequencies well beyond the bubble resonance frequency.

Tikhonov regularization and linear programming techniques are employed to solve the inverse problem of determining the particle volume fraction distribution from the attenuation versus frequency data. Although these techniques are successful in solving the inverse problem in several cases, it is also shown that the results are very sensitive to the choice of frequency range, the physical properties of the particles, and the nature of the particle volume fraction distribution (unimodal, bimodal, etc.). Since the same techniques were shown to work quite well for bubbly liquids, the failures in the technique results which did occur are attributed to the complex resonance behavior of the slurries.

\subsection{Recommendations for Future Work:}

The work which is recommended to further the progress of this area of study can be divided into three categories. The first category consists of work which will advance the development of the acoustic probe within the current scope of its development. The second category is work which is recommended to broaden the scope of the acoustic probe development project. The final category is work which is recommended to enhance the performance of the photomicrographic imaging system.

The current status of efforts to solve the inverse problem for solid-liquid slurries indicates that the inverse problem for these systems is far more difficult to solve than for other systems, such as bubbly liquids. This difficulty arises due to the complicated and 
multiple resonances exhibited by the solid particles. It is found that satisfactory inverse problem results can only be obtained for solid-liquid systems where the solid phase is of a somewhat elastic material, such as polystyrene. The lack of distinct separation between the various modes of resonance in glass particles makes satisfactory solution of the inverse problem impossible in those systems. Therefore, it is most desirable to obtain solid-liquid-gas attenuation data for systems where the solid phase consists of a more elastic material, such as polystyrene particles. The success in solution of the inverse problem for solid-liquid systems of this nature indicates that similar systems should be ideal for investigating the possibility of solving the inverse problem for solid-gas-liquid systems of similar composition. It is recommended that solid-gas-liquid attenuation experiments be attempted in systems containing particles of another material, such as polymethylmethacrylate (PMMA) or another system where flotation problems will be avoided. With a density of $1.19 \mathrm{~g} / \mathrm{cm}^{3}$ (as opposed to $1.05 \mathrm{~g} / \mathrm{cm}^{3}$ for polystyrene), the PMMA particles are less likely to be carried to the surface by the gas bubbles; yet the density is still close enough to that of water to facilitate homogeneous suspension. Also, the PMMA is a polymeric material which should be more elastic than glass. Therefore, there should be the desired separation between the resonances of the individual modes of oscillation which made the solution of the inverse problem for polystyrene/water solidliquid systems possible.

A fluidized bed or other flow system apparatus allows for better control of the slurry properties. A system of this type is preferable if more economical solid particles could be acquired to facilitate such an apparatus. 
Another area where further investigation is recommended is in the use of lower frequency transducers. Now that initial investigation has shown that the currently used methods of bubble generation create bubbles with resonance frequencies in the range of 0.03 to $0.5 \mathrm{MHz}$, it would be advisable to employ transducers which operate in these frequency ranges. Acquisition of solid-gas-liquid data down to these frequency ranges would provide information about the bubble resonance peaks which would better delineate the contributions of the gas bubbles to the solid-gas-liquid attenuation behavior. Also, implementation of these lower frequency transducers may also provide a means for collecting more reliable bubbly liquid attenuation data.

In regard to expansion of the scope of the acoustic probe development program, it is recommended that solid-liquid experimentation be revisited for the purpose of examining the acoustic behavior of very dilute (less than $1 \%$ solids by volume) solidliquid suspensions.

Another possible direction in which the probe development may proceed in the future could be an investigation of the propagation of acoustic energy in foams. These foams could be both gas-liquid only and, as has been observed in this study, gas-liquid with entrained solids. Another method to characterize such systems could be useful.

The final area where a considerable amount of future work can be done is in regard to the photomicrographic video imaging system. The current status of the imaging system allows for making multiple frame "movies" of bubbles and particles.

Measurement of bubble sizes can be performed and recorded manually with reasonable accuracy. However, the combined hardware and software capabilities of this system offer 
the potential for far more accurate and efficient bubble and particle size measurements through automation.

The Image SXM software package allows for many manipulations of an acquired image and has the capability to provide an automated measurement of bubble or particle size distributions. However, completion of these tasks will require the creation of userwritten "macros", or short program codes which instruct the computer software to perform operations which would normally be performed manually at the keyboard. A user will need to create "macros" to perform the necessary thresholding, conversion to binary image, bubble/particle distribution measurement, and presentation of data, and/or writing of data to data files for computer storage.

Another issue in regard to the imaging system is determining the proper lighting which should be used under experimental conditions to obtain the clearest photographs of the bubbles or particles with the best resolution of the bubble/particle interface with the liquid, or what is referred to as a large edge gradient (Bongiovanni et al. 1997). Indeed, there may not be a single lighting arrangement which will allow for optimum photo resolution of both the bubbles and particles in a solid-gas-liquid system. It is recommended that a study be performed to examine, in detail, the advantages and disadvantages of different lighting configurations as described in the literature in order to construct an overall imaging system which will provide for optimum use of the existing equipment: 


\section{References}

Alba, E., "Method and Apparatus for Determining Particle Size Distribution and Concentration in a Suspension Using Ultrasonics," US Patent No. $5,121,629$ (1992).

Allegra, J. R. and Hawley, S. A., "Attenuation of Sound in Suspensions and Emulsions: Theory and Experiments," J. Acoust. Soc. Am. 51, 1545 (1972).

Atkinson, C. M., "Acoustic Wave Propagation and Non-Intrusive Velocity Measurements in Highly Concentrated Suspensions," D.Sc. Thesis, Mass. Inst. Technol., Cambridge (1991).

Atkinson, C. M., and Kytomaa, H. K., "Acoustic Wavespeed and Attenuation in Suspensions," Int. J. Multiphase Flow. 18, 577 (1993).

Bapat, P. M., "Mass Transfer in a Liquid-Liquid Continuous-Flow Stirred-Tank Reactor," Ph.D. Thesis, Ill. Inst. Technol., Chicago (1982).

Barrett, S., Image SXM v1.61-7 Image Analysis Software, University of Liverpool, England (1998).

Bolz, R. E., CRC Handbook of Tables for Applied Engineering Science (CRC Press, Boca Raton, FL, USA, 1973).

Bongiovanni, C., Chevaillier, J. Ph., and Fabre, J., "Sizing of Bubbles by Incoherent Imaging: Defocus Bias," Experiments in Fluids 23, 209 (1997).

Bonnet, J. C. and Tavlarides, L., L., "Ultrasonic Technique for Dispersed Phase Hold-Up Measurements," I \& EC Res. 26, 811 (1987).

Burns, S. E., Yiacoumi, S., and Tsouris, C., "Microbubble Generation for Environmental and Industrial Separations," Separation and Purification Technology 11, 221 (1997).

Carstensen, E. L. and Foldy, L. L., "Propagation of Sound Through a Liquid Containing Bubbles," J. Acoust. Soc. Am. 19, 481 (1947).

Chivers, R. C. and Anson, L. W., "Calculations of the Backscattering and Radiation Force Functions of Spherical Targets for Use in Ultrasonic Beam Assessment," Ultrasonics 20, 25 (1982). 
Commander, K. W. and McDonald, R. J., "Finite-element Analysis Solution of the Inverse Problem in Bubble Swarm Acoustics," J. Acoust. Soc. Am. 89, 592 (1991).

Commander, K. W. and Prosperetti, A., "Linear Pressure Waves in Bubbly Liquids: Comparison Between Theory and Experiment," J. Acoust. Soc. Am. 85, 732 (1989).

Delves, L. M. and Mohamed, J. L., Computational Methods for Integral Equations (Cambridge University Press, Cambridge, England, 1985).

Dodd, T. L., Hammer, D. A., Sangani, A. S. and Koch, D. L., "Numerical Simulations of the Effect of Hydrodynamic Interactions on Diffusivities of Integral Membrane Proteins”, J. Fluid Mech., 293, 147 (1995).

Duraiswami, R., "Bubble Density Measurement Using an Inverse Acoustic Scattering Technique," ASME Cavitation and Multiphase Forum, Washington, DC, edited by O. Furuya, FED vol. 153, p. 67, (ASME, New York, 1993).

Duraiswami, R., Prabhukumar, S. and Chahine, G. L., "Bubble Counting Using an Inverse Acoustic Scattering Method," J. Acoust. Soc. Am., 104, 2699 (1998).

Epstein, P. S. and Carhart, R. R., "The Absorption of Sound in Suspensions and Emulsions. I. Water Fog in Air," J. Acoust. Soc. Am. 25, 553 (1953).

Faran, J. J., "Sound Scattering by Solid Cylinders and Spheres," J. Acoust. Soc. Am. 23, 405 (1951).

Foldy, L. L., "The Multiple Scattering of Waves," Phys. Rev. 67, 107 (1945).

Garr, D., "Too Hot to Handle," Popular Science 52, August 1992.

Gradshteyn, I. S., and Ryszhik, I. M., Tables of Integrals, Series, and Products, $5^{\text {th }}$ Edition, Academic Press (1994).

Greenwood, M. S., Mai, J. L., and Good, M. S., "Attenuation Measurements of Ultrasound in a Kaolin-Water Slurry: A Linear Dependence Upon Frequency," J. Acoust. Soc. Am. 94, 908 (1993).

Happel, J. and Brenner, H., Low Reynolds Number Hydrodynamics, Martinus Nijhoff Publishers, Dordrecht, The Netherlands, (1973). 
Jeffrey, D. J., "Conduction Through a Random Suspension of Spheres", Proc. $R$. Soc. Lond. A, 335, 355 (1973).

Kessler, L. W., Hawley, S. A., and Dunn, F., "Semiautomatic Determination of Ultrasonic Velocity and Absorption," Acoustica 24, 105 (1971).

Kim, S., and Russel, W. B., "Modeling of Porous Media by Renormalization of the Stress Equations", J. Fluid Mech., 154, 269 (1995).

Kinsler, L. E., Frey, A. R., Coppens, A. B., and Sanders, J. V., Fundamentals of Acoustics (John Wiley \& Sons, Inc., New York, NY, USA, 1982).

Kirou, V. I., Tavlarides, L. L., Bonnet, J. C., and Tsouris, C., "Flooding, Holdup, and Drop Size Measurement in a Multistage Column Extractor," AIChE Journal 34, 283 (1988).

Kol'tsova, I. S., Krynskii, L. O., Mikhalov, I. G. and Pokrovskaya, I. E., "Attenuation of Ultrasonic Waves in Low-Viscosity Liquids Containing Gas Bubbles," Akust. Zh. 25, 725 (1979) [English Translation: Sov. Phys. Acoust. 25, 409 (1979)].

Kress, R., Linear Integral Equations (Springer, Berlin, Germany, 1989).

Kuster, G., and Toksoz, M. N., "Velocity and Attenuation of Seismic Waves Waves in Two-Phase Media: Part II. Experimental Results," Geophysics, 39, 607 (1974).

Kuwabara, S., "The Forces Experienced by Randomly Distributed Parallel Circular Cylinders or Spheres in a Viscous Flow at Small Reynolds Numbers", J. Phys. Soc. Japan, 14, 527 (1959).

Kytomaa, H. K., "Theory of Sound Propagation in Suspensions: A Guide to Particle Size and Concentration Characterization," Powder Technol. 82, 115 (1995).

Ladd, A. J. C., "Hydrodynamic Transport Coefficients of Random Dispersions of Hard Spheres", J. Chem. Phys, 93, 3484 (1990).

Landau, L. D. and Lifshitz, E. M., Theory of Elasticity, $3^{\text {rd }}$ Edition, Pergamon Press (1986).

Levi, B. G., "Hanford Seeks Short- and Long-term Solutions to Its Legacy of Waste," Physics Today, 17 March 1992. 
Lighthill, M. J., "Viscosity Effects in Sound Waves of Finite Amplitude," in Surveys in Mechanics, edited by G. K. Batchelor and R. M. Davies (Cambridge University Press, Cambridge, England, 1956).

Ma, Y., Varadan, V. K. and Varadan, V. V., "Comments on Ultrasonic Propagation in Suspensions,” J. Acoust. Soc. Am. 87, 2779 (1990).

Martin, J., Rakotomalala, N., and Salin, D., "Hydrodynamic Dispersion of Noncolloidal Suspensions: Measurement form Einstein's Argument," Phys. Rev. Lett. 74, 1347 (1995).

McClements, D. J., "Comparison of Multiple Scattering Theories with Experimental Measurements in Emulsions," J. Acoust. Soc. Am. 91, 849 (1992).

McLeod, F., "Directional Doppler Flowmeter," Intl. Conf. Med. Bio. Eng. Stockholm, Sweden 213 (1967).

Mo, G., and Sangani, A. S., "A Method for Computing Stokes Flow Interactions Among Spherical Objects and Its Application to Suspensions of Drops and Porous Particles", Phys. Fluids, 6, 1637 (1994).

Oja, T. and Alba E., "Acoustic Attenuation Spectroscopy for Particle Sizing of High Concentration Dispersions," presentation at the NIST International Workshop on Ultrasonic and Dielectric Characterization Techniques for Suspended Particulates. (Gaithersburg, MD, USA, 1997).

Okamura, S., Uchida, S., Katsumata, T., and Iida, K., "Measurement of Solids Holdup in a Three-Phase Fluidized Bed by an Ultrasonic Technique," Chem. Eng. Sci. 44, 196 (1989).

Pacek, A. W., Moore, I. P. T., Nienow, A. W., and Calabrese, R. V., "Video Technique for Measuring Dynamics of Liquid-Liquid Dispersion During Phase Inversion," AIChE Journal 40, 1940 (1994).

Perry, R. H., Green, D. W., and Maloney, J. O., (Eds.), Perry's Chemical Engineer's Handbook, (McGraw-Hill, New York, NY, USA; 1984).

Ramirez, R. W., The FFT - Fundamentals and Concepts (Prentice Hall, Englewood Cliffs, NJ, USA, 1985).

Sangani, A. S., "A Pairwise Interaction Theory for Determining the Linear Acoustic Properties of Dilute Bubbly Liquids," J. Fluid Mech. 232, 
221 (1991).

Sangani, A. S., Zhang, D. Z., and Prosperetti, A., "The Added Mass, Basset, and Viscous Drag Coefficients in Nondilute Bubbly Liquids Undergoing Small-Amplitude Oscillatory Motion," Phys. Fluids A 3, 2955 (1991).

Sangani, A. S. and Mo, G., "Elastic Interactions in Particulate Composites with Perfect as Well as Imperfect Interfaces", J. Mech. Phys. Solids 45, 2001 (1997).

Shin, W-T, Yiacoumi, S., and Tsouris, C. "Experiments on Electrostatic Dispersion of Air in Water," I \& EC Res. 36, 3647 (1997).

Silberman, E., "Sound Velocity and Attenuation in Bubbly Mixtures Measured in Standing Wave Tubes," J. Acoust. Soc. Am. 29, 925 (1957).

Soong, Y., Blackwell, A. G., Schehl, R. R., Zarochak, M. F., and Rayne, J. A., "Ultrasonic Characterization of Three-Phase Slurries", Chem. Eng. Comm., 138, 213 (1995).

Soong, Y., Gamwo, I. K., Blackwell, A. G., Harke, F. W., Schehl, R. R., and Zarochak, M. F., "Ultrasonic Characterization of Slurries in an Autoclave Reactor at Elevated Temperature", I \& EC Res., 35, 1807 (1996).

Spelt, P. D. M., Norato, M. A., Sangani, A. S., and Tavlarides, L. L., "Determination of Particle Size Distributions from Acoustic Wave Propagation Measurements", accepted for publication in Phys. Fluids, January (1999).

Spelt, P. D. M., Norato, M. A., Sangani, A. S., Tavlarides, L. L., and Greenwood, M. S., "Attenuation of Sound Waves in Concentrated Slurries: An Effective-Medium Theory and Experiments", in preparation (1999).

Strout, T. A., "Attenuation of Sound in High-Concentration Suspensions:

Development and Application of an Oscillatory Cell Model," Ph.D.

Thesis, University of Maine, Orono (1991).

Tavlarides, L. L., and Bonnet, J. C., "An Ultrasonic Technique for Dispersed

Phase Holdup Measurements," US Patent No. 4,726,221 (1988).

Tsouris, C., and Tavlarides, L. L., "Comments on 'Model for Hold-Up Measurements in Liquid Dispersions Using an Ultrasonic Technique'," I \& EC Res. 29, 2170 (1990).

Tsouris, C., and Tavlarides, L. L., "Volume Fraction Measurements of Water in Oil by an Ultrasonic Technique," I \& EC Res. 32, 998 (1993). 
Tsouris, C., Norato, M. A., and Tavlarides, L. L., "A Pulse-Echo Ultrasonic Probe for Local Volume Fraction Measurements in Liquid-Liquid Dispersions," I \& EC Res. 34, 3154 (1995).

Tsouris, C., DePaoli, D. W., Feng, J. Q., and Scott, T. C., "Experimental Investigation of Electrostatic Dispersion of Nonconductive Fluids into Conductive Fluids," I \& EC Res. 34, 1394 (1995).

Turner, J. C. R., "Two-Phase Conductivity, the Electrical Conductance of Liquid Fluidized Beds of Spheres", Chem. Eng, Sci. 31, 487 (1976).

Uchida, S., Okamura, S., and Katsumata, T., "Measurement of Longitudinal Distribution of Solids Holdup in a Three-Phase Fluidized Bed by Ultrasonic Technique," Can. J. Chem. Eng. 67, 166 (1989).

Urick, R. J., "Sound Velocity Method for Determining the Compressibility of Finely Divided Substances," J. Appl. Physics 18, 983 (1947).

Urick, R. J., "The Absorption of Sound in Suspensions of Irregular Particles," J. Acoust. Soc. Am. 20, 283 (1948).

Varadan, V. K., Bringi, V. N., Varadan, V. V., and Ma, Y, "Coherent Attenuation of Acoustic Waves by Pair-Correlated Random Distribution of Scatterers with Uniform and Gaussian Size Distributions," J. Acoust. Soc. Am. 73, 1941 (1983).

Wang, W. and Sangani, A. S., "Nusselt Number for Flow Perpendicular to Arrays of Cylinders in the Limit of Small Reynolds and Large Peclet Numbers", Phys. Fluids, 9, 1529 (1997).

Weast, R. C., Astle, M. J., and Beyer, W. H., (Eds.), CRC Handbook of Chemistry and Physics, (CRC Press, Boca Raton, FL, USA 1984).

Yi, J. H., and Tavlarides, L. L., "Model for Hold-Up Measurements in Liquid-Liquid Dispersions Using an Ultrasonic Technique," I \& EC Res. 29, 475 (1990). 


\section{Appendix A: Boundary Conditions from Epstein and Carhart (1953) and Allegra and Hawley(1972)}

Given in this Appendix is a set of linear equations for unknowns which include the coefficients, $A_{n}$, required to calculate the attenuations from equations (2.59) and (2.66) through (2.68), or (2.69). These equations are derived from the boundary

conditions on the surface of a test particle. In addition to the coefficients, $A_{n}$, and $\widetilde{A}_{n}$, of the solution of equation (2.46) outside and inside the particle, respectively; similar coefficients arise due to the solutions of equations (2.47) and (2.48), denoted by $B_{n}$ and $C_{n}$, respectively. It should be noted that equation $(2.48)$ is an equation for the vector $\mathbf{A}$, rather than for a scalar velocity potential, but only the azimuthal component of $\mathbf{A}$ is non-zero. Therefore, the solution of equation (2.48) has only the scalar coefficient, $C_{n}$. The following notations are relevant to the equations presented here: $z_{c}=k_{c} a$, $z_{T}=k_{T} a, z_{s}=k_{s} a$

$$
\begin{gathered}
z_{c} j_{n}^{\prime}\left(z_{c}\right)+A_{n} z_{c} h_{n}^{\prime}\left(z_{c}\right)+B_{n} z_{T} h_{n}^{\prime}\left(z_{T}\right)-C_{n} n(n+1) h_{n}\left(z_{s}\right)= \\
\widetilde{A}_{n} \widetilde{z}_{c} j_{n}^{\prime}\left(\widetilde{z}_{c}\right)+\widetilde{B}_{n} \widetilde{z}_{T} j_{n}^{\prime}\left(\widetilde{z}_{T}\right)-\widetilde{C}_{n} n(n+1) j_{n}\left(\widetilde{z}_{s}\right)
\end{gathered}
$$




$$
\begin{aligned}
& j_{n}\left(z_{c}\right)+A_{n} h_{n}\left(z_{c}\right)+B_{n} h_{n}\left(z_{T}\right)-C_{n}\left(h_{n}\left(z_{s}\right)+z_{s} h_{n}^{\prime}\left(z_{s}\right)\right)= \\
& \quad \widetilde{A}_{n} j_{n}\left(\widetilde{z}_{c}\right)+\widetilde{B}_{n} j_{n}\left(\widetilde{z}_{T}\right)-\widetilde{C}_{n}\left(j_{n}\left(\widetilde{z}_{s}\right)+\widetilde{z}_{s} j_{n}^{\prime}\left(\widetilde{z}_{s}\right)\right) \\
& b_{c}\left[j_{n}\left(z_{c}\right)+A_{n} h_{n}\left(z_{c}\right)\right]+B_{n} b_{T} h_{n}\left(z_{T}\right)=\widetilde{A}_{n} \widetilde{b}_{c} j_{n}\left(\widetilde{z}_{c}\right)+\widetilde{B}_{n} \widetilde{b}_{T} j_{n}\left(\widetilde{z}_{T}\right) \\
& \tau\left(z_{c} b_{c}\left[j_{n}^{\prime}\left(z_{c}\right)+A_{n} h_{n}^{\prime}\left(z_{c}\right)\right]+B_{n} b_{T} z_{T} h_{n}^{\prime}\left(z_{T}\right)\right)= \\
& \quad \widetilde{\tau}\left(\widetilde{A}_{n} \widetilde{b}_{c} \widetilde{z}_{c} j_{n}^{\prime}\left(\widetilde{z}_{c}\right)+\widetilde{B}_{n} \widetilde{b}_{T} \widetilde{z}_{T} j_{n}^{\prime}\left(\widetilde{z}_{T}\right)\right) \\
& (-i \omega \mu)\left(\left[\left(z_{s}^{2}-2 z_{c}^{2}\right) j_{n}\left(z_{c}\right)-2 z_{c}^{2} j_{n}^{\prime \prime}\left(z_{c}\right)\right]+\right. \\
& A_{n}\left[\left(z_{s}^{2}-2 z_{c}^{2}\right) h_{n}\left(z_{c}\right)-2 z_{c}^{2} h_{n}^{\prime \prime}\left(z_{c}\right)\right]+ \\
& B_{n}\left[\left(z_{s}^{2}-2 z_{T}^{2}\right) h_{n}\left(z_{T}\right)-2 z_{T}^{2} h_{n}^{\prime \prime}\left(z_{T}\right)\right]+ \\
& \left.C_{n} 2 n(n+1)\left[z_{s} h_{n}^{\prime}\left(z_{s}\right)-h_{n}\left(z_{s}\right)\right]\right)= \\
& \widetilde{A}_{n}\left[\left(\omega^{2} \widetilde{\rho} a^{2}-2 \widetilde{\mu} \widetilde{z}_{c}^{2}\right) j_{n}\left(\widetilde{z}_{c}\right)-2 \widetilde{\mu} \widetilde{z}_{c}^{2} j_{n}^{\prime \prime}\left(\widetilde{z}_{c}\right)\right]+ \\
& \widetilde{B}_{n}\left[\left(\omega^{2} \widetilde{\rho} a^{2}-2 \widetilde{\mu} \widetilde{z}_{T}^{2}\right) j_{n}\left(\widetilde{z}_{T}\right)-2 \widetilde{\mu} \widetilde{z}_{T}^{2} j_{n}^{\prime \prime}\left(\widetilde{z}_{T}\right)\right]+ \\
& \widetilde{C}_{n} 2 \widetilde{\mu} n(n+1)\left[\widetilde{z}_{s} j_{n}^{\prime}\left(\widetilde{z}_{s}\right)-j_{n}\left(\widetilde{z}_{s}\right)\right]
\end{aligned}
$$




$$
\begin{aligned}
& (-i \omega \mu)\left(z_{c} j_{n}^{\prime}\left(z_{c}\right)-j_{n}\left(z_{c}\right)+A_{n}\left[z_{c} h_{n}^{\prime}\left(z_{c}\right)-h_{n}\left(z_{c}\right)\right]+\right. \\
& B_{n}\left[z_{T} h_{n}^{\prime}\left(z_{T}\right)-h_{n}\left(z_{T}\right)\right]- \\
& \left(C_{n} / 2\right)\left[z_{s}^{2} h_{n}^{\prime \prime}\left(z_{s}\right)+\left(n^{2}+n-2\right) h_{n}\left(z_{s}\right)\right]= \\
& \widetilde{\mu}\left(\widetilde{A}_{n}\left[\widetilde{z}_{c} j_{n}^{\prime}\left(\widetilde{z}_{c}\right)-j_{n}\left(\widetilde{z}_{c}\right)\right]+\widetilde{B}_{n}\left[\widetilde{z}_{T} j_{n}^{\prime}\left(\widetilde{z}_{T}\right)-j_{n}\left(\widetilde{z}_{T}\right)\right]-\right. \\
& \left(\widetilde{C}_{n} / 2\right)\left[\widetilde{z}_{s}^{2} j_{n}^{\prime \prime}\left(\widetilde{z}_{s}\right)+\left(n^{2}+n-2\right) j_{n}\left(\widetilde{z}_{s}\right)\right] .
\end{aligned}
$$

Here, $b_{c}$ and $b_{T}$ are given by

$$
b_{c}=\frac{(1-\gamma) \omega^{2}}{\beta c^{2}}, \quad b_{T}=-\frac{\gamma}{c_{1}^{2} \beta}\left[\omega^{2}-\left(\frac{c_{1}^{2}}{\gamma}-\frac{4 i \omega \mu}{3 \rho}\right) k_{T}^{2}\right]
$$

where $\beta$ is the thermal expansion coefficient and $c_{l}$ is the liquid equivalent speed of sound for spherical compressional waves in an elastic isotropic solid given by $\widetilde{c}_{1}=\sqrt{(\tilde{\lambda}+2 \tilde{\mu} / 3) / \widetilde{\rho}}$. The Lame constant, $\tilde{\lambda}$, is not really needed when the speed of sound, $c$, of the longitudinal compressional waves is specified, as one can also write $\widetilde{c}_{1}^{2}=c^{2}\left(1-4 \tilde{\mu} /\left(3 \rho c^{2}\right)\right)$. The above equations have also been given by Epstein and Carhart (1953) and Allegra and Hawley (1972). However, in both there are typographical errors. In Epstein and Carhart (1953), the last $j_{n}^{\prime}\left(\widetilde{z}_{s}\right)$ in equation (A.2) is erroneously replaced by $h_{n}^{\prime}\left(\widetilde{z}_{s}\right)$. In Allegra and Hawley (1972), the signs of both $\left(n^{2}+n-1\right)$ terms are wrong, and the $h_{n}\left(z_{s}\right)$ term on the left-hand side of equation (A.6) has an argument $\widetilde{z}$ instead. Also, the first $z_{s}$ after $C_{n}$ is replaced by $\widetilde{z}_{s}$. Not 
correcting the typographical errors in Allegra and Hawley (1972) would alter the results significantly. 


\section{Appendix B: Experimental Procedures}

\section{B.1 Slurry Attenuation Measurement Procedure:}

\section{B.1.1 General Considerations:}

In all attenuation measurement experiments, regardless of which type of vessel or cell is being used, it is imperative that the separation distance between each transducer pair be measured once the transducers are installed. The vessel or test cell should be clean and dry. The electronics and instrumentation should be set up as per Figures 3.2 and 3.4 , depending upon whether the Toneburst or FFT/Pulse Technique is being employed. It is recommended that, whenever possible, data should be acquired by both techniques as agreement between results of the two techniques provides reproducibility of results. Also, the Toneburst Technique provides slightly better results at higher frequencies and larger attenuations where significant noise in the data may interfere with FFT calculations. Conversely, the FFT/Pulse Technique seems to provide slightly better results at lower frequencies and lower attenuations where the error the difference between the slurry and reference water voltage signals is so small that the error in the measurement is of the same order of magnitude as the measurement, itself. This is the trend that was seen in earlier attenuation experiments; however, later toneburst measurements were performed by having the oscilloscope make the peak-to-peak voltage measurement on a waveform averaged over 50 sweeps, rather than making manual peakto-peak voltage measurements in real time. Allowing the oscilloscope to make the 
measurements does improve the data quality for the Toneburst Technique at lower frequencies and attenuations.

It should also be noted that earlier attenuation measurements refer to a "Standard" Toneburst Technique. This technique is just the toneburst technique described in Section 3.1.2, with the received signal going through an attenuator box, to the receiver/amplifier of the TB-1000 card, then on to the oscilloscope. This variation of the Toneburst Technique has been all but abandoned as it is sometimes difficult to achieve consistent receiver/amplifier performance. Secondly, in most cases it was found that the use of the receiver/amplifier was unnecessary, and better (more accurate and reproducible) results are achieved when the received signal is input directly to the oscilloscope. This variation of the Toneburst Technique is the "Direct" Toneburst Technique. The "Direct" method is the preferred Toneburst procedure, and it is the method which has been (and should be) employed, unless noted otherwise.

In regard to the operation of the electronic instrumentation, it is not the objective of this work to reproduce information which is available in the various user's manuals. The objective is, rather, to document information which is conducive to obtaining quality experimental results, yet is NOT available or clear in the user's manuals. The contents of this work should not be construed as to obviate the use of user's manuals.

\section{B.1.2 Operation of the Matec TB-1000 Toneburst Generator and}

\section{Panametrics 5052 PR Pulser/Receiver:}




\section{B.1.2.1 Matec TB-1000 Toneburst Generator:}

The Matec TB-1000 toneburst generator is a digital synthesizer card installed in the Gateway 2000 P5-66 data acquisition computer. It is used as the signal generator for all measurements pertaining to the Toneburst Technique. The operation of the TB-1000 is fairly straightforward as the software is entirely menu driven. However, there is also a user's manual available in the laboratory.

The TB-1000 is accessed by closing Windows and obtaining a DOS prompt. Change the directory to the TB-1000 directory by typing CDITB1000 at the $\mathrm{C}: 1>$ prompt. Once in the TB-1000 directory, the driver software may be started by typing MATEC at the $C: \mid T B 1000>$ prompt. The software will now start running and the toneburst card can be turned on by choosing the TB-1000 menu choice. As the "Direct" toneburst method will usually be the method used, the user need only adjust the frequency of the output signal and the pulse width to suit experimental conditions. More detailed operation instructions are provided in the user's manual if necessary.

The overall experimental set up is shown schematically in Figure 3.2.

Measurements should be made at frequencies spaced fairly evenly over the operational range of each transducer pair. It is usually preferable to turn on the toneburst card approximately one half hour prior to making measurements so that the card has time to "warm up". Also, it is advisable to always have some transducer connected to the "PULSE OUT" connector on the back of the computer so that the toneburst card is always "seeing" some load while the power is on. If the measurement transducers are 
disconnected and the toneburst generator is on, an old transducer can be connected in place of the measurement transducers. IT IS ALSO IMPORTANT TO TURN OFF THE VOLTAGE TO THE TONEBURST CARD (via the software menu) BEFORE CONNECTING OR DISCONNECTING ANY CABLES. The TB-1000 outputs several hundred volts.

\section{B.1.2.2 Panametrics 5052PR Pulser/Receiver:}

All signals used in the FFT/Pulse technique are generated by the Panametrics 50502PR Pulser/Receiver. It is a relatively simple instrument to operate, and it also has a user's manual available in the laboratory. The pulser/receiver is always operated in the "pitch-catch" mode. This implies that the transmitter transducer will be connected at the $T / R$ connector, and the receiver transducer will be connected at the RCVR connector. The general experimental set up is shown schematically in Figure 3.4 The pulser control settings (as they appear on the Pulser/Receiver unit) which have been determined to yield the best results for attenuation measurements are as follows:

$$
\begin{array}{ll}
\text { REP. RATE }= & 0 \quad \text { (Lowest setting possible WITHOUT being on MIN/EXT) } \\
\text { ENERGY }= & 4 \\
\text { ATTEN }(\mathrm{dB})= & \text { Variable: Setting which is appropriate for experimental } \\
& \text { conditions. } \\
\text { H.P. FILTER = } & \text { OUT } \\
\text { DAMPING = } & \text { Variable: Setting depends upon transducer pair used. }
\end{array}
$$


The $1 \mathrm{MHz}$ transducers operate best at a setting of about

4. The $2.25 \mathrm{MHz}, 5 \mathrm{MHz}, 7.5 \mathrm{MHz}$, and $10 \mathrm{MHz}$ transducers operate best at a setting of 10 .

$\operatorname{GAIN}(\mathrm{dB})=40$

\section{B.1.3 Operation of the LeCroy 9310A Dual $400 \mathrm{MHz}$ (Digital)}

\section{Oscilloscope:}

All acoustic signals obtained in this study are acquired by a LeCroy $9310 \mathrm{~A}$ digital oscilloscope. When compared to earlier models of digital oscilloscopes from various manufacturers, the LeCroy $9310 \mathrm{~A}$ is rather simple and intuitive to operate. All of the functions of which the oscilloscope are capable are menu driven, and a clear and concise user's manual is available in the laboratory. It is recommended that anyone who is not familiar with this particular model of oscilloscope take the time to perform the tutorial provided by the manufacturer in the user's manual. This tutorial demonstrates all of the major capabilities of the oscilloscope.

An important detail about proper operation of the oscilloscope is that all input channels (Channels 1 and 2) should be set for a coupling impedance of $50 \Omega \mathrm{DC}$. It is also important to make sure that the oscilloscope is being triggered properly by either the toneburst card (coaxial cable connecting "TRIGGER" connector on toneburst card and "EXT" connector on the front of the oscilloscope) or the pulser/receiver (coaxial cable connecting "+SYNC" connector on pulser/receiver and "EXT" connector on the 
oscilloscope). The trigger should be set at positive slope, external trigger, DC coupling at $1 \mathrm{M} \Omega$; and the trigger level is set at $500 \mathrm{mV}$.

\section{B.1.3.1 Saving Waveforms to Floppy Diskette:}

The oscilloscope is equipped with a 3.5" floppy diskette drive allowing that waveforms and FFT spectra can be saved as computer files for use in calculations using other software packages or for later display and manipulation by the oscilloscope. It should be noted that when files (waveforms/FFT spectra) are being saved diskette, the must be saved as BINARY files IF they are to be retrieved and displayed on the oscilloscope at a later time. This information IS NOT provided in the user's manual. If the waveforms/spectra are to be saved as data files for later importation into a software package for the performance of calculations, then they should be saved in an ASCII format.

All the attenuation calculations in this study are performed in MATLAB ${ }^{\mathrm{TM}}$. Although there is a menu choice for storing files in an ASCII format suitable for MATLAB ${ }^{T M}$, the files do not store correctly on diskette and MATLAB ${ }^{T M}$ will encounter errors when reading the files to load them. This problem is rectified by instead choosing the MATHCAD ${ }^{\text {TM }}$ ASCII file format when storing a waveform/spectrum to diskette. This format is one which, once the files are opened in the DOS EDIT file editor and the text headers are deleted leaving only data, MATLAB ${ }^{T M}$ will accept without error. These minor modifications to the data files are performed after they have been saved to diskette by the oscilloscope. 
The procedure for storing a waveform/spectrum to diskette, the aforementioned details not withstanding, is described in the user's manual.

\section{B.1.3.2 Oscilloscope Set up for Toneburst Measurements:}

When making toneburst measurements, the oscilloscope should be set up with a standard single channel display. The "Persistence" should be turned off, and the "Dot Join" should be turned on. The waveform intensity may be adjusted as per the comfort of the experimenter. Under the CURSOR/MEASURE option, the oscilloscope should be set to measure "Parameters" in the "Std. Voltage" mode, and the "Statistics" should be turned off. Once a signal is acquired on Channel 1, it may be shut off and the 50 sweep average of Channel 1 may be displayed by activating "TRACE A". It should be noted that the procedure for acquiring an average waveform is described in the user's manual. After an average waveform is obtained, the peak-to-peak voltage is read from the "pkpk (A)" line of the display, and recorded in the laboratory notebook. The time duration and scaling of the waveform is variable depending upon experimental conditions and user preference.

\section{B.1.3.3 Oscilloscope Set up for FFT/Pulse Measurements:}

When making FFT/Pulse measurements, the oscilloscope should be set up with a four channel display, although only three of the four channels are actually used. The procedure for obtain the four channel display is described in the user's manual. The "live" time domain waveform is displayed in Channel 1. The 50 sweep average of the 
"live" waveform is displayed in "TRACE A" on the second channel, and the FFT magnitude spectrum of "TRACE A" (average waveform) is displayed in "TRACE B" on the third channel. The procedure for obtaining an FFT magnitude spectrum of a waveform is also described in the user's manual.

The frequency range of the FFT spectrum is determined by the scaling of the time domain signal. The maximum frequency of the FFT magnitude spectrum is determined the Nyquist frequency. It is not the objective of this study to make an in-depth study of signal processing, and an excellent discussion of the FFT is given by Ramirez (1985) and by the LeCroy Operator's Manual. Suffice to say that the Nyquist frequency is equal to one half of the effective sampling frequency (after decimation), and the effective sampling frequency is determined by the scaling of the time domain waveform. It is recommended that the time domain scaling be set so that the Nyquist frequency is 5.00 $\mathrm{MHz}$ for the $1.0 \mathrm{MHz}$ and $2.25 \mathrm{MHz}$ transducers, and that the Nyquist frequency is 12.5 $\mathrm{MHz}$ for the $5.0 \mathrm{MHz}, 7.5 \mathrm{MHz}$, and $10.0 \mathrm{MHz}$ transducers. It should be noted that there is also a useful discussion and exercise on the use of the FFT and decimation by Dr. Margaret Greenwood of the Battelle Pacific Northwest National Laboratory beginning on page 38 of the laboratory notebook numbered APD-005. It is recommended that this discussion and exercise be studied by any user not familiar with the use of FFT's.

It is preferable to use a rectangular sampling window for the FFT/Pulse measurements. The time domain waveform (pulse) should be centered as well as possible in the window. This should be done as the FFT algorithm assumes periodicity of the waveform, and waveform position in the window can affect FFT results (cf. Ramirez 
1985). The amplitude scaling of the time domain signal should be set according to experimental conditions.

To perform the FFT analysis, a time domain waveform of the received pulse should be acquired and displayed in Channel 1. The amplitude (voltage) scaling should be adjusted, together with the attenuation settings on the Pulser/Receiver, if necessary, to obtain a distinct received pulse waveform. An average waveform should then be acquired in "TRACE A". The averages are generally performed over 50 sweeps, the number of sweeps can be changed if experimental conditions; i.e., rapidly separating systems, dictate. Once the average has been performed and displayed by the oscilloscope, the sample acquisition in the "live" waveform should be stopped by pressing the "STOP" button on the control panel of the oscilloscope. The FFT magnitude spectrum of the average waveform should be performed as described in the user's manual. Once the FFT magnitude spectrum has been acquired, the spectrum should be saved to diskette in an ASCII MATHCAD ${ }^{\text {TM }}$ format as described in Section B.1.3.1. It should be noted that a "hard copy" of the oscilloscope display should be made by pressing the "SCREEN DUMP" button on the control panel of the oscilloscope.

\section{B.1.4 Operation of the Photomicrographic Video Imaging System:}

Bubble size measurements and volume fraction estimates are obtained by using the video imaging system described in Section 3.1.3. Video Images are obtained using the Company 7 "Questar" QM-100 long distance microscope which is coupled, as a single unit, to a Cohu Model 4810 monochrome CCD video camera. There is a user's 
manual for both the microscope and video camera available in the laboratory which describe procedures as to the care and maintenance of these instruments. It should be noted that the camera is "on" whenever the AC power adapter is plugged in. The microscope/camera unit is mounted on a sliding track which allows for variability of the working distance; i.e., the distance between the microscope objective and object to be studied. In the experiments performed in this study, it was found that a working distance of $17.8 \mathrm{~cm}$ (7") allowed for the widest range of bubble size measurements without changing the focus of the microscope. Achieving the widest range of measurements is important in that any time the focus or working of the microscope is changed, the calibration must be performed to reset the pixel to distance scale in the image analysis software.

Backlighting is provided by a strobotach directly behind the sample cell at a distance of $61 \mathrm{~cm}\left(24^{\prime \prime}\right)$. The strobotach is synchronized to the video camera via the wire lead protruding from the back of the camera. This wire is connected to the vertical update "pin" on the "U8" integrated circuit chip in the camera. The schematic diagram of this chip is available in the laboratory in the Installation and Operation Instructions manual for the camera. The synchronization lead wire is connected by a coaxial cable to the “GATE/TRIG IN" connector on the Global Specialties Corporation 40001 Pulse Generator. The pulse generator is used to trigger the strobotach. The vertical update signal from the camera has a frequency which is actually twice the frame/second rate of the camera. The camera updates at twice the frame/second rate because the odd numbered lines on the $\mathrm{CCD}$ chip are acquired first, then the even numbered lines are 
"filled in". Therefore, the vertical update rate of the camera $(60 \mathrm{~Hz})$ is twice the frame/second rate $(30 \mathrm{~Hz})$, and the vertical update signal must be effectively divided by two by the pulse generator in order to synchronize the strobotach such that moving bubbles will be "frozen" in the acquired images. The pulse generator is then connected to the strobotach by connecting the "VAR OUT" connector on the pulse generator, via coaxial cable, to the "INPUT" connector on the strobotach. The amplitude of the pulse generator signal should be adjusted, by trial and error, to be sufficient in amplitude to trigger the strobotach.

Calibration of the microscope/camera unit must be performed each time the focus or working distance of the microscope is changed. This calibration is performed using a reticle mounted on a Plexiglas plate. The reticle has a series of linear and circular scales the dimensions of which are marked on the reticle. The reticle is rated by the manufacturer (Edmund Scientific Company, Barrington, NJ) to be accurate to within 2 $\mu \mathrm{m}$. Calibration is performed by immersing the reticle in the liquid in which the sample is suspended. The focus is set so that the image of the reticle is as clear as possible, and then the focus is NOT to be changed during bubble measurements without recalibrating. It is preferable to focus on the $0.005^{\prime \prime}(127 \mu \mathrm{m})$ circle on the reticle as it is the smallest calibration feature. Once the inage is focused, a one or two frame "movie" should be acquired using the image analysis software and saved to be added to stacks of movie frames of bubbles (the procedure for making movies will be discussed in the next paragraph). An example image of the reticle used in calibration is shown in Figure B.1. 


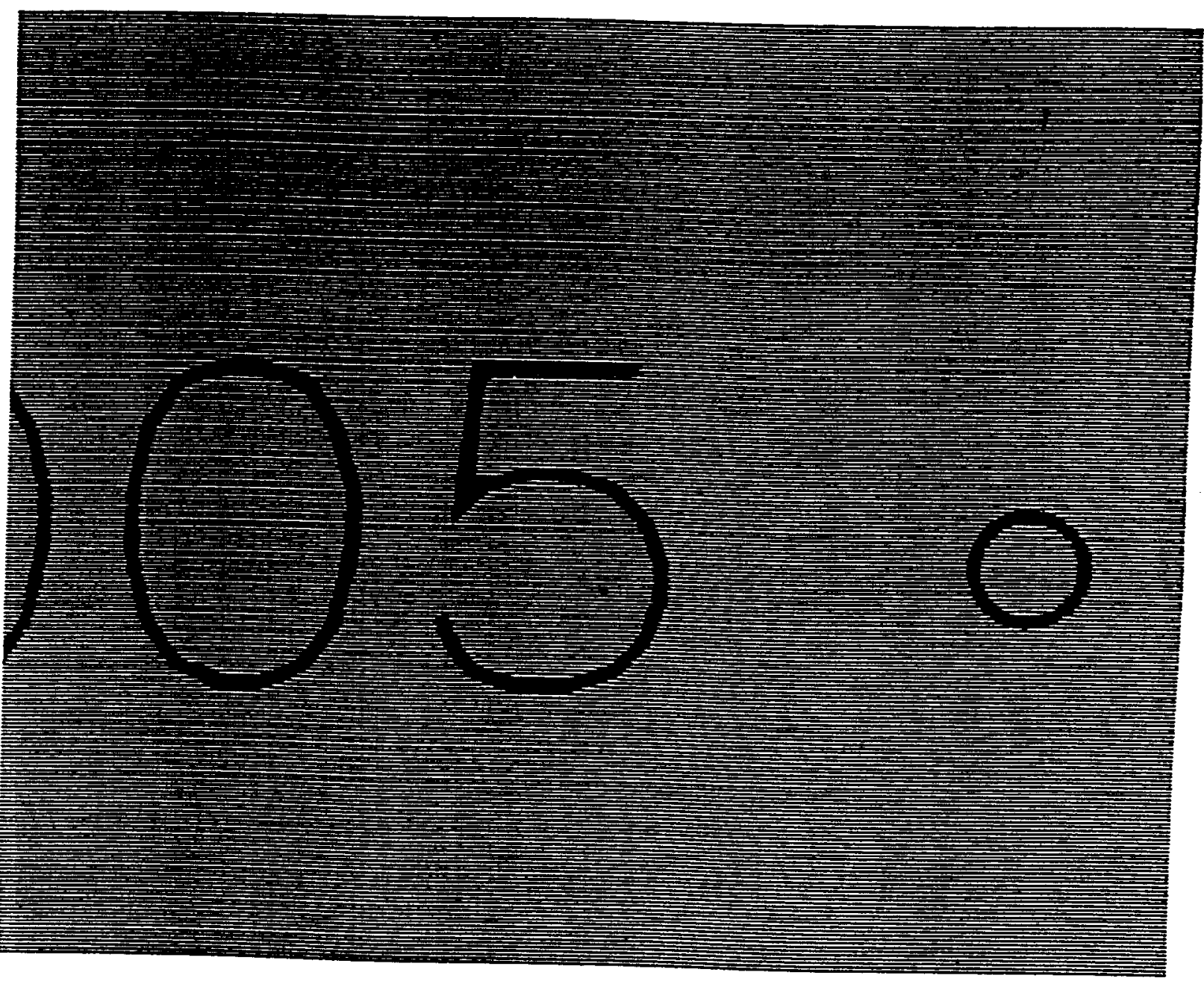

Figure B.1: Photograph of the 0.005 " diameter circle on the reticle used in calibrating the photomicrographic video imaging system. 
The movie frames containing the reticle image will then be measured to set the pixel to length scale in the image analysis software.

The images acquired by the microscope/camera unit are analyzed using the Image $S X M$ image analysis software package installed on the Apple Power Macintosh G3 computer which is interfaced with the microscope/camera unit. The Image SXM image analysis software package does not have a user's manual per se, but it is almost identical to the NIH Image image analysis software package available through the National Institutes of Health. The NIH Image image analysis software package has an online manual available at the NIH Internet site at http://rsb.info.nih.gov/nihimage/manual/Contents.html. Images are obtained by opening Image SXM on the desktop of the Power Macintosh. When the Image SXM folder opens, open the program by double clicking on the Image SXM icon. When the program is open, a live video image can be obtained by clicking on the "Special" menu choice and choosing the "Start Capturing" option. Movies are acquired by selecting the entire image screen and then choosing the "Stacks" menu choice. Choose "Make Movie" option and follow the menu options which appear. It is usually desirable to acquire about 40 frames in each movie, but this number can be adjusted to match experimental conditions. An example bubble image is shown in Figure B.2. The details of these procedures are described in the online manual, and the manual should be examined by user's not familiar with this software package.

When a movie has been acquired, frames which do not have any images should be deleted from the "stack" as per the user's manual to conserve disk space on the computer. 


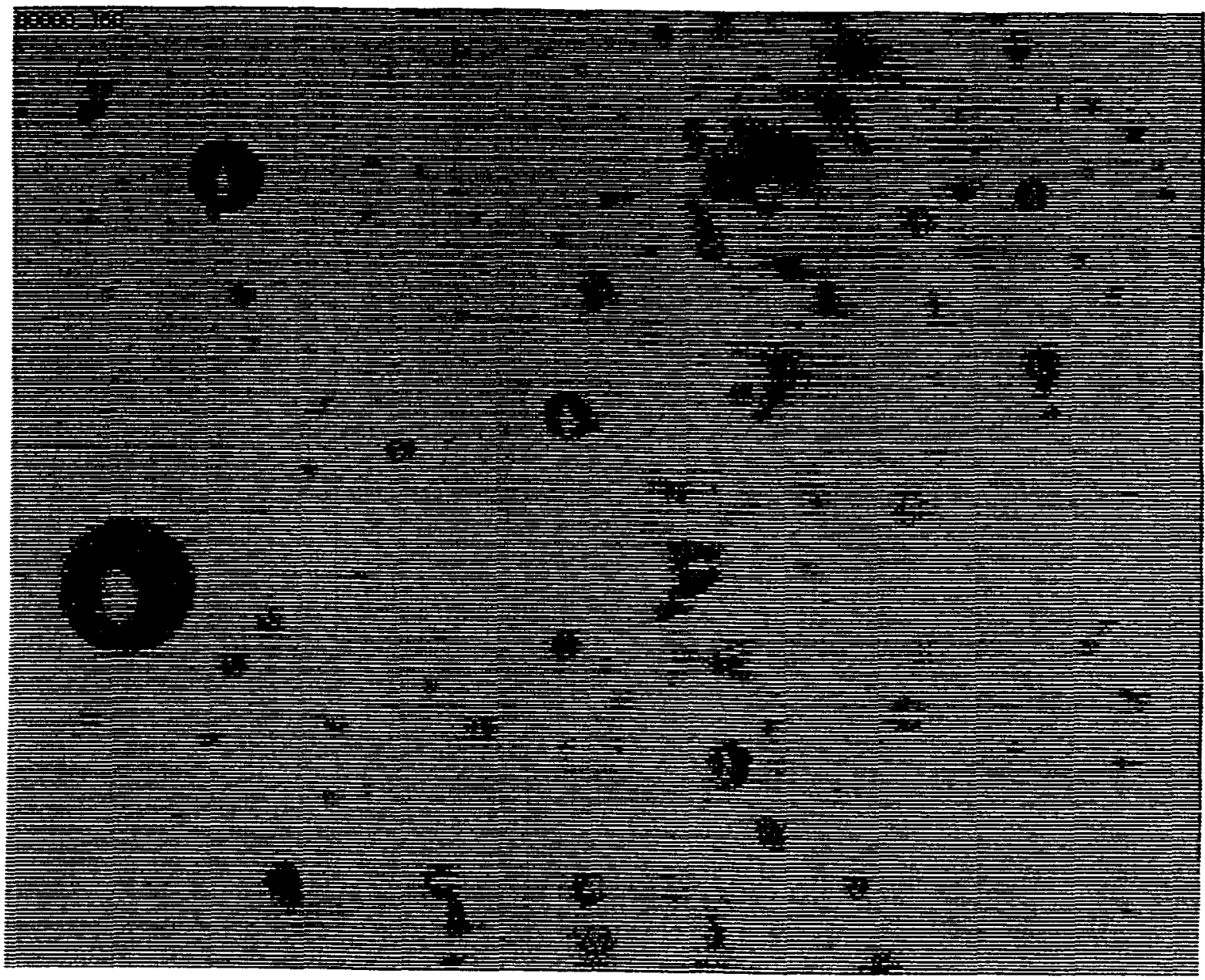

Figure B.2: Photograph of a electrolytically generated "bubble stream". The bubbles are are generated at $40 \mathrm{~V}, 6$ to $8 \mathrm{~mA},<1 \mathrm{~W}$. 
Also, the image frames of the reticle should be added to the image stack and saved with the movie. Calibration is performed by using the straight line tool to draw a diameter across the reticle circle. Then choose the "Analyze" menu choice and choose the "Set Scale" option. Enter the known distance in the appropriate units and click on the "OK" choice. The pixel to length scale is now set. It is usually a good idea to measure the reticle circle diameter vertically, horizontally, and on each diagonal and average the reported pixel/ $\mu \mathrm{m}$ conversion factor to be used in scaling. This average value can then be entered manually in the set scale procedure instead of the known distance of the reticle diameter.

Once the scale has been set, size measurements can be made on images by using a similar technique. The straight line tool is used to draw a diameter across a bubble of interest, and the "Analyze" menu choice is chosen. Now the "Measure" option should be chosen and the length of the line drawn will be displayed in microns in the "Info" display box. The measurement should be recorded. Measurement should only be made on bubbles which are in focus and are not touching other bubbles or the edge of the image frame. The backlighting will cause some error due to shadowing in the bubble size measurement. Therefore, a calibration curve of measured versus true size of polystyrene optical standard particles is shown in Figure B.3.

One set of bubble size measurements is performed for bubbles generated in water both by electrolysis and aeration. Photographs are made of these bubbles using the above technique, and size mesurements are performed using the above procedure. The results of these measurements are used to estimate a bubble size distribution for bubbles generated 


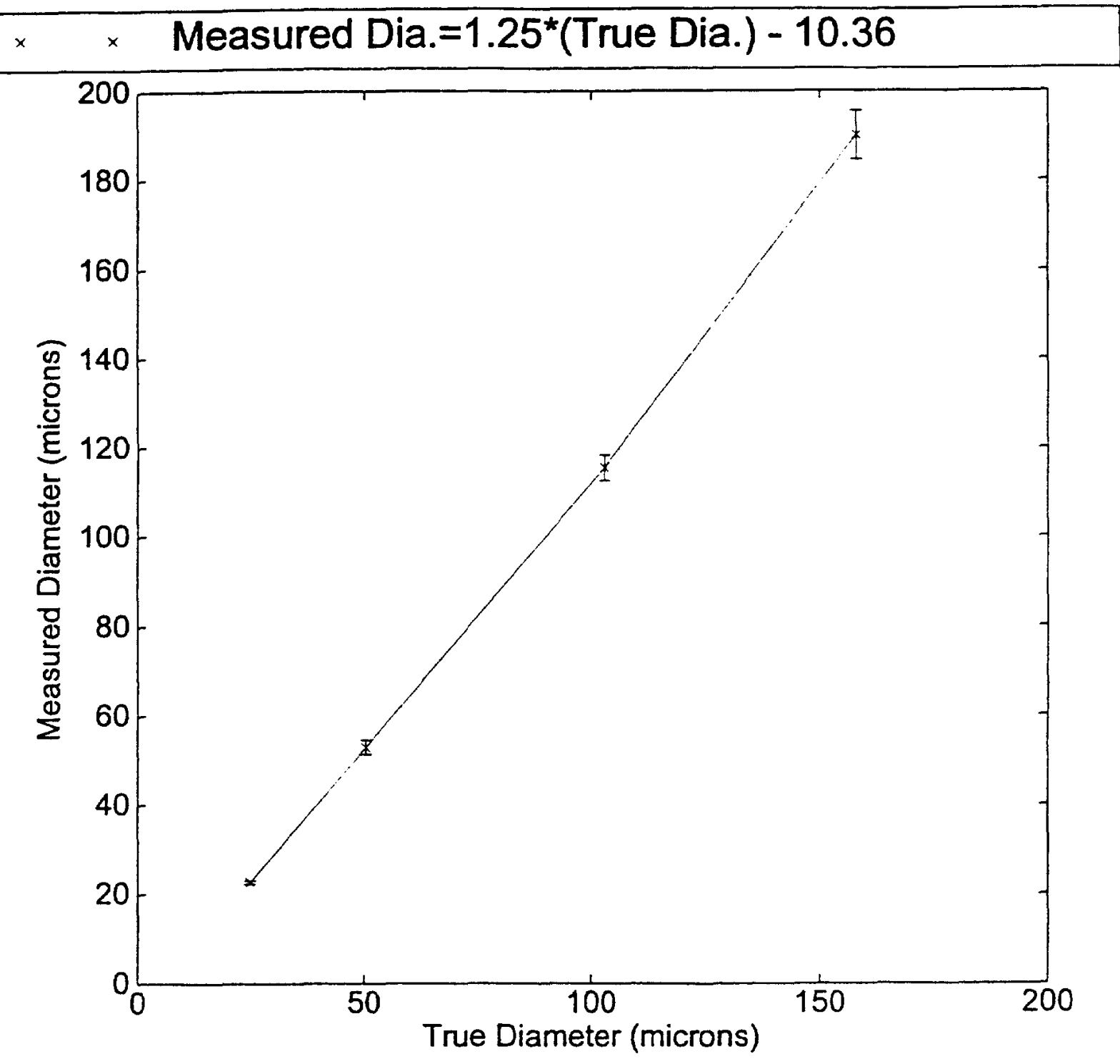

Figure B.3: Calibration plot relating measured particle diameters to the NIST certified diameters of polystyrene particle standards. 
electrolytically at $40 \mathrm{~V} ; 6$ to $8 \mathrm{~mA} ;<1 \mathrm{~W}$. This size distribution is determined to have a mean diameter of $51 \mu \mathrm{m}$ and a standard deviation of $26 \mu \mathrm{m}$. A log-normal size distribution is fitted to these data, and the results are shown in Figure B.4.

Volume fraction estimation is obtained from knowledge of the field of view and depth of field of the microscope at a particular working distance, and the number of bubbles in a given frame averaged over the number of frames in the movie stack. This estimation, of course, assumes that the bubbly liquid is well mixed, and the image field is representative of conditions throughout the sample cell. 


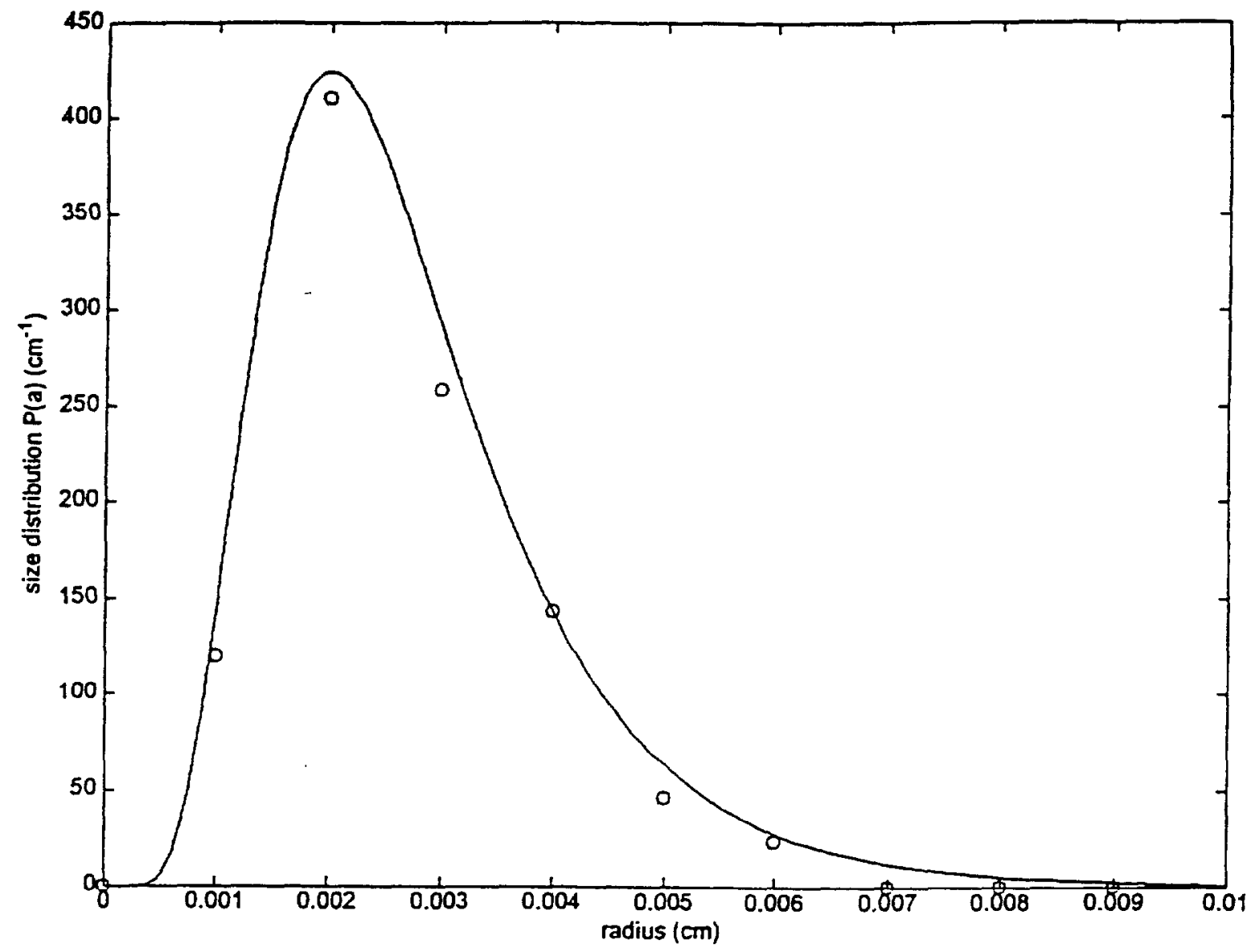

Figure B.4: Bubble size distribution determined by the microphotographic video imaging system and the log-normal size distribution fitted to the data. 


\section{Appendix C: MATLAB ${ }^{\mathrm{TM}}$ Routines}

The following pages contain some of the various MATLAB ${ }^{\text {TM }}$ routines used to calculate attenuation from raw voltage data in slurries and then plot calculated attenuation as either a function of frequency or solids concentration.

Not all the existing MATLAB ${ }^{\mathrm{TM}}$ routines are shown in this appendix. The reason for this is because many of the routines are simply variations of one-another. Therefore, for the sake of brevity, only the most important "watershed" routines, upon which all the others are derived, are shown.

The routines are annotated with comment lines to explain the progression of data manipulation within the routines. Each version of MATLAB ${ }^{T M}$ is provided with a user's manual, and the later versions available in our laboratories also have on-line help through Netscape $^{T M}$. The User's Guide (black cover book) from the earliest version (v.4.2c.1) of MATLAB ${ }^{\text {TM }}$ is available in the laboratory. Although, new versions of MATLAB ${ }^{\text {TM }}$ have been installed on the laboratory computers, this user's manual contains a tutorial on the basic functions and capabilities of MATLAB ${ }^{T M}$. It is recommended that anyone who is not familiar with MATLAB ${ }^{\text {TM }}$ take the time to read and follow the tutorial to gain experience in using this software package. 
$\%$ This program calculates attenuation vs frequency in slurries of \%Soda-lime glass beads using Direct

$\%$ (Toneburst) Oscilloscope Data

$\%$ (called DIRECT.m)

\%Load $0.5 \mathrm{MHz}$ water data

load s1wdp5.dat

fwp $5=[\operatorname{siwdp} 5(:, 1)]$;

vwp5 $=[\operatorname{siwdp} 5(:, 2)]$;

\%Load $0.5 \mathrm{MHz}$ slurry data

load s1sdp5.dat

fsp $5=[\operatorname{sisdp} 5(:, 1)]$;

vsp $5=[\operatorname{sisdp} 5(:, 2)]$;

\%Load 1.0 MHz water data

load slwdl.dat

fw $1=[\operatorname{siwdl}(:, 1)]$;

vw $1=[\operatorname{slwd} 1(:, 2)]$;

$\%$ Load 1.0 MHz slurry data

load slsd 1.dat

$\mathrm{fs} 1=[\operatorname{sisd} 1(:, 1)]$;

vs $1=[\operatorname{sisd} 1(:, 2)]$;

\%Load 2.25 MHz water data

load s1wd2.dat

fw $2=[\mathrm{s} 1 \mathrm{wd} 2(:, 1)]$;

vw2 $=[\operatorname{siwd} 2(:, 2)]$;

\%Load 2.25 MHz slurry data load s1sd2.dat

fs $2=[\operatorname{s} 1 \mathrm{sd} 2(:, 1)]$;

vs $2=[\operatorname{sisd} 2(:, 2)]$;

\%Load 5.0 MHz water data

load s1wd5.dat

fw5 $=[\operatorname{siwd5}(:, 1)]$;

vw5 $=[\operatorname{siwd5}(:, 2)]$;

$\%$ Load 5.0 $\mathrm{MHz}$ slurry data

load slsd5.dat

fs $5=[\mathrm{s} 1 \mathrm{sd} 5(:, 1)]$;

vs $5=[\operatorname{sisd} 5(:, 2)]$; 
\%Load 7.5 MHz water data

load slwd7.dat

fw7 $=[\operatorname{siwd} 7(:, 1)]$;

vw $7=[\operatorname{siwd7}(:, 2)]$;

\%Load 7.5 MHz slurry data

load s1 sd7.dat

fs $7=[\operatorname{sisd} 7(:, 1)]$;

vs $7=[\operatorname{sisd} 7(:, 2)]$;

$\%$ Load $10.0 \mathrm{MHz}$ water data

load s1wd10.dat

fw $10=[\operatorname{siwd10}(:, 1)]$;

vw10 $=[\operatorname{siwd10}(:, 2)]$;

$\%$ Load 10.0 MHz slurry data

load s1sd10.dat

fs $10=[\operatorname{sisd} 10(:, 1)]$;

vs $10=[\operatorname{sisd} 10(:, 2)]$;

\%Input Transducer Separation Distances in $\mathrm{cm}$

$\mathrm{dp} 5=4.006 * 2.54$;

$\mathrm{d} 1=4.006 * 2.54$;

$\mathrm{d} 2=3.9875 * 2.54$;

d5 $=4.072 * 2.54$;

$\mathrm{d} 7=1.986 * 2.54$

$\mathrm{d} 10=2.040 * 2.54$;

$\%$ Calculate Slurry to Water voltage ratios

vгp5=vsp5./vwp5;

$\mathrm{vr} 1=\mathrm{vs} 1 . / \mathrm{vw} 1$;

vr2=vs $2 . / v w 2 ;$

$\mathrm{vr} 5=\mathrm{vs} 5 . / \mathrm{vw} 5$

$\mathrm{vr} 7=\mathrm{vs} 7 . / \mathrm{vw} 7$;

vr10=vs $10 . /$ vw 10 ;

$\%$ Calculate logs of voltage ratios

$\operatorname{lvrp5}=\log 10(\mathrm{vrp} 5)$;

$\operatorname{lvrl}=\log 10(\mathrm{vr} 1)$;

$\operatorname{lvr} 2=\log 10(\mathrm{vr} 2)$;

$\operatorname{lvr} 5=\log 10(\mathrm{vr} 5)$;

$\operatorname{lvr} 7=\log 10(\mathrm{vr} 7)$; 
$\operatorname{lvr} 10=\log 10(\mathrm{vr} 10)$

$\%$ Calculate attenuation

ap $5=-1.0 *(\operatorname{lvp} 5 / \mathrm{dp} 5)$

$\mathrm{a} 1=-1.0 *(\operatorname{lvr} 1 / \mathrm{d} 1)$

$\mathrm{a} 2=-1.0 *(\operatorname{lvr} 2 / \mathrm{d} 2)$

a5 $=-1.0 *(\operatorname{lvr} 5 / \mathrm{d} 5)$

a $7=-1.0 *(\operatorname{lvr} 7 / \mathrm{d} 7)$

a $10=-1.0 *(\operatorname{lvr} 10 / \mathrm{d} 10)$

$\%$ Rectify with theory (put in Np/cm using natural logarithms)

ap5 $=$ ap $5 * 2.302$;

al $=\mathrm{al} * 2.302$;

$\mathrm{a} 2=\mathrm{a} 2 * 2.302$;

a5 $=\mathrm{a} 5 * 2.302$;

$\mathrm{a} 7=\mathrm{a} 7 * 2.302$

a $10=\mathrm{a} 10 * 2.302$

$\%$ Use one set of frequencies per transducer to plot

$\mathrm{fp} 5=\mathrm{fwp} 5$;

$\mathrm{fl}=\mathrm{fw} 1$

$\mathrm{f} 2=\mathrm{fw} 2$;

$\mathrm{f} 5=\mathrm{fw} 5$

$\mathrm{f} 7=\mathrm{fw} 7$;

f10 $=$ fw 10 ;

\%Convert Frequency into $\mathrm{Hz}$

$\mathrm{fp} 5=1.0 \mathrm{e} 6 * \mathrm{fp} 5$

$\mathrm{fl}=1.0 \mathrm{e} 6 * \mathrm{fl}$

$\mathrm{f} 2=1.0 \mathrm{e} 6^{*} \mathrm{f} 2$

$\mathrm{f} 5=1.0 \mathrm{e} 6 * \mathrm{f} 5$

$\mathrm{f} 7=1.0 \mathrm{e} 6 * \mathrm{f7}$

fl $0=1.0 \mathrm{e} 6 * \mathrm{f} 10$

$\%$ Plot results

$\log \log \left(\mathrm{fp} 5\right.$, ap $\left.5,,^{\prime}\right)$

$\%$ title('Direct Oscilloscope Measurements-5\% glass ( 5 micron)')

ylabel('Attenuation $\left.(\mathrm{dB} / \mathrm{cm})^{\prime}\right)$

xlabel('Frequency $\left.(\mathrm{Hz})^{\prime}\right)$

hold on

$\log \log \left(\mathrm{fl}, \mathrm{a} 1, \mathrm{\prime}^{\prime}\right)$

hold on

$\log \log \left(\mathrm{f} 2, \mathrm{a} 2,,^{\prime}\right)$

hold on 
$\log \log \left(\mathrm{f} 5, \mathrm{a} 5, \mathrm{\prime}^{\prime}\right)$

hold on

$\log \log (\mathrm{f} 7, \mathrm{a} 7, . ')$

hold on

$\log \log \left(\mathrm{fl} 0, \mathrm{a} 10, \mathbf{\prime}^{\prime}\right)$

hold on

$\%$ Program to plot average attenuation vs concentration at various fixed $\%$ frequencies for Dense Potters Beads Slurries in Glycerol/water Mixture $\%$ (Called AVSPHI.m)

$\%$ Concentration (volume fraction) arrays

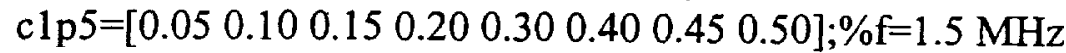

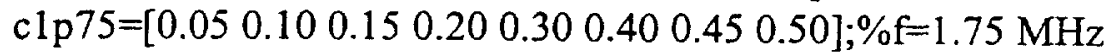

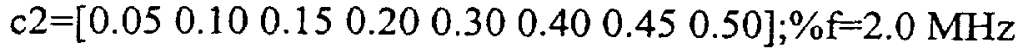

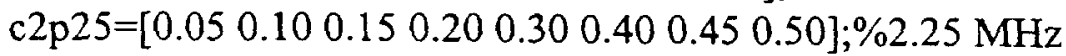

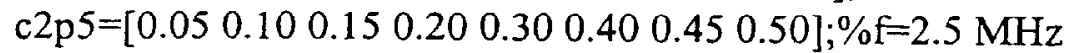

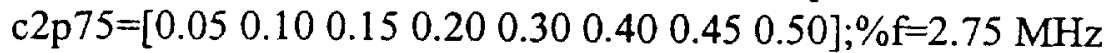

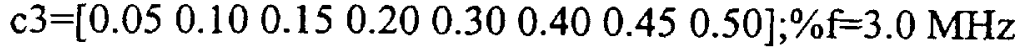

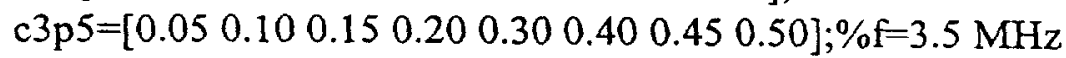

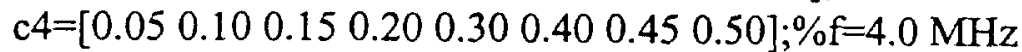

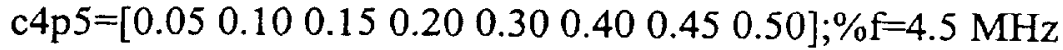

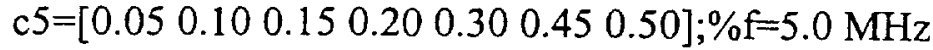

\%Attenuation Arrays--Attenuations are average values at freqs. where transducers $\%$ overlap and data from multiple experiments is available.

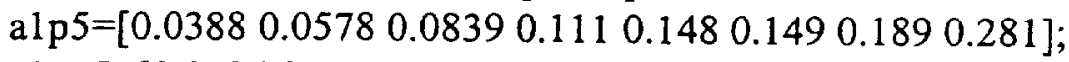

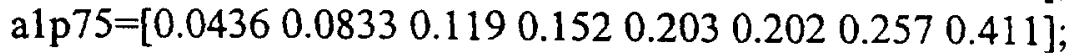

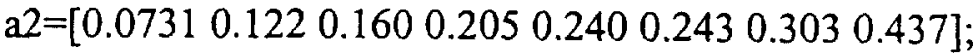

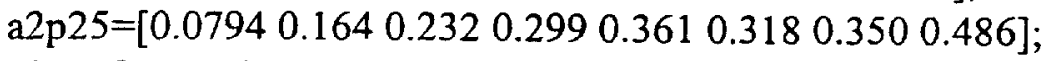
a2p $5=\left[\begin{array}{llllllll}0.135 & 0.232 & 0.319 & 0.393 & 0.502 & 0.449 & 0.431 & 0.459\end{array}\right]$;

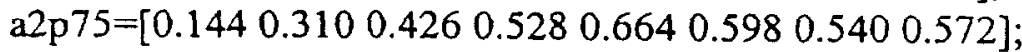
a3 $=\left[\begin{array}{llllllll}0.195 & 0.414 & 0.543 & 0.660 & 0.847 & 0.778 & 0.792 & 0.748\end{array}\right]$; a3p5 $=\left[\begin{array}{llllllll}0.321 & 0.596 & 0.679 & 0.872 & 1.20 & 1.36 & 1.40 & 1.28\end{array}\right]$;

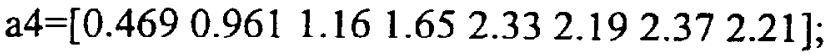
a4p5 $=\left[\begin{array}{lllllllll}0.613 & 1.16 & 1.56 & 2.44 & 3.32 & 2.91 & 3.57 & 3.74\end{array}\right]$;

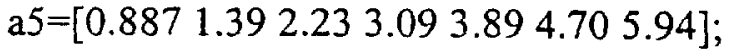

$\%$ Plot Results semilogy(clp5,alp5,'r-') 


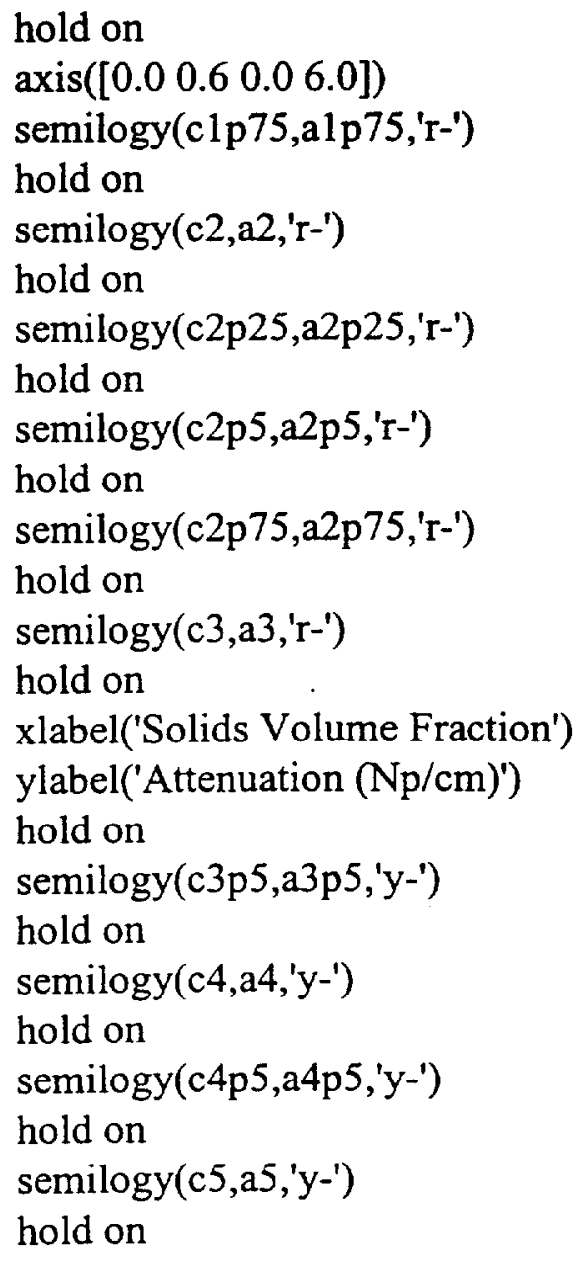

$* * * * * * * * * * * * * * * * * * * * * * * * * \mathrm{END}$ OF AVSPHI.DOC

\%Program to calculate attenuation by FFT

$\%$ of three phase systems of soda lime glass beads

$\%$ in water at vol. fract. $\sim 0.10$

$\%$ with air generated bubbles at $100 \mathrm{ml} / \mathrm{min}$ (called sgll.m)

\%Load FFT magnitude data files

$\%$ Load $5 \mathrm{MHz}$ water data

load cont5w.dat

$\%$ choose only the frequencies in the effective $x$-ducer operating range

$\%$ and disregard the rest..do for all $\mathrm{x}$-ducer pairs.

$\mathrm{n} 5 \mathrm{w}=\max (\operatorname{size}(\operatorname{cont} 5 \mathrm{w}))$;

fw $5=\left[\operatorname{cont} 5 \mathrm{w}\left(0.20^{*} \mathrm{n} 5 \mathrm{w}: 0.5^{*} \mathrm{n} 5 \mathrm{w}, 1\right)\right]$

v5wag $=\left[\operatorname{cont} 5 \mathrm{w}\left(0.20 * \mathrm{n} 5 \mathrm{w}: 0.5 *^{\mathrm{n}} 5 \mathrm{w}, 2\right)\right]$;

$\%$ Load $5 \mathrm{MHz}$ slurry w/out bubbles data 
load cont5s.dat

$\mathrm{n} 5 \mathrm{~s}=\max (\operatorname{size}(\operatorname{cont} 5 \mathrm{~s}))$;

fs $5=[\operatorname{cont} 5 \mathrm{~s}(0.20 * \mathrm{n} 5 \mathrm{~s}: 0.5 * \mathrm{n} 5 \mathrm{~s}, 1)]$;

$\mathrm{v} 5 \mathrm{sag}=\left[\operatorname{cont} 5 \mathrm{~s}\left(0.20^{*} \mathrm{n} 5 \mathrm{~s}: 0.5^{*} \mathrm{n} 5 \mathrm{~s}, 2\right)\right]$;

\%Load $5 \mathrm{MHz}$ slurry w/ bubble data

load cont5b.dat

$\mathrm{n} 5 \mathrm{~b}=\max (\operatorname{size}(\operatorname{con} \mathrm{t} 5 \mathrm{~b}))$;

$f s 5 b=[\operatorname{cont} 5 b(0.20 * n 5 b: 0.5 * n 5 b, 1)]$;

v5sagb $=[\operatorname{cont} 5 b(0.20 * n 5 b: 0.5 * n 5 b, 2)]$;

\%Load 2.25 MHz water data

load cont $2 w$.dat

$\mathrm{n} 2 \mathrm{w}=\max (\operatorname{size}(\operatorname{con} \mathrm{2} 2 \mathrm{w}))$;

fw $2=\left[\operatorname{cont} 2 \mathrm{w}\left(0.25^{*} \mathrm{n} 2 \mathrm{w}: 0.5^{*} \mathrm{n} 2 \mathrm{w}, 1\right)\right]$;

$\mathrm{v} 2 \mathrm{wag}=\left[\operatorname{cont} 2 \mathrm{w}\left(0.25^{*} \mathrm{n} 2 \mathrm{w}: 0.5^{*} \mathrm{n} 2 \mathrm{w}, 2\right)\right]$;

\%Load 2.25 MHz slurry w/out bubbles data

load cont 2 s.dat

$\mathrm{n} 2 \mathrm{~s}=\max (\operatorname{size}(\operatorname{cont} 2 \mathrm{~s}))$;

fs $2=\left[\operatorname{cont} 2 \mathrm{~s}\left(0.25{ }^{*} \mathrm{n} 2 \mathrm{~s}: 0.5 * \mathrm{n} 2 \mathrm{~s}, 1\right)\right]$

$\mathrm{v} 2 \mathrm{sag}=\left[\operatorname{cont} 2 \mathrm{~s}\left(0.25^{*} \mathrm{n} 2 \mathrm{~s}: 0.5^{*} \mathrm{n} 2 \mathrm{~s}, 2\right)\right]$;

$\%$ Load 2.25 MHz slurry with bubble bubble data

load cont 2 b.dat

$\mathrm{n} 2 \mathrm{~b}=\max (\operatorname{size}(\operatorname{con} \mathrm{t} 2 \mathrm{~b}))$;

fs $2 b=\left[\operatorname{cont} 2 b\left(0.25 *^{*} 2 b: 0.5 *_{n} 2 b, 1\right)\right]$;

$\mathrm{v} 2 \mathrm{bag}=\left[\operatorname{cont} 2 \mathrm{~b}\left(0.25^{*} \mathrm{n} 2 \mathrm{~b}: 0.5^{*} \mathrm{n} 2 \mathrm{~b}, 2\right)\right]$;

$\%$ Load $1.0 \mathrm{MHz}$ water data

load cont $1 \mathrm{w}$. dat

$\mathrm{n} 1 \mathrm{w}=\max (\operatorname{size}(\operatorname{cont} \mathrm{l} \mathrm{w}))$;

fw $1=\left[\operatorname{cont} 1 \mathrm{w}\left(0.14^{*} \mathrm{n} 1 \mathrm{w}: 0.25 * \mathrm{n} 1 \mathrm{w}, 1\right)\right]$;

$\mathrm{vlwag}=\left[\operatorname{cont} 1 \mathrm{w}\left(0.14^{*} \mathrm{n} 1 \mathrm{w}: 0.25^{*} \mathrm{n} 1 \mathrm{w}, 2\right)\right]$;

$\%$ Load 1.0 MHz slurry w/out bubbles data

load cont 1 s.dat

$\mathrm{n} 1 \mathrm{~s}=\max (\operatorname{size}(\operatorname{cont} 1 \mathrm{~s}))$;

fs $1=\left[\operatorname{cont} 1 \mathrm{~s}\left(0.14^{*} \mathrm{n} 1 \mathrm{~s}: 0.25^{*} \mathrm{n} 1 \mathrm{~s}, 1\right)\right]$;

$\mathrm{v} 1 \mathrm{sag}=[\operatorname{cont} 1 \mathrm{~s}(0.14 * \mathrm{n} 1 \mathrm{~s}: 0.25 * \mathrm{n} 1 \mathrm{~s}, 2)]$;

\%Load 1.0 MHz bubble data

load contlb.dat

$\mathrm{n} 1 \mathrm{~b}=\max (\operatorname{size}(\operatorname{cont} \mathrm{lb}))$; 
fs $1 b=[\operatorname{cont} 1 b(0.14 * n 1 b: 0.25 * n 1 b, 1)]$;

vlbag $=\left[\operatorname{contlb}\left(0.14^{*}\right.\right.$ nlb:0.25*nlb,2)];

\%Load $0.5 \mathrm{MHz}$ water data

load thruyp 5 w.dat

np5w=max (size(thruyp5w));

fwp5=[thruyp 5w $\left.\left(0.03^{*} n p 5 w: 0.07^{*} n p 5 w, 1\right)\right]$;

vp5wag=[thruyp $5 w(0.03 * n p 5 w: 0.07 * n p 5 w, 2)]$;

\%Load $0.5 \mathrm{MHz}$ slurry w/out bubbles data

load thruyp5s.dat

$\mathrm{np} 5 \mathrm{~s}=\max ($ size(thruyp5s));

fsp5 $=[$ thruyp5s $(0.03 *$ np5s:0.07*np5s, 1)];

vp5sag=[thruyp5s $(0.03 * n p 5 s: 0.07 * n p 5 s, 2)]$;

\%Load $0.5 \mathrm{MHz}$ slurry with bubble bubble data

load thruyp5b.dat

np $5 b=\max (\operatorname{size}($ thruyp $5 b)$ );

fsp $5 b=[$ thruyp $5 b(0.03 * n p 5 b: 0.07 * n p 5 b, 1)]$;

vp5bag=[thruyp5b(0.03*np5b:0.07*np5b,2)];

\%Load 7.5 MHz water data

load cont7w.dat

$\mathrm{n} 7 \mathrm{w}=\max (\operatorname{size}(\operatorname{cont} 7 \mathrm{w}))$;

fw $7=[\operatorname{cont} 7 w(0.3 * n 7 w: 0.4 * n 7 w, 1)]$;

$\mathrm{v} 7 \mathrm{wag}=\left[\operatorname{cont} 7 \mathrm{w}\left(0.3^{*} \mathrm{n} 7 \mathrm{w}: 0.4 * \mathrm{n} 7 \mathrm{w}, 2\right)\right]$;

\%Load 7.5 MHz slurry w/out bubbles data

load cont 7 s.dat

$\mathrm{n} 7 \mathrm{~s}=\max (\operatorname{size}(\operatorname{cont} 7 \mathrm{~s}))$;

fs $7=[\operatorname{cont} 7 \mathrm{~s}(0.3 * \mathrm{n} 7 \mathrm{~s}: 0.4 * \mathrm{n} 7 \mathrm{~s}, 1)]$;

v7sag $=\left[\operatorname{cont} 7 \mathrm{~s}\left(0.3^{*} \mathrm{n} 7 \mathrm{~s}: 0.4^{*} \mathrm{n} 7 \mathrm{~s}, 2\right)\right]$;

\%Load 7.5 MHz slurry w/ bubble data

load cont $7 \mathrm{~b}$.dat

$\mathrm{n} 7 \mathrm{~b}=\max (\operatorname{size}(\operatorname{cont} 7 \mathrm{~b}))$;

fs $7 b=\left[\operatorname{cont} 7 b\left(0.3 *_{n} 7 b: 0.4 * n 7 b, 1\right)\right]$;

v7sagb $=\left[\operatorname{cont7b}\left(0.3^{*} \mathrm{n} 7 \mathrm{~b}: 0.4^{*} \mathrm{n} 7 \mathrm{~b}, 2\right)\right]$;

$\%$ Load $10 \mathrm{MHz}$ water data

load cont $10 \mathrm{w}$.dat

$\mathrm{n} 10 \mathrm{w}=\max (\operatorname{size}(\operatorname{cont} 10 \mathrm{w}))$;

fw $10=\left[\operatorname{cont} 10 \mathrm{w}\left(0.35^{*} \mathrm{n} 10 \mathrm{w}: 0.8 * \mathrm{n} 10 \mathrm{w}, 1\right)\right]$;

$\mathrm{v} 10 \mathrm{wag}=\left[\operatorname{cont} 10 \mathrm{w}\left(0.35^{*} \mathrm{n} 10 \mathrm{w}: 0.8 * \mathrm{n} 10 \mathrm{w}, 2\right)\right]$; 
$\%$ Load $10 \mathrm{MHz}$ polysty slurry w/out bubbles data

load cont 10 s.dat

$\mathrm{n} 10 \mathrm{~s}=\max (\operatorname{size}(\operatorname{cont} 10 \mathrm{~s}))$;

fs $10=\left[\operatorname{cont} 10 \mathrm{~s}\left(0.35^{*} \mathrm{n} 10 \mathrm{~s}: 0.8 * \mathrm{n} 10 \mathrm{~s}, 1\right)\right]$;

$\mathrm{v} 10 \mathrm{sag}=\left[\operatorname{cont} 10 \mathrm{~s}\left(0.35^{*} \mathrm{n} 10 \mathrm{~s}: 0.8 * \mathrm{n} 10 \mathrm{~s}, 2\right)\right]$;

$\%$ Load $10 \mathrm{MHz}$ slurry w/ bubble data

load cont $10 \mathrm{~b}$.dat

$\mathrm{n} 10 \mathrm{~b}=\max (\operatorname{size}(\operatorname{cont} 10 \mathrm{~b}))$;

fs $10 \mathrm{~b}=\left[\operatorname{cont} 10 \mathrm{~b}\left(0.35 *^{*} \mathrm{n} 10 \mathrm{~b}: 0.8 * \mathrm{n} 10 \mathrm{~b}, 1\right)\right]$;

$\mathrm{v} 10$ sagb $=\left[\right.$ cont $\left.10 \mathrm{~b}\left(0.35{ }^{*} \mathrm{n} 10 \mathrm{~b}: 0.8 * \mathrm{n} 10 \mathrm{~b}, 2\right)\right]$;

$\%$ Correct Voltage Values for Receiver Attenuation and gain

v5wg=v5wag./(10.^((40-54)/20));

$\mathrm{v} 5 \mathrm{sgb}=\mathrm{v} 5 \mathrm{sagb} . /\left(10 .^{\wedge}((40-46) / 20)\right)$;

$\mathrm{v} 2 \mathrm{sg}=\mathrm{v} 2 \mathrm{sag} . /\left(10\right.$. $\left.^{\wedge}((40-44) / 20)\right)$;

v2bg=v2bag. $/\left(10 \wedge^{\wedge}((40-44) / 20)\right)$;

v5sg-v5sag./(10.^((40-46)/20));

$\mathrm{v} 2 \mathrm{wg}=\mathrm{v} 2 \mathrm{wag} . /\left(10 .^{\wedge}((40-46) / 20)\right)$;

$\mathrm{vlwg}=\mathrm{vl}$ wag. $/\left(10\right.$ ^ $\left.^{\wedge}((40-50) / 20)\right)$;

v1sg=v1sag./(10.^((40-50)/20));

$\mathrm{vlbg}=\mathrm{v}$ lbag. $/\left(10 .^{\wedge}((40-50) / 20)\right)$;

vp5wg=vp5wag./(10.^((40-46)/20));

vp5sg=vp5sag./(10.^((40-46)/20));

vp5bg=vp5bag./(10.^((40-46)/20));

$\mathrm{v} 7 \mathrm{wg}=\mathrm{v} 7 \mathrm{wag} . /\left(10 .^{\wedge}((40-48) / 20)\right)$;

$\mathrm{v} 7 \mathrm{sgb}=\mathrm{v} 7 \mathrm{sagb} . /\left(10{ }^{\wedge}((40-34) / 20)\right)$;

$\mathrm{v} 7 \mathrm{sg}=\mathrm{v} 7 \mathrm{sag} . /\left(10 \mathrm{.}^{\wedge}((40-34) / 20)\right)$;

v10wg=vl0wag. $/\left(10 .^{\wedge}((40-38) / 20)\right)$;

v10sgb=v10sagb. $/\left(10 \wedge^{\wedge}((40-24) / 20)\right)$;

v10sg=v10sag./(10.^((40-24)/20));

$\%$ Renaming variables to be less confusing

$v 5 w=v 5 w g ;$

$\mathrm{v} 5 \mathrm{sb}=\mathrm{v} 5 \mathrm{sgb}$

$\mathrm{v} 2 \mathrm{~s}=\mathrm{v} 2 \mathrm{sg}$;

$\mathrm{v} 2 \mathrm{~b}=\mathrm{v} 2 \mathrm{bg}$;

$\mathrm{v} 5 \mathrm{~s}=\mathrm{v} 5 \mathrm{sg}$

$\mathrm{v} 2 \mathrm{w}=\mathrm{v} 2 \mathrm{wg}$;

$\mathrm{vlw}=\mathrm{vlwg}$;

$\mathrm{v} 1 \mathrm{~s}=\mathrm{v} 1 \mathrm{sg}$;

$\mathrm{vlb}=\mathrm{vlbg}$;

vp $5 w=v p 5 w g$; 


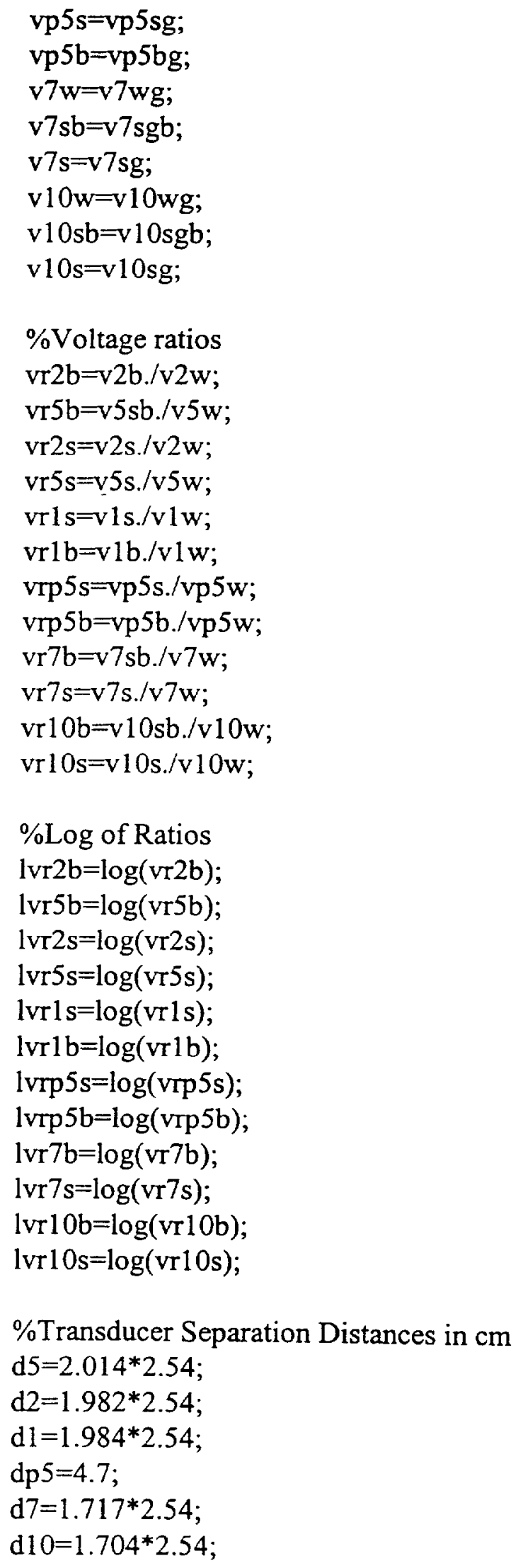




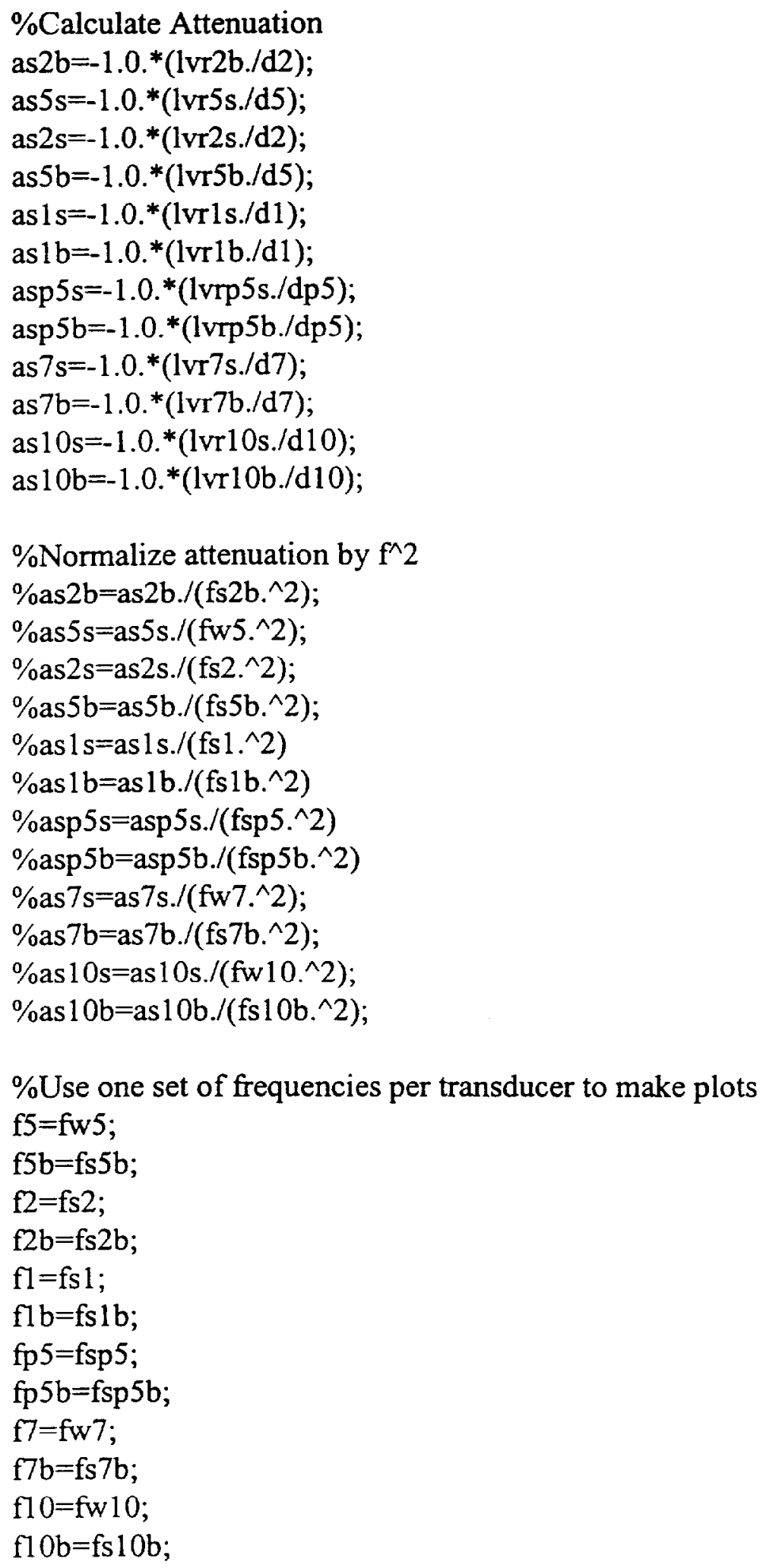

\%Plot Results 


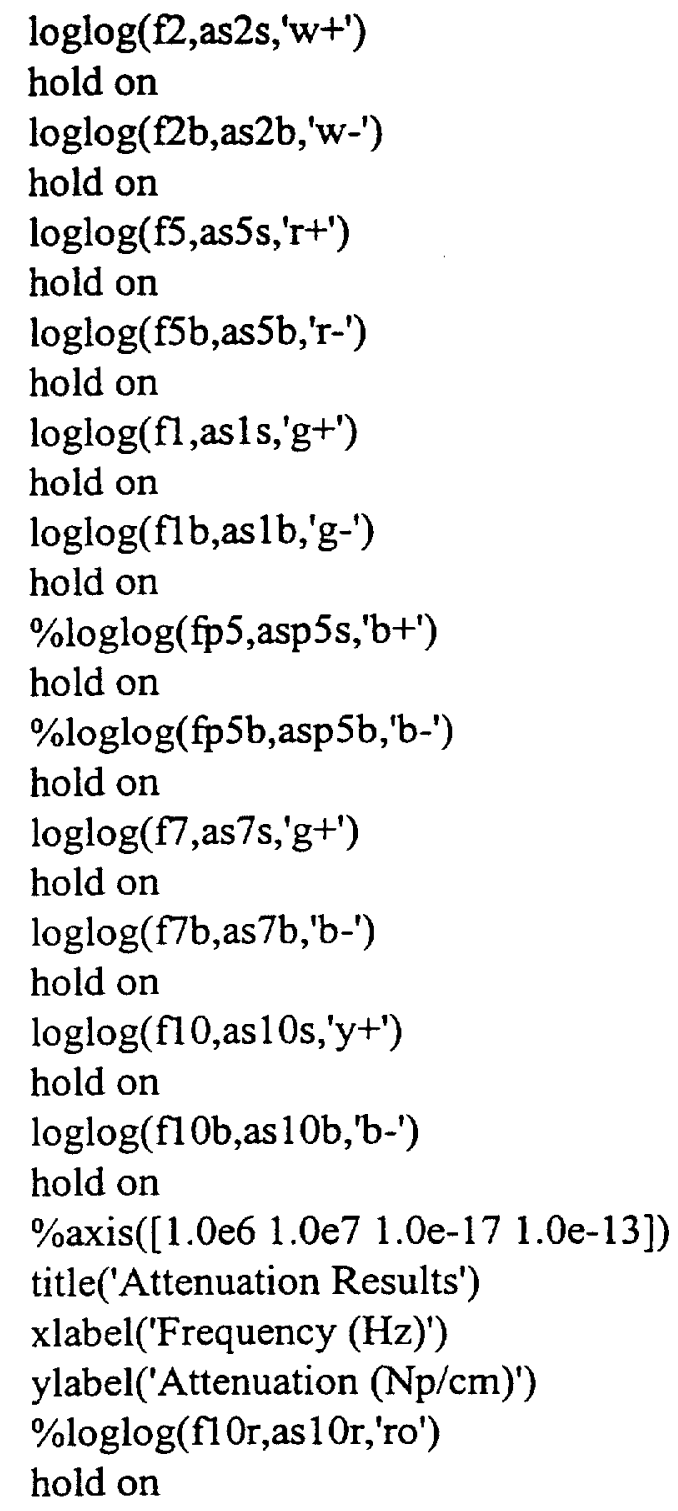

$* * * * * * * * * * * * * * * * * * * * * * * * \mathrm{END}$ OF SGL 1.M

$\%$ Program to calculate attenuation by FFT

$\%$ of a $5 \%$ Solution

$\%$ (158 micron diameter polystyrene beads)

$\% 2 "$ in plexiglas test cell

$\%$ This program plots FFT and toneburst results

$\%$ (called STYRENE5.m)

$\%$ Load $0.5 \mathrm{MHz}$ water data

$\%$ load s3wp5.dat

$\%$ fwp $5=[\mathrm{s} 3 \mathrm{wp} 5(:, 1)]$;

$\%$ dbmp $5 \mathrm{w}=[\mathrm{s} 3 \mathrm{wp} 5(:, 2)]$; 
$\%$ Load $0.5 \mathrm{MHz}$ slurry data

\%load s3sp5.dat

$\%$ fsp5=[s3sp5(:,1)];

$\%$ dbmp5s $=[$ s3sp5(:,2)];

$\%$ Load $1.0 \mathrm{MHz}$ water data

load sty51w.dat

$\mathrm{n} 1 \mathrm{w}=\max (\operatorname{size}(\operatorname{sty} 51 \mathrm{w}))$;

fw $1=\left[\operatorname{sty} 51 \mathrm{w}\left(0.18^{*} \mathrm{n} 1 \mathrm{w}: 0.3^{*} \mathrm{n} 1 \mathrm{w}, 1\right)\right]$;

$\mathrm{v} 1 \mathrm{wag}=\left[\operatorname{sty} 51 \mathrm{w}\left(0.18^{*} \mathrm{n} 1 \mathrm{w}: 0.3 * \mathrm{n} 1 \mathrm{w}, 2\right)\right]$;

\%Load 1.0 MHz slurry data

load sty 51 s.dat

$\mathrm{n} 1 \mathrm{~s}=\max (\operatorname{size}(\mathrm{sty} 51 \mathrm{~s})$ );

$\mathrm{fs} 1=\left[\operatorname{sty} 51 \mathrm{~s}\left(0.18^{*} \mathrm{n} 1 \mathrm{~s}: 0.3^{*} \mathrm{n} 1 \mathrm{~s}, 1\right)\right]$;

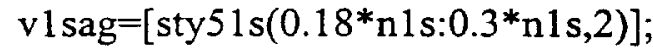

\%Load 2.25 MHz water data

load sty $52 \mathrm{w}$.dat

$\mathrm{n} 2 \mathrm{w}=\max (\operatorname{size}(\operatorname{sty} 52 \mathrm{w}))$;

$\mathrm{fw} 2=\left[\operatorname{sty} 52 \mathrm{w}\left(0.3 *_{\mathrm{n}} 2 \mathrm{w}: 0.5{ }^{*} \mathrm{n} 2 \mathrm{w}, 1\right)\right]$;

$\mathrm{v} 2 \mathrm{wag}=\left[\operatorname{sty} 52 \mathrm{w}\left(0.3^{*} \mathrm{n} 2 \mathrm{w}: 0.5^{*} \mathrm{n} 2 \mathrm{w}, 2\right)\right]$;

\%Load 2.25 MHz slurry data

load sty 52 s.dat

$\mathrm{n} 2 \mathrm{~s}=\max (\operatorname{size}(\operatorname{sty} 52 \mathrm{~s}))$;

$\mathrm{fs} 2=\left[\operatorname{sty} 52 \mathrm{~s}\left(0.3^{*} \mathrm{n} 2 \mathrm{~s}: 0.5^{*} \mathrm{n} 2 \mathrm{~s}, 1\right)\right]$;

$\mathrm{v} 2 \mathrm{sag}=[\operatorname{sty} 52 \mathrm{~s}(0.3 * \mathrm{n} 2 \mathrm{~s}: 0.5 * \mathrm{n} 2 \mathrm{~s}, 2)]$;

\%Load 5.0 MHz water data

load sty $55 \mathrm{w}$.dat

$\mathrm{n} 5 \mathrm{w}=\max (\operatorname{size}($ sty $55 \mathrm{w}))$;

fw5 $=\left[\operatorname{sty} 55 \mathrm{w}\left(0.25^{*} \mathrm{n} 5 \mathrm{w}: 0.5^{*} \mathrm{n} 5 \mathrm{w}, 1\right)\right]$;

$\mathrm{v} 5 \mathrm{wag}=\left[\operatorname{sty} 55 \mathrm{w}\left(0.25{ }^{*} \mathrm{n} 5 \mathrm{w}: 0.5{ }^{*} \mathrm{n} 5 \mathrm{w}, 2\right)\right]$;

\%Load 5.0 MHz slurry data

load sty 55 s.dat

$\mathrm{n} 5 \mathrm{~s}=\max (\operatorname{size}(\mathrm{sty} 55 \mathrm{~s}))$;

fs $5=[\operatorname{sty} 55 \mathrm{~s}(0.25 * \mathrm{n} 5 \mathrm{~s}: 0.5 * \mathrm{n} 5 \mathrm{~s}, 1)]$;

v $5 \mathrm{sag}=\left[\mathrm{sty} 55 \mathrm{~s}\left(0.25^{*} \mathrm{n} 5 \mathrm{~s}: 0.5^{*} \mathrm{n} 5 \mathrm{~s}, 2\right)\right]$;

$\%$ Load 5.0 MHz (repeat) water data

$\%$ load sty55w.dat 
$\%$ n5wr-max (size(sty55w));

$\%$ fw5r $=[\operatorname{tc} 305 w r(0.25 * n 5 w r: 0.5 * n 5 w r, 1)]$;

$\%$ v5wagr $=\left[\operatorname{tc} 305 \mathrm{wr}\left(0.25 * \mathrm{n} 5 \mathrm{wr}: 0.5{ }^{*} \mathrm{n} 5 \mathrm{wr}, 2\right)\right]$;

$\%$ Load 5.0 $\mathrm{MHz}$ (repeat) slurry data

$\%$ load tc 305 sr.dat

$\%$ n5sr=max $(\operatorname{size}(t c 305 \mathrm{sr}))$;

$\% \mathrm{fs} 5 \mathrm{r}=[\operatorname{tc} 305 \mathrm{sr}(0.25 * \mathrm{n} 5 \mathrm{sr}: 0.5 * \mathrm{n} 5 \mathrm{sr}, 1)]$;

$\%$ v5sagr $=[\operatorname{tc} 305 \mathrm{sr}(0.25 * \mathrm{n} 5 \mathrm{sr}: 0.5 * \mathrm{n} 5 \mathrm{sr}, 2)]$;

\%Load 7.5 MHz water data

load sty $57 \mathrm{w}$.dat

$\mathrm{n} 7 \mathrm{w}=\max (\operatorname{size}($ sty $57 \mathrm{w})$ );

fw $7=\left[\operatorname{sty} 57 w\left(0.40^{*} n 7 w: 0.80^{*} n 7 w, 1\right)\right]$;

$\mathrm{v} 7 \mathrm{wag}=\left[\operatorname{sty} 57 \mathrm{w}\left(0.40^{*} \mathrm{n} 7 \mathrm{w}: 0.80^{*} \mathrm{n} 7 \mathrm{w}, 2\right)\right]$;

\%Load 7.5 $\mathrm{MHz}$ slurry data

load sty 57 s.dat

$\mathrm{n} 7 \mathrm{~s}=\max (\operatorname{size}($ sty $57 \mathrm{~s}))$;

fs $7=\left[\right.$ sty $\left.57 \mathrm{~s}\left(0.40^{*} \mathrm{n} 7 \mathrm{~s}: 0.80^{*} \mathrm{n} 7 \mathrm{~s}, 1\right)\right]$;

v7sag $=[\operatorname{sty} 57 \mathrm{~s}(0.40 * \mathrm{n} 7 \mathrm{~s}: 0.80 * \mathrm{n} 7 \mathrm{~s}, 2)]$;

$\%$ Load $10.0 \mathrm{MHz}$ water data

load sty $510 \mathrm{w}$. dat

$\mathrm{n} 10 \mathrm{w}=\max (\operatorname{size}(\operatorname{sty} 510 \mathrm{w}))$

fw $10=[\operatorname{sty} 510 \mathrm{w}(0.60 * \mathrm{n} 10 \mathrm{w}: 0.98 * \mathrm{n} 10 \mathrm{w}, 1)]$;

$\mathrm{vl0wag}=[\operatorname{sty} 510 \mathrm{w}(0.60 * \mathrm{n} 10 \mathrm{w}: 0.98 * \mathrm{n} 10 \mathrm{w}, 2)]$;

$\%$ Load 10.0 MHz slurry data

load sty510s.dat

$\mathrm{n} 10 \mathrm{~s}=\max (\operatorname{size}(\mathrm{sty} 510 \mathrm{~s}))$;

fs $10=[\operatorname{sty} 510 \mathrm{~s}(0.60 * \mathrm{n} 10 \mathrm{~s}: 0.98 * \mathrm{n} 10 \mathrm{~s}, 1)]$;

v10sag $=[\operatorname{sty} 510 \mathrm{~s}(0.60 * \mathrm{n} 10 \mathrm{~s}: 0.98 * \mathrm{n} 10 \mathrm{~s}, 2)]$;

$\%$ Correct Voltages for Receiver Attenuation and gain

vlwg=vlwag./(10.^((40-52)/20));

v7wg=v7wag./(10.^((40-48)/20));

v5wg=v5wag. $/\left(10 .^{\wedge}((40-44) / 20)\right)$;

$\%$ vp5wg=vp5wag./(10.^((40-36)/20));

v2wg=v2wag./(10.^((40-52)/20));

$\%$ v5wgr=v5wagr./(10.^((40-50)/20));

v10wg=v10wag./(10.^((40-40)/20));

$\%$ v10wgr=v10wagr./(10.^((40-40)/20)); 


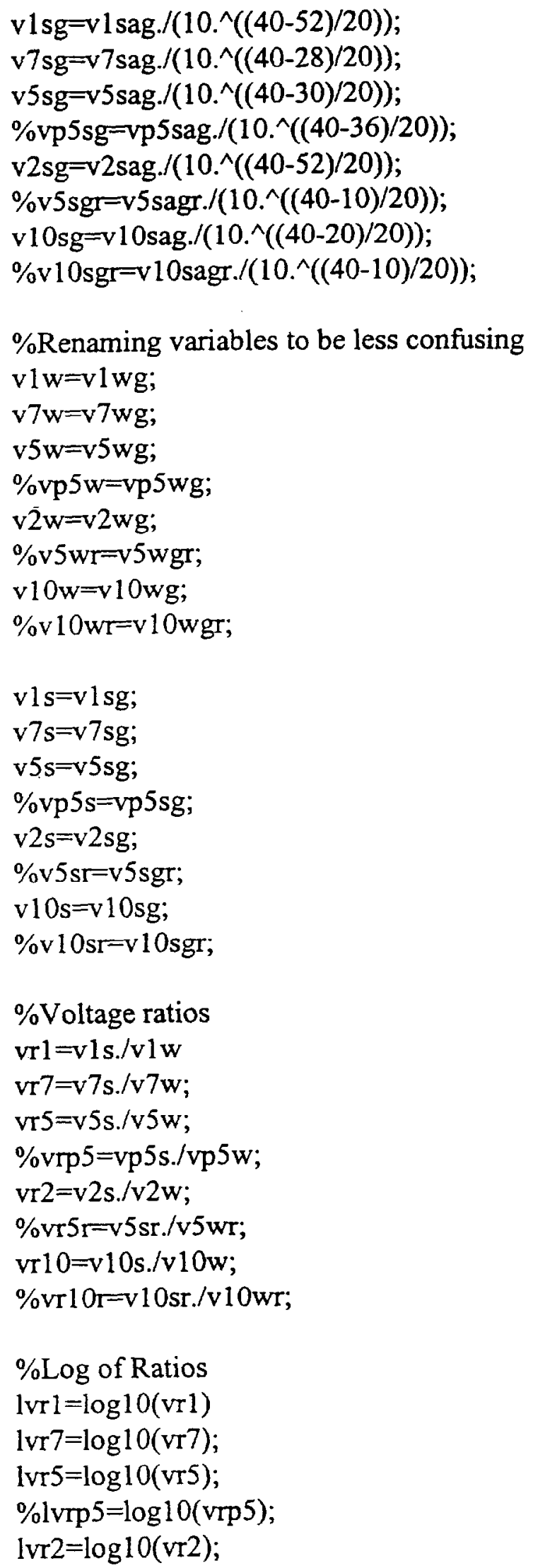


$\% \operatorname{lvr} 5 r=\log 10(v r 5 r)$

$\operatorname{lvr} 10=\log 10(v r 10)$;

$\% \operatorname{lvr} 10 \mathrm{r}=\log 10(\mathrm{vr} 10 \mathrm{r})$;

\%Transducer Separation Distances in cm

$\%$ dp5 $=4.941 * 2.54$;

$\mathrm{d} 1=2.008 * 2.54$

$\mathrm{d} 2=1.995 * 2.54$

$\% \mathrm{~d} 5 \mathrm{r}=1.998 * 2.54$;

$\mathrm{d} 5=1.995 * 2.54$

$\mathrm{d} 7=1.719 * 2.54$

$\mathrm{d} 10=1.700 * 2.54$;

$\% \mathrm{~d} 10 \mathrm{r}=1.770 * 2.54$;

\%Calculate Attenuation

as $1=-1.0 . *(\operatorname{lvr} 1 . / \mathrm{d} 1)$;

as $7=-1.0{ }^{*}(\operatorname{lvr} 7 . / \mathrm{d} 7)$;

as $5=-1.0{ }^{*}(\operatorname{lvr} 5 . / \mathrm{d} 5)$;

$\%$ asp5 $=-1.0 . *(\operatorname{lvrp} 5 . / \mathrm{dp} 5)$;

as $2=-1.0{ }^{*}(\operatorname{lvr} 2 . / \mathrm{d} 2)$;

$\%$ as $5 \mathrm{r}=-1.0 . *(\operatorname{lvr} 5 \mathrm{r} . \mathrm{d} 5 \mathrm{r})$;

as $10=-1.0 . *(\operatorname{lvr} 10 . / \mathrm{d} 10)$;

$\% a s 10 \mathrm{r}=-1.0{ }^{*}(\operatorname{lvr} 10 \mathrm{r} . \mathrm{d} 10 \mathrm{r})$;

$\%$ Multiply by 2.302 to rectify with natural $\log$ calculation, i.e. put in $\mathrm{Np} / \mathrm{cm}$ as $1=2.302 . *$ as 1

as $7=2.302 . *$ as 7 ;

as $5=2.302 . *$ as 5 ;

$\%$ asp $5=2.302 . *$ asp 5

as $2=2.302 .{ }^{*}$ as 2

$\%$ as $5 \mathrm{r}=2.302 . *$ as $5 \mathrm{r}$;

as $10=2.302 . *$ as 10 ;

$\%$ as $10 \mathrm{r}=2.302 . *$ as $10 \mathrm{r}$;

$\%$ Use one set of frequencies per transducer to make plots

$\%$ fp5 $=$ fwp 5 ;

$\mathrm{fl}=\mathrm{fwl}$

$\mathrm{f} 2=\mathrm{fw} 2$;

$\mathrm{f} 5=\mathrm{fw} 5$

$\mathrm{f} 7=\mathrm{fw} 7$;

fl $0=$ fw 10 ;

$\% \mathrm{f} 5 \mathrm{r}=\mathrm{fw} 5 \mathrm{r}$;

$\%$ fl0r=fw $10 \mathrm{r}$; 
\%Load simulation data

load theo.dat

$\mathrm{ff}=[$ theo(:,1)];

af $=[$ theo $(:, 2)]$;

$\% \mathrm{ff}=\mathrm{ff}^{*} 1.0 \mathrm{e} 6$;

\%Toneburst results (for comparison) entered as arrays. The "fb\#" stands for $\%$ Frequency of toneBurst for \# MHz transducer. The "ab\#" stands for Attenuation $\%$ of toneBurst for \# $\mathrm{MHz}$ transducer.

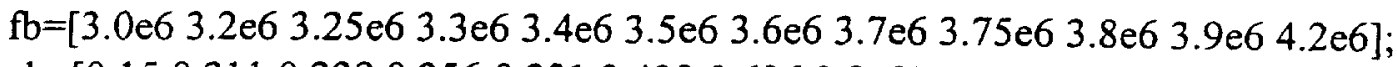

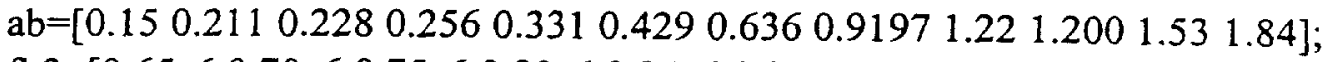

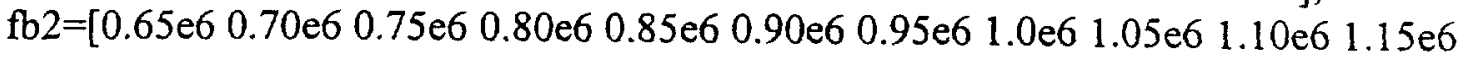
$1.20 \mathrm{e} 6]$

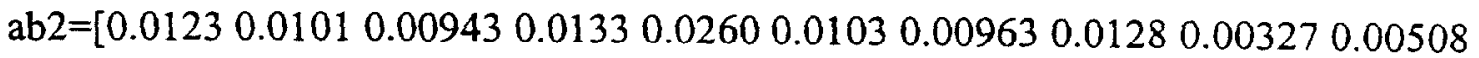
$0.008840 .00696]$;

$\mathrm{fb} 3=[3.00 \mathrm{e} 63.25 \mathrm{e} 63.50 \mathrm{e} 63.75 \mathrm{e} 63.80 \mathrm{e} 63.85 \mathrm{e} 63.90 \mathrm{e} 63.95 \mathrm{e} 6]$;

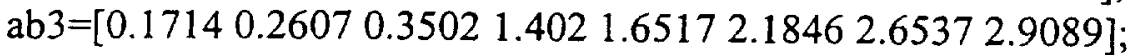

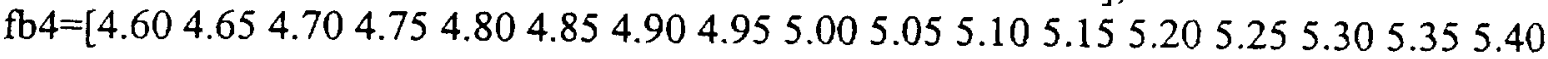

$5.455 .505 .555 .605 .655 .705 .755 .805 .855 .905 .956 .006 .056 .106 .156 .20]$;

$\mathrm{fb} 4=\mathrm{fb} 4 * 1.0 \mathrm{e} 6$;

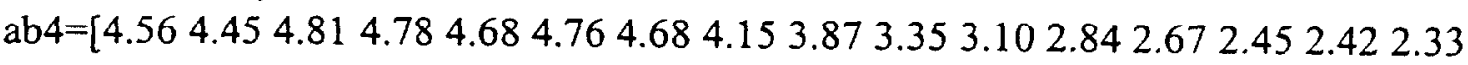

2.262 .242 .202 .162 .202 .332 .412 .542 .742 .943 .213 .514 .054 .564 .995 .00 5.20];

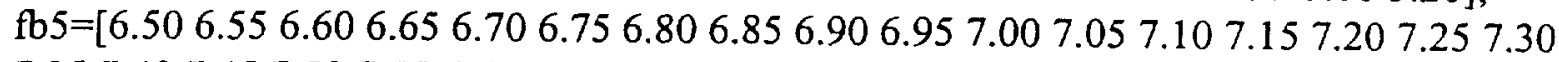

7.357 .407 .457 .507 .557 .607 .657 .707 .757 .807 .857 .907 .95 8.00];

$\mathrm{fb} 5=\mathrm{fb} 5 * 1.0 \mathrm{e} 6$;

ab5=[5.002 4.969 4.9334.986 5.0375.096 4.956 4.856 4.834 4.7154.601 4.5034.326

4.2393 .7143 .6743 .4043 .4443 .1753 .3553 .3343 .3463 .2783 .3333 .4843 .5933 .903

$4.1194 .6485 .2515 .338]$;

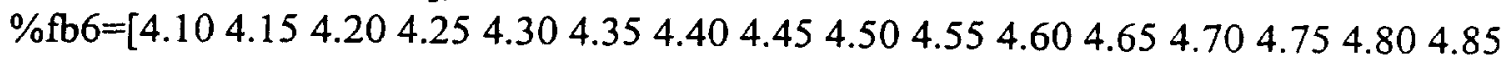

$4.904 .955 .00]$;

$\% \mathrm{fb} 6=\mathrm{fb} 6 * 1.0 \mathrm{e} 6$;

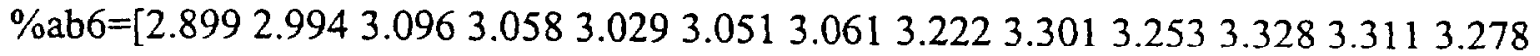

$3.4523 .4573 .4093 .4653 .4273 .371]$;

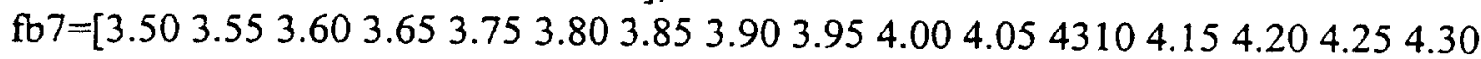

$4.354 .404 .454 .50]$;

$\mathrm{fb} 7=\mathrm{fb} 7 * 1.0 \mathrm{e} 6$;

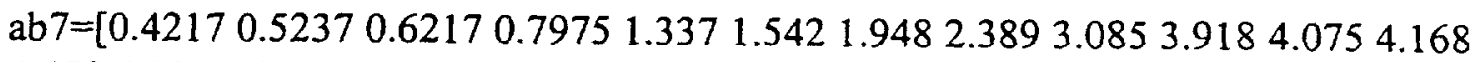

4.478 4.398 4.225 4.418 4.566 4.301 4.478 4.656];

$\%$ Plot Results

$\% \log \log (\mathrm{fp} 5$,asp5,'ro')

\%hold on

$\log \log (\mathrm{fl}, \mathrm{as} 1, \mathrm{~b}+\mathrm{\prime})$

hold on 


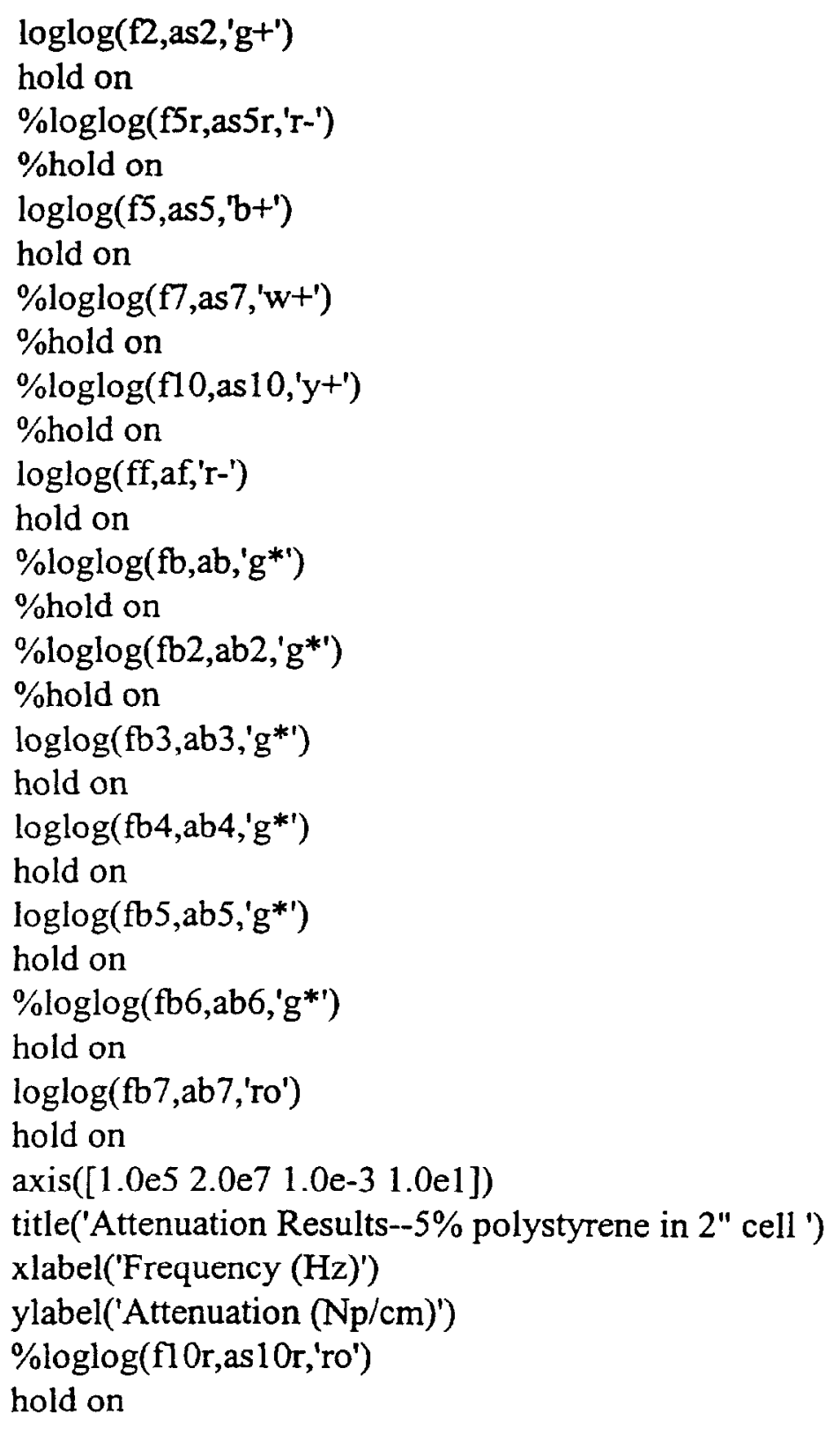

$* * * * * * * * * * * * * * * * * * * * * * *$ END OF STYRENES.M

$\%$ Program to calculate the mean and standard deviation of \%the size measurements made on bubbles(generated both by $\%$ aerator stone and electrolysis)using the video imaging systems. $\%$ These measurements are then used to calculate a size distribution. $\%$ Program is called "bublsize.m"

$\%$ Enter the aerator bubble measurements as arrays (bubble stream data) 
daerstrm=[179 189171183154156150157558558550552414427410419452454 45144122822822122425272625196201183190588603580584134141137 125213213210216199204194 196];

maerstrm=mean (daerstrm); sigaerstrm=std(daerstrm, 1 );

$\%$ data for aerator bubbles mixed

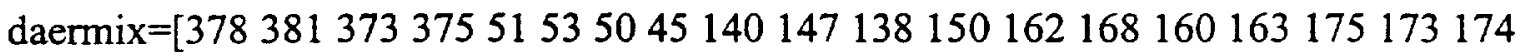
18020419919220116616914916117116115916816415615559171164166166 $106113101102164166160157]$;

maermix $=$ mean(daermix)

sigaermix $=\operatorname{std}($ daermix, 1$)$

$\%$ Enter the electrolysis bubble measurements as arrays (bubble stream data) delestrm=[154 585252605863633439403636343534343636401719212356 5658503641363828323229494752485652535345454749495252496467 $686667717066263032325460555734363935]$;

melestrm=mean(delestrm); sigelestrm=std (delestrm,1); $\%$ Data for electrolysis mixed in the cell

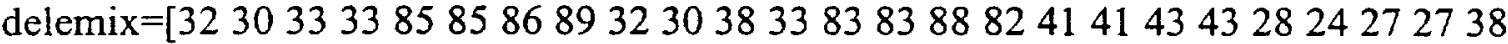
3840406868656224262826434548469490979064646764454747473236 3533535150515653515226262424494947491201181211184343444236 3440357375707166717468383636337168687136343741616161626165 636630303129302627297781797910910310910561576157107111107 107575761612026242487858887404041395757555232323634464850 4469656970262427254646464628303132535357524440434471736970 8789909355554752464240374844484461615959403841383434383634 34333375736773 ;

melemix $=$ mean (delemix)

sigelemix $=$ std(delemix, 1$)$

\%delemix2=[6161616261656366303031293026272977817979109103109 10561576157107111107107575761612026242487858887404041395757 5552323236344648504469656970262427254646464628303132535357 5244404344717369708789909355554752464240374844484461615959 40384138343438363434333375736773 ; ;

$\%$ melemix $2=$ mean (delemix 2$)$

$\%$ sigelemix $2=$ std(delemix 2,1$)$

$\%$ Calculate Bubble Size distribution

$\%$ Set up "bins" such that all bubbles in a bin are within 10 micron of bin diameter

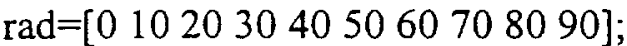

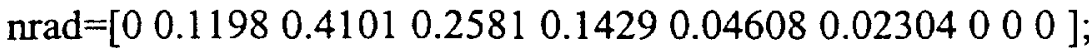


$\%$ Plot results

plot(rad, nrad,'-.')

ylabel('number fraction of bubbles')

xlabel('radius (micron)')

$\operatorname{axis}\left(\left[\begin{array}{llll}0 & 100 & 0 & 0.5\end{array}\right]\right)$ 


\section{Appendix D: Experimental Parameter Series Coding}

The attenuation data presented in this study are obtained using a number of different combinations of transducers, mixing vessels, data acquisition methods, and slurry types. It is not obvious, upon examination of a given set of attenuation data, that it may have been collected from a slurry placed in more than one vessel (different acoustic - pathlengths) and interrogated by more than one set of transducers. Therefore, a coding system is established to facilitate the acquisition of this information for every experimentally obtained set of data in this work. Each experimental figure has an experimental parameter number at the end of the figure caption. The experimental code has the following format:

$$
\mathrm{E}(\mathrm{I})(\mathrm{II})(\mathrm{III})(\mathrm{IV}) \text {. }
$$

All codes begin with the letter "E" (for Experimental Parameter Series) followed by four digits, shown above by the Roman numerals I through IV. Each Roman numeral refers to an experimental set up parameter category shown in Tables D1 to D4.. Each category in Tables D1 through D4 has all the combinations of that parameter used in this study marked by a specific Arabic numeral. Thus the sequence of Arabic numerals following the "E" in the experimental code gives the pertinent information about the mixing vessels, transducers, etc. employed in obtaining that data set. 


\begin{tabular}{|lll|}
\hline & Category I & Transducer Combinations \\
\hline 1 & & \\
\hline 2 & -- & $0.5 \mathrm{MHz}, 1.0 \mathrm{MHz}, 2.25 \mathrm{MHz}, 5.0 \mathrm{MHz}, 7.5 \mathrm{MHz}$, and $10.0 \mathrm{MHz}$ \\
\hline 3 & $-\cdots$ & $1.0 \mathrm{MHz}, 2.25 \mathrm{MHz}, 5.0 \mathrm{MHz}, 7.5 \mathrm{MHz}$, and $10.0 \mathrm{MHz}$ \\
\hline 4 & $-\cdots$ & $1.0 \mathrm{MHz}, 2.25 \mathrm{MHz}, 5.0 \mathrm{MHz}$ \\
\hline 5 & $-\cdots--$ & $1.0 \mathrm{MHz}, 2.25 \mathrm{MHz}, 5.0 \mathrm{MHz}, 7.5 \mathrm{MHz}$ \\
\hline 6 & $-\cdots$ & $2.25 \mathrm{MHz}, 5.0 \mathrm{MHz}$ \\
\hline
\end{tabular}

Table D.1: Transducer combinations employed to obtain attenuation data in the various slurries investigated in this study.

\begin{tabular}{|c|c|c|}
\hline & Category II - & Experimental Method Combinations \\
\hline 1 & ---- Toneburst Method & \\
\hline 2 & ---- Pulse/FFT Method & \\
\hline & ---- Both Techniques & \\
\hline
\end{tabular}

Table D.2: Experimental method combinations employed to obtain attenuation data in the various slurries investigated in this study.

\begin{tabular}{|c|}
\hline $\begin{array}{l}\text { Category III - - } \\
\text { Combinations }\end{array}$ \\
\hline $1 \quad----1 / 2 "(1.27 \mathrm{~cm})$ Plexiglas Test Cell \\
\hline 2 ---- 1" $(2.54 \mathrm{~cm})$ Plexiglas Test Cell \\
\hline 3 --- 2" $(5.08 \mathrm{~cm})$ Plexiglas Test Cell \\
\hline $4 \quad----4$ 4" $(10.2 \mathrm{~cm})$ Plexiglas Test Cell \\
\hline 5 - $-\cdots-1 / 2$ " and 1" Plexiglas Test Cells \\
\hline
\end{tabular}




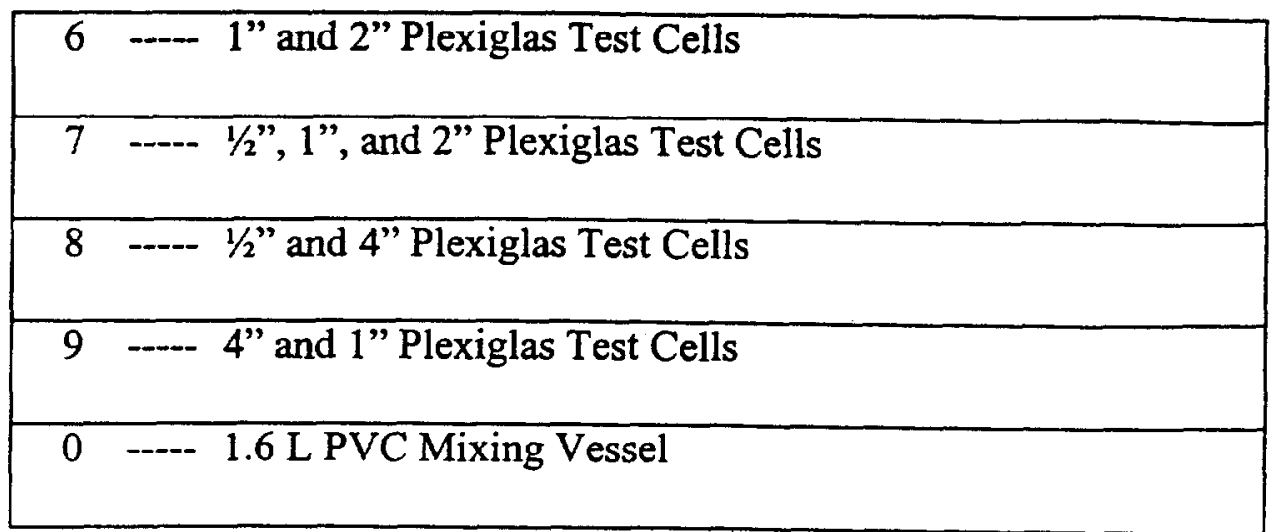

Table D.3: Experimental mixing vessel/test cell combinations employed to obtain attenuation data in the various slurries investigated in this study.

\begin{tabular}{|lll|}
\hline \multicolumn{3}{|c|}{ Category IV } \\
\hline 1 & $\cdots-$ & Soda-Lime Glass Beads (small beads) in Water \\
\hline 2 & $\cdots-$ & Potter's Beads in Glycerin/Water \\
\hline 3 & $\cdots--$ & Polystyrene Beads in Water \\
\hline
\end{tabular}

Table D.4: Various slurries investigated in this study.

An example of the interpretation of this coding system could be in the case shown in Figure 4.2, where we have a set of data labeled as E2363. This code implies the Experimental set up where the $1.0,2.25,5.0,7.5$, and $10.0 \mathrm{MHz}$ transducers are used (Table D.1, item \#2) in both the Toneburst and Pulse/FFT techniques (Table D.2, item \#3) to obtain attenuation data in the 1" and 2" Plexiglas test cells (Table D.3, item \#6) for a slurry of polystyrene beads in water (Table D.4, item \#3). This coding system can be . used to determine the experimental parameters relevant to each data set shown in this work. 


\section{Appendix E: Discussion of Transducer Bandwidth}

A parameter which is often discussed in reference to a given ultrasonic transducer is the transducer bandwidth. The bandwidth is a term which describes the frequency range over which a given transducer will function effectively. It is usually referred to as the "- 6 $\mathrm{dB}$ Bandwidth" and is described in terms of a percentage of the "peak frequency" of the transducer. The peak frequency is the frequency at which the FFT frequency spectrum of a received pulse (transducer response) displays a maximum. A signal which $-6 \mathrm{~dB}$ from the peak frequency signal has one half of the peak frequency amplitude. As the transducer response is usually a bell shaped curve, there will be two frequencies which are $-6 \mathrm{~dB}$ from the peak frequency. One frequency is greater than the center frequency, and one is less than the center frequency. The $-6 \mathrm{~dB}$ bandwidth is the difference between those two frequencies divided by the center frequency, times $100 \%$. That is

$$
-6 \mathrm{~dB} \text { Bandwidth }=\frac{f_{-6 \mathrm{~dB}}^{+}-f_{-6 \mathrm{~dB}}^{-}}{f_{c}} \times 100 \% \text {, }
$$

where $f_{-6 \mathrm{~dB}}^{+}$and $f_{-6 \mathrm{~dB}}^{-}$are the frequencies at $-6 \mathrm{~dB}$ from the center frequency which are greater and less than the center frequency, respectively, and $f_{c}$ is the transducer center frequency. For example, a $0.50 \mathrm{MHz}$ transducer with a reported center frequency of $0.457 \mathrm{MHz}$ and $-6 \mathrm{~dB}$ frequencies of $0.61 \mathrm{MHz}$ and $0.305 \mathrm{MHz}$ will have a bandwidth of $66.7 \%$. 


\section{Notation}

\section{Latin Characters:}

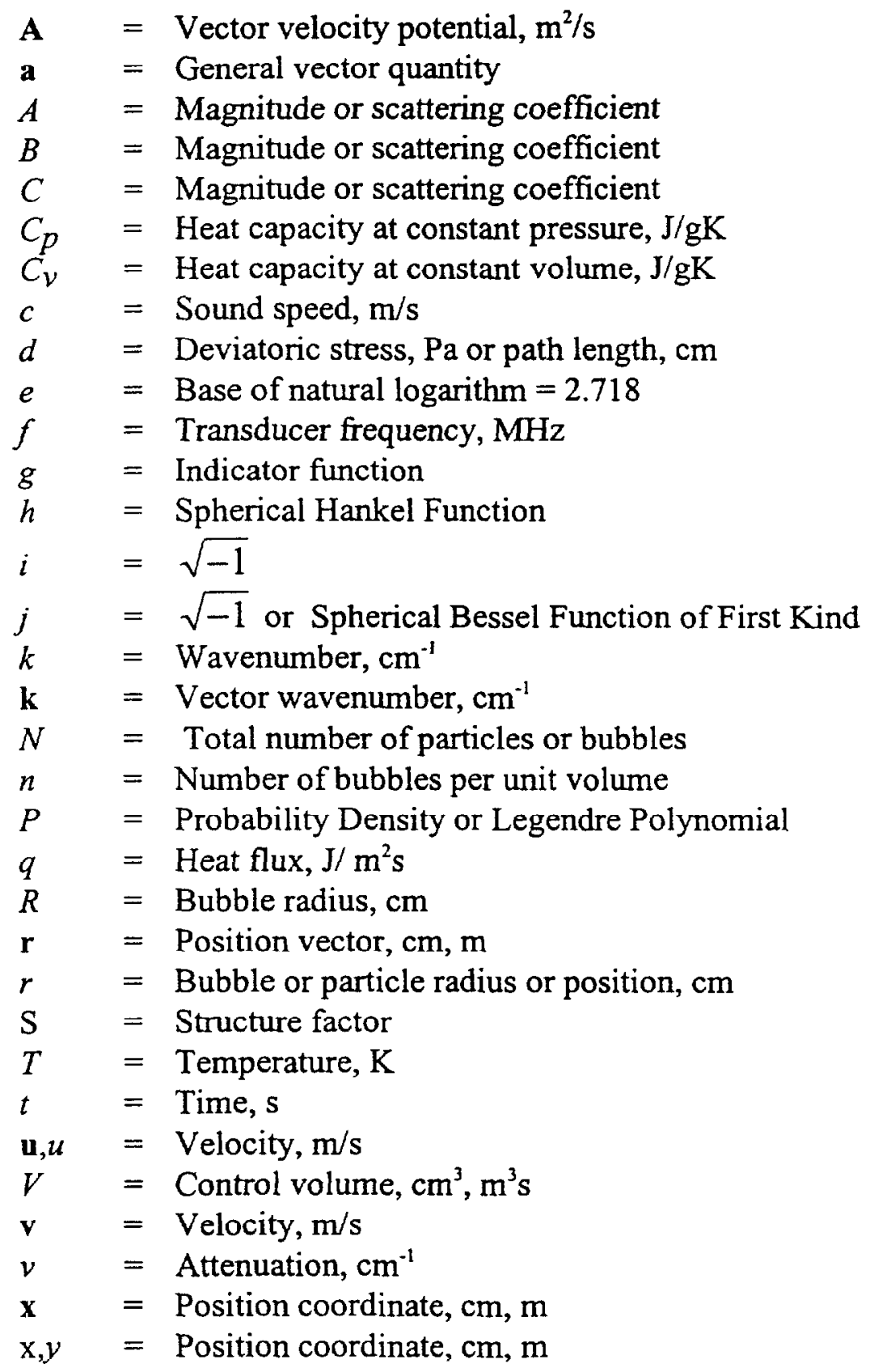




\section{Greek Characters:}

$\alpha=$ Attenuation coefficient, $\mathrm{cm}^{-1}$

$\beta=$ Bubble volume fraction or thermal expansion coefficient, $1 / \mathrm{K}$

$\gamma \quad=\quad$ Ratio of specific heats $=C_{p} / C_{\nu}$

$\varepsilon=$ Error parameter

$\delta=$ Deviatoric stress, Pa or damping coefficiènt or Dirac delta or Kronecker delta

$\eta \quad=$ Parameter

$\theta=$ Radial angle, rad

$\lambda=$ Parameter or Lamè constant

$\mu \quad=$ Viscosity, $\mathrm{kg} / \mathrm{ms}$ or Lamè constant

$\rho \quad=$ Density, $\mathrm{g} / \mathrm{cm}^{3}$

$\pi=3.14159$

$\Phi=$ Scalar velocity potential, $\mathrm{m}^{2} / \mathrm{s}$

$\varphi=$ Azimuthal angle, rad

$\phi \quad=$ Solids volume fraction or scalar velocity potential, $\mathrm{m}^{2} / \mathrm{s}$

$\omega=$ Angular Frequency, $\mathrm{MHz}$

$\sigma=$ Stress tensor, $\mathrm{Pa}$ or surface tension, $\mathrm{dyn} / \mathrm{cm}$

$\tau=$ Thermal Conductivity, $\mathrm{J} / \mathrm{Kcms}$

\section{Superscripts and Subscripts:}

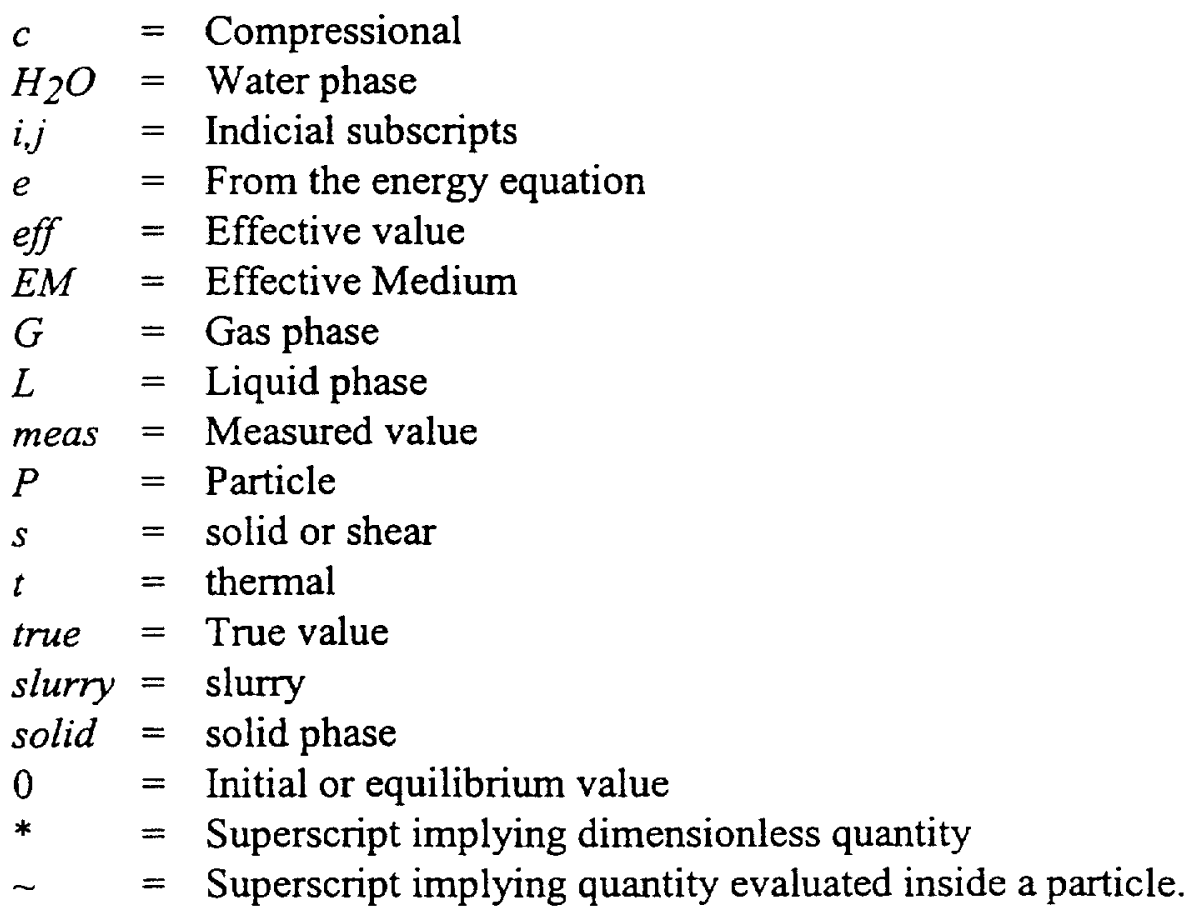




\section{Vita}

NAME OF AUTHOR: Michael Anthony Norato

DATE OF BIRTH: January 25, 1969

PLACE OF BIRTH: Philadelphia, Pennsylvania, USA

GRADUATE AND UNDERGRADUATE SCHOOLS ATTENDED:

Bachelor of Science, 1991;

Department of Chemical Engineering and Materials Science, Syracuse University, Syracuse, New York, USA.

Master of Science, 1994;

Department of Chemical Engineering and Materials Science, Syracuse University, Syracuse, New York, USA.

\section{PROFESSIONAL EXPERIENCE:}

Department of Chemical Engineering and Materials Science, Syracuse University: Teaching Assistant, 1991, 1995, 1996, \& 1997.

Research Assistant, 1991-Present.

Associated Western Universities/Battelle Pacific Northwest National Laboratory: Graduate Laboratory Fellow, 1996. 